\title{
Optimierung der Exploration, Gewinnung und Materialcharakterisierung von Naturwerksteinen
}

\author{
Dissertation \\ zur Erlangung des Doktorgrades \\ der Mathematisch-Naturwissenschaftlichen Fakultäten \\ der Georg-August-Universität Göttingen
}

vorgelegt von

Stephan Mosch

aus Unterlüß 
D 7

Referent: $\quad$ Prof. Dr. Siegfried Siegesmund

Geowissenschaftliches Zentrum der Universität Göttingen

Korreferent: $\quad$ Prof. Dr. Klaus Germann

Technische Universität Berlin

Tag der mündlichen Prüfung: 27.01.2009 


\section{Kurzfassung}

Rohstoff Naturwerkstein - seit Alters her die Verkörperung des Kostbaren und Langlebigen. Aufgrund der breiten Palette an Farben und Strukturen erfreut sich Naturwerkstein in den letzten Jahrzehnten einer zunehmenden Beliebtheit und findet eine vielfache Anwendung als Bau- und Ornamentgestein. Resultierend aus einer rasanten Entwicklung der abbau- und verarbeitungstechnischen Industrie, den damit verbundenen Forderungen an die auszubringende Rohblockware sowie der weltweit steigenden Nachfrage ist sowohl eine geowissenschaftliche Charakterisierung der potenziellen Verwendbarkeit einer Lagerstätte als auch die Definition der bautechnischen Anwendungsmöglichkeit eines Gesteins unabdingbar. Das Ziel muss eine sowohl aus wirtschaftlicher als auch ökologischer Sicht optimale Nutzung der natürlichen Ressource Naturwerkstein sein.

Im ersten Teil der vorliegenden Arbeit werden methodische Grundlagen zur bestmöglichen Nutzung von Naturwerksteinen detailliert dargestellt. Aus der Analyse des Trennflächensystems sowie dessen Auswirkungen auf die Zergliederung eines Gesteinskörpers, werden Möglichkeiten erarbeitet, den Abbau zum Ausbringen werksteingerechter Rohblöcke an die gegebene geologische Situation in optimierter Weise anzupassen. An theoretischen und praktischen Beispielen konnte herausgestellt werden, dass die lagerstättengeologische Erfassung dazu beitragen kann, die Effektivität bei der Gewinnung und Aufarbeitung des Gesteins zu steigern und damit die Wirtschaftlichkeit einer Lagerstätte positiv zu beeinflussen. Im Weiteren sind detaillierte Kenntnisse der spezifischen physikalischen und technischen Gesteinseigenschaften notwendig, da diese das Materialverhalten der Gesteine in der bautechnischen Anwendung diktieren und somit als ein entscheidendes Qualitätskriterium eines Werksteins zu beachten sind. Aus der statistischen Auswertung recherchierter Daten zu rund 2300 Werksteinvarietäten wurden detailliert lithologietypische Wertebereiche verwitterungsrelevanter Parameter herausgearbeitet. Ferner wurden statistische Hilfsmittel zur qualitativen und quantitativen Bewertung experimenteller Daten und zur prognostischen Einschätzung der potenziellen bautechnischen Verwendbarkeit einer bisher ungenutzten Werksteinressource aus einfach und kostengünstig zu ermittelnden Parametern entwickelt.

Der zweite Teil der Arbeit konzentriert sich auf die Umsetzung der aus den methodischen Grundlagen erarbeiteten Erkenntnisse in einer überregionalen und einer regionalen Erfassung und Erkundung von Werksteinlagerstätten. Die Fallbeispiele stellen exemplarisch unterschiedliche Stadien der Prospektion bzw. Exploration dar. Mit der überregionalen Erkundung des Werksteinpotenzials Argentiniens wird das Ziel einer Katalogisierung vorhandener Abbaue in magmatischen, metamorphen und sedimentären Lagerstätten mit der Erfassung der jeweiligen generellen Lagerstättensituation verfolgt. Insgesamt wird damit die Basis zur Entscheidungsfindung im weiteren Entwicklungsprozess der nationalen Natursteinindustrie gestellt. Ferner konnte durch den Vergleich prognostischer und experimenteller bautechnisch relevanter Daten gezeigt werden, dass bereits in der Frühphase der Exploration eine weiterreichende Kenntnis über die Materialeigenschaften unter Beachtung der gesteinsspezifischen Gefüge erreicht werden kann. Im regionalen Beispiel einer Lagerstätte in Tansania erfolgte die exemplarische Erfassung und Bewertung eines Dolomitmarmorvorkommens nach marktwirtschaftlichen Kriterien. Dafür werden die Grundlagen der Trennflächenanalyse und Gesteinscharakterisierung mit weiteren Faktoren zur Qualität des Rohstoffs und deren Veränderlichkeit innerhalb der Lagerstätte als auch im Hinblick auf spezifische Gefügeoder Strukturelemente zusammengeführt.

Die vorliegende Arbeit zeigt, dass eine detaillierte geowissenschaftliche Basis dazu beitragen muss, die Effektivität in der Exploration, Gewinnung und Nutzung des Rohstoffs Naturwerkstein zu steigern. 


\section{Abstract}

Since antiquity dimension stones have represented the embodiment of values like preciousness and longevity. The last several decades were characterised by a continuous growth in the use of dimension stones for building and ornamentation because of the diversity in the colors and structures. Due to the rapid development of the extraction and processing technologies for dimension stones, special requirements concerning the quality and dimensions of the extracted raw block material exist. Detailed geological investigations for characterising potential dimension stone deposits and the optimised utilisation of the resources have to be the aim when regarding both the economical as well as the ecological aspects.

The first part of this research describes the methodological basics for optimising the quarrying and utilisation of dimension stones. Based on detailed studies of the discontinuity systems and its effect on the fragmentation of the rock masses, possibilities have been developed to adjust the excavation of the raw blocks to the prevailing geologic setting. Using theoretical and practical examples it could be demonstrated, that a fundamental geological investigation has to be carried out to increase the effectiveness of quarrying and processing of rock material, which also exerts a positive influence on the profitability of a deposit. Moreover, detailed knowledge of specific physical and technical properties is essential, since they control the behavior of the rock when being utilised for different applications, and are thus a contributing factor to the quality criteria of cut stone. Statistical analyses have been carried out on data collected from more than 2,300 rock varieties, where it was possible to define specific domains for most of the important parameters in the different lithologies. To evaluate the experimental data in a qualitative as well as quantitative way, statistical means have been developed and applied. The statistical methods are used for the prediction of the technical behaviour, which is based on parameters that can be determined in an easy and cost-effective way.

The second part of this research applies the basic methods to two case studies concerning the assessment and evaluation of dimension stone deposits at different stages of exploration. Dimension stone deposits consisting of magmatic, metamorphic and sedimentary rocks in Argentina were studied in detail with respect to their depositional environment. The objective of the study is to create a general catalogue of commercial grades, whereby the assessments were mainly done by lithological, structural and geotechnical analyses. Investigations such as these are useful for the decision-making process and for the continuing development of a national dimension stone industry. For the rocks investigated the experimental data of the geotechnical properties are compared with the predictive values derived from the statistical analyses. Statistical approaches show that a preliminary estimate of the rock properties can be made, however specific structural elements have to be taken into consideration. In southern Tanzania, the second study deals with a dimension stone deposit, where the exemplary investigation and evaluation of a dolomite marble exposure is assessed within the framework of market-based criteria. The detailed studies of discontinuity system and rock properties at this location were combined to include more factors related to the quality of raw material and its variability in the deposit as well as to specific rock structure elements.

The present work shows that detailed structural and lithological investigations based on geoscientific methods are essential in order to increase the effectiveness of exploration, extraction and utilisation of resource dimension stones. 


\section{Inhaltsverzeichnis}

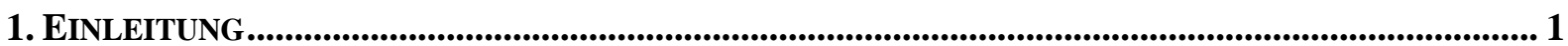

2. DIE RELEVANZ DES TRENNFLÄCHENSYSTEMS BEI DER GEWINNUNG

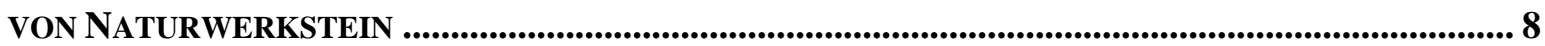

2.1 Anforderungen im Abbau und der Verarbeitung von Naturwerkstein .................................... 8

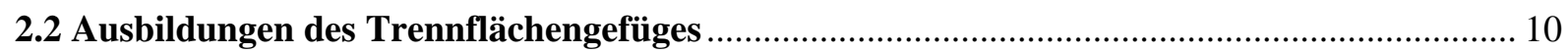

2.3 Methodik der Trennflächenanalyse und Darstellungsmöglichkeiten .................................... 16

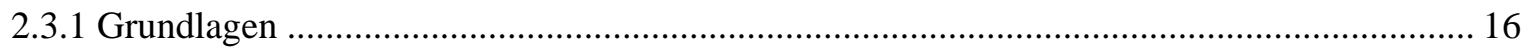

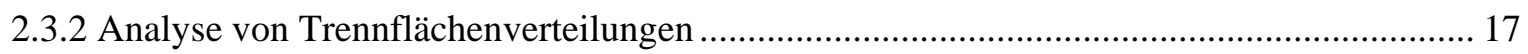

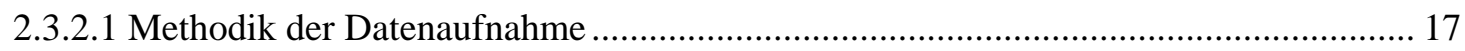

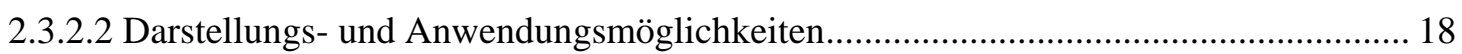

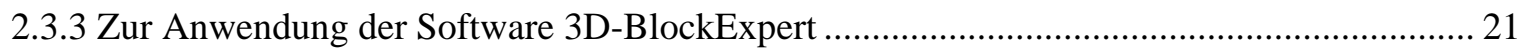

2.3.3.1 Datenaufnahme und -aufbereitung zur Anwendung der Software .............................. 21

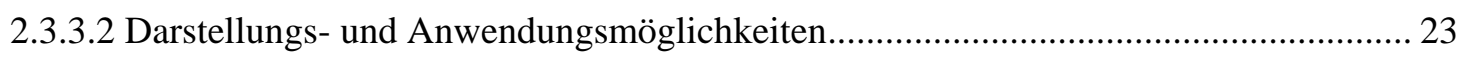

2.4 Auswirkungen des Trennflächengefüges auf die Werksteingewinnung................................... 25

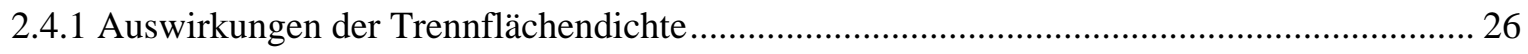

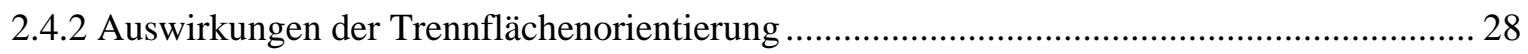

2.5 Möglichkeiten der Optimierung im Abbau von Naturwerkstein............................................. 32

2.6 Zusammenfassende Bewertung zur Erfassung des Trennflächengefüges.............................. 41

3. STATISTISCHES VERHALTEN PHYSIKALISCHER UND TECHNISCHER EIGENSCHAFTEN

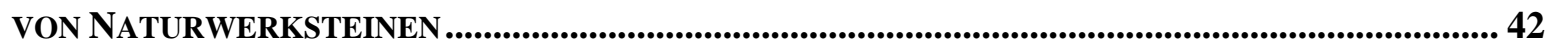

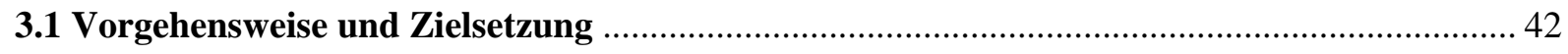

3.2 Problematik der Vergleichbarkeit von Datensätzen ................................................................ 44

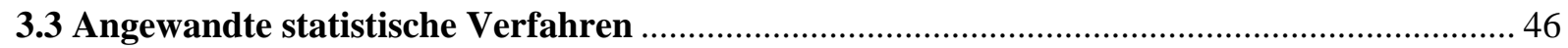

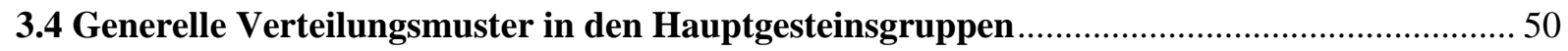

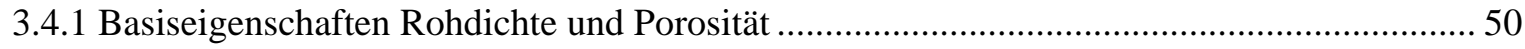

3.4.2 Technische Eigenschaften Druck-, Biege- und Spaltzugfestigkeit ......................................... 52

3.4.3 Basiseigenschaften in Korrelation mit mechanischen Festigkeiten ....................................... 54

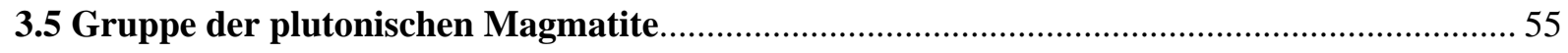

3.5.1 Basiseigenschaften Rohdichte und Porosität ......................................................................... 56

3.5.2 Technische Eigenschaften Druck-, Biege- und Spaltzugfestigkeit ......................................... 57

3.5.3 Basiseigenschaften in Korrelation mit mechanischen Festigkeiten ....................................... 60

3.5.4 Beziehungen zwischen dem mittleren Atomgewicht und den Festigkeitseigenschaften........ 61

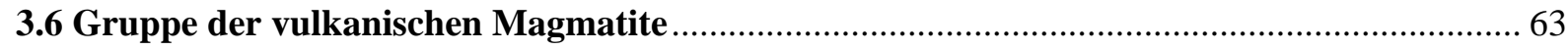

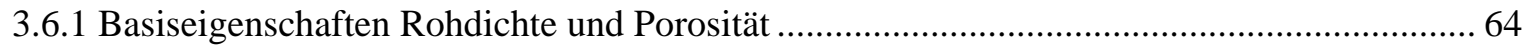

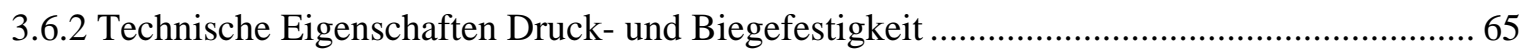

3.6.3 Basiseigenschaften in Korrelation mit mechanischen Festigkeiten ..................................... 67 
3.7.1 Basiseigenschaften Rohdichte und Porosität

3.7.2 Technische Eigenschaften Druck-, Biege- und Spaltzugfestigkeit ...................................... 75

3.7.3 Basiseigenschaften in Korrelation mit mechanischen Festigkeiten ........................................ 78

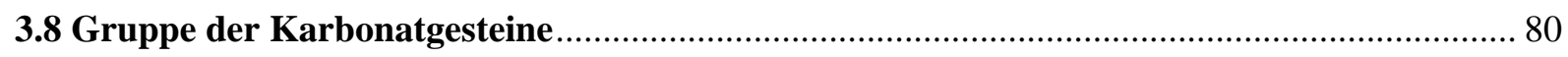

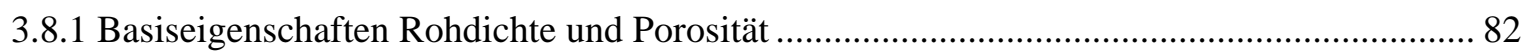

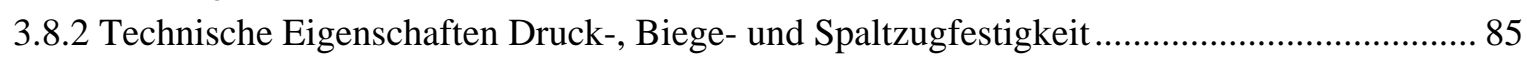

3.8.3 Basiseigenschaften in Korrelation mit mechanischen Festigkeiten ..................................... 87

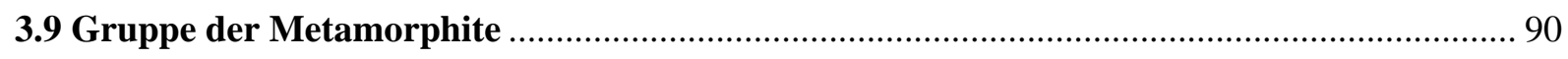

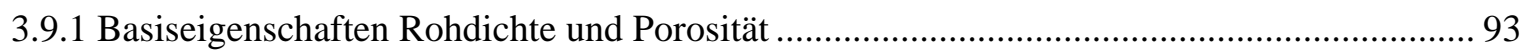

3.9.2 Technische Eigenschaften Druck-, Biege- und Spaltzugfestigkeit ......................................... 94

3.9.3 Basiseigenschaften in Korrelation mit mechanischen Festigkeiten ...................................... 98

3.10 Abschätzungen bivariater Aufenthaltswahrscheinlichkeiten................................................ 99

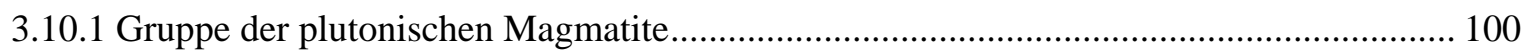

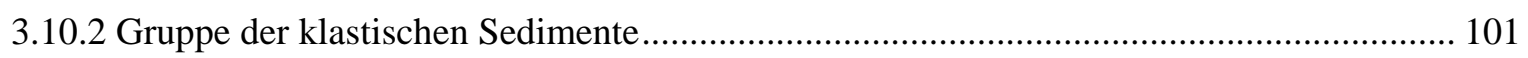

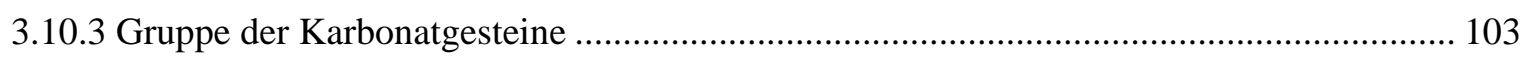

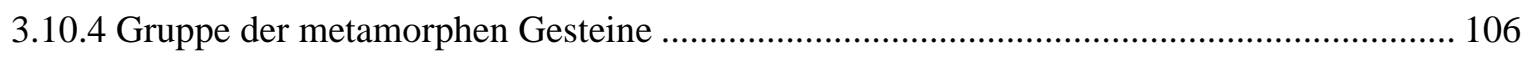

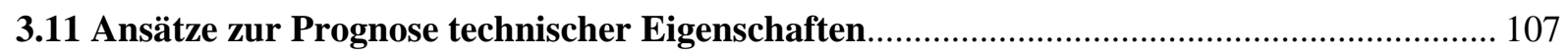

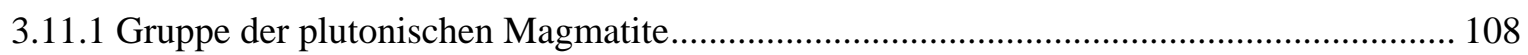

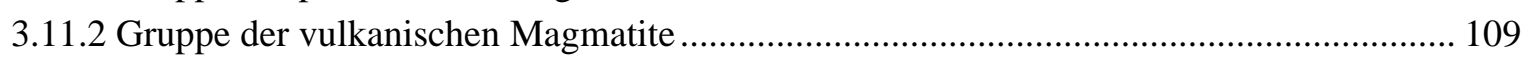

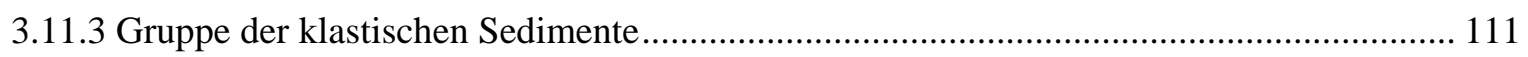

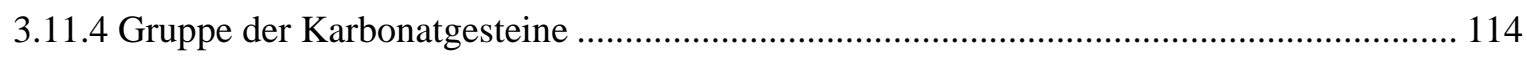

3.12 Ultraschallanalytik - Korrelationen zu den elastischen Eigenschaften ............................... 115

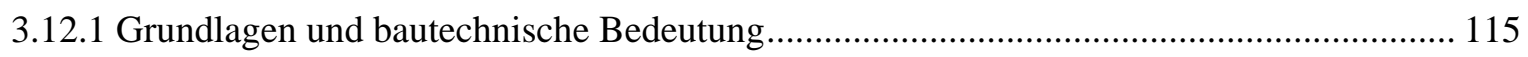

3.12.2 Gruppenspezifische Verteilungen und Beziehungen zu den Basiseigenschaften ............... 116

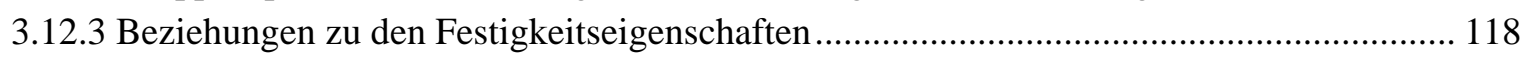

3.12.4 Ansätze zu Prognosen aus der Ultraschallanalytik ........................................................ 119

3.13 Feuchtehaushalt - Korrelationen zur Wasseraufnahmefähigkeit ...................................... 126

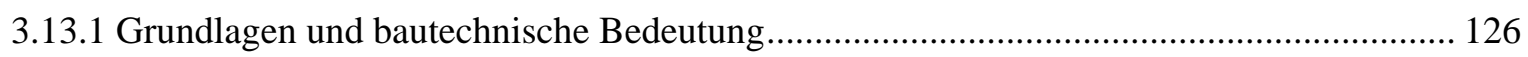

3.13.2 Gruppenspezifische Verteilungen und Beziehungen zu den Basiseigenschaften .............. 128

3.13.3 Abschätzung bivariater Aufenthaltswahrscheinlichkeiten.............................................. 132

3.13.4 Sättigungsbeiwert - Zusammenhang zwischen freiwilliger und erzwungener

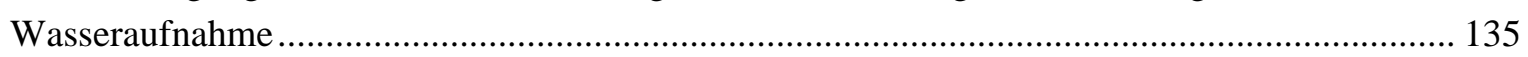

3.13.5 Ansätze zur Prognose der freiwilligen Wasseraufnahme .............................................. 137

3.14 Zusammenfassende Betrachtung zur statistischen Untersuchung

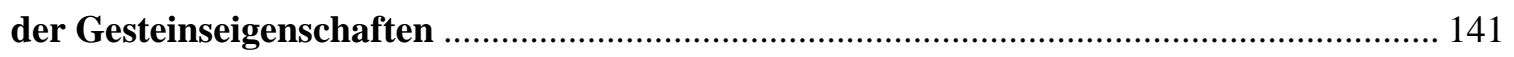

4. ÜBERREGIONALE ERFASSUNG VON NATURWERKSTEINEN:

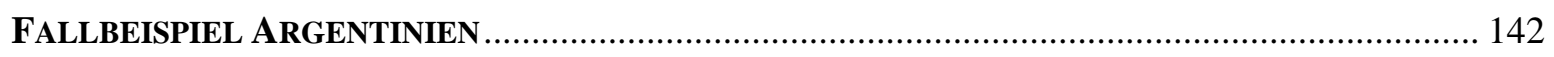

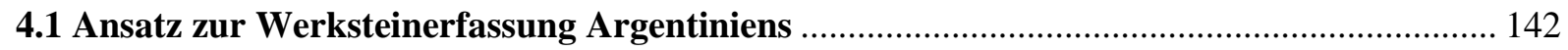

4.2 Wirtschaftliche Situation des argentinischen Natursteinsektors .......................................... 143 
4.4 Geologischer Überblick über die für Naturwerkstein relevanten Regionen.......................... 145

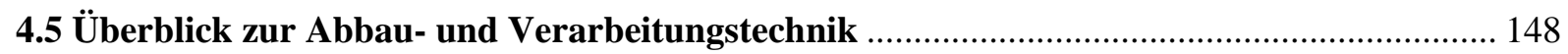

4.6 Lithologische und lagerstättengeologische Charakterisierung des Werksteinpotenzials..... 150

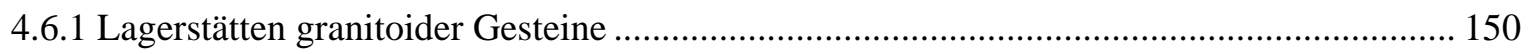

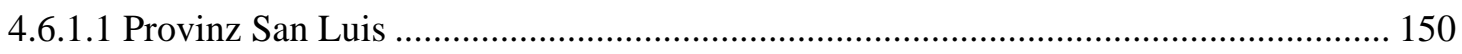

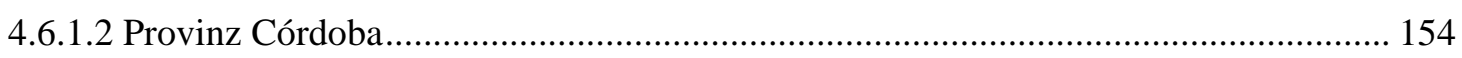

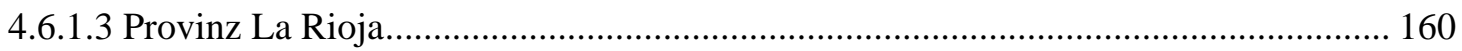

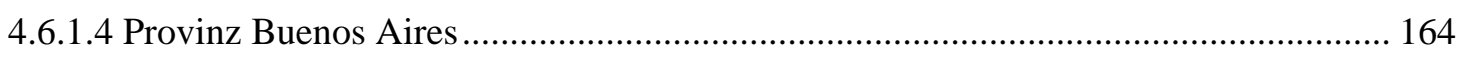

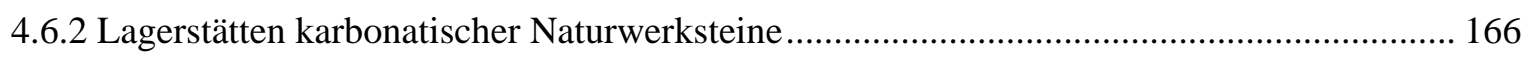

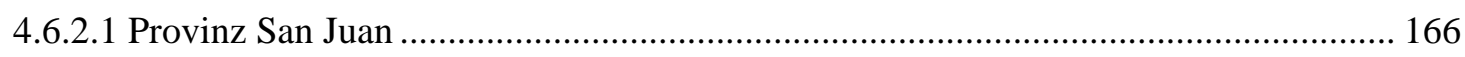

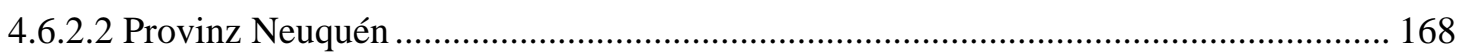

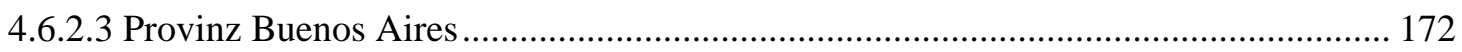

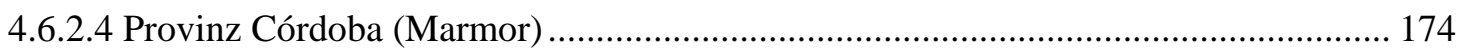

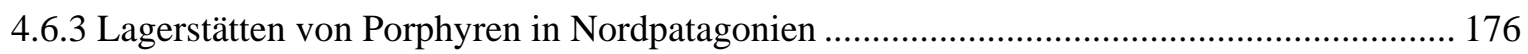

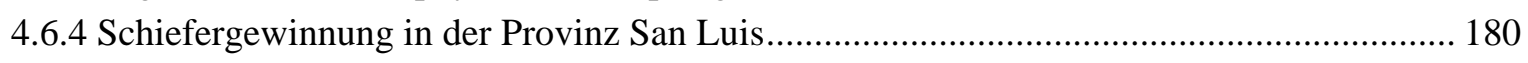

4.7 Physikalische und technische Charakterisierung der aufgenommenen Lithologien ............ 182

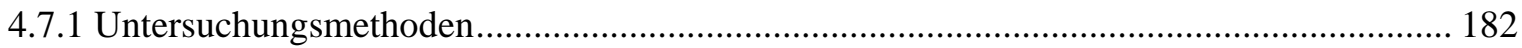

4.7.1.1 Dichten, Porenraum und Wasseraufnahmeverhalten ................................................. 182

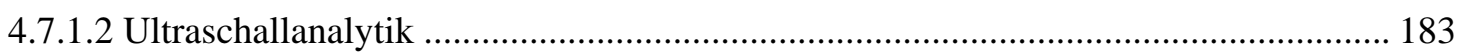

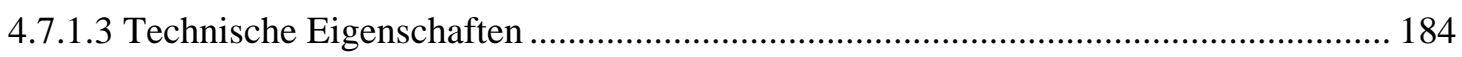

4.7.2 Physikalische Eigenschaften des Probenmaterials.............................................................. 185

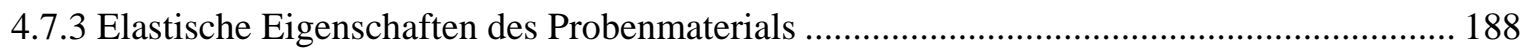

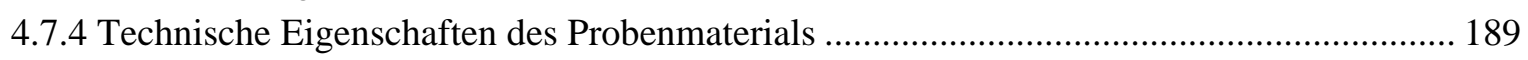

4.8 Exemplarischer Vergleich experimenteller und prognostizierter Daten ............................... 192

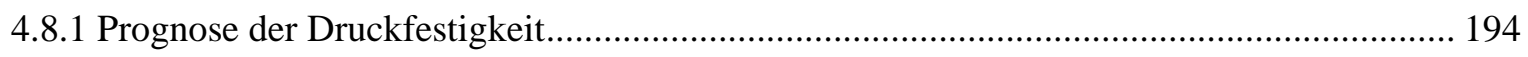

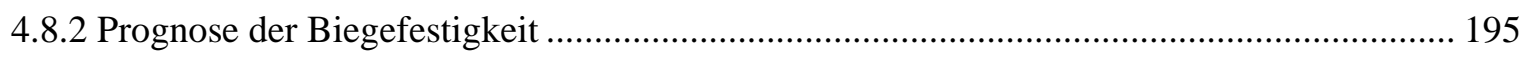

4.9 Zusammenfassende Bewertung des Werksteinpotenzials und Schlussfolgerungen .............. 200

5. REGIONALE ERFASSUNG VON NATURWERKSTEIN:

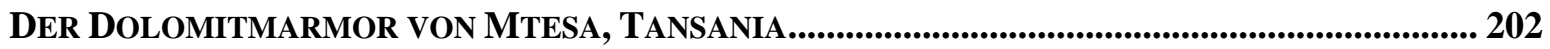

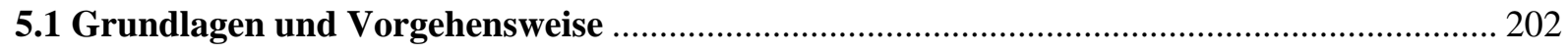

5.2 Geographische Einordnung und infrastrukturelle Anbindung des Arbeitsgebietes............. 203

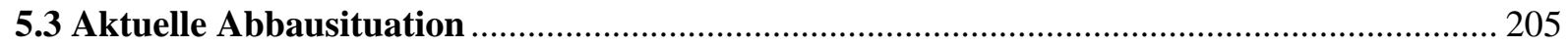

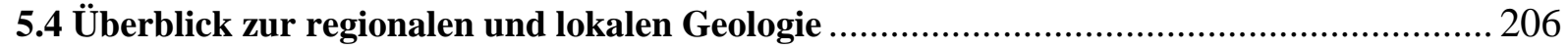

5.5 Lithologische Charakterisierung der auftretenden Gesteine im Vorkommen ..................... 207

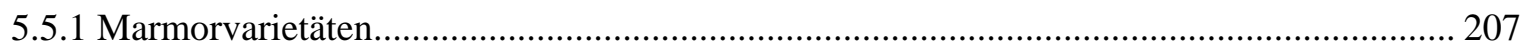

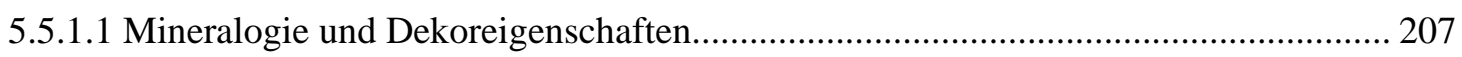

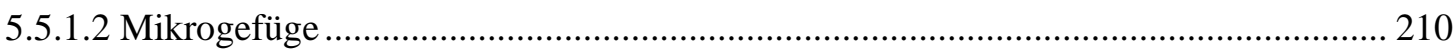

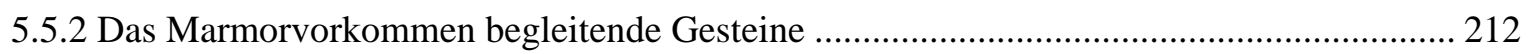

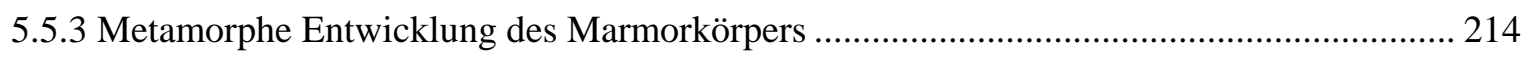




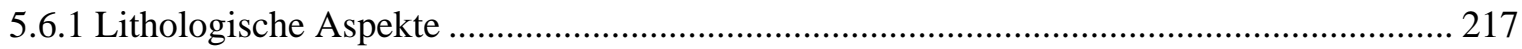

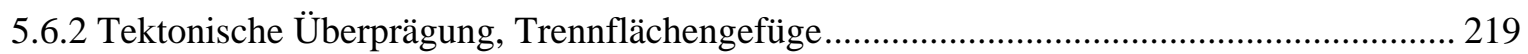

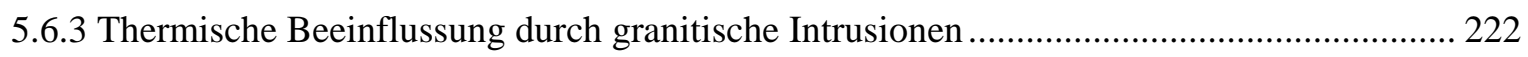

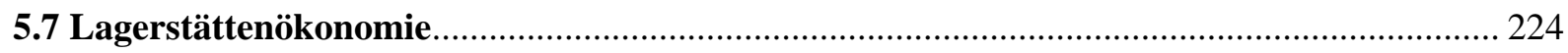

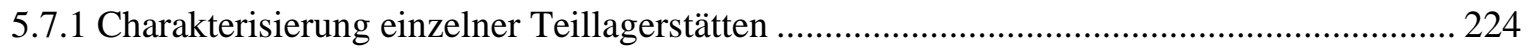

5.7.2 Abschätzung der Gesamtvorratslage des Dolomitmarmors................................................. 227

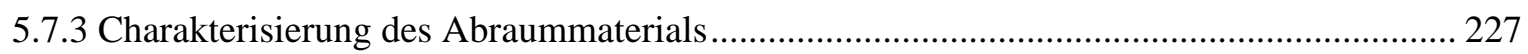

5.8 Physikalische und technische Charakterisierung des Werksteinpotenzials......................... 230

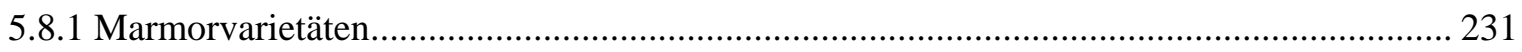

5.8.1.1 Dichten, Porenraumeigenschaften und Wasseraufnahmeverhalten............................ 231

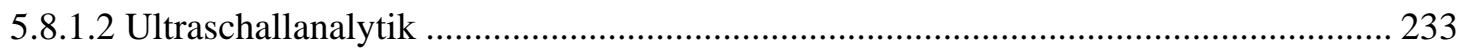

5.8.1.3 Thermische und thermohygrische Dehnungseigenschaft ........................................ 234

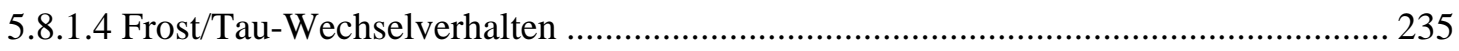

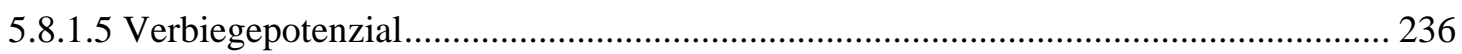

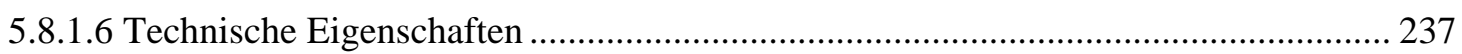

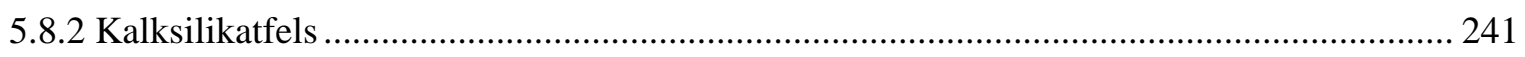

5.9 Zusammenfassende Bewertung des Lagerstättenpotenzials................................................ 241

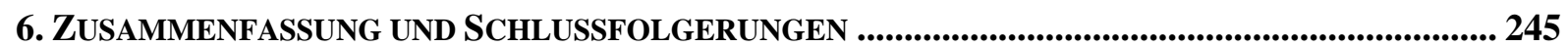

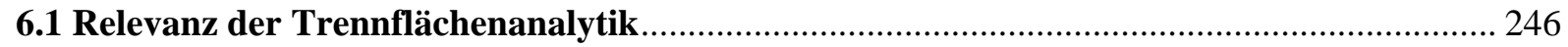

6.2 Relevanz der physikalischen und technischen Gesteinscharakterisierung .......................... 247

6.3 Erkenntnisse aus der Zusammenführung der methodischen Grundlagen ........................... 253

7. LITERATURVERZEICHNIS ................................................................................................................. 262

\section{Danksagung}

Lebenslauf 


\section{EINLEITUNG}

Die Nutzung von Naturwerkstein als Baurohstoff ist eng mit der Entwicklung der menschlichen Zivilisation verbunden. Die Reichhaltigkeit der Gesteine in Färbung und Ornamentierung führte zu einer vielfachen Anwendung als Bau- und Monumentgestein. Während in der Vergangenheit überwiegend Massivmauerwerke und Säulen aber auch Dachziegel aus Naturwerksteinen hergestellt wurden, hat die Entwicklung der Verarbeitungstechnologie eine erhebliche Erweiterung der Einsatzmöglichkeiten bedingt. So wurde bereits vor einigen Jahrzehnten die Verkleidung von Fassaden mit Plattenstärken im Bereich von 3 bis $5 \mathrm{~cm}$ ein Standardverfahren zur ästhetischen Gestaltung auch großdimensionierter Gebäude. Neueste technologische Entwicklungen ermöglichen die Fertigung von Fassadenplatten mit Dicken unter $3 \mathrm{~cm}$ oder gar Dünnschichtpaneele in Stärken von 0,1 bis $10 \mathrm{~mm}$. Als Folge dieser dynamischen Veränderungen durch die Entwicklung immer neuer Fertigungstechniken zusammen mit der sich intensivierenden Globalisierung der Naturwerksteinindustrie ist ein zunehmender Einfluss der Material- und Geowissenschaften auf den Sektor der Werksteinindustrie unabdingbar.

Die Wirtschaftlichkeit einer Lagerstätte wird, neben der generellen Eignung des Gesteins und dem potenziell gewinnbaren Volumen, durch das Verhältnis von gewinn- und verwertbarem Rohsteinaufkommen (Wertgestein) zu nicht verwertbarem Gestein (Abraum) bestimmt. Es ist davon auszugehen, dass bei der Gewinnung von Naturwerkstein im weltweiten Mittel rund $51 \%$ des im Abbau bewegten Materials als Abraumanteil durch die Rohblockgewinnung anfallen (Abb. 1.1). Weitere etwa $20 \%$ Verlust sind als Verschnittmenge mit der industriellen Weiterverarbeitung verbunden. Somit gelangt lediglich ein knappes Drittel des gewonnenen Rohgesteins als Fertigprodukte in den weltweiten Handel. Dieser relativ geringe Anteil unterliegt einer weiteren Einschränkung. Aufgrund unzureichender Kenntnisse über das spezifische Materialverhalten kommt es nicht selten schon während der üblichen Nutzungsdauer zu irreversiblen Schäden.

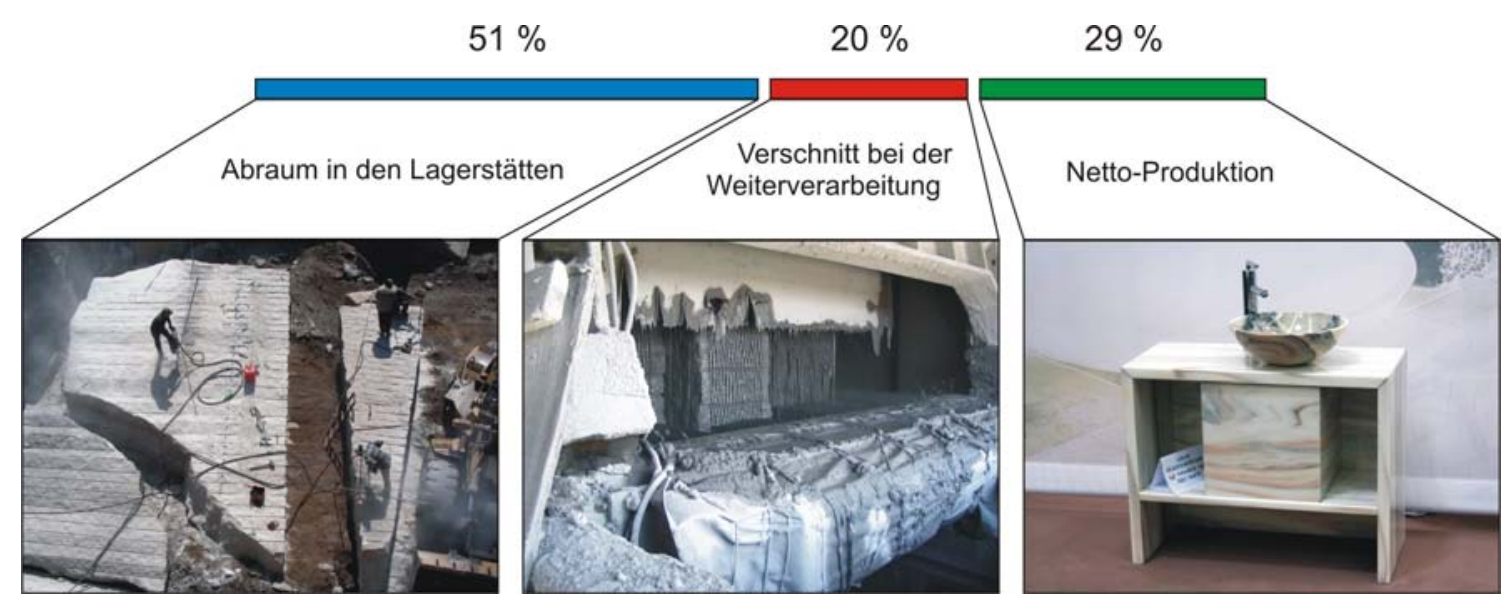

Abb. 1.1: Lediglich rund ein Drittel des weltweit gewonnenen Rohstoffs Naturwerkstein geht als Fertigware (Netto-Produktion) in den Handel ein (Montani 2003). 
Um eine optimierte Ausnutzung des Rohstoffs und damit verbunden auch einen nachhaltigen Ressourcenschutz zu erreichen, bedarf es zweier auf eine wissenschaftliche Basis gestellter Aspekte:

1) Die Erfassung der geologischen Situation eines Vorkommens und den sich daraus ergebenden lagerstätten-kontrollierenden Faktoren mit dem Ziel eines optimierten Abbaus des Wertgesteins.

2) Die umfassende Charakterisierung der physikalischen und technischen Eigenschaften nach modernen Prüfbestimmungen sowie der Verwitterungsanfälligkeit eines Gesteins mit dem Ziel einer Bewertung der bautechnischen Eignung.

Auf dem Weg zum fertigen Endprodukt stellt der Rohblock, als zugerichteter, annähernd quaderförmiger Block, das erste Zwischenprodukt im Prozess der Werksteingewinnung und -verarbeitung dar. Durch die eingangs genannte dynamische Entwicklung in der verarbeitenden Industrie werden an die Steinbruchbetreiber Forderungen gestellt. Diese beziehen sich vor allem auf die Größe und Abmessungen der Rohblöcke, um eine maximale Auslastung des Fertigungsinventars zu gewährleisten. So können beispielsweise nach Selonen \& Suominen (2003) in modernen Sägeeinrichtungen lediglich Blöcke mit Dimensionen von 2,4-3,0 m (Länge) x 1,3-1,9 m (Höhe) x 1,0-1,4 m (Breite) aufgearbeitet werden. Außerdem ist eine rechtwinklige Form der Rohblöcke gefordert, um unnötige Leerlaufzeiten der Sägegatter und unnötige Schnittabfälle zu vermeiden. Des Weiteren müssen die zugerichteten Rohblöcke frei von Defekten, wie Haarrissen oder Abweichungen vom definierten Dekorund Farbbild einer Handelssorte, sein. Die mögliche Blockdimension und die Blockqualität sind dabei eng mit der geologischen Situation eines Abbaugebietes verknüpft. Ausdruck findet diese in einer Vielzahl an lagerstätten-kontrollierenden Elementen. Also den Faktoren, die sich auf den Werkstein selbst und den möglichen Abbau auswirken und im Endeffekt den auszubringenden Teil gut dimensionierter, defektfreier Rohblöcke in Qualität und Quantität bestimmen. In vielen Fällen fehlt jedoch bereits bei der Gewinnung ein ausreichendes Maß an geowissenschaftlichen Grundlagen über ein Vorkommen. Dabei kommt dem Trennflächengefüge eine dominante Rolle zu, also der Gesamtheit aller flächigen Elemente, die durch gegenseitiges Schneiden die Bildung von individuellen Blöcken, den so genannten in-situ Blöcken (Lu \& Latham 1999), im Gesteinskörper hervorrufen. Aus der Nichtbeachtung resultiert nicht selten ein übermäßiger und dabei häufig vermeidbarer Verlust im Abbau. Neben der rein wirtschaftlichen Komponente sind damit auch Umweltschäden durch die unkontrollierte Anlage eines Abbaus oder auch die Erweiterungen eines Abbauareals zu beachten, die sicherlich nicht dazu beitragen, das positive Image, das der Rohstoff Werkstein als voll recyclebares Naturprodukt besitzt, zu tragen und zu fördern. Die Beachtung aller geologischen Vorgaben gilt somit als Grundvoraussetzung zur gezielten Nutzung der Ressource, wobei vor allem die Optimierung des Abbau der Lagerstätten von Relevanz ist (z.B. Singewald 1992, Palmström 1995, Primavori 1999, Prissang et al. 2007). 
Somit ist eine geowissenschaftlich unterstützte Planungsgrundlage bei der Gewinnung von Werkstein unumgänglich, da aus der kritischen Betrachtung von Blockgrößen und -verteilungen eine positive Beeinflussung der wirtschaftlichen Bilanz einer Lagerstätte erfolgen kann. Im ersten Teil der Dissertation (Kap. 2, Abb. 1.2) werden daher theoretische und methodische Grundlagen zur detaillierten Aufnahme und Analyse des Trennflächensystems bei der Erkundung, Abbauplanung und Abbauführung von Werksteinlagerstätten aufgezeigt. Entscheidende Kriterien sind einerseits die grundsätzlichen Belange der Werksteinindustrie sowie andererseits die lithologieabhängige Ausbildung des Trennflächengefüges in seiner Orientierung und Abstandsverteilung. Anhand verschiedener ausgewählter Lagerstätten werden die Auswirkungen der natürlichen Vorgaben der geologischen Situation eines Vorkommens auf die Möglichkeit der Werksteingewinnung dargestellt. Dafür wurden Steinbrüche unterschiedlicher Lithologien im In- und Ausland befahren. Die Erfassung und Analyse der spezifischen Trennflächengefüge sollen vor allem im Hinblick auf eine möglichst optimale Anpassung der Abbauführung an die geologischen Konditionen kritisch betrachtet werden. Theoretische Modelle möglicher Abbausituationen unterstützen dabei die Darstellung primär positiver aber auch negativer Auswirkungen, die sich aus der Veränderung der Abbauführung ergeben können.

Der zweite Aspekt bezieht sich auf die Anwendung des Rohstoffs Naturwerkstein, sei es beispielsweise als tragendes, konstruktives Bauelement oder als reines Schmuck- und Dekorelement z.B. in Form sichtbarer Hüllflächen beziehungsweise Verkleidungen. In der DIN EN 12670 ist Naturwerkstein als ein natürlich vorkommendes Gestein definiert, das im Bauwesen und für Denkmäler verwendet wird. Am internationalen Natursteinmarkt ist eine Vielzahl von Handelssorten vertreten, wobei mit der fortschreitenden Globalisierung ein ständiger Zuwachs zu verzeichnen ist. So haben z.B. Börner \& Hill (2007) 13677 verschiedene Handelssorten erfasst. Aus dieser Flut resultiert die Tatsache, dass eine Vielfalt an Gesteinen dem Markt zur Verfügung steht, die eine immense Bandbreite an Farben und Dekoren abdecken. Eine grundlegende Problematik, die sich aus der Mannigfaltigkeit des angebotenen Materials ergibt, ist die häufig willkürliche Wahl der Handelsbezeichnung eines Gesteins ohne jeden Bezug zu Herkunft oder lithologischer Zuordnung (z.B. Hill 2007). So resultiert aus der Namensgebung zum Teil eine vollständige Verfälschung eines Gesteins. Bestes Beispiel dafür ist ein bekannter mikritischer Kalkstein aus Belgien, der unter der Handelsbezeichnung BELGISCH GRANIT geführt wird. Nicht selten treten Gesteine auch unter verschiedensten Namen im Handel auf. Börner \& Hill (2007) zählen beispielsweise für einen derzeitig am Markt häufig angebotenen chinesischen Granit 67 synonyme Handelsbezeichnungen auf. Diese Verwirrungen führen dazu, dass, möglicherweise auch beabsichtigt, eine Verzerrung des Marktes herbeigeführt wird. So ist es häufig schwer nachvollziehbar, ob das für ein Bauvorhaben tatsächlich gelieferte Produkt dem in der Planungsphase anhand vorgelegter Musterplatten ausgewählten Gestein entspricht. Dies wird umso schwieriger, da gemäß der DIN 18332 Farb-, Struktur- und Texturschwankungen innerhalb eines Werksteinvorkommens ausdrücklich zulässig sind, sofern sie nicht außerhalb der erfassten Bemusterung liegen. Vor allem aus gestalterischer Sicht werden diese Faktoren, 
also die rein optische Wahrnehmung, meist zur Entscheidungsfindung herangezogen. Dabei bleibt vielfach die Einbeziehung materialspezifischer Eigenschaften, die das Verhalten eines Gesteins in der bautechnischen Anwendung bestimmen, unbeachtet. Dies führt nicht selten zu einer unpassenden oder gar fehlerhaften Anwendung des Materials, sei es in Form baukonstruktiver Fehler oder einer optischen Beeinträchtigung durch z.B. verwitterungsbedingte Verfärbungen. Als direkte Folge davon können erhebliche Schäden entstehen, die, abgesehen von der wirtschaftlichen Komponente, auch die ästhetische Schönheit des Werksteins beeinträchtigen. Aus dieser Sachlage heraus resultiert eine zunehmende Unsicherheit am Markt und auch, bei der Aufstellung entsprechender Mängellisten, eine zunehmende Unzufriedenheit der Verbraucher. Unumgänglich ist daher eine wissenschaftlich fundierte Erfassung der spezifischen Materialcharakteristika, die neben den Farben und Strukturen, also den ästhetischen Gesichtspunkten, vor allem den mineralogischen, physikalischen und technischen Parametern Rechnung trägt. Weiterhin ist als ein wesentlicher Punkt die Erfassung des Materialverhaltens in Bezug auf verschiedene Verwitterungsprozesse von eminenter Bedeutung, denn neben den Auswirkungen einer rein statischen Belastung unterliegen viele Gesteine vor allem auch verwitterungsbedingten Veränderungen. In diesem Zusammenhang sind Gefügeänderungen zu erfassen, die aus Kräften resultieren, welche aus der Vielzahl an Prozessen der physikalischen, chemischen und biologischen Verwitterung hervorgehen können.

Für die einheitliche und dadurch vergleichbare Ermittlung der verschiedenen physikalischen und technischen Gesteinsparameter existieren für den Natursteinsektor rund 50 Normen (EN bzw. DIN)(Lepper 2007). Entsprechend genormte Richtlinien sind mittlerweile auch für die verschiedenen Anwendungen von Naturwerksteinen verfasst, in denen die wesentlichen Anforderungen an die unterschiedlichen Produktgruppen enthalten sind. Für verschiedene Einsatzbereiche ist zudem seit kurzem eine CE-Kennzeichnung verpflichtend, womit die internationale Vergleichbarkeit zum technischen Untersuchungsstand der Produkte sowie der Nachweis bezüglich der Übereinstimmung mit den jeweils relevanten Normen gewährleistet werden soll. Entscheidend ist aber, dass mit der CE-Kennzeichnung eines Gesteins keine Aussage hinsichtlich einer generellen technischen Eignung gemacht wird (Brändlein \& Deppisch 2007) beziehungsweise darüber, wie die an einem Gestein normgerecht ermittelten Eigenschaften bewertend betrachtet werden können.

An diesem Punkt setzt der zweite methodische Teil der Arbeit an (Kap. 3). Auf einer breiten Datenbasis werden die Eigenschaften Rohdichte und effektive Porosität, Parameter der mechanischen Stabilität, namentlich Druck-, Biege- und Spaltzugfestigkeit, die elastischen Eigenschaften anhand der Kompressionswellengeschwindigkeit sowie Parameter zum Feuchtehaushalt der Gesteine mit einfachen statistischen Mitteln betrachtet (Abb. 1.2). Primär wird eine explorative Datenauswertung durchgeführt, um aus dem mehr als 2300 Handelssorten umfassenden Datensatz lithologietypische Wertebereiche $\mathrm{zu}$ definieren. Basierend auf statistischen Kenndaten der verschiedenen Datenreihen werden darauf aufbauend für verschiedene Korrelationen Bereiche der jeweils theoretischen 
Grundgesamtheit mit dem Ausdruck graphisch dargestellter Bereiche der 80 \%-igen Aufenthaltswahrscheinlichkeit berechnet. Damit wird ein Werkzeug gegeben, das einen direkten, bewertenden Vergleich einzelner Datensätze oder auch von Einzeldaten ermöglicht.

Eine umfassende physikalische und bautechnische Materialcharakterisierung nach den geltenden europäischen und nationalen Normen erfordert insgesamt ein hohes Probenvolumen. Zudem ist auch die laborgestützte Ermittlung mit einem hohen Kostenaufwand verbunden, was gerade in der Ersterkundung von Vorkommen dazu führt, dass, wenn überhaupt, lediglich ein stark eingeschränktes Wissen über die materialspezifischen Eigenschaften gewonnen werden kann. Um dem entgegenzutreten, werden im Weiteren Prognosemöglichkeiten vorgestellt, mit dem Ziel, ausgehend von einfach und kostengünstig zu bestimmenden Eigenschaften, wie der Rohdichte oder der Kompressionswellengeschwindigkeit, zu einer erweiterten Materialkenntnis zu gelangen.

Mit der kritischen Betrachtung des Trennflächengefüges im Hinblick auf die generelle Möglichkeit und optimale Anpassung einer Werksteingewinnung sowie mit der umfassenden Materialcharakterisierung nach dem aktuellen Stand von Wissenschaft und Technik und den daraus abzuleitenden Einsatzmöglichkeiten eines Gesteins, sind zwei wesentliche Grundpfeiler der möglichen Einflussnahme der Geo- und Materialwissenschaften auf die Werksteinnutzung umrissen. Vorrangiges Ziel ist es, diese Aspekte methodisch aufzuarbeiten. Die daraus gewonnenen Grundlagen und Erkenntnisse werden anschließend in zwei ausgewählte Beispiele zur Erfassung, Erkundung und Bewertung von Werksteinlagerstätten eingebracht. Diese Fallstudien stellen beispielhaft unterschiedliche Erkundungsstadien dar.

Im ersten Schritt soll eine überregionale Erfassung von Werksteinlagerstätten in Argentinien erfolgen (Kap. 4). Aufgrund der komplexen geologischen Verhältnisse des südamerikanischen Kontinents ist eine weite Bandbreite an struktur- und dekorreichen Gesteinen vorhanden, die in der ersten Betrachtung ein hohes wirtschaftliches Potenzial darstellen. Bestes Beispiel dafür gibt der direkte Nachbar Brasilien, dessen Produktionszahlen vergleichbar mit Ländern wie Indien und China einem starken Wachstum unterliegen (Merke 2007). Die argentinische Wirtschaft durchlief dagegen in den Jahren 1998/99 und 2001/02 eine tiefe Rezession, von der auch der Werksteinsektor empfindlich getroffen wurde. Zurzeit ist eine leichte Erholung mit schwach steigenden Produktionszahlen zu beobachten. Der Hauptanteil des ausgebrachten Rohstoffs geht dabei in den nationalen Markt ein, wodurch auch für viele Varietäten ein nur geringer Informationsstand zu den physikalischen und technischen Gesteinseigenschaften vorliegt. Bei dieser überregionalen Erfassung liegt der Fokus auf der Katalogisierung und Regionalisierung auftretender Werksteinvarietäten, von denen sich der Großteil zum Zeitpunkt der Untersuchung im Abbau befand. Die Befahrung von Steinbrüchen in einer Vielzahl von Lagerstätten ermöglichte weiterhin die Aufnahme der jeweiligen lagerstätten-kontrollierenden Elemente und damit eine Einschätzung der wirtschaftlichen Nutzbarkeit. Zudem sollen die aus der statistischen Datenauswertung gewonnenen Erkenntnisse über regressive Zusammenhänge genutzt werden, um zu einer wie oben beschriebenen, erweiterten Kenntnis der Gesteinseigenschaften zu gelangen. Da im 
Rahmen dieser überregionalen Erfassung eine ausreichende Beprobung der Lagerstätten möglich war, ist somit auch der direkte Vergleich der prognostischen Daten mit experimentell ermittelten Daten möglich.

Die überregionale Erfassung basiert somit auf dem Kenntnisstand, dass aktive Abbaue zur Gewinnung von Werksteinrohblöcken vorhanden sind und das ausgebrachte Material voranging in den regionalen und nationalen sowie zu geringeren Anteilen auch in den internationalen Natursteinmarkt eingeht. Ausgangssituation für das zweite Fallbeispiel, den Dolomitmarmor von Mtesa, Tansania (Kap. 5), ist dagegen lediglich die Kenntnis über ein mögliches Marmorvorkommen anhand weniger Handstücke sowie durch Informationen über einen aktiven Kleinstabbau zur Gewinnung von Zuschlagstoffen für den regionalen Bedarf. Die Befahrung des Vorkommens hat somit in diesem Fall die detaillierte, lagerstättengeologische Erfassung zum Ziel. Dabei steht primär die Frage der wirtschaftlichen Nutzbarkeit als Werkstein im Vordergrund. Neben der generellen petrologischen Erfassung der auftretenden Gesteine im Vorkommen werden dafür offene Fragen zum Trennflächengefüge, zu Lagerungsverhältnissen sowie auch beispielsweise zur Verteilung möglicher Farbvarianzen des Marmors beantwortet. Im Weiteren soll in diesem Rahmen beispielhaft eine umfassende Charakterisierung des Materials hinsichtlich der möglichen bautechnischen Verwendung nach den Standards für Naturwerkstein entsprechend dem Stand der Technik erfolgen. Zu ausgewählten Fragestellungen, hier insbesondere der bei vielen Marmoren auftretenden mangelnden Formbeständigkeit vor allem bei der Verwendung als Fassadenelement, werden detaillierte Analysen nach dem Stand der Wissenschaft durchgeführt. In der Gesamtheit sollen die Ergebnisse der regionalen Erfassung zu einer Einschätzung des wirtschaftlichen Potenzials einer Lagerstätte, ausgerichtet an der „Internationale Rahmen-Vorratsklassifikation der Vereinten Nationen / UNFCR“ (UNO 1997), zusammengeführt werden.

Zusammenfassend ist das Ziel der vorliegenden Dissertation, die Aspekte der Abbauoptimierung und Materialcharakterisierung aus geo- und materialwissenschaftlicher Sicht methodisch aufzuarbeiten. Die daraus entwickelten Grundlagen und gewonnenen Erkenntnisse werden in zwei ausgewählten Beispielen zur Erfassung, Erkundung und Bewertung von Werksteinlagerstätten eingebracht. Unter Beachtung der methodischen Grundlagen werden final Aspekte der industriellen beziehungsweise wirtschaftlichen Umsetzbarkeit diskutiert. 


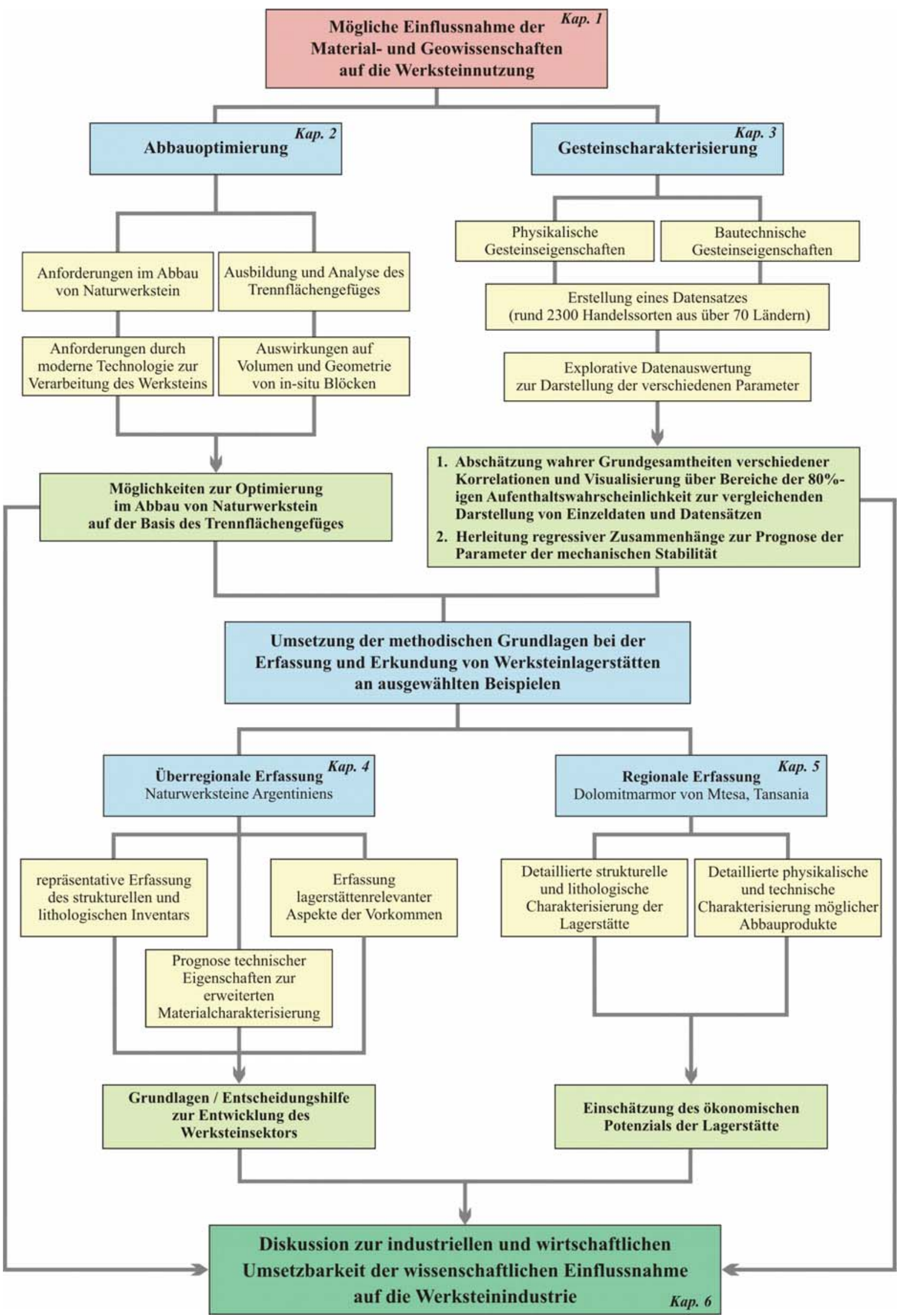

Abb. 1.2: Schematisches Grundkonzept zum Aufbau der vorliegenden Dissertation mit methodischen Grundlagen zur Abbauoptimierung und Materialcharakterisierung von Werkstein, der praktischen Umsetzung an ausgewählten Beispielen und der finalen Zusammenführung der gewonnenen Erkenntnisse. 


\section{DiE RELEVANZ DES TRENNFLÄCHENSYSTEMS BEI DER GEWINNUNG VON NATURWERKSTEIN}

\subsection{Anforderungen im Abbau und der Verarbeitung von Naturwerkstein}

Bei allen Lagerstätten mineralischer Rohstoffe sind genaue Kenntnisse der Ressourcenbeschaffenheit und -qualität notwendig. Dabei nehmen Naturwerksteine eine Sonderstellung ein, da im gesamten Prozess von der Gewinnung, über die Verarbeitung bis zum Einsatz als Baustoff keine stoffliche Umwandlung des Materials erfolgt (Peschel 1983). Die Qualität des Gesteins wird somit ausschließlich von den geologischen Voraussetzungen diktiert. Dies bezieht sich zum einen auf das Erscheinungsbild und die physikalischen und technischen Eigenschaften eines Gesteins sowie dem daraus resultierenden Verhalten in der bautechnischen Anwendung. Zum anderen lassen sich aus der lagerstättengeologischen Betrachtung eines Vorkommens verschiedene Rückschlüsse ziehen, die vor allem einen entscheidenden Einfluss auf die Möglichkeit und technische Umsetzbarkeit der dimensionsgerechten Ausbringung von Rohblöcken ausüben. Da die Bildung von individuellen Blöcken, den so genannten in-situ Blöcken ( $\mathrm{Lu}$ \& Latham 1999), in einem intakten Gesteinskörper auf das gegenseitige Schneiden von verschieden gerichteten Trennflächensystemen zurückgeht, gilt somit das Trennflächengefüge in seiner Abstandsverteilung und Orientierung als eines der wesentlichsten Elemente bei der Bewertung eines Werksteinvorkommens. Eine weitere Problematik infolge einer erhöhten Trennflächendichte ergibt sich zudem im Bereich der untertägigen Gewinnung von Werkstein, der vor allem im Abbau von Marmor eine zunehmende Rolle zukommt, durch eine reduzierte Stabilität aufzufahrender Hohlräume (Koch-Moeck 2005).

Ein entscheidendes Kriterium für Werksteinlagerstätten ist durch das Verhältnis von Abfall- zu Werksteinaufkommen gegeben. Dabei sind bestimmte Rohblockabmessungen erforderlich, um moderne Verarbeitungstechnologien effizient und wirtschaftlich einsetzen zu können (Selonen \& Suominen 2003). Primär ist aber der Dimensionierung der Rohblöcke durch die logistischen Möglichkeiten eine Grenze gesetzt. Primavori (1999) nennt für Rohblöcke optimale Bedingungen bei einem Volumen von $6-8 \mathrm{~m}^{3}$. Jedoch müssen auch Mindestkantenlängen gegeben sein, um die Kippstabilität in den Prozessen der Verarbeitung, so z.B. in modernen Großgattersägen, zu gewährleisten. Detailliertere Rahmenwerte werden in den Richtlinien der Vereinten Nationen (1976) gegeben. Danach ist für zu exportierende Natursteinblöcke ein Mindestvolumen von 1 bis $6 \mathrm{~m}^{3}$ bei Abmessungen zwischen $2 \mathrm{x} 1 \mathrm{x}$ $0,5 \mathrm{~m}$ und $3 \times 2 \times 1 \mathrm{~m}$ erforderlich. Anhand der Befahrung einer Vielzahl von Steinbrüchen und weiterverarbeitenden Betrieben definiert Singewald (1992) dagegen einen Mindestblock, der ein Volumen von > 0,5 $\mathrm{m}^{3}$ bei Abmessungen von mindestens 0,4 x 1,0 x 1,0 m aufweist. Zudem ist neben der Blockgröße bzw. dem Volumen die Form von entscheidender Bedeutung bei der maschinellen Aufarbeitung. Aus rein wirtschaftlicher Perspektive sollen die Rohblöcke im Idealfall nicht bzw. nur sehr unwesentlich von einer quaderförmigen Geometrie abweichen, da ansonsten das Ansägen in Gattersägen deutlich zeit- und damit auch kostenintensiver ausfällt. Außerdem sollte zwischen dem Rohblock und dem Nettoblock, der das tatsächlich nutzbare Gesteinsvolumen eines Rohblocks beschreibt (Abb. 2.1), ein ideales 
Verhältnis bestehen. Der bei der Aufarbeitung des Rohblocks anfallende Rauabfall steigt mit abnehmender Rohblockgröße potenziell an, so dass er bei einem Volumen von rund $1 \mathrm{~m}^{3}$ bereits annähernd 30 \% ausmacht (Singewald 1992). Gleiches gilt auch für die Formatierung der Rohblöcke aus den in-situ Blöcken, so dass im Idealfall bereits annähernd orthogonale insitu Blöcke gemäß den geologischen Vorraussetzungen vorliegen sollten. Auf diesen Aspekt wird in Kapitel 2.4.2 detaillierter eingegangen.
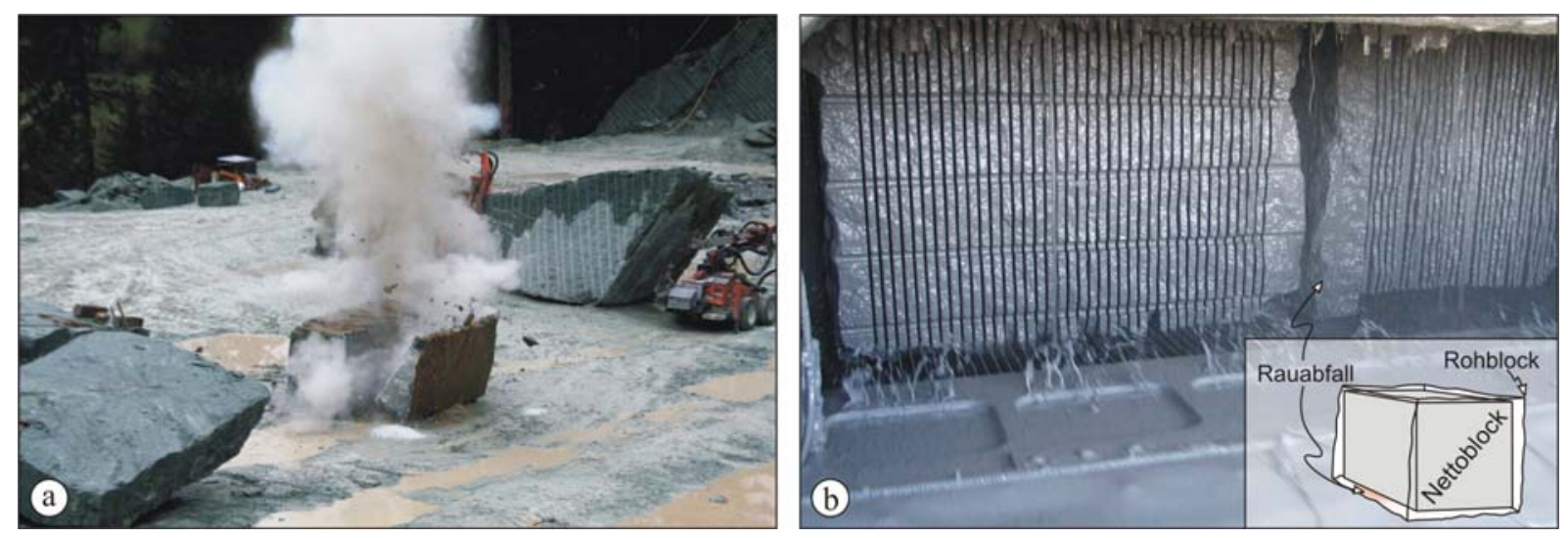

Abb. 2.1: a) Formatierung eines Rohblocks (VERDE ANDEER, Orthogneis, Schweiz): Die seitliche Abtrennung erfolgt über eng gesetzte, durchgehende Bohrungen, in die Sprengschnüre eingebracht werden; b) Aufsägen von Rohblöcken im Gatter zu 3 cm starken Rohplatten (GRIS PERLA, Granit, Argentinien).

Wie bereits erwähnt, muss die Dimensionierung der Rohblöcke auch an die jeweiligen Bedürfnisse bzw. die jeweilig gewünschten Fertigprodukte und somit an das gegebene technische Inventar zur Weiterverarbeitung angepasst sein. In vielen Fällen erfolgt das primäre Aufschneiden der Rohblöcke in Gattersägen, so dass zumeist großformatige Unmaßtafeln als Zwischenprodukt vorliegen. Dabei bestimmt die Länge und Höhe des Blocks deren Größe, während die Blockbreite die mögliche Anzahl an Platten vorgibt. Dieser Umstand muss vor allem bei strukturreichen Werksteinvarietäten bereits im Abbau Beachtung finden, da sich die interessanteste Ornamentierung auf der Längsseite der Blöcke befinden muss, um sie in der Anwendung voll zur Geltung bringen zu können. Anders verhält es sich z.B. beim Einsatz von Mehrblattsägen zur Produktion von Wand- und Bodenfliesen, wobei häufig flachere Blöcke verwendet werden. Dabei kommt dann jedoch unter Umständen der negative Effekt des erhöhten Rauabfallanteils bei abnehmenden Blockvolumina zum Tragen. Andere Vorraussetzungen ergeben sich zwangsläufig bei der Fertigung von Trockenmauerwerksteinen, Pflastersteinen oder Polygonalplatten für den Garten- und Wegebau. Hierbei können zum Teil freie Abmessungen der Rohblöcke gegeben sein. So erfolgt die Fertigung entsprechender Elemente auch häufig als Nebenprodukt im Abbau von Werksteinrohblöcken. Trotz der häufig manuellen, und damit zeitintensiven Aufarbeitung, kann somit das finale Verhältnis zwischen Abraum und Wertgestein positiv beeinflusst und folglich die wirtschaftliche Effizienz einer Lagerstätte gesteigert werden. Zusammengefasst ist es im Abbau von Naturwerkstein in hohem Maße erforderlich, aus den natürlichen Vorgaben Konsequenzen im Hinblick auf technisch-wirtschaftliche Aspekte zu ziehen. 


\subsection{Ausbildungen des Trennflächengefüges}

Das Trennflächengefüge eines geologischen Körpers umfasst sämtliche flächigen Elemente, die eine Zergliederung des Gesteinskörpers zur Folge haben. Als wesentliche Grundtypen sind Störungen, Klüfte, Risse, Brüche, Haarrisse, Schichtflächen und Scherflächen zu nennen, die nach Palmström (1995) ihrer räumlichen Ausdehnung folgend definiert werden können (Abb. 2.2). Im Allgemeinen treten Klüfte dabei als dominierendes Element des Trennflächengefüges eines Lagerstättenkörpers auf.

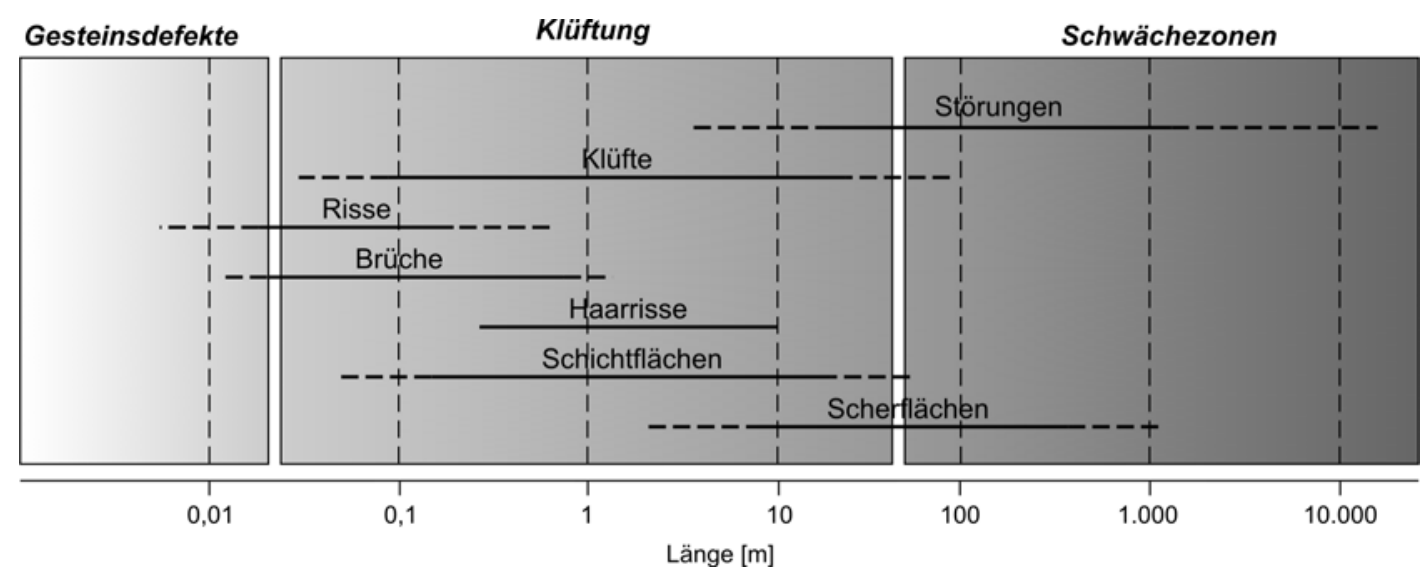

Abb. 2.2: Definition der Trennflächen nach ihrer räumlichen Ausdehnung (nach Palmström 1995).

Die natürlichen Bruch- oder Rissstrukturen können auf kinematische oder epirogenetische Prozesse zurückgeführt werden. Infolge einer Überschreitung der Zugfestigkeit des Gesteins kommt es zu einem bruchhaften Versagen, womit der Zusammenhalt des Festgesteins aufgehoben ist (Davis \& Reynolds 1996). Eisbacher (1991) unterscheidet dabei zwischen Extensionsbrüchen nicht-tektonischen und tektonischen Ursprungs.

Zu den nicht-tektonischen Brüchen zählen vor allem Abkühlungs- und Entlastungsklüfte. Die Ausbildung von Abkühlungsklüften basiert auf thermischen Gradienten, wie sie z.B. innerhalb eines magmatischen Körpers zwischen den Randbereichen und dem Kern in der Phase der Kristallisation auftreten. Dabei breiten sich in der Regel die Abkühlungsklüfte, die aus einer räumlich differenzierten Volumenreduzierung hervorgehen, senkrecht zu den oberflächenparallelen Isothermen aus. Aufgrund der relativ schnellen Temperaturabnahme im Falle vulkanischer Gesteine ist hier diese Klüftung oft besonders ausgeprägt vorhanden (Abb. 2.3). Dabei kann zusätzlich ein Zusammenhang zwischen der Abkühlungsgeschwindigkeit und den Kluftabständen hergestellt werden. Eine rasche Abkühlung, wie sie zum Beispiel an der Oberseite vulkanischer Fließdecken stattfindet, hat eine sehr engständige Klüftung zur Folge. Im tieferen Bereich ist hingegen häufig ein Wechsel zu weitständigeren Trennflächen zu beobachten (Abb. $2.3 \mathrm{a}$ ). 

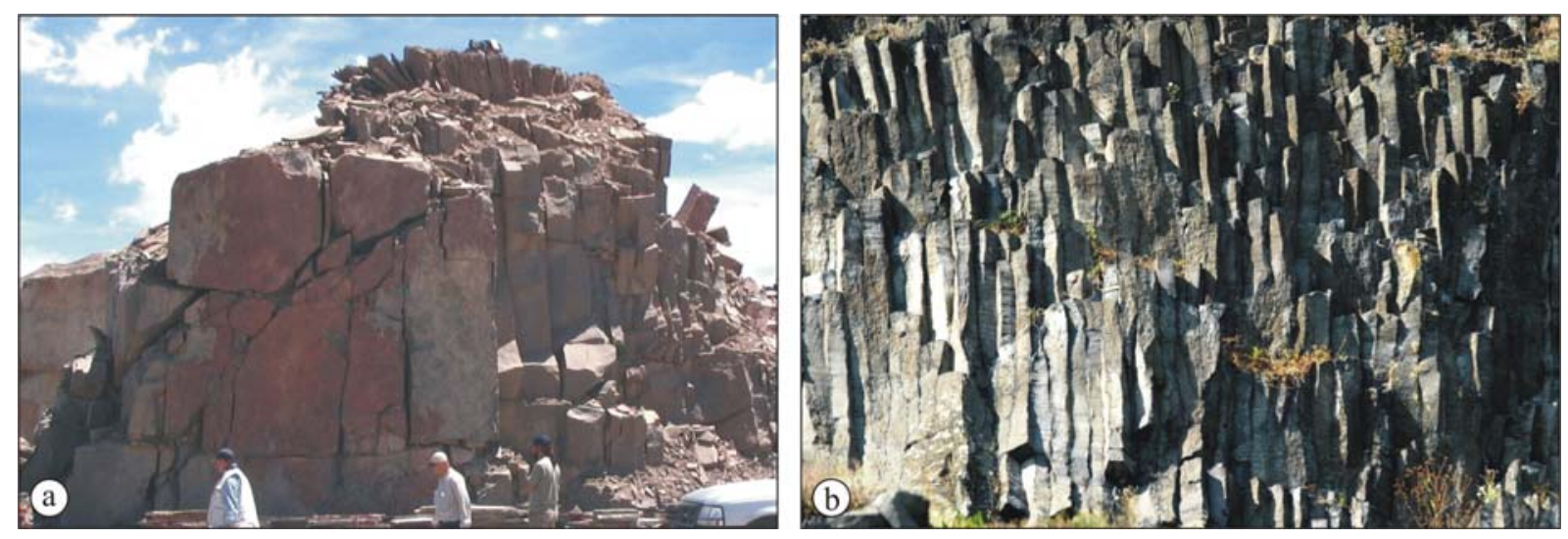

Abb. 2.3: Ausbildung von charakteristischen Abkühlungsklüften senkrecht zu oberflächenparallelen Isothermen in a) Porphyr (Provinz Chubut, Argentinien) mit einer Zunahme der Kluftabstände in tieferen Bereichen und b) Basalt (Bayern, Deutschland) mit typischen, hexagonalen Abkühlungssäulen.

Des Weiteren treten bei einer erosiven Entlastung eines Gesteinskörpers oberflächenparallele Klüfte, die so genannten Entlastungsklüfte, in Erscheinung (Abb. 2.4 a). Im Allgemeinen lassen sich diese Klüfte bis in eine Tiefe von rund 20 bis $50 \mathrm{~m}$ verfolgen (Eisbacher 1991). Diese Kluftscharen sind zwar erst in einem vertikalen Anschnitt erkennbar, deuten aber auf eine nicht unerhebliche Entspannung und Lockerung eines sonst massiven Körpers hin. In der Folge sind somit mehrere Kluftscharen ausgebildet, die nicht selten senkrecht zueinander ausgerichtet sind. Bei Granitoiden resultiert bei einsetzender Verwitterung daraus die Bildung quaderartiger Körper, die im weiteren Verlauf durch eine verstärkte Kantenabrundung nicht selten das typische, oft eine Landschaft prägende Erscheinungsbild der „Wollsack-Verwitterung“ ergeben (Abb. 2.4 b). Dabei ist die Größe der durch die Zergliederung und anschließende Verwitterung gebildeten Blöcke direkt auf die Verteilungsdichte innerhalb der Kluftscharen zurückzuführen. Aus der Oberflächensituation ist eine Abschätzung der Tiefenwirkung der Trennflächen zumeist nur schwer einzuschätzen. In manchen Fällen kann jedoch an der Oberfläche eine erhebliche Zergliederung gegeben sein, während bereits in geringer Tiefe ein relativ kompakter Gesteinskörper ansteht (Abb. 2.5).
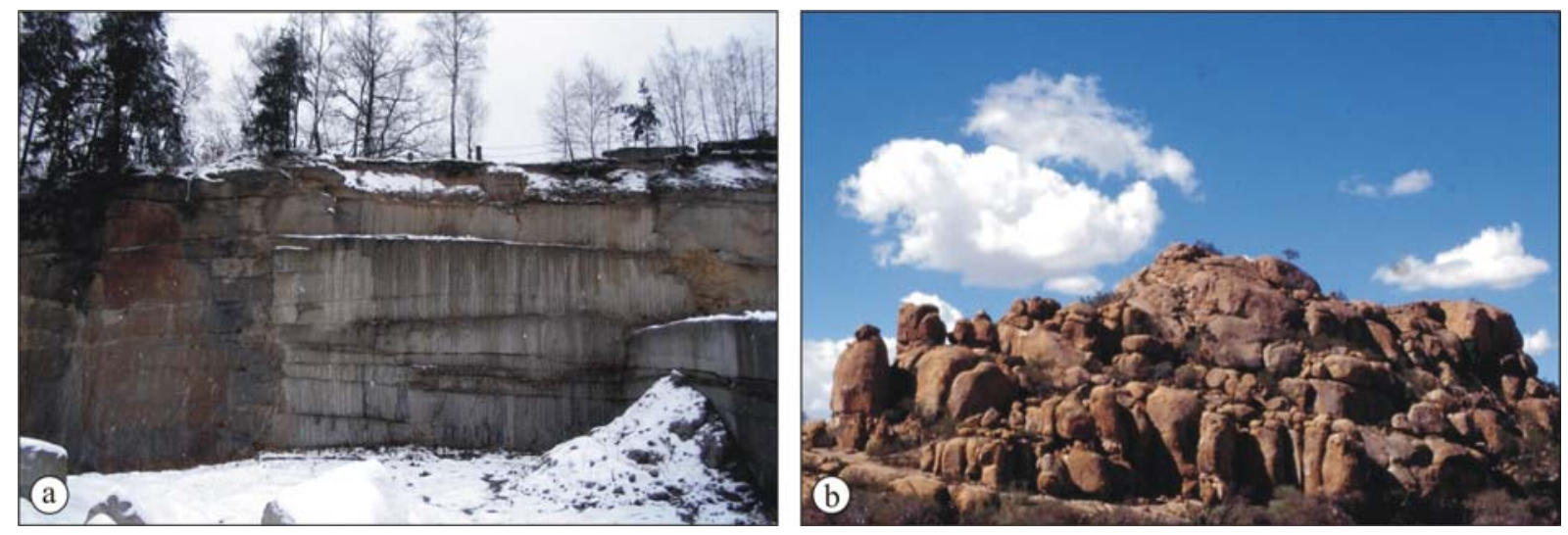

Abb. 2.4: Oberflächenparallele Entlastungsklüfte in Granit (Steinbruch Flossenbürg, Deutschland); d) typische Wollsack-Verwitterung von Granitkomplexen (Namibia). 


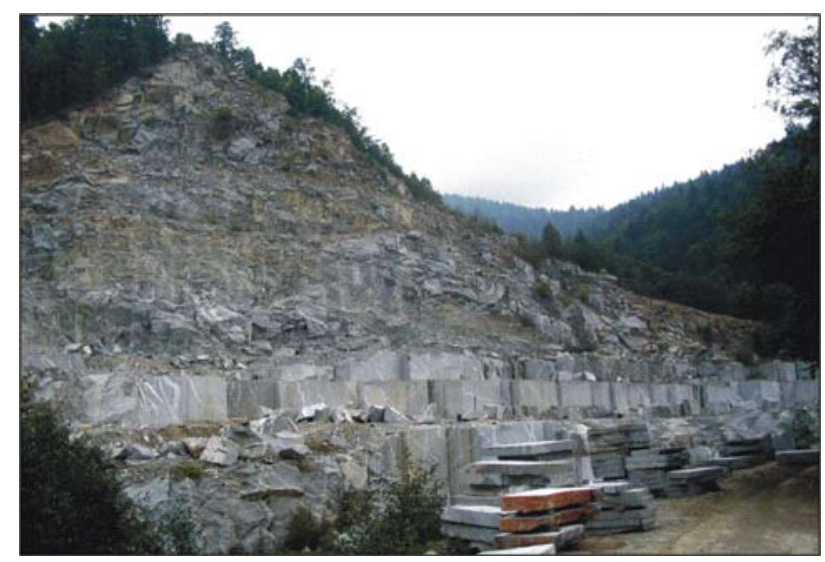

Abb. 2.5: Steinbruch der Handelssorte CEZLAK GRANODIORIT (Slowenien): Im Hangenden ist eine erhebliche Zergliederung gegeben, während im unteren Bereich ein kompakter Gesteinskörper ansteht.

Entlastungsklüfte sind vor allem auch in Sedimentgesteinen ausgebildet. Dabei kommt es häufig zu einer Öffnung der Schichtung, da mit dem Materialwechsel auch meist ein Wechsel im mechanischen Verhalten der aufeinander gelagerten Schichten verbunden ist. Parallel erfolgt eine Zergliederung der einzelnen Bänke innerhalb einer Schichtenfolge bis zum Zustand der sogenannten Kluftsättigung (Wu \& Pollard 1995). Dabei tritt eine gesetzmäßige Abhängigkeit zwischen Schichtmächtigkeit und Kluftabstand auf (Abb. 2.6), wobei in der Literatur sowohl lineare als auch nicht-lineare Zusammenhänge diskutiert werden (z.B. Pollard \& Aydin 1988, Bock 1971, Ladeira \& Price 1981, Huang \& Angelier 1989, Gross et al. 1995). Die innerhalb einer Schichtenfolge auftretenden Kluftabstände sind von den spezifischen gesteinstechnischen Eigenschaften abhängig. Dabei neigen Gesteine, die eine hohe Steifigkeit aufweisen, eher zu einer verstärkten Kluftbildung und somit zu geringeren Kluftabständen (Meier \& Kronberg 1989).

Vor allem für poröse Gesteine gilt außerdem der Porenflüssigkeitsdruck als elementarer Parameter der Kluftentstehung (z.B. Hubert \& Rubey 1959, Secor 1965). Die Porenflüssigkeiten, so z.B. wässrige Lösungen im Porenraum, tragen einen Teil der auf einen Gesteinskörper wirkenden Kräfte, wodurch das Korngerüst einen verringerten Anteil der Spannungen aufnehmen muss. Es ergibt sich ein gesetzmäßiger Zusammenhang, nach dem mit zunehmendem Fluiddruck der einwirkende lithostatische Druck vermindert oder gar aufgehoben wird. In der Folge kann allein durch den Porenflüssigkeitsdruck die mechanische Stabilität eines Gesteins überschritten werden, womit es zur Ausbildung einer Bruchfläche kommen kann (Meier \& Kronberg 1989). Unter bestimmten Bedingungen können Klüfte so auch in Tiefen von einigen 1000 m gebildet werden (Secor 1965).

Eine andere sowohl für klastische als auch chemisch-biogene Sedimente typische Kluftform sind Trocknungsklüfte (Abb. 2.7 a), die, vergleichbar mit Abkühlungsrissen, auf Spannungen im Gestein infolge einer Volumenreduzierung zurückzuführen sind. Dabei kann gerade bei Karbonatgesteinen neben der Hauptklüftung ein mehr oder weniger ausgeprägtes Risssystem vorhanden sein, dass auf einen erheblichen Wechsel des sedimentierten Materials oder aber auf eingeregelte Schalenfragmente o.ä. zurückzuführen ist. 

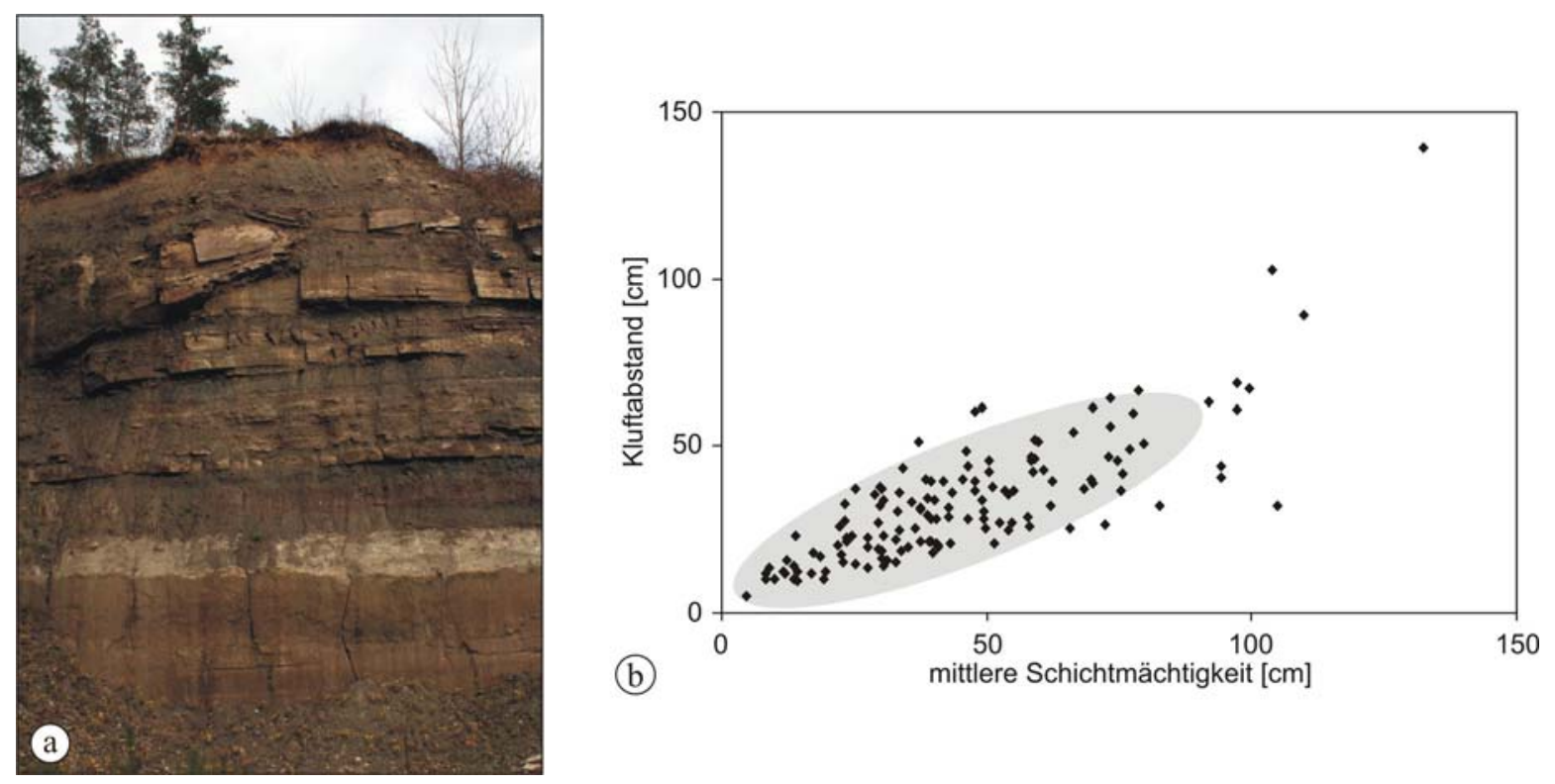

Abb. 2.6: Zusammenhang zwischen Schichtmächtigkeit und Kluftabstand: a) unterschiedliche Bankmächtigkeiten im Sandsteinbruch Schönbrunn (Bayern, Deutschland); b) Darstellung einer Korrelation aus Schichtmächtigkeit und Kluftabstand am Beispiel des Sandsteinbruchs Lohbach, Niedersachsen (Deutschland).

$\mathrm{Zu}$ den tektonisch bedingten Extensionsbrüchen zählen alle Bruchstrukturen, die auf regionale kinematische Prozesse zurückzuführen sind. Eine häufige Form bilden die sogenannten Fiederbrüche oder Fiederklüfte (Abb. 2.7 b). Diese Gefüge sind durch ihre staffelförmig angeordneten (,en echelon“) Bruchstrukturen gekennzeichnet. Häufig ist zu beobachten, dass die Öffnungsbereiche von Mineralsubstanz sekundär verfüllt sind. Dies gilt vor allem für nicht metamorphe Karbonatgesteine, wohingegen in Marmoren eine derartige Mineralisation dieser Extensionsbrüche relativ selten zu beobachten ist (Eisbacher 1991).
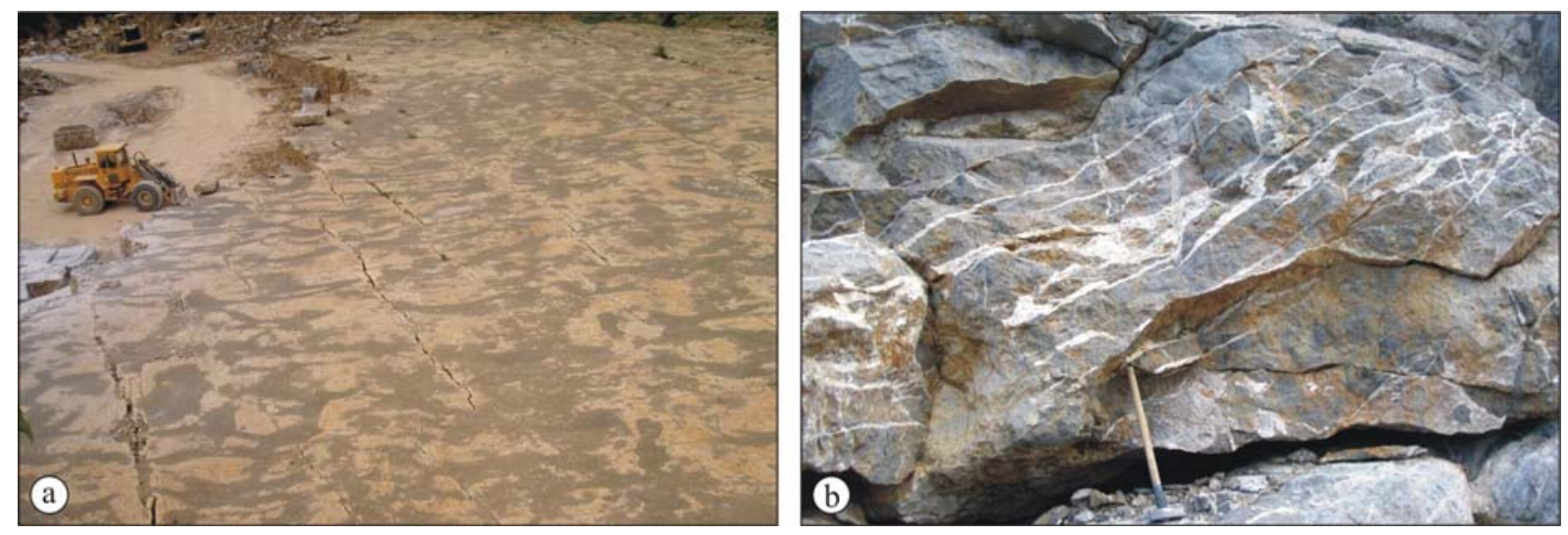

Abb. 2.7: a) Orthogonales System von Trocknungsklüften in söhlig gelagertem Kalkstein (Kirchheim, Deutschland), b) mineralisierte Fiederklüfte in Kalkstein (Provinz Neuquén, Argentinien).

Auch wenn für verschiedene Gesteinsarten gewisse Regelmäßigkeiten hinsichtlich des Trennflächensystems vermutet werden können, muss doch jede Lagerstätte individuell betrachtet werden. Dieser Umstand zeigt sich insbesondere in metamorphen Lagerstätten, so vor allem bei Marmoren. Dabei liegt eine besondere Schwierigkeit in der häufig sehr heterogenen Ausbildung der Trennflächen innerhalb eines Marmorkörpers. Ursächlich dafür ist die möglicherweise wechselhafte tektonische Entwicklung, die z.B. mehrere 
Faltungsphasen unterschiedlicher Einengungsrichtungen beinhalten kann. Darauf basierend können entsprechende Lagerstätten sehr komplex gebaut sein. Neben möglichen Variationen hinsichtlich Dekor und Mineralogie auf kurzen Distanzen muss im Abbau metamorpher Komplexe zu jedem Zeitpunkt mit einem Wechsel der strukturellen Gegebenheiten in Folge von z.B. Faltenstrukturen, dem Ausdünnen im Bereich von Faltenschenkel, bruchhaften Versätzen o.ä. gerechnet werden (z.B. Primavori 1999, Abb. 2.8). Hingegen sind viele sedimentäre und magmatische Lagerstätten in ihrer räumlichen Ausdehnung zumeist relativ homogen ausgebildet und deutlich abgegrenzt.
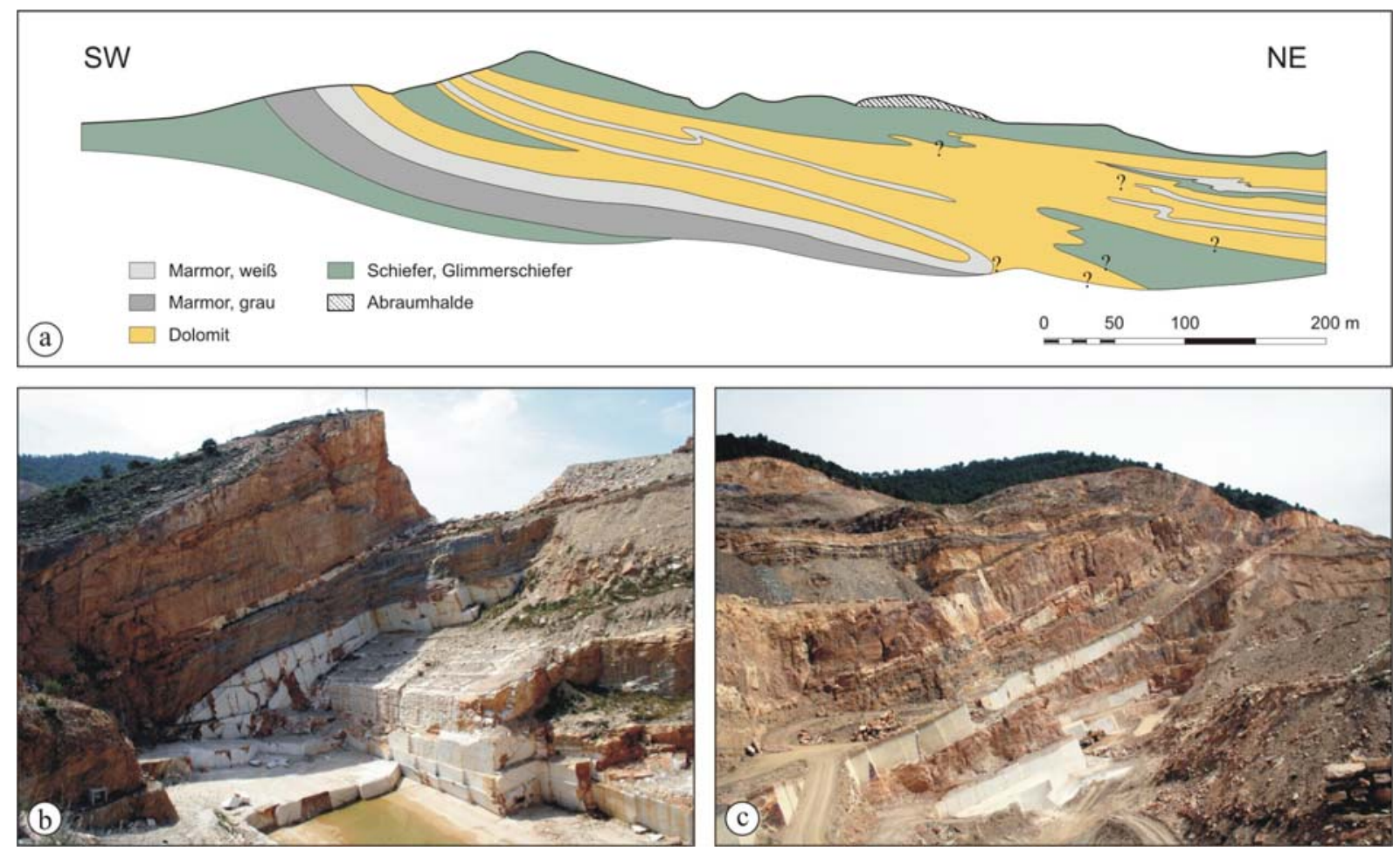

Abb. 2.8: Provinz Macaél (Spanien): a) Das geologische Profil durch das Marmorvorkommen (O.T.C. 2006) verdeutlicht die häufigen Gesteinswechsel mit b) erheblichen Schwankungen der Dichte und Orientierung der Trennflächen im Bereich von Faltenscharnieren und c) dem möglichen Auslaufen bauwürdiger Bänke. In der Folge ergeben sich lokal unterschiedliche und zum Teil sehr hohe Abraummengen im Abbau des Marmors.

Infolge der möglicherweise wechselhaften metamorphen und strukturellen Entwicklung kann auch die Ausbildung der Elemente des Trennflächeninventars von sehr heterogenem Charakter hinsichtlich der räumlichen Orientierung und der Intensität sein. In anderen Fällen, so z.B. mehrfach im mediterranen Raum, liegen auch Marmorkörper vor, die von ausgeprägten, orthogonalen Kluftsystemen zergliedert sind (pers. Mitt. Prof. Dr. K. Germann). Doch ist es nicht selten, dass in Marmorkörpern durchschlagende Hauptklüfte kaum existent sind. Vielmehr können dann lediglich Trends zur Orientierung von Kluftscharen beobachtet werden, die dabei intern stark gegliedert sein können (Abb. 2.9). So zeigt sich oft ein Verspringen der Klüfte, ein fiederartiges Auslaufen oder auch extrem unregelmäßig verlaufende Trennflächen, die häufig scheinbar auslaufen, sich dann aber wieder fortsetzen (Abb. 2.10 a). Gleiche Situationen können auch in nicht oder nur schwach metamorph umgewandelten Karbonatgesteinen auftreten, wobei hier noch zumeist eine Hauptkluftrichtung durch die sedimentäre Schichtung gegeben ist (Abb. 2.10 b). In allen 
karbonatischen Gesteinen können zudem sekundäre Lösungsprozesse stattfinden. Dabei kommt es zur Aufweitung der Trennflächen bis hin zu kavernenartigen Hohlräumen (Karst), die kaum vorhersehbar im Gestein auftreten können (Abb. 2.11).

Für alle Lithologien gilt es, im Rahmen der geologischen Erkundung eines Vorkommens vor allem auch größere Diskontinuitäten wie Störungen oder Scherbahnen zu erfassen. Entsprechende tektonische Elemente haben einen erheblichen Einfluss auf die Trennflächendichte, die wiederum das Maß der Zerlegung eines Gesteinskörpers bestimmt. Diese Zergliederung ist im Hinblick auf die potenzielle Nutzung eines Gesteinskomplexes von erstrangiger Bedeutung, da die Möglichkeit zur Herstellung von Werksteinbauelementen davon abhängt. Dieser Aspekt wird in Kapitel 2.4 detailliert betrachtet.
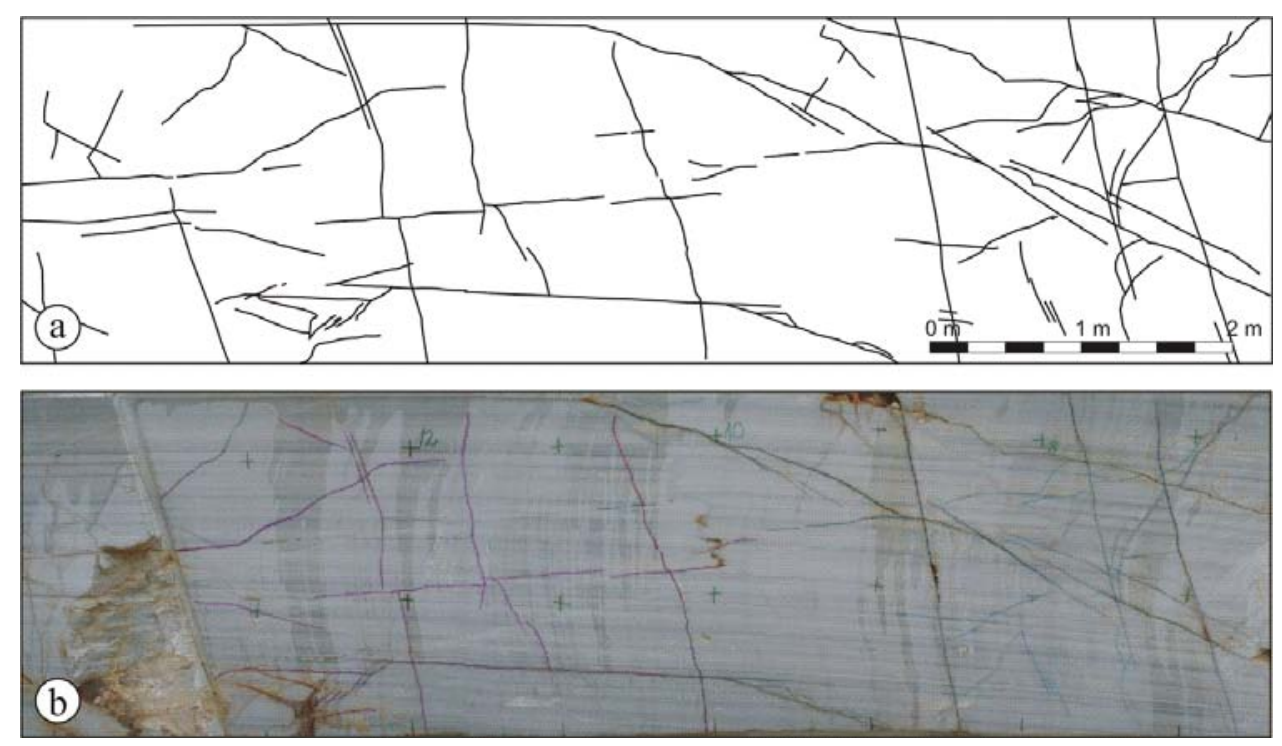

Abb. 2.9: a) Die schematische Darstellung des Verlaufs der Trennflächen auf der in b) abgebildeten Abbauwand eines Marmorsteinbruchs lässt trotz erheblicher Unregelmäßigkeit eine generelle Vorzugsrichtung (senkrecht) erkennen (Provinz Macaél, Spanien).
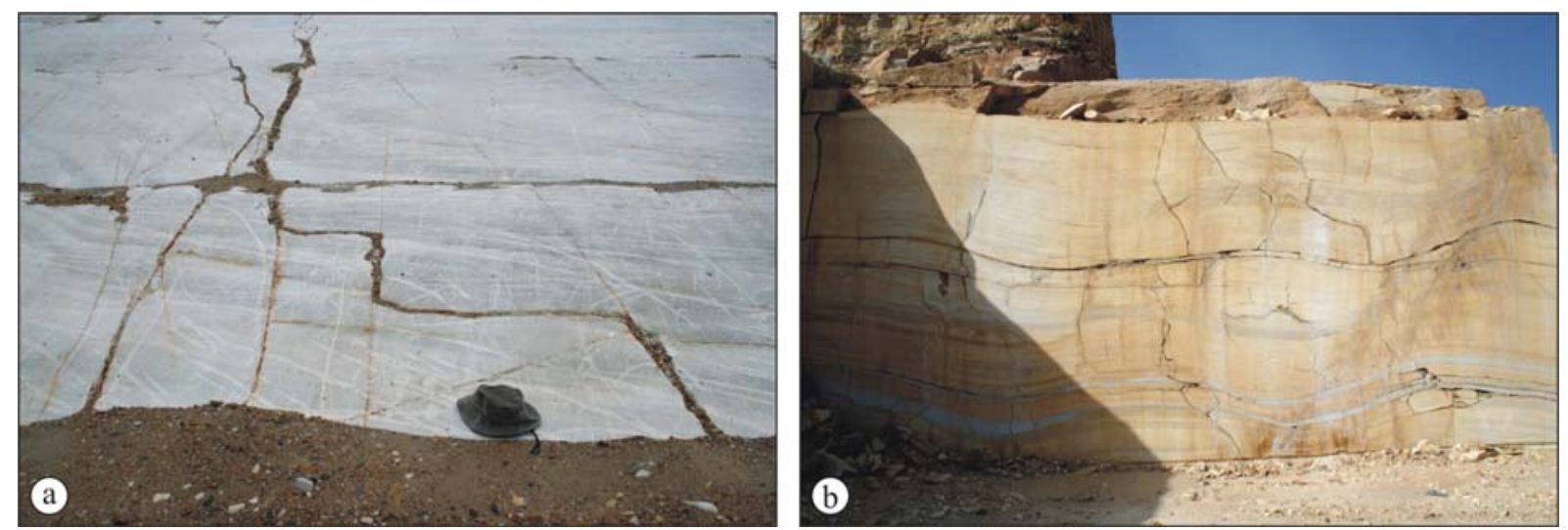

Abb. 2.10: a) Unregelmäßiger Kluftverlauf in Marmor mit häufigem Verspringen oder Auslaufen der Trennflächen; b) wellenförmige Ausbildung der sedimentären Lagerklüfte in Dolomit (Provinz Macaél, Spanien). 

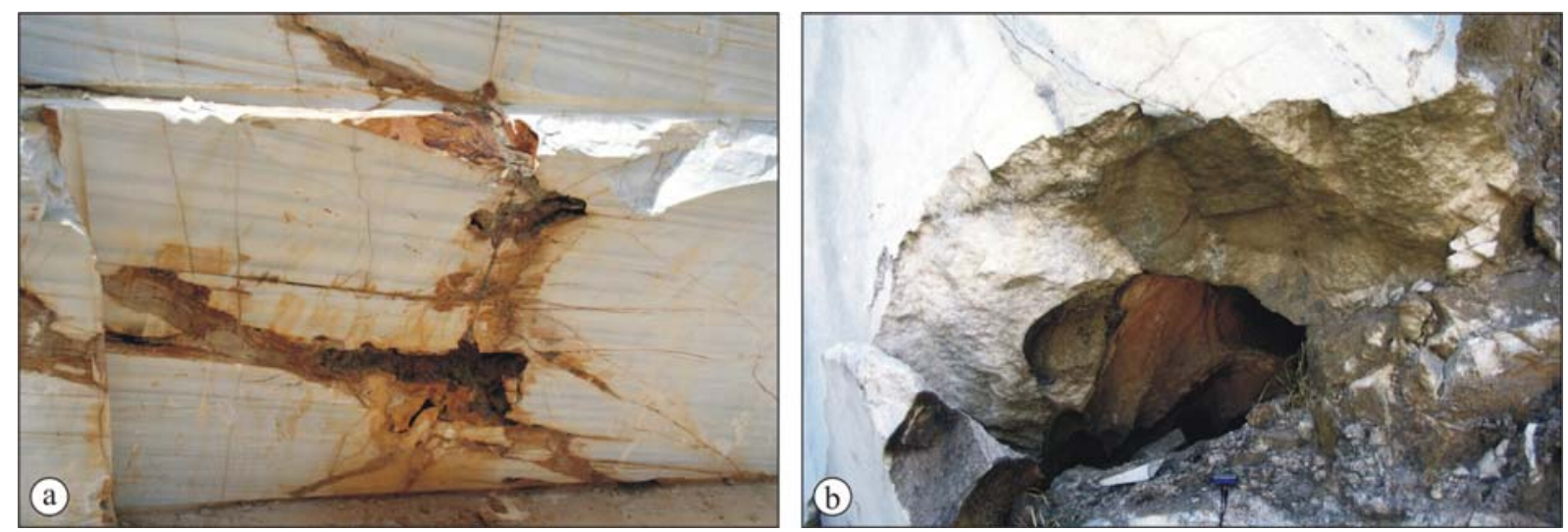

Abb. 2.11: Karstbildungen in Karbonatgesteinen, hier Marmor, sind auf sekundäre Lösungsprozesse zurückzuführen, die entlang von Trennflächen im Gestein ansetzen; a) Provinz Macaél (Spanien); b) Provinz Córdoba (Argentinien).

\subsection{Methodik der Trennflächenanalyse und Darstellungsmöglichkeiten}

\subsubsection{Grundlagen}

Im Folgenden soll die Klüftung als dominantes Element des Trennflächengefüges betrachtet werden, da auf der spezifischen Ausbildung des Kluft- bzw. Risssystems die Form und die Größe der generierten in-situ Blöcke beruht und die Wirtschaftlichkeit einer Werkstein-Lagerstätte davon kontrolliert wird. Um diese Wirtschaftlichkeit greifbar zu machen, definierte Singewald (1992) die so genannte Rohblockhöffigkeit. Sie beschreibt den Anteil an gewinnbaren Rohblöcken, die den Anforderungen des oben beschriebenen Mindestblocks (Kap. 2.1) entsprechen. Die Berechnung basiert auf der Abstandsverteilung innerhalb der auftretenden Kluftscharen und deren jeweiligen Verknüpfungen zu dreidimensionalen Körpern. Nachteilig hierbei ist, dass für die jeweiligen Abstandsverteilungen ein orthogonales Bezugssystem zugrunde gelegt wird, einer möglicherweise abweichenden Orientierung der Trennflächen somit also keine Rechnung getragen wird. Daher kann die Rohblockhöffigkeit zwar als vergleichendes Instrument und zur generellen Eignungseinschätzung einer Lagerstätte eingesetzt werden, erlaubt jedoch keine Aussagen über die Formen der in-situ Blöcke.

Gerade in den letzten drei Jahrzehnten wurden verschiedenste Ansätze zur Vorhersage von Blockgrößen und -formen sowie deren Verteilung in einem Gesteinskörper unternommen. Eine diesbezügliche aktuelle Zusammenstellung der Quellen ist z.B. in Hoffmann (2006) gegeben. In vielen Fällen wurde dabei versucht, Parameter aus montangeologischen und felsmechanischen Aspekten der Stabilitätsberechnung auf die lagerstättengeologischen Bedürfnisse zu übertragen bzw. zu erweitern. Entscheidend ist jedoch, dass die meisten definierten Parameter zwar Informationen über eine durchschnittliche Blockgröße aber keine direkten Aussagen über die tatsächliche Höffigkeit oder die räumliche Anordnung der in-situ Blöcke erlauben.

Erst mit dem Aufkommen moderner Computersoftware konnten Fortschritte verzeichnet werden. So wurden verschiedene computergestützte Systeme entwickelt, die eine Detektion 
defektfreier Gesteinspartien und somit eine Konzentration auf möglichst hochwertiges Rohgestein ermöglichen. Diese stützen sich entweder auf geophysikalische Messmethoden wie dem Bodenradar (Prissang et al. 2007) oder aber auf konventionelle Methoden der Datenermittlung zur Abstandsverteilung und -orientierung des Trennflächensystems (Nikolayev et al. 2007). Die Grundzüge des von Nikolayev et al. (2007) erarbeiteten Programms 3D-BlockExpert sollen auch in dieser Arbeit zur zwei- und dreidimensionalen Darstellung einzelner Lagerstättenbereiche verwendet werden.

\subsubsection{Analyse von Trennflächenverteilungen}

\subsubsection{Methodik der Datenaufnahme}

Die Trennflächenanalyse umfasst also die Lokalisierung, Aufnahme und Bewertung von u.a. Klüften, Schichtgrenzen oder Störungszonen. Dafür stehen verschiedene geophysikalische Methoden wie beispielsweise Georadar (z.B. Singewald 1992) zur Verfügung. In der praktischen Bohrlochgeophysik kommen z.B. Schichtneigungsmessungen im Diplog-Verfahren zum Einsatz. Dabei wird die Bohrlochwand von azimutal versetzten Mikrowiderstandsmesssystemen abgetastet, die beim Passieren von Trennflächen eine Widerstandsänderung registrieren. Aus der Verknüpfung der Widerstandskurven können dann Aussagen über die räumliche Lage der Trennflächen gewonnen werden (Abb. 2.12). Auch werden verschiedentlich bildgebende Verfahren wie optische Fernseher oder akustische Bohrlochfernseher eingesetzt (Fricke \& Schön 1999).

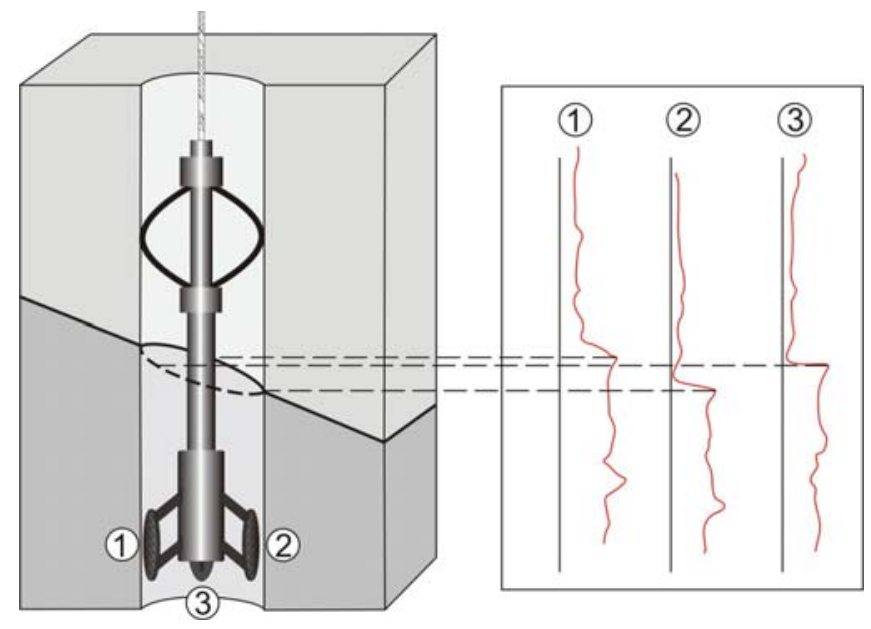

Abb. 2.12: Trennflächenanalyse mittels 3-ArmDiplog-Messanordnung im Bohrloch mit den entsprechenden Widerstandskurven an einer geneigten Trennfläche (nach Fricke \& Schön 1999).

Die beschriebenen Verfahren erfordern jedoch im Allgemeinen einen hohen technischen und finanziellen Aufwand. Daher kann die Aufnahme der Trennflächenabstände auch mittels direkter Längenmessung im Aufschluss bzw. in einem Bruch erfolgen. Die Raumlage der Fläche wird dabei konventionell mit einem Gefügekompass ermittelt. Als Grundproblematik tritt dabei das Erkennen der Trennflächen auf. Zum einen können diese durch eine sedimentäre Bedeckung oder auch Abraummaterial verdeckt sein, so dass über den eigentlichen Zielbereich, so z.B. einen eventuell abzubauenden Bereich, kaum eine direkte 
Aussage gemacht werden kann. Andererseits können feinere Rissstrukturen häufig erst nach einem Benässen der Flächen erkannt werden.

Im Idealfall sollte die Kluftaufnahme an senkrechten Lagerstättenwänden erfolgen. Bei der so genannten „window sampling“ oder „scanline sampling technique“ (ISRM 1978, Priest 1993) müssen an einer Wand mindestens je eine Messlinie vertikal und horizontal angelegt werden, so dass dabei zwei Kluftscharen erfasst werden können (Abb. 2.13: Wand 1, Kluftscharen A, C). Um ein vollständiges Bild der Kluftverteilung im Gesteinskörper zu erlangen, ist es notwendig, weitere Messungen an einer im Idealfall rechtwinklig versetzten Wand entsprechend den Vorgaben durchzuführen (Abb. 2.13: Wand 2, Kluftschar B). Diese Vorgehensweise kann für die Aufnahme des Kluftsystems in unterschiedlichsten Gesteinsvarietäten problemlos eingesetzt werden. Voraussetzung ist jedoch eine Orientierung der Kluftscharen zueinander, die nur unwesentlich von der Orthogonalität abweicht. Einzige auftretende Einschränkung ist dabei durch das Terminationsverhalten der Klüfte gegeben. Trennflächen, deren Ausstreichen im Anschnitt einer Wand noch gegeben sein kann, können bereits nach wenigen Zentimetern in der Tiefe des Gesteins auslaufen und/oder verspringen. Somit können im Messfenster zu kleine oder zu große Kluftabstände registriert werden. In der Folge kann es bei der Beurteilung einer abzubauenden Gesteinspartie von Nutzen sein, auch die Oberfläche des Körpers weitestgehend zu reinigen und somit die Spur der an den Wänden aufgenommenen Trennflächen verfolgen zu können und dadurch das Gesamtbild durch die dreidimensionale Betrachtung zu vervollständigen.

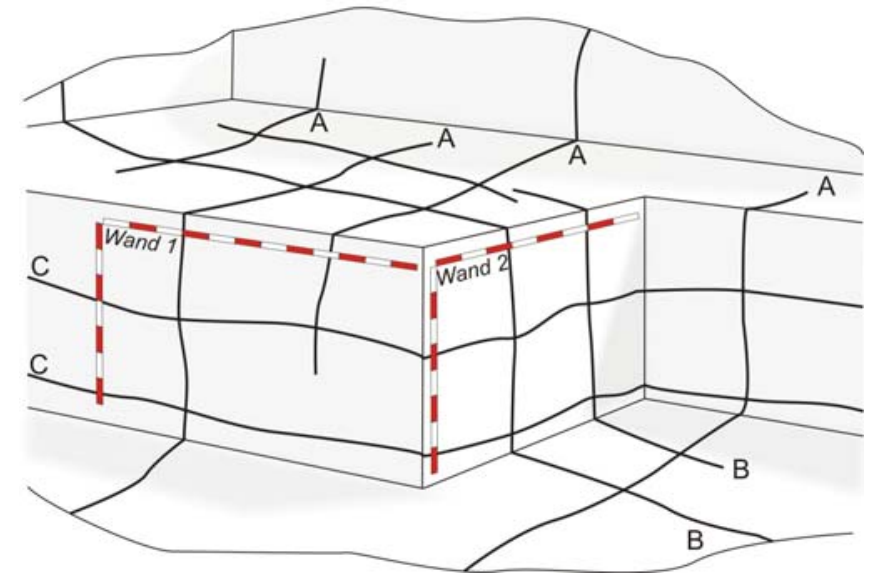

Abb. 2.13: Darstellung der Anlage von Messbereichen zur Bestimmung der Kluftverteilung (Kluftscharen A, B, C) in einem abzubauenden Bereich. Nähere Erläuterungen im Text.

\subsubsection{Darstellungs- und Anwendungsmöglichkeiten}

Zur Darstellung der aus der Trennflächenmessung gewonnenen Daten zur Raumstellung der Klüfte oder Kluftscharen hat sich die Anwendung von Isoliniendiagrammen im Schmidtschen Netz bewährt. Diese spiegeln eine relative Dichteverteilung der Polpunkte, die die Flächennormalen zu den eingemessenen Kluftflächen darstellen, wider. Damit ergibt sich die Möglichkeit, neben dem generellen Streichen vorhandener Kluftscharen auch deren Einfallen zu beschreiben. Die Auswertung dieser Verteilungsdichtediagramme erfordert jedoch eine gewisse Übung. Für fachfremde Personen ist daher unter Umständen eine Darstellung des Trennflächengefüges über einfache Kluftrosendiagramme übersichtlicher. 
Diese zeigen jedoch lediglich eine relative Häufigkeitsverteilung der Streichrichtungen der Kluftflächen an, nicht jedoch deren Einfallswinkel. Das Beispiel in Abbildung 2.14 stellt die verschiedenartig ausgebildete Trennflächenorientierung in zwei Bereichen eines Steinbruchs dar. Aus Abbildung 2.14 a gehen zwei steil stehende Kluftscharen hervor, deren Streichrichtungen annähernd rechtwinklig zueinander stehen. In der Folge ergibt sich eine recht gleichmäßige Zergliederung des Gesteinskörpers in rechtwinklige Quader. Dagegen ist aus der Trennflächenverteilung in Abbildung 2.14 b zwar auch eine Vorzugsorientierung mit Streichrichtung E-W zu erkennen. Insgesamt ist jedoch eine deutlich heterogenere Verteilung der Klüfte gegeben, aus der eine stärkere Fragmentierung des Gesteins hervorgeht.
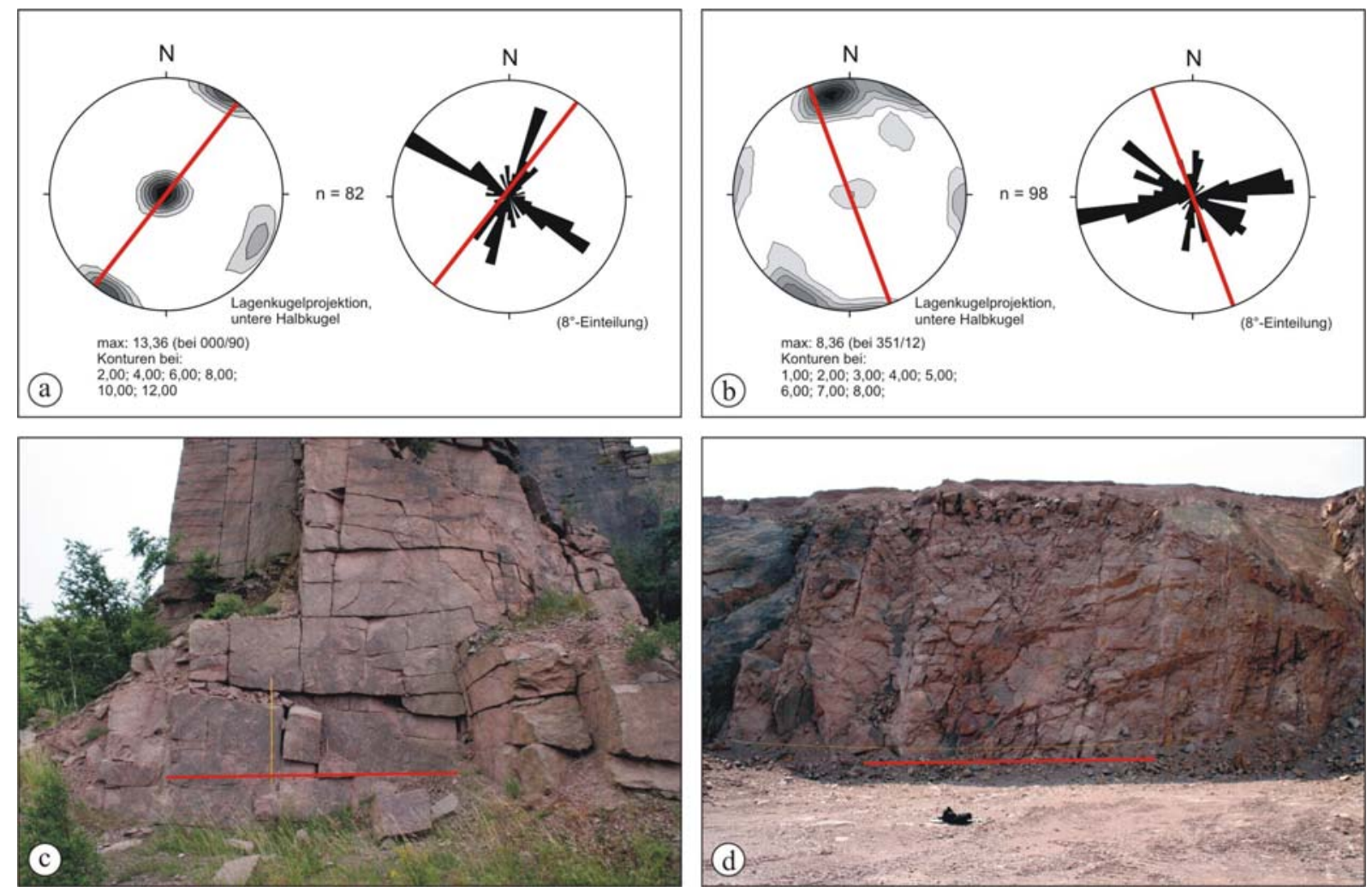

Abb. 2.14: Darstellung der Trennflächenorientierung über Verteilungsdichtediagramme im Schmidtschen Netz (Einfallsrichtung und -winkel, jeweils links in a und b) und Kluftrosendiagramme (Streichrichtung, jeweils rechts in a und b) mit Bezug zur Ausrichtung der Hauptabbauwand (rote Linien); Beispiel: Löbejüner Qz-Porphyr (Sachsen-Anhalt, Deutschland); Kluftaufnahme a) im Bruchbereich eines ehemaligen Werksteinabbaus (c) und b) im Bereich eines aktiven Schotterabbaus (d).

Zur Darstellung der Abstandsverteilung im Trennflächensystem einer Lagerstätte sind von verschiedenen Autoren unterschiedliche Verfahren beschrieben worden, die jedoch nicht die unterschiedliche Orientierung von Kluftscharen einbeziehen und zum Teil auch recht unübersichtlich erscheinen (z.B. Schöne \& Peschel 1974). Eine klare Darstellung der Häufigkeitsverteilung von Kluftabständen bietet die Datenauswertung mittels Histogrammen (z.B. Singewald 1992). So ergibt sich zum einen die Möglichkeit einer überschaubaren Darstellung der richtungsbedingten Differenzen verschieden orientierter Kluft- bzw. Trennflächenscharen. Gleichzeitig ist über die Betrachtung der Mittelwerte der Trennflächenabstände die Volumenkalkulation eines so genannten „Durchschnittsblocks“ $(\overline{\mathrm{x}} \cdot \overline{\mathrm{y}} \cdot \overline{\mathrm{z}}=\overline{\mathrm{V}}$; Singewald 1992) möglich. Zur generellen Bewertung einer Lagerstätte oder 
aber zu Vergleichszwecken ist dieser Parameter besonders im Bereich sedimentärer, stratiformer Lagerstättentypen durchaus zu verwenden. Weicht das Trennflächengefüge jedoch deutlich von der Orthogonalität ab bzw. zeigen eine oder mehrere Hauptkluftscharen eine ausgeprägtere Streuung, können keine fundierten Aussagen über mögliche Rohblockgrößen daraus gezogen werden. Zum anderen kann somit eine Abschätzung der Eignung eines betrachteten Bereichs hinsichtlich des Werksteinabbaus erfolgen. Abbildung 2.15 zeigt eine vergleichende Gegenüberstellung der zuvor beschriebenen zwei Bereiche eines Steinbruchs (vgl. Abb. 2.14). Die Partie, die durch eine stärkere Variation im Einfallen und Streichen der Klüfte zeigt, weist insgesamt auch engere Kluftabstände auf und wird daher für den Abbau von Bruchmaterial genutzt. Das engständigere Kluftsystem wird dabei vor allem anhand der auf einer horizontalen Messlinie aufgenommen Abstände deutlich. Auf einer Messstrecke von rund $30 \mathrm{~m}$ entfallen dabei über $60 \%$ auf Abstände unter 0,5 m.
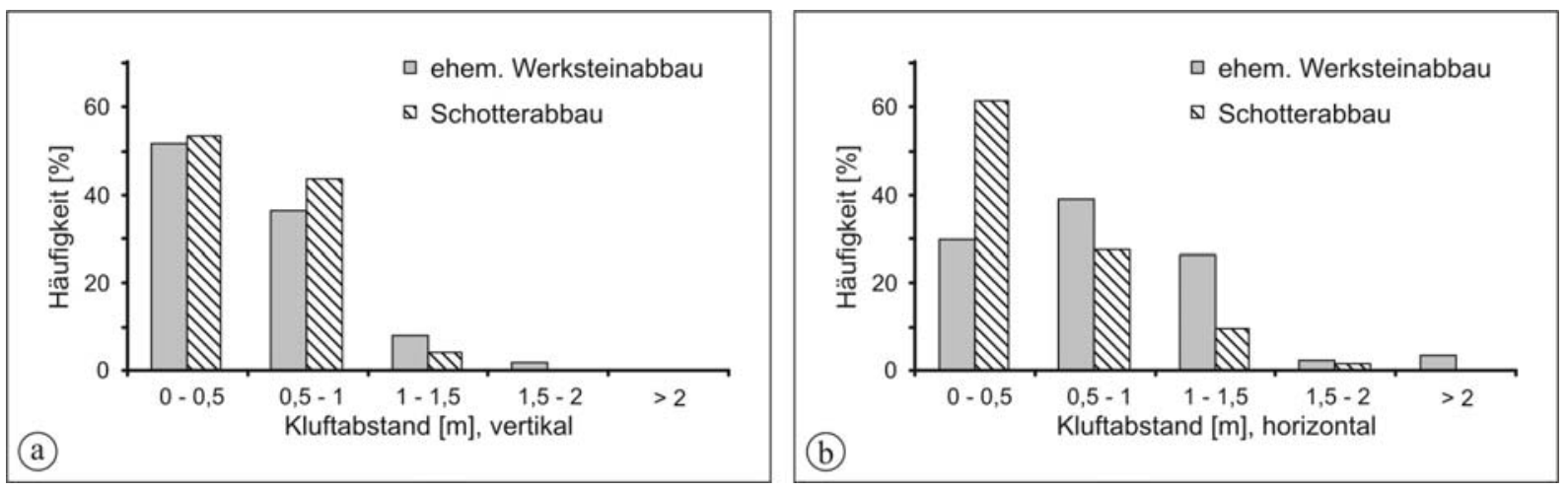

Abb. 2.15: Vergleich zweier a) vertikalen und b) horizontalen Kluftabstandsverteilungen im Qz-Porphyrbruch Löbejün (Sachsen-Anhalt, Deutschland, vgl. Abb. 2.14). Der Bereich des aktiven Schotterabbaus weist eine deutlich engständigere Kluftabstandsverteilung auf, als der Bereich eines ehemaligen Werksteinabbaus mit Kluftabständen, die zum Teil über 2 m erreichen.

Somit erlaubt die zusammenfassende Betrachtung von Orientierung und Abstandsverteilung im Trennflächengefüge eine generelle Einschätzung über den Grad der Zergliederung eines Gesteinskörpers und der damit verbundenen Nutzungsmöglichkeit.

Einschränkungen ergeben sich jedoch bei der Bewertung der Abstandsverteilung unter Anwendung von Histogrammen durch erhebliche Abweichungen von der Orthogonalität bzw. bei stark schwankenden Einfallsrichtungen der Klüfte. Besonders in diesen Fällen kann die Wahl der jeweiligen Messlinie einen Einfluss auf die relative Häufigkeitsverteilung haben (Abb. 2.16). Daraus können dann wiederum auch Abweichungen z.B. bei der Berechnung des durchschnittlichen Blockvolumens resultieren. Daher erscheint es zweckmäßig, mehrere Messfenster anzulegen oder aber weiterreichende Methoden, so z.B. computergestützte Modellierungen, anzuwenden.

Es ist generell zu beachten, dass die Ergebnisse der Trennflächenanalyse in Abhängigkeit von der Lithologie oder den tektonischen Gegebenheiten gewissen Schwankungen unterliegen können. Ein Phänomen, das z.B. häufig bei Sandsteinen auftritt, ist ein wellenförmiger Verlauf der Schichtung, woraus sich starke Veränderungen der Bankmächtigkeit ergeben. Gleiche Beobachtungen können auch zur Klüftung in granitoiden Gesteinen gemacht werden 
(vgl. Abb. 4.18, Kap. 4.6.1.4). Daher ist bei der Auswertung einer Abstandsverteilung stets eine gewisse Vorsicht geboten.

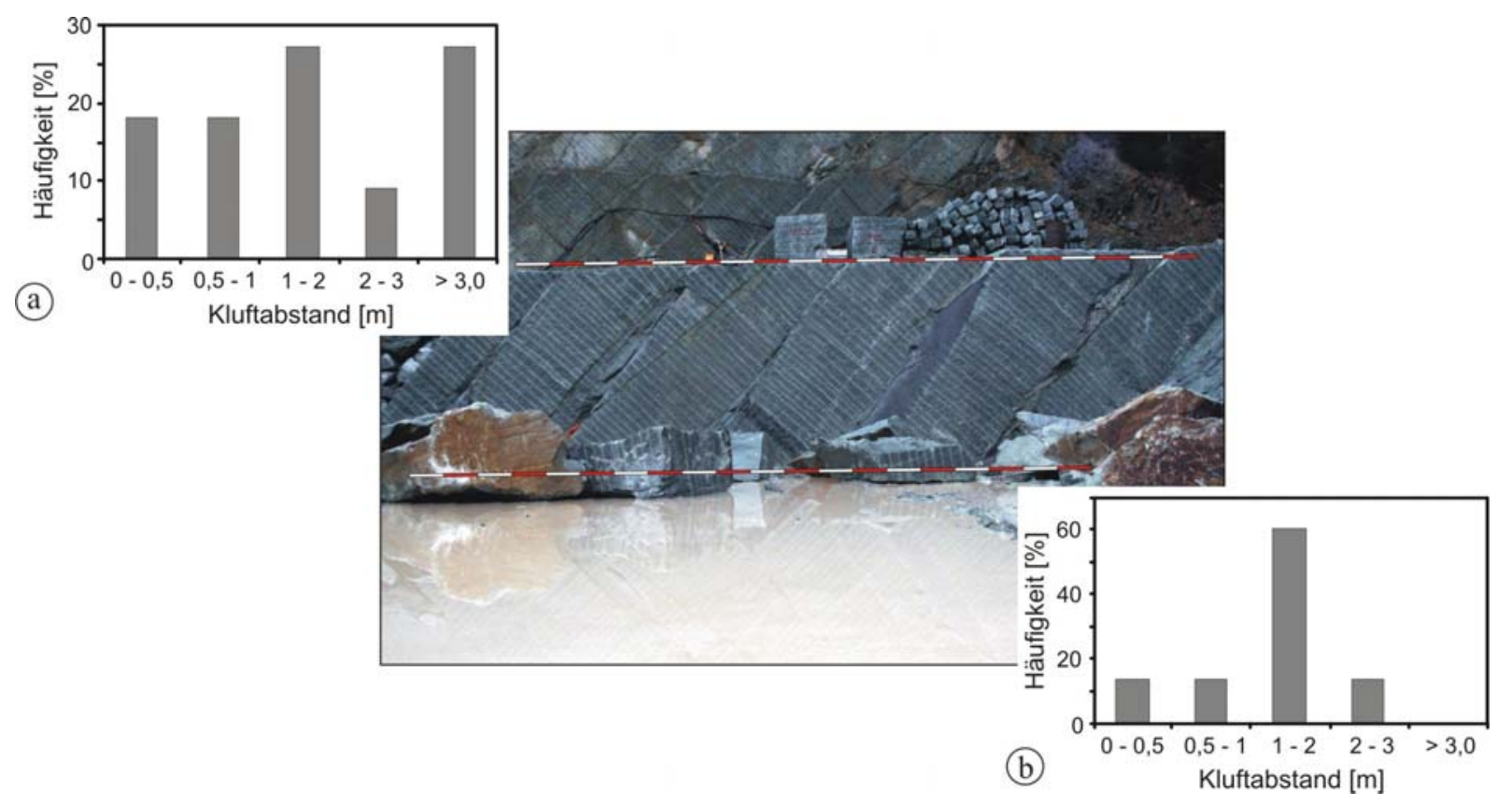

Abb. 2.16: Abweichungen in der Abstandsverteilung der Trennflächen an zwei parallel zueinander angeordneten Messlinien: a) oberes und b) unteres Profil (VERDE ANDEER, Orthogneis, Schweiz).

\subsubsection{Zur Anwendung der Software 3D-BlockExpert}

Das Computerprogramm 3D-BlockExpert (Nikolayev et al. 2007) ermöglicht über eine primäre Dateneingabe die Darstellung von Kluftverteilungssituationen. Dabei können direkte Informationen über das Volumen der resultierenden in-situ Blöcke eines aufgenommenen Lagerstättenkörpers gewonnen werden. Gleichzeitig besteht die Möglichkeit, die entsprechende Gesteinspartie in frei wählbaren zweidimensionalen Schnitten oder auch in dreidimensionalen Simulationen zu visualisieren, um so zu Aussagen hinsichtlich der Verteilung und der Geometrie einzelner Blöcke zu gelangen. Die Software erlaubt die Verarbeitung aller Raumlagen von Trennflächen und ist somit nicht auf ein orthogonales Trennflächensystem angewiesen. Es ist jedoch $\mathrm{zu}$ beachten, dass im aktuellen Entwicklungsstand Klüfte stets als durchgehende Trennflächen umgesetzt werden. Daraus resultiert zwangsweise eine Einschränkung in der Darstellungsmöglichkeit und der Aussagekraft der Ergebnisse.

\subsubsection{Datenaufnahme und -aufbereitung zur Anwendung der Software}

Mathematisch kann eine Fläche in einem 3-achsigen Koordinatensystem x, y, z über zwei Wege exakt definiert werden (Abb. 2.17 a). Zum einen über die Angabe von drei Punkten (1, 2, 3), deren Position jeweils über die drei Parameter $\mathrm{x}, \mathrm{y}$ und $\mathrm{z}$ beschrieben ist. Diese drei Punkte spannen dann eine Fläche auf. Zum anderen besteht die Möglichkeit von einem Punkt (A) auszugehen und von diesem aus zwei Winkel $(\alpha, \beta)$ abzutragen, wobei die resultierenden Geraden die Fläche definieren. 

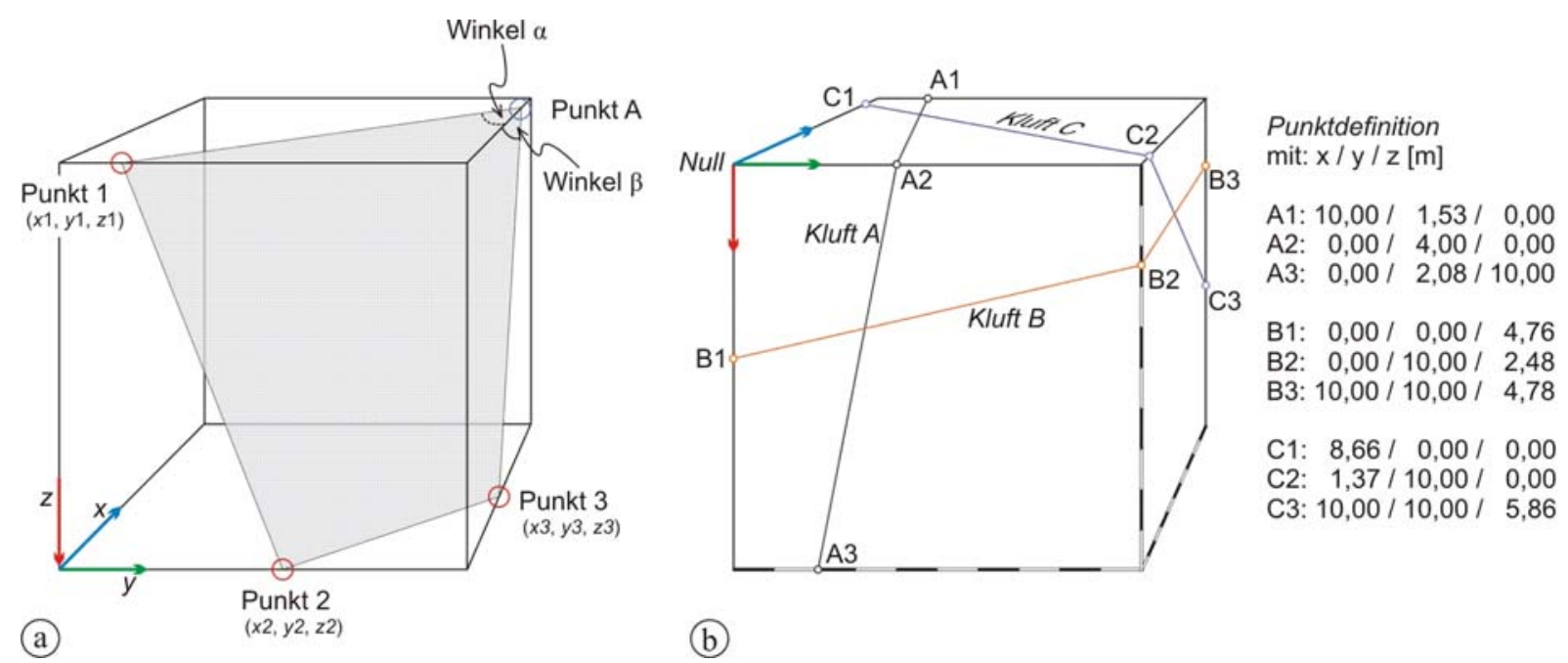

(b)

Abb. 2.17: Datenaufbereitung zur Anwendung der Software 3D-BlockExpert: a) Flächendefinition über drei Punkte (1, 2, 3) oder einen Punkt (A) und zwei Winkel $(\alpha, \beta)$; b) Exemplarische Flächendefinition über drei Punkte (Maßstab in Meter).

In der Praxis hat sich herausgestellt, dass, wenn es die Aufschlusslage erlaubt, der ersteren Variante zur Dateneingabe in die Software der Vorzug zu geben ist (Abb. 2.17 b). Bei Eingabe von Winkeldaten hat sich gezeigt, dass zwischen den daraus resultierenden theoretischen Kluftabständen und den tatsächlich gemessenen nicht selten erhebliche Abweichungen bestehen. Naturgemäß tritt dies besonders in Erscheinung, wenn das Trennflächengefüge deutlich von der Orthogonalität abweicht. Dementsprechend muss die Datenaufnahme nach dem „scanline sampling“-Verfahren derart erweitert werden, dass zusätzlich parallel verschobene Messreihen angelegt werden, so dass auftretende Trennflächen nach Möglichkeit auf beiden Messlinien dokumentiert und somit eindeutig definiert sind. Das heißt, dass auf den zu untersuchenden Wänden bzw. Flächen einer Lagerstätte Messfenster angelegt werden müssen, an denen mindestens zwei Messreihen pro Raumrichtung (horizontal, vertikal) aufgenommen werden.

Oft stellt sich in Lagerstätten das Problem, dass die Abbauwände nicht im rechten Winkel zueinander stehen oder aber die Abbauwand selber nicht geradlinig verläuft, sondern Sprünge im Verlauf aufweist (Abb. 2.18). In diesen Fällen ist es möglich, die Punktdefinition über eine geometrische Extrapolation der Trennflächen zu erlangen. Das gleiche Verfahren kann verwendet werden, wenn z.B. lediglich ein 2-dimensionaler Anschnitt, das heißt nur eine Abbauwand oder eine Geländeoberfläche, für die Kluftaufnahme zur Verfügung steht. Dabei müssen jedoch zwei Dinge beachtet werden. Zum einen sind in den daraus gebildeten Modellen keine direkten Informationen über eventuelle Trennflächen in der dritten Dimension enthalten. Mögliche Lagerstättensituationen können in diesem Fall durch eine theoretische Kluftverteilung ergänzt werden, die z.B. auf dem Gesamtkluftbild eines Vorkommens beruhen kann. Zum anderen ist bei der zeichnerischen Umsetzung der aufgenommenen Klüfte dem scheinbaren Einfallen Rechnung zu tragen, das sich ergibt, wenn die Profilrichtung nicht senkrecht zum Streichen der jeweiligen Trennfläche liegt (z.B. Flick et al. 1991). 


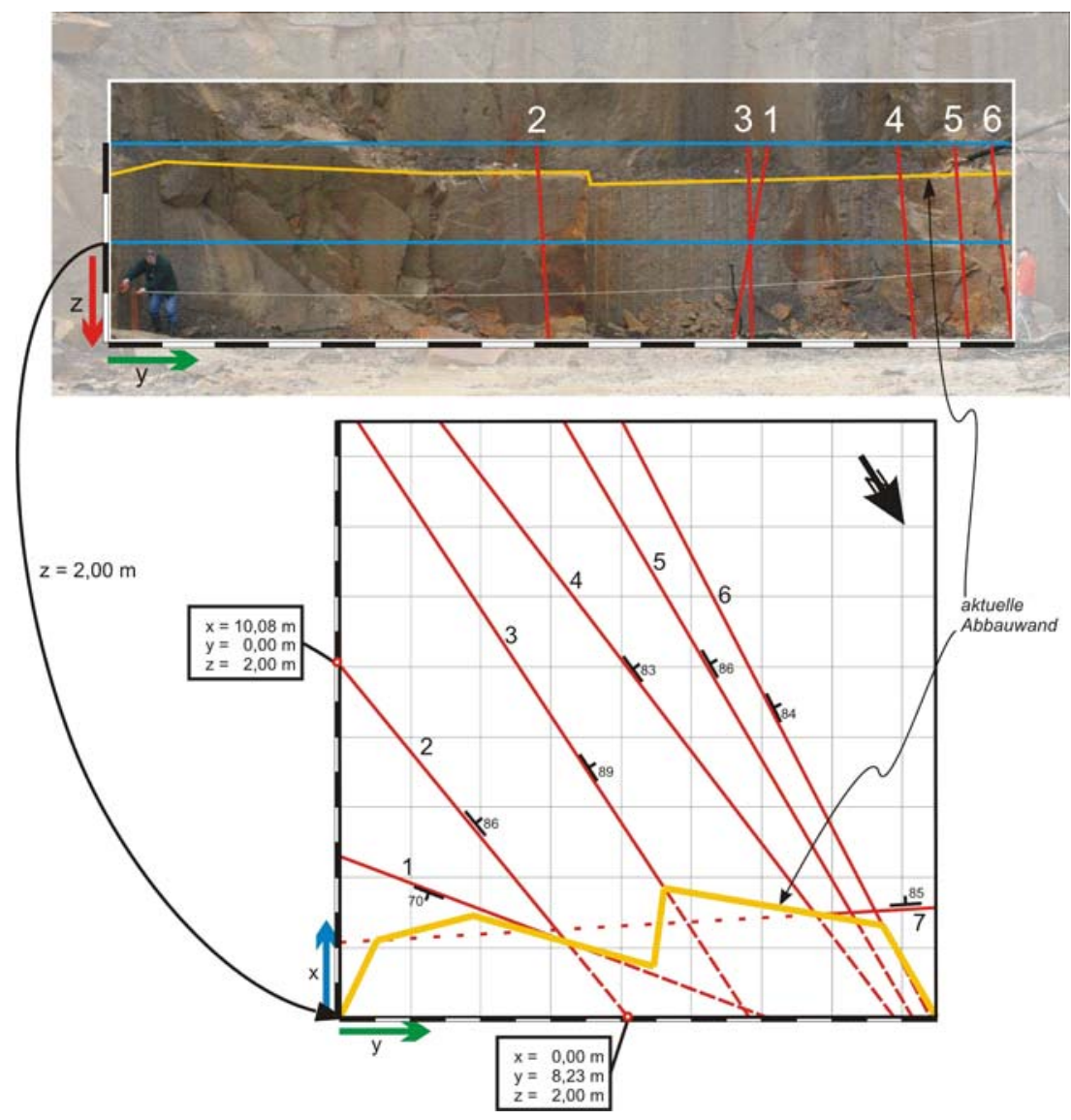

Abb. 2.18: Datenermittlung zur Anwendung der Software 3D-BlockExpert durch geometrische Extrapolation auf der Basis der Kluftaufnahme an einer versetzten Abbauwand (Sandsteinbruch Buch, Bayern, Deutschland). Die schematisch dargestellte Fläche beschreibt den Kluftverlauf auf einer horizontalen Ebene in 2 m Höhe über der Abbausohle. Nähere Erläuterungen im Text.

\subsubsection{Darstellungs- und Anwendungsmöglichkeiten}

Der darzustellende Bereich einer Lagerstätte wird in der Software als geometrischer Körper betrachtet, der aus sechs paarweise in parallelen Ebenen liegenden Parallelogrammen gebildet wird. Dieser Körper setzt sich aus gleichdimensionierten Voxeln (Voxel: Volumetric Pixel) zusammen. Nach der gewählten Voxelanzahl richtet sich dementsprechend auch die Auflösung der Darstellungen, wobei zu beachten ist, dass die benötigte Rechenzeit über eine Quadratfunktion mit der Erhöhung der Voxelanzahl verbunden ist (Nikolayev et al. 2007). Somit kann aus einer geringen Erhöhung der Anzahl eine enorme Verlängerung der Rechenzeit resultieren. Durch die Integration der Trennflächendaten in das mathematische Modell erfolgt eine dreidimensionale Segmentierung des Großkörpers. Basierend auf einem iterativen Prozess, der auf einen numerischen Algorithmus zurückgeht (Nikolayev et al. 2007), wird den zu einem in-situ Block zugehörigen Voxeln eine Farbe zugeordnet, so dass die Zergliederung übersichtlich coloriert dargestellt wird.

In einem ersten Schritt können mit der Software frei wählbare Schnittflächen senkrecht zu den Außenflächen dargestellt werden. In Abbildung 2.19 sind verschiedene Schnittlagen für das in Abbildung $2.17 \mathrm{~b}$ gegebene Beispiel dargestellt. 

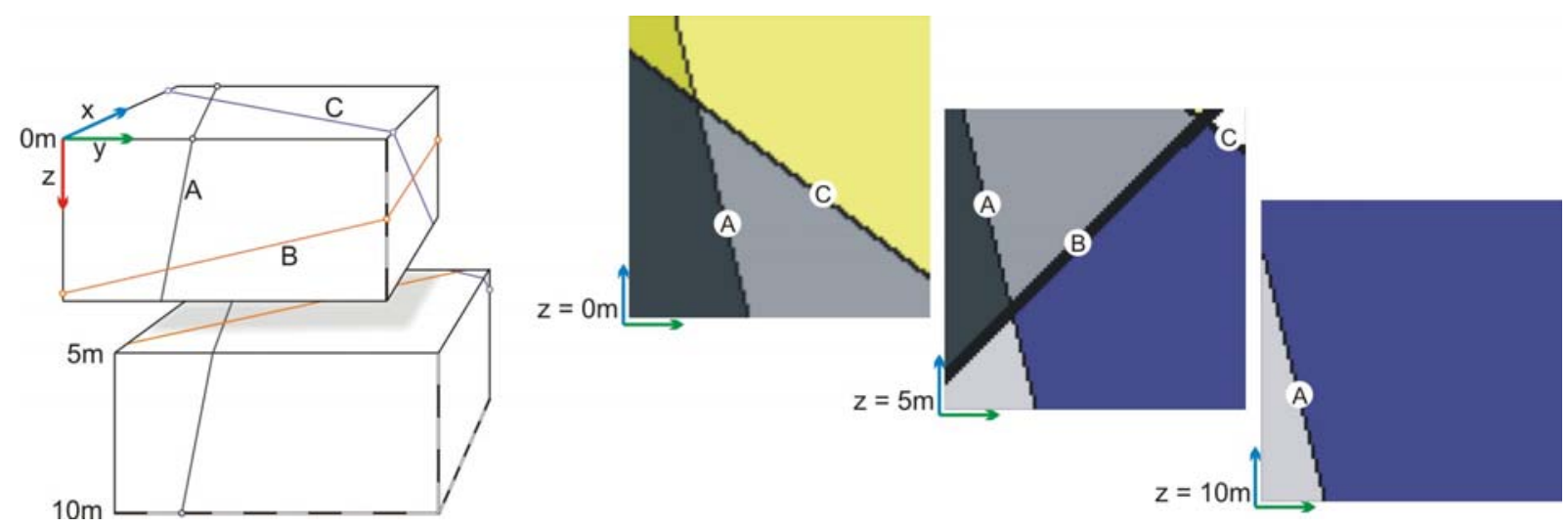

Abb. 2.19: Trennflächenverlauf auf modellierten Schnittflächen parallel zur Oberfläche (xy-Fläche, vgl. Abb. 2.17 b); gleich gefärbte Bereiche beschreiben einen in-situ Block.

Für die Modellierung der zweidimensionalen Schnitte wurde im Beispiel eine Voxelanzahl von 85 je Raumrichtung gewählt, was hier einer Auflösung von rund $12 \mathrm{~cm}$ entspricht. In Anbetracht der Tatsache, dass es sich um die Darstellung natürlicher Trennflächen handelt, die gewissen Schwankungen unterliegen, und zudem in den weiteren Schritten der Verarbeitung von Werkstein ohnehin ein gewisser Rauabfall einbezogen werden muss (vgl. Abb. 2.1), ist ein Fehlerbereich dieser Art problemlos vertretbar. Ein weiterer relevanter Punkt ist, dass real zu jedem Zeitpunkt mit dem Auftreten von Trennflächen gerechnet werden muss, die nicht auf den direkt beobachtbaren Flächen ausstreichen.

Die Darstellung der Schnittflächen kann somit zum Beispiel genutzt werden, einen Einblick über den Verlauf und damit über eine generelle Verteilung der Trennflächen im fortschreitenden Abbau zu erlangen (Abb. 2.20). So wird im Beispiel deutlich, dass nach dem Bild der Sektionen x1 und x2 im tieferen Bereich des Lagerstättenkörpers ein großvolumiger in-situ Block (A) angeschnitten wird, der auf der aktuellen Abbauwand (Sektion x0) noch nicht in Erscheinung tritt. Daher sollte eine selektive Abbauführung auf den rechten Bereich konzentriert werden.
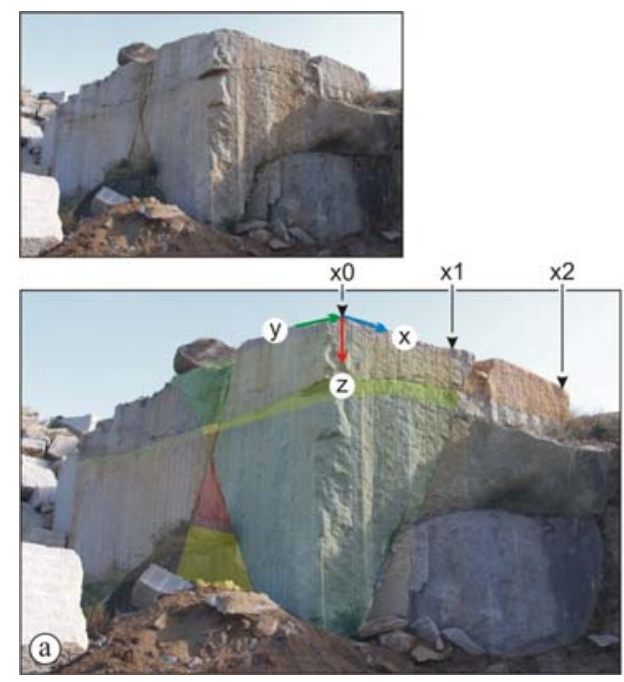

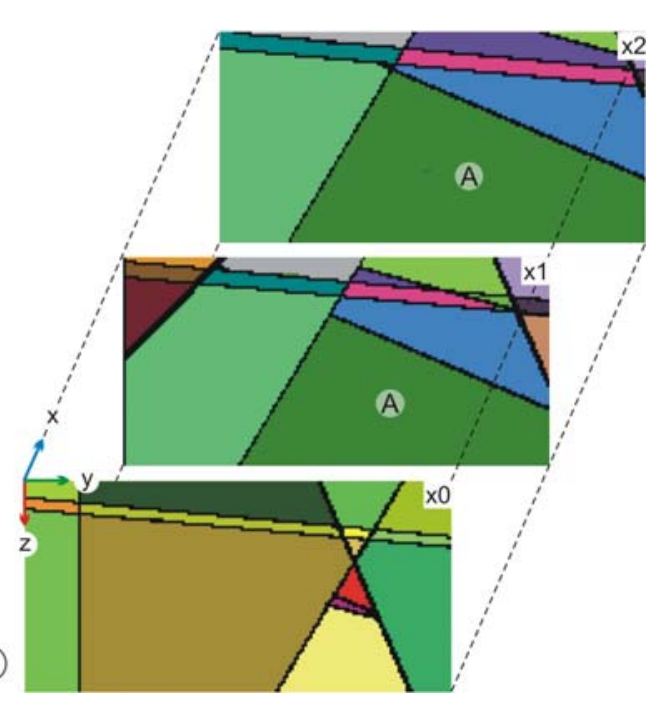

(b)

Abb. 2.20: a) Zergliederung eines Lagerstättenbereichs in mehrere in-situ Blöcke (Steinbruch „Rigo di San Antonio“, Granit, Sardinien); b) Modellierte Schnittflächen parallel der Abbaufront (yz-Fläche) zeigen einen großvolumigen in-situ Block (A), der auf der aktuellen Abbauwand (x0) noch nicht aufgeschlossen ist. 
Parallel zur Darstellung der frei wählbaren Schnittlagen erstellt die Software einen Datensatz, der in ein dreidimensionales Modell umgesetzt werden kann (Abb. 2.21). Die dafür verwendete Software (Intel ${ }^{\circledR}$ Array Viewer) gibt die Möglichkeit zu einer multivariablen Modellierung eines segmentierten Körpers. Durch eine freie Verschiebung der Schnittflächen parallel zu den Außenflächen können direkt Rückschlüsse auf den Verlauf der verschiedenen Grenzflächen gezogen werden. Darüber hinaus kann der betrachtete Körper frei rotiert werden, so dass in der Gesamtheit eine visuelle Beurteilung der geometrischen Gegebenheiten einzelner in-situ Blöcke erfolgen kann.

(a)

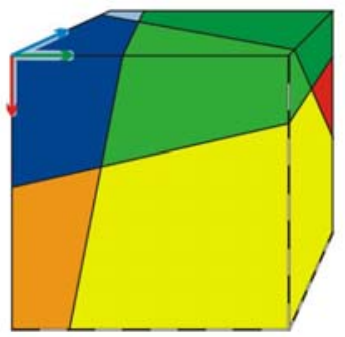

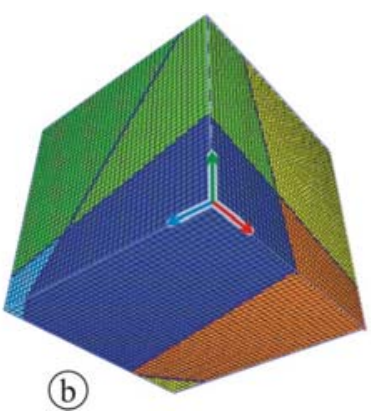

(b)

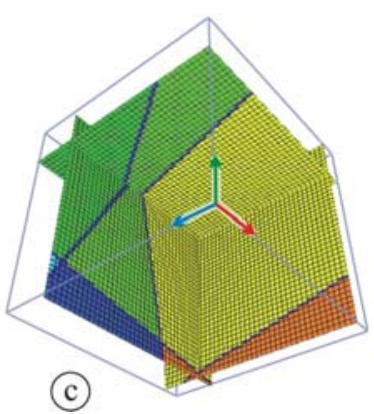

(c)

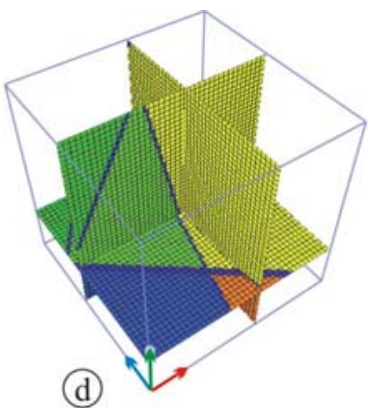

(d)

Abb. 2.21: Möglichkeiten der Darstellung aus den Daten der Software 3D-BlockExpert: a) regellos zergliederter Block (vgl. Abb. 2.17 und 2.19); b - d) eine freie Rotation mit freier Auswahl von Schnittflächen parallel zu den Außenflächen ermöglicht Rückschlüsse auf den Verlauf der verschiedenen Grenzflächen im Block.

\subsection{Auswirkungen des Trennflächengefüges auf die Werksteingewinnung}

Generell ist das Auftreten von Trennflächen für die Gewinnung von Naturstein erwünscht, da dadurch im Prozess der Ausbringung im Allgemeinen ein geringerer Energieeinsatz erforderlich ist. Besonders deutlich wird dies im Abbau von Massenrohstoffen, so z.B. Hartgestein zur Schotter- und Splittproduktion. Zum einen ist hier eine Beachtung geometrischer Vorgaben für das auszubringende Material im Allgemeinen nicht notwendig. Zum anderen kann durch ein verstärktes Auftreten von Klüften die Bohrbarkeit bzw. die Vortriebsgeschwindigkeit positiv beeinflusst werden (Abb. 2.22 a, Thuro 1996). Damit tritt wieder der wirtschaftliche Faktor Zeit in den Vordergrund. Auch im Abbau von Werkstein ist im Idealfall eine deutliche Zergliederung des Gesteins gegeben, so dass eventuell sogar Bohrarbeiten und ein Einsatz von Explosiva o.ä. nicht notwendig sind, um das Material zu brechen (Abb. 2.22 b). Gleichzeitig ist jedoch ein möglichst weitständiges Trennflächensystem erwünscht, um optimale Rohblockgrößen zu erreichen. Dabei ist zudem die Orientierung der Trennflächenscharen zueinander von Relevanz, da durch diese die Geometrie der in-situ Blöcke definiert ist. Folglich ist in der Exploration sowie auch im laufenden Abbau eine Analyse des Trennflächensystems unerlässlich.

Im Hinblick auf Werksteinlagerstätten müssen zudem neben den oben beschriebenen Elementen auch Diskontinuitäten im Gestein beachtet werden, die zwar keine räumliche Abtrennung einzelner in-situ Blöcke zur Folge haben, jedoch eine Unterbrechung im generellen Erscheinungsbild verursachen. Zwar wird in manchen Fällen das charakteristische Dekor eines Gesteins durch die gegebenen Farbdifferenzen geprägt (Abb. 2.23 a). Durch ein unregelmäßiges Auftreten von z.B. deutlich abgesetzten Aplitgängen können jedoch 
mechanisch intakte Gesteinspartien im Hinblick auf die Werksteinnutzung weiter zergliedert werden, da die zumeist erforderliche Farb- und Dekorstabilität empfindlich gestört sein kann. Dies gilt insbesondere, wenn damit auch ein deutlicher Wechsel in der mechanischen Stabilität einhergeht, wie es zum Beispiel bei grobkristallinen Calcitgängen in mikritischen Karbonatgesteinen oder aber einer erheblichen parallelen Akkumulation von z.B. Glimmermineralen der Fall sein kann. Zudem kann das Vorhandensein von z.B. mineralisierten Klüften (Abb. 2.23 b) die Qualität eines Rohblocks deutlich herabsetzen. Auch wenn entsprechende potenzielle Schwächezonen primär nicht zum Versagen des Gesteinsverbandes führen müssen, kann dies in den weiteren Schritten der Produktion der Fall sein.
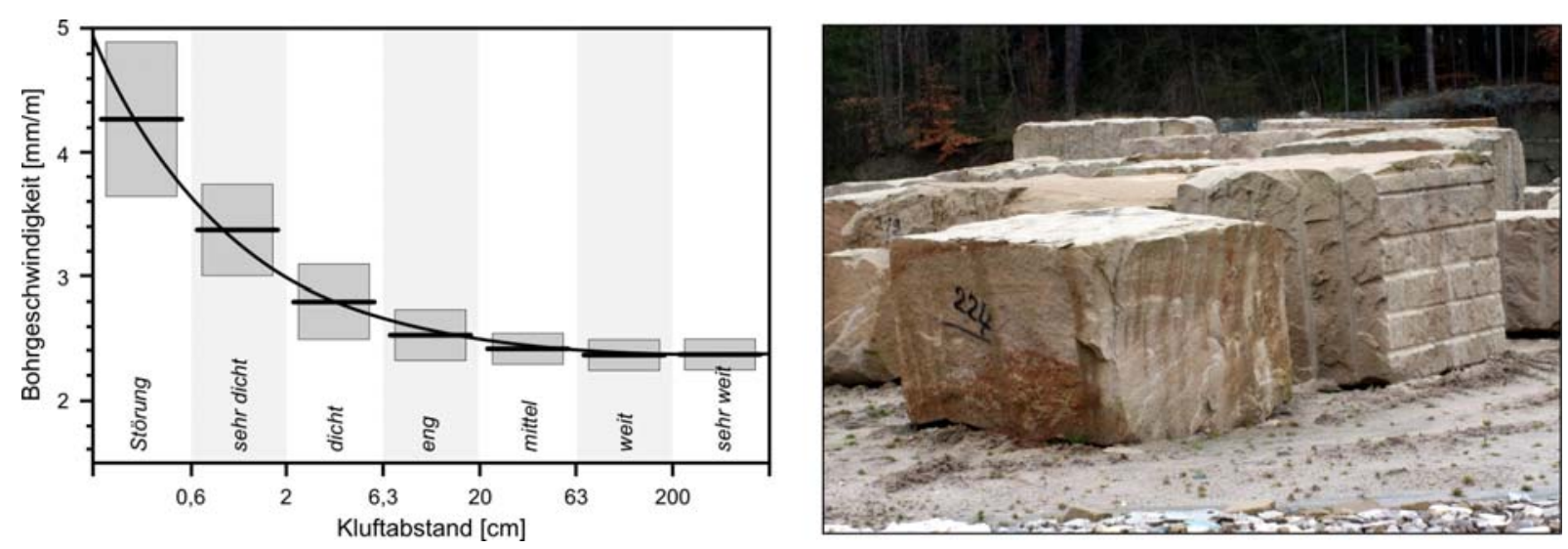

Abb. 2.22: a) Zunahme der Bohrgeschwindigkeit im Verhältnis zum Kluftabstand (nach Thuro 1996); b) Schönbrunner Sandstein: Das natürliche Trennflächengefüge erlaubt zum Teil ein Ausbringen von Rohblöcken ohne eine maschinelle Bearbeitung (Block 224).
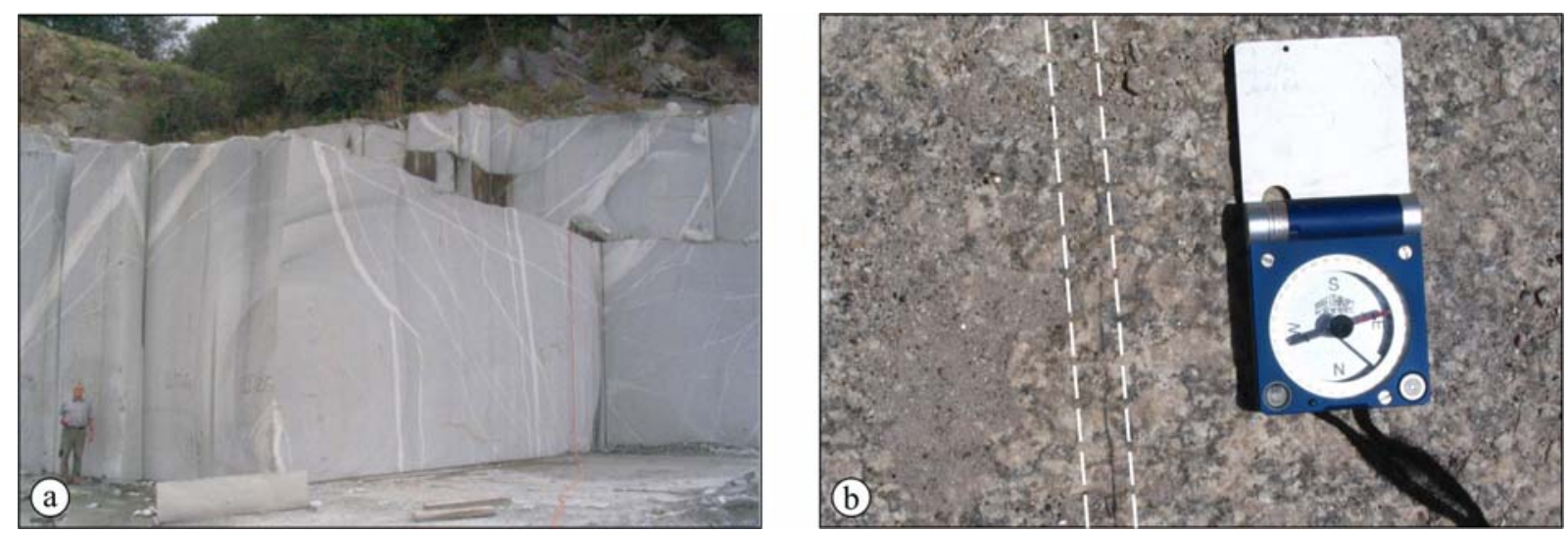

Abb. 2.23: a) CEZLAK GRANODIORIT (Slowenien): farblich abgesetzte Aplitgänge bestimmen das charakteristische Dekor des Gesteins; b) ROSA DEL SALTO (Granit, Argentinien): potenzielle Schwächezone durch mineralisch geschlossene Klüfte als zu beachtendes Element des Trennflächengefüges.

\subsubsection{Auswirkungen der Trennflächendichte}

Zur generellen Betrachtung der Auswirkungen der Abstandsverteilung innerhalb des Trennflächensystems soll an dieser Stelle von einer annähernd orthogonal ausgerichteten Klüftung ausgegangen werden. Dabei wird die Größe und somit das Volumen der zu erwartenden Rohblöcke primär durch die auftretenden Distanzen zwischen den einzelnen Trennflächen, also der Trennflächendichte, bestimmt. Voraussetzung dafür ist jedoch ein 
homogenes Erscheinungsbild des Gesteins zwischen den individuellen Diskontinuitäten. Ein generelles Klassifikationsschema zur Beschreibung der mittleren Abstandsverteilung eines Trennflächengefüges wird z.B. von Smith (1999) gegeben, womit eine Vergleichbarkeit verschiedener Lagerstätten oder Bruchbereiche möglich ist.

Generell sind hohe Abstände als für eine Werksteinlagerstätte günstigste Voraussetzung anzusehen. Im Idealfall können so direkt Rohblöcke in entsprechenden Dimensionen entnommen werden, ohne dass eine Abvierung notwendig ist. Wird ein in zwei Dimensionen weitständiges Kluftsystem durch ein drittes, engständiges System zergliedert, weisen die Rohblöcke natürlicherweise eine eher flache Form auf. Dadurch kann es bereits zu Einschränkungen hinsichtlich der möglichen Verwendung beziehungsweise Verarbeitung kommen. Ist lediglich in einer Dimension ein weitständiges Kluftsystem ausgebildet oder treten in allen Raumrichtungen sehr geringe Abstände auf, können lediglich kleinvolumige Rohblöcke gewonnen werden. Bei einer derart starken Zergliederung des Gesteinskörpers ist lediglich die Produktion von Kleinblöcken für den Garten- und Landschaftsbau oder im Extremfall die Gewinnung von Brechmaterial denkbar.

Sehr weitständige Kluftsysteme können jedoch auch Probleme im Abbau von Werkstein mit sich bringen. Derart kompakte Gesteinskörper verlangen einen erhöhten technischen Einsatz, um zu bewegbaren und formatierbaren Großblöcken zu gelangen. Dabei können Probleme aus residualen Spannungen im Gestein zu Tage treten, da durch den Abbau die Spannungsverhältnisse innerhalb kürzester Zeit verändert werden. In der Folge kann es zu schlagartigen Entspannungen mit der Bildung von Brüchen kommen. Dieses „Bergdrücken“ kann innerhalb weniger Tage oder auch Stunden geschehen (Singewald 1992). Im Extremfall bauen sich diese Restspannungen erst beim Aufschneiden von Rohblöcken ab, was das Zerbrechen der Platten zur Folge haben kann. Daher ist es notwendig, so früh wie möglich eine Entlastung herbeizuführen. Dies geschieht über breiter ausgelegte Trennschnitte, den so genannten Entlastungsschlitzen, die durch Schlitzbohrungen oder aber im Flammenschneidverfahren angelegt werden können (Singewald 1992). Dabei wird der Mineralverband des Gesteins durch eine Propangas-Flamme gesprengt. Eine Tiefenwirkung hinsichtlich einer weiteren Gefügeauflockerung wird dabei jedoch nicht verursacht, wie es z.T. bei der unsachgemäßen Anwendung von Sprengstoffen beobachtet werden kann. In anderen Fällen kann schon das Abtragen des aufliegenden Abraummaterials eine ausreichende Entlastung des Gesteinskörpers mit sich bringen.

Ein weiterer Aspekt hinsichtlich der zu erwartenden Abraummengen ist die Homogenität der Verteilung der eine Gesteinspartie zergliedernden Trennflächen. Bei wiederholt auftretenden engständigeren Kluftscharen ist für die entsprechenden Bereiche eine wie oben beschriebene Nutzungseinschränkung gegeben. Gleichzeitig kann sich die heterogene Verteilung parallel angelegter Diskontinuitäten auch auf die Möglichkeit zur Ausbringung gleichmäßig dimensionierter Rohblöcke ohne zusätzliche Materialverluste negativ auswirken.

Die Möglichkeit zur Abschätzung der zu erwartenden Blockgrößen aus der Abstandsverteilung zeigt sich auch bei einem Vergleich der daraus errechneten mittleren Blockgröße mit den tatsächlich ausgebrachten Rohblöcken einer Lagerstätte, wie es in 
Abbildung 2.24 am Beispiel des Granits GRIS PERLA (Argentinien) dargestellt ist. Die geringeren mittleren Blockgrößen des tatsächlich ausgebrachten Materials resultieren dabei aus den Vorgaben der Blockdimensionen. Dabei ist im gegebenen Beispiel eine Blockgröße zwischen 2,4 x 1,4 x 0,6 m und 3,4 x 2,0 x 1,4 m von Seiten des verarbeitenden Betriebes gefordert. Aus der Abstandsverteilung errechnet sich dagegen eine mittlere Blockdimension von 2,2 x 1,7 x 1,5 m, womit zwar ein größeres Volumen erreicht wird, die geforderten Abmessungen jedoch nicht gegeben sind.
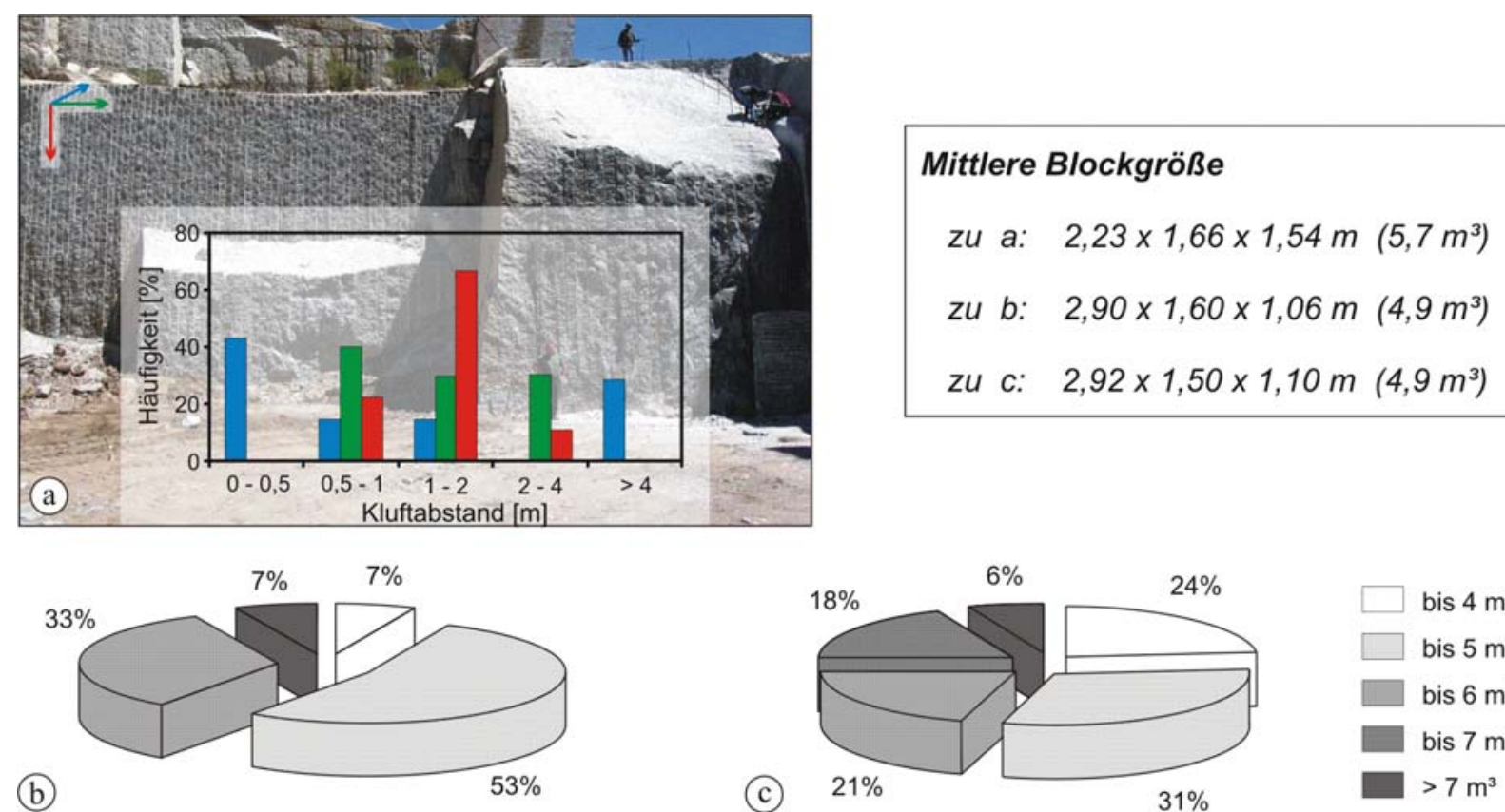

(c)

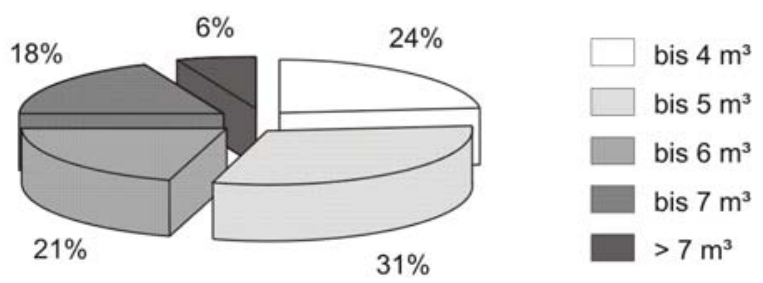

Abb. 2.24: Granit GRIS PERLA (Provinz San Luis, Argentinien): Vergleich der mittleren Blockgrößen errechnet aus a) der Trennflächenverteilung eines Bruchbereichs, b) 15 Rohblöcke formatiert aus einer Abbauscheibe ( 12 $\mathrm{x} 10 \mathrm{x} 1,7 \mathrm{~m}$ ) und c) der Vermessung von 77 gelagerten Rohblöcken, die aus verschiedenen Bereichen des Bruchs stammen.

\subsubsection{Auswirkungen der Trennflächenorientierung}

Das Ziel im Abbau von Werkstein ist ein rechtwinkliger Rohblock zur industriellen Weiterverarbeitung (vgl. Kap. 2.1). In vielen Fällen ist eine direkte Gewinnung entsprechender Blöcke durch ein Abweichen des Trennflächengefüges von der Rechtwinkligkeit nicht möglich. Daher resultiert allein aus dem Abbau und der Formatierung der in-situ Blöcke im weltweiten Durchschnitt ein Abraumanteil von 51 \% (Montani 2003). Häufig liegt dieser Wert noch deutlich höher, womit die Frage der Qualität eines Gesteins und der damit verbundenen Marktfähigkeit bzw. des möglichen Preissegments in den Vordergrund rückt. Bei Abraumraten von 80 \% oder mehr im Abbau eines z.B. grauen Granits ist die Wirtschaftlichkeit der Lagerstätte stark in Frage zu stellen.

Abbildung 2.25 zeigt modellhaft die Veränderungen, die, ausgehend von einem orthogonal angelegten System an Diskontinuitäten (Abb. 2.25 a), mit der Rotation einer (Abb. 2.25 b) und beider Trennflächengruppen (Abb. 2.25 c) einhergeht. Dabei ist zu beachten, dass ein Vergleich der Abstandsverteilung der Trennflächen in allen drei Fällen zum gleichen Ergebnis führt. Es wird deutlich, dass nicht nur eine deutliche Zunahme des Materialverlustes, sondern 
auch gleichzeitig eine Abnahme der mittleren Blockgröße zu verzeichnen ist. Ein Abbau in derart zergliederten Lagerstätten ist konsequenterweise mit vergleichsweise niedrigen Ertragszahlen gekennzeichnet, was auch in einer vergleichenden Bewertung der Trennflächenverteilung in magmatischen Lagerstätten Thailands gezeigt werden konnte (Hoffmann 2006).

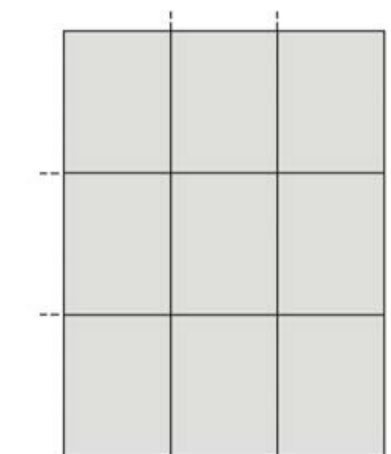

(a)

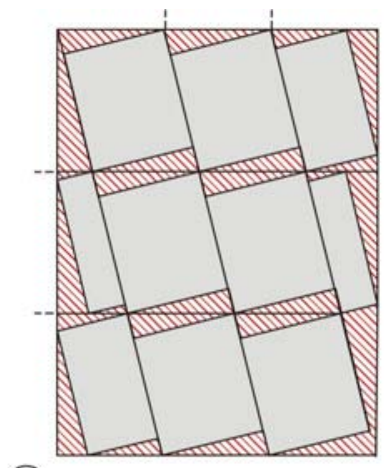

(b)

$\sim 25 \%$ Verlust

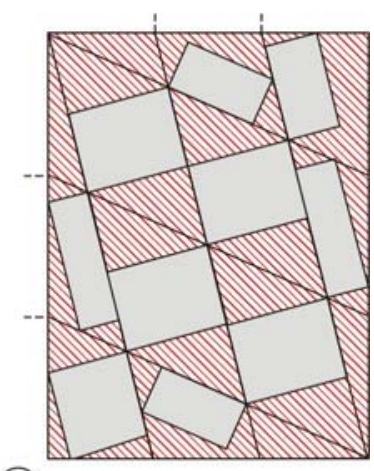

(c)

$\sim 50 \%$ Verlust

Abb. 2.25: Zunahme der Abraummenge bei gleichzeitiger Abnahme der mittleren Blockgröße durch von der Orthogonalität abweichende Trennflächensysteme bei gleich bleibender Abstandsverteilung.

Abweichend vom oben beschriebenen Modell, handelt es sich bei einer Lagerstätte um einen dreidimensionalen Gesteinskomplex. Das bedeutet, dass bei einer Orientierung der Trennflächen, wie sie z.B. in Abb. 2.25 b skizziert ist, die Flächen nur in Ausnahmefällen lotrecht ausgerichtet sind. Dadurch ergibt sich eine weitere Herabsetzung der Ausbringungsrate, die auf der Winkelabweichung aller einen in-situ Block definierenden Flächen beruht (Abb. 2.26).

(a)

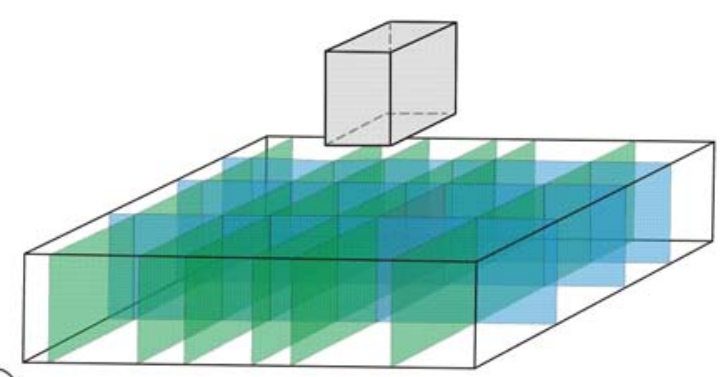

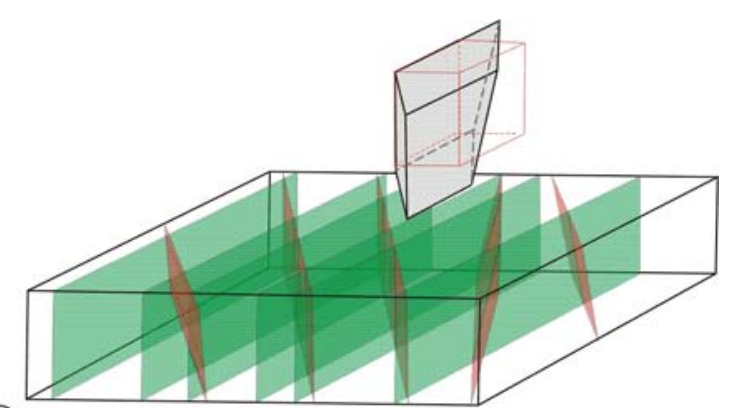

(b)

Abb. 2.26: a) Orthogonales Trennflächensystem; b) Zunahme der Spitzwinkligkeit von in-situ Blöcken durch Trennflächenverteilungen mit Abweichungen von der Orthogonalität im Streichen und Einfallen.

Die Auswirkungen dieser Winkelabweichung werden am Beispiel des Bruches NEGRO GRAPESA (Qz-Bt-Diorit, Spanien, Abb. 2.27) deutlich. Das Kluftrosendiagramm (Abb. 2.27 b) weist ein insgesamt relativ heterogen verteiltes Streichen der Klüfte aus. Dabei zeichnen sich zwei Vorzugsrichtungen ab, die sich in einem Winkel von etwa $60^{\circ}$ bis $70^{\circ}$ schneiden (Abb. 2.27 c). Die Anlage der Sohle bzw. der Abbauwände folgt nur in geringem Maße dem generellen Trend der Trennflächen, was vermutlich in der Heterogenität ihrer Verteilung begründet ist. Als Folge davon zeigen sich in vielen Bereichen Winkelausbrüche an den Abbauwänden in kleinerem und größerem Maßstab (Abb. 2.27 a). Außerdem ist mehrfach zu beobachten, dass zwischen Trennflächen und der Schnittführung ein spitzer Winkel 
aufgespannt ist (Abb. 2.27 d). Konsequenterweise finden sich im Abraum viele abgewinkelte Restblöcke, die sich aus der Abvierung der in-situ Blöcke ergeben haben (Abb. 2.27 e). In Lagerstätten, die eine entsprechende Verteilung der Diskontinuitäten aufweisen, ist es dabei unumgänglich, zumindest eine Richtung der natürlichen Trennflächen im Gestein zu nutzen und den Abbau daran zu leiten.
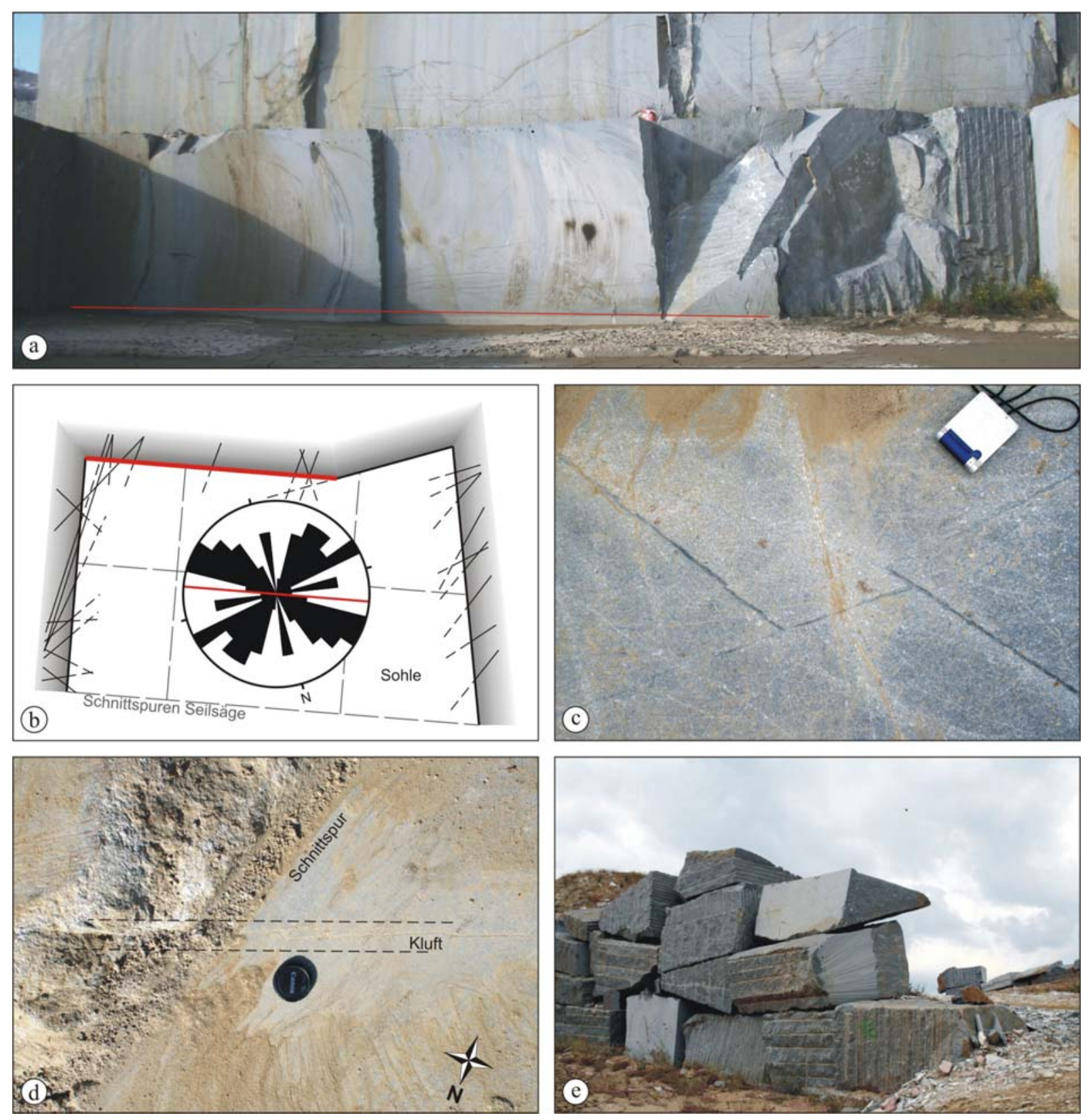

Abb. 2.27: Auswirkung eines nicht orthogonalen Trennflächengefüges: a) Steinbruch NEGRO GRAPESA (QzBt-Diorit, Spanien); b) Sohle zur Abbauwand mit Kluftrosendiagramm und Darstellung der direkt im Sohlenbereich messbaren Klüfte (schwarze Striche) sowie gesetzten Seilsägeschnitte; c) spitzwinkliges Schneiden von Klüften auf der Sohle; d) spitzwinklig zur NE-SW streichenden Kluftschar ausgeführter Schnitt; e) Abraum mit einer hohen Anzahl nicht rechtwinkliger Restblöcke. Nähere Erläuterungen im Text.

Neben der Abstandsverteilung und der Orientierung der Diskontinuitäten stellt somit auch die Homogenität der Trennflächen und ihrer Verteilung einen wesentlichen Aspekt für die Werksteinnutzung dar. Besonders häufig ist, wie erwähnt, die Klüftung in Lagerstätten von Marmor oder auch anderen metamorphen Gesteinen von stark heterogenem Charakter. Daraus 
können sich bei der Beurteilung des Trennflächeninventars hinsichtlich Orientierung und Abstandsverteilung erhebliche Schwierigkeiten ergeben. In Abbildung 2.28 a ist exemplarisch die Kluftverteilung einer Abbauwand in einem Bruch der Marmorprovinz Macaél (Spanien) dargestellt (vgl. Abb. 2.9, 2.10, 2.11 a). Aus den in diesem Lagerstättenabschnitt aufgenommenen Klüften lassen sich zwei Hauptstreichrichtungen erkennen (Abb. 2.28 b). Somit ist auch die Anlage der Wand annähernd dem Trennflächengefüge angepasst, wobei wiederum das Problem der Spitzwinkligkeit gegeben ist. Erheblich vermindert wird die Ausbringrate gut dimensionierter, formgerechter Rohblöcke hier jedoch zusätzlich durch starke Schwankungen im Einfallen der Klüfte. Dabei ist ein breites Spektrum zwischen $5^{\circ}$ und $88^{\circ}$ in relativ gleichmäßiger Verteilung abgedeckt. Das NNE-SSW streichende Trennflächensystem unterliegt in seiner Gesamtheit einer geringeren Schwankung im Einfallen und ist etwas steiler anzusetzen. Durch die Orientierung dieser Abbauwand ist eine Einschätzung einer möglichen Ausbringungsrate stark eingeschränkt, da die Trennflächenschar, die verstärkt Schwankungen im Einfallen aufweist, kaum direkt beobachtbar ist. Dazu müsste eine weitere Wand parallel des NW-SE streichenden Kluftsystem zugänglich sein. Doch allein aus der Abstandsverteilung des hier dargestellten zweidimensionalen Anschnitts der Lagerstätte wird die stark eingeschränkte Aussagekraft durch die heterogene Ausbildung des Trennflächengefüges deutlich (Abb. 2.28 c).
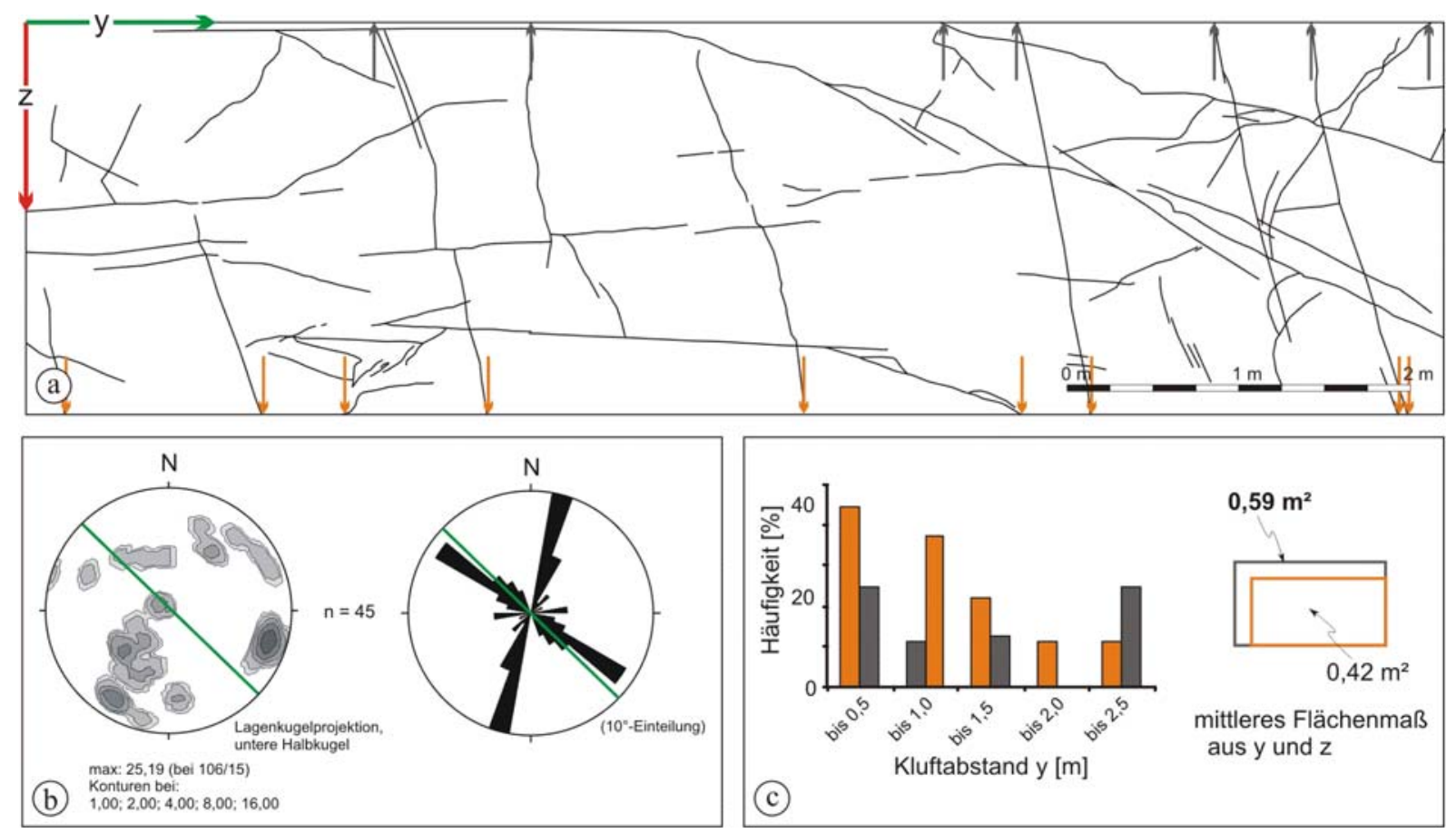

Abb. 2.28: a) Darstellung des Trennflächenverlauf auf einer Bruchwand (Marmor, BIANCO MACAEL, Spanien, vgl. Abb. 2.8); b) Darstellung der Kluftverteilung im entsprechenden Lagerstättenbereich; c) verschiedene Messlinien bei der Aufnahme der Abstandsverteilung (orange - unten, grau - oben, jeweils bezogen auf Teilabbildung a) führen zu unterschiedlichen mittleren Flächenmaßen. Nähere Erläuterungen im Text. 


\subsection{Möglichkeiten der Optimierung im Abbau von Naturwerkstein auf der Basis der Trennflächenanalyse}

Unter Optimierung ist die Verbesserung eines Vorgangs hinsichtlich bestimmter Ziele zu verstehen. Dabei müssen konsequenterweise mit dem Vorgang zusammenhängende Prozesse gegeneinander abgewogen werden, um im Endeffekt das bestmögliche Resultat zu erreichen. In der Werksteingewinnung ist dabei vor allem der Arbeits- und Betriebsmittelaufwand sowie auch die Preiskategorie des möglichen Produkts von Relevanz.

Die Betrachtungen zum Trennflächengefüge in Werksteinlagerstätten haben gezeigt, dass nur in wenigen Fällen gut dimensionierte Rohblöcke direkt aus der Zergliederung einer Lagerstätte resultieren. Somit ist es im Abbau notwendig, das Material in optimierter Weise zu nutzen. Dabei müssen neben den Maßvorgaben für Rohblöcke vor allem auch verschiedene weitere Faktoren berücksichtigt werden, die die Wirtschaftlichkeit eines Abbaus beeinflussen. Dazu zählen besonders die möglichst positiv zu gestaltende Bilanz zwischen Wertgestein und Abraum sowie der aufzubringende Maschinen- bzw. Arbeitseinsatz. Letzteres ist in einer Kostenkalkulation aufzurechnen, die u.a. Betriebsstoffe und Materialabnutzung der Geräte als auch den Zeitaufwand mit einbezieht (z.B. Singewald 1992).

An dieser Stelle soll vor allem auf Möglichkeiten eingegangen werden, die zu einer Ertragssteigerung im Abbau von Naturwerkstein beitragen können. Generell ist in diesem Zusammenhang eine möglichst vollständige Verwertung des ausgebrachten Materials anzustreben. Je nach der physikalischen und technischen Charakteristik eines Gesteins sollten so in jedem Fall Überlegungen angestellt werden, wie auch Abraummaterial, das bei der Gewinnung von Rohblöcken anfällt, sinnvoll genutzt werden kann. In vielen Hartgesteinslagerstätten könnte so z.B. die Produktion von Bruchmaterial die Abraumrate deutlich herabsetzen. Andere Möglichkeiten bieten sich durch die Produktion von Massivmauersteinen oder anderen Elementen für den Garten-, Landschafts- und Wegebau an.

Wie oben genannt, muss das Trennflächengefüge in seiner räumlichen Verteilung bereits bei der Anlage eines Steinbruchs als auch im laufenden Abbau unbedingte Beachtung finden. Wie bereits am Beispiel des spanischen Diorits NEGRO GRAPESA (Abb. 2.27) ansatzweise zu erkennen ist, kann aus Abweichungen zwischen der Orientierung des Trennflächengefüges und der Abbaurichtung ein überhöhter Abraumanteil resultieren. Dementsprechend kann bereits die Rotation der Abbaurichtung eine Reduktion der Abraummenge bewirken, da die Geometrie der in-situ Blöcke besser genutzt wird. In Abbildung 2.29 ist eine zweidimensionale Modellierung der Sohle eines Sandsteinbruchbereichs dargestellt (vgl. Abb. 2.18), die mit fortschreitendem Abbau zu erwarten ist. Aus der durchgeführten Trennflächenanalyse ist bekannt, dass die Klüftung mit einem mittleren Einfallen von etwa $88^{\circ}$ annähernd saiger gestellt ist. Die Kluftrose zeigt ein deutliches Maximum bei NS streichenden Klüften. Die zweite Hauptkluftschar ist dagegen breiter gefächert. Die Schichtung des Gesteins, die hier annähernd horizontal gelagert ist, wurde aufgrund der speziellen Fragestellung nicht in die Trennflächenanalyse mit einbezogen. Es wird deutlich, dass die Ausrichtung der aktuellen Abbauwand in nur geringem Maße der 
Trennflächenorientierung angepasst ist. So zeigt sich auch ein ständiger Wechsel von gebohrten Abschnitten und natürlichen Bruchflächen an der Abbaufront. Auch wenn für den rückwärtigen Bereich keine direkten Informationen über die räumliche Lage weiterer Trennflächen aus dem Geländebefund vorliegen, sollte in diesem Beispiel die Abbauwand im rechten Winkel zur Hauptkluftschar, also in E-W-Richtung, angelegt werden.
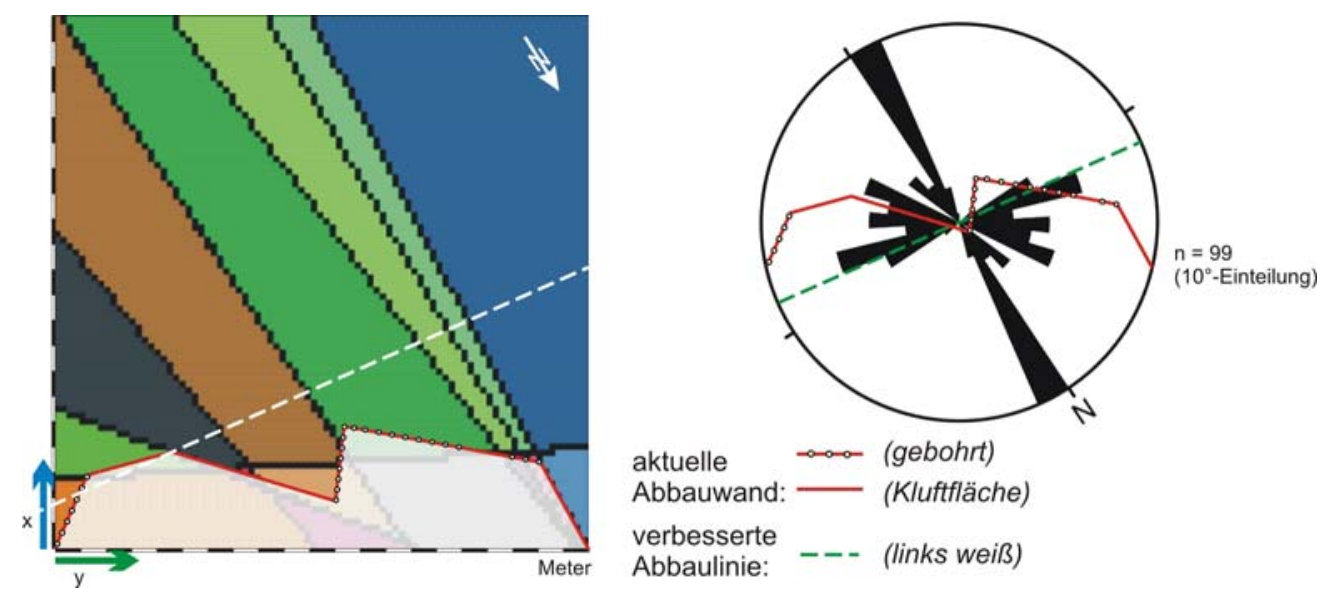

Abb. 2.29: Darstellung einer modellierten Schnittfläche, die der bei fortschreitendem Abbau zu erwartenden Sohle eines Sandsteinbruchs entspricht (vgl. Abb. 2.18). Zur optimierten Gewinnung von Rohblöcken sollte eine Rotation im Abbau durchgeführt werden, um die Front rechtwinklig zur Hauptkluftschar auszurichten. Nähere Erläuterungen im Text.

Ähnliche Möglichkeiten zur Optimierung ergeben sich bereits bei insgesamt kompakten, sehr weitständig geklüfteten und homogenen Gesteinskörpern, wenn nur wenige Diskontinuitäten, so z.B. farblich prägnant abgesetzte Gänge o.ä., regellos im Gestein auftreten. Entsprechen diese Elemente nicht dem charakteristischen Dekor eines Gesteins, können daraus erhebliche Materialverluste resultieren, die sich bis zum Aufschneiden der Rohblöcke fortsetzen können (Abb. 2.30).

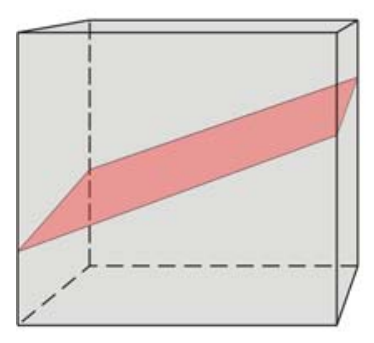

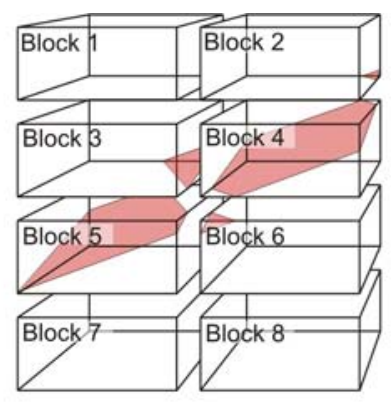

Block 4, Block 5 Abraum (25\%)

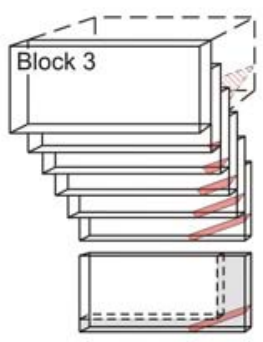

bis $30 \%$ Verschnitt je Rohplatte

Abb. 2.30: Theoretische Abraumanteile, die bei der Formatierung und dem Aufschneiden von Rohblöcken aus einer einfach zerteilten Abbauscheibe resultieren können.

Hier können durch eine Rotation der auszubringenden Rohblöcke im Gesteinskörper übermäßige Abraumanteile vermieden werden. Abbildung 2.31 zeigt eine schematische Darstellung eines Steinbruchs, der von derartigen Bändern durchzogen ist. Hierbei kann durch die angesetzte Tiefe der Abbauscheiben eine positive Beeinflussung des Abraumanteils erreicht werden. Die Rotation bezieht sich dabei auf die Ausrichtung der Längsachse der 
Rohblöcke. In Abbildung 2.30 sind die theoretischen Anteile der voll verwertbaren Rohblöcke gegeben, die sich bei der Lage der Längsachse quer zur Abbaurichtung (Abb. 2.31 a) und parallel zur Abbaurichtung (Abb. 2.31 b) für die aufeinander folgenden Abbauschritte ergeben. Die Stärke der Abbauscheiben definiert im zweiten Fall die Länge der Rohblöcke. Daher kann einzig die Bewegbarkeit der gelösten Partien als limitierender Faktor auftreten. Dem könnte jedoch durch eine Herabsetzung der Abstufungshöhe entgegengewirkt werden.

Es zeigt sich, dass sowohl die Ausrichtung der Abbaufront als auch die direkten Planungsschritte im laufenden Abbau, so z.B. die Mächtigkeit der Abbauscheiben oder Wandhöhen, an die gegebenen geologischen beziehungsweise tektonischen Verhältnisse einer Lagerstätte oder auch in separaten Teilbereichen angepasst werden müssen. Gleichzeitig gilt es, die natürliche Teilbarkeit des Gesteins zu nutzen, um zeit- und kostenintensive Trennarbeit durch Bohrungen oder Sägeschnitte möglichst gering zu halten.
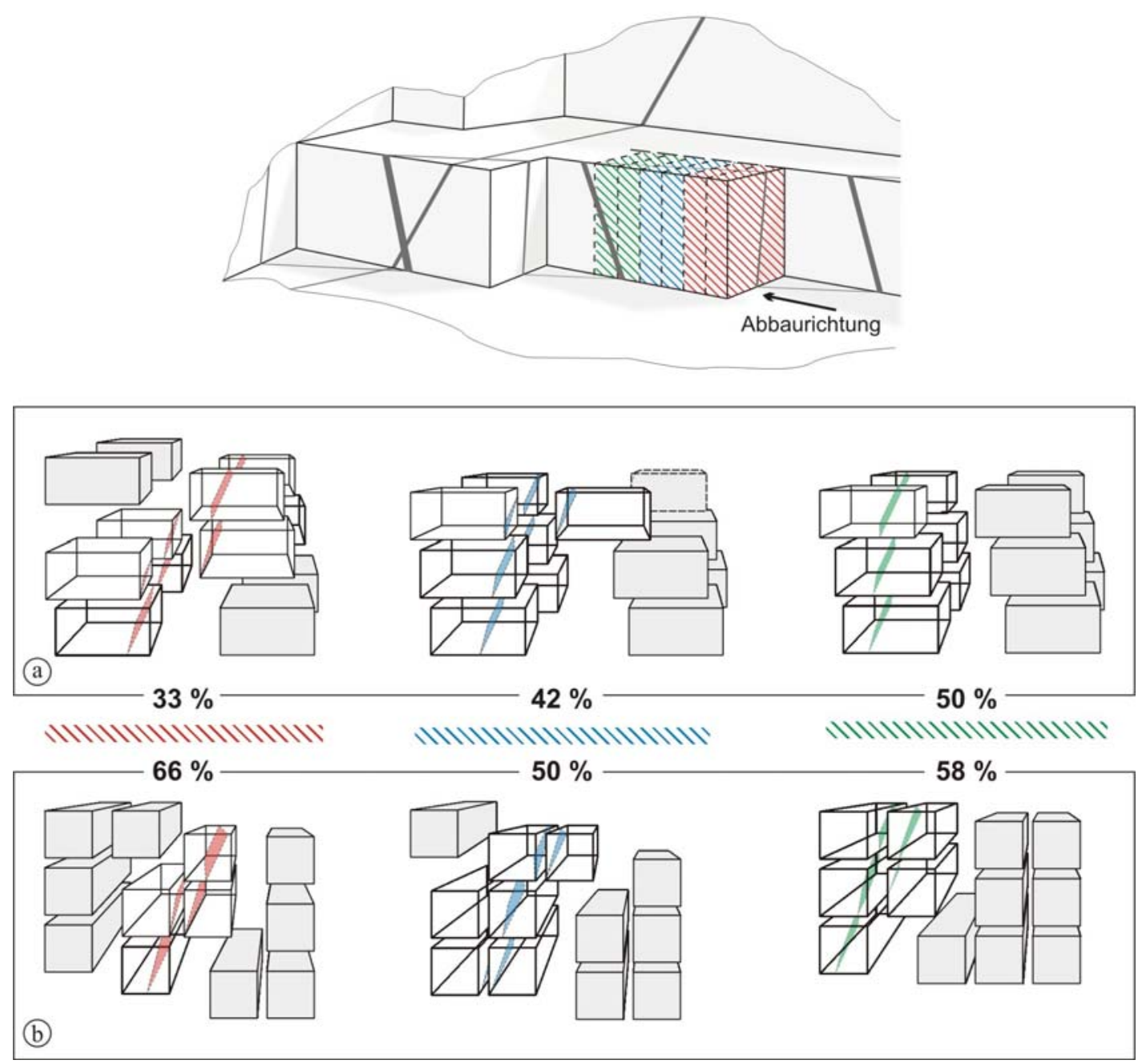

Abb. 2.31: Schematische Darstellung eines insgesamt homogenen und kompakten Gesteinskörpers, der von wenigen, regellosen Diskontinuitäten durchzogen ist. Eine Anpassung der Stärke der Abbauscheiben und damit der Ausrichtung der auszubringenden Rohblöcke bewirkt eine positive Beeinflussung der Wertgestein-AbraumBilanz. Die in Abbaurichtung aufeinander folgenden Schritte sind farblich verschieden markiert. Ausrichtung der Längsseite der Rohblöcke: a) in Abbaurichtung und b) quer zur Abbaurichtung. Prozentwerte beschreiben den Anteil voll verwertbarer Rohblöcke. 
An diesem Punkt können auch Profilschnitte parallel zur Abbaufront hilfreich sein, wie sie mit der Software 3D-BlockExpert aus der detaillierten Aufnahme des Trennflächengefüges erstellt werden können. Die jeweiligen Schnittflächen sind dabei so zu wählen, dass sie Vorder- und Rückseite einer potenziellen Abbauscheibe wiedergeben, deren Dicke gleichzeitig eine Dimension der finalen Rohblöcke darstellt. Daraus kann über einfache geometrische Beziehungen die Form der sich ergebenden in-situ Blöcke konstruiert werden und damit der primäre Abraum, der aus dem senkrechten Abstechen der Seitenflächen resultiert, abgeschätzt werden (Abb. 2.32, vgl. Abb. 2.20). In der Folge ergibt sich die Möglichkeit, das tatsächlich nutzbare Material im Zweidimensionalen hinsichtlich der besten Flächenausnutzung auszufüllen. Dieser Vorgang könnte auch über mathematische Beziehungen automatisiert werden, wie es z.B. in der Automobilindustrie beim Ausstechen von Einzelteilen aus Metallplatten Standard ist.

In Abbildung 2.32 ist eine derartige Optimierung der Rohblockformatierung dargestellt. Dabei sind aus einer Vielzahl von Möglichkeiten lediglich drei exemplarisch herausgegriffen. Es wir jedoch deutlich, dass entsprechende Verschiebungen in der Anordnung der Rohblöcke sowohl deren mögliche Anzahl als auch die dafür notwendigen Trennarbeiten beeinflussen. Dabei müssen wiederum auch rein wirtschaftliche Faktoren beachtet werden. So ist bei der Anordnungsmöglichkeit C zwar die höchste Blockzahl der gegebenen Beispiele realisiert, doch ist deren Formatierung mit einem erhöhten Arbeitsaufwand (relative Schnittlänge) gegenüber der Anordnungsmöglichkeit B verbunden. In diesem Fall ist dass zu erwartende Preissegment eines Gesteins in Betracht zu ziehen und der zusätzliche Ertrag gegen die Produktionsmehrkosten abzuwägen. Durch eine entsprechende Beurteilung aufeinander folgender Abbauscheiben können zusätzlich auch prognostische Aussagen über Anzahl und Volumen von Rohblöcken in folgenden Abbaupartien gewonnen werden. Neben der direkten Abbauplanung ergibt sich so zudem ein Werkzeug, um z.B. weitere Schritte der Verarbeitung zu planen oder auch gezielt auf Anfragen und Bedürfnisse des Marktes reagieren zu können.

Vorrausetzung für eine derartige Nutzungsoptimierung ist selbstverständlich, dass der betrachtete Bereich frei von Defekten ist, die die Verwendung der jeweiligen Gesteinsvarietät als Werkstein ausschließen. So müssen z.B. auch Bereiche einer deutlichen Farbveränderung o.ä. von vornherein aus der Flächenbetrachtung ausgeschlossen werden, wenn diese nicht dem definierten Sortenstandard entsprechen (vgl. Abb. 2.23). Eine gewisse Problematik, die sich zudem ergeben kann, stellt sich bei Gesteinsvarietäten, deren Dekor sich auf gerichtete Strukturelemente stützt und daher eine bestimmte Ausrichtung der Rohblöcke in der Abbauscheibe verlangt. 


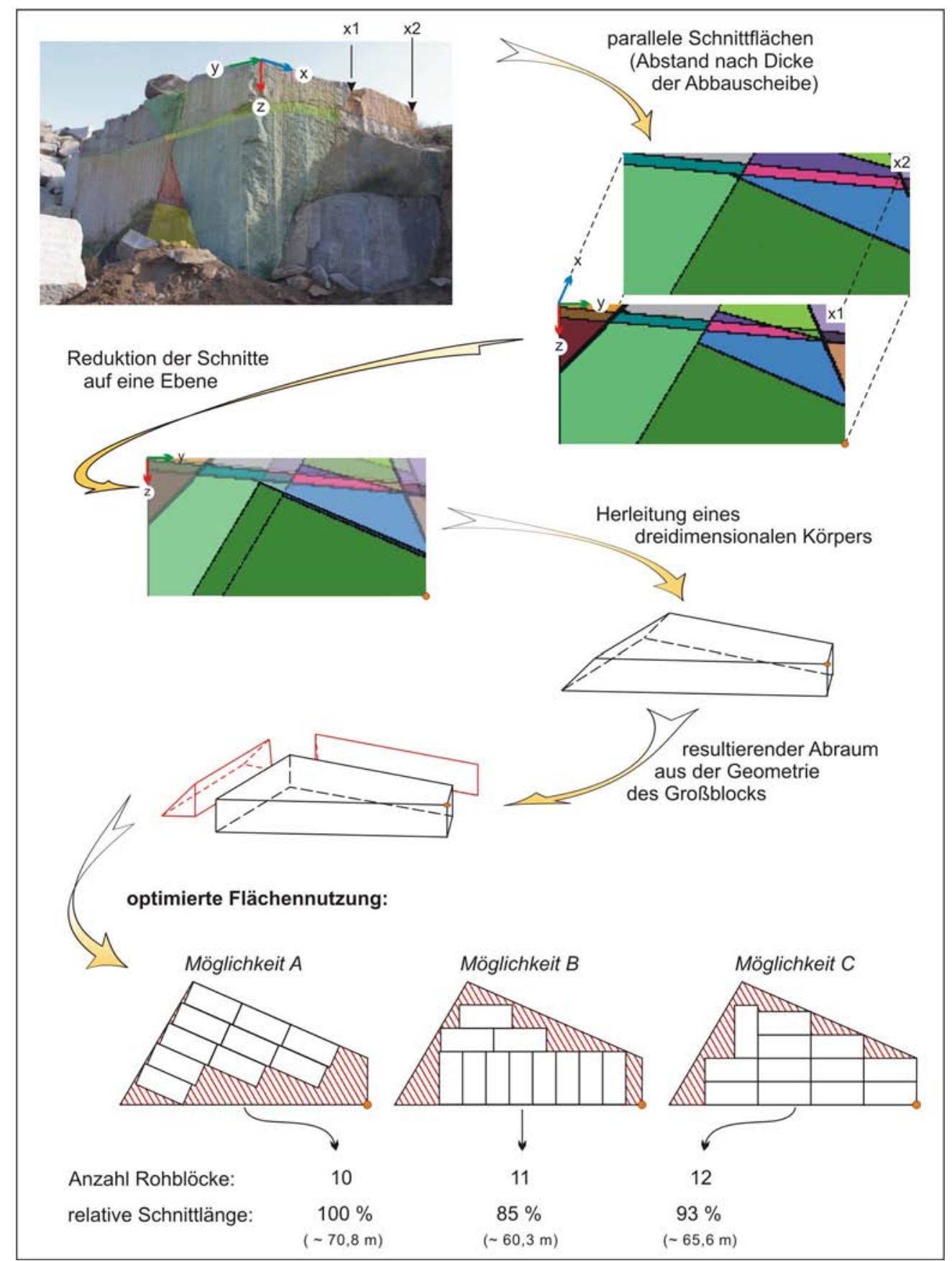

Abb. 2.32: Optimierung der Rohblockausbringung unter Zuhilfenahme der mit der Software 3D-BlockExpert aus der Trennflächenanalyse erstellten, parallelen Schnittflächen. Zur optimierten Flächennutzung sind aus einer Vielzahl von Möglichkeiten drei beispielhaft dargestellt. Nähere Erläuterungen im Text.

Eine ideale Abstimmung des Abbauvorgangs auf die Elemente des Trennflächengefüges konnte im Steinbruch Bärenburg (Schweiz) bei der Gewinnung der Varietät VERDE ANDEER erreicht werden (Abb. 2.33, vgl. Abb. 2.16). In diesem Gneiskomplex sind neben einer annähernd horizontal ausgerichteten Klüftung (Kluftschar A), die bei der Trennflächenanalyse infolge der eindeutigen Orientierung und gleichmäßig hoher Abstände nur schematisch dargestellt ist, zwei weitere Hauptkluftscharen ausgebildet. Zum einen ist dies ein NNW-SSE streichendes System von Klüften mit Einfallswinkeln von meist über $80^{\circ}$ (Kluftschar B), die häufig durch die Mineralisation von Turmalin verheilt sind (Abb. 2.33 b). Insgesamt sind diese Kluftschließungen mechanisch von geringer Stabilität, womit potenzielle 
Trennflächen gegeben sind, die sich bereits im Prozess des Abbaus öffnen. Dieses verheilte Kluftsystem wird von einer weiteren Kluftschar C in der Streichrichtung in einem Winkel von annähernd $90^{\circ}$ geschnitten, deren mittlerer Einfallswinkel etwa $50^{\circ}$ nach NNW beträgt. Wie auch die horizontalen Klüfte weisen diese Trennflächen zumeist einen geringen mechanischen Zusammenhalt auf und können durch wenige Bohrungen in Abständen von über 1,5 m und dem schonenden Einsatz von Sprengschnüren gelöst werden.

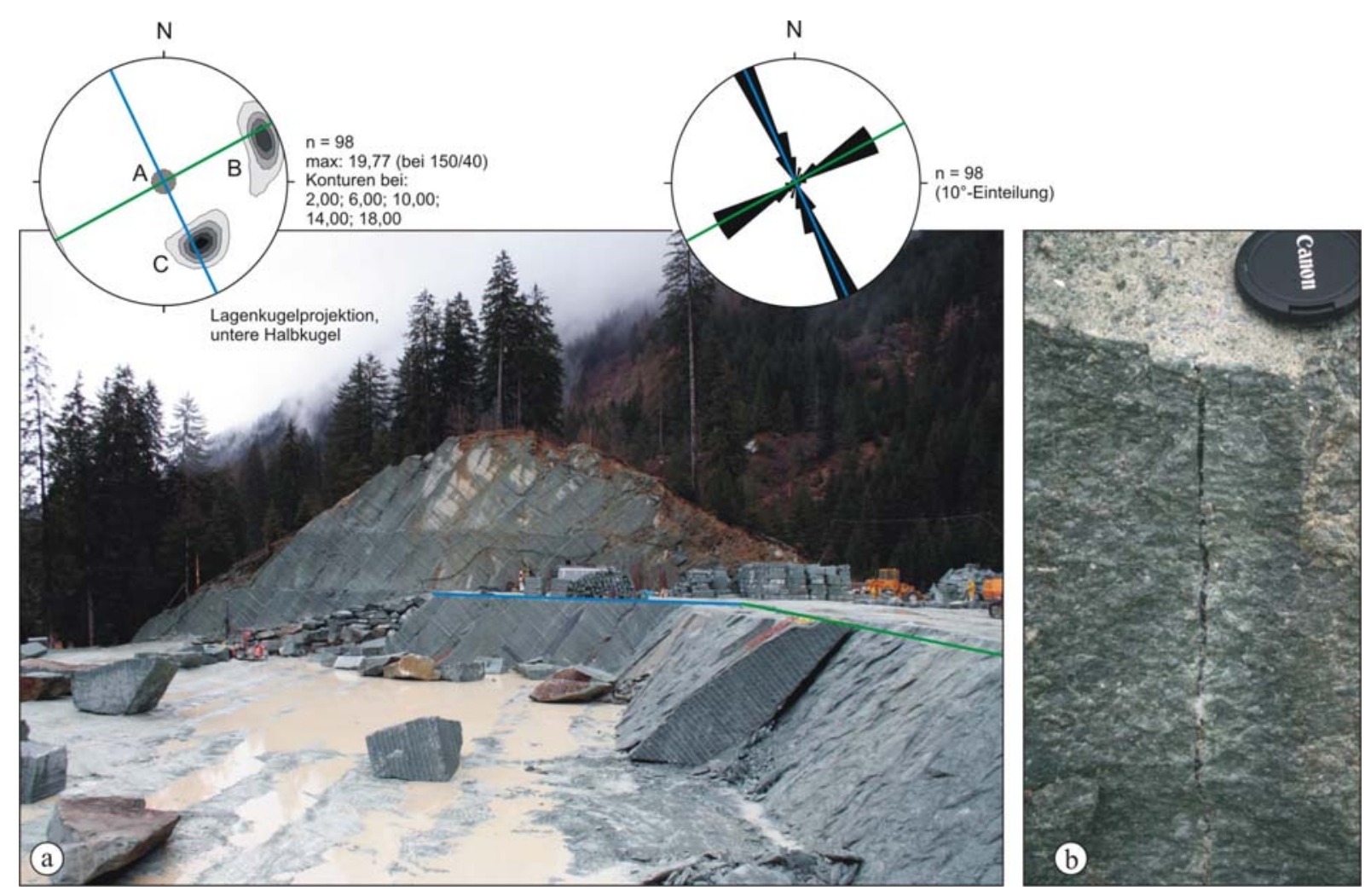

Abb. 2.33: Abbau der Varietät VERDE ANDEER (Orthogneis, Schweiz): a) Trennflächenverteilung im Steinbruch Bärenburg / E-Teil (Fa. Toscano AG); b) mit Turmalin mineralisierte Klüfte stellen potenzielle Trennflächen im Gestein dar. Nähere Erläuterungen im Text.

Insgesamt ergibt sich somit ein Trennflächengefüge, in welchem zwei Hauptscharen annähernd senkrecht zueinander ausgerichtet sind (Kluftscharen A und B) und durch ein diagonales System (Kluftschar C) geschnitten werden (Abb. 2.34 a und b). Der Abbau ist in der Längserstreckung am Streichen der Kluftschar C ausgerichtet, während die jeweilige Abbausohle durch die horizontale Lagerklüftung gegeben ist, die im angeschnittenen Bereich Abstände von etwa 6,5 m aufweist. Die Ausbringung erfolgt im ersten Schritt durch eng gesetzte Bohrungen, die senkrecht zur Diagonalklüftung niedergebracht werden. Dabei wird jeweils nur eine Bank durchstoßen. In einem zweiten Schritt erfolgt das Aufbrechen der jeweiligen Kluft C, im Falle des Bruchbereichs in Abbildung 2.34 auf einer Länge von rund $40 \mathrm{~m}$. Dabei öffnen sich auch die zumeist mineralisierten Klüfte der Kluftschar B. In der Folge ergeben sich schräge Formate der in-situ Blöcke, womit naturgemäß ein Abraumanteil durch die Abvierung gegeben ist (Abb. 2.34 c). 
(b)
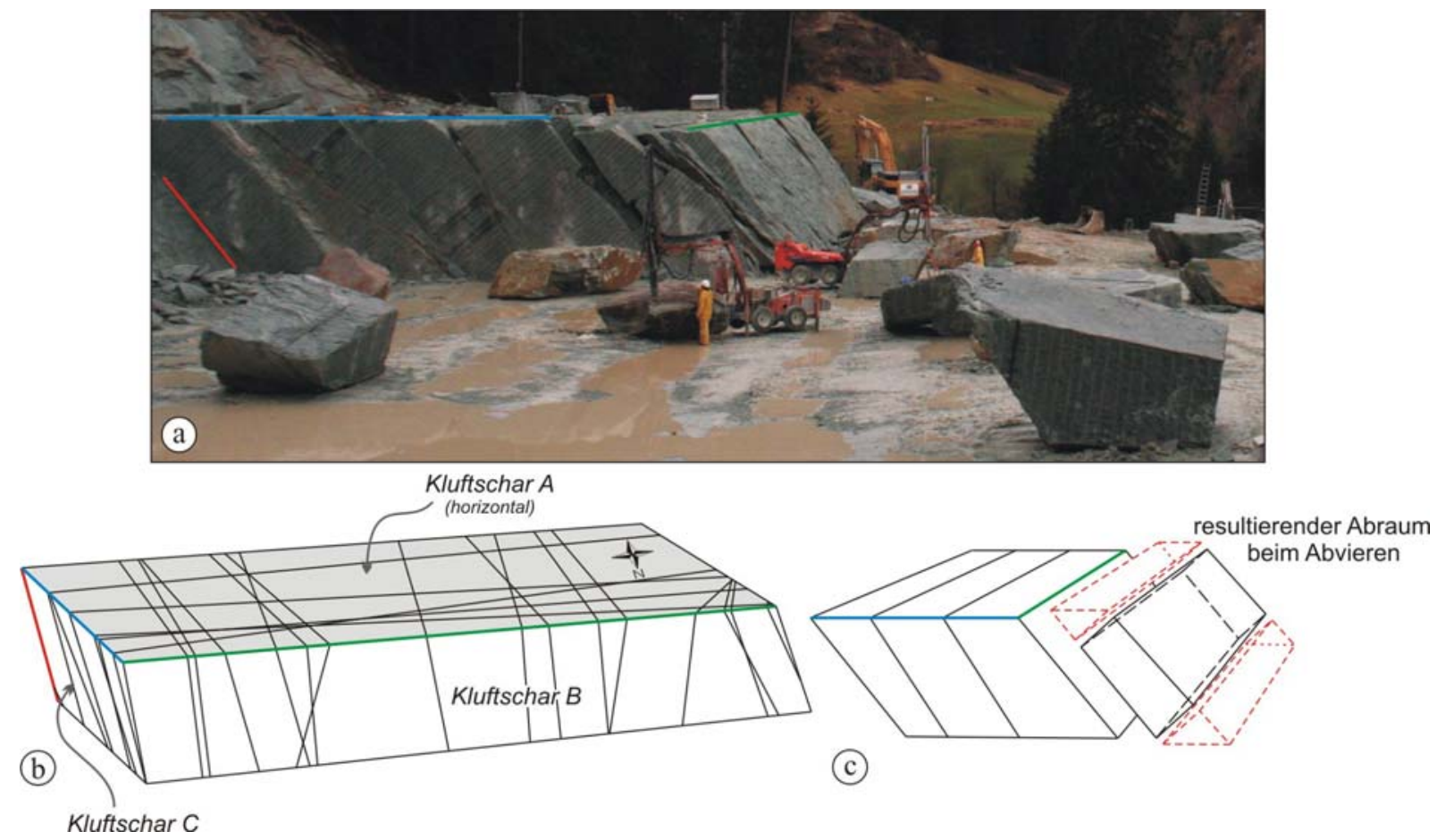

Abb. 2.34: a) Abbau der Varietät VERDE ANDEER (Orthogneis, Schweiz) im Steinbruch Bärenburg / W-Teil (Fa. Toscano AG); b) Schematische Darstellung des Trennflächengefüges des Bruchbereichs in a; c) Der generell spitze Winkel zwischen den Kluftscharen A und C hat schräge in-situ Blöcke zur Folge.

Singewald (1992) beschreibt ähnliche Auswirkungen einer schräg gestellten Klüftung für den Kalksteinbruch Marching (Deutschland). Dabei ist die schräge Form der Blöcke jedoch auf einen fehlerhaften Abbau zurückzuführen, der sich nicht an die Orientierung der Trennflächen hält. Aufgrund einer fehlenden intensiveren Aufnahme und Interpretation des Trennflächengefüges wurden senkrechte Wände in Streichrichtung der schräg einfallenden Klüftung angelegt (Abb. 2.35). Neben den ungünstigen Blockformaten besteht zudem auch die Gefahr, dass einzelne Gesteinspartien abgleiten können, wodurch ein enormes Gefahrenpotenzial im Abbau gegeben ist. Da in diesem Vorkommen zudem keine Lagerklüftung ausgebildet ist, könnte eine Änderung der Abbauführung, wie es in Abbildung 2.35 dargestellt ist, den Abraumanteil deutlich verringern. Daraus würde zwar eine nicht befahrbare Sohle entstehen, doch kann für das Abheben der Blöcke ein Derrick eingesetzt werden. Aufgrund der tektonischen Gegebenheiten im Steinbruch Bärenburg (Abb. 2.16, 2.33, 2.34) kann dagegen auch durch entsprechende Veränderungen der Abbauführung der Abraum beim Formatieren der gelösten in-situ Blöcke nicht vermieden werden kann. 


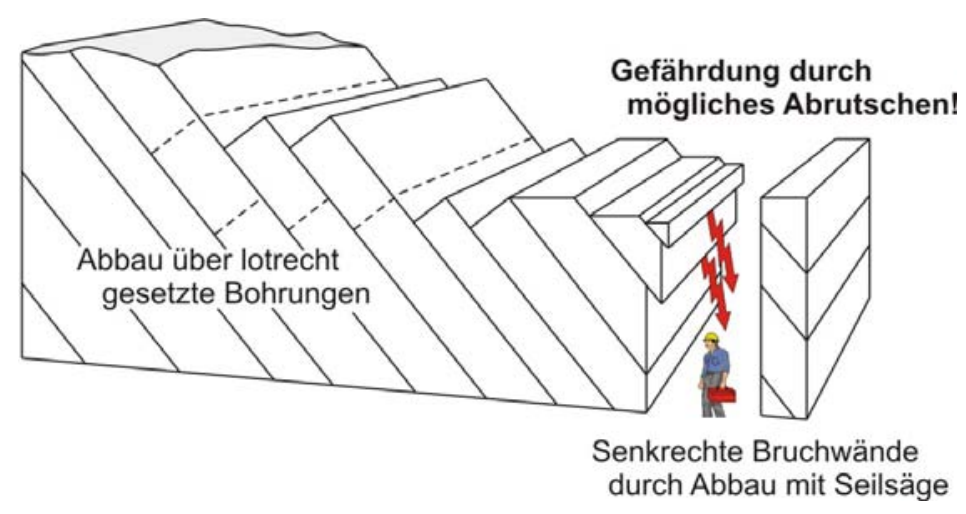

Abb. 2.35: Mögliche Änderung der Abbauführung bei schräg einfallender Trennflächenorientierung (verändert nach Singewald 1992). Nähere Erläuterungen im Text.

Die vorherigen Betrachtungen setzen zumeist eine relativ gut ausgebildete Regelmäßigkeit in der Trennflächenverteilung voraus. Wie schon in Kapitel 2.2 und 2.4.2 am Beispiel von Marmor beschrieben, können in metamorphen Gesteinskomplexen infolge einer mehrfachen tektonischen Überprägung zum Teil sehr heterogen ausgeprägte Trennflächengefüge vorherrschen. Diese Komplexität kann dazu führen, dass mit der Öffnung eines Steinbruchs beziehungsweise der Definition einer Abbaurichtung erhebliche Schwierigkeiten verbunden sein können (Primavori 1999). Deutlich wird dies anhand des Werksteinbruchs der Varietät AZUL TANGO (Cdr-Gt-Gneis, Argentinien, Abb. 2.36 a). Sowohl die Streichrichtung als auch die Einfallswinkel der auftretenden Trennflächen unterliegen deutlichen Schwankungen (Abb. 2.36 b). Aus der Dichteverteilung ist eine Vorzugsorientierung mit steil einfallenden Klüften und E-W gerichtetem Streichen feststellbar. Trotz annähernd umlaufender Streichrichtungen im Kluftrosendiagramm sind hier jedoch zwei Hauptkluftscharen zu vermuten, die sich in einem Winkel von etwa $60^{\circ}$ bis $70^{\circ}$ schneiden. Zusätzlich beschreiben die Abstandsverteilungsdiagramme ein insgesamt engständiges Kluftsystem mit mittleren Abständen von jeweils etwa $1 \mathrm{~m}$ in allen Raumrichtungen (Abb. 2.36 c). Die Berechnung der theoretischen mittleren Blockgröße aus der Abstandverteilung ergibt lediglich ein Volumen von etwa $0,9 \mathrm{~m}^{3}$. Aus der Gesamtheit der Faktoren ist eine erhebliche und unregelmäßige Zergliederung des Lagerstättenkörpers abzuleiten. Dieses heterogene Trennflächensystem führte schließlich auch zur Einstellung der Abbautätigkeiten in dieser Lagerstätte.

Sowohl der Abbau als auch die Formatierung zu Rohblöcken erfolgte über Bohrungen und den Einsatz von Explosiva. An mehreren Abschnitten der Abbaustufen wird deutlich, dass der Abbau nicht oder nur unvollständig den geologischen Vorgaben angepasst wurde. Ohne Beachtung des Kluftsystems erfolgte die Abteufung senkrechter Bohrungen, wobei Trennflächen durchstoßen wurden (Abb. 2.37 a). Dadurch wurden auch möglicherweise größere in-situ Blöcke unkontrolliert zertrennt, was an zahlreichen Rohblöcken im Lager an zum Teil erheblichen Defekten erkennbar ist. Diese reichen von mineralisierten bis hin zu deutlich geöffneten Trennflächen oder auch erheblichen Abweichungen von der Orthogonalität (Abb. 2.37 b). Trotz dessen lag nach Auskunft der Anteil ausgebrachter Rohblöcke bei nicht mehr als 10 bis $20 \%$.

Sinnvoller wäre es hier gewesen, konsequent einen selektiven Abbau voranzutreiben, der sich auf singuläre Großblöcke bezieht. Grundvoraussetzung dafür ist wiederum das Erkennen 
der Vorzugsorientierung der Klüfte, um eine entsprechende Anlage des Abbaus parallel zur Hauptstreichrichtung ( 100 - 280) vornehmen zu können. Außerdem sollten aufliegende Abraumbereiche vollständig geräumt werden, um so im Abbau zu einer besseren Übersicht über den Verlauf der Trennflächen zu gelangen. Für jeden weiteren Abbauschritt muss dann eine detaillierte Aufnahme und Beurteilung des Trennflächengefüges für einzelne Gesteinspartien vorgenommen werden, um so individuelle Großblöcke im Gesteinskörper zu detektieren. Diese müssten durch ein schonendes Heranarbeiten, das heißt unter Ausnutzung der natürlichen Teilbarkeit des Gesteins, freigelegt und separat aufgearbeitet werden.
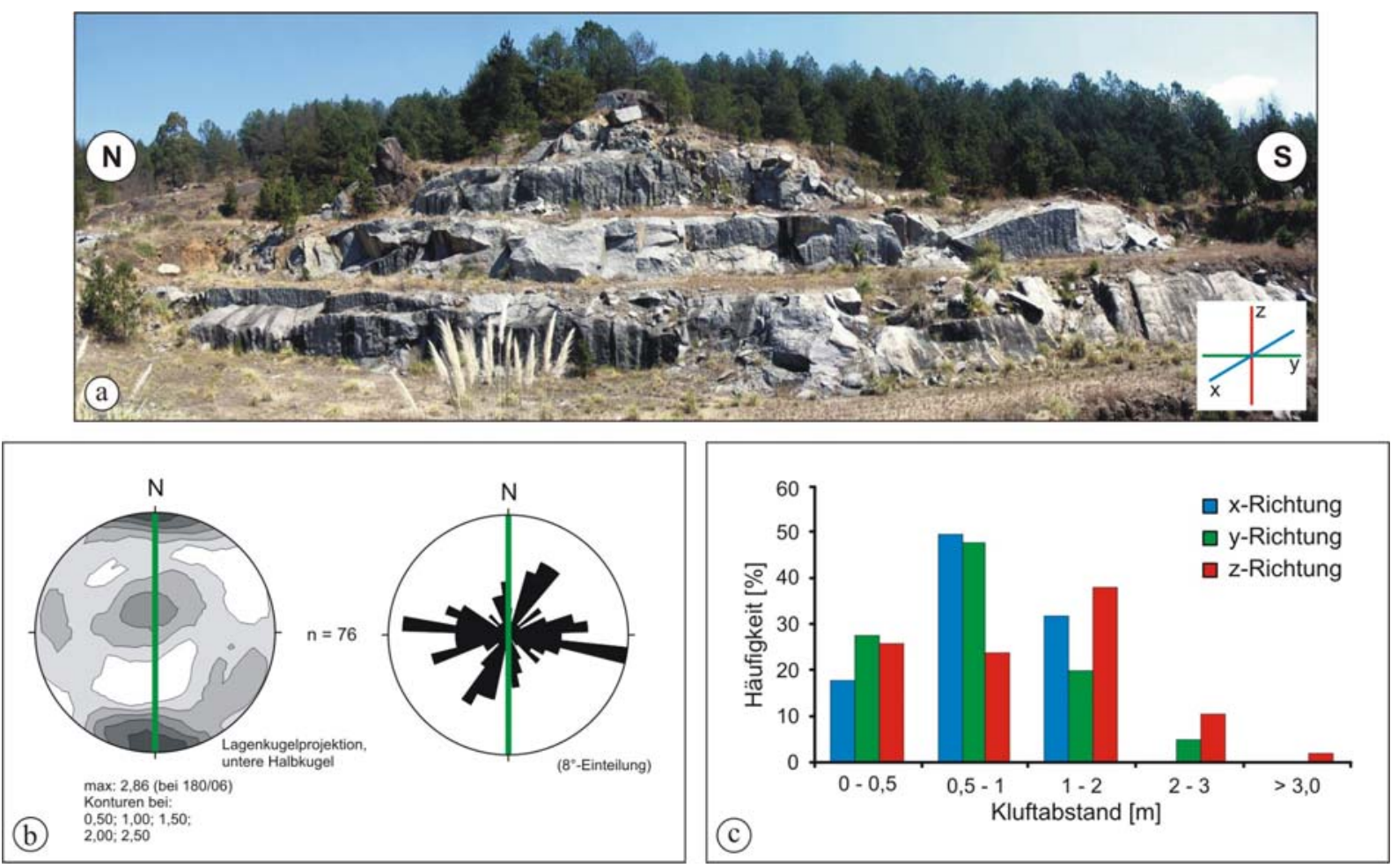

Abb. 2.36: a) Steinbruch „La Cantera Kinzigita“ (AZUL TANGO, Cdr-Gt-Gneis, Provinz Córdoba, Argentinien): Die b) Kluft- und c) Abstandsverteilungsdiagramme beschreiben ein insgesamt engständiges und annähernd richtungsloses Trennflächensystem.
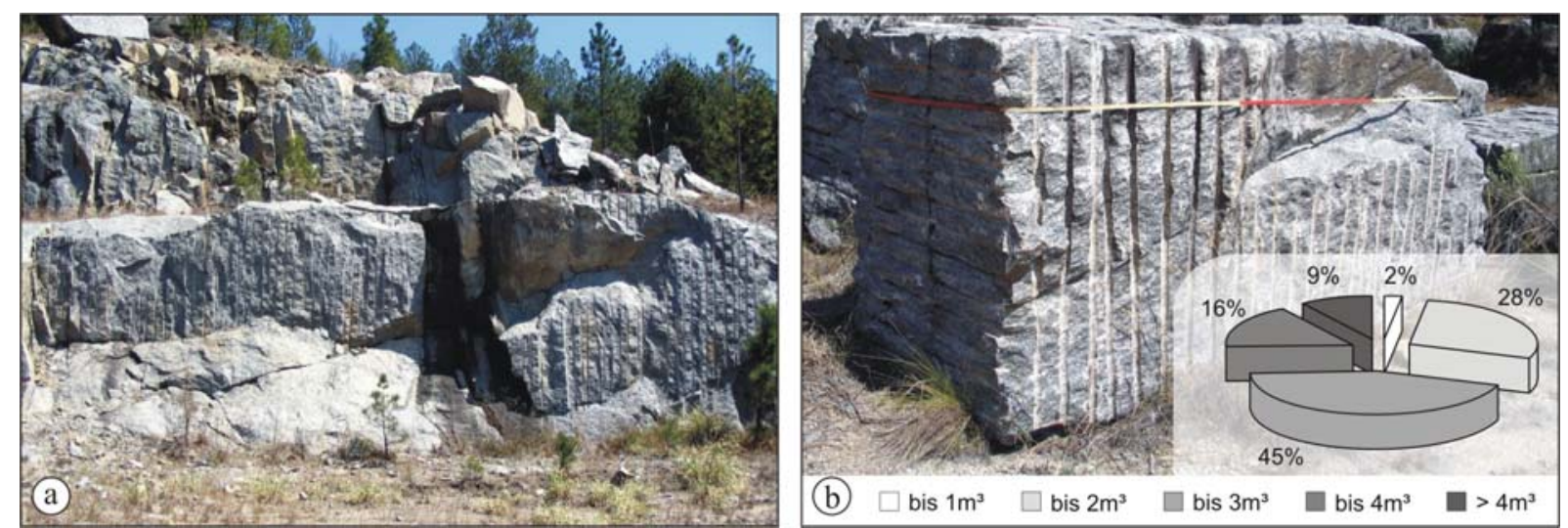

Abb. 2.37: Steinbruch „La Cantera Kinzigita“ (AZUL TANGO, Cdr-Gt-Gneis, Argentinien): a) senkrechte Bohrungen ohne Beachtung des Kluftsystem (Absatzhöhe etwa 4 m); b) Viele Blöcke weisen erhebliche Mängel in Form von Trennflächen oder geometrischer Abweichungen auf. Diese sind nicht in die Berechnung der Volumenverteilung aus dem Aufmaß ausgebrachter Rohblöcken ( $\mathrm{n}=43, \varnothing$ 2,51 m³) eingegangen. 
In Teilbereichen des Steinbruchs ist zu erkennen, dass versucht wurde, eine ähnliche Strategie zu verfolgen (Abb. 2.36, mittlere Abbaustufe links). Dementsprechend konnte aus dem vorhandenen Blocklager ein mittleres Rohblockvolumen von 2,51 $\mathrm{m}^{3}$ (Abb. 2.36 b) bei einer mittleren Kantenlänge von 2,10 x 1,25 x 0,95 m bestimmt werden. Trotz der geringen mittleren Blockgröße aus der Abstandsverteilung kann somit vermutet werden, dass großvolumigere in-situ Blöcke im Lagerstättenkörper vorhanden sind und diese gezielt abgebaut werden könnten. Da es sich um ein qualitativ hochwertiges Material handelt, sollte daher ein erhöhter Abraumanteil, der mit dem Freilegen einzelner Großboulder einhergehen würde, bei der Gewinnung dimensionsgerechter und defektfreier Rohblöcke eventuell hingenommen werden können.

\subsection{Zusammenfassende Bewertung zur Erfassung des Trennflächengefüges}

Zusammenfassend ermöglicht eine systematische und detaillierte Aufnahme des Trennflächengefüges eines Lagerstättenkörpers oder -bereichs:

- die Einschätzung bzw. Bewertung der Verwendungsmöglichkeit eines Lagerstättenkörpers bzw. -bereichs.

- die Detektion nicht abbauwürdiger Bereiche und damit die Konzentration auf qualitativ hochwertigen Rohstoff.

- die optimierte Anpassung der Abbauführung.

- die Optimierung im Prozess der Rohblockformatierung.

Unter Beachtung der Abstandsverteilung und Orientierung der auftretenden Trennflächen kann somit:

$$
\begin{aligned}
& \Rightarrow \quad \text { eine positive Beeinflussung des Verhältnisses zwischen } \\
& \text { Abraum und Wertgestein erreicht werden. } \\
& \Rightarrow \quad \text { die Blockausbeute optimiert und damit die Wirtschaftlichkeit } \\
& \text { einer Werksteinlagerstätte positiv beeinflusst werden. } \\
& \Rightarrow \quad \text { die Effektivität in der Gewinnung und Nutzung des Rohstoffs } \\
& \text { Naturwerkstein gesteigert werden. }
\end{aligned}
$$

Neben der rein wirtschaftlichen Komponente sind zudem auch unnötige Umweltschäden, die mit einer unkontrollierten Anlage oder Erweiterung eines Abbauareals einhergehen, vermeidbar. Somit kann auch ein Beitrag zum nachhaltigen, ökologischen Ressourcenschutz geleistet werden.

Aus der Feststellung der generellen Nutzbarkeit eines Vorkommens resultiert die Frage der möglichen bautechnischen Anwendung eines Gesteins. Daher ist eine genaue Kenntnis der physikalischen und technischen Eigenschaften des Materials erforderlich. Diesem Aspekt soll im folgenden Kapitel mit einer statistischen Auswertung recherchierter Daten zu mehr als 2300 verschiedenen Werksteinvarietäten Rechnung getragen werden. 


\section{STATISTISCHES VERHALTEN PHYSIKALISCHER UND TECHNISCHER EIGENSCHAFTEN VON NATURWERKSTEINEN}

Die Möglichkeit einer bautechnischen Anwendung eines Gesteins ist eng mit den spezifischen physikalischen und technischen Eigenschaften eines Materials verbunden. Daher sind fundierte Kenntnisse über die verschiedenen Parameter unumgänglich, da diese ausschlaggebend für die Stabilität und Langlebigkeit eines Gesteins bei der Verwendung als Baurohstoff sind. In der Literatur findet sich zudem eine Vielzahl von Beiträgen zu technischen Gesteinsdaten und ihrer Korrelation mit einfach zu bestimmenden Eigenschaften wie der Dichte oder der Porosität (z.B. Peschel 1983, Winkler 1994, Strohmeyer 2003). Das grundlegende Ziel der meisten Quellen ist es, aus der Extrapolation der Werte eine indirekte Einschätzung zur technischen Eignung eines Gesteins vorzunehmen, ohne die Vielzahl der physikalischen und technischen Eigenschaften experimentell bestimmen zu müssen. Die zugrunde liegenden Daten dieser Zusammenfassungen sind jedoch aus verschiedenen Gründen häufig nicht repräsentativ. Dies kann z.B. darauf beruhen, dass sich Datensätze auf eine bestimmte Lokalität beziehen oder nur von geringem Umfang sind. Im Rahmen der vorliegenden Arbeit wurden Ergebnisse gesteinstechnischer Untersuchungen von 2.335 verschiedenen Handelsvarietäten von Gesteinen aus über 70 Ländern zusammengetragen und ausgewertet (vgl. Mosch \& Siegesmund 2007). Die Zusammenstellung erfolgte durch eine umfangreiche Recherche sowohl in fachbezogenen Büchern, Publikationen und Analyseprotokollen als auch in Prospekten und dem Internet. Eine derartig breit gefächerte Datenzusammenstellung kann gewisse Problematiken nach sich ziehen, auf die in Kapitel 3.2 näher eingegangen wird.

\subsection{Vorgehensweise und Zielsetzung}

Grundlegendes Ziel ist es, das statistische Verhalten verschiedener physikalischer (Dichten, effektive Porosität) und technischer Parameter (Druck-, Biege-, Spaltzugfestigkeit) von Naturwerksteinen und ihrer Beziehungen untereinander zu beschreiben. Eine primäre explorative Datenauswertung im Hinblick auf die verschiedenen Eigenschaften erfolgt zunächst nach einer übergeordneten Gesteinsklassifikation in die Gruppen Plutonite, Vulkanite, Sandsteine, Karbonatgesteine und Metamorphite. Unter Verwendung weiterreichender Klassifikationen (Abb. 3.1) werden dann spezifische Verteilungsmuster detaillierter betrachtet.

Das hohe Datenaufkommen kann dabei leicht zu unübersichtlichen Darstellungen führen. Daher soll für die verschiedenen Korrelationen die Berechnung bivariater Aufenthaltswahrscheinlichkeiten der unterschiedlichen Eigenschaftspaare aus den statistischen Kenndaten erfolgen. Diese dienen der Abschätzung der wahren Grundgesamtheit der verschiedenen Datenpaare und stellen eine übersichtliche Visualisierung der Datenbeziehungen dar. Somit sind eine vergleichende Betrachtung unterschiedlicher Gesteinsgruppen sowie auch die Einpassung von Einzeldaten innerhalb einer Gruppe 
möglich. In einem weiteren Schritt sollen dann Möglichkeiten zur Prognose der Festigkeitsparameter aus den einfach zu bestimmenden Eigenschaften Rohdichte und Porosität dargestellt und die entsprechenden Verknüpfungen über lineare beziehungsweise nichtlineare Funktionstypen beschrieben werden.

\begin{tabular}{|c|c|c|c|}
\hline & $\underset{\text { (Kap. 3.4) }}{\text { Hauptgruppen }}$ & $\begin{array}{c}\text { Untergruppen } \\
\text { (Kap. } 3.5 \text { bis } 3.9 \text { ) }\end{array}$ & $\begin{array}{l}\text { geschätzter Anteil } \\
\text { an der Weltproduktion* }\end{array}$ \\
\hline \multirow{2}{*}{ Magmatische Gesteine } & Plutonite $(n=618)$ & $\begin{array}{l}\text { Granit } \\
\text { Granodiorit, Tonalit } \\
\text { Monzonit, Syenit } \\
\text { Gabbro, Diorit }\end{array}$ & $\sim 25 \%$ \\
\hline & Vulkanite $(n=71)$ & $\begin{array}{l}\text { Andesit, Basalt } \\
\text { Rhyolith, Dazit } \\
\text { Trachyt } \\
\text { Tuff }\end{array}$ & $\sim 5 \%$ \\
\hline \multirow[b]{2}{*}{ Sedimentäre Gesteine } & $\begin{array}{l}\quad \text { (Kap. 3.7) } \\
\text { Sandsteine }(n=507) \\
\text { (klastische Sedimente) }\end{array}$ & $\begin{array}{l}\text { Porositätsklasse I (<13,4 Vol.-\%) } \\
\text { Porositätsklasse II (13,4 - 20,5 Vol.-\%) } \\
\text { Porositätsklasse III (>20,5 Vol.-\%) }\end{array}$ & $\sim 5 \%$ \\
\hline & $\begin{array}{r}\text { (Kap. 3.8) } \\
\text { Karbonatgesteine }(n=674) \\
\text { (chemische-biogene Sed.) }\end{array}$ & $\begin{array}{l}\text { Kalkstein } \\
\text { Kalkbrekzie } \\
\text { Kalkoolith } \\
\text { Dolomit } \\
\text { Travertin }\end{array}$ & $\sim 35 \%$ \\
\hline Metamorphe Gesteine & $\begin{aligned} \quad(\text { Kap. 3.9) } \\
(n=465)\end{aligned}$ & $\begin{array}{l}\text { Gneis, Migmatit } \\
\text { Marmor } \\
\text { Quarzit } \\
\text { Schiefer } \\
\text { Serpentinit }\end{array}$ & $\sim 30 \%$ \\
\hline
\end{tabular}

Abb. 3.1: Klassifikation der Gesteine in Haupt- und Untergruppen zur spezifischen Darstellung der physikalischen und technischen Eigenschaften ( $n=$ Anzahl der Werksteinvarietäten im Datensatz). Die Herleitung der Untergruppen ist in den jeweiligen Kapiteln aufgeführt.

Daran anschließend werden die verschiedenen Eigenschaften auf ihr Verhalten in Korrelation mit der Kompressionswellengeschwindigkeit untersucht, die im Allgemeinen mit den Eigenschaften Porosität sowie Rohdichte, und somit auch der Mineralogie, variiert. Zudem stellt die Ultraschalldiagnostik ein Verfahren der zerstörungsfreien Materialprüfung dar. Anhand der verschiedenen Korrelationen sollen auch hier Ansätze zu Prognosemöglichkeiten der physikalischen und technischen Gesteinseigenschaften dargestellt werden.

Abschließend sollen verschiedene Aspekte des Feuchtehaushaltes der Gesteine betrachtet werden. Zahlreiche Verwitterungsphänomene von Naturwerksteinen sind auf die materialspezifische Charakteristik des Wasseraufnahmeverhaltens zurückzuführen. Die relevanten Mechanismen für den Feuchtetransport und die Feuchtespeicherung werden dabei durch die stoffliche Zusammensetzung und die Gefügemerkmale eines Gesteins kontrolliert. In einem weiteren Kapitel werden gruppenspezifische Verteilungsmuster zur freiwilligen, kapillaren Wasseraufnahme aufgezeigt und dabei die relevanten Kenndaten zum Feuchtehaushalt dargestellt. Abschließend werden auch für die freiwillige Wasseraufnahme 
eines Gesteins Prognoseansätze ausgehend von den Basiseigenschaften sowie der Ultraschallanalytik beleuchtet.

In der Gesamtheit sollen die statistischen Untersuchungen dazu beitragen, die verschiedenen gesteinstypischen Charaktere hinsichtlich des physikalischen und technischen Verhaltens strukturiert darzustellen. Die Berechnung der wahren Grundgesamtheit mit dem Ausdruck bivariater Aufenthaltswahrscheinlichkeiten (Büttgenbach 1990) kann so die vergleichende Einschätzung tatsächlich vorliegender Eigenschaftsdaten eines Gestein und dessen abschätzende Einpassung in die jeweilige Gesteinsgruppe ermöglichen. Des Weiteren wird das Ziel verfolgt, auch bei dem Vorliegen einer nur geringen Probenmenge über die Prognose von Gesteinseigenschaften aus relativ einfach und kostengünstig zu bestimmenden Eigenschaften im Mindesten zu einer einschätzenden Charakterisierung eines Gesteins zu gelangen.

Der Großteil der Gesteinseigenschaften ist physikalischer Natur. Um eine übersichtliche Gruppierung bei der Beschreibung der verschiedenen Gesteinseigenschaften zu erlangen, soll im Folgenden eine Nomenklatur verwendet werden, wie sie in Abbildung 3.2 gegeben ist. Wird weiterhin im Text ein Mittelwert genannt, ist dieser durch das Zeichen $\varnothing$ in Verbindung mit dem jeweiligen Eigenschaftskürzel kenntlich gemacht.

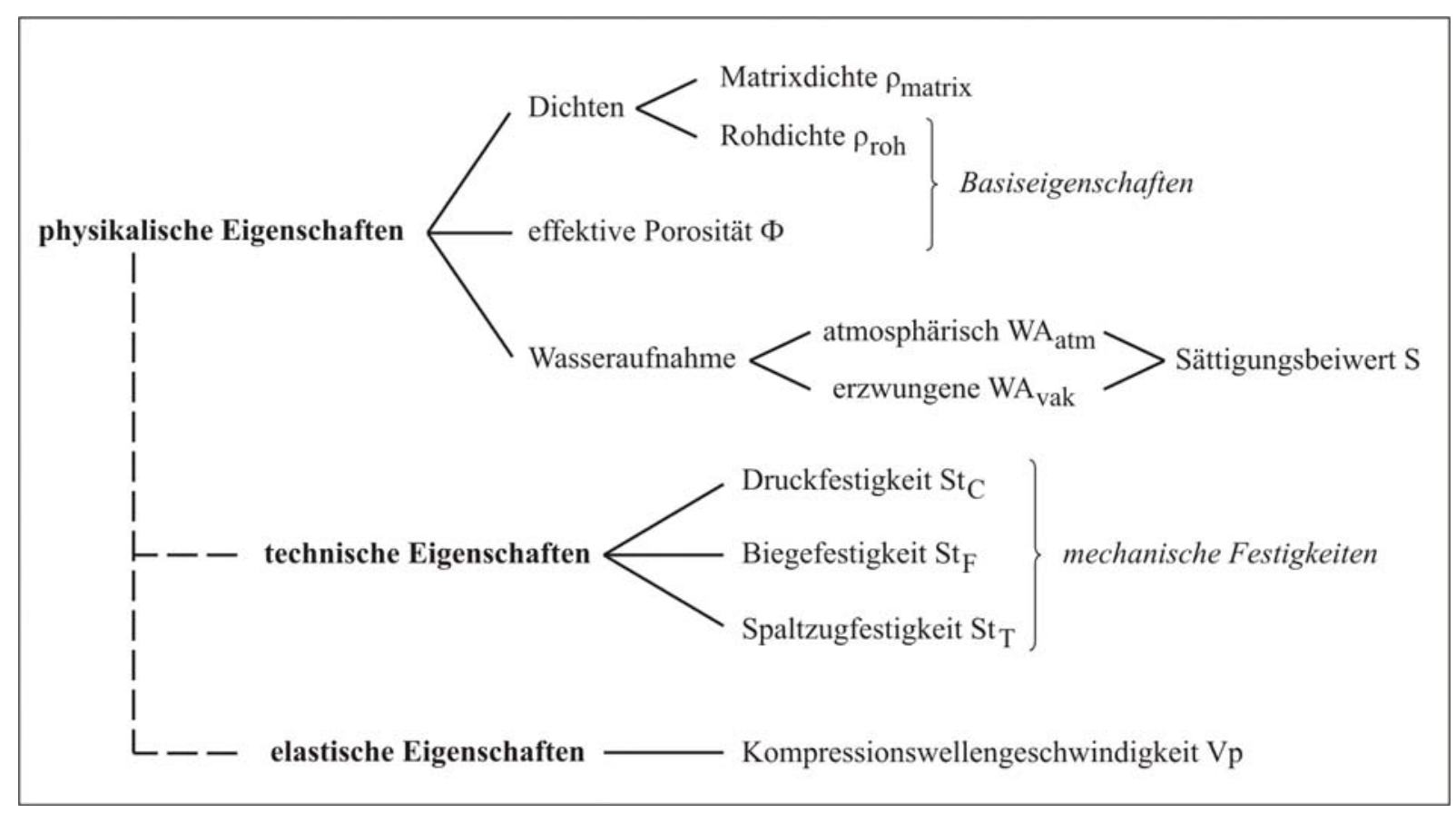

Abb. 3.2: In dieser Arbeit verwendete Nomenklatur für die verschiedenen Gesteinseigenschaften.

\subsection{Problematik der Vergleichbarkeit von Datensätzen}

Die Ermittlung der technischen Parameter erfolgt nach unterschiedlichen Richtlinien und Normen. Zwar sind die aktuellen deutschen (DIN) und besonders die europäischen (EN) Normen bereits weit verbreitet und etabliert, doch in vielen Ländern basieren die 
Versuchsdurchführungen auf anderen Richtlinien. Zum Teil finden sich leicht abweichende Angaben in den nationalen Standards, so z.B. in den Richtlinien der American Society for Testing and Materials (ASTM) oder der französischen Association Française de Normalisation (AFNOR). Für türkische Naturwerksteine liegen auch noch häufig Daten vor, deren Ermittlung auf Verfahren aus der Zeit des Deutschen Reiches aufbaut, und die somit zumindest in Teilen vermutlich nicht dem aktuellen Stand von Forschung und Technik entsprechen. Diese Differenzen können die Vergleichbarkeit der Daten beeinflussen. Peschel (1983) z.B. beschreibt eine Erniedrigung der Druckfestigkeit um 10 bis 20 \% bei Zylindern mit einem Schlankheitsgrad h/d $>2$ im Gegensatz zu einer Zylinderprobe mit einem Schlankheitsgrad von $\mathrm{h} / \mathrm{d}=1$, wie sie die aktuelle DIN-EN 1926 vorsieht. Weiterhin beeinflusst z.B. auch die Probenform die Druckfestigkeit. In der Regel werden bei der Würfeldruckfestigkeit höhere Werte erreicht als bei zylinderförmigen Proben.

Aus der zusammenfassenden statistischen Auswertung weltweit existierender Daten können daher aus verschiedenen Gründen Verzerrungen herrühren. So kann bereits aus dem unterschiedlichen Umfang von Datensätzen bestimmter Fundorte eine verstärkte Streuung hervorgehen (Büttgenbach 1990). Eine ebenso hohe Bedeutung kommt der Versuchsdurchführung, wie etwa der Belastungsgeschwindigkeit bei Festigkeitsprüfungen, zu. Auch die verschiedenen Laborbedingungen, unter denen die Messungen durchgeführt werden, können als ein Einflussfaktor angesehen werden. Gerade bei stärker porösen Gesteinen kann beispielsweise ein erhöhter Feuchtigkeitsgehalt zum Prozess der so genannten Erweichung führen, der eine Festigkeitsminderung nach sich zieht (Vilwock 1966, Peschel 1983, Morales Demarco et al. 2007).

Grundsätzlich ergeben sich somit schon oft beträchtliche Unterschiede bei der Betrachtung von Einzelprüfergebnissen nach den standardisierten Prüfverfahren. Die Streuung der Daten ist dabei einerseits auf die natürlichen Eigenschaften des Gesteins (Inhomogenität, Gefügeanisotropie) zurückzuführen, andererseits können auch systematische Fehler, also Abweichungen der apparativen und methodischen Versuchsbedingungen, eine entscheidende Rolle spielen (Rac \& Chernyshev 1970).

Eine weitere Problematik der Datensammlung, gerade aus Prospekten und dem Internet, besteht oftmals auch in der Erfassung und Aufbereitung der Daten für das jeweilige Gestein. So können oftmals zu einem Gestein in unterschiedlichen Quellen abweichende Angaben über seine technischen Eigenschaften gefunden werden, die in einfachsten Fällen auf Übertragungs- oder Übersetzungsfehler zurückzuführen sind (Abb. 3.3). Vor allem aber findet häufig die Anisotropie eines Gesteinsgefüges, die möglicherweise zu unterschiedlichen Werten bei der Ermittlung technischer Eigenschaften parallel und senkrecht zu einem Lagenbau o.ä. führt, bei der Präsentation der Daten keine Beachtung. Doch gerade im Bereich der Sedimentgesteine oder verschiedener metamorpher Gesteine kann die Gefügeanisotropie bedeutsame Abweichungen der technischen Eigenschaften bewirken (Strohmeyer 2003). Sie kann leicht Anlass zu einer Fehleinschätzung der möglichen Eignung oder Verwendung eines Gesteins sein. Im Rahmen der statistischen Auswertungen der hier zusammengestellten Daten konnte diesem Aspekt keine vollständige Beachtung geschenkt werden, da in den meisten Fällen lediglich Einzeldaten vorliegen. 
Methodisch kann die häufig vorgenommene Teilung einer einzelnen Großprobe in mehrere Teilproben zur Charakterisierung der physikalischen und technischen Parameter eines Gesteins eine schlechtere Alternative zu einer Anzahl repräsentativer, über das gesamte Vorkommen verteilter Proben sein (Reinsch 1991). Entscheidend dabei ist auch die unterschiedlich ausgebildete, lithologisch bedingte Heterogenität geologischer Körper. So können beispielsweise die technischen und physikalischen Eigenschaften eines Sandsteins im horizontalen Verlauf (also parallel zur Schichtung) über weite Strecken recht stabil sein, dagegen senkrecht zur Schichtung infolge von z.B. Material- und/oder Fazieswechseln einer relativ hohen Variabilität unterliegen.

Wenn jedoch die behandelte Datenmenge im statistischen Sinne ohne weiteres als eine Stichprobe der Grundgesamtheit angesehen werden kann, werden diese Probleme zumindest teilweise eliminiert. Weiterhin kann angenommen werden, dass bei Gesteinen, die aufgrund ihres Gefüges eine extreme Anisotropie hinsichtlich der mechanischen Festigkeitseigenschaften aufweisen, wie z.B. Schiefer, die minimalen Werte, wie sie beim Bruch parallel zur Schieferungsfläche auftreten, in der Datenzusammenstellung in den seltensten Fällen auftreten. Dies kann jedoch unberücksichtigt bleiben, da das spezielle Gesteinsgefüge die mögliche Nutzung des Gesteins von vornherein bestimmt.

\begin{tabular}{|c|c|}
\hline $\begin{array}{l}\text { CARATERISTICAS TÉCTNICAS } \\
\text { TECHNICAL CHARACTERISTICS } \\
\text { CARACTÉRITIQUES TECHNIQUES } \\
\text { TECHNISCHE EIGENSCHAFTEN }\end{array}$ & $\begin{array}{c}\text { RESULTADOS OBTENIDOS } \\
\text { RESULTS OBTAINED } \\
\text { RÉSULTATS OBTENUS } \\
\text { RESULTATE HERRSCHTEN VOR }\end{array}$ \\
\hline $\begin{array}{l}\text { Resistencia al desgaste / Resistance to wear } \\
\text { Réstistance à / 'usure / Widerstand gegen das Tragen }\end{array}$ & $2,8 \mathrm{~mm}$ \\
\hline $\begin{array}{l}\text { Coeficiente de absorción / Coefficient of absorption } \\
\text { Coefficient d'absorption / Koeffizient der absorption }\end{array}$ & $24,356 \mathrm{~g} / \mathrm{cm}^{2} \cdot \mathrm{s}^{0,5}$ \\
\hline $\begin{array}{l}\text { Resistancia a la compressión / Resistance to the compression } \\
\text { Résistance à la compression / Widerstand gegen die Verdichtung }\end{array}$ & $104 \mathrm{MPa}$ \\
\hline $\begin{array}{l}\text { Densidad real / Royal density } \\
\text { Densité royale / Königliche Dichte }\end{array}$ & $2747{\mathrm{~kg} / \mathrm{cm}^{3}}^{3}$ \\
\hline Porosidad / Porosity / Porosité / Porosität & $12,9 \%$ \\
\hline $\begin{array}{l}\text { Resitencia a la heladicidad / Ice resistance } \\
\text { Résistance au heladicidad / Widerstand gegen den heladicidad }\end{array}$ & $-0,1 \%$ \\
\hline $\begin{array}{l}\text { Resistencia a flexión / Flexural resistance } \\
\text { Résistance à flexion / Widerstand gegen Beugung }\end{array}$ & 4,5 $\mathrm{MPa}$ \\
\hline $\begin{array}{l}\text { Resistaencia al impacto / Resistance to the impact } \\
\text { Résistance à l'impact / Widerstand gegen den Einfluß }\end{array}$ & $45 \mathrm{~cm}$ \\
\hline
\end{tabular}

Abb. 3.3: Beispiel einer missverständlichen Ausweisung physikalischer und technischer Daten auf einem Muster eines italienischen Sandsteins aufgrund von Übersetzungsfehlern.

\subsection{Angewandte statistische Verfahren}

Insgesamt umfasst der vorliegende Datensatz rund 10.000 Einzeldaten zu den verschiedenen Gesteinseigenschaften. Um die einzelnen Parameter in ihrer Verteilung darzustellen, werden Box-Whisker-Plots (kurz: Boxplot) angewendet (Abb. 3.4 a). Die 
graphische Darstellung der „Häufigkeitsverteilung“ einer Variablen fasst Median, Spannweite und die Symmetrieeigenschaften der Verteilung (inkl. möglicher Ausreißer) in einer Grafik zusammen (z.B. Schlittgen 2000). Der verwendete Medianwert hat im Vergleich mit dem arithmetischen Mittel den Vorteil, robuster gegenüber statistischen Ausreißern zu sein, also geringeren Störungen durch Extremwerte zu unterliegen (Kürzl 1988). Durch die relative Lage des Medians innerhalb der Box wird auch gleichzeitig ein Eindruck über die Schiefe beziehungsweise Symmetrie der den Daten zugrunde liegenden Verteilung vermittelt (Abb. 3.4 b). Die Box, begrenzt durch den $25 \%$ - und 75 \%-Quartilwert, gilt ebenfalls als unabhängig von extremen Werten und stellt somit ein resistentes Streuungsmaß einer Datenmenge dar. Als „Whiskers“ werden die horizontalen Linien bezeichnet, für deren Abgrenzung es verschiedene Grundlagen gibt. Bei der geläufigsten und auch hier verwendeten Variante beträgt die Länge der Whiskers maximal das 1,5-fache des Interquartilsabstands (R Development Core Team 2005), wobei die Endpunkte immer durch Werte aus der Datenmenge gegeben werden. Darüber beziehungsweise darunter liegende Werte werden als Ausreißer bezeichnet.
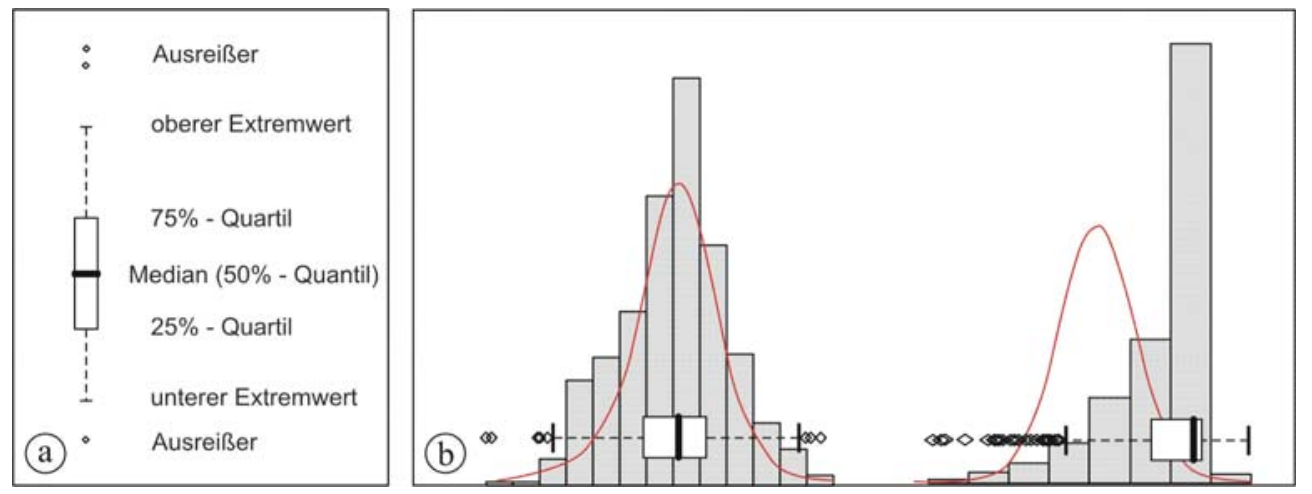

Abb. 3.4: a) Konstruktion der Boxplots zur explorativen Datenauswertung nach der Ermittlung statistischer Kenngrößen; b) Beispielhafte Darstellung der Anpassung einer Datenmenge an eine Normalverteilung.

Zur Darstellung der Korrelationen der Eigenschaftspaare werden primär Punktdiagramme (im Weiteren auch als Korrelationsdiagramme bezeichnet) genutzt. Zur besseren Übersicht wurde die Punktanzahl in einigen Darstellungen reduziert, nicht jedoch die Anzahl der den Berechnungen zugrunde liegenden Datenpaare. In diesen Fällen erfolgt eine entsprechende Ausweisung in der jeweiligen Legende ( $\mathrm{x}$ von $\mathrm{n}$ Daten: $\mathrm{x} / \mathrm{n}$ ). In den Beschreibungen dient der Korrelationskoeffizient $r$ (Bravais-Pearson-Korrelationskoeffizient, z.B. Bamberg \& Baur 2002), als Maßzahl für den wechselseitigen Zusammenhang zweier Merkmale. Die Berechnung erfolgt nach:

$$
\begin{gathered}
r=\frac{\sum_{i=1}^{n}\left(x_{i}-\bar{x}\right)\left(y_{i}-\bar{y}\right)}{\sqrt{\sum_{i=1}^{n}\left(x_{i}-\bar{x}\right)^{2} \sum_{i=1}^{n}\left(y_{i}-\bar{y}\right)^{2}}} \\
\text { mit } \quad x_{i}: \text { Einzeldaten } x_{1} \text { bis } x_{n} \\
\bar{x}: \text { arithmetisches Mittel des Merkmals } x \\
y_{i}: \text { Einzeldaten } y_{1} \text { bis } y_{n} \\
\bar{y}: \text { arithmetisches Mittel des Merkmals } y
\end{gathered}
$$


Der Korrelationskoeffizient ist auf einen Wertebereich von +1 bis -1 eingegrenzt. Dabei beschreibt ein positiver Wert einen deterministischen linearen Zusammenhang mit positivem Anstieg, ein negativer Wert entsprechend einen Zusammenhang mit negativem Anstieg (Abb. 3.5).
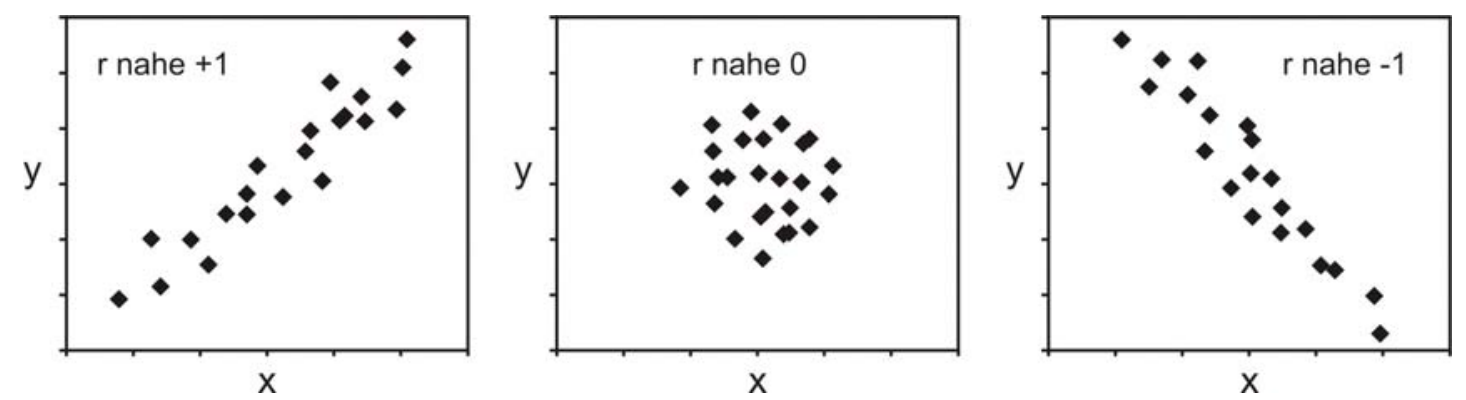

Abb. 3.5: Abhängigkeit des Bravais-Pearson-Korrelationskoeffizienten $r$ von der Ausbildung des Streu- / bzw. Punktdiagramms in der Korrelation zweier Parameter x und y.

Der Korrelationskoeffizient $r$, der zwei Merkmale symmetrisch behandelt, beschreibt somit lediglich die Stärke des Zusammenhangs zwischen diesen (Interdependenzanalyse),. Im Gegensatz dazu erlaubt der Regressionskoeffizient $b_{x y}$ eine Hypothese bezüglich eines korrelativen Zusammenhangs in eine Wirkungsrichtung zu bewerten (z.B. Bamberg \& Baur 2002). Eine Variable (traditionellerweise die $y$-Variable; Kriteriumsvariable) wird dabei als die von der $x$-Variablen (Prädiktorvariablen) abhängige betrachtet. Die Grundlage der linearen Regressionsrechung ist eine Regressionsgerade $\hat{y}_{i}$, welche die Lage der Punkte $x_{i} y_{i}$ in einem Diagramm bestmöglich repräsentiert. Mathematisch wird die Regressionsgerade durch

$$
\begin{gathered}
\hat{y}_{i}=a_{y x}+b_{x y} \cdot x_{i} \\
\text { häufig vereinfacht zu: } \quad \hat{y}=f(x)=a+b x
\end{gathered}
$$

beschrieben. Der Wert $a_{y x}$ beschreibt dabei die Stelle, an der die Regressionsgerade die y-Achse schneidet beziehungsweise die Eigenschaft $x$ den Wert 0 hat. Die Konstante $b_{y x}$ (Regressionskoeffizient) gibt die Steigung der Geraden und somit die Änderung der $y$-Variablen bei Änderung der $x$-Variablen an. Dieser Regressionskoeffizient $b_{x y}$ bestimmt sich nach dem Prinzip der kleinsten Fehlerquadrate aus:

$$
\begin{array}{cc}
\hat{b}=\frac{\sum_{i=1}^{n}\left(x_{i}-\bar{x}\right)\left(y_{i}-\bar{y}\right)}{\sum_{i=1}^{n}\left(x_{i}-\bar{x}\right)^{2}} \\
\text { mit } \quad x_{i}: \text { Einzeldaten } x_{1} \text { bis } x_{n} \\
\quad \bar{x}: \text { arithmetisches Mittel des Merkmals } x \\
y_{i}: \text { Einzeldaten } y_{1} \text { bis } y_{n} \\
\bar{y}: \text { arithmetisches Mittel des Merkmals } y
\end{array}
$$

Das Regressionsmodell erlaubt die Prognose einer Variablen $y$ aus einer Variablen $x$ unter der Voraussetzung des Vorliegens eines korrelativen Zusammenhanges zwischen diesen 
beiden Variablen. Die Qualität der Anpassung der Regressionsgeraden an die Verteilung der Datenpaare $x_{i} y_{i}$ wird durch das Bestimmtheitsmaß $\mathrm{R}^{2}$ (Wertebereich $0 \leq \mathrm{R}^{2} \leq 1$ ) beschrieben, welches sich aus der Quadrierung des Korrelationskoeffizienten $r$ ergibt. Je größer $\mathrm{R}^{2}$ ist, umso größer ist die „Erklärungskraft“ des ermittelten Regressionsmodells. $\mathrm{R}^{2}=1$ bedeutet folglich, dass alle Merkmalspaare auf dem entsprechenden Funktionsgraphen liegen.

In der Anpassung statistischer Analyseverfahren zur Prognose soll nach Möglichkeit Modellen linearen Charakters der Vorzug gegeben werden, da die lineare Regressionsanalyse aufgrund ihrer relativ einfachen Struktur als eines der am häufigsten verwendeten Analyseverfahren gilt (Hair et al. 1992, Backhaus et al. 1996). Bei einer linearen Regression kann zudem die Standardabweichung der dem Modell zugrunde liegenden Datenmenge zur Kriteriumsvariablen (hier: z.B. Parameter der Festigkeit) als Maß für die Streuung der geschätzten Anpassung betrachtet werden. Nichtlineare Modelle in Form exponentieller Anpassung (unter Einbeziehung der Eulerschen Zahl $e=2,71828 . .$.$) kommen lediglich in der$ Gruppe der Karbonatgesteine zur Anwendung, da hier häufig mit einer Veränderung der Prädiktorvariablen (hier: Rohdichte, Porosität, Vp) ein deutlich weiteres Datenspektrum hinsichtlich der in Abhängigkeit gesetzten zweiten Variablen zu beobachten ist. Hier ist jedoch eine mathematische Fehlerbereichserfassung nur schwer durchführbar, da dieser infolge der Logarithmierung des zu schätzenden Parameters niveauabhängig ausfällt. Daher sei zur Fehlerabschätzung auf die jeweiligen Diagrammdarstellungen verwiesen.

Unter der Annahme, dass die Daten der Basiseigenschaften sowie der verschiedenen Festigkeitseigenschaften annähernd normalverteilt sind, erfolgt unter Nutzung der Mittelwerte, der Standardabweichungen sowie der jeweiligen Korrelationskoeffizienten der entsprechenden Merkmalspaare die Berechnung bivariater Aufenthaltswahrscheinlichkeiten (z.B. Kreyszig 1977, Bortz 1985) für die theoretische Grundgesamtheit einzelner betrachteter Gesteinsgruppen. Die mathematische Herleitung ist detailliert von Büttgenbach (1990) gegeben, der die Abschätzung von Aufenthaltswahrscheinlichkeiten auf die Geschwindigkeits-Dichte-Relation in kristallinen Gesteinen anwendet. In dieser Arbeit werden für die graphische Darstellung jeweils Bereiche berechnet, innerhalb derer die betrachteten Parameterwerte der Grundgesamtheit mit einer Wahrscheinlichkeit von 80 \% zu finden sind (80\%-ige Aufenthaltswahrscheinlichkeit). Aus der verwendeten Anpassung an lineare Modelle resultieren in manchen Darstellungen nicht realisierbare Datenbereiche (z.B. negative Porositäten, Abb. 3.6), da einer der Parameter eine Lognormalverteilung aufweist. In diesen Fällen kann die entsprechende Datenreihe durch eine Logarithmierung einer Normalverteilung (Transformation) angepasst werden, worauf dann die Berechnung der Aufenthaltswahrscheinlichkeiten erfolgt. Bei der Rücktransformation der berechneten Datenpunkte zur Darstellung der Ellipse und der Einpassung in das ursprüngliche Korrelationsdiagramm ergeben sich deformierte, zweidimensionale Flächen. Diese Methode stellt ein in der Statistik standardmäßig verwendetes Verfahren dar, bei dem der zu beschreibende Wertebereich keine Einschränkungen oder Veränderungen erfährt (pers. Mitt. Dr. O. Nenadić).

Für die statistische Bearbeitung der Daten wurde das Statistikpaket „R“ (R Development Core Team 2005) verwendet. 

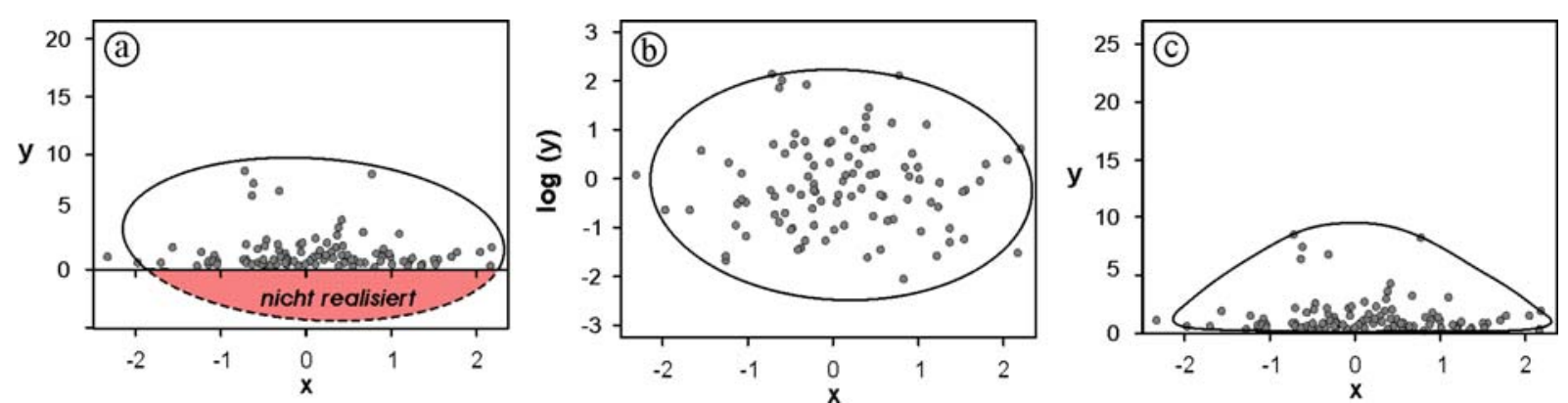

Abb. 3.6: Exemplarische Darstellung des bivariaten Aufenthaltswahrscheinlichkeitsbereichs der Parameter $\mathrm{x}$ (normalverteilt, z.B. Druckfestigkeit) und y (lognormalverteilt, z.B. Porosität): a) Das primär auf der Annahme einer bivariaten Normalverteilung errechnete Ellipsoid erstreckt sich über nicht realisierte Datenbereiche (hier: negative Porosität). b) Durch die Logarithmierung der Datenreihe y wird diese in eine Normalverteilung eingepasst und c) das so errechnete Ellipsoid durch Rücktransformation angepasst.

\subsection{Generelle Verteilungsmuster in den Hauptgesteinsgruppen}

\subsubsection{Basiseigenschaften Rohdichte und Porosität}

Die Rohdichte $\rho_{\text {roh }}$ und die effektive Porosität $\Phi$ beschreiben das Gesamtvolumen eines Gesteins, also Mineraldichten und Hohlraumanteil, und gelten als Standardparameter zur Charakterisierung von Gesteinen. Viele physikalische und technische Eigenschaften, wie z.B. die Wasseraufnahme oder auch Festigkeiten, stehen in einer engen Beziehung zu den Basiseigenschaften. Die Matrixdichte $\rho_{\text {matrix }}$ (Reindichte) dagegen bezieht sich lediglich auf die spezifische Mineraldichte der gesteinsbildenden Komponenten beziehungsweise deren Volumenanteile und schließt den Porenraum aus. So können beispielsweise calcitische und dolomitische Marmore basierend auf der spezifischen Mineraldichte von Calcit und Dolomit unterschieden werden. Nach DIN-EN 1936 errechnen sich Rohdichte, Matrixdichte und Porosität nach:

$$
\begin{array}{rll}
\rho_{\text {roh }}=\frac{m_{d}}{m_{s}-m_{h}} & \multicolumn{1}{c}{\rho_{\text {matrix }}=\frac{m_{d}}{m_{d}-m_{h}} \quad \Phi=\frac{m_{s}-m_{d}}{m_{s}-m_{h}} \cdot 100} \\
\text { mit } \quad \rho_{\text {roh }}= & \text { Rohdichte }\left[\mathrm{g} / \mathrm{cm}^{3}\right] \\
\rho_{\text {matrx }}= & \text { Matrix- (Rein-)dichte }\left[\mathrm{g} / \mathrm{cm}^{3}\right] \\
\Phi & = & \text { Porosität [Vol.-\%] } \\
\mathrm{m}_{\mathrm{s}}= & \text { Masse des wassergesättigten Probekörpers } \\
\mathrm{m}_{\mathrm{d}}= & \text { Trockenmasse des Probekörpers } \\
\mathrm{m}_{\mathrm{h}}= & \text { Masse des in Wasser eingetauchten Probekörpers } \\
& & \text { (Auftriebsgewicht) }
\end{array}
$$

Die Rohdichten und Porositäten der hier behandelten Gesteine spiegeln im Allgemeinen gruppentypische Wertebereiche, dargestellt durch die 50 \%-Boxen, wider (Abb. 3.7). Größere Boxen und damit größere Streubereiche finden sich jeweils bei den Vulkaniten, Sandsteinen und Karbonatgesteinen. Auffällig ist die deutliche Schiefe in den gruppeninternen 
Verteilungen der Plutonite und der Karbonatgesteine. Dabei wird ein Teil der Daten innerhalb der jeweiligen Gruppe als Ausreißer identifiziert. Im Falle der Plutonite $\left(\varnothing \rho_{\text {roh }}=2,68 \mathrm{~g} / \mathrm{cm}^{3}\right)$ beziehen sich diese auf die Gabbros und Diorite, die mit ihrer höheren Dichte $\left(\varnothing \rho_{\text {roh }}=2,88\right.$ $\left.\mathrm{cm}^{3}\right)$ aus der Gruppe ausscheren. Bei den Karbonatgesteinen $\left(\varnothing \rho_{\text {roh }}=2,55 \mathrm{~g} / \mathrm{cm}^{3}\right)$ fallen Gesteine mit einer geringeren Dichte und auch solche einer geringeren Porosität aus der Gruppe heraus. Der Vergleich beider Gruppen verdeutlicht, dass sich das Hohlraumvolumen im Falle der Plutonite und auch Metamorphite nur gering auf die Rohdichte auswirkt und somit diese primär über den Mineralbestand kontrolliert wird. Die Porosität liegt hier zumeist als feine Rissporosität vor und übt in seiner Volumengesamtheit einen nur untergeordneten Einfluss auf die Rohdichte aus (Abb. 3.8, Strohmeyer 2003). Bei den sedimentären Gesteinen ist eine deutliche Abnahme der Rohdichte mit zunehmendem Hohlraumanteil zu verzeichnen.
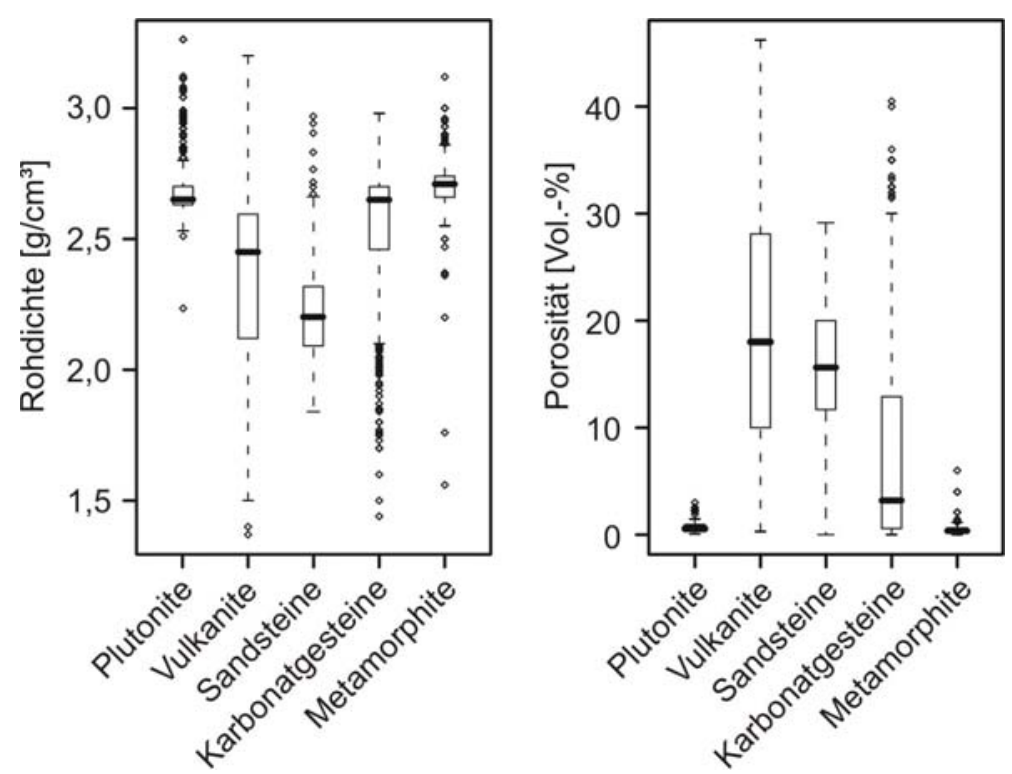

Abb. 3.7: Rohdichten und Porositäten der fünf Hauptgesteinsgruppen.

Durch diese verschiedenartige Einflussnahme der Porosität auf die Rohdichte können auch die sehr weiten Datenspektren in der Gruppe der Vulkanite $\left(\varnothing \rho_{\text {roh }}=2,25 \mathrm{~cm}^{3}\right.$; $\varnothing \Phi=22,43$ Vol.-\%) erklärt werden. Die Dichtewerte liegen hier bei $1,37 \mathrm{~g} / \mathrm{cm}^{3}$ bei einer Porosität von 46,21 Vol.-\% (WEIBERNER TUFF) einerseits und bei 3,20 g/ $\mathrm{cm}^{3}$ bei einer Porosität von 0,48 Vol.-\% (Basalt, SS FEIN, Schweden) andererseits. Sandsteine zeigen im Allgemeinen eine ausgeglichene Verteilung der Dichtewerte zwischen 1,69 g/cm ${ }^{3}$ und 2,96 g/cm³ (Ø 2,23 g/ $\mathrm{cm}^{3}$ ) bei Porositäten zwischen 30 Vol.-\% und annähernd 0,10 Vol.-\% (Ø 15,73 Vol.-\%).

Diese Relationen zwischen der Rohdichte und der Porosität drücken sich auch in den Korrelationskoeffizienten für den linearen Zusammenhang aus. Bei Plutoniten und Metamorphiten fällt dieser sehr gering aus, wohingegen die Koeffizienten in den verbleibenden Gesteinsgruppen einen deutlich ausgeprägten Zusammenhang widerspiegeln (s. Tab. in Abb. 3.8). 


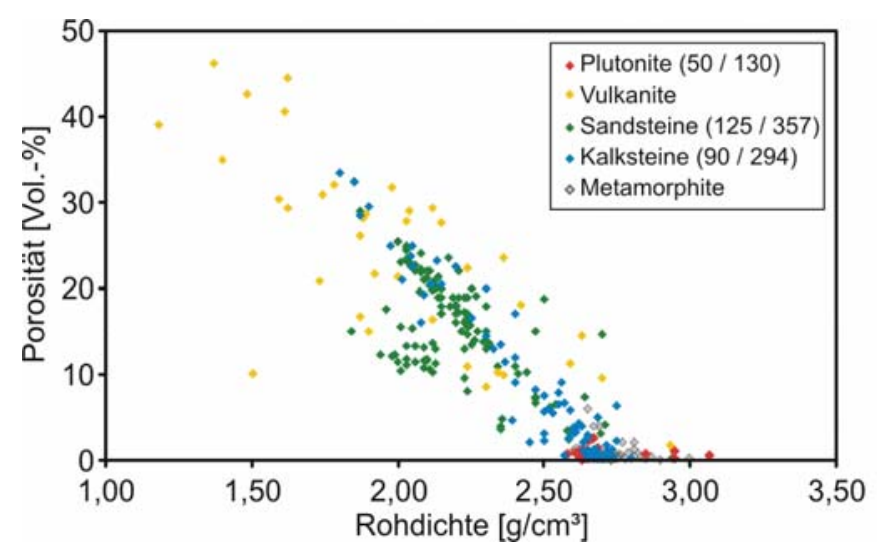

\begin{tabular}{lcc}
\hline & \multicolumn{2}{c}{ linearer Korrelationskoeffizient r } \\
& für $\rho_{\text {roh }} / \Phi$ & $n$ \\
\hline Plutonite & $-0,162$ & 130 \\
Vulkanite & $-0,655$ & 39 \\
Sandsteine & $-0,619$ & 357 \\
Karbonatgesteine & $-0,923$ & 294 \\
Metamorphite & $-0,136$ & 87 \\
\hline
\end{tabular}

Abb. 3.8: Korrelationsdiagramm der Parameter Rohdichte $\rho_{\text {roh }}$ und Porosität $\Phi$ mit den Korrelationskoeffizienten für den jeweiligen linearen Zusammenhang.

\subsubsection{Technische Eigenschaften Druck-, Biege- und Spaltzugfestigkeit}

Die technischen Eigenschaften eines Gesteins sind bei der Beurteilung der Einsatzmöglichkeit zur Anwendung als Baustoff von elementarer Bedeutung. Dabei ist die mechanische Stabilität primär vom oft sehr heterogenen Gefügeverband der Gesteine und weniger von den individuellen Eigenschaften der beteiligten Mineralkomponenten abhängig.

Abbildung 3.9 beschreibt die Wertebereiche der drei wesentlichen Festigkeitseigenschaften (Druck- $\quad\left(\mathrm{St}_{\mathrm{C}}\right)$, Biege $\left(\mathrm{St}_{\mathrm{F}}\right)^{-}$, Spaltzugfestigkeit $\left(\mathrm{St}_{\mathrm{T}}\right)$ ) für die verschiedenen Hauptgesteinsgruppen. Die jeweiligen Datenmengen zeigen zumeist eine symmetrische Verteilung, wobei hinsichtlich der Druck- und Biegefestigkeit die Gruppe der Sandsteine die geringsten Werte aufweist. Bemerkenswert ist dabei, dass hier auch relativ geringe Datenspannweiten auftreten. Für die Spaltzugfestigkeit ergeben sich für Plutonite und Metamorphite vergleichbare Medianwerte, wohingegen für die Gruppe der Sandsteine und Karbonatgesteine eine deutlich geringere Spaltzugfestigkeit festzustellen ist. Die im Mittel geringsten Werte sind in der Gruppe der Karbonatgesteine beschrieben, was mit der generell guten Spaltbarkeit der Karbonatkristalle in Verbindung gebracht werden kann.

Ausreißer sind, mit Ausnahme der Gruppe der Vulkanite, für alle weiteren Gruppen in unterschiedlicher Anzahl vorhanden. Primär kann die Ausweisung verschiedener Extremwerte möglicherweise auch mit einer fehlerhaften Datenauswertung (Ausweisung einzelner Extremwerte als geltender Eigenschaftswert) oder aber auch mit der Auswirkung eines extremen Richtungsgefüges in Verbindung gebracht werden.

Hinsichtlich der Biegefestigkeit in der Gruppe der Metamorphite werden, mit einer Ausnahme, alle Werte über $40 \mathrm{MPa}$ von Schiefern erreicht, die zumeist eine z.T. extrem ausgeprägte Gefügeanisotropie aufweisen. Die Ausnahme wird hier durch einen italienischen Quarzit (BARGIOLINA) gestellt, der mit einem Wert von $\mathrm{St}_{\mathrm{F}}=40,5 \mathrm{MPa}$ aber noch durchaus in die angegebenen Wertebereiche verschiedener Quellen (Peschel 1983, Müller 2001) eingefügt werden kann. 

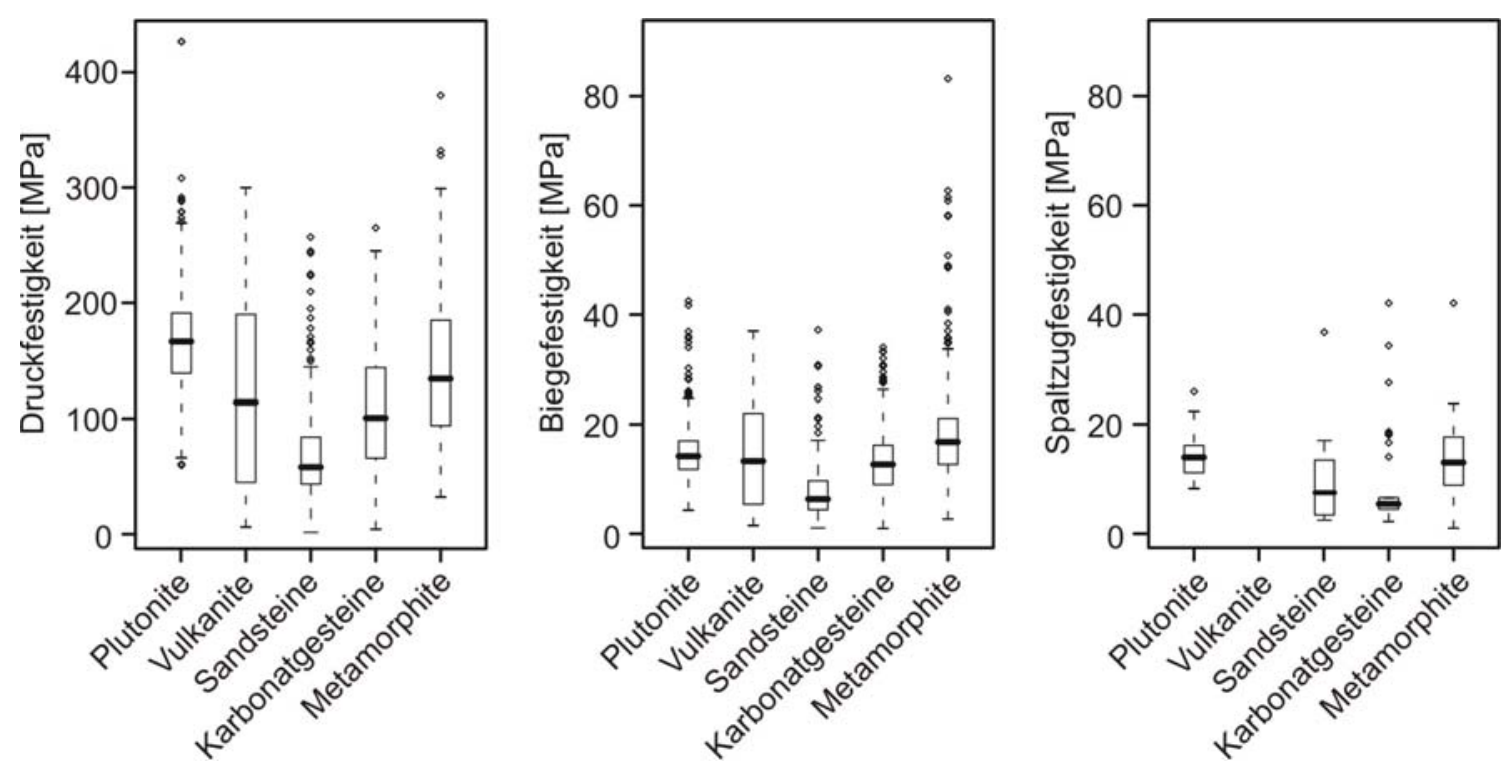

Abb. 3.9: Druck- (links), Biege- (Mitte), und Spaltzugfestigkeit (rechts) der fünf Hauptgesteinsgruppen.

In Abbildung 3.10 sind die Korrelationen der drei möglichen Eigenschaftspaare der hier betrachteten Festigkeitsparameter dargestellt. Zahlreiche Quellen verweisen auf die Beziehungen mechanischer Festigkeiten untereinander. So beschreibt Hirschwald (1912) ein relativ gleich bleibendes Verhältnis von etwa 1 : 10 bis 15 zwischen der direkten Zugfestigkeit und der Druckfestigkeit. Untersuchungen zur Richtungsabhängigkeit technischer Gesteinseigenschaften (Strohmeyer 2003) ergaben, dass die im Allgemeinen linearen Verknüpfungen zwischen den Festigkeitsparametern besonders bei Betrachtung der Mittelwerte aller gefügeorientierten Probekörper durch ein hohes Bestimmtheitsmaß ausgezeichnet sind. Durch die Verwendung der Mittelwerte wird praktisch der Einfluss der Gefügeanisotropie auf das statistische Verhalten der jeweiligen Eigenschaften verringert, und somit das Bestimmtheitsmaß R² größer. Entsprechend wird bei einer separaten Betrachtung richtungsorientierter Körper einer Probe die Relevanz des Gefüges durch eine Herabsetzung des Bestimmtheitsmaßes entsprechender Korrelationen deutlich.

In allen drei hier dargestellten Beziehungen wird ein mehr oder weniger gleich bleibendes Verhältnis der Festigkeiten zueinander deutlich. Für den Zusammenhang zwischen der Druckund der Biegefestigkeit ergibt sich in der Gesamtheit ein Verhältnis von etwa $10: 1$. Hinsichtlich der Verhältnisse zwischen der Druck- und der Spaltzugfestigkeit zeichnet sich ein vergleichbares Bild ab, wobei hier aber eine deutlich geringere Datenbasis zur Verfügung steht (Abb. 3.3.4). In der Korrelation zur Biegefestigkeit zeigt sich bei den Metamorphiten im Druckfestigkeitsbereich bis etwa 180 MPa eine etwas abweichende Datenverteilung, die eher einen Trend zu einem geringeren Verhältniswert angibt. Auf dieses Phänomen wird in Kapitel 3.9 näher eingegangen.

Übereinstimmend mit eigenen Beobachtungen nennt Peschel (1983) für das Verhältnis zwischen der Biege- und der Spaltzugfestigkeit einen Faktor von etwa 2 bis 2,5 : 1 für Naturwerksteine im Allgemeinen. Gruppenspezifisch zeigen die hier betrachteten Daten dabei für Plutonite und Metamorphite ein Verhältnis von etwa $1: 1$, Karbonatgesteine dagegen ein Verhältnis von rund $3: 1$. Es ist anzunehmen, dass dieser erhöhte Faktor aus der erhöhten 
Spaltbarkeit der Karbonatkristalle resultiert. Aussagen über die verbleibenden Gesteinsgruppen können hier infolge des zu geringen Datenaufkommens über Spaltzugfestigkeiten nicht getroffen werden.
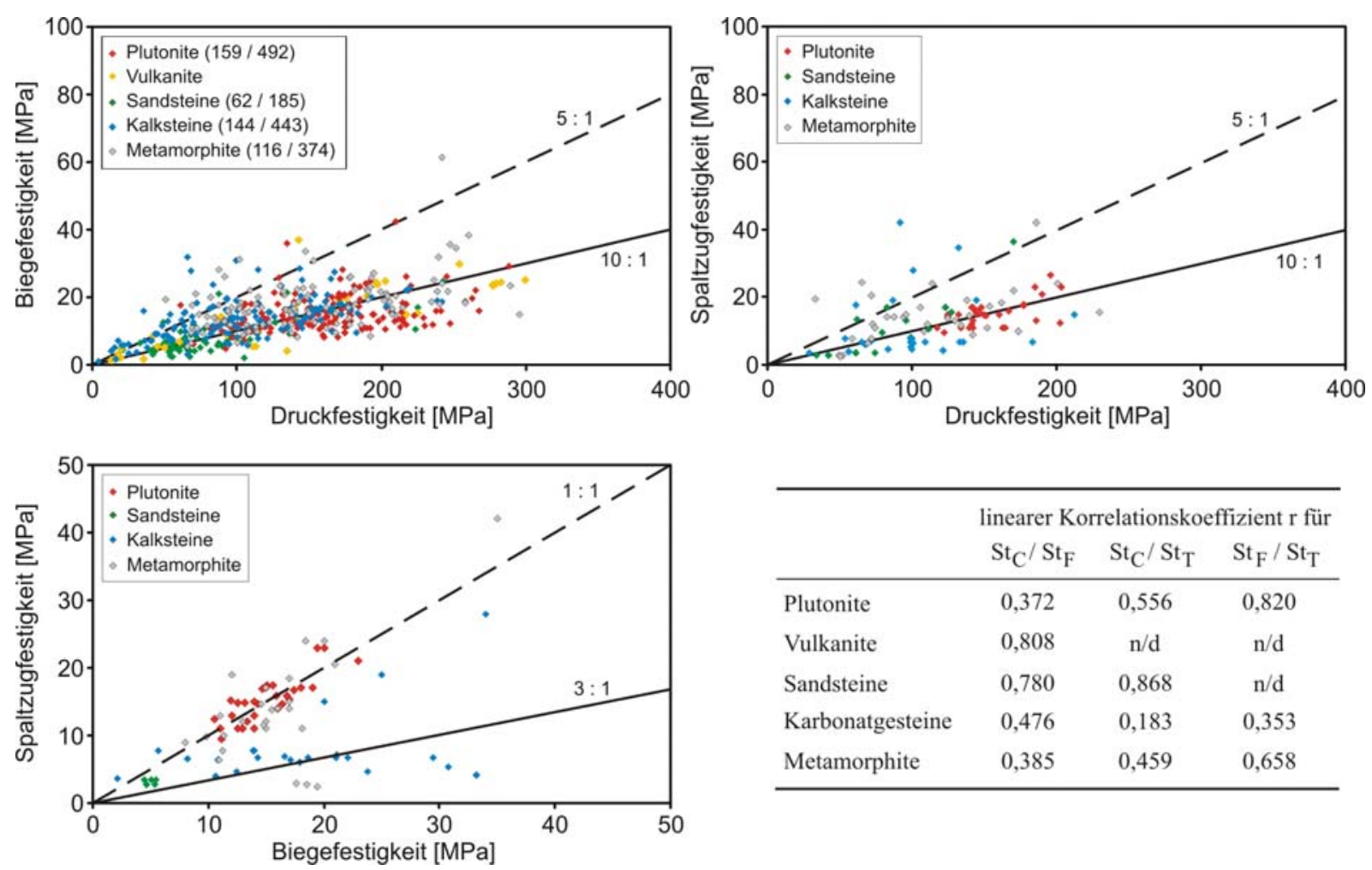

\begin{tabular}{lccc}
\hline & \multicolumn{3}{c}{ linearer Korrelationskoeffizient $\mathrm{r}$ für } \\
& $\mathrm{St}_{\mathrm{C}} / \mathrm{St}_{\mathrm{F}}$ & $\mathrm{St}_{\mathrm{C}} / \mathrm{St}_{\mathrm{T}}$ & $\mathrm{St}_{\mathrm{F}} / \mathrm{St}_{\mathrm{T}}$ \\
\hline Plutonite & 0,372 & 0,556 & 0,820 \\
Vulkanite & 0,808 & $\mathrm{n} / \mathrm{d}$ & $\mathrm{n} / \mathrm{d}$ \\
Sandsteine & 0,780 & 0,868 & $\mathrm{n} / \mathrm{d}$ \\
Karbonatgesteine & 0,476 & 0,183 & 0,353 \\
Metamorphite & 0,385 & 0,459 & 0,658 \\
\hline
\end{tabular}

Abb. 3.10: Korrelationsdiagramme der technischen Gesteinseigenschaften $\left(\mathrm{St}_{\mathrm{C}}=\mathrm{Druck}_{\mathrm{r}}, \mathrm{St}_{\mathrm{F}}=\mathrm{Biege}, \mathrm{St}_{\mathrm{T}}=\right.$ Spaltzugfestigkeit) untereinander sowie die Korrelationskoeffizienten für den jeweiligen linearen Zusammenhang in den fünf Hauptgesteinsgruppen. Zusätzlich sind idealisierte Trends dargestellt.

\subsubsection{Basiseigenschaften in Korrelation mit mechanischen Festigkeiten}

Im Allgemeinen werden niedrige Rohdichten mit höheren Porositäten verknüpft. Gleichzeitig werden hohe Rohdichten mit hohen Werten für die Festigkeitseigenschaften in Verbindung gebracht (u.a. Peschel 1983). Gegenläufig verhält sich grundsätzlich lediglich der Masseverlust durch abrasive Kräfte. Während die hier behandelten statischen Festigkeitseigenschaften auf das bruchhafte Versagen eines Gesteins bei Überschreitung einer bestimmten Maximalkraft basieren, ist der Versagensmechanismus der Abriebsfestigkeit eher in einem komplexen Zusammenwirken aus statischen und dynamischen Kräften zu begründen, deren Mechanismen bisher nur ansatzweise erkannt werden konnten (Strohmeyer 2003).

Die Annahmen bezüglich der Zusammenhänge zwischen den Basiseigenschaften und den Festigkeitsparametern bestätigen sich weitestgehend in den Punktverteilungen der Abbildung 3.11. Dabei zeigen die verschiedenen Punktwolken gruppenspezifische Unterschiede. Für Gesteine, die eine hohe Variabilität in der Rohdichte beziehungsweise der Porosität aufweisen, ist ein Trend erkennbar. Dagegen ist für Plutonite und Metamorphite bei relativ geringen Wertebereichen der Rohdichte und der Porosität jeweils eine große Spannweite der technischen Eigenschaften ausgewiesen. Die unterschiedlichen Verteilungsmuster der 
verschiedenen Punktwolken sollen in den folgenden gruppenbezogenen Kapiteln näher betrachtet werden.
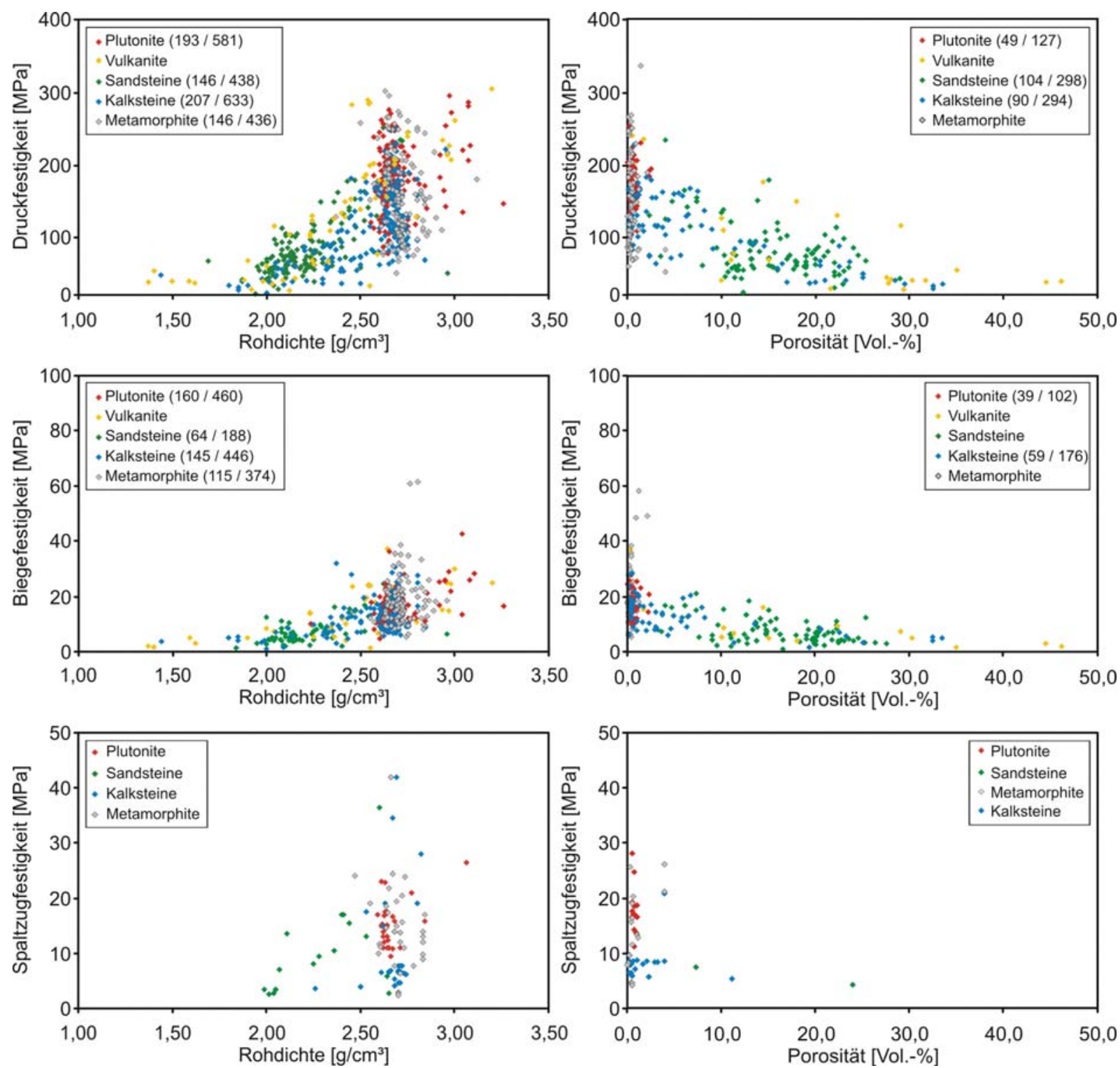

Abb. 3.11: Korrelationsdiagramme der Basiseigenschaften Rohdichte bzw. Porosität und den technischen Eigenschaften Druck-, Biege- und Spaltzugfestigkeit in den fünf Hauptgesteinsgruppen.

\subsection{Gruppe der plutonischen Magmatite}

Häufig typisch für Plutonite ist eine gleichmäßige, meist xenomorphe Struktur, wobei die Korngröße von der Kristallisationsgeschwindigkeit abhängig ist. Verschiedene Plutonite weisen eine porphyrische Struktur auf, die durch große, bevorzugt idiomorphe Phänokristalle (häufig Feldspat) in einer relativ feinkörnigeren Matrix gekennzeichnet ist. Vielfach ist keine Vorzugsorientierung der mineralischen Bestandteile erkennbar, so dass das Gestein massig erscheint. Daher wurde früher für diese Gesteinsklasse auch der Begriff „Massengestein“ verwendet (Wimmenauer 1985). Doch auch in makroskopisch richtungslosen Gesteinen kann eine kristallographische Vorzugsorientierung, so z.B. durch eine parallele Einregelung von 
Glimmern, ausgebildet sein, woraus auch richtungsbedingte Anisotropien im physikalischen und mechanischen Verhalten der Materialien resultieren können.

Die hier betrachteten plutonischen Magmatite wurden nach der Streckeisen-Klassifikation (Streckeisen 1974, 1980) in vier Untergruppen unterteilt: /Granit/ (Felder 2-3), /Granodiorit, Tonalit/ (Felder 4-5), /Monzonit, Syenit/ (Felder 6-8), /Gabbro, Diorit/ (Felder 9-10) (Abb. 3.12).
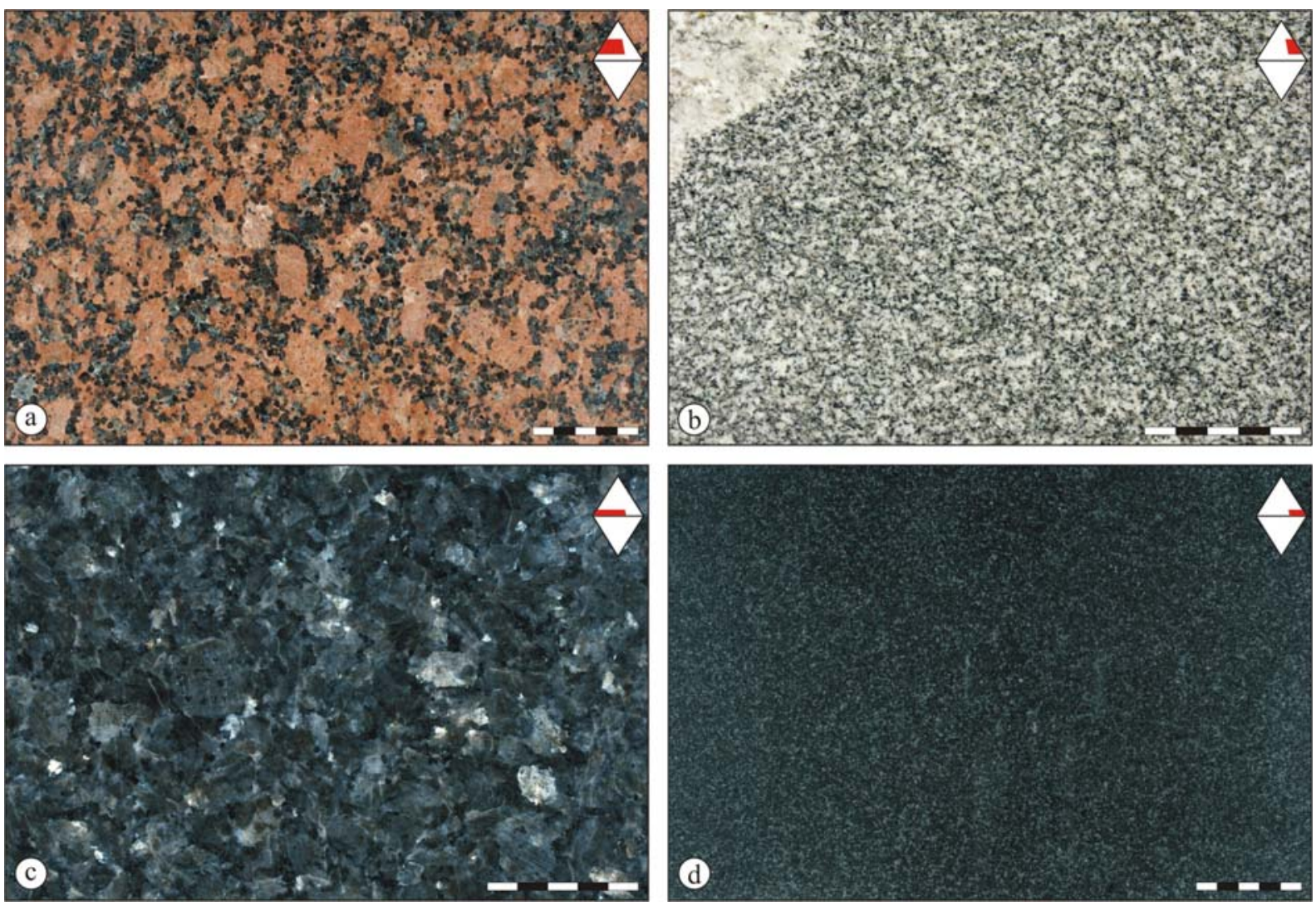

Abb. 3.12: Varietäten plutonischer Naturwerksteine zu den vier definierten Untergruppen: a) RED BALMORAL (Finnland), Granit; b) CEZLAK GRANODIORITE (Slowenien); c) BLUE PEARL DUNKEL (Norwegen), Larvikit/Syenit; d) NEGRO ORIENTAL (Uruguay), Gabbro/Dolerit (polierte Oberflächen, Maßstäbe in cm).

\subsubsection{Basiseigenschaften Rohdichte und Porosität}

Die Porositäten der plutonischen Gesteine liegen, exklusive der ermittelten Ausreißer, im Bereich zwischen 0,05 und 1,65 Vol.-\% (Abb. 3.13). Diese geringen Beträge resultieren daraus, dass es sich weitestgehend um Rissporositäten handelt, die in der Gesamtheit lediglich ein geringes Volumen ergeben (vgl. Kap. 3.4.1). Die Rohdichten der hier behandelten Gesteine belaufen sich exklusive weniger Ausreißer auf Werte von 2,55 g/cm bis 3,26 g/ $/ \mathrm{cm}^{3}$. Die Aneinanderreihung der Boxplots (Abb. 3.13) zeigt, dass bezüglich der Daten eine Zweiteilung möglich ist. Überschneiden sich die zentralen Segmente der Boxplots nicht, so sind die entsprechenden Verteilungen auf dem 5 \%-Niveau signifikant unterschiedlich (Popp 1994). Somit können die drei Untergruppen /Granit/, /Granodiorit, Tonalit/ und /Monzonit, Syenit/ aus statistischer Sicht zusammengefasst betrachtet werden. 

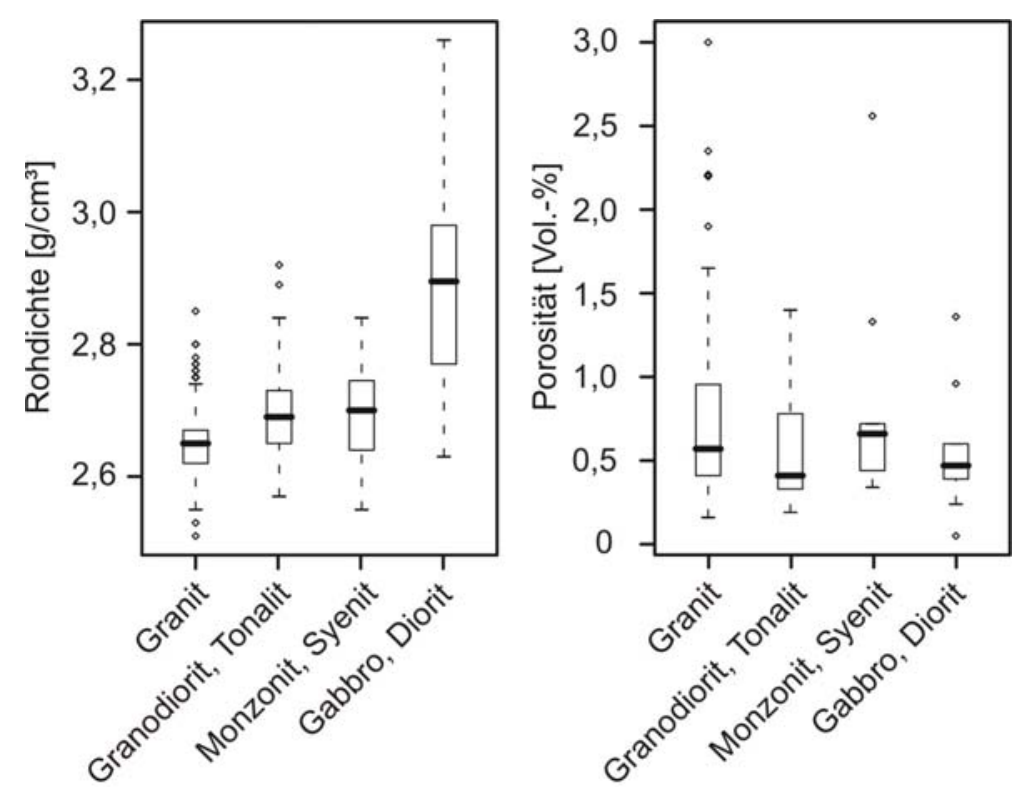

Abb. 3.13: Rohdichten und Porositäten in den Untergruppen der plutonischen Gesteine.

Basierend darauf, dass die Rohdichte im Bereich der hochkompakten Gesteine primär durch die mineralogische Zusammensetzung und lediglich stark untergeordnet durch die Gesamtporosität beeinflusst wird, ist die Korrelation der Basiseigenschaften nur sehr schwach ausgebildet (Abb. 3.14). Deutlich wird dies an einem exemplarischen Vergleich der Handelssorten KÖSSEINE (Granit, Deutschland, $\rho_{\text {roh }}=2,67 \mathrm{~g} / \mathrm{cm}^{3}, \Phi=0,17$ Vol.-\%, Daten aus Strohmeyer 2003) und BAN TAK BLACK (Diorit, Thailand, $\rho_{\text {roh }}=2,87$, $\Phi=0,39$ Vol.-\%, Daten aus Hoffmann 2006). Die relative höhere Rohdichte der thailändischen Varietät geht hier lediglich auf einen Amphibolanteil von 24 Vol.-\% am Mineralbestand zurück. Der Granit besteht dagegen aus dominant Quarz und Feldspat sowie einer Beimengung von rund 10 Vol.-\% Biotit, weist aber insgesamt eine kompaktere Struktur auf, die sich in einer relativ geringeren Porosität äußert.

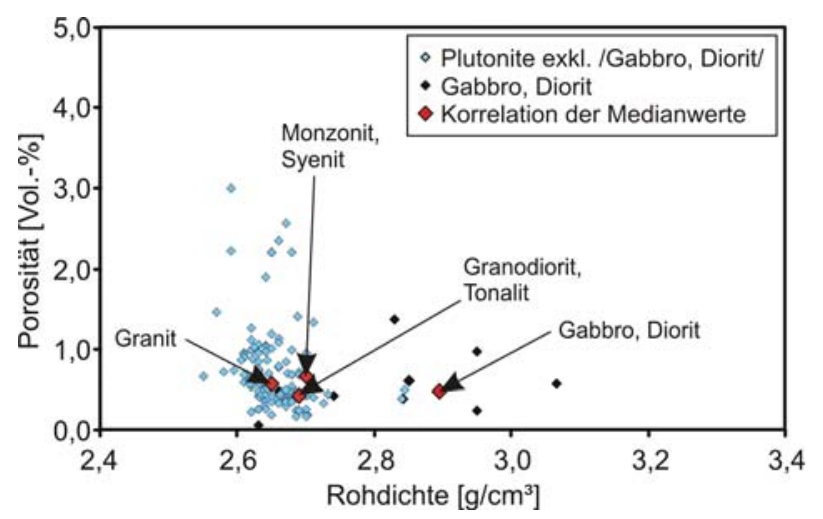

\begin{tabular}{lcc}
\hline & linearer Korrelationskoeffizient r \\
& für $\rho_{\text {roh }} / \Phi$ & $n$ \\
\hline Plutonite gesamt & $-0,162$ & 129 \\
Plutonite exkl. /Gabbro, Diorit/ & $-0,250$ & 120 \\
Gabbro, Diorit & 0,214 & 9 \\
\hline
\end{tabular}

Abb. 3.14: Korrelationsdiagramm der Parameter Rohdichte $\rho_{\text {roh }}$ und Porosität $\Phi$ sowie die Korrelationskoeffizienten für den jeweiligen linearen Zusammenhang für die Untergruppen der Plutonite.

\subsubsection{Technische Eigenschaften Druck-, Biege- und Spaltzugfestigkeit}

Die einaxialen Druckfestigkeiten liegen in einem Wertebereich von $60 \mathrm{MPa}$ bis 292,3 MPa (Abb. 3.15), womit ein großer Teil der für plutonische Naturwerksteine dokumentierten Druckfestigkeitsdaten abgedeckt wird (Peschel 1983, Winkler 1994, Müller, 2001). Ein noch 
höherer Wert von 427,7 MPa wird für den Gabbro AFRICAN BLACK (Südafrika) angegeben, wobei dieser Wert jedoch als fragwürdig, auf jeden Fall aber als extremer Ausreißer einzustufen ist. Die Biegefestigkeiten werden mit Werten im Bereich von 5,1 MPa bis 30,23 MPa dokumentiert (Abb. 3.15). Auch hier zeigen sich extreme Ausreißer mit Maximalwerten bis über $40 \mathrm{MPa}$, wie sie auch in manchen Quellen genannt werden (z.B. Peschel 1983). Bei anderen Autoren (z.B. Müller 2001) werden Biegefestigkeiten > $30 \mathrm{MPa}$ lediglich kristallinen Schiefern zugeordnet.

Auffällig ist, dass die Gruppe /Gabbro, Diorit/ bezüglich beider Festigkeitseigenschaften den größten Interquartilsabstand aufweist, was auf eine weite Streuung der Daten im Vergleich zu den übrigen Gruppen hindeutet. Gleiches ist auch bezüglich der Rohdichte zu beobachten (Abb. 3.13). Für die Spaltzugfestigkeiten der Gesteine (Abb. 3.15) stehen relativ wenige Daten zur Verfügung. Diese sind vom Betrag her mit den Werten zur Biegefestigkeit vergleichbar.
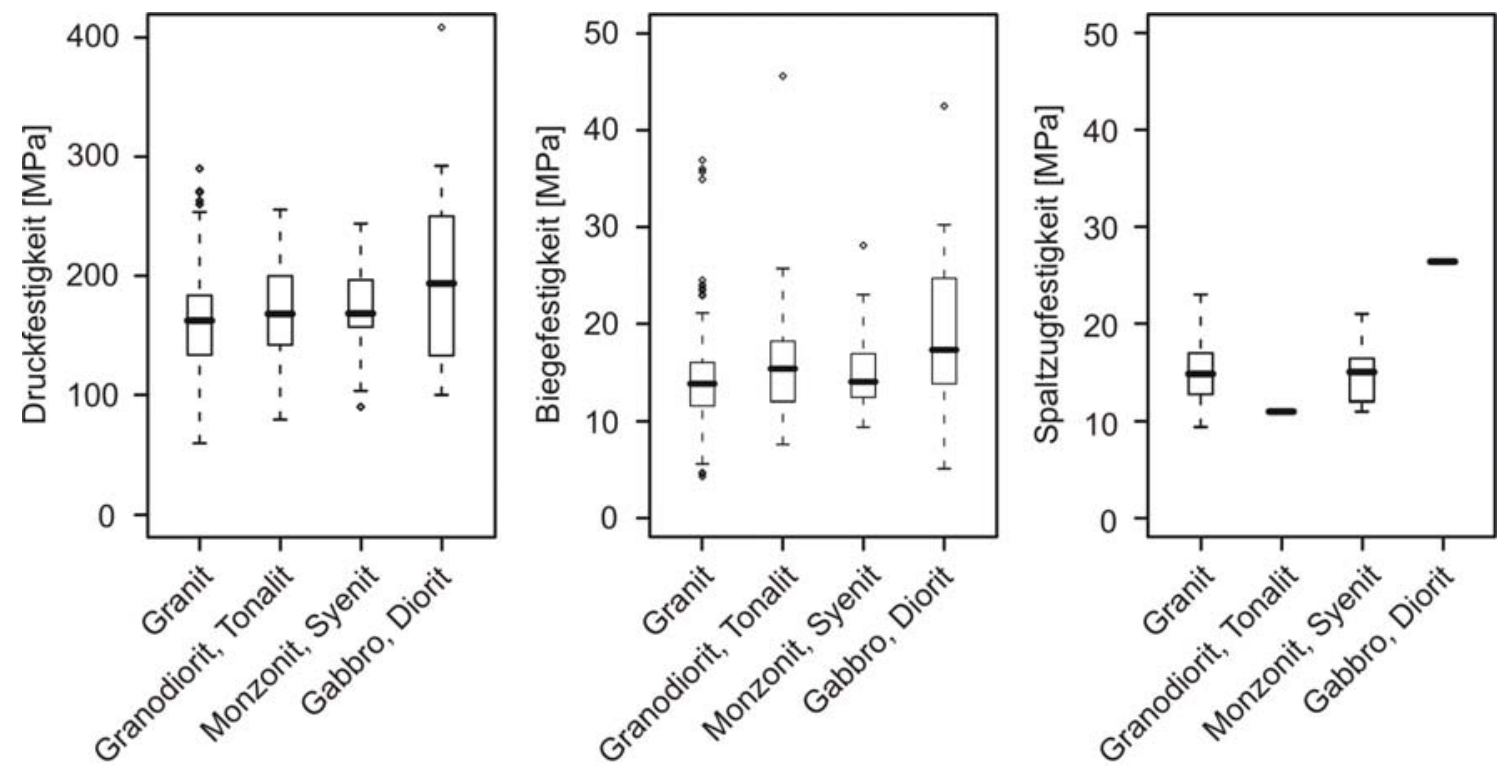

Abb. 3.15: Druck- (links), Biege- (Mitte), und Spaltzugfestigkeiten (rechts) in den Untergruppen der Plutonite. Infolge des sehr geringe Datenaufkommen hinsichtlich der Spaltzugfestigkeit in den Untergruppen /Granodiorit, Tonalit/ und /Gabbro, Diorit/ ist eine Datenauswertung mittels Boxplots nicht möglich.

Zwischen den verschiedenen Festigkeitseigenschaften können gruppenübergreifend gleich bleibende Verhältnisse festgestellt werden (Abb. 3.16). Für die Beziehung zwischen der Druck- und der Biegefestigkeit ergeben sich Faktoren zwischen 9,1 und 14,2 (25 \%- und 75 \%-Quartil). Auch hier wird die Korrelation durch die Gruppe /Gabbro, Diorit/ geprägt. Der Eindruck, dass sich der Verhältnisfaktor hinsichtlich der übrigen Gruppen mit ansteigenden Druckfestigkeiten leicht erhöht, bestätigt sich nicht. Aus den recherchierten Daten ergibt sich für die Beziehung zwischen der Druckfestigkeit und der Spaltzugfestigkeit ein vergleichbares Bild mit einem Verhältnis von rund $10: 1$. Gleichzeitig ist die Spaltzugfestigkeit augenscheinlich mit der Biegefestigkeit annähernd gleichzusetzen. Aktuelle Untersuchungen der technischen Eigenschaften (ausgeführt nach DIN bzw. EN) granitoider Naturwerksteine aus Thailand (Hoffmann 2006) und Argentinien (vgl. Kap. 4) zeigen jedoch, dass für die Spaltzugfestigkeiten tatsächlich geringere Werte angesetzt werden sollten, wodurch sich der 
Faktor zur Druck- als auch zur Biegefestigkeit erhöht. Als Folge davon kann vermutet werden, dass einige der betrachteten Daten zur Biegefestigkeit, resultierend aus einer mehrdeutigen bzw. verwirrenden Bezeichnung der Analyseverfahren (vgl. Abb. 3.3), tatsächlich der Spaltzugfestigkeit zuzuordnen sind.
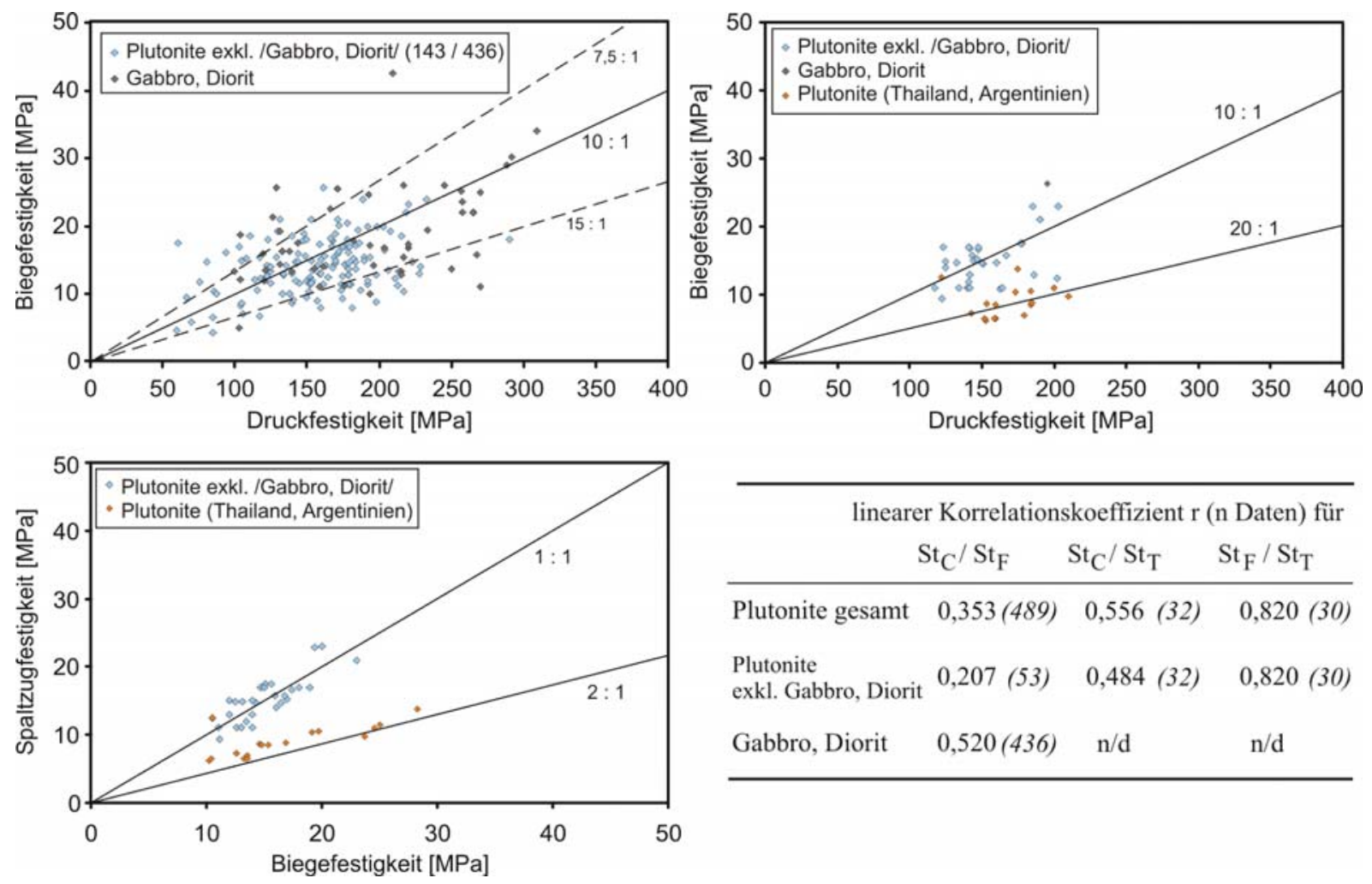

\begin{tabular}{|c|c|c|c|}
\hline \multicolumn{4}{|c|}{ linearer Korrelationskoeffizient $\mathrm{r}$ ( $\mathrm{n}$ Daten) für } \\
\hline & $\mathrm{St}_{\mathrm{C}} / \mathrm{St}_{\mathrm{F}}$ & $\mathrm{St}_{\mathrm{C}} / \mathrm{St}_{\mathrm{T}}$ & $\mathrm{St}_{\mathrm{F}} / \mathrm{St}_{\mathrm{T}}$ \\
\hline Plutonite gesamt & $0,353(489)$ & $0,556(32)$ & $0,820(30)$ \\
\hline $\begin{array}{l}\text { Plutonite } \\
\text { exkl. Gabbro, Diorit }\end{array}$ & $0,207(53)$ & $0,484(32)$ & $0,820(30)$ \\
\hline Gabbro, Diorit & $0,520(436)$ & $\mathrm{n} / \mathrm{d}$ & $\mathrm{n} / \mathrm{d}$ \\
\hline
\end{tabular}

Abb. 3.16: Korrelationsdiagramme der technischen Gesteinseigenschaften $\left(\mathrm{St}_{\mathrm{C}}=\right.$ Druck-, $\mathrm{St}_{\mathrm{F}}=$ Biege-, $\mathrm{St}_{\mathrm{T}}=$ Spaltzugfestigkeit) untereinander sowie die Korrelationskoeffizienten für den jeweiligen linearen Zusammenhang in den Untergruppen der plutonischen Gesteine. Zusätzlich sind idealisierte Trends dargestellt. Die Daten zur Spaltzugfestigkeit wurden um aktuelle Untersuchungsergebnisse an granitoiden Naturwerksteinen aus Thailand (Hoffmann 2006) und Argentinien (vgl. Kap. 4) erweitert.

Tab. 3.1: Richtwerte für die Basiseigenschaften Rohdichte [g/ $\left./ \mathrm{cm}^{3}\right]$ und effektive Porosität [Vol.-\%] sowie für die Parameter Druck-, Biege- und Spaltzugfestigkeit [jeweils MPa] in den Untergruppen der plutonischen Gesteine. Die Minimum- bzw. Maximumwerte entsprechen den statistischen Kennwerten oberer und unterer Extremwert aus der explorativen Datenauswertung mittels Boxplots. Ausgewiesene Ausreißer sind folglich nicht in die Übersicht mit eingegangen.

\begin{tabular}{|c|c|c|c|c|c|c|c|c|c|}
\hline Gruppe & $\begin{array}{l}\text { Rohd } \\
\text { min }\end{array}$ & $\begin{array}{l}\text { ichte } \\
\max \end{array}$ & $\begin{array}{l}\text { Poros } \\
\text { min }\end{array}$ & $\begin{array}{l}\text { sität } \\
\max \end{array}$ & $\begin{array}{c}\text { Druckfe } \\
\text { min }\end{array}$ & $\begin{array}{l}\text { stigkeit } \\
\max \end{array}$ & $\begin{array}{c}\text { Biegefe } \\
\text { min }\end{array}$ & $\begin{array}{l}\text { stigkeit } \\
\max \end{array}$ & $\begin{array}{c}\text { Spaltzugfestigkeit } \\
\min \max \end{array}$ \\
\hline Granit & 2,55 & 2,74 & 0,16 & 1,65 & 60,0 & 253,5 & 5,6 & 21,1 & $9,4 \quad 23,0$ \\
\hline Granodiorit, Tonalit & 2,57 & 2,84 & 0,19 & 1,40 & 79,9 & 255,5 & 7,6 & 25,7 & 11,0 \\
\hline Monzonit, Syenit & 2,55 & 2,84 & 0,34 & 0,72 & 103,4 & 243,8 & 7,6 & 25,7 & $11,0 \quad 21,0$ \\
\hline Gabbro, Diorit & 2,63 & 3,26 & 0,24 & 0,69 & 100,0 & 292,3 & 5,1 & 30,2 & 26,4 \\
\hline
\end{tabular}




\subsubsection{Basiseigenschaften in Korrelation mit mechanischen Festigkeiten}

Allgemein wird die mechanische Stabilität häufig mit der spezifischen Härte der aufbauenden Minerale sowie vor allem auch der Korngröße und -verzahnung in Zusammenhang gebracht. Im Hinblick auf einen linearen Zusammenhang zwischen der Rohdichte und der Druck-, der Biege- beziehungsweise der Spaltzugfestigkeit wird jeweils lediglich ein schwach positiver Trend beschrieben (Abb. 3.18). Im Dichtebereich von $2,6 \mathrm{~g} / \mathrm{cm}^{3}$ bis $2,7 \mathrm{~g} / \mathrm{cm}^{3}$ ist eine deutliche Punkthäufung erkennbar, was besonders bei der Betrachtung der relativen Häufigkeitsverteilungen deutlich wird (Abb. 3.17). Geprägt wird demnach der grundsätzliche Trend primär durch die Datenpunkte der Untergruppe /Gabbro, Diorit/. Die unterschiedlich starke Ausprägung der Verteilungsdichte in der bivariaten Häufigkeitsverteilung ist dabei lediglich auf die Anzahl der eingeflossenen Daten zurückzuführen. Aufgrund der geringen Datenmenge zur Spaltzugfestigkeit besonders für die Gruppe /Gabbro, Diorit/ kann hier keine nähere Betrachtung erfolgen. Es ist jedoch zu vermuten, dass relativ gleiche Verhältnisse anzunehmen sind, wie sie durch die Beziehungen zwischen der Rohdichte und den übrigen Parametern der Festigkeit beschrieben sind.

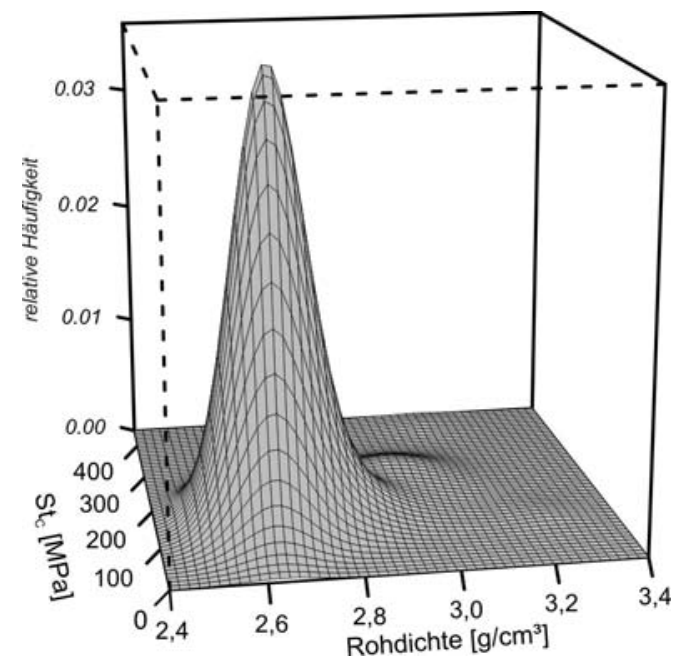

Abb. 3.17: Exemplarische Darstellung der relativen Häufigkeitsverteilung der Korrelation Rohdichte und Druckfestigkeit $\left(\mathrm{St}_{\mathrm{C}}\right)$. Neben dem dominierenden Datencluster im Bereich um 2,65 g/ $\mathrm{cm}^{3}$ wird eine weitere Datenhäufung deutlich, die höhere Dichten mit höheren Werten zur Festigkeiten verknüpft. Die Gruppe /Gabbro, Diorit/ prägt somit eine Linearität in der Gesamtheit der Plutonite. Die extrem unterschiedliche Ausprägung ist auf die Anzahl der einbezogenen Datenpaare zurückzuführen.

Hinsichtlich eines linearen Zusammenhanges zwischen der Porosität und den Festigkeitseigenschaften ergibt sich zwar ein maximaler Korrelationskoeffizient von $r=-0,575$ (Gruppe /Monzonit, Syenit/; $\Phi / \mathrm{St}_{\mathrm{F}}$ ), wobei dieser Wert bei nur 5 berücksichtigten Datenpaaren für die Gesamtheit eher angezweifelt werden muss. Verdeutlicht wird der fehlende Zusammenhang zwischen der Porosität und den Festigkeitseigenschaften besonders durch die Verteilung der in Abbildung 3.18 eingetragenen Medianwerte.

Insgesamt ist $\mathrm{zu}$ vermerken, dass für die Gruppe der Plutonite zwischen den Basiseigenschaften und den Parametern der Festigkeit kein oder nur ein sehr schwacher linearer Trend vermutet werden kann. 

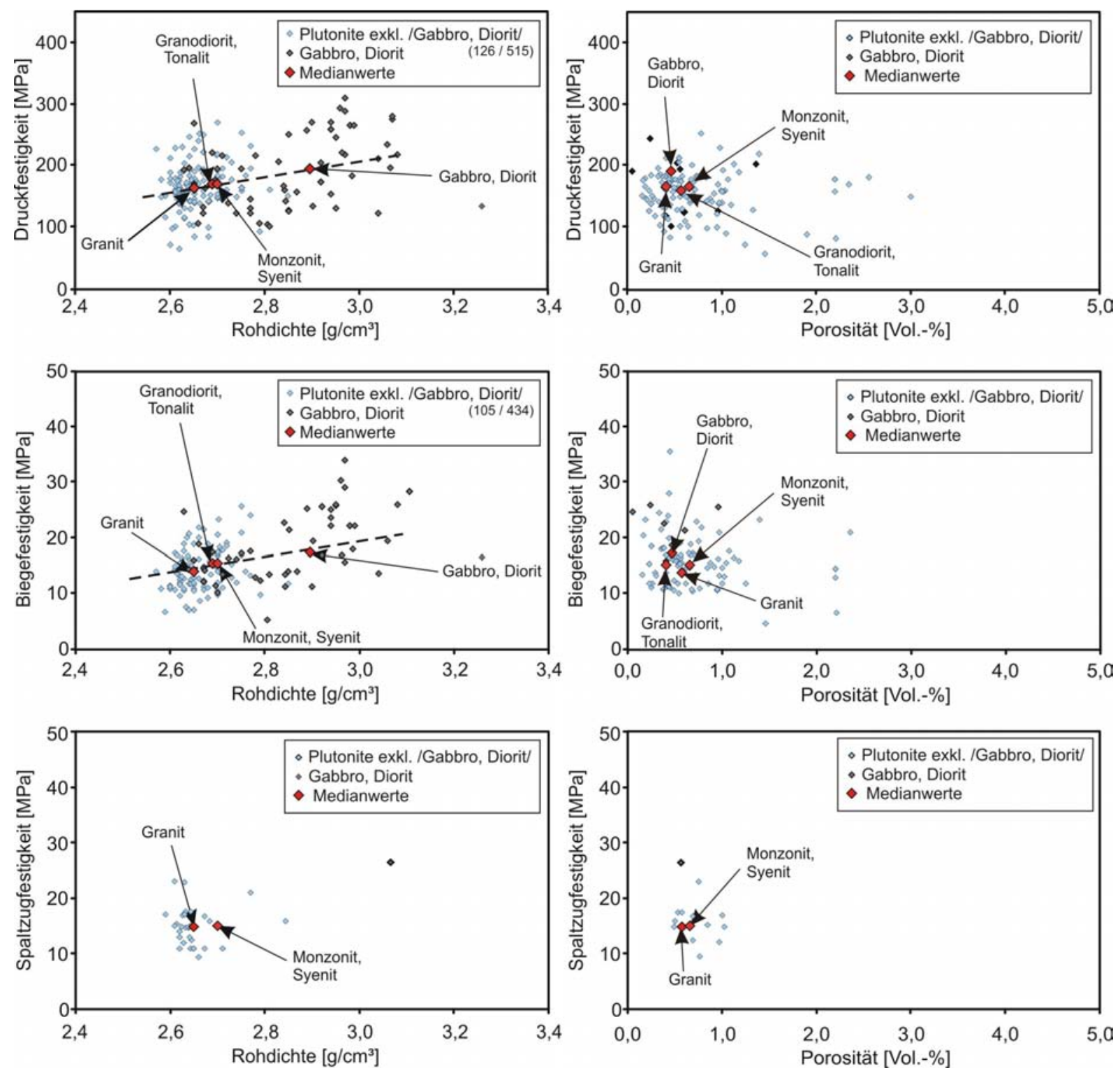

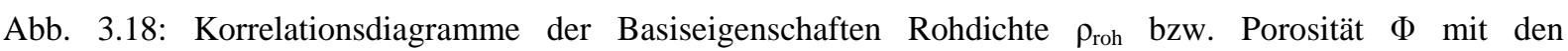
mechanischen Parametern Druck- $\left(\mathrm{St}_{\mathrm{C}}\right)$, Biege- $\left(\mathrm{St}_{\mathrm{F}}\right)$ sowie Spaltzugfestigkeit $\left(\mathrm{St}_{\mathrm{T}}\right)$ bei Plutoniten. Rote Punkte: Datenpaare der Medianwerte in den vier definierten Untergruppen. Die relativ ausgeprägte positive Korrelation wird in den Fällen $\rho_{\text {roh }} / \mathrm{St}_{\mathrm{C}}$ sowie $\rho_{\text {roh }} / \mathrm{St}_{\mathrm{F}}$ durch die Untergruppe /Gabbro, Diorit/ diktiert.

\subsubsection{Beziehungen zwischen dem mittleren Atomgewicht und den Festigkeitseigenschaften}

Bei den hier behandelten niedrigporösen Gesteinen wird die Rohdichte primär durch die spezifische Dichte des Mineralbestands bedingt (Kap. 3.4.1). Infolge des wenn auch schwachen Zusammenhanges zwischen der Rohdichte und den mechanischen Festigkeiten, kann auch ein Zusammenhang zwischen der Mineralogie der Gesteine mit den Festigkeitseigenschaften vermutet werden. Als stellvertretender Parameter für die komplexe chemische Zusammensetzung gilt das richtungsunabhängige mittlere Atomgewicht $\mathrm{M}_{\mathrm{G}}$, das in enger Beziehung zur Rohdichte steht (z.B. Birch 1961, Siegesmund 1989, Abb. 3.19). Das mittlere Atomgewicht $\mathrm{M}_{\mathrm{G}}$ errechnet sich aus den prozentualen Anteilen der Hauptelementoxide: 


$$
M_{G}=\left(\sum_{i=1}^{n} x_{i} / M_{i}\right)^{-1}
$$

mit: $\quad \mathrm{M}_{\mathrm{G}}$ : mittleres Atomgewicht;

$\mathrm{x}_{\mathrm{i}}$ : Anteile des i-ten Oxids [Gew.\%];

$\mathrm{M}_{\mathrm{i}}$ : mittleres Atomgewicht des i-ten Oxids;

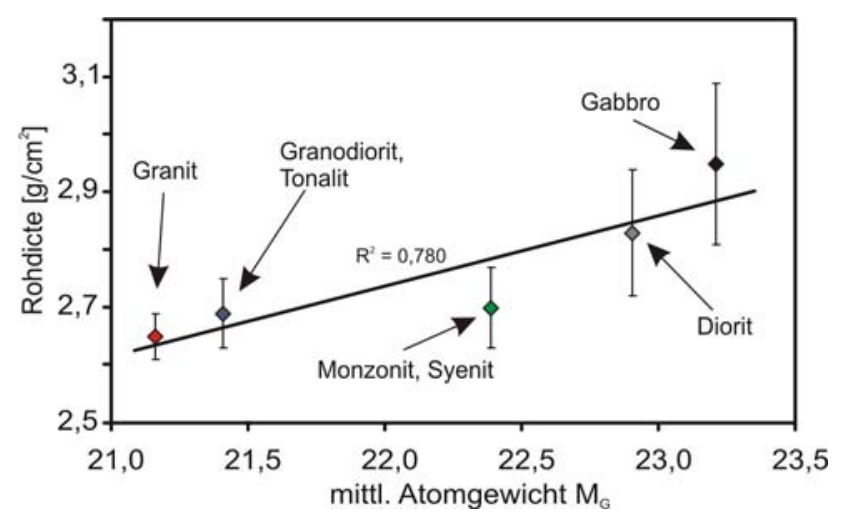

Abb. 3.19: Das mittlere Atomgewicht $\mathrm{M}_{\mathrm{G}}$, basierend auf der mineralogischen Komposition der Gesteine, steht in engem Zusammenhang mit der Rohdichte.

Um einen generellen Überblick über den Zusammenhang des mittleren Atomgewichts $\mathrm{M}_{\mathrm{G}}$ mit der Druck- bzw. Biegefestigkeit zu bekommen, erfolgt die Berechnung von $\mathrm{M}_{\mathrm{G}}$ primär aus einer chemischen Durchschnittszusammensetzung nach Nockolds (1954). Dem gegenübergestellt sind die aus der vorliegenden Datenbank für die Plutonit-Gruppen ermittelten Medianwerte der technischen Eigenschaft mit Standardabweichung (Abb. 3.20). Hierbei wurde zusätzlich die Gruppe /Gabbro, Diorit/ aufgrund des deutlichen Unterschieds im $\mathrm{SiO}_{2}$-Gehalt aufgeteilt. Es zeigt sich, dass sich mit zunehmendem mittleren Atomgewicht $\mathrm{M}_{\mathrm{G}}$ für $\mathrm{St}_{\mathrm{C}}$ und $\mathrm{St}_{\mathrm{F}}$ ein Trend $\mathrm{zu}$ höheren Werten, gleichbedeutend mit dem Anstieg der Festigkeiten von der Gruppe /Granit/ zur Gruppe /Gabbro, Diorit/, abzeichnet. Dies ist auch mit der Mineralogie in Einklang zu bringen, da gerade Minerale wie Pyroxene und Olivin, als Hauptkomponenten eines Gabbros, durch hohe mittlere Atomgewichte gekennzeichnet sind. Auch für die Spaltzugfestigkeit wird eine negative Korrelation zum $\mathrm{SiO}_{2}$-Gehalt beschrieben (Peschel 1983).

Im Vergleich zu den Relationen zwischen der Rohdichte und den Festigkeitseigenschaften zeigt sich hier, dass durch die relative Verschiebung insbesondere der Gruppe /Monzonit, Syenit/ die jeweilige geschätzte Regressionsgerade eine deutlich bessere Abdeckung erfährt. Im Hinblick auf die korrespondierenden recht hohen Determinationskoeffizienten (Abb. 3.20) ist jedoch zu beachten, dass jeweils nur fünf Wertepaare beschrieben sind. Die 49 Datenpaare aus dem zugrunde liegenden Datensatz geben die berechneten Bereiche dagegen nur in Ansätzen wieder.

Es wird jedoch deutlich, dass basische Gesteine zumeist eine höhere Festigkeit aufweisen als dies bei sauren Gesteinen der Fall ist. Ausschlaggebend dafür ist wiederum der Anteil an mechanisch schwachen Glimmermineralen. Für höhere Festigkeiten der basischen Gesteine spricht zudem das im Allgemeinen feinkörnigere und homogenere Gefüge. 

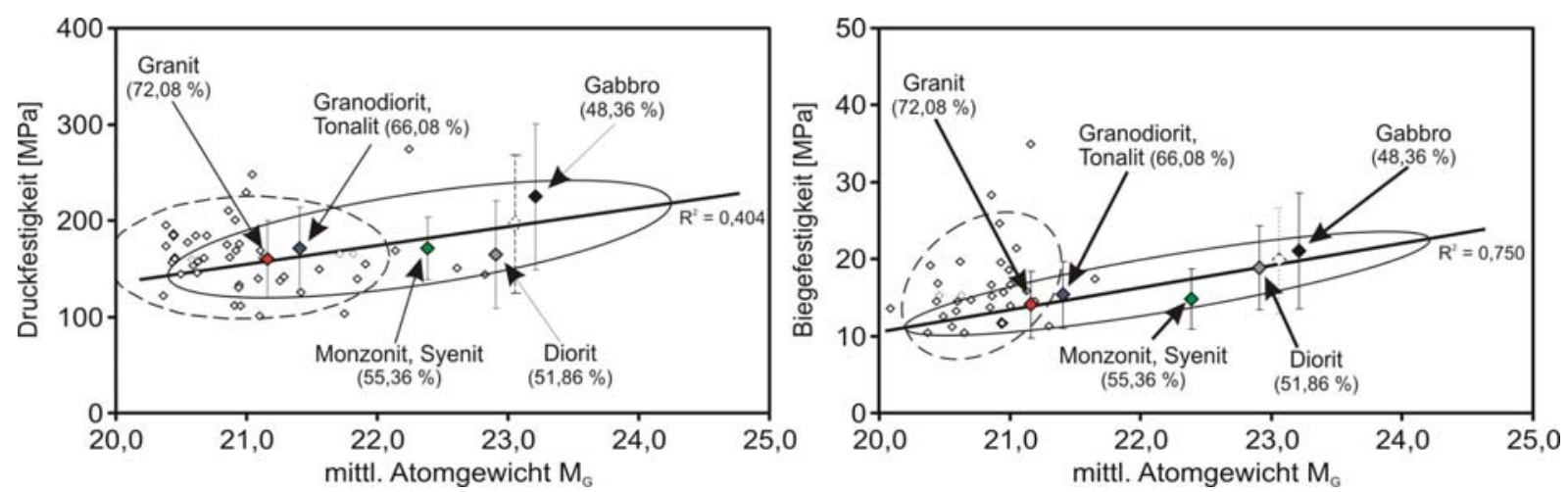

Abb. 3.20: Korrelationsdiagramme des mittleren Atomgewichts $\mathrm{M}_{\mathrm{G}}$ und der Druck- (links) bzw. Biegefestigkeit (rechts). Die mittleren Atomgewichte der Gruppen (große Datenpunkte) basieren auf der durchschnittlichen chemischen Zusammensetzung der Gesteine nach Nockolds (1954). Angegeben sind ferner der entsprechende $\mathrm{SiO}_{2}$-Gehalt sowie die Determinationskoeffizienten für den jeweiligen linearen Zusammenhang. Die Werte zur Festigkeit entsprechen den Medianwerten aus der Datensammlung, die y-Fehler der jeweiligen Standardabweichung. Die durchgezogen eingetragenen Ellipsoide beschreiben jeweils die 80 \%-ige Aufenthaltswahrscheinlichkeit. Die nicht gefüllten Punkte entsprechen der für diese Arbeit zusammengestellten Daten und geben die Grundlage für die gestrichelt eingetragenen Aufenthaltswahrscheinlichkeitsbereiche.

\subsection{Gruppe der vulkanischen Magmatite}

Seit dem Altertum finden auch verschiedene Vulkanite in weiter Verbreitung als Werkstein Verwendung. Infolge der relativ leichten Bearbeitbarkeit des Materials sind vor allem Tuffe in der Werksteinverarbeitung etabliert. Nachteilig hingegen ist die auf den gesteinsphysikalischen Eigenschaften beruhende zum Teil erheblich reduzierte Verwitterungsresistenz im Vergleich mit anderen Naturwerksteinen (z.B. Eggloffstein 1998, Fitzner \& Basten 1994).

Im vorliegenden Datensatz sind 71 vulkanische Werksteinvarietäten enthalten, die in vier Untergruppen gegliedert werden: /Andesit, Basalt/, /Rhyolith, Dazit/, /Trachyt/, /Tuff/ (Abb. 3.21). Die Gliederung basiert hinsichtlich der ersten drei Untergruppen auf der Streckeisen-Klassifikation (Streckeisen 1974, 1980). Die Tuffe, für die die größte Anzahl an Varietäten im Datensatz enthalten ist, sollen als eine separate Untergruppe betrachtet werden. Entgegen den übrigen Effusivgesteinen bestehen diese aus Schlacken, Aschepartikeln und Gesteinsbruckstücken, die bei explosiv verlaufenden Eruptionen abgelagert und anschließend kompaktiert und verschmolzen werden. Daher werden auch häufig die Begriffe Vulkanoklastit (Fisher 1961, 1966) oder pyroklastisches Gestein verwendet. 

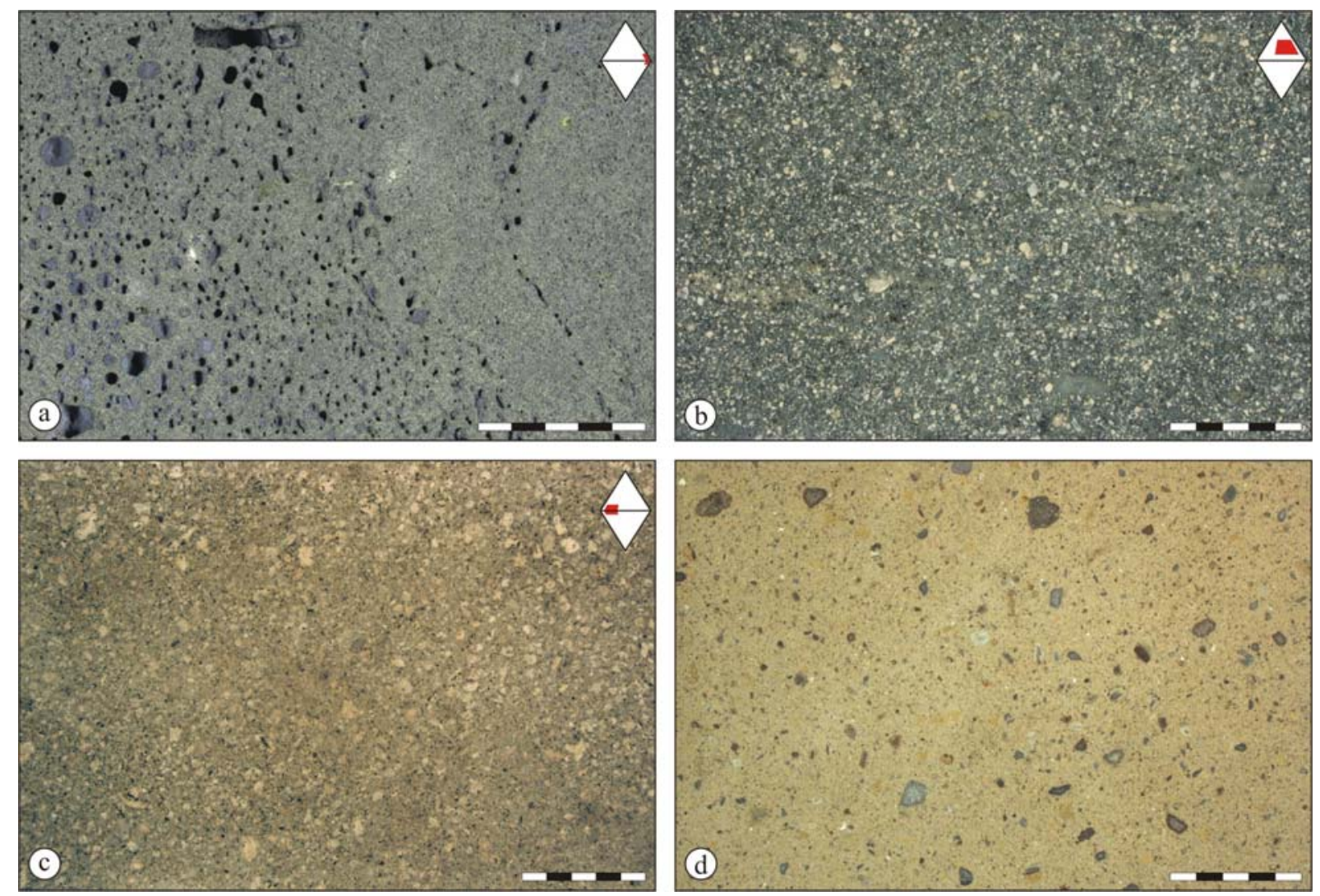

Abb. 3.21: Varietäten vulkanischer Naturwerksteine zu den vier definierten Untergruppen: a) LONDORFER BASALTLAVA (Deutschland) mit ausgeprägt heterogener Porosität; b) STARDUST GREY (Rhyolith, Argentinien); c) MONTEMERLO (Trachyt, Italien); d) WEIBERNER TUFF (Deutschland), (in a, c, d sägeraue Oberflächen, in b polierte Oberfläche, Maßstäbe in cm).

\subsubsection{Basiseigenschaften Rohdichte und Porosität}

In den Untergruppen /Andesit, Basalt/ und /Tuff/ existieren für die Parameter Rohdichte und Porosität jeweils weite Datenspannen (Abb. 3.22). Für beide Untergruppen ist dabei eine gute Korrelation der Basiseigenschaften ausgebildet (Abb. 3.23). Bei Tuffen kann bereits die Matrixdichte zwischen 1,90 g/ $\mathrm{cm}^{3}$ und 2,80 g/ $\mathrm{cm}^{3}$ schwanken (Auras \& Steindlberger 2005). Dies ist durch den Chemismus des Stammmagmas, der anteilsmäßigen Zusammensetzung von aufbauenden Komponenten und Bindemittel sowie auch der Ab- und Umlagerungsbedingungen bedingt.

Bei vielen basaltischen Naturwerksteinen ist eine ausgesprochen unregelmäßige Verteilung und Ausformung des Porenraumes zu beobachten. Mit zunehmendem Aufstieg des schnell fließenden Magmas kommt es infolge veränderter Druckbedingungen zur Entgasung, die sich dann bei rascher Abkühlung in einem ausgeprägten Blasengefüge äußern kann (vgl. Abb. 3.21 a). Diese häufig anzutreffende Gefügeinhomogenität erklärt die weiten Datenspannen der Basiseigenschaften der Basalte und die gute Korrelation der Parameter.

Durch den höheren Kieselsäuregehalt sind rhyolithische und dazitische Laven dagegen deutlich zähflüssiger. Dadurch tritt eine abrupte Bildung größerer Hohlräume durch eine Entgasung eher selten auf. Gleichzeitig kommt es infolge einer langsameren Abkühlung zu einem Kristallwachstum. Daher weisen als Werkstein verwendete Rhyolithe oft 
charakteristische Merkmale eines Porphyrits auf. Häufig sind dabei in die zum Teil hyaline Matrix gewachsene Kristalle oder xenolithische Fragmente im mm-Maßstab eingeschlossen. Infolge dieses Gefüges ergeben sich relativ hohe Werte der Rohdichte und entsprechend geringe der Porosität.
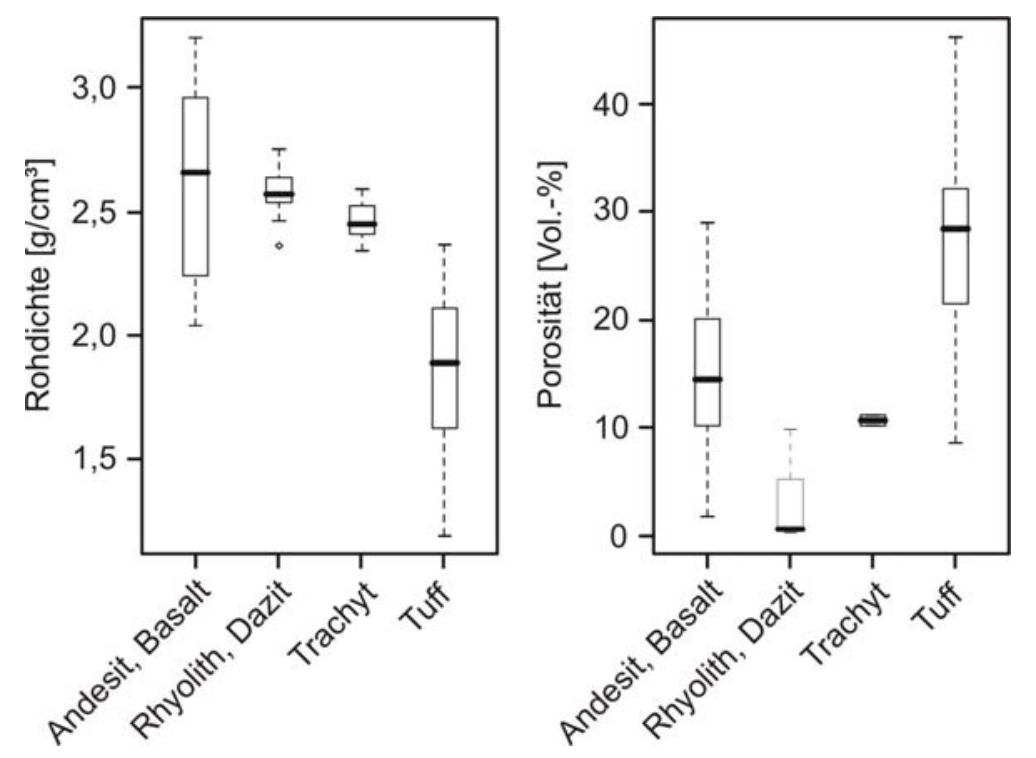

Abb. 3.22: Rohdichten (links) und Porositäten (rechts) in den Untergruppen der vulkanischen Gesteine.

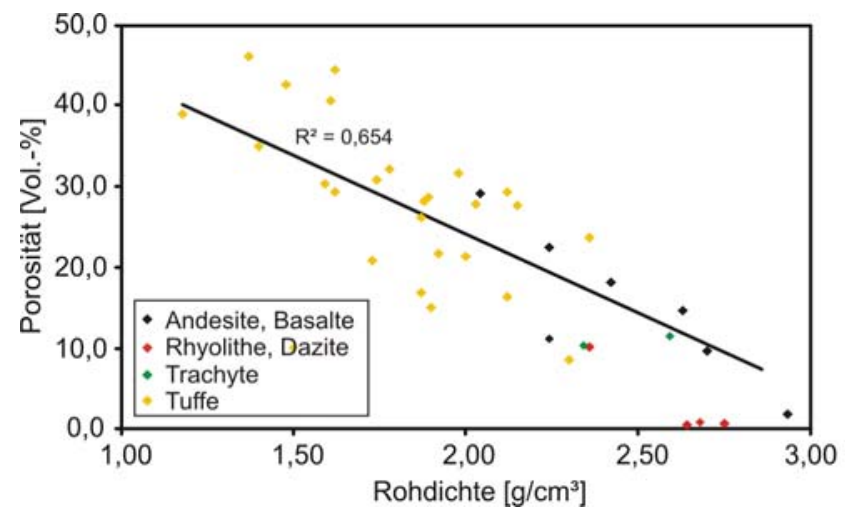

\begin{tabular}{lcc}
\hline & \multicolumn{2}{c}{ linearer Korrelationskoeffizient r } \\
& für $\rho_{\text {roh }} / \Phi$ & $n$ \\
\hline Andesite, Basalte & $-0,860$ & 7 \\
Rhyolithe, Dazite & $-0,960$ & 4 \\
Trachyte & - & 2 \\
Tuffe & $-0,573$ & 26 \\
\hline
\end{tabular}

Abb. 3.23: Korrelationsdiagramm der Parameter Rohdichte $\rho_{\text {roh }}$ und Porosität $\Phi$ mit den Korrelationskoeffizienten des jeweiligen linearen Zusammenhangs für die Untergruppen der vulkanischen Gesteine. Das angegebene Bestimmtheitsmaß bezieht sich auf die Gesamtheit der Vulkanite.

\subsubsection{Technische Eigenschaften Druck- und Biegefestigkeit}

In der Gruppe der vulkanischen Gesteine zeigen sich hinsichtlich der mechanischen Stabilität erhebliche Unterschiede (Abb. 3.24). Exklusive identifizierter Ausreißer ist für die Untergruppe /Andesit, Basalt/ sowohl für die Druck- als auch die Biegefestigkeit die größte Datenspannweite beschrieben, was auf der zum Teil extremen Heterogenität der Porosität beruht. So sind z.B. für die Druckfestigkeit Werte zwischen etwa 50 und 300 MPa realisiert. Daher ist eine Unterscheidung von kompakten Basalten und porösen Basaltlaven zu beachten, womit für die mögliche Verwendung dieses Materials folglich die Bildungsbedingungen beziehungsweise die Erstarrungsbedingungen von Bedeutung sind. Dichte und feinkörnige Basalte stehen der Naturwerksteinindustrie in entsprechend dimensionierten Rohblöcken 
infolge der charakteristisch säuligen Erstarrungsstruktur (vgl. Kap. 2.2, Abb. 2.3 b) nur selten zur Verfügung. Dieses Material wird daher eher im Massenbergbau als Zuschlag- und Bettungsstoff gewonnen (Peschel 1983).

Die Untergruppe /Rhyolith, Dazit/ überspannt in etwa einen Druckfestigkeitsbereich, der den kompakten Andesiten bzw. Basalten entspricht. Daran schließt sich mit einem Wertebereich von rund 60 bis $180 \mathrm{MPa}$ die Untergruppe der Trachyte an. Die geringsten Werte der Stabilität gegen einfache Kompression weisen erwartungsgemäß, infolge der beschriebenen Basiseigenschaften, die Tuffe auf. Eine relativ gleiche Verteilung der Wertebereiche ist für die Biegefestigkeiten gegeben. Die wenigen Ausreißer können eventuell auf gefügebedingte Anisotropien zurückgeführt werden, die bei der Datenausweisung nicht weiter benannt wurden. So sind Lagerstätten von rhyolitischen Porphyren häufig durch eine engständige Klüftung im oberflächennahen Bereich gekennzeichnet. Infolgedessen erfolgt ein Abbau auch zumeist plattenweise und nur in wenigen Ausnahme ist die Gewinnung von Großblöcken möglich (z.B. Puerto Madryn, Argentinien, vgl. Kap. 4.6.3). Dieses Charakteristikum ist auf Abkühlungsklüfte zurückzuführen, die bei einer rascheren Abkühlung im oberflächennahen Bereich des Magmaflusses engständiger auftreten (vgl. Kap. 2.2).
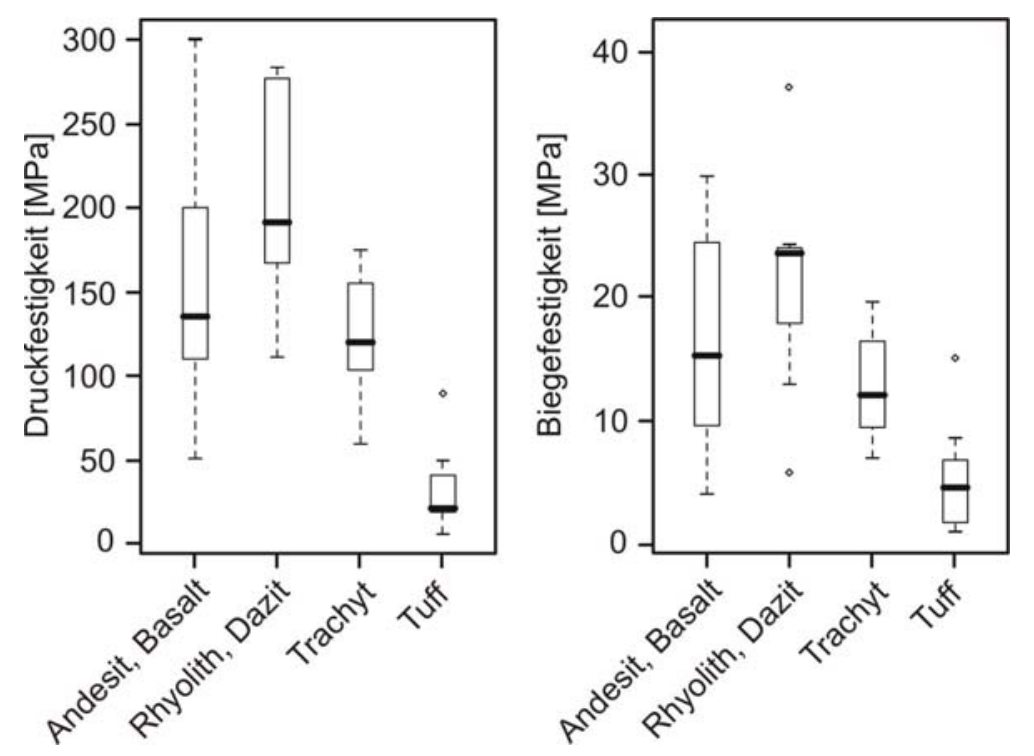

Abb. 3.24: Druck- (links), und Biegefestigkeiten (rechts) in den Untergruppen der vulkanischen Gesteine.

Hinsichtlich der Beziehung zwischen den beiden Parameter Druck- und Biegefestigkeit ergibt sich im Mittel ein Verhältnis von etwa $1: 11$ (Abb. 3.25). Dabei verringert sich der Faktor ausgehend von der Untergruppe /Andesit, Basalt/ $(13,5)$ über /Rhyolith, Dazit/ $(11,2)$ und /Trachyt/ $(9,9)$ bis zu /Tuff/ $(8,7)$, wobei die Einzelgruppen mit nur relativ wenigen Datenpaaren dokumentiert sind. Ob sich die Faktoren bei einer größeren Datenmenge verifizieren würden, ist daher fraglich. Daher kann gruppenübergreifend ein gleich bleibendes Verhältnis zwischen etwa 8,5 : 1 bis 12 : 1 (25 \%- und 75 \%-Quartil) angenommen werden. 


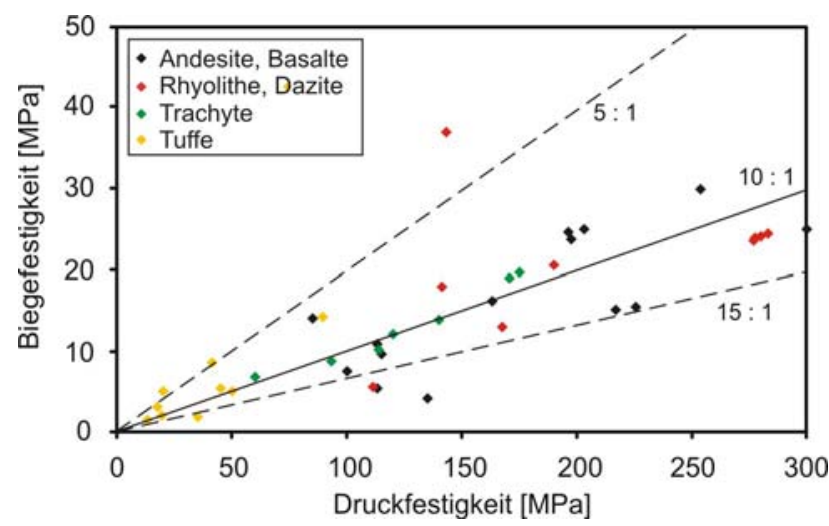

Abb. 3.25: Korrelationsdiagramm der technischen Eigenschaften Druck- und Biegefestigkeit in den Untergruppen der vulkanischen Gesteine. Zusätzlich sind idealisierte Trends dargestellt.

Tab. 3.2: Richtwerte für die Basiseigenschaften Rohdichte $\left[\mathrm{g} / \mathrm{cm}^{3}\right]$ und Porosität [Vol.-\%] sowie für die Parameter Druck- und Biegefestigkeit [jeweils MPa] in den Untergruppen der vulkanischen Gesteine. Minimumbzw. Maximumwerte entsprechen den statistischen Kennwerten oberer und unterer Extremwert aus der explorativen Datenauswertung mittels Boxplots. Ausgewiesene Ausreißer sind folglich nicht in die Übersicht mit eingegangen.

\begin{tabular}{lccccrlrl}
\hline \multirow{2}{*}{ Gruppe } & \multicolumn{2}{c}{ Rohdichte } & \multicolumn{2}{c}{ Porosität } & \multicolumn{2}{c}{ Druckfestigkeit } & \multicolumn{2}{c}{ Biegefestigkeit } \\
& $\min$ & $\max$ & $\min \max$ & $\min$ & $\max$ & $\min$ & $\max$ \\
\hline Andesit, Basalt & 2,04 & 3,20 & 1,75 & 29,06 & 87,7 & 300,0 & 4,0 & 29,9 \\
Rhyolith, Dazit & 2,46 & 2,75 & 0,31 & 9,98 & 111,0 & 283,0 & 12,9 & 24,3 \\
Trachyt & 2,34 & 2,59 & & & 60,0 & 175,2 & 6,5 & 19,6 \\
Tuff & 1,18 & 2,36 & 8,50 & 46,21 & 6,3 & 50,0 & 1,5 & 8,5 \\
\hline
\end{tabular}

\subsubsection{Basiseigenschaften in Korrelation mit mechanischen Festigkeiten}

Auch bezüglich des Zusammenhangs zwischen der Rohdichte und den technischen Eigenschaften lassen sich die vulkanischen Gesteine aus statistischer Sicht in zwei Grundtypen, d.h. einerseits die Untergruppen /Andesit, Basalt/, /Rhyolith, Dazit/ und /Trachyt/ und andererseits die Untergruppe der Tuffe, aufgliedern. Zwar ergibt die Anpassung der Gesamtdatenmenge an ein nichtlineares, exponentielles Modell einen relativ hohen Determinationskoeffizienten, doch erscheint eine separate Anpassung an lineare Modelle sinnvoller (Abb. 3.26). Hinsichtlich der Tuffe ist demnach jedoch keine fundierte Aussage über eine regressive Verknüpfung zu erwarten $\left(\mathrm{R}^{2}\right.$ nahe Null), was vermutlich mit der insgesamt sehr geringen Festigkeit des Gesteins zu begründen ist. Abnorm erscheint aber eine Verknüpfung des Rohdichtewertes 2,55 g/ $\mathrm{cm}^{3}$ mit einer Druckfestigkeit von $13 \mathrm{MPa}$. Hier ist eher anzunehmen, dass ein Wert zur Matrixdichte des Gesteins gegeben ist. Für die übrigen vulkanischen Gesteine kann in ihrer Gesamtheit nach dem vorliegenden Datensatz ein gerichteter linearer Zusammenhang vermutet werden. Aussagen hinsichtlich der Beziehung zwischen der Porosität und den Parametern der Festigkeit können an dieser Stelle infolge der geringen Anzahl an beschreibenden Datenpaaren kaum erfolgen. Deutlich wird jedoch, dass für die Untergruppe /Tuff/, vor allem im Vergleich mit der Untergruppe /Andesit, Basalt/, kein Zusammenhang erkannt werden kann. 

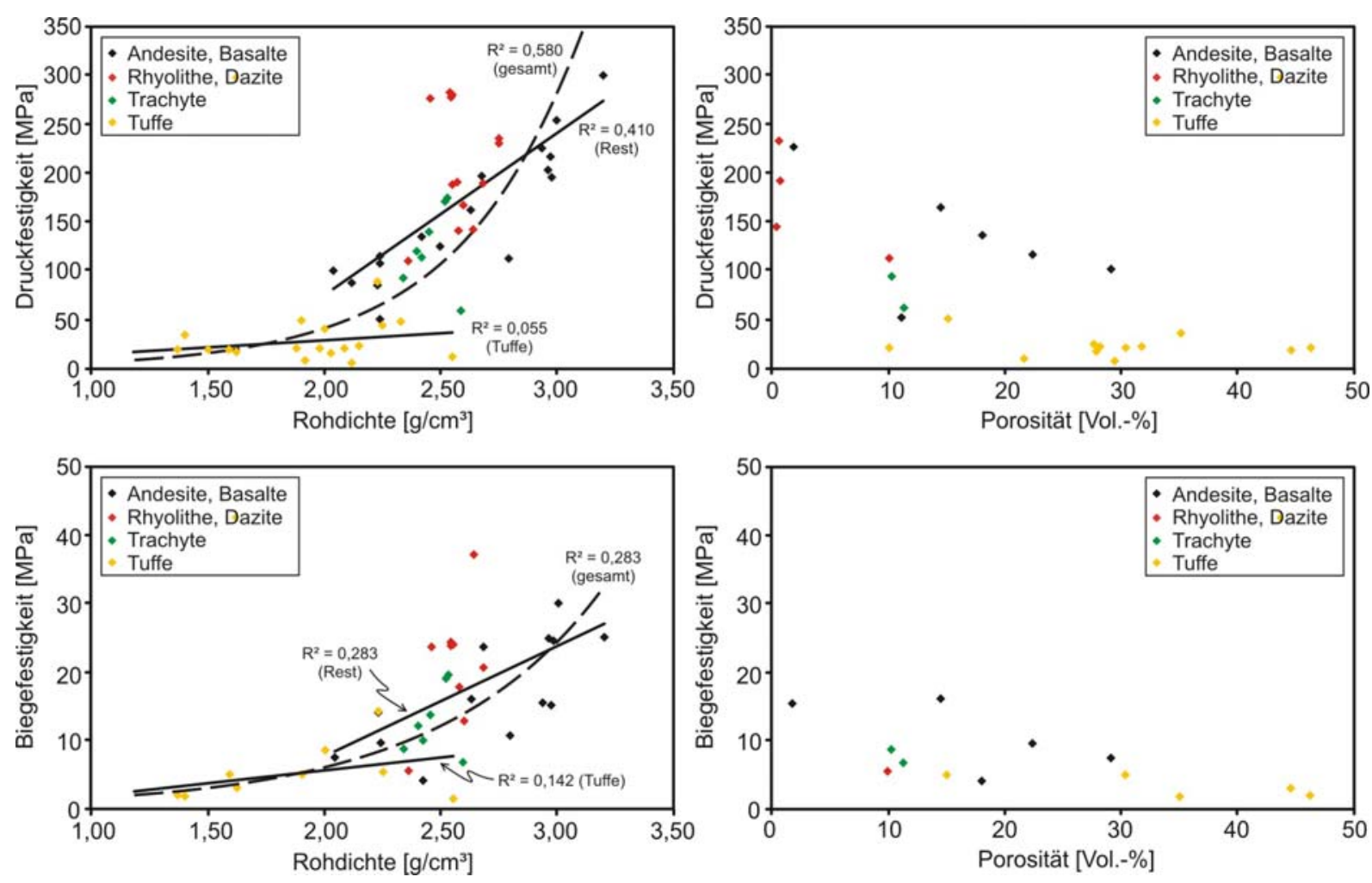

Abb. 3.26: Korrelationsdiagramme der Basiseigenschaften Rohdichte bzw. Porosität mit den technischen Eigenschaften Druck- und Biegfestigkeit bei vulkanischen Gesteinen. Zusätzlich dargestellt sind angepasste gerichtete Zusammenhänge linearen und nichtlinearen Charakters.

\subsection{Gruppe der klastischen Sedimente}

In der langen historischen Verwendung von Naturstein als Baustoff kommt gerade den Sandsteinen in Deutschland eine besondere Bedeutung zu (Abb. 3.27). Im Rahmen dieser Arbeit konnten etwa 500 verschiedene internationale Varietäten zusammengestellt werden, für die in unterschiedlichem Umfang Daten recherchiert werden konnten.

Mit etwa 70 \% entfällt dabei der größte Teil auf deutsche Sandsteinvarietäten, für die zum Teil sehr detaillierte Angaben der physikalischen und technischen Kenndaten vorliegen (z.B. Fitzner 1988, Grimm 1990, Wenzel \& Häfner 2003, Katzschmann et al. 2006). In diesen verschiedenen Quellen erfolgt zumeist auch eine wissenschaftliche Klassifikation, die sich z.B. nach Füchtbauer (1959), McBride (1963) oder Pettijohn et al. (1973) richtet. Dabei werden aber nur in wenigen Fällen die relevanten Daten zur Zusammensetzung angegeben (Abb. 3.28). Oftmals ist eine Klassifikation gar nicht genannt oder aber stark anzuzweifeln beziehungsweise als falsch zu betrachten, was im Folgenden noch an detaillierten Beispielen verdeutlicht wird. 

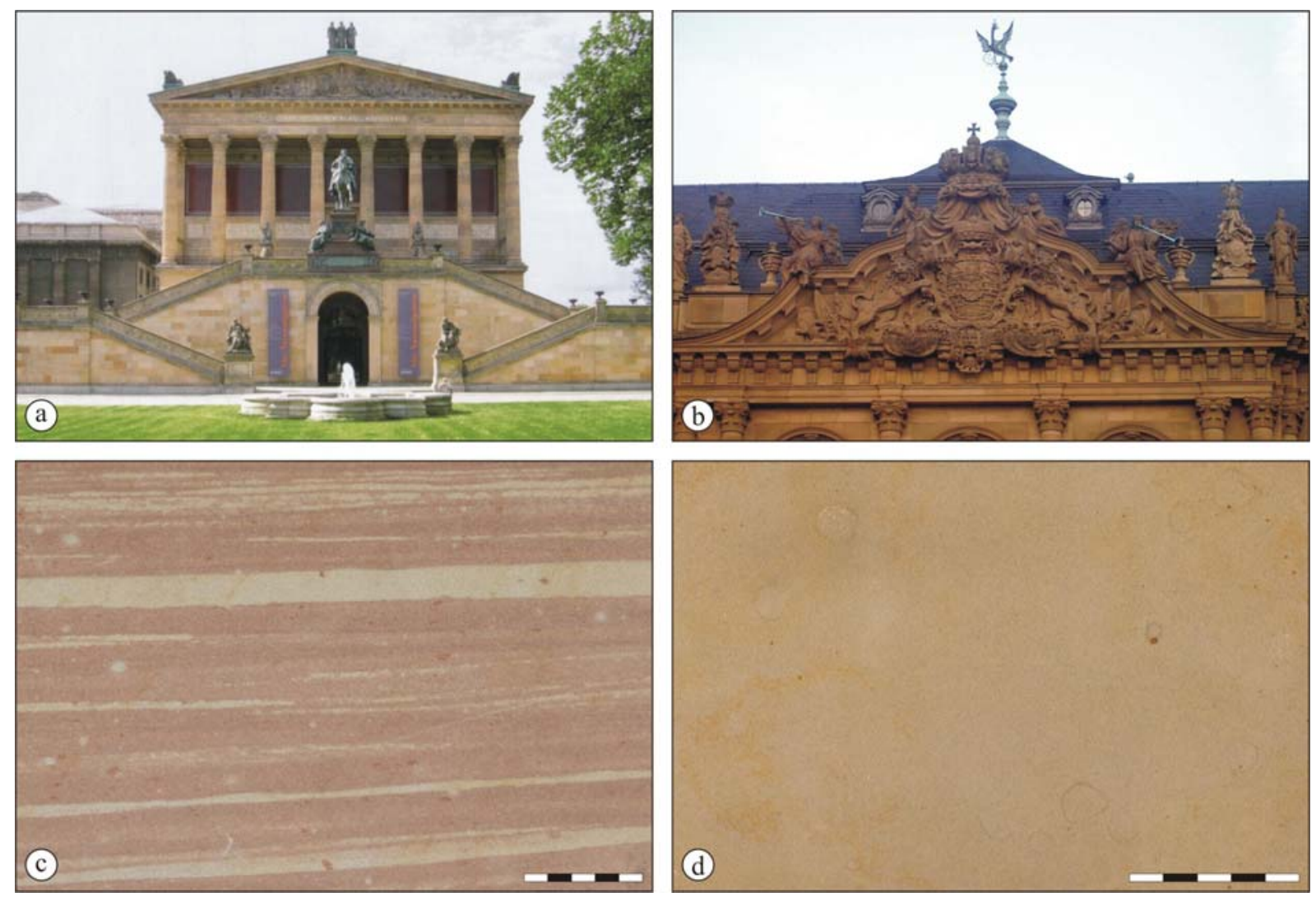

Abb. 3.27: Varietäten deutscher Sandsteine: a) Nationalgalerie in Berlin, NEBRAER S. (Adamini et al. 2005). b) Fassadendetail der Hofresidenz in Würzburg, Keuper-Sandstein, Franken. c) MILTENBERGER S. mit charakteristischem Farbwechsel aufgrund chemischer Unterschiede. d) OBERNKIRCHNER S. (Quelle: Deutsches Natursteinarchiv); Maßstäbe in cm.

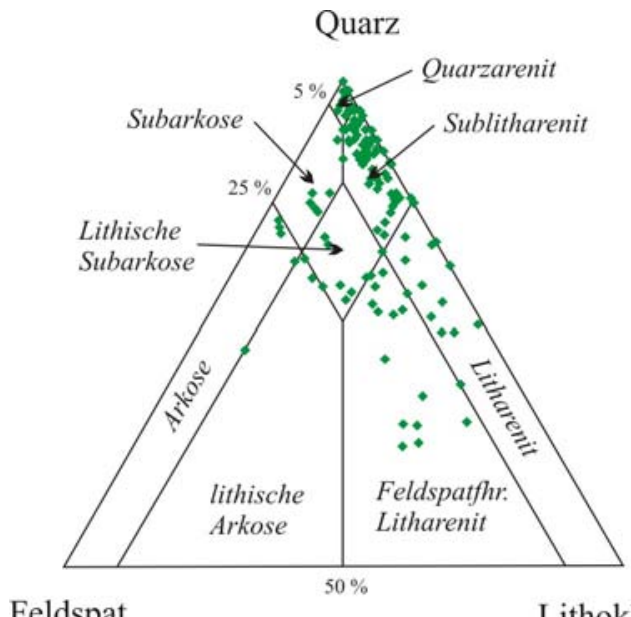

Feldspat
Lithoklasten
Abb. 3.28: Klassifikation der Sandsteine nach McBride (1963). Eingetragen sind alle Varietäten der hier behandelten Datensammlung, für die eine eindeutige Klassifikation möglich ist $(27 \%$ der Gesamtdaten Sandsteine i.w.S.).

\subsubsection{Basiseigenschaften Rohdichte und Porosität}

Im Gegensatz zu den niedrigporösen plutonischen Gesteinen, bei denen im Allgemeinen die Rohdichte mit der Matrixdichte infolge einer vernachlässigbaren Porosität gleichgesetzt werden kann (vgl. Kap. 3.5.1), ist bei Sandsteinen eine Differenzierung zwischen der Matrixund der Rohdichte notwendig. Hier ist eine erhebliche Beeinflussung der Rohdichte durch das 
Hohlraumvolumen gegeben (Abb. 3.29). Hinsichtlich der Eigenschaften Rohdichte und effektive Porosität ist eine symmetrische Verteilung der Daten ausgebildet. Bei einer mittleren Porosität von etwa $15 \%$ ergibt sich für die Rohdichte ein Mittelwert um 2,2 g/ $\mathrm{cm}^{3}$. Für die Matrixdichte ergibt sich ein mittlerer Wert von $2,67 \mathrm{~g} / \mathrm{cm}^{3}$, was der mineralspezifischen Dichte von Quarz, als wesentliche Mineralphase der meisten Sandsteine, entspricht.

Anhand der Sandsteine, stellvertretend für alle höher porösen Gesteine, wird in der Gesamtheit die Relevanz der eindeutigen Unterscheidung von Roh- und Matrixdichte bei der Ausweisung der physikalischen Eigenschaften deutlich.
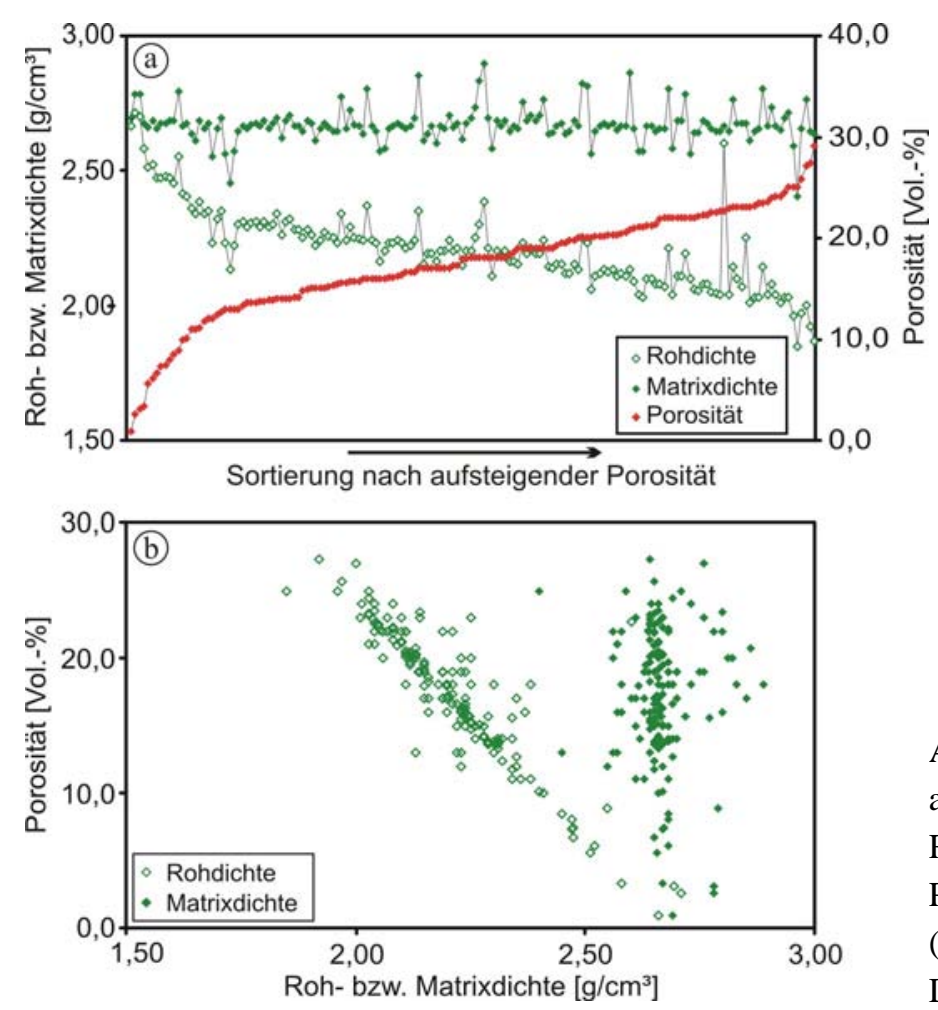

Abb. 3.29: a) Sandsteine sortiert nach aufsteigender Porosität mit verbundener Roh- und Matrixdichte; b) Korrelation der Porosität und der Roh- bzw. Matrixdichte (Daten aus Fitzner 1988, Grimm 1990, David 2006).

Der Porenraum bei Sandsteinen resultiert primär aus den Korngrößen und der Kornsortierung eines Sediments. Die texturelle Reife eines Sediments ist dabei weitgehend abhängig vom Ablagerungsprozess, obwohl sie untergeordnet auch durch diagenetische Faktoren beeinflusst wird (Tucker 1985). Ausdruck dafür ist im verfestigten Sediment vor allem die Sortierung, als Maß für die Streuung der Korngrößenverteilung. Sandsteine, die eine mäßige bis schlechte Sortierung und zudem eckige Kornformen aufweisen (texturell unreife Sedimente), besitzen in der Regel eine geringere Porosität, da durch die unterschiedlichen Korngrößen eine günstigere Raumnutzung erreicht wird. Ebenso sind sehr feinkörnige Varietäten zumeist durch geringe Porositätswerte gekennzeichnet. Dagegen weisen texturell reife Sedimente, charakterisiert durch angerundete bis gerundete Körner und eine gute bis sehr gute Sortierung, zumeist hohe Porositäten auf. Ein relevanter Aspekt ist dabei der Eintrag an kinetischer Energie, der zur Ablagerung führt. So weisen schnell abgelagerte Sedimente, wie z.B. aus Schlammströmen, generell eine schlechte Sortierung und damit auch zumeist eine deutlich geringere Porosität auf, als z.B. sandige Sedimente, die äolisch oder fluviatil über einen längeren Zeitraum auf- und umgearbeitet werden (Tucker 1985). 
Eine weitere wichtige Kenngröße der Porosität, auch in anderen Gesteinsgruppen, ist die Porengröße und die prozentualen Anteile der verschiedenen Porenradienklassen (Abb. 3.30). Die Verteilung der daraus klassifizierten Makro-, Kapillar- und Mikroporen (Klopfer 1985) gibt ein relevantes Kriterium besonders zur Beurteilung hygrischer Eigenschaften (Mirwald 1991).
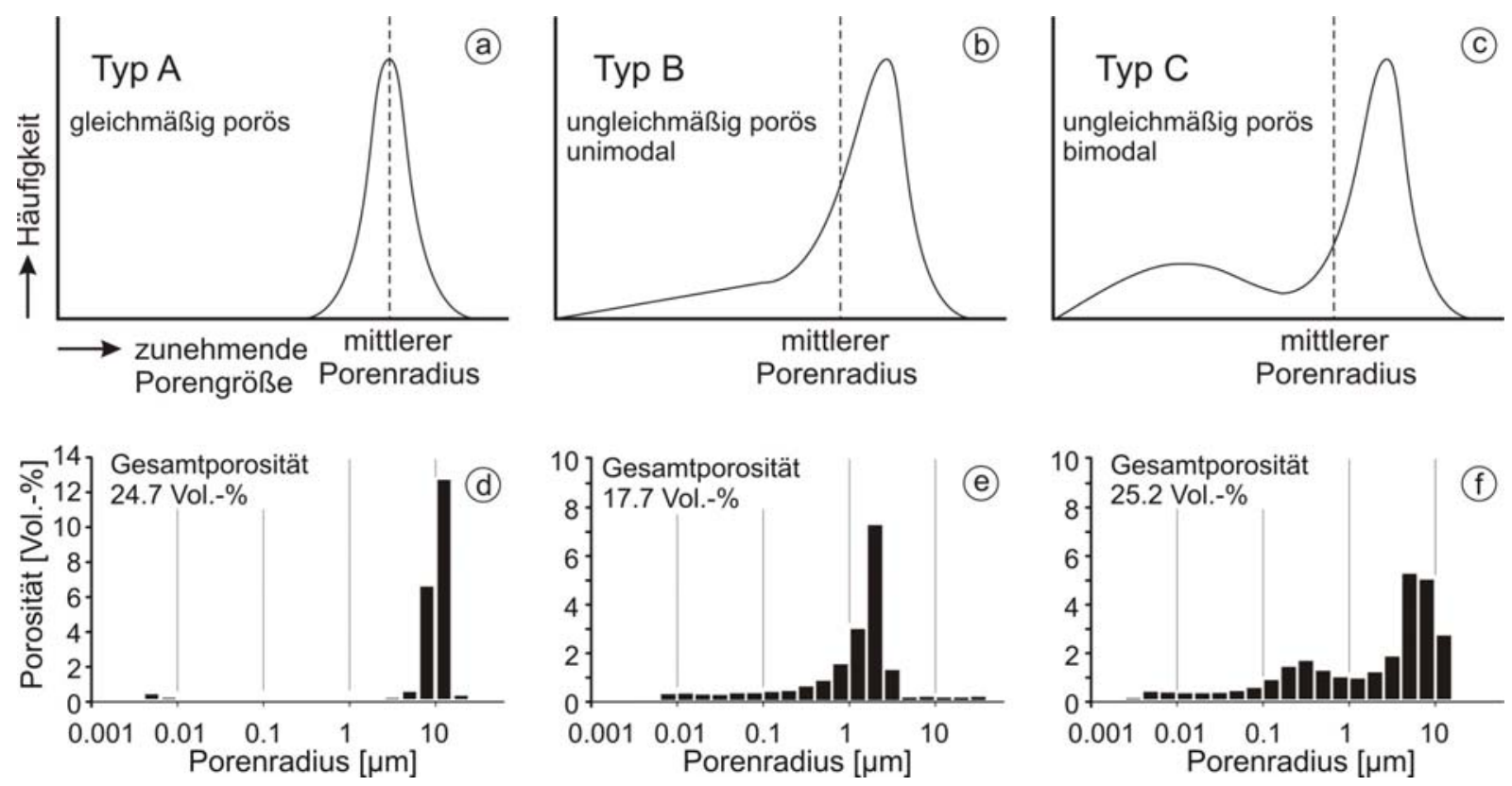

Abb. 3.30: Verschiedene idealisierte Typen der Porenradienverteilung bei Sandsteinen (a - c) und entsprechende Beispiele (d: BAD BENTHEIMER S., e: OBERNKIRCHNER S., f: COTTA S.; nach Rüdrich \& Siegesmund 2006).

Mit diesem Hintergrund ist bei den hier behandelten Daten zu beachten, dass es sich nicht um eine generelle Zusammenstellung von verschiedenen Sandsteintypen handelt, sondern lediglich um solche, die als Naturwerkstein genutzt werden oder wurden. Daraus ergibt sich zweifelsohne eine Vorauswahl, die auf verschiedenen Aspekten beruht. Diese beziehen sich sowohl auf die bautechnische Eignung und die Bearbeitbarkeit eines Gesteins als auch auf ästhetische Gesichtspunkte.

Da die Porosität ein entscheidender Einflussfaktor für verschiedene Verwitterungsphänomene von Sandsteinen ist, soll in der Folge die Beschreibung der Rohdichte in einer groben Klassifikation in Porositätsklassen erfolgen. Diese basiert auf der explorativen Datenauswertung von 360 Sandsteinvarietäten, wobei die Grenzwerte der Klassen durch den 25 \%- bzw. 75 \%-Quartilwert definiert werden (Abb. 3.31). 

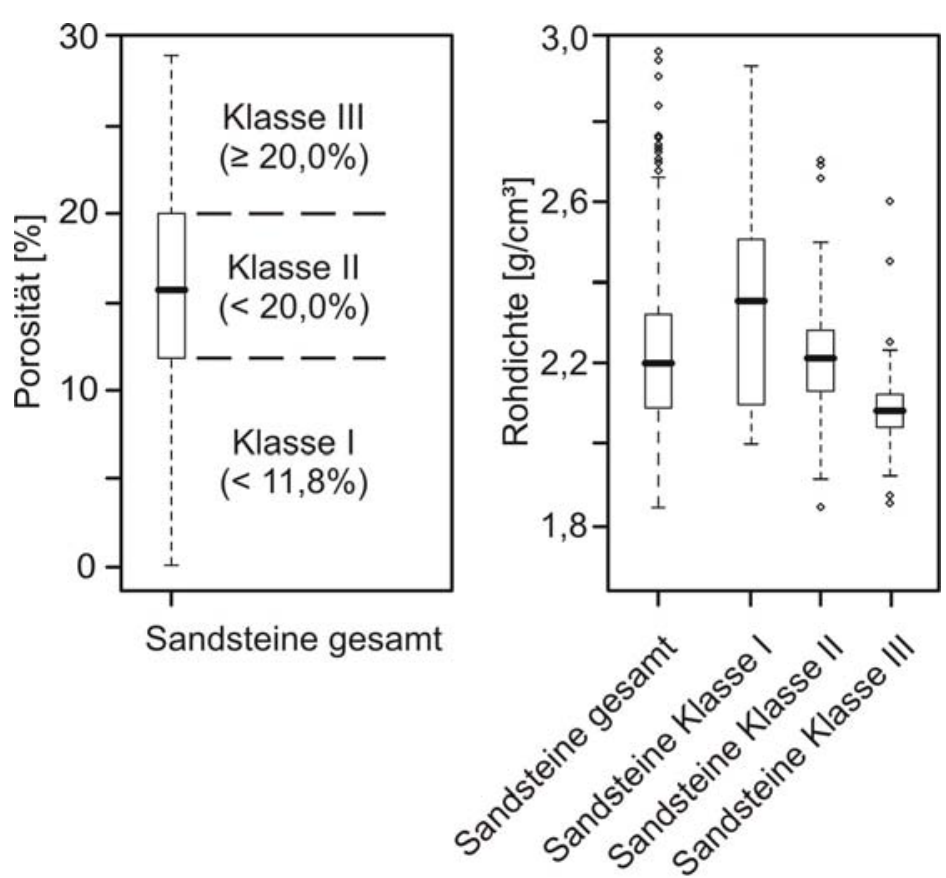

Abb. 3.31: Klassifikation der Sandsteine nach der Porosität (links); Rohdichten in den unterschiedenen Gruppen (rechts). Fehlende Ausreißerwerte bei den Boxplots zur Darstellung der klassifizierten Sandsteine beruhen darauf, dass nicht für alle Varietäten Werte zur Porosität, wohl aber zur Rohdichte vorliegen.

Insgesamt reicht die Spannweite der Porosität für die als Sandsteine i.w.S. bezeichneten Gesteine von 0,1 Vol.-\% bis 29,0 Vol.-\% (Abb. 3.31). Die zentrale Lage des Medianwertes in Verbindung mit dem Nichtauftreten von Ausreißern beschreibt eine gute Annäherung an eine Normalverteilung, wie sie auch aus Abbildung $3.29 \mathrm{zu}$ vermuten war. Für die Rohdichte existieren Werte zwischen 1,69 g/ $\mathrm{cm}^{3}$ und 2,96 g/cm. Die Werte, die deutlich über 2,65 g/cm ${ }^{3}$ (Mineraldichte Quarz) liegen, sind mit einer gewissen Skepsis zu betrachten, obwohl sie in der Porositätsklasse I nicht als Ausreißer identifiziert werden. Die separate Betrachtung der Rohdichte innerhalb der Porositätsklassen zeigt, dass scheinbar vor allem der obere Extremwert die Klassifikation beeinflusst. Folglich kann in der Porositätklasse I ( $<11,8 \%)$ die größte Variabilität der Rohdichtedaten beobachtet werden. Ein signifikanter Unterschied ist lediglich zwischen den Porositätsklassen II und III gegeben.

Abbildung 3.32 zeigt die Punktverteilung der Korrelation der Basiseigenschaften. Darin sind zwei Gruppen von Merkmalspaaren deutlich erkennbar. Sämtliche Datenpaare, die eine relativ geringe Porosität mit einer ebenfalls geringen Rohdichte verknüpfen, stammen aus einer Datenquelle (Wenzel \& Häfner 2003). Diese Arbeit behandelt Werksandsteine aus der Westpfalz. Die Darstellung des Bereichs der 80 \%-igen Aufenthaltswahrscheinlichkeit unter Ausschluss dieser Datenquelle verdeutlicht deren Sonderstellung. Auch bei einem sich ergebenden Koeffizienten von $r=0,681$ für den linearen Zusammenhang innerhalb dieses Datensatzes wird die Gesamtkorrelation erheblich gestört. Die Verbindung einer geringen Rohdichte mit einer relativ geringen Porosität wäre eventuell durch eine sekundäre Verfüllung des Porenraumes durch sehr leichte Minerale realisierbar. Dies ist natürlicherweise aber kaum zu erklären. Die Unregelmäßigkeit dieser Daten wird auch in einem Vergleich mit regionaläquivalenten Daten (Grimm 1990) deutlich (Tab. 3.3). 


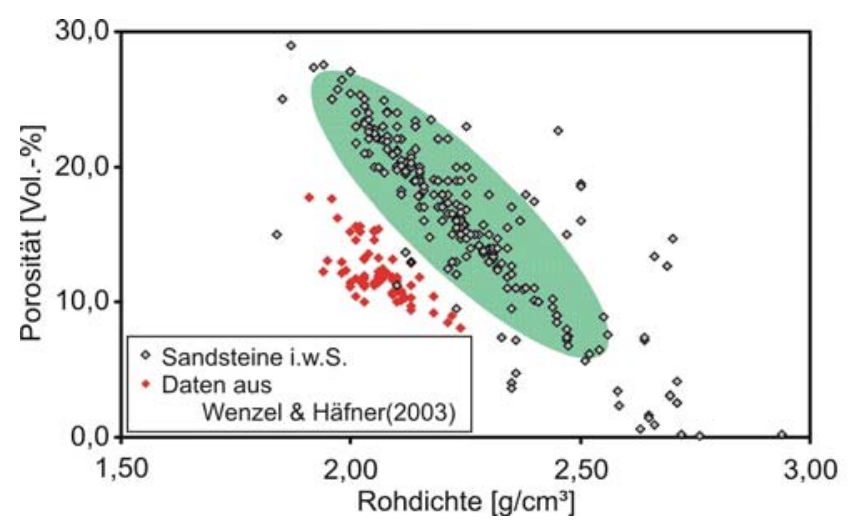

\begin{tabular}{lcc}
\hline & \multicolumn{2}{c}{ linearer Korrelationskoeffizient r } \\
& für $\rho_{\text {roh }} / \Phi$ & $n$ \\
\hline Sandstein i.w.S. & $-0,620$ & 357 \\
$\begin{array}{l}\text { Sandsteine } \\
\text { (aus Wenzel \& Häfner 2003) }\end{array}$ & $-0,681$ & 69 \\
$\begin{array}{l}\text { Sandstein i.w.S. } \\
\text { (exkl. Wenzel \& Häfner 2003) }\end{array}$ & $-0,859$ & 288 \\
\hline
\end{tabular}

Abb. 3.32: Korrelationsdiagramm der Parameter Rohdichte $\rho_{\text {roh }}$ und Porosität $\Phi$ mit den Korrelationskoeffizienten für den jeweiligen linearen Zusammenhang in der Gruppe der Sandsteine. Die Darstellung der 80 \%-igen Aufenthaltswahrscheinlichkeit unter Ausschluss des Datensatzes aus Wenzel \& Häfner (2003) verdeutlicht die Sonderstellung dieser Daten.

Tab. 3.3: Vergleich der Daten zur Rohdichte und Porosität nach Wenzel \& Häfner (2003) mit lokal äquivalenten Sandsteinen nach Grimm (1990). Hervorgehoben sind die jeweiligen Medianwerte mit den entsprechenden Standardabweichungen (in Klammern).

\begin{tabular}{lcccc}
\hline & \multicolumn{3}{c}{ Rohdichte $\left[\mathrm{g} / \mathrm{cm}^{3}\right]$} & \multicolumn{2}{c}{ Porosität [Vol.-\%] } \\
\hline Daten Wenzel \& Häfner (2003) & $\mathbf{2 , 1 0}$ & $(0,06)$ & $\mathbf{1 0 , 8 2}$ & $(3,50)$ \\
Vergleichsdaten Westpfalz & $\mathbf{2 , 1 7}$ & $(0,09)$ & $\mathbf{1 8 , 1 5}$ & $(3,60)$ \\
Neustadt-Haardter Sandstein & 2,32 & & 12,38 & \\
Obersulzbacher Sandstein & 2,13 & & 20,19 & \\
Olsbrücker Sandstein & 2,21 & & 16,56 & \\
Schweinsthaler Sandstein & 2,12 & & 19,74 & \\
\hline
\end{tabular}

Demnach sind die Sandsteinvarietäten aus Wenzel \& Häfner (2003), die in die Porositätsklasse I einzuordnen wären (vgl. Abb. 3.31), ihrer Rohdichte nach eher mit den Gesteinen der Porositätsklasse II oder III konform. Dabei weisen sie bei einer deutlich geringeren Porosität im Vergleich mit den vier lokaläquivalenten Sandsteinen einen sogar noch geringeren Dichte-Medianwert auf. Alle Untersuchungen werden in Verbindung mit der geltenden DIN-EN 1936 (vgl. Kap. 3.4.1) gesetzt. Daher kann die Abweichung lediglich auf eine in Teilen ungenaue Durchführung der Eigenschaftsermittlung zurückgeführt werden. Eine nicht ausreichende Wassersättigung der Probe, z.B. infolge eines unvollständigen Vakuums, kann dazu führen, dass Lufteinschlüsse im Probekörper verbleiben. Bei den anschließenden Wägungen resultiert daraus sowohl ein zu geringes Sättigungsgewicht $\mathrm{m}_{\mathrm{s}}$ als auch ein zu geringes Auftriebsgewicht $\mathrm{m}_{\mathrm{h}}$. Daraus ergibt sich in der Berechnung bei einer relativ gleichen Abnahme der beiden Parameter $m_{h}$ und $m_{s}$ bei gleich bleibender Rohdichte eine deutlich geringere Porosität. Eine ähnliche Vermutung kann für den ausgewiesenen Ausreißer LOCHARBRIGGS SANDSTONE angenommen werden. Hier ist eine hohe Porosität ( $\Phi=24,90$ Vol.-\%) mit einer relativ hohen Rohdichte $\left(\rho=2,45 \mathrm{~g} / \mathrm{cm}^{3}\right)$ verknüpft, was nur aus einem zu hohen Wert für das Auftriebsgewicht resultieren kann, insofern es sich tatsächlich um die Roh- und nicht die Matrixdichte handelt. 
Durch die Daten aus Wenzel \& Häfner (2003) werden nicht nur die definierten Grenzwerte für eine Klassifikation nach der Porosität verschoben, sondern auch die Datenverteilungen zur Rohdichte in der Gesamtheit und innerhalb der Gruppen beeinflusst (Abb. 3.33).
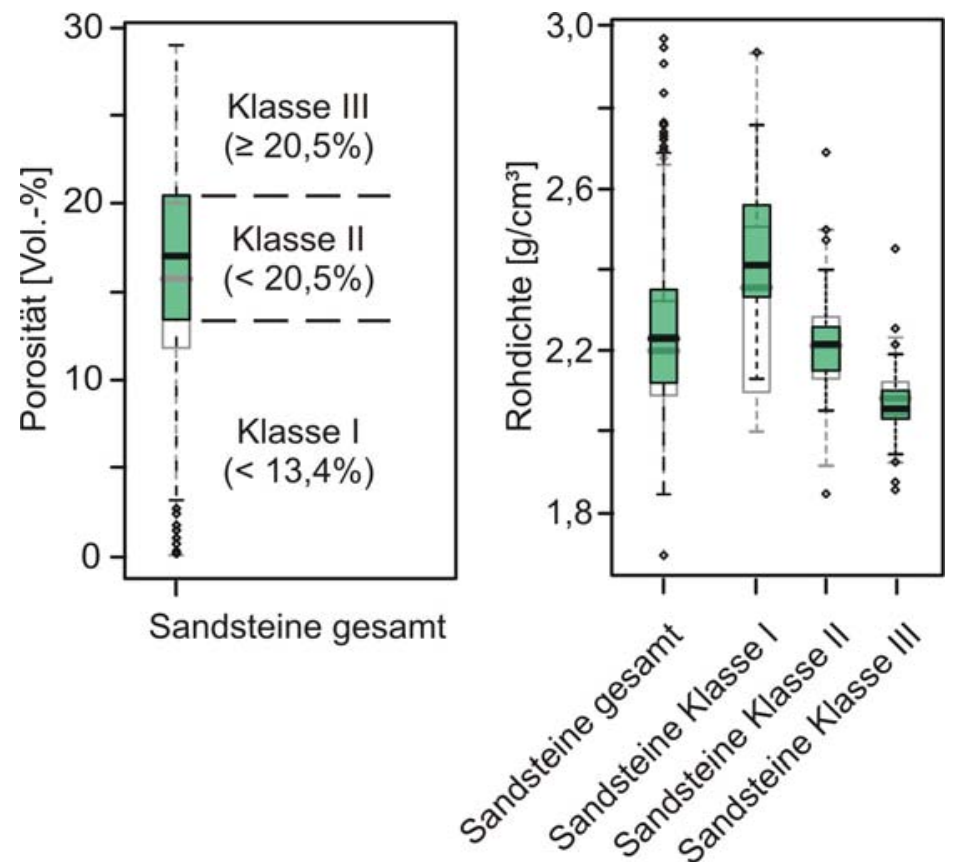

Abb. 3.33: Verbesserte Klassifikation der Sandsteine i.w.S. nach Ausschluss des Datensatzes Wenzel \& Häfner (2003). In grau ist die primäre Klassifikation zum Vergleich eingetragen. Fehlende Ausreißerwerte bei den Boxplots zur Darstellung der klassifizierten Sandsteine beruhen darauf, dass nicht für alle Varietäten Werte zur Porosität, wohl aber zur Rohdichte vorliegen.

Der Vergleich der Rohdichten in den Porositätsklassen zeigt nach der Datenfilterung nun auch drei signifikant unterschiedliche Verteilungen. Die Verteilung innerhalb der Gruppen ist im Vergleich mit der primären Grenzwertsetzung zur Klassifikation vor allem in der Porositätsklasse I deutlich gleichmäßiger. Die größte Datenspannweite besteht jedoch weiterhin in dieser Gruppe.

Als extreme Ausreißer sind maximal vier Werte einzustufen (in Klasse I: $\rho_{\text {roh }}=2,94 \mathrm{~g} / \mathrm{cm}^{3}$; in Klasse II: $\rho_{\text {roh }}=1,84 \mathrm{~g} / \mathrm{cm}^{3}$ und $\rho_{\text {roh }}=2,70 \mathrm{~g} / \mathrm{cm}^{3}$; in Klasse III: $\left.\rho_{\text {roh }}=2,45 \mathrm{~g} / \mathrm{cm}^{3}\right)$. Exemplarisch soll die brasilianische Handelssorte MARINACE betrachtet werden, die durch mehrere Auffälligkeiten hervortritt. Dies beginnt bei der Vielfalt der Handelsnamen für diese Varietät: VERDE AMAZONIA, VERDE MARINACE, NERO MARINACE, PALLADIUM, PALLADIO, VESUVIO, VEZUVIA, VEZUVIO, MARINACHI. Klassifiziert wird das Gestein als Konglomerat oder Grobklastit, manchmal lediglich als Sandstein. Zum Teil zeigt sich hier auch die willkürliche Klassifikation mancher Gesteine: „Like the red variety, this material is a hard Conglomerate. Because of the extreme hardness we classified it as granite." (www.graniteland.com). Zur Porosität finden sich Werte zwischen 0,10 Vol.-\% und 0,22 Vol.-\%, für die (Roh-?)dichte Werte von 2,76 g $/ \mathrm{cm}^{3}$ bis 2,94 g/ $\mathrm{cm}^{3}$. Im Hinblick auf das Gefüge konnten, außer verschiedenen Abbildungen (Abb. 3.34), keine weiteren Details ermittelt werden. Doch auch die Abbildungen lassen Zweifel an der korrekten Bezeichnung bzw. Beschreibung der Handelssorte aufkommen. 

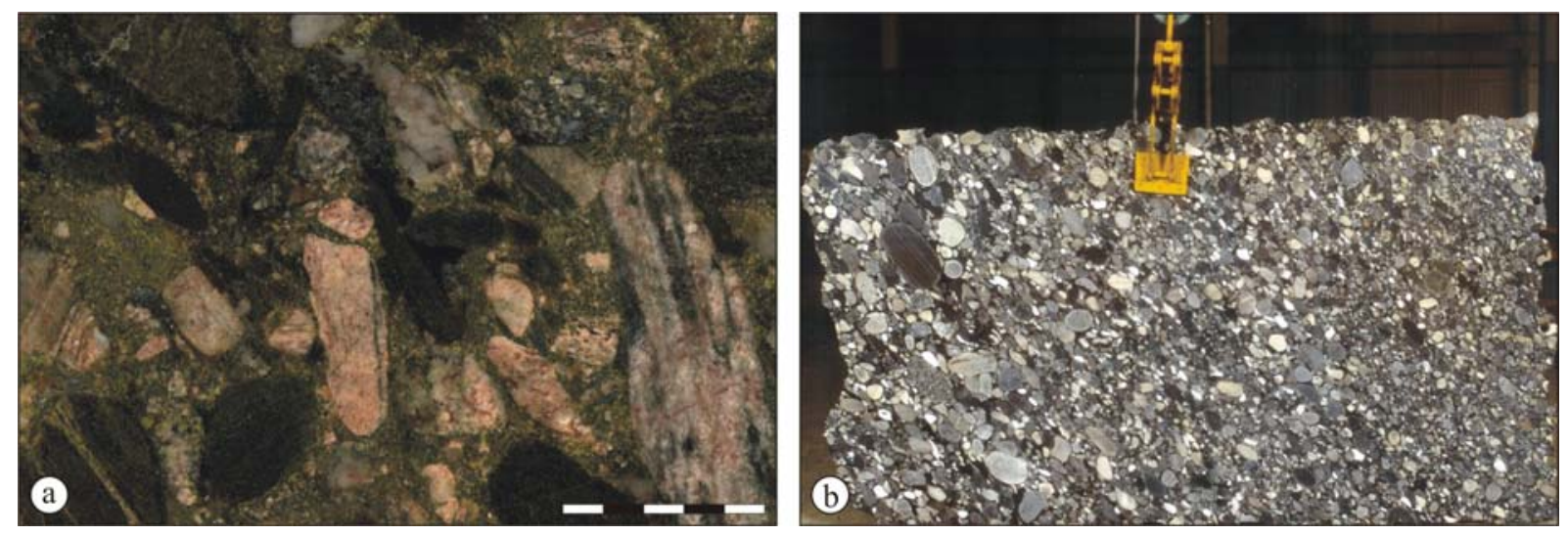

Abb. 3.34: Handelssorte MARINACE, Brasilien: a) VERDE MARINACE (Maßstab in cm, polierte Oberfläche; Quelle: Deutsches Natursteinarchiv), b) NERO MARINACE, polierte Unmaßtafel (Quelle: www.nouna.fr).

Anhand der Abbildung 3.34 wird die enorme Relevanz einer detaillierten bzw. fundierten Gesteinsbeschreibung sichtbar. Es wird deutlich, dass einzelne Klasten Größen von mindestens $30 \mathrm{~cm}$ aufweisen können. Die polymikte Zusammensetzung verschieden großer Komponenten erschwert dabei erheblich eine repräsentative Beprobung des Materials, was ebenfalls zu einer Fehleinschätzung der petrophysikalischen Eigenschaften führen kann. Darüber hinaus ist auch ein Richtungsgefüge erkennbar. Eventuell steht das Gefüge mit einer leichten Metamorphose im Zusammenhang, da die Matrix grünschieferfaziell überprägt ist (pers. Mitt. J. Stein). Eine Vergleichbarkeit innerhalb der Gruppe Sandsteine i.w.S. ist daher für diese Varietät sicherlich nicht gegeben.

\subsubsection{Technische Eigenschaften Druck-, Biege- und Spaltzugfestigkeit}

Aus der Betrachtung der Hauptgesteinsgruppen (Kap. 3.4) ist bekannt, dass die mechanische Stabilität eines Gesteins im Allgemeinen mit der Zunahme des relativen Hohlraumanteils reduziert ist. Daher sollen die Festigkeitsparameter in der Gruppe der Sandsteine im Folgenden primär nach den definierten Porositätsklassen gegliedert betrachtet werden. Für die Spaltzugfestigkeit liegen nur wenige Werte vor, so dass eine separierte Darstellung hier nicht möglich ist.

Alle Datenmengen, sowohl in der Gesamtheit als auch nach den Porositätsklassen, lassen visuell auf mehr oder weniger normalverteilte Daten schließen (Abb. 3.35). Einzige Ausnahme bildet dabei die Biegefestigkeit in der Porositätsklasse I. Der generelle Trend bleibt aber auch hier erhalten. Die einaxialen Druckfestigkeiten weisen in ihrer Gesamtheit Werte zwischen 10,01 MPa und 257 MPa auf. Dabei entfällt der höchste Wert auf die bereits dargestellte Varietät MARINACE. Auch der höchste Wert für die Biegefestigkeit (37,2 MPa) ist für ein Konglomerat gegeben (LAKSEFJORD, Norwegen), das, vergleichbar mit der Handelssorte MARINACE, grünschieferfaziell überprägt ist. Allerdings unterlag das norwegische Konglomerat einer Dynamometamorphose, welche ein intensives Richtungsgefüge sowohl der Matrix als auch der Klasten hervorgerufen hat (pers. Mitt. J. Stein). In der Folge ist bei der Ermittlung der Festigkeit eine erhöhte Anisotropie zu erwarten. 
Durch die Klassifikation nach der Porosität wird hier bereits die Abhängigkeit der mechanischen Festigkeiten vom Gesamthohlraumvolumen deutlich (Abb. 3.35). Dabei ist dieser Trend neben den Medianwerten vor allem an den oberen Extremwerten ablesbar. Geringere Festigkeiten (für $\mathrm{St}_{\mathrm{C}}<30 \mathrm{MPa}$, für $\mathrm{St}_{\mathrm{F}}<3,5 \mathrm{MPa}$ ) sind dagegen in allen definierten Porositätsklassen realisiert. Folglich kann die Porosität nicht als einziger Einflussfaktor für die Festigkeit eines Gesteins gelten. Es wird aber deutlich, dass eine Angabe von Festigkeitsbereichen für Sandsteine ohne Beachtung der Porosität als unzureichend anzusehen ist, da die Spannweite zwischen unterem und oberem Extremwert für die Sandsteine i.w.S. nur einen Ausschnitt der tatsächlichen Wertebereiche darstellt.
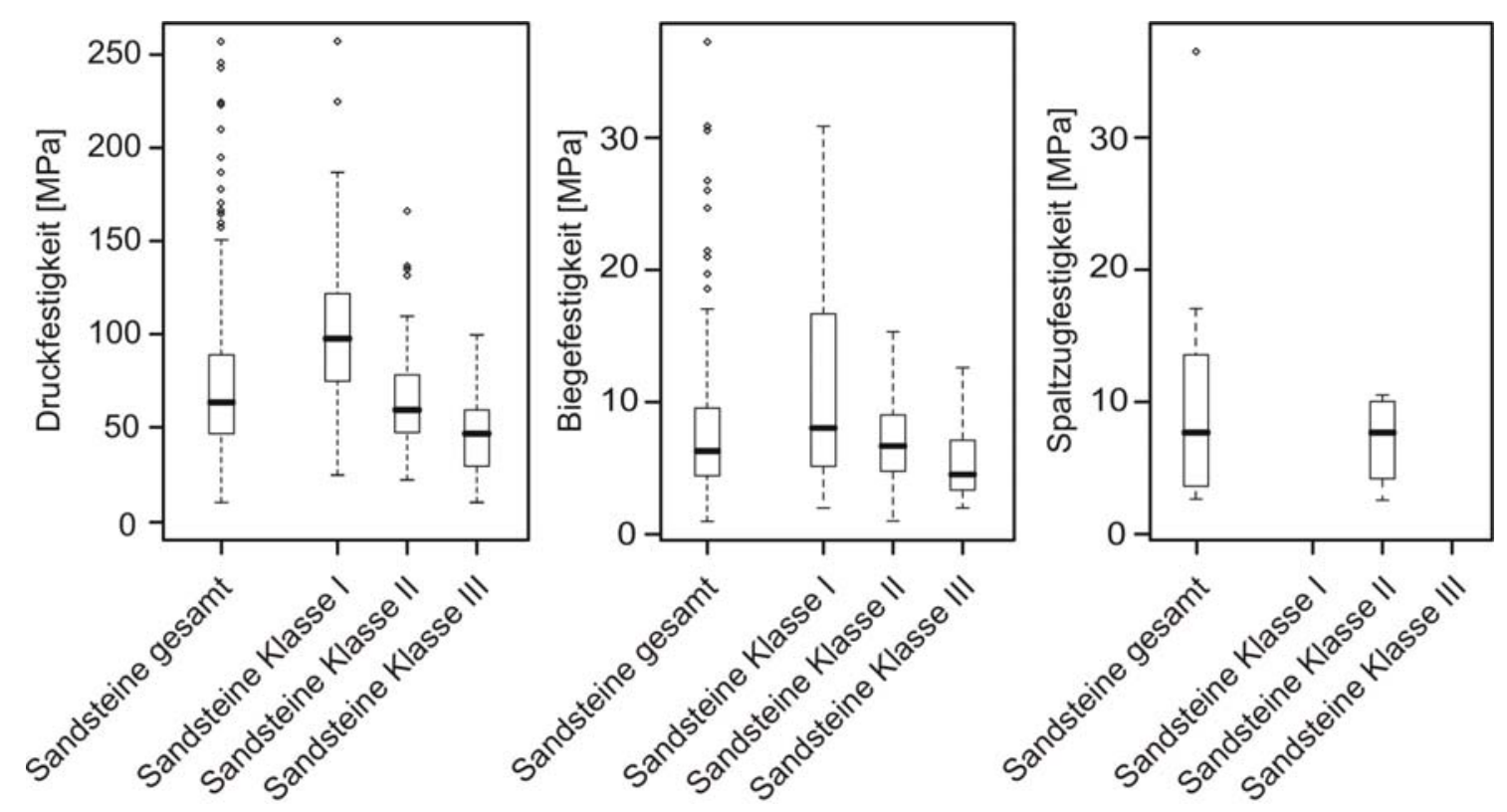

Abb. 3.35: Druck- (links), Biege- (Mitte), und Spaltzugfestigkeiten (rechts) in der Gesamtheit und in den definierten Porositätsklassen der Sandsteine (I < 13,4 \%, II < 20,5 \%, III $\geq 20,5$ \%). Fehlende Ausreißerwerte bei den Boxplots zur Darstellung der klassifizierten Sandsteine beruhen darauf, dass nicht für alle Varietäten Werte zur Porosität vorliegen. Infolge des sehr geringen Datenaufkommens hinsichtlich der Spaltzugfestigkeit ist eine Auswertung mittels Boxplots innerhalb der Porositätsklassen nicht möglich.

Für die Spaltzugfestigkeit wird ein Wert $\left(\mathrm{St}_{\mathrm{T}}=36,5 \mathrm{MPa}\right)$ als Ausreißer identifiziert. Es handelt sich um die indische Varietät MODAK, der als mittel- bis grobkörnig beschrieben wird und ein leicht lagiges Gefüge aufweisen soll. Bekannt ist, dass der Stein in Plattenstärken von 15-25 mm mit spaltrauer Oberfläche im Handel angeboten wird (Abb. 3.36) Für die Spaltzugfestigkeit ist ohne explizite Nennung einer Prüfrichtung mit Bezug auf das Gefüge ein Datenbereich von $\mathrm{St}_{\mathrm{Tmin}}=34 \mathrm{MPa}$ bis $\mathrm{St}_{\mathrm{Tmax}}=39 \mathrm{MPa}$ angegeben. Vermutlich beziehen sich die Daten lediglich auf die Spaltzugfestigkeit gegen den Lagenbau, da sonst eine höhere Anisotropie zu erwarten wäre. An einem vergleichbaren Sandstein aus Thailand (PAKCHONG GREEN, Arkose) konnte z.B. eine Anisotropie von bis zu $40 \%$ ermittelt werden (Hoffmann 2006). Da auch Pflastersteine mit bis zu $15 \mathrm{~cm}$ Kantenlänge angeboten werden, ist die Ermittlung der Festigkeitseigenschaft bzw. die Darstellung der Ergebnisse in diesem Fall als nicht ausreichend zu betrachten. 

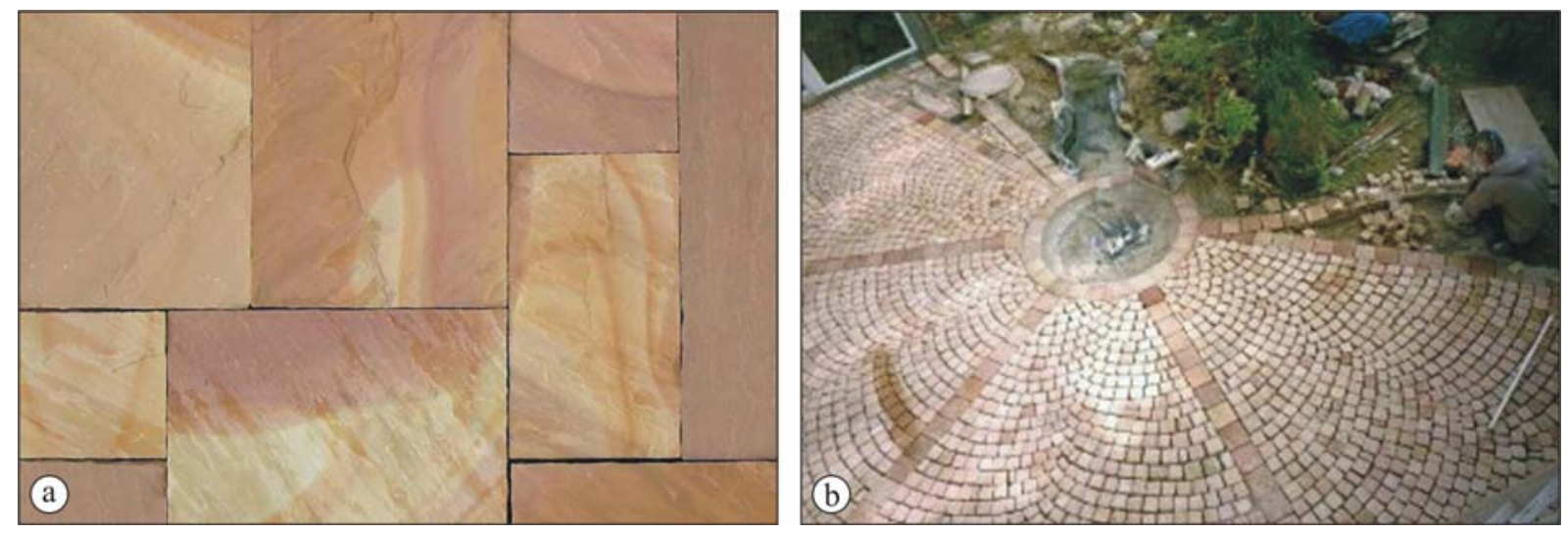

Abb. 3.36: Sandstein-Varietät MODAK (Indien). a) deutlich sichtbar wird die Verarbeitung als Bodenplatte mit spaltrauer Oberfläche, was als Indiz für ein ausgeprägteres Richtungsgefüge gelten kann. b) Terrasse aus MODAK-Kleinstein (6/8 cm) mit Bänderung aus MODAK-Pflasterplatten $(15 / 15 \quad \mathrm{~cm})$ (www.michaelkrueger.de).

Für die Gesamtheit der Daten konzentrieren sich die Verhältniswerte für die Beziehung Druck- zu Biegefestigkeit (Abb. 3.37) um den Faktor 10,5 : 1 (25 \%-Quartil: 8,4; 75 \%-Quartil: 14,3). Faktoren über 22,5 werden statistisch als Ausreißer identifiziert. Auch innerhalb der einzelnen Porositätsklassen verändern sich die Verhältnisse nur unwesentlich (Klasse I: 9,33; Klasse II: 11,25; Klasse III: 10,22). Dabei ist zwar eine Abnahme des Korrelationskoeffizienten von $\mathrm{r}=0,739$ (Porositätsklasse I) auf $\mathrm{r}=0,563$ (Porositätsklasse III) zu verzeichnen, dennoch ist auch bei hohen Porositäten eine gute Korrelation gegeben.

Für die Korrelation von Druck- und Spaltzugfestigkeit ergibt sich mit einem Verhältnis von 10,62 : 1 ein entsprechendes Bild, wobei lediglich 17 Datenpaare in die Berechnung eingehen. Daraus wäre abzuleiten, das die Biege- und die Spaltzugfestigkeit in etwa die gleichen Werte annehmen. Tatsächlich ergibt sich aber aus den wenigen zur Verfügung stehenden Merkmalspaaren $(n=11)$ ein Verhältnis von etwa 1,7 : 1 .

Die ermittelten Richtwerte für die Basiseigenschaften und die Festigkeitsparameter sind in Tabelle 3.4 zusammengefasst.

Tab. 3.4: Richtwerte für die Basiseigenschaften Rohdichte $\left[\mathrm{g} / \mathrm{cm}^{3}\right.$ ] und Porosität [Vol.-\%] sowie für die technischen Eigenschaften Druck-, Biege- und Spaltzugfestigkeit [jeweils in MPa] der Sandsteine unterteilt in Porositätsklassen. Die Minimum- bzw. Maximumwerte entsprechen den statistischen Kennwerten oberer und unterer Extremwert aus der explorativen Datenauswertung mittels Boxplot. Ausreißer sind folglich nicht in die Übersicht mit eingegangen.

\begin{tabular}{|c|c|c|c|c|c|c|c|c|}
\hline Gruppe & $\begin{array}{l}\text { Rohd } \\
\text { min }\end{array}$ & $\begin{array}{l}\text { lichte } \\
\max \end{array}$ & $\begin{array}{l}\text { Porosität } \\
\min \text { Max }\end{array}$ & $\begin{array}{c}\text { Druckfe } \\
\text { min }\end{array}$ & $\begin{array}{l}\text { stigkeit } \\
\max \end{array}$ & $\begin{array}{l}\text { Biegefe } \\
\text { min }\end{array}$ & $\begin{array}{l}\text { stigkeit } \\
\max \end{array}$ & $\begin{array}{c}\text { Spaltzugfestigkeit } \\
\min \max \end{array}$ \\
\hline Sandsteine i.w.S. & 1,84 & 2,69 & $3,1 \quad 29,0$ & 10,0 & 151,0 & 1,0 & 17,0 & $2,6 \quad 17,0$ \\
\hline Porositätsklasse I & 2,13 & 2,76 & $<13,4$ & 25,0 & 186,9 & 2,0 & 30,8 & $\mathrm{n} / \mathrm{d}$ \\
\hline Porositätsklasse II & 2,05 & 2,40 & $13,4<20,5$ & 21,9 & 110,0 & 1,0 & 15,3 & $\mathrm{n} / \mathrm{d}$ \\
\hline Porositätsklasse III & 1,94 & 2,19 & $\geq 20,5$ & 10,0 & 100,0 & 2,0 & 12,6 & $\mathrm{n} / \mathrm{d}$ \\
\hline
\end{tabular}



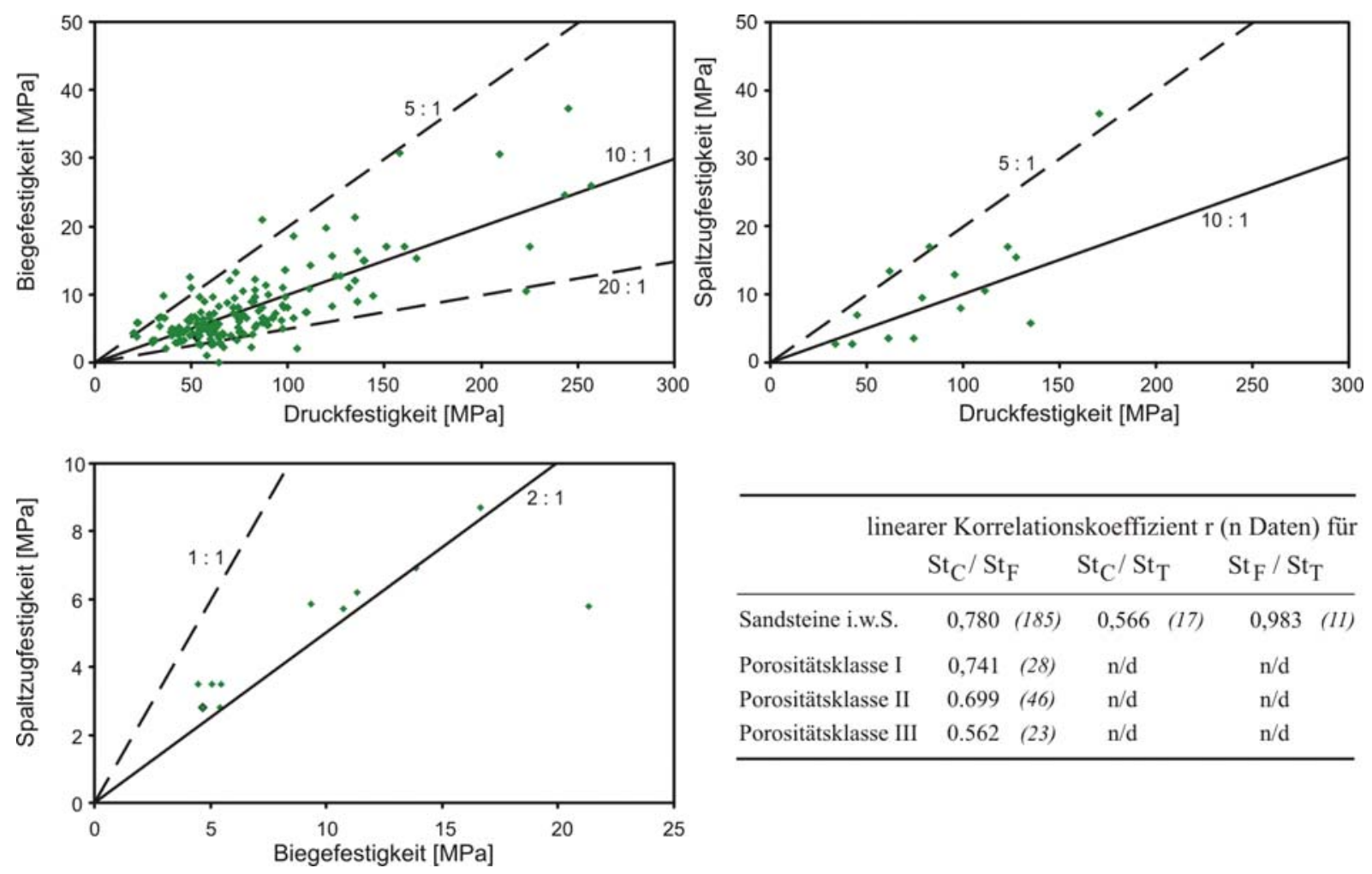

\begin{tabular}{lccccc}
\hline \multicolumn{5}{c}{ linearer Korrelationskoeffizient $\mathrm{r}$ (n Daten) für } \\
& $\mathrm{St}_{\mathrm{C}} / \mathrm{St}_{\mathrm{F}}$ & $\mathrm{St}_{\mathrm{C}} / \mathrm{St}_{\mathrm{T}}$ & $\mathrm{St}_{\mathrm{F}} / \mathrm{St}_{\mathrm{T}}$ \\
\hline Sandsteine i.w.S. & 0,780 & $(185)$ & 0,566 & $(17)$ & 0,983 \\
Porositätsklasse I & 0,741 & $(28)$ & $\mathrm{n} / \mathrm{d}$ & $\mathrm{n} / \mathrm{d}$ \\
Porositätsklasse II & 0.699 & $(46)$ & $\mathrm{n} / \mathrm{d}$ & $\mathrm{n} / \mathrm{d}$ \\
Porositätsklasse III & 0.562 & $(23)$ & $\mathrm{n} / \mathrm{d}$ & $\mathrm{n} / \mathrm{d}$ \\
\hline
\end{tabular}

Abb. 3.37: Korrelationsdiagramme der technischen Gesteinseigenschaften $\left(\mathrm{St}_{\mathrm{C}}=\right.$ Druck-, $\mathrm{St}_{\mathrm{F}}=$ Biege-, $\mathrm{St}_{\mathrm{T}}=$ Spaltzugfestigkeit) untereinander sowie die Korrelationskoeffizienten für den jeweiligen linearen Zusammenhang in der Gruppe der Sandsteine. Zusätzlich sind idealisierte Trends dargestellt.

\subsubsection{Basiseigenschaften in Korrelation mit mechanischen Festigkeiten}

Insgesamt ist zwischen den Basiseigenschaften und den Festigkeitsparametern jeweils ein deutlicher Zusammenhang gegeben (Abb. 3.38). Hier bestätigt sich auch die Annahme, dass die Spannweite beider Festigkeitsdaten mit ansteigender Rohdichte beziehungsweise abnehmender Porosität leicht zunimmt. Detaillierte Auswertungen zur Spaltzugfestigkeit sind hier infolge der zu geringen Datenmenge nicht möglich.

In der Korrelation zur Druckfestigkeit bestätigt sich, dass der Datensatz aus Wenzel \& Häfner (2003) hinsichtlich der Porosität mit einer gewissen Unsicherheit behaftet ist. Während die Daten zur Rohdichte eine nur sehr geringe Auswirkung auf das Verhalten der Gesamtdatenmenge haben, wird die Korrelation zwischen der Porosität und der Druckfestigkeit deutlich beeinflusst. Angaben zur Biegefestigkeit sind in dem genannten Datensatz nicht vorhanden. 

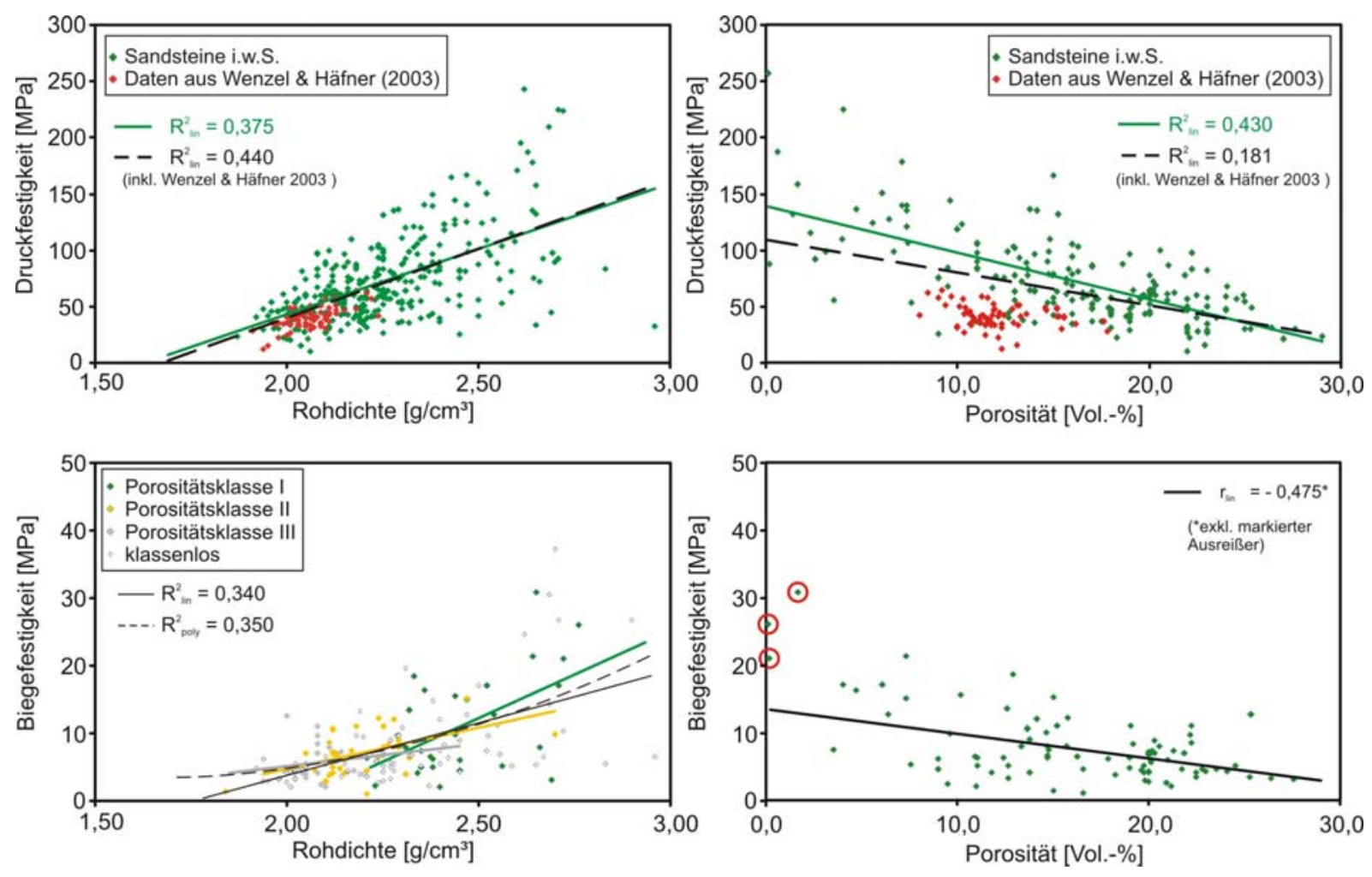

Abb. 3.38: Punktverteilungen der Korrelationen zwischen den Basiseigenschaften und den mechanischen Festigkeiten Druck- (oben) und Biegefestigkeit (unten) in der Gruppe der klastischen Sedimente. Dargestellt sind weiterhin die geschätzten Determinationskoeffizienten für die jeweiligen gerichteten Zusammenhänge. Nähere Erläuterungen im Text.

In der Korrelation der Rohdichte mit der Biegefestigkeit (Abb. 3.38) ist exemplarisch der Einfluss der Porositätsklassen durch die jeweilige Regressionsgerade (I = grün, II = gelb, III = grau) dargestellt. Die unterschiedlichen Verläufe sind relativ auf die Beziehung zwischen der Rohdichte und der Druckfestigkeit übertragbar. Es zeigt sich, dass mit ansteigender Rohdichte die Steigung der jeweiligen Regressionsgeraden zunimmt. Daraus wäre folglich eine nichtlineare Zunahme der jeweiligen Festigkeit zu erwarten. Tatsächlich aber ergibt eine Verknüpfung über einen nichtlinearen Ansatz keine wesentliche Verbesserung für die Beschreibung des gerichteten Zusammenhangs.

Auch die Porosität ist scheinbar sowohl mit der Druck- als auch mit der Biegefestigkeit über eine Linearfunktion verknüpft. David (2006) beschreibt für Bausandsteine im Raum Marburg (Unterer und Oberer Buntsandstein) eine Verknüpfung der Porosität mit der Druckfestigkeit über eine Potenzfunktion. Eine zunehmende Quarz-Zementation der Sandkörner, einhergehend mit einer Abnahme der Porosität, führt hier zu einem Anstieg der Festigkeit. Es wird beschrieben, dass der Anstieg der Festigkeit nicht linear, sondern potenziell erfolgt, da die Entwicklung dieser Anwachssäume in allen drei Dimensionen stattfindet. Eine lineare Verknüpfung der Parameter wird dagegen von Chitsazian (1985) für die niedersächsischen Wealdon-Sandsteine als auch von Schuh (1987) für Rote Mainsandsteine (Oberer Buntsandstein) postuliert. Allerdings beruhen die Angaben der zwei letztgenannten Autoren auf einer sehr geringen Datenbasis ( $n=9$ bzw. $n=7$ ). Schuh (1987) schlussfolgert, dass die Druckfestigkeit in erheblichem Maße von der Porosität beeinflusst wird und nur ein untergeordneter Zusammenhang zum sedimentären Gefüge und zum 
Bindemittel besteht. Auch wenn die Untersuchungen von David (2006) auf einer deutlich besseren Datenbasis fundieren $(n=65)$, zeigt sich die Relevanz einer überregionalen statistischen Betrachtung, da scheinbar lokalspezifische Details einen Einfluss auf die Zusammenhänge der verschiedenen Parameter haben.

\subsection{Gruppe der Karbonatgesteine}

Die karbonatischen Sedimentgesteine unterliegen einer sehr großen Variationsbreite hinsichtlich ihrer Genese und folglich auch ihrer Gefügeeigenschaften und Porositäten. So können die frisch abgelagerten Sedimente in vielfältiger Weise aus klastischen, chemischen und/oder organogenen Anteilen bestehen. Dieser polygene Charakter ist auch durch die häufig unsortierte Vermischung ungleich großer Partikel geprägt (Abb. 3.39). So kann es synsedimentär zur Vermengung von gröberen Komponenten mit feinkörnigem Karbonatschlamm oder einer sekundären Zementation von Hohlräumen durch Auskristallisation kommen (Clemens et al. 1990). Der Gefügecharakter wird zudem stark durch diagenetische Prozesse beeinflusst (z.B. Friedman 1972, Bebout et al. 1979, Moore 1989).

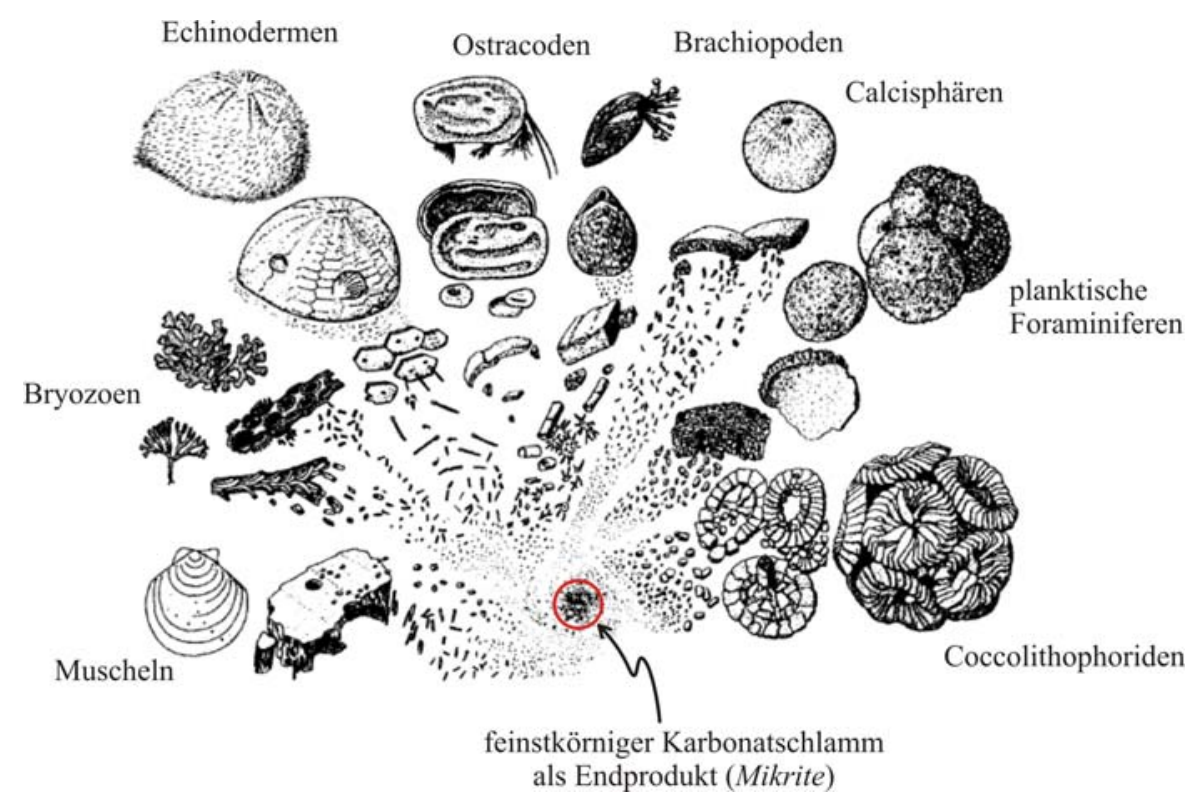

Abb. 3.39: Zerfallsstadien biogenen Detritus’ verschiedener Organismen (Beispiel aus der Schreibkreide). Zu jedem Zeitpunkt kann es zur Sedimentation und Kompaktion der Partikel kommen, was eine erhebliche Heterogenität des entstehenden Primärsedimentes nach sich ziehen kann (aus Pätzold et al. 2004).

Die Bildung von Karbonatgesteinen ist überwiegend an marine Ablagerungsräume, wie z.B. Lagunen, Riffe, Vorriffe, Schelfbereiche, gebunden (Koch \& Sobott 2005). Im Folgenden sollen fünf verschiedene Grundtypen differenziert betrachtet werden.

Als vorherrschendes chemisch-biogenes Sediment ist der Kalkstein (Abb. 3.40 a, b), als diagenetisches Produkt von Karbonatsedimenten, anzusehen. Die Partikelgrößen des aufbauenden Schalenmaterials kann von mehreren Zentimetern Größe bis zu mikroskopisch 
feinen Partikeln reichen. Folglich können sich daraus auch unterschiedliche Gefüge mit unterschiedlichen Porenraumvolumina ergeben.

Davon abgegrenzt stehen Kalkbrekzien (Abb. 3.40 c), welche durch eine sekundäre Aufarbeitung und erneuter diagenetischer Verfestigung karbonatischer Sedimente entstehen.

Kalkoolithe (Abb. 3.40 d) bestehen aus konzentrisch-schaligen, bei der Sedimentation bereits verfestigten Kalkkugeln (Ooide), die zumeist kalkig, teilweise auch tonig-sandig verkittet sind. Dadurch ergibt sich ein charakteristisches Gefügebild, das nur selten eine deutliche Schichtung aufweist. Die einzelnen Ooide können Durchmesser von bis zu mehreren Millimetern aufweisen.

Dolomite (Abb. 3.40 e) entstehen durch die sekundäre Dolomitisierung (Ersatz von Ca durch Mg) von Kalkschlämmen. Die Umkristallisation während oder kurz nach der Sedimentation bewirkt zumeist, dass fossile Spuren verloren gehen. Bei Dolomit-Gehalten unter $90 \%$ wird das Gestein als dolomitischer Kalkstein bezeichnet.

Neben diesen marin gebildeten Karbonatgesteinen kann die Bildung von Kalziumkarbonat in Form dünner Krusten oder Lagen im terrestrischen Milieu beziehungsweise in oder an heißen Quellen erfolgen. Hier erfolgt die Bildung des Karbonats entweder durch den Entzug von $\mathrm{CO}_{2}$ aus dem Wasser infolge der Assimilation durch Algen oder zumeist durch eine rein chemische Ausfällung. Karbonatablagerungen dieses Charakters werden als Travertin bezeichnet (Abb. $3.40 \mathrm{f}$ ). 

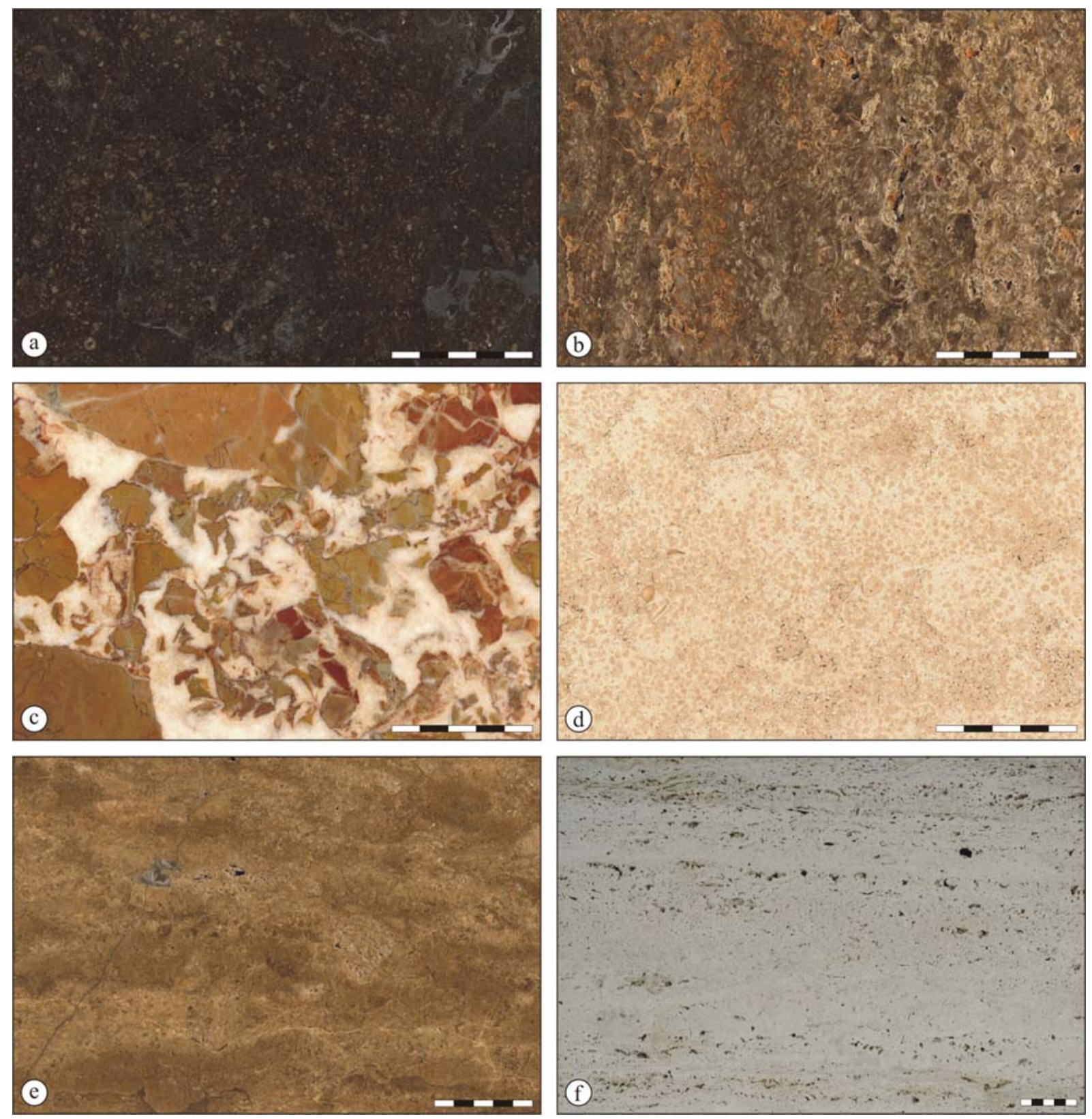

Abb. 3.40: Auswahl verschiedener Varietäten an Karbonatgesteinen: a) BELGISCH GRANIT (Belgien), massiver mikritischer Kalkstein; b) MOOS (Deutschland), Muschelkalk mit hohem Fossilgehalt; c) ZILLER (Kroatien), Kalkbrekzie; d) PIERRE DE VILHONNEUR (Frankreich), Kalkoolith; e) WACHENZELL (Deutschland), massiver Dolomit; f) ROMANO CLASSICO (Italien), Travertin. Maßstäbe in cm (Quelle Abb. a bis e: Deutsches Natursteinarchiv); (in a - e polierte Oberflächen, in f sägeraue Oberfläche, Maßstäbe in cm).

\subsubsection{Basiseigenschaften Rohdichte und Porosität}

Wie bei den Sandsteinen ist auch bei Karbonatgesteinen eine Unterscheidung von Matrixund Rohdichte unabdingbar (Abb. 3.41). Infolge ihrer lithologischen Eigenschaften aus der Genese und der kompositionellen Zusammensetzung ist in der Gruppe der Karbonatgesteine eine hohe Variabilität bezüglich der Porosität und damit einhergehend der Rohdichte gegeben. Folglich ist zwischen beiden Parametern ein direkter Zusammenhang ausgebildet. 

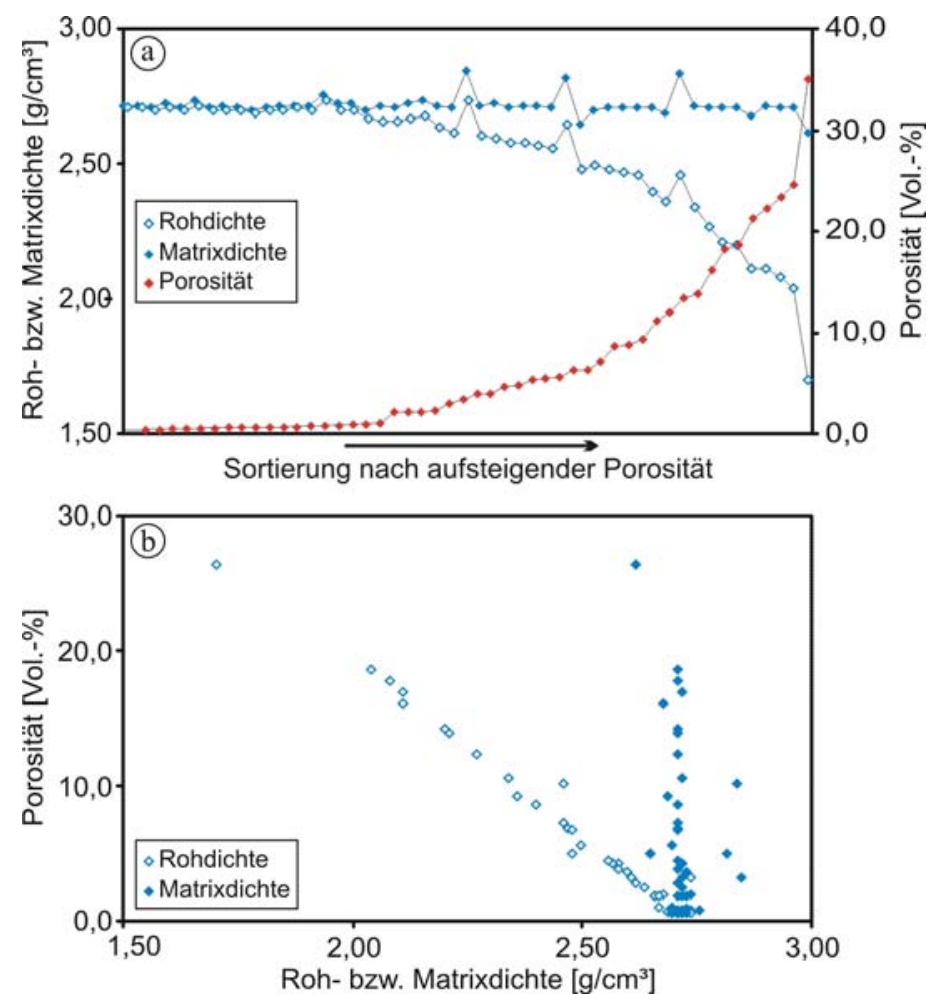

Abb. 3.41: a) Karbonatgesteine sortiert nach aufsteigender Porosität mit verbundenen Werten zur Roh- und Matrixdichte. b) Korrelation der Porosität und der Roh- bzw. Matrixdichte (Daten aus Grimm 1990).

Die Problematik hinsichtlich der für diese Arbeit zusammengestellten Daten ergibt sich nun wiederum aus einer häufig zu ungenauen bzw. unzureichenden Charakterisierung des im Handel angebotenen Materials. Aus dem Vergleich der Abbildung 3.41 mit der Abbildung 3.43 kann jedoch davon ausgegangen werden, dass in den verschiedenen Datenquellen zumeist die Rohdichte angegeben worden ist.

Die höchsten Medianwerte zur Rohdichte sind für die Untergruppen /Dolomit/ und /Kalkbrekzie/ gegeben (Abb. 3.42). Die Gruppe /Kalkstein/ zeigt zwar einen vergleichbar hohen Medianwert, weist jedoch gleichzeitig eine erhebliche Spannweite mit einer Vielzahl von Ausreißerwerten auf. Die im Mittel geringsten Werte sind für die Untergruppe /Kalkoolith/ dokumentiert, wobei hier bei einer ebenfalls weiten Datenspanne eine gleichmäßige Verteilung ausgebildet ist. Die Höhe und Verteilung der Porosität in den Untergruppen ist gegenläufig zur Rohdichte ausgebildet. Dass heißt geringe Porositäten mit enger Spannweite in den Untergruppen /Dolomit/ und /Kalkbrekzie/ sowie große Wertebereiche in den Untergruppen /Kalkoolith/ und /Kalkstein/. Auch hier sind bei den Kalksteinen zahlreiche statistische Ausreißer identifiziert (Abb. 3.42). Nach der Klassifikation von Quervain (1967) decken die beiden letztgenannten Untergruppen den gesamten Bereich von sehr kompakten (<1,0 Vol.-\%) bis sehr stark porigen (> 20 Vol.-\%) Gesteinen ab. Dadurch wird auch die Korrelation für die Gesamtheit der Karbonatgesteine, die mit einem Koeffizienten $r=-0,973$ beschrieben ist, geprägt. Die verbleibenden Untergruppen zeigen ebenfalls den Trend der Rohdichteabnahme mit zunehmender Porosität, wenn sich auch relativ geringere Koeffizienten ergeben (Tab. in Abb. 3.43). 

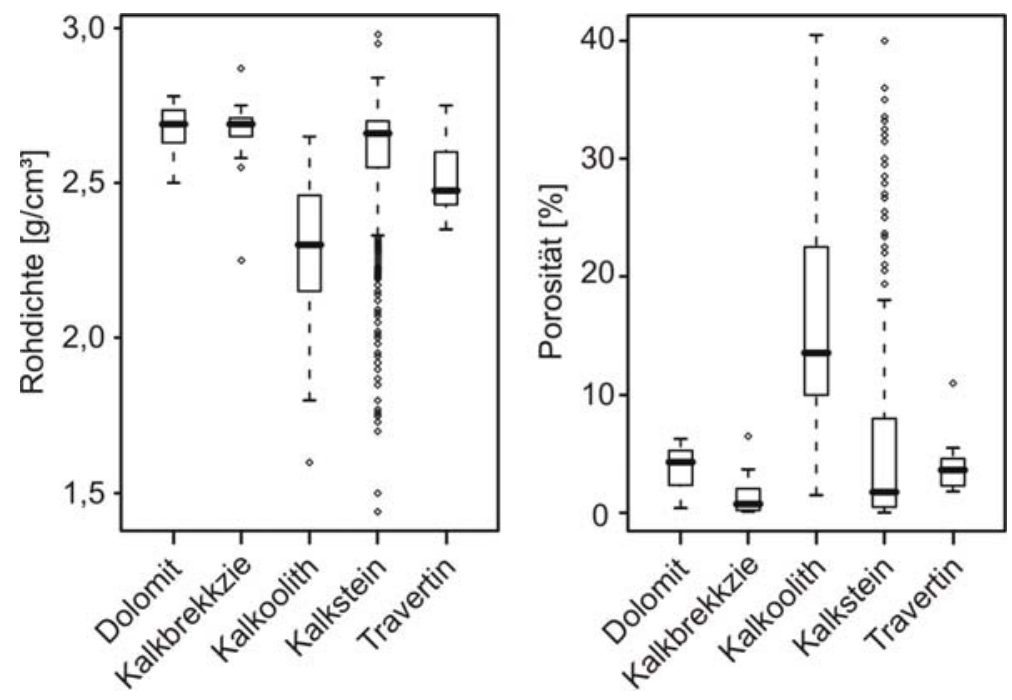

Abb. 3.42: Rohdichten (links) und Porositäten (rechts) in den Untergruppen der Karbonatgesteine.

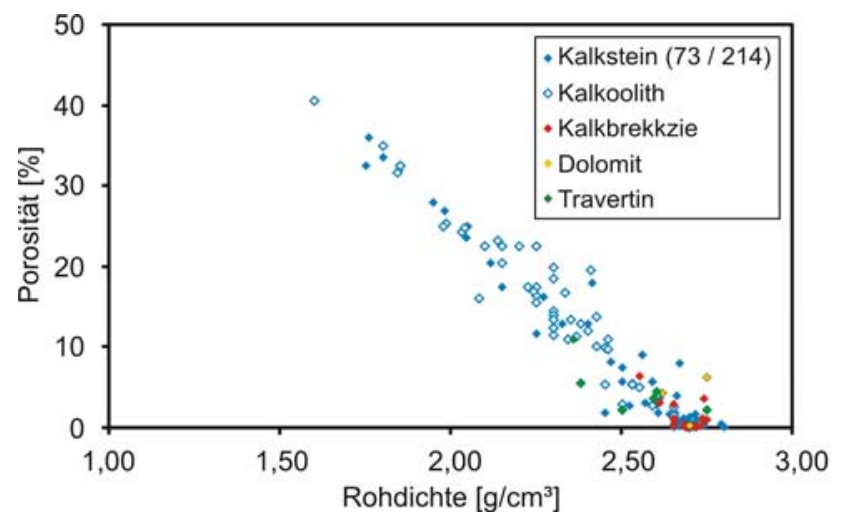

\begin{tabular}{lcc}
\hline \multicolumn{3}{c}{ linearer Korrelationskoeffizient $\mathrm{r}$} \\
& für $\rho_{\text {roh }} / \Phi$ & $n$ \\
\hline Kalkstein & $-0,971$ & 214 \\
Kalkbrekkzie & $-0,610$ & 16 \\
Kalkoolith & $-0,985$ & 53 \\
Dolomit & 0,202 & 3 \\
Travertin & $-0,656$ & 8 \\
\hline
\end{tabular}

Abb. 3.43: Korrelationsdiagramm der Parameter Rohdichte $\rho_{\text {roh }}$ und Porosität $\Phi$ mit den Korrelationskoeffizienten des jeweiligen linearen Zusammenhangs für die Untergruppen der Karbonatgesteine. Der positive Koeffizient für die Gruppe /Dolomit/ ist infolge der geringen Datenbasis als nicht repräsentativ zu betrachten.

Wie beschrieben, unterliegen die in etwa vergleichbar großen Spannweiten innerhalb der Kalkoolithe und Kalksteine einer deutlich unterschiedlichen Verteilungssymmetrie. So weisen die Boxplots für die Datenmenge der Gruppe /Kalkstein/ jeweils eine deutliche Verteilungsschiefe, gleichzeitig einen relativ engen Interquartilsabstand sowie eine hohe Anzahl an Ausreißern auf. Der Dichte-Medianwert für die Gruppe /Kalkstein/ liegt bei 2,66 g/ $\mathrm{cm}^{3}$ und beschreibt somit annähernd die mineralspezifische Dichte von Calcit (Matthes 1990). Dagegen ist die Gruppe /Kalkoolith/ hinsichtlich beider Basiseigenschaften durch eine recht symmetrische Datenverteilung gekennzeichnet. Folglich liegt der Medianwert für die Dichte unter und der Medianwert der Porosität über den entsprechenden Werten für die Gruppe /Kalkstein/. Begründet werden kann diese Verschiedenheit mit den unterschiedlichen Gefügemustern. Die Größe der die Oolithe aufbauenden kugeligen Körper ist innerhalb eines Vorkommens zumeist recht konstant, unterliegt aber lokal, je nach den Bildungsbedingungen der Ooide, deutlichen Schwankungen. Die unterschiedlichen Handelssorten weisen somit verschiedenste Korngrößen bei im Allgemeinen gut sortierten Gefügen auf. Vergleichbar ist dieser Effekt mit den Auswirkungen der Korngrößenverteilung sowie -sortierung bei Sandsteinen. Auch hier sind in der Regel gut sortierte, grobkörnig-gerundete Varietäten mit 
relativ hohen Porositäten assoziiert (z.B. Füchtbauer 1959, Tucker 1985, vgl. Kap. 3.7.1). Die Auswirkungen einer sekundären Verfüllung des Porenraumes sind bei dieser Betrachtung jedoch nicht berücksichtigt.

Für die Gruppe /Travertin/ sind relativ hohe Rohdichten sowie relativ niedrige Porositäten bei jeweils geringen Datenspannweiten beschrieben. Erwartungsgemäß sollten hier, infolge der Genese auch deutlich höhere Werte für die Porosität bzw. deutlich geringere Dichten auftreten. Peschel (1983) z.B. nennt als Obergrenze für die Porosität von Travertinen (inkl. Kalktuff) einen Wert von 60 \%. Da Travertine hinsichtlich der Porosität bzw. der Verteilung des Porenraumes häufig eine z.T. extreme Heterogenität aufweisen können (Clemens et al. 1990, Abb. 3.44), ist zu folgern, dass das aus der hier betrachteten relativ geringen Datenmenge (Rohdichte: $n=39$; Porosität: $n=9$ ) hervorgehende obere Extremum bei weitem nicht als Grenzwert anzunehmen ist. Beispielsweise ergaben aktuelle Untersuchungen am Travertin ROMANO CLASSICO (Abb. 3.40 f) effektive Porositäten zwischen 7 Vol-\% und 15 Vol-\%, was sich aus der unterschiedlichen Ausprägung des Porenraumes im Lagengefüge ergibt.

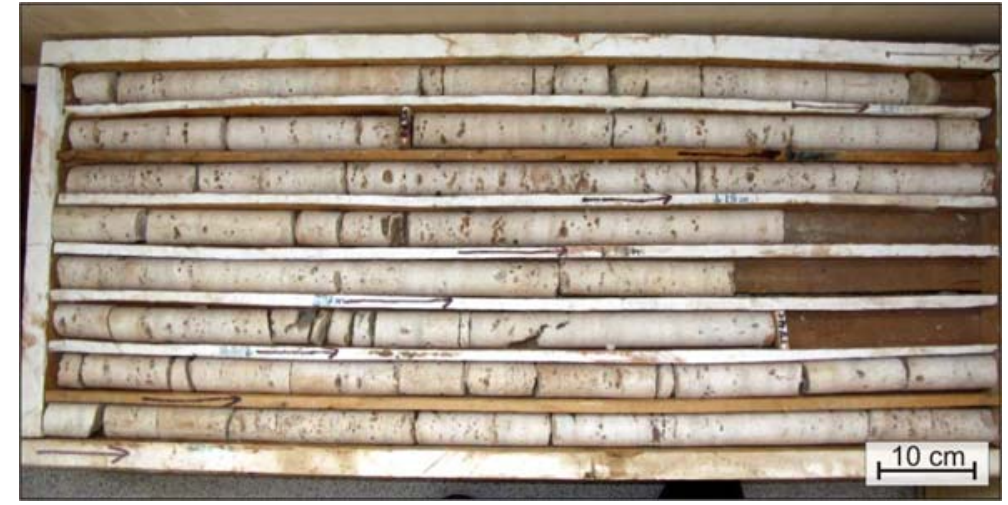

Abb. 3.44: Vertikaler Bohrkern aus einer Tavertin-Lagerstätte in der Provinz San Juan, Argentinien. Deutlich ist der lagenweise z.T. sehr heterogene Charakter des Porenraumes zu erkennen.

\subsubsection{Technische Eigenschaften Druck-, Biege- und Spaltzugfestigkeit}

Die verschiedenen Festigkeitseigenschaften zeigen in nahezu allen hier differenzierten Untergruppen der Karbonatgesteine, besonders in der Untergruppe /Kalkstein/, zumeist relativ große Spannweiten der Datenverteilungen, wobei diese bis auf wenige Ausnahmen scheinbar annähernd normalverteilt vorliegen (Abb. 3.45, Tab. 3.5).

Die einaxialen Druckfestigkeiten weisen insgesamt Werte zwischen 4,4 MPa und 265,0 MPa auf. Damit ist durch die Karbonatgesteine nahezu der gesamte, für Naturwerksteine dokumentierte Druckfestigkeitsbereich abgedeckt. Hinsichtlich der Höhe der Medianwerte ergeben sich aus den 5 Untergruppen zwei generelle Wertebereiche: i) $\mathrm{St}_{\mathrm{C}} \approx 80$ MPa für /Dolomit/, /Kalkbrekzie/, /Kalkstein/ und ii) $\mathrm{St}_{\mathrm{C}} \approx 50 \mathrm{MPa}$ für /Kalkoolith/, /Travertin/. Gruppenübergreifend sind lediglich zwei Werte als Ausreißer klassifiziert. Der Ausreißer in der Gruppe /Kalkoolith/ überrascht dadurch, dass die entsprechende Varietät PIERRE DE LANGRES (Frankreich) als mittel- bis grobkörnig beschrieben wird (Gargi et al. 1998). Im Allgemeinen wird eine Zunahme der Korn- bzw. Partikelgröße jedoch mit einer Reduktion der Festigkeit in Verbindung gebracht. 
Auch hinsichtlich der Biegefestigkeit überspannen die Karbonatgesteine mit Werten zwischen 1,0 MPa und 34,0 MPa annähernd den gesamten, für Naturwerksteine beschriebenen Datenbereich. Im Hinblick auf die relative Höhe der Medianwerte ergibt sich im Gruppenvergleich ein den Druckfestigkeitswerten annähernd ähnliches Bild. Davon abweichend ist für Travertine mit einem Medianwert von rund 11,5 MPa eine überraschend hohe Biegefestigkeit beschrieben. Zudem treten in der Gruppe /Kalkstein/ mehrerer Ausreißer auf, wobei diese jedoch lediglich $3 \%$ der gesamten Datenmenge dieser Untergruppe ausmachen.

Zur Spaltzugfestigkeit konnten nur wenige Daten dokumentiert werden, so dass eindeutige Aussagen zu den Untergruppen nicht möglich sind. Auffällig ist jedoch die Asymmetrie in der Gruppe /Kalkstein/. Allein durch die zwei klassifizierten Ausreißer kann diese nicht erklärt werden, da der Medianwert gegenüber Ausreißern als relativ robust gilt (Kürzl 1988). Bei den zugehörigen Gesteinen handelt es sich um zwei Farbvarietäten des indischen Kalksteins KOTHA (BLUE, BROWN), der als homogen und feinkörnig beschrieben wird. Gleichzeitig ist eine sehr geringe Wasseraufnahme angegeben, was wiederum mit einer geringen Porosität assoziiert werden kann (Peschel 1983, vgl. Kap. 3.13.2). Demzufolge sollten auch hohe Werte für die Druckfestigkeit gegeben sein. Tatsächlich aber liegt zumindest einer der Werte, wenn auch nur gering, unterhalb des errechneten Medianwertes. Es ist anzunehmen, dass das Gestein ein ausgeprägtes Richtungsgefüge aufweist, da nach Firmeninformation der Stein mit spaltrauer Oberfläche im Handel angeboten wird. Dafür ist jedoch das Vorhandensein eines ausgeprägten Lagenbaus notwendig. Hinsichtlich der Spaltzugfestigkeit sind daher vermutlich nur Werte angegeben, die senkrecht zum Lagenbau ermittelt wurden.
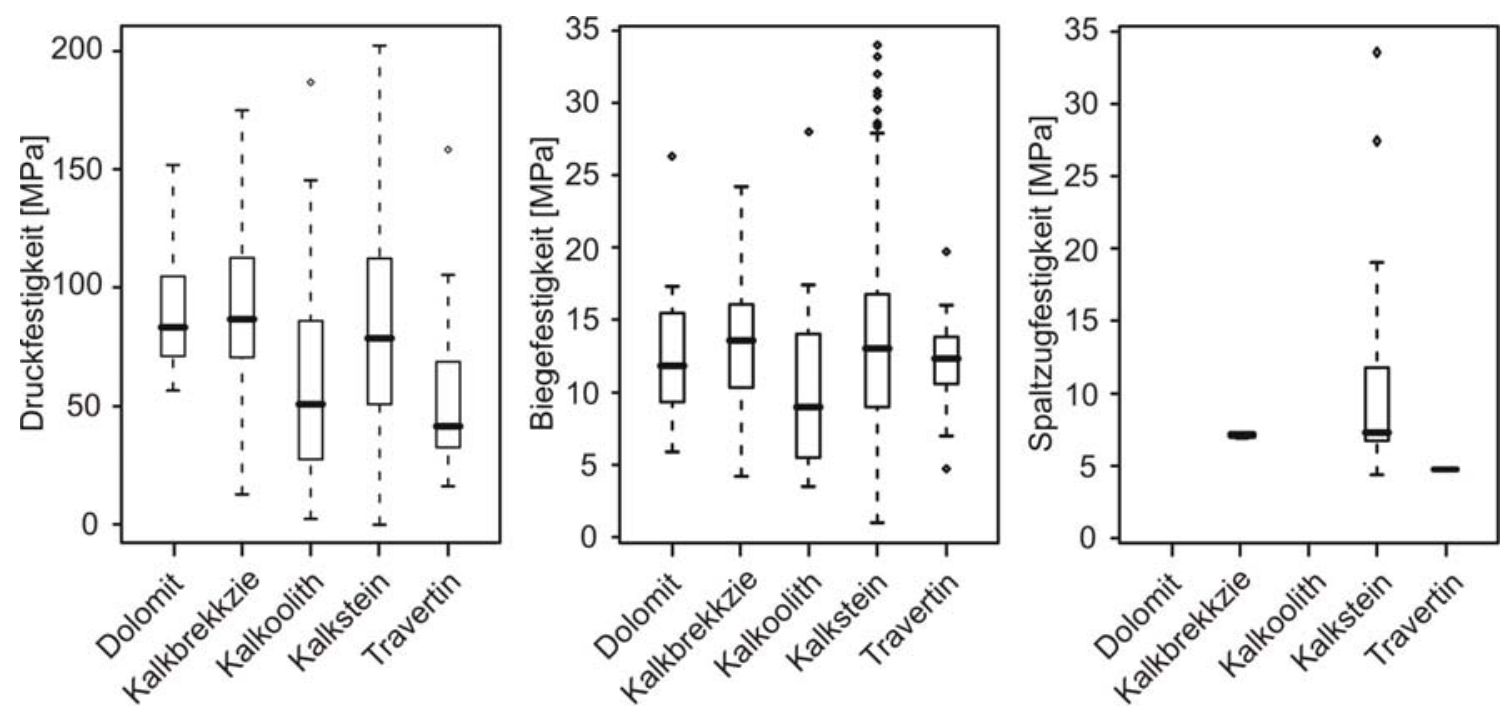

Abb. 3.45: Druck- (links), Biege- (Mitte), und Spaltzugfestigkeiten (rechts) in den Untergruppen der Karbonatgesteine. Infolge des sehr geringen Datenaufkommens hinsichtlich der Spaltzugfestigkeit mit Ausnahme der Gruppe /Kalkstein/ ist eine Datenauswertung mittels Boxplot nicht möglich.

Das Verhältnis zwischen der Druckfestigkeit $\mathrm{St}_{\mathrm{C}}$ und der Biegefestigkeit $\mathrm{St}_{\mathrm{F}}$ ist durch eine relativ hohe Variabilität geprägt. So sind für die Beziehung $\mathrm{St}_{\mathrm{C}}: \mathrm{St}_{\mathrm{F}}$ Verhältnisse zwischen $36: 1$ und 1,3: 1 (im Mittel 9,1:1) realisiert (Abb. 3.46). Bei Druckfestigkeiten über 100 MPa sind die jeweiligen Verhältnisse um die $10: 1$-Gerade konzentriert. Unterhalb dieses 
Wertes errechnen sich zumeist geringere Verhältnisse. Bezüglich der verbleibenden zwei Korrelationen ist eine fundierte Aussage infolge der geringen Datenbasis nicht möglich.
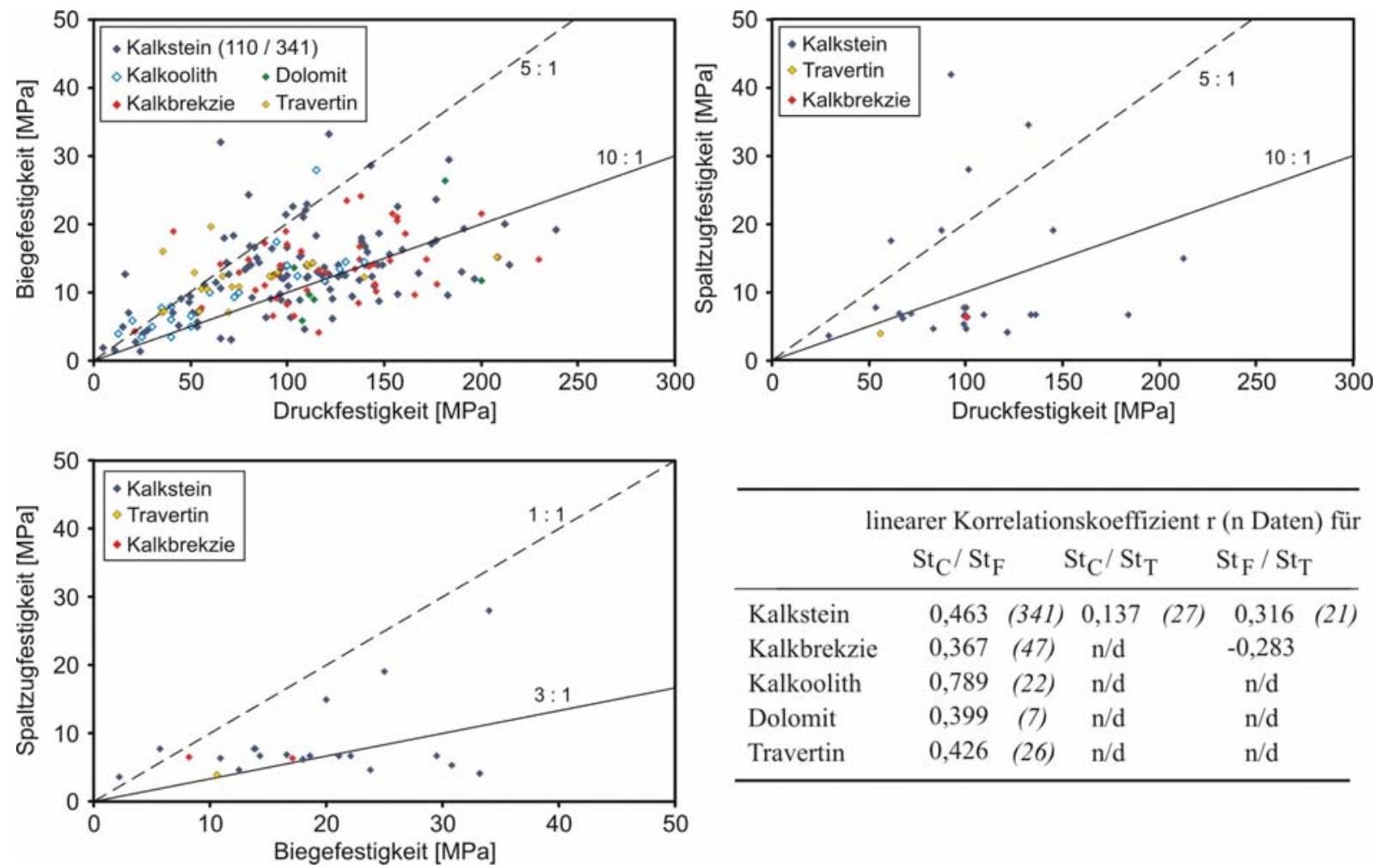

\begin{tabular}{|c|c|c|c|c|}
\hline \multicolumn{5}{|c|}{ linearer Korrelationskoeffizient $\mathrm{r}$ ( $\mathrm{n}$ Daten) für } \\
\hline & $\mathrm{St}_{\mathrm{C}} / \mathrm{St}_{\mathrm{F}}$ & & $\mathrm{St}_{\mathrm{C}} / \mathrm{St}_{\mathrm{T}}$ & $\mathrm{St}_{\mathrm{F}} / \mathrm{St}_{\mathrm{T}}$ \\
\hline Kalkstein & 0,463 & $(341)$ & 0,137 & $0,316(21)$ \\
\hline Kalkbrekzie & 0,367 & $(47)$ & $\mathrm{n} / \mathrm{d}$ & $-0,283$ \\
\hline Kalkoolith & 0,789 & $(22)$ & $\mathrm{n} / \mathrm{d}$ & $\mathrm{n} / \mathrm{d}$ \\
\hline Dolomit & 0,399 & (7) & $\mathrm{n} / \mathrm{d}$ & $\mathrm{n} / \mathrm{d}$ \\
\hline Travertin & 0,426 & (26) & $\mathrm{n} / \mathrm{d}$ & $\mathrm{n} / \mathrm{d}$ \\
\hline
\end{tabular}

Abb. 3.46: Korrelationsdiagramme der technischen Gesteinseigenschaften $\left(\mathrm{St}_{\mathrm{C}}=\mathrm{Druck}_{\mathrm{r}}, \mathrm{St}_{\mathrm{F}}=\mathrm{Biege}, \mathrm{St}_{\mathrm{T}}=\right.$ Spaltzugfestigkeit) untereinander sowie die Korrelationskoeffizienten für den jeweiligen linearen Zusammenhang in den Untergruppen der Karbonatgesteine. Zusätzlich sind idealisierte Trends dargestellt.

Tab. 3.5: Richtwerte für die Basiseigenschaften Rohdichte $\left[\mathrm{g} / \mathrm{cm}^{3}\right.$ ] und Porosität [Vol.- \%] sowie für die Parameter Druck- , Biege- und Spaltzugfestigkeit [jeweils in MPa] der Untergruppen der Karbonatgesteine. Die Minimum- bzw. Maximumwerte entsprechen den statistischen Kennwerten oberer und unterer Extremwert aus der explorativen Datenauswertung mittels Boxplots. Ausreißer sind folglich nicht in die Übersicht mit eingegangen.

\begin{tabular}{|c|c|c|c|c|c|c|c|c|c|}
\hline \multirow{2}{*}{ Gruppe } & \multicolumn{2}{|c|}{ Rohdichte } & \multicolumn{2}{|c|}{ Porosität } & \multicolumn{2}{|c|}{ Druckfestigkeit } & \multicolumn{2}{|c|}{ Biegefestigkeit } & \multirow{2}{*}{$\begin{array}{c}\text { Spaltzugfestigkeit } \\
\min \max \end{array}$} \\
\hline & $\min$ & $\max$ & $\min$ & $\max$ & $\min$ & $\max$ & $\min$ & $\max$ & \\
\hline Kalkstein & 2,28 & 2,95 & 0,02 & 19,36 & 4,4 & 265,0 & 1,0 & 27,9 & $3,6 \quad 19,1$ \\
\hline Kalkbrekzie & 2,58 & 2,75 & 0,11 & 3,69 & 20,1 & 208,5 & 4,2 & 24,2 & 6,6 \\
\hline Kalkoolith & 1,80 & 2,65 & 1,50 & 40,50 & 7,5 & 191,6 & 3,5 & 17,4 & $\mathrm{n} / \mathrm{d}$ \\
\hline Dolomit & 2,50 & 2,78 & 0,40 & 6,27 & 33,0 & 200,0 & 5,9 & 17,3 & $\mathrm{n} / \mathrm{d}$ \\
\hline Travertin & 2,35 & 2,75 & 1,80 & 5,51 & 25,3 & 140,0 & 7,0 & 16,0 & 4,4 \\
\hline
\end{tabular}

\subsubsection{Basiseigenschaften in Korrelation mit mechanischen Festigkeiten}

Für Karbonatgesteine besteht zwischen der Druck- bzw. der Biegefestigkeit ein deutlicher Zusammenhang mit den Basiseigenschaften (Abb. 3.47). Im Falle der Rohdichte ist dabei eine positive, bezüglich der Porosität eine negative Korrelation ausgebildet. In beiden Fällen deutet sich eine Zweiteilung der Datensätze an. Während um die aus der explorativen Datenauswertung hervorgegangenen Medianwerte von $\rho_{\text {roh }}=2,65 \mathrm{~g} / \mathrm{cm}^{3}$ für die Rohdichte 
bzw. $\Phi=3,20 \%$ für die Porosität jeweils eine weite Spannweite der Festigkeitswerte dokumentiert ist, ergibt sich mit geringerer Rohdichte bzw. zunehmender Porosität ein deutlich regressiver Zusammenhang.
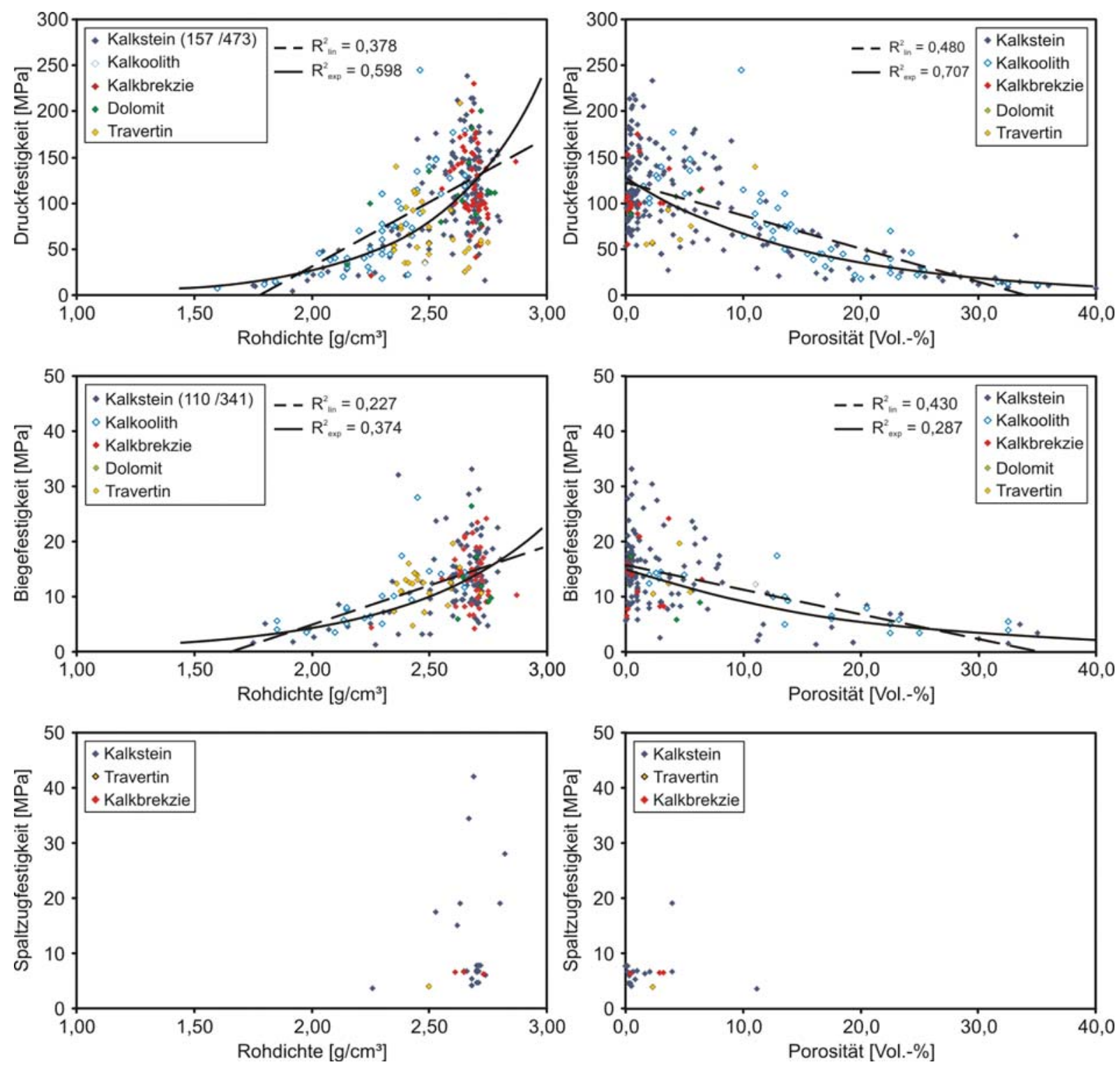

Abb. 3.47: Korrelationsdiagramme der Basiseigenschaften Rohdichte $\rho_{\text {roh }}$ bzw. Porosität $\Phi$ und den mechanischen Festigkeiten in den Untergruppen der Karbonatgesteine. Angegeben sind die Determinationskoeffizienten für den jeweiligen linearen und exponentiellen Zusammenhang für die Gesamtheit der Karbonatgesteine. Die geringe Datenanzahl zur Spaltzugfestigkeit schränkt die Möglichkeit zur statistischen Datenauswertung stark ein.

Dieser bimodale Charakter ist ausgeprägt in der Gruppe /Kalkstein/ zu beobachten. In den anderen Untergruppen, markant in der Gruppe /Kalkbrekzie/, kommt es eher zur Bildung eines Datenclusters. Die größte Datenspannweite der Festigkeitsparameter ist noch unterhalb des Medianwertes der Porosität gegeben. Für sehr kompakte Gesteine ( $\Phi<1,0$ Vol.-\%) ist dabei für die Druckfestigkeit ein Bereich zwischen etwa $50 \mathrm{MPa}$ und gut $220 \mathrm{MPa}$ und für die Biegefestigkeit zwischen etwa 5 MPa und 35 MPa beschrieben. Für die Spaltzugfestigkeit sind etwas geringere Werte dokumentiert, was auch aus dem Verhältnis zur Biegefestigkeit zu erwarten ist. Erstaunlich sind die zum Teil extremen Unterschiede in der Festigkeit bei 
gleichen Basiseigenschaften. Deutlich wird dies beim Vergleich der drei Gesteine AEGEAN BROWN $^{1)}$ (Kalkbrekzie, Türkei), BURDUR BEIGE ${ }^{2)}$ (Kalkstein, Türkei) und MARBRE PALOMA $^{3)}$ (Kalkstein, Frankreich). Bei gleicher Rohdichte $\left(2,71 \mathrm{~g} / \mathrm{cm}^{3}\right)$ und gleicher Porosität (0,18 Vol.-\%) sind Druckfestigkeiten von 55,5 $\mathrm{MPa}^{1)}$, 99,0 $\mathrm{MPa}^{2)}$ sowie 150,3 $\mathrm{MPa}^{3)}$ realisiert. Die Herabsetzung der Festigkeit ist dabei unter anderem auf die lithologische Differenzierung der begleitenden Mineralparagenesen zurückzuführen. Die Varietät AEGEAN BROWN ist hinsichtlich ihrer stofflichen Komponenten durch einen hohen Anteil an Tonmineralen gekennzeichnet (Abb. 3.48 a). Entweder treten diese in Bändern bzw. in Lagen akkumuliert auf oder einzelne härtere Gesteinspartikel sind von einem tonigen Überzug umschlossen. Letzteres ist gerade durch den brekziösen Charakter dieser Kalksteinvarietät gegeben. Diese Bereiche können im Falle einer Belastung durch Druck- oder Biegekräfte als Schwächezonen die Gesamtfestigkeit eines Gesteins erheblich negativ beeinflussen.
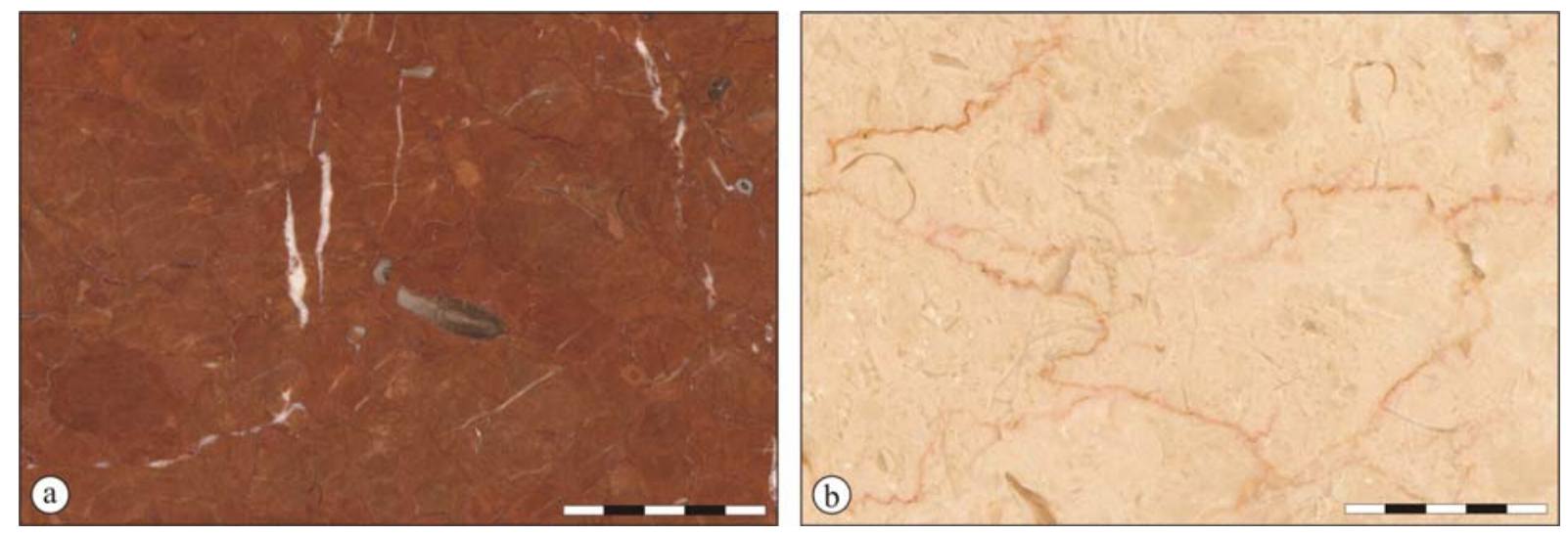

Abb. 3.48: Herabgesetzte Festigkeit durch a) erhöhte Tongehalte und einen brekziösen Charakter des Gefüges (AEGEAN BROWN, Türkei) und b) deutlich ausgebildete Stylolithenbahnen (ROSALIA LIGHT, Türkei); (Quelle: Deutsches Natursteinarchiv); (polierte Oberflächen, Maßstäbe in cm).

Auch das vermehrte Auftreten von Stylolithen kann den gleichen Effekt hervorbringen (Abb. 3.48 b). Diese Strukturen, die auf die flächige Auflösung des Kalksteins aufgrund von Gebirgsdruck oder tektonischem Druck zurückzuführen sind (z.B. Wanless 1979), bestehen aus dünnen Säumen von Ton und schwer löslichen Materialien (Tucker 1985). Durch ihren meist parallel zur Schichtung orientierten Verlauf können sie neben einer generellen Herabsetzung der Gesteinsfestigkeit auch eine höhere Anisotropie im mechanischen Verhalten hervorrufen. Für die Varietäten BURDUR BEIGE und MARBRE PALOMA ist visuell nur ein sehr geringer Tongehalt anzunehmen. Darüber hinaus erscheint das Gefüge insgesamt homogener und massiger. Das Gefüge des BURDUR BEIGE ist dabei von feinen Calcitadern durchzogen, die eventuell für die geringere Festigkeit im Vergleich mit der Varietät MARBRE PALOMA verantwortlich sein können.

Es wird grundsätzlich deutlich, dass im unteren Porositäts- bzw. im oberen Rohdichtebereich kein deutlicher Trend zwischen den Festigkeitsparametern und den Basiseigenschaften zu erwarten ist. Mit zunehmender Porosität bzw. abnehmender Rohdichte ist dagegen zumeist ein deutlicher Zusammenhang regressiven Charakters ausgebildet. Anhand der Determinationskoeffizienten erscheint dabei in allen betrachteten Eigenschaftspaaren ein nichtlinearer Ansatz (über Exponentialfunktionen) eher für eine 
Prognose geeignet, als ein linearer (Abb. 3.47). Als Besonderheit ergibt sich für die Gruppe /Travertin/ sowohl hinsichtlich der Rohdichte als auch der Porosität trotz relativ großer Datenspannweiten kein deutlicher Trend in Bezug zu den Festigkeitsparametern.

Aufgrund der extremen Verteilungsschiefe in den Basiseigenschaften und auch den daraus hervorgehenden Abweichungen in den Parametern der mechanischen Stabilität wird die Untergruppe /Kalkstein/ in den Kapiteln 3.10 bis 3.13 in zwei separaten Untergruppen behandelt. Als Grenzwert ist ein Rohdichtewert von 2,6 g/ $\mathrm{cm}^{3}$ definiert. Diese Trennung bewirkt zudem eine deutlich gleichmäßigere Verteilung der Daten innerhalb der neu gebildeten Gruppen, was vor allem an der Reduzierung der Ausreißer sowie an der deutlich zentraleren Lage der Medianwerte der Basiseigenschaften erkennbar ist. Abbildung 3.49 zeigt beispielhaft die Korrelation zwischen der Rohdichte und der Druckfestigkeit nach Aufteilung der Gruppe /Kalkstein/. Parallel dazu ist auf der Sekundärachse die Porosität aufgetragen. Mit der Neugruppierung kann eine markante Verbesserung im Hinblick auf eine Normalverteilung erreicht werden, was zeigt, dass die schiefe Verteilung primär durch die kompakten bis sehr kompakten Gesteine geprägt wird. Das gleiche Datenverhalten ist hinsichtlich der Biegefestigkeit festzustellen, wodurch sich auch die Übertragbarkeit auf die Spaltzugfestigkeit vermuten lässt. Infolge des geringen Datenpools und der damit verbundenen statistischen Unsicherheit kann eine entsprechende Überprüfung nicht erfolgen.

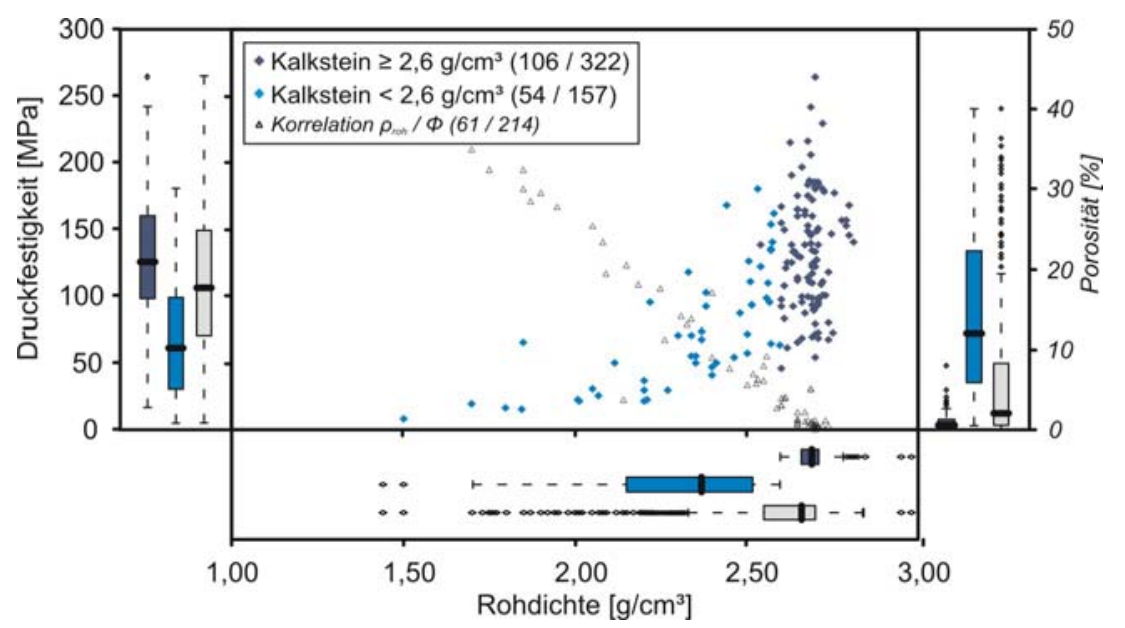

Abb. 3.49: Korrelation der Basiseigenschaften und der Druckfestigkeit nach Bildung zweier Datengruppen ( $\rho_{\text {roh }} \geq 2,6 \mathrm{~g} / \mathrm{cm}^{3}$ und $<2,6 \mathrm{~g} / \mathrm{cm}^{3}$ ) innerhalb der Untergruppe /Kalkstein/. Die jeweilige explorative Datenauswertung mittels Boxplot (grau: Kalkstein gesamt) zeigt die bessere Annäherung an eine Normalverteilung, als Vorraussetzung der Darstellung bivariater Aufenthaltswahrscheinlichkeitsbereiche.

\subsection{Gruppe der Metamorphite}

Die als Werkstein verwendeten metamorphen Gesteine decken ein weites Spektrum in Dekor, Farbe, Struktur etc. ab. Sie entstehen durch die Umwandlung von Gesteinen aller Kategorien unter Bedingungen, die von denen ihrer ursprünglichen Bildung verschieden sind (Temperatur, Druck, Bewegung). Diese differenten Bedingungen bewirken eine Veränderung am Mineralbestand und am Gefüge, die im Wesentlichen auf Reaktionen im festen Zustand beruhen. Der primäre Chemismus der Gesteine bleibt dabei im Allgemeinen erhalten (z.B. 
Wimmenauer 1985). Die Vielzahl der Varietäten infolge geologischer, metamorphosebedingter Details erschwert eine eindeutige Klassifikation der metamorphen Gesteine. Für die folgenden Betrachtungen sind die Gesteine in fünf Untergruppen gegliedert.

Gneise (Abb. 3.50 a) sind metamorphe Gesteine, die wesentliche Anteile an Feldspäten (meist mehr als 20-30\%) und zumeist auch Quarz aufweisen. Die Bezeichnung Orthogneis bezieht sich auf eine magmatische, Paragneis dagegen auf eine sedimentäre Herkunft des Ausgangsgesteins. Vorherrschend sind mittel- bis grobkörnige Gefüge ausgebildet. Sehr häufig treten lineare Paralleltexturen auf, wobei sich quarz- und feldspatreiche Lagen von glimmerhaltigen oder mafischen Lagen separieren. Daraus können bevorzugte, meist regelmäßige Bruchflächen entstehen. Auf dieser Gefüge- bzw. Bruchcharakteristik basiert auch die ältere Bezeichnung „Kristalline Schiefer“ (Wimmenauer 1985). Mit einbezogen in diese Untergruppe sind migmatische Gesteine (Migmatite, Abb. 3.50 b), also solche, die infolge einer Anatexis sowohl gneisartige, metamorphe Merkmale (Paläosom) als auch magmatische Bereiche (Leukosom) aufweisen.

Marmore (Abb. 3.50 c) stellen das metamorphe Äquivalent zu Kalksteinen dar. Fälschlicherweise werden dagegen in der Natursteinindustrie sehr häufig alle dichten und polierfähigen Kalksteine als Marmore bezeichnet. Nach der petrographischen Nomenklatur weisen Marmore im eigentlichen Sinne einen Karbonatgehalt von > $90 \%$ auf. Dem gegenüber stehen die stärker quarz- und silikathaltigeren Silikatmarmore, die Volumenanteile zwischen $50 \%$ und $90 \%$ aufweisen (Wimmenauer 1985). Im Allgemeinen gilt Calcit als druck- und temperaturstabil. Daher finden in Calcitmarmoren sehr selten Reaktionen statt. Das Gestein erfährt jedoch häufig weitgehende Gefügeänderungen bei der Rekristallisation des Calcits hin zu groben Korngrößen mit häufig bevorzugten Orientierungen (Yardley 1997).

Die Genese von Quarziten (Abb. 3.50 d) ist an eine Metamorphose von Sandsteinen unter Hochdruck- und Hochtemperaturbedingungen gebunden. Durch eine Sammelkristallisation der ursprünglich klastischen Quarzkörner und des Bindemittels wird das primäre Korngefüge überprägt und es resultiert eine dicht vernetzte Struktur (z.B. Matthes 1990). Die farbliche Varietät wird durch die Art und den Gehalt der Nebengemengteile kontrolliert (z.B. grau Graphit, grün - Fuchsit, blau - Dumortierit). In seltenen Fällen können auch primäre Sedimentationsstrukturen oder auch Fossilien erhalten bleiben.

Als Schiefer (Abb. 3.50 e) werden niedrigmetamorphe Gesteine mit sedimentärem Ursprung bezeichnet, die ein z.T. extrem ausgeprägtes Flächengefüge (Schieferungsebene) aufweisen und dadurch in mm- bis cm-dicke Platten aufspalten. Gerade diese ausgezeichnete Spaltbarkeit (besonders bei Tonschiefern) begünstigt die Verwendung als plattenförmige Bauelemente. Die Klassifikation beruht auf dem normativen Mineralbestand (z.B. Peschel 1983). Dabei werden Tonschiefer eher in eine Übergangsphase zwischen Diagenese und Metamorphose (Anchimetamorphose, Matthes 1990) gestellt. Infolge des gleichartigen technischen Verhaltens basierend auf dem extremen Richtungsgefüge werden jedoch die Gesteinstypen Glimmer-/Chloritschiefer, Phyllite und Tonschiefer im Folgenden zusammengefasst betrachtet. 
Als Serpentinite (Abb. $3.50 \mathrm{f}$ ) werden Gesteine bezeichnet, die überwiegend aus Serpentinmineralen bestehen. Sie bilden sich bei der Hydratation olivinreicher, ultrabasischer Gesteine (Yardley 1997). Zumeist weisen Serpentinite ein feinkörniges, dichtes und massiges, seltener ein schiefriges Gefüge auf (Matthes 1990). Charakteristisch ist häufig eine fleckige oder streifige Textur mit unterschiedlichen Farbtönungen und auch Durchaderungen mit anderen Mineralen, was zu Reliefunterschieden bei der Verwitterung führen kann (Wimmenauer 1985).
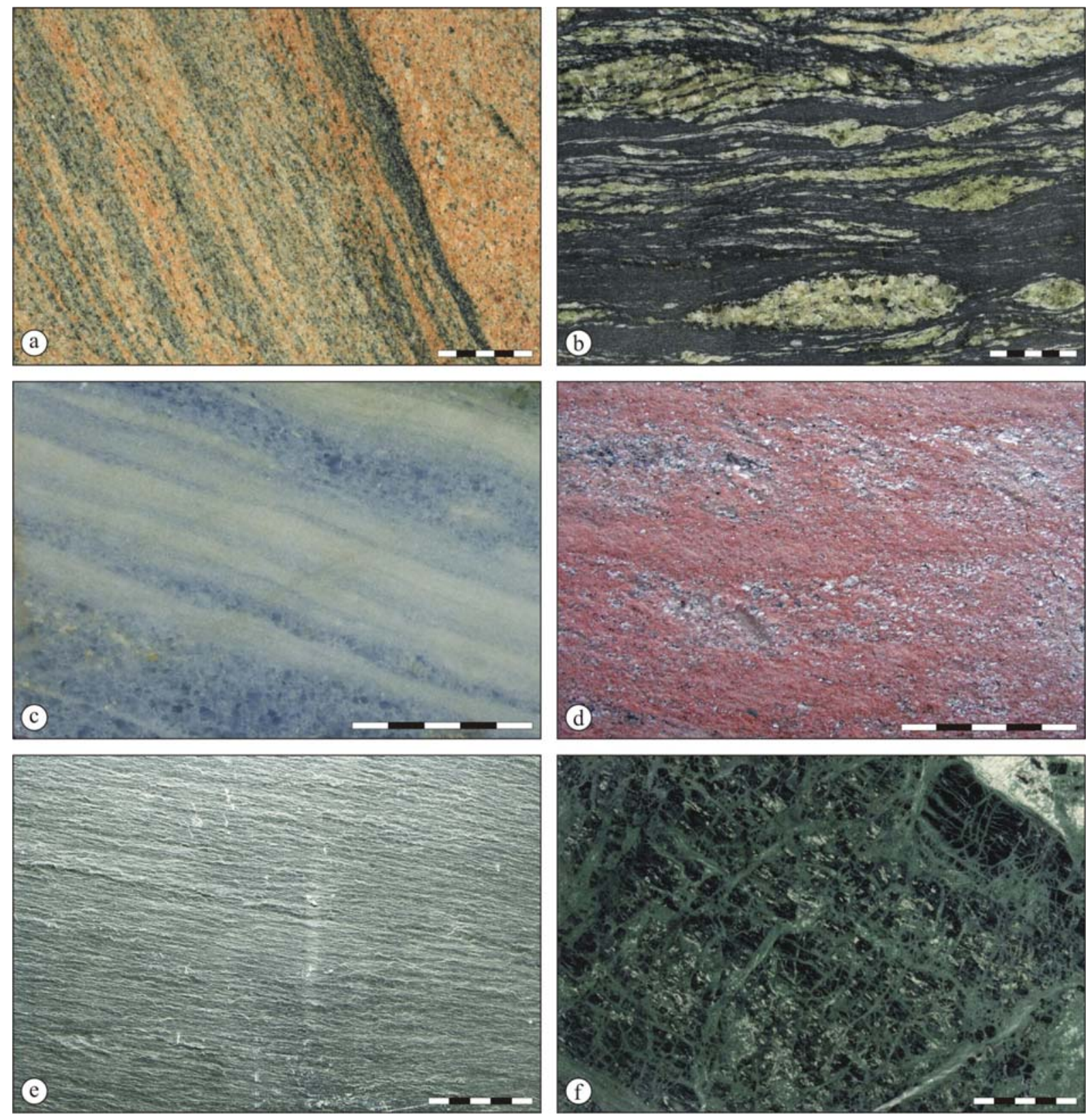

Abb. 3.50: Auswahl verschiedener Varietäten metamorpher Gesteine: a) INDIANA JUPARANA (Indien); Paragneis; b) PORTOROSA (Portugal), Migmatit / Gneis; c) AZUL CIELO (Argentinien), Calcitmarmor; d) Quarzit von Natitingou, (Benin); e) RIVERSTONE (Argentinien), Schiefer; f) GUATEMALA VERDE (Guatemala), Serpentinit. (in a, b, c, f polierte Oberflächen, in d, e spaltraue Oberfläche, Maßstäbe in cm). 


\subsubsection{Basiseigenschaften Rohdichte und Porosität}

Hinsichtlich der Rohdichte zeigen sich in allen hier unterschiedenen Untergruppen keine sehr hohen Datenspannweiten (Abb. 3.51). Der höchste Medianwert ist für die Gruppe /Schiefer/ (2,75 g/cm $\left.{ }^{3}\right)$ dokumentiert. In den Gruppen /Marmor/ (2,71 g/cm³), /Gneis, Migmatit/ (2,67 $\left.\mathrm{g} / \mathrm{cm}^{3}\right)$ und /Quarzit/ $\left(2,65 \mathrm{~g} / \mathrm{cm}^{3}\right)$ beschreibt der jeweilige Medianwert in etwa die mineralspezifische Dichte der Hauptmineralphasen. Dabei können Marmore und Quarzite als annähernd monomineralisch angesehen werde. Die Gneise lassen sich mit Kenndaten der Plutonite vergleichen, da in beiden Fällen im Allgemeinen Feldspat und Quarz als Hauptminerale auftreten. Der Medianwert der Untergruppe /Serpentinit/ liegt bei $2,68 \mathrm{~g} / \mathrm{cm}^{3}$. Zudem zeigt sich hier insgesamt eine etwas breitere Datenspannweite. Dies ist auf das nicht seltene Auftreten relativ hochdichter Minerale aus dem vorangegangenen Peridotitstadium zurückzuführen.

Für die Porosität sind nur in sehr wenigen Fällen Werte über 1 Vol.-\% beschrieben. Diese werden in der explorativen Datenauswertung zumeist als Ausreißer identifiziert (Abb. 3.51). Daher können für metamorphe Gesteine im Allgemeinen Rissporositäten angenommen werden. Im Falle der Untergruppen /Schiefer/ und /Serpentinit/ ist der Datenpool mit $\mathrm{n}=6$ beziehungsweise $n=4$ für eine fundierte Aussage deutlich zu gering.
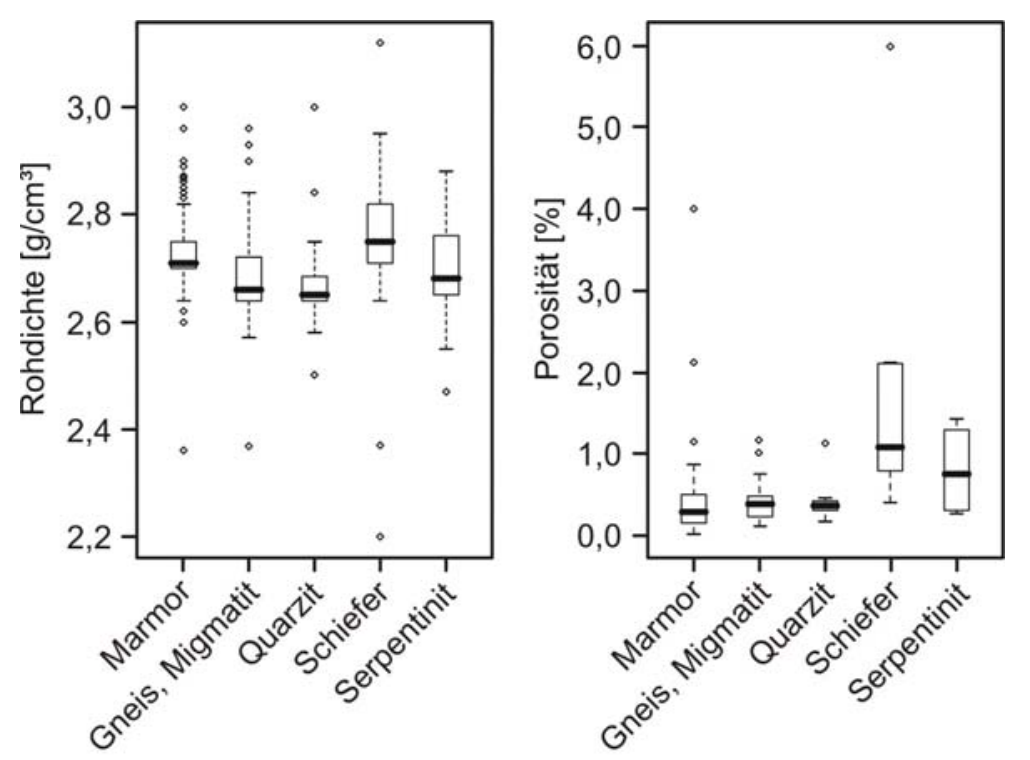

Abb. 3.51: Rohdichten (links) und Porositäten (rechts) in den Untergruppen der metamorphen Gesteine.

Infolge der sehr geringen Datenspannweiten von Porosität und Rohdichte ist auch der korrelative Zusammenhang, vergleichbar mit der Gruppe der Plutonite, mit relativ geringen Koeffizienten beschrieben (Abb. 3.52). Ein erhöhter Hohlraumanteil ist hier, aufgrund einer in unterschiedlichem Maße durchgreifenden Umstrukturierung des Gesteins, nicht zu erwarten. Besonders deutlich wird diese entscheidende Veränderung durch die Metamorphose anhand der Marmore. So können unter sehr hohen Temperaturen zum Teil annähernd monomineralische Calcitmarmore entstehen, die im frischen Zustand eine Porosität von nicht mehr als 0,1 Vol.-\% aufweisen. 
Zusammengefasst ergibt sich aus der Betrachtung der Basiseigenschaften Rohdichte und Porosität in den Untergruppen der metamorphen Gesteine ein mit den plutonischen Magmatiten vergleichbares statistisches Verhalten.

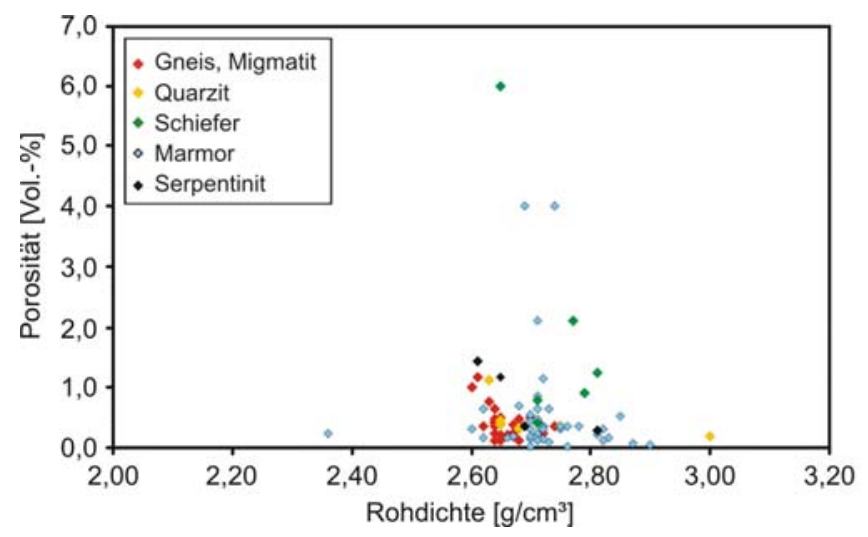

\begin{tabular}{lcc}
\hline & \multicolumn{2}{c}{ linearer Korrelationskoeffizient $\mathrm{r}$} \\
& für $\rho_{\text {roh }} / \Phi$ & $n$ \\
\hline Gneis, Migmatit & $-0,449$ & 25 \\
Quarzit & $-0,493$ & 8 \\
Schiefer & $-0,605$ & 6 \\
Marmor & 0,205 & 42 \\
Serpentinit & $-0,493$ & 4 \\
\hline
\end{tabular}

Abb. 3.52: Korrelationsdiagramm der Parameter Rohdichte $\rho_{\text {roh }}$ und Porosität $\Phi$ sowie die Korrelationskoeffizienten für den jeweiligen linearen Zusammenhang für die Untergruppen der metamorphen Gesteine.

\subsubsection{Technische Eigenschaften Druck-, Biege- und Spaltzugfestigkeit}

Gerade bei metamorphen Gesteinen ist häufig eine erhebliche Richtungsanisotropie der technischen Eigenschaften in Abhängigkeit vom Gefüge gegeben. Extrem ausgeprägt tritt dieses Phänomen vor allem bei verschiedenen Schiefern, insbesondere bei der Prüfung der Biege- und Spaltzugfestigkeit, auf. Dabei können die gegen das Lagengefüge ermittelten Festigkeitswerte die parallel dazu ermittelten ohne weiteres um das 10- oder 20fache übersteigen. Aber auch Marmore, bei denen oftmals ein makroskopischer Nachweis einer kristallographischen Vorzugsorientierung nur bedingt möglich ist, können erhebliche Unterschiede im mechanischen Verhalten aufweisen. So ergab z.B. die Druckfestigkeitsprüfung am thailändischen Marmor PHRAN KRATAI GREY, der eine massive Gesamtstruktur ohne ein ausgeprägtes Lagengefüge aufweist, einen Anisotropiewert von 47 \% (Hoffmann 2006).

Infolge dieser gefügeabhängigen mechanischen Stabilität ergeben sich für die verschiedenen Festigkeitsparameter zumeist weite Datenspektren (Abb. 3.53). Die geringsten Medianwerte sind jeweils für Marmore dokumentiert (in MPa: St $t_{\mathrm{C}}$ 102,1; $\mathrm{St}_{\mathrm{F}}$ 15,8; $\mathrm{St}_{\mathrm{T}}$ 11,8). Der maximale Medianwert für die Druckfestigkeit ist für die Gruppe /Quarzit/ (St ${ }_{C}$ 237,6 $\mathrm{MPa}$ ) dokumentiert. Die Medianwerte zur Druckfestigkeit der übrigen Gruppen belaufen sich auf (in MPa): /Gneis, Migmatit/ St 184,4 ; /Schiefer/ St $\mathrm{C}_{\mathrm{C}}$ 158,5; /Serpentinit/ St Ct $_{\mathrm{C}}$ 159,3. Hinsichtlich der Biegefestigkeit ist für die Untergruppe /Schiefer/ ( $\mathrm{St}_{\mathrm{F}}$ 33,4 MPa) der maximale Medianwert beschrieben. Gleichzeitig ist eine sehr hohe Datenspannweite mit Werten zwischen 5,9 MPa und 83,2 MPa gegeben. Dieser Umstand ist auf das extrem ausgeprägte Parallelgefüge von Schiefer zurückzuführen.

Die relativ geringe Festigkeit verschiedener Marmore steht in engem Zusammenhang mit dem nahezu monomineralischen Charakter, sowie dem Gefügeinventar und der 
Korngrößenverteilung (Brosch et al. 2000). Verschiedene Marmorvarietäten sind durch ein equigranulares, polygonales Korngefüge gekennzeichnet. Dabei können die Korngrenzen infolge einer Minimierung der Kornoberflächen durch Prozesse wie die Korngrenzenflächenreduktion (Passchier \& Trouw 1996) nahezu vollständig equilibriert sein. Infolgedessen können die Festigkeiten solcher Marmorvarietäten, im Gegensatz zu solchen mit stärker verzahnten, interlobaten Korngrenzgefügen, deutlich reduziert sein. Generell weisen auch verschiedene andere Autoren auf die geringe Zugfestigkeit selbst unverwitterter Marmore im Vergleich mit anderen kristallinen Gesteinen hin (z.B. Fleischer 2002, Strohmeyer \& Siegesmund 2002).

Hinsichtlich der Spaltzugfestigkeit übersteigt der Medianwert der Gruppe /Gneis, Migmatit/ $\left(\mathrm{St}_{\mathrm{T}}\right.$ 15,1 MPa) nur leicht den der Marmore ( $\mathrm{St}_{\mathrm{F}}$ 12,0 MPa), bei denen jedoch eine größere Spannweite der Daten dokumentiert ist. Auffällig sind zudem die hohen Werte in der Untergruppe /Serpentinit/. Der Maximalwert von $42 \mathrm{MPa}$ wurde bereits in der Gesamtdatenmenge der Metamorphite als Ausreißer identifiziert (vgl. Kap. 3.4.2). Im Allgemeinen weisen Serpentinite ein dichtes, richtungsloses Gefüge auf. Die Wahrscheinlichkeit einer erhöhten Anisotropie hinsichtlich der Festigkeitsparameter wird dadurch herabgesetzt, da diese vom Grad der Gefügeprägung abhängig ist. Teilweise kann aber auch ein massig-schiefriges Gefüge dominieren (Matthes 1990). Eventuell ist auch die im Gestein auftretende Strukturvarietät der Serpentinminerale von Bedeutung dafür. Zwei sehr häufige Serpentinminerale (zweischichtige Phyllosilikate) sind Chrysotil und Antigorit. Während das erstgenannte infolge einer Krümmung und Einrollung der Zweischichtstruktur einen eher nadeligen Habitus besitzt, bildet Antigorit eine wellenartige Struktur aus. Dadurch ist hier eine blättrige Spaltbarkeit entwickelt. Dieses gerichtete Flächengefüge könnte im Zusammenhang mit Mineralrelikten aus dem Peridotitstadium in einer erhöhten Festigkeit senkrecht zum Gefüge resultieren.
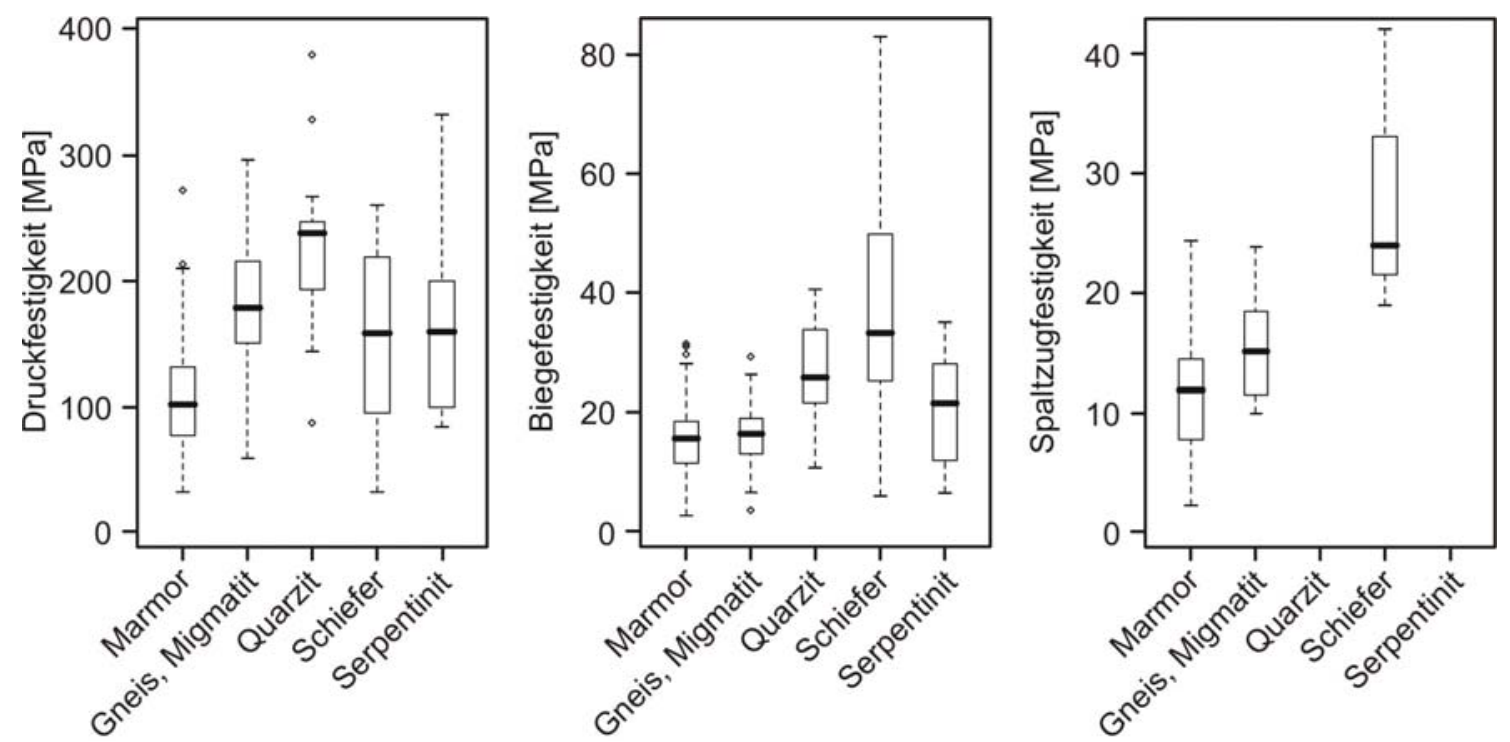

Abb. 3.53: Druck- (links), Biege- (Mitte), und Spaltzugfestigkeiten (rechts) in den Untergruppen der metamorphen Gesteine. 
Im Verhältnis der Kennwerte Druck- und Biegefestigkeit ist eine unterschiedliche Punktverteilung in den Untergruppen /Marmor/ und /Gneis, Migmatit/ ausgebildet (Abb. 3.54). Während sich die Wertepaare der Untergruppe der Gneise mit einem Verhältnis von etwa 1 : 11 in den Trend der Gesamtheit aller Naturwerksteine einpassen lassen, ist die Untergruppe /Marmor/ durch einen geringeren Verhältniswert gekennzeichnet (vgl. Kap. 3.4.2). Für die Festigkeit niedrigporöser Gesteine sind die Minerale und Gefügeelemente mit der geringsten mechanischen Stabilität entscheidend. Dabei gilt Calcit (als Hauptbestandteil der meisten Marmore im vorliegenden Datensatz) als ein potenziell mechanisch sehr schwaches Mineral, wobei gleichzeitig ein nahezu isotropes Verhalten ausgebildet ist (Strohmeyer 2003). Glimmerminerale, die sehr häufig im Mineralbestand von Gneisen in unterschiedlicher Menge mit zumeist deutlich ausgeprägter Orientierung auftreten, weisen ebenfalls ein mechanisch relativ schwaches, dabei jedoch deutlich anisotropes Verhalten auf. Daher können die unterschiedlichen Versagensmechanismen der Bruchbildung (Zugrisse bei der Biegefestigkeit; Kombination aus Zug- und Scherrissen bei der Druckfestigkeit) im Falle der Biegefestigkeit bei Gneisen ausgeprägter zur Wirkung kommen.

In den Verhältnissen der mechanischen Festigkeiten untereinander spiegeln sich insgesamt die grundsätzlichen Tendenzen der Ausgangsgesteine wieder. So kann die Gruppe der Marmore in etwa mit den karbonatischen Gesteinen verglichen werden, während Gneise in ihren Festigkeitsverhältnissen eine gewisse Verwandtschaft mit der Gruppe der Plutonite aufweisen. Der Einfluss der Gefügeelemente nicht nur auf die Höhe einzelner Festigkeitseigenschaften, sondern auch auf das hier betrachtete Verhältnis von Druck- und Biegefestigkeit, hat sich auch bei der Untersuchung thailändischer Marmore gezeigt (Hoffmann 2006). Während Marmorvarietäten, die durch ein polygonales Korngefüge mit geraden Korngrenzen gekennzeichnet sind, ein Verhältnis $\mathrm{St}_{\mathrm{C}}: \mathrm{St}_{\mathrm{F}}$ von etwa $8: 1$ aufweisen, ergibt sich bei einer durch ein interlobates Korngefüge (suturierte Korngrenzen) geprägten Varietät trotz einer schwachen Richtungsanisotropie lediglich ein Verhältnis $\mathrm{St}_{\mathrm{C}}: \mathrm{St}_{\mathrm{F}}$ von etwa $6: 1$.

Tab. 3.6: Richtwerte für die Basiseigenschaften Rohdichte $\left[\mathrm{g} / \mathrm{cm}^{3}\right.$ ] und Porosität [Vol.-\%] sowie für die Parameter Druck-, Biege- und Spaltzugfestigkeit [jeweils in MPa] in den Untergruppen der metamorphen Gesteine. Die Minimum- bzw. Maximumwerte entsprechen den statistischen Kennwerten oberer und unterer Extremwert aus der explorativen Datenauswertung mittels Boxplots. Ausgewiesene Ausreißer sind folglich nicht in die Übersicht mit eingegangen.

\begin{tabular}{lcccccccccc}
\hline \multirow{2}{*}{ Gruppe } & \multicolumn{2}{c}{ Rohdichte } & \multicolumn{2}{c}{ Porosität } & \multicolumn{2}{c}{ Druckfestigkeit } & \multicolumn{2}{c}{ Biegefestigkeit } & \multicolumn{3}{c}{ Spaltzugfestigkeit } \\
& $\min$ & $\max$ & $\min$ & $\max$ & $\min$ & $\max$ & $\min$ & $\max$ & $\min$ & $\max$ \\
\hline Gneis, Migmatit & 2,57 & 2,84 & 0,11 & 0,76 & 59,0 & 296,0 & 6,5 & 26,3 & 10,0 & 23,9 \\
Quarzit & 2,58 & 2,75 & 0,18 & 0,46 & 143,9 & 267,0 & 10,7 & 40,5 & $\mathrm{n} / \mathrm{d}$ & $\mathrm{n} / \mathrm{d}$ \\
Schiefer & 2,64 & 2,95 & 0,40 & 2,10 & 32,2 & 260,8 & 5,9 & 83,2 & 19,0 & 42,0 \\
Marmor & 2,64 & 2,82 & 0,02 & 0,87 & 32,7 & 210,0 & 2,7 & 28,0 & 2,4 & 24,4 \\
Serpentinit & 2,55 & 2,88 & 0,28 & 1,44 & 84,4 & 332,0 & 6,6 & 35,0 & $\mathrm{n} / \mathrm{d}$ & $\mathrm{n} / \mathrm{d}$ \\
\hline
\end{tabular}



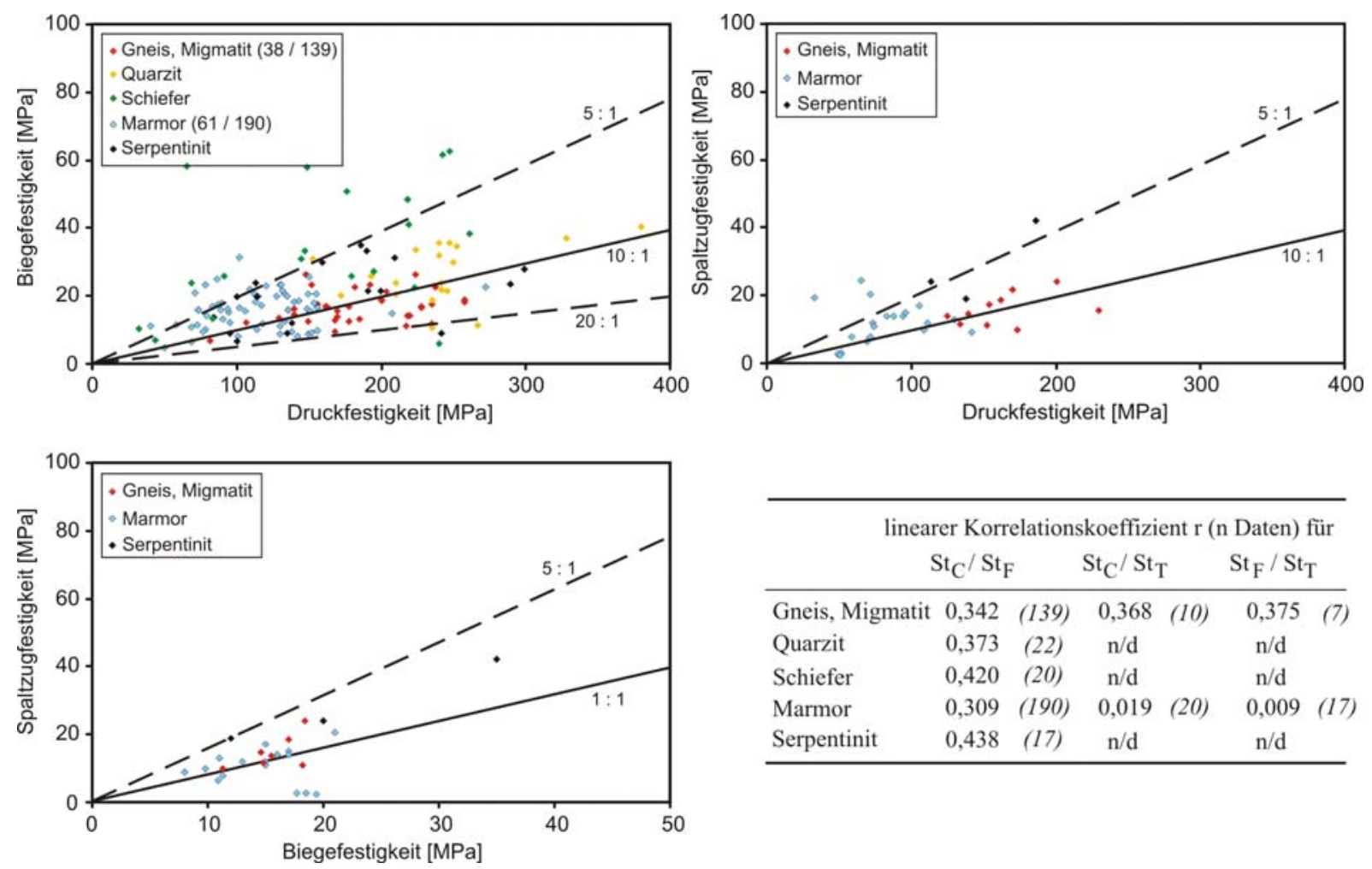

\begin{tabular}{lcccccc}
\hline \multicolumn{5}{c}{ linearer Korrelationskoeffizient $\mathrm{r}(\mathrm{n}$ Daten) für } \\
& $\mathrm{St}_{\mathrm{C}} / \mathrm{St}_{\mathrm{F}}$ & $\mathrm{St}_{\mathrm{C}} / \mathrm{St}_{\mathrm{T}}$ & $\mathrm{St}_{\mathrm{F}} / \mathrm{St}_{\mathrm{T}}$ \\
\hline Gneis, Migmatit & 0,342 & $(139)$ & 0,368 & $(10)$ & 0,375 & $(7)$ \\
Quarzit & 0,373 & $(22)$ & $\mathrm{n} / \mathrm{d}$ & $\mathrm{n} / \mathrm{d}$ & \\
Schiefer & 0,420 & $(20)$ & $\mathrm{n} / \mathrm{d}$ & $\mathrm{n} / \mathrm{d}$ & \\
Marmor & 0,309 & $(190)$ & 0,019 & $(20)$ & 0,009 & $(17)$ \\
Serpentinit & 0,438 & $(17)$ & $\mathrm{n} / \mathrm{d}$ & $\mathrm{n} / \mathrm{d}$ & \\
\hline
\end{tabular}

Abb. 3.54: Korrelationsdiagramme der technischen Gesteinseigenschaften $\left(\mathrm{St}_{\mathrm{C}}=\right.$ Druck-, $\mathrm{St}_{\mathrm{F}}=$ Biege-, $\mathrm{St}_{\mathrm{T}}=$ Spaltzugfestigkeit) untereinander sowie die Korrelationskoeffizienten für den jeweiligen linearen Zusammenhang in den Untergruppen der metamorphen Gesteine. Zusätzlich sind idealisierte Trends dargestellt.

Wie bereits erwähnt, wird gerade anhand der metamorphen Gesteine die enorme Relevanz einer gefügeabhängigen Probennahme und Ermittlung der technischen Eigenschaften deutlich. In speziellen Fällen (z.B. Dachschiefer) kann zwar unter Umständen auf eine vollständige Prüfung (d.h. einer Ermittlung der mechanischen Festigkeiten senkrecht und parallel zum Hauptgefügeelement) verzichtet werden. Dann ist jedoch eine explizite Beschreibung der Datenermittlung beziehungsweise eine Zielsetzung der Prüfung hinsichtlich einer bestimmten Verwendung des betroffenen Gesteins unumgänglich.

Ein erhebliches Problem kann aus der zum Teil hohen Variabilität im Richtungsgefüge gerade bei Gneisen und Migmatiten resultieren. Auch bei der Verwendung eines orthogonalen Referenzsystems (vgl. Kap. 4.7.1) kann nicht die exakte Bestimmung der richtungsabhängigen Festigkeitsparameter erfolgen, wie es etwa bei Sandsteinen der Fall ist. In dem in Abbildung 3.55 gezeigten Beispiel der argentinischen Handelsvarietät FRANCO VETEADO (Migmatit, vgl. Kap. 4.6.1.2) ergibt sich in der Prüfung der Biegefestigkeit (nach DIN EN 12372) im Mittel zwar lediglich ein Anisotropiewert von 17,3 \%, doch ergeben sich in den Einzelprüfungen deutlich extremere Werte. So werden allein in der z-Richtung (senkrecht zur generellen Orientierung des Hauptgefüges) infolge der hohen Variabilität im Dezimeterbereich Werte zwischen 9,1 MPa und 15,6 MPa erreicht. Diese Spannweite resultiert einerseits aus dem unregelmäßigen Wechsel von Bereichen mit deutlicher Trennung von Leukosom und Paläosom und hier der starken Anreicherung von lagig gerichteten Biotitmineral. Andererseits sind Bereiche einer verstärkten Anatexis ausgebildet, die aufgrund der Kristallisation einen eher magmatischen Charakter aufweisen. Dabei ist im Gegensatz zu 
einem regelmäßigen Gefüge, wie es z.B. durch einen sedimentären Lagenbau gegeben sein kann, bei gleicher Belastungsrichtung ein ständiger Wechsel der mechanischen Auswirkungen gegeben. Resultierend daraus können sich auf kurzer Distanz Minimal- und Maximalwerte der Stabilität ergeben, wodurch in unregelmäßiger Verteilung potenzielle Schwächebereiche innerhalb einer Gesteinsplatte ausgebildet sein können (Abb. 3.56). So können sich in der Anwendung, z.B. bei Treppenstufen, Probleme ergeben, die mit baukonstruktiven Lösungen durch unterstützende Tragelemente umgangen werden müssen.
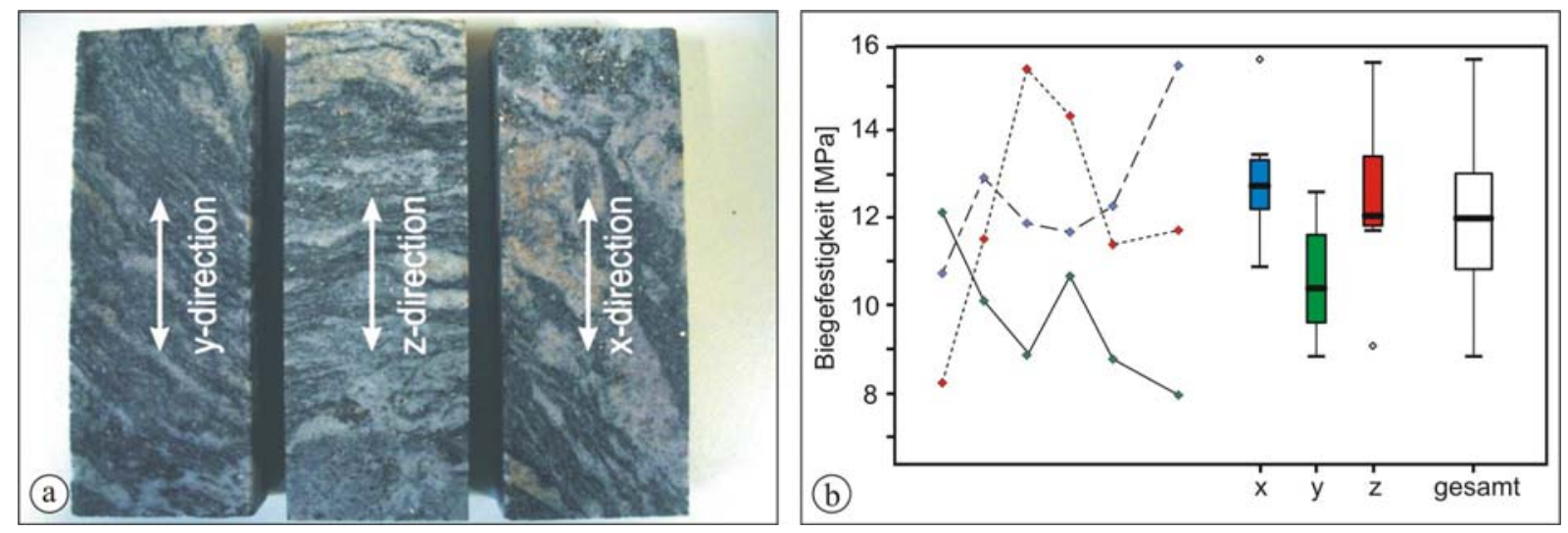

Abb. 3.55: a) Prüfkörper zur Ermittlung der Biegefestigkeit nach DIN EN 12372: Problematik der stark variablen Textur bei Gneisen und Migmatiten (hier: FRANCO VETEADO, Migmatit, Argentinien) mit b) den entsprechenden Einzelwerten in einer Testserie von 18 Probekörpern.
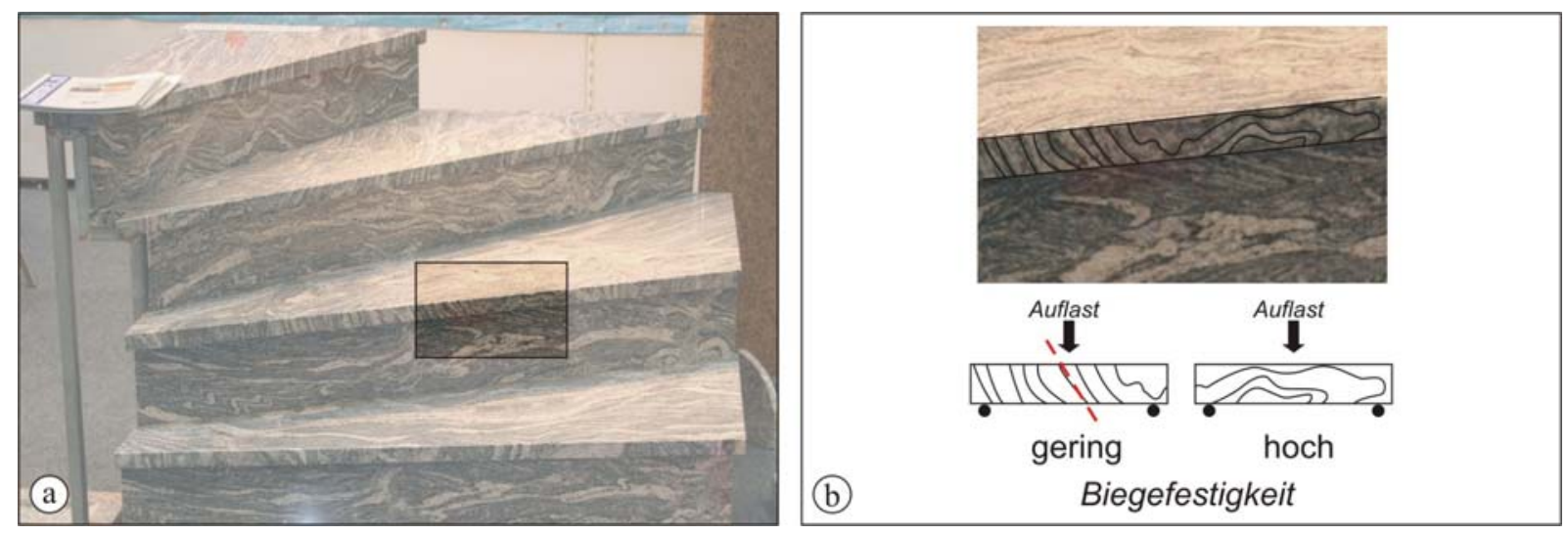

Abb. 3.56: Variabilität des Richtungsgefüges in Gneisen oder Migmatiten mit den Auswirkungen auf eine häufig wechselnde mechanische Stabilität von Werkstücken bei gleich bleibender Belastungsrichtung (Indischer Gneis).

\subsubsection{Basiseigenschaften in Korrelation mit mechanischen Festigkeiten}

Basierend auf einer relativen Gleichheit von Roh- und Matrixdichte infolge sehr geringer Porositäten der metamorphen Gesteine errechnen sich für die Verteilungsdiagramme der verschiedenen Merkmalspaare lediglich geringe bis sehr geringe Korrelationskoeffizienten. In Abbildung 3.57 sind exemplarisch für die Untergruppen /Marmor/ und /Gneis, Migmatit/ die geschätzten Regressionsgeraden sowie die Determinationskoeffizienten für den jeweiligen gerichteten linearen Zusammenhang zwischen der Rohdichte und der Druck- sowie der Biegefestigkeiten angegeben. Alle Koeffizienten gehen gegen Null, womit eine mögliche regressive Verknüpfung der Parameter als sehr unwahrscheinlich angesehen werden kann. 

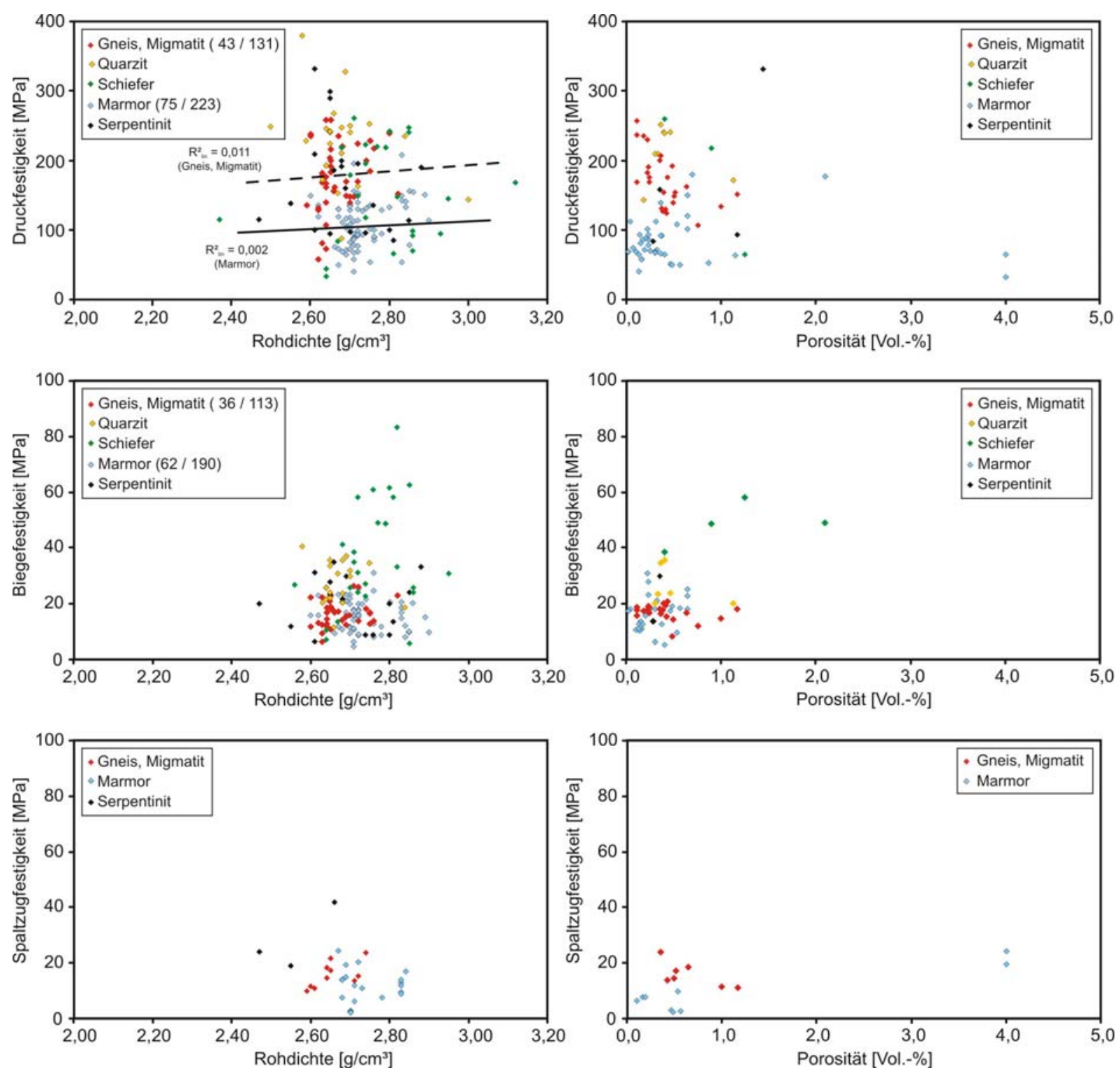

Abb. 3.57: Korrelationsdiagramme der Basiseigenschaften Rohdichte bzw. Porosität mit den Parametern Druck-, Biege- sowie Spaltzugfestigkeit in den Untergruppen der metamorphen Gesteine. Angegeben sind die Determinationskoeffizienten für den jeweiligen linearen Zusammenhang für die Untergruppen /Marmor/ und /Gneis, Migmatit/.

\subsection{Abschätzungen bivariater Aufenthaltswahrscheinlichkeiten}

Die Aufenthaltswahrscheinlichkeit einer Korrelation beschreibt einen Bereich, in dem die Daten einer theoretischen Grundgesamtheit der betrachteten Merkmalspaare auftreten (z.B. Kreyszig 1977, Bortz 1985). In den folgenden Unterkapiteln werden die Bereiche dargestellt, in denen die Parameter der Festigkeit in Korrelation zu den Basiseigenschaften mit einer Wahrscheinlichkeit von 80 \% auftreten. Die Berechnung der Aufenthaltswahrscheinlichkeit beruht auf statistischen Kennwerten (Mittelwert, Standardabweichung, Korrelationskoeffizient), die jeweils tabellarisch zusammengefasst wiedergegeben sind. Die Gruppe der vulkanischen Gesteine ist hierbei infolge der geringen Datenbasis nicht berücksichtigt. Ebenso konnten für die übrigen Gesteinsgruppen die Beziehungen zwischen den Basiseigenschaften und der Spaltzugfestigkeit nicht mit einbezogen werden. 
Einleitend ist zu sagen, dass die Berechnung der bivariaten Aufenthaltswahrscheinlichkeit zweier Merkmale respektive die Darstellung entsprechender Bereiche mathematisch keine Möglichkeit der Prognose, sondern lediglich eine Visualisierung der theoretischen Grundgesamtheit darstellt. Damit ist ein praktikables Werkzeug gegeben, um Datensätze oder auch Einzeldaten hinsichtlich ihrer Richtigkeit beziehungsweise Genauigkeit abschätzend zu bewerten (vgl. Kap. 3.7.1, Abb. 3.32).

\subsubsection{Gruppe der plutonischen Magmatite}

Die statistischen Kenndaten zur Berechnung der bivariaten Aufenthaltswahrscheinlichkeiten für die Gruppe der Plutonite (Abb. 3.58) sind in den Tabellen 3.7 und 3.8 zusammengefasst. Die Visualisierung der entsprechenden Bereiche verdeutlicht, dass zwischen der Porosität und den Festigkeitseigenschaften in der Gruppe der Plutonite kein wechselseitiger Zusammenhang besteht. Die Bereiche der einzelnen Untergruppen unterscheiden sich hinsichtlich Lage und Ausformung nur unwesentlich voneinander. Damit bestätigt sich, dass die Festigkeit beziehungsweise die mechanische Stabilität der niedrigporösen Gesteine dominant durch das mechanische Verhalten der Minerale beziehungsweise durch das Mineralgefüge kontrolliert wird.

Die Beziehungen zwischen der Rohdichte und den Festigkeitsparametern zeigen dagegen ein deutlich andersartiges Bild. Hier hebt sich die Gruppe /Gabbro, Diorit/ ab. Die verbleibenden Untergruppen ergeben wiederum vergleichbare Bereiche, die hinsichtlich der jeweiligen Festigkeit eine deutlich größere Spannweite in Relation zum Rohdichtespektrum aufweisen. Dadurch sind die Korrelationen mit entsprechend niedrigen Koeffizienten beschrieben. Hier bestätigt sich somit die Zusammenfassung der Untergruppen, die nach der explorativen Datenauswertung mittels Boxplot definiert wurden.

Tab. 3.7: Datengrundlage zur Berechnung der Bereiche der bivariaten Aufenthaltswahrscheinlichkeit für die Untergruppen der Plutonite: Lineare Korrelationskoeffizienten der Wechselbeziehungen zwischen den

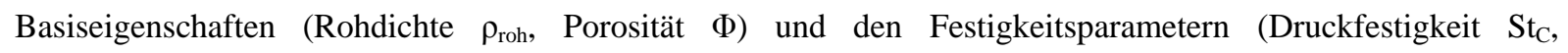
Biegefestigkeit $\mathrm{St}_{\mathrm{F}}$ ).

\begin{tabular}{|c|c|c|c|c|c|c|c|c|}
\hline \multirow{2}{*}{ Gruppe } & \multicolumn{8}{|c|}{ linearer Korrelationskoeffizient r für } \\
\hline & $\rho_{\text {roh }} / \mathrm{St}_{\mathrm{C}}$ & $n$ & $\rho_{\text {roh }} / \mathrm{St}_{\mathrm{F}}$ & $n$ & $\Phi / \mathrm{St}_{\mathrm{C}}$ & $n$ & $\Phi / \mathrm{St}_{\mathrm{F}}$ & $n$ \\
\hline Gabbro, Diorit & 0,391 & 62 & 0,452 & 53 & $-0,077$ & 9 & 0,003 & 7 \\
\hline Plutonite exkl. Gabbro, Diorit & 0,104 & 516 & 0,218 & 434 & $-0,061$ & 113 & $-0,152$ & 95 \\
\hline Granit & 0,120 & 392 & 0,192 & 342 & $-0,103$ & 97 & $-0,181$ & 81 \\
\hline Granodiorit, Tonalit & $-0,128$ & 77 & 0,262 & 61 & 0,555 & 11 & 0,388 & 9 \\
\hline Monzonit, Syenit & 0,128 & 47 & 0,159 & 31 & 0,060 & 5 & $-0,575$ & 5 \\
\hline
\end{tabular}


Tab. 3.8: Datengrundlage zur Berechnung der Bereiche der bivariaten Aufenthaltswahrscheinlichkeit für die Untergruppen der Plutonite: Mittelwerte und Standardabweichungen $(\sigma)$ der Basiseigenschaften (Rohdichte $\rho_{\text {roh }}$ [g/cm³], Porosität $\Phi$ [Vol.-\%]) und der Festigkeitsparameter (jeweils in MPa).

\begin{tabular}{lcccccccc}
\hline \multicolumn{1}{c}{ Gruppe } & \multicolumn{2}{c}{ Rohdichte } & \multicolumn{2}{c}{ Porosität } & \multicolumn{2}{c}{ Druckfestigkeit } & \multicolumn{2}{c}{ Biegefestigkeit } \\
& $\rho_{\text {roh }}$ & $\sigma$ & $\Phi$ & $\sigma$ & $\mathrm{St}_{\mathrm{C}}$ & $\sigma$ & $\mathrm{St}_{\mathrm{F}}$ & $\sigma$ \\
\hline Gabbro, Diorit & 2,88 & 0,15 & 0,56 & 0,39 & 198,64 & 71,75 & 19,22 & 6,93 \\
Plutonite exkl. Gabbro, Diorit & 2,66 & 0,05 & 0,73 & 0,52 & 162,79 & 40,14 & 14,31 & 4,32 \\
Granit & 2,65 & 0,05 & 0,73 & 0,52 & 160,17 & 40,10 & 14,07 & 4,33 \\
Granodiorit, Tonalit & 2,69 & 0,06 & 0,59 & 0,39 & 170,99 & 43,18 & 15,37 & 4,34 \\
Monzonit, Syenit & 2,70 & 0,07 & 0,83 & 0,67 & 171,60 & 32,26 & 14,83 & 3,95 \\
\hline
\end{tabular}
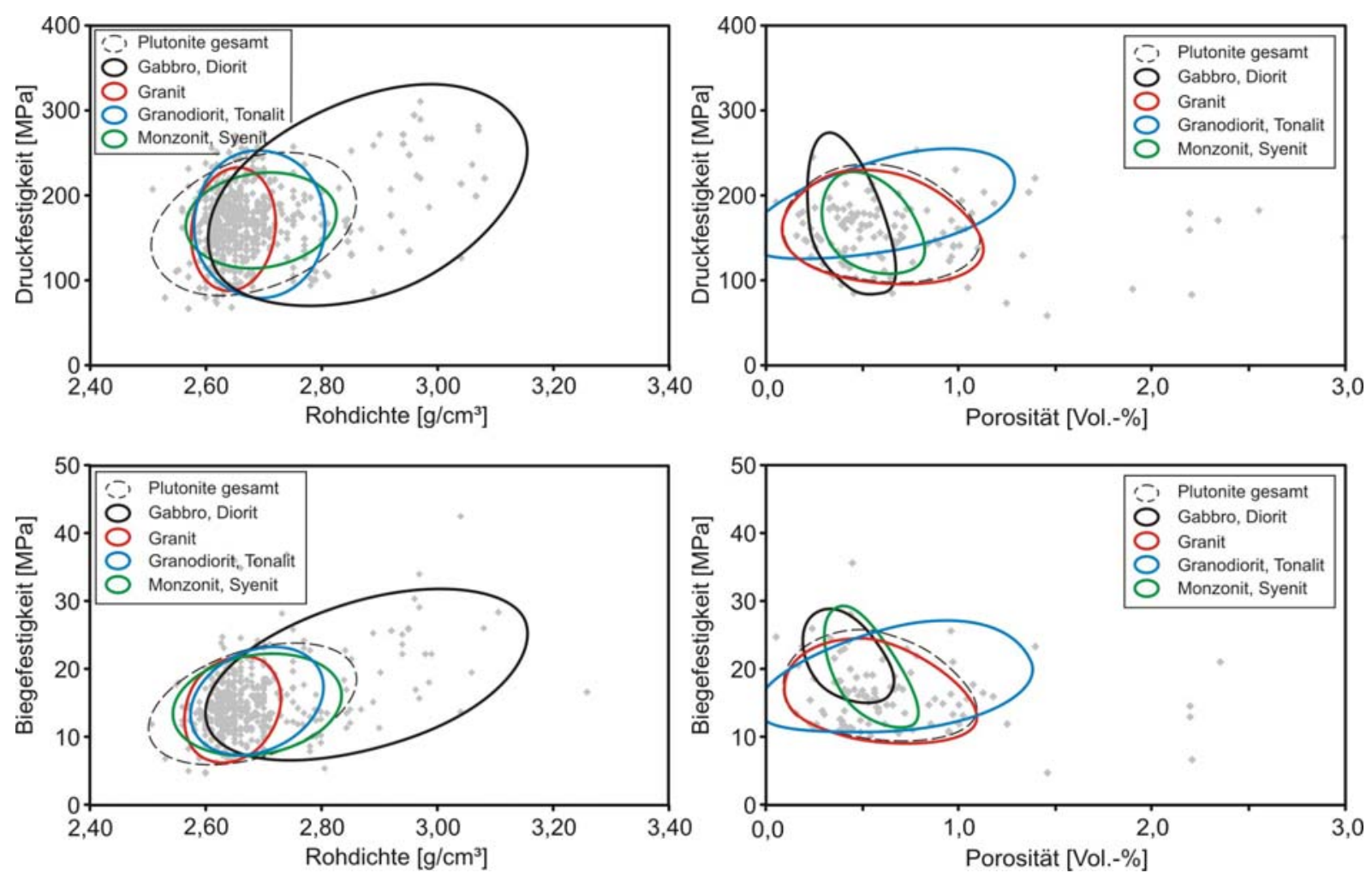

Abb. 3.58 Aufenthaltsbereiche für die verschiedenen Korrelationen der Basiseigenschaften mit den Festigkeitsparametern für die Untergruppen der Plutonite. Die Bereiche wurden aus den Daten der Tabellen 3.7 und 3.8 berechnet und beschreiben 80 \% der zu erwartenden Werte für die jeweilige Grundgesamtheit.

\subsubsection{Gruppe der klastischen Sedimente}

In den Tabellen 3.9 und 3.10 sind die statistischen Kenndaten zur Berechnung der Bereiche der bivariaten Aufenthaltswahrscheinlichkeit für die Gruppe der klastischen Sedimente (Abb. 3.59) zusammengefasst. Der Datensatz aus Wenzel \& Häfner (2003) wurde aufgrund der Anomalien, die damit verbunden sind (vgl. Kap. 3.7.1) nicht in die Berechnungen mit einbezogen. Gleiches gilt für die beschriebenen Konglomerate.

Die Beschreibung der Druckfestigkeit in Korrelation mit den Basiseigenschaften ergibt ein homogenes Bild. Deutlich ist der lineare Charakter der jeweiligen Beziehung erkennbar. Hinsichtlich der Biegefestigkeit fallen dagegen die Daten der Porositätsklasse I recht markant aus dem allgemeinen Trend heraus. Auch wenn hier im Vergleich mit der Druckfestigkeit 
weniger Korrelationspaare auftreten, ist zu vermuten, dass die Unregelmäßigkeit als Folge einer Nichtbeachtung von vorhandenen Gefügen auftritt. Bei verschiedenen Sandsteinvarietäten ist die Verarbeitungsmöglichkeit aufgrund eines ausgeprägten Lagenbaus auf spaltraue Platten im cm-Bereich beschränkt. Daher werden hier wahrscheinlich auch nur entsprechende Untersuchungen in den für die Verwendung relevanten Gefügerichtungen vorgenommen beziehungsweise entsprechende Ergebnisse präsentiert. Für eine allgemeine Einschätzung einer Gesteinsfestigkeit muss jedoch auch eine mögliche Anisotropie Beachtung finden. So bewirkt schon die Eliminierung der beiden Werte über $30 \mathrm{MPa}$ für die Biegefestigkeit eine deutliche Veränderung des resultierenden Bereichs der $80 \%$-igen Aufenthaltswahrscheinlichkeit der Grundgesamtheit in der Porositätsklasse I.

Am Beispiel der in dieser Gruppe dokumentierten Daten ist die Verwendbarkeit der Darstellung von Aufenthaltswahrscheinlichkeiten als Bewertungsinstrument deutlich zu erkennen. Die eindeutige Zuordnung der Daten aus Wenzel \& Häfner (2003) in die Porositätsklasse III aus der Korrelation Rohdichte zu Druckfestigkeit bestätigt sich in der Korrelation mit der Porosität nicht, womit eine Unsicherheit in diesem Datensatz belegt ist.

Tab. 3.9: Datengrundlage zur Berechnung der Bereiche der bivariaten Aufenthaltswahrscheinlichkeit für die Gruppe der Sandsteine: Lineare Korrelationskoeffizienten der Wechselbeziehungen zwischen den

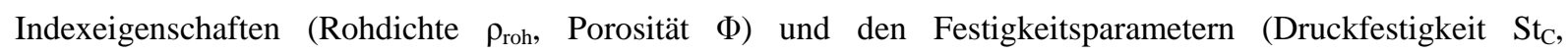
Biegefestigkeit $\mathrm{St}_{\mathrm{F}}$ ).

\begin{tabular}{lcccccccc}
\hline \multicolumn{1}{c}{ Gruppe } & \multicolumn{9}{c}{ linearer Korrelationskoeffizient $\mathrm{r}$ für } \\
& $\rho_{\mathrm{roh}} / \mathrm{St}_{\mathrm{C}}$ & $n$ & $\rho_{\text {roh }} / \mathrm{St}_{\mathrm{F}}$ & $n$ & $\Phi / \mathrm{St}_{\mathrm{C}}$ & $n$ & $\Phi / \mathrm{St}_{\mathrm{F}}$ & $n$ \\
\hline Sandsteine i.w.S. & 0,584 & 423 & 0,527 & 174 & $-0,667$ & 297 & $-0,522$ & 95 \\
Porositätsklasse I & 0,440 & 59 & 0,407 & 27 & $-0,529$ & 59 & $-0,523$ & 27 \\
Porositätsklasse II & 0,275 & 115 & 0,485 & 45 & $-0,430$ & 115 & $-0,530$ & 45 \\
Porositätsklasse III & 0,175 & 54 & 0,126 & 23 & $-0,330$ & 54 & $-0,091$ & 23 \\
\hline
\end{tabular}

Tab. 3.10: Datengrundlage zur Berechnung der Bereiche der bivariaten Aufenthaltswahrscheinlichkeit für die Gruppe der Sandsteine: Mittelwerte und Standardabweichungen $(\sigma)$ der Basiseigenschaften (Rohdichte $\rho_{\text {roh }}$ [g/cm³]; Porosität $\Phi$ [Vol.-\%]) und Festigkeitsparameter (jeweils in MPa).

\begin{tabular}{cccccccccc}
\hline \multirow{2}{*}{ Gruppe } & \multicolumn{2}{c}{ Rohdichte } & \multicolumn{2}{c}{ Porosität } & \multicolumn{2}{c}{ Druckfestigkeit } & \multicolumn{3}{c}{ Biegefestigkeit } \\
& $\rho_{\text {roh }}$ & $\sigma$ & $\Phi$ & $\sigma$ & St $_{\mathrm{C}}$ & $\sigma$ & St $_{\mathrm{F}}$ & $\sigma$ \\
\hline Sandsteine i.w.S. & 2,23 & 0,19 & 17,00 & 5,68 & 63,70 & 36,15 & 6,20 & 4,91 \\
Porositätsklasse I & 2,41 & 0,15 & 9,50 & 3,77 & 97,99 & 37,14 & 8,00 & 6,97 \\
Porositätsklasse II & 2,21 & 0,12 & 17,20 & 2,24 & 62,50 & 26,18 & 6,60 & 3,05 \\
Porositätsklasse III & 2,07 & 0,09 & 22,90 & 1,59 & 48,11 & 22,46 & 4,50 & 2,86 \\
\hline
\end{tabular}



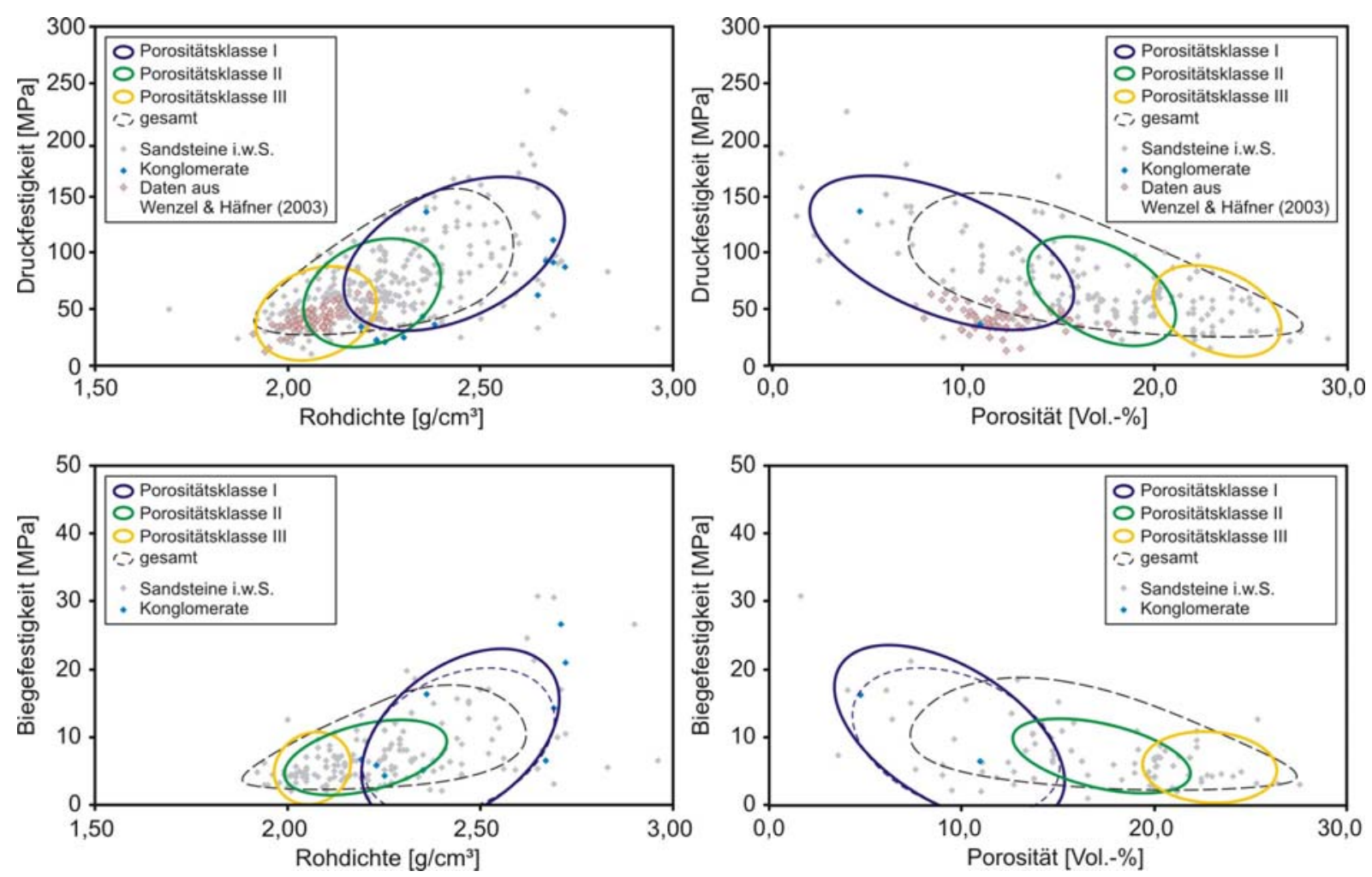

Abb. 3.59: Aufenthaltsbereiche für die verschiedenen Korrelationen der Basiseigenschaften mit den Festigkeitsparametern für die definierten Porositätsklassen der Sandsteine. Die Bereiche wurden aus den Daten der Tabellen 3.9 und 3.10 berechnet und beschreiben $80 \%$ der zu erwartenden Werte für die jeweilige Grundgesamtheit. Die eng gepunkteten Ellipsen stellen die Aufenthaltsbereiche der Porositätsklasse I exkl. der Varietäten mit $\mathrm{St}_{\mathrm{F}}>30 \mathrm{MPa}$ dar.

\subsubsection{Gruppe der Karbonatgesteine}

Bei den Karbonatgesteinen ergeben sich lediglich in den Untergruppen, die sowohl hinsichtlich der Basis- als auch der Festigkeitseigenschaften relativ hohe Datenspannweiten bei einer gleichmäßigen Verteilung aufweisen, hohe Korrelationskoeffizienten (Tab. 3.11 und 3.12). Besonders deutlich wird dies anhand der beschriebenen Neugruppierung innerhalb der Gruppe /Kalkstein/ (vgl. Kap. 3.8.3). Während für die Gesteine mit einer Rohdichte $\rho_{\text {roh }} \geq 2,6 \mathrm{~g} / \mathrm{cm}^{3}$ jeweils ein annähernd fehlender Zusammenhang beschrieben ist, kann für die Gesteine mit $\rho_{\text {roh }}<2,6 \mathrm{~g} / \mathrm{cm}^{3}$ ein deutlicher Trend erkannt werden. Für die Gesamtgruppe /Kalkstein/ ist offensichtlich eine nur unzureichende Beschreibung der Datenpaare möglich. Dies kann durch die Auftrennung der Daten nach der Rohdichte in erheblichem Maße kompensiert werden. Als Ergebnis ergeben sich zwei Bereiche (80 \%-ige Aufenthaltswahrscheinlichkeit), die im Resultat eine deutlich bessere Abschätzung der wahren Gesamtheit der Korrelationsdaten liefern, als die Betrachtung der Gesamtgruppe /Kalkstein/ (Abb. 3.60). Die Überschneidung der beiden Bereiche basiert dabei auf der mathematischen Herleitung über die statistischen Kennwerte der Datensätze.

Die sich für die verschiedenen Untergruppen der Karbonatgesteine ergebenden Bereiche der 80 \%-igen Aufenthaltswahrscheinlichkeit sind in Abbildung 3.61 dargestellt. 


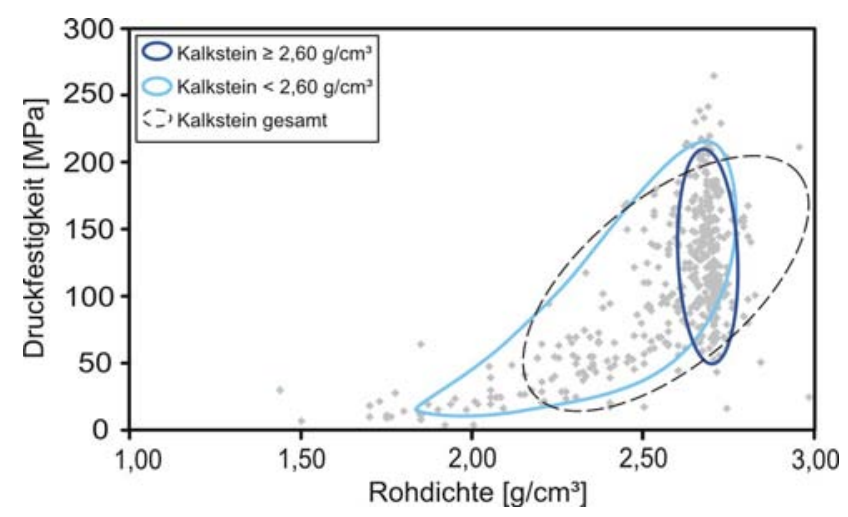

Abb. 3.60: Beispielhafte Darstellung der Auswirkung einer Datenauftrennung nach der Rohdichte bei logarithmischer Anpassung der Daten $/$ Kalkstein $<2,6 \mathrm{~g} / \mathrm{cm}^{3} /$. Nähere Erläuterungen siehe Text.

Tab. 3.11: Datengrundlage zur Berechnung der Bereiche der bivariaten Aufenthaltswahrscheinlichkeit für die Untergruppen der Karbonatgesteine: Lineare Korrelationskoeffizienten der Wechselbeziehungen zwischen den Indexeigenschaften (Rohdichte $\rho_{\text {roh}}$, Porosität $\Phi$ ) und den Festigkeitsparametern (Druckfestigkeit St $\mathrm{S}_{\mathrm{C}}$, Biegefestigkeit $\mathrm{St}_{\mathrm{F}}$ ).

\begin{tabular}{lcccccccc}
\hline \multicolumn{1}{c}{ Gruppe } & \multicolumn{9}{c}{ linearer Korrelationskoeffizient $\mathrm{r}$ für } \\
& $\rho_{\text {roh }} / \mathrm{St}_{\mathrm{C}}$ & $n$ & $\rho_{\text {roh }} / \mathrm{St}_{\mathrm{F}}$ & $n$ & $\Phi / \mathrm{St}_{\mathrm{C}}$ & $n$ & $\Phi / \mathrm{St}_{\mathrm{F}}$ & $n$ \\
\hline Kalkstein $\geq 2,6 \mathrm{~g} / \mathrm{cm}^{3}$ & $-0,124$ & 322 & 0,164 & 249 & 0,035 & 128 & $-0,126$ & 93 \\
Kalkstein $<2,6 \mathrm{~g} / \mathrm{cm}^{3}$ & 0,700 & 151 & 0,598 & 92 & $-0,739$ & 86 & $-0,632$ & 41 \\
Kalkbrekzie & 0,123 & 54 & 0,179 & 47 & 0,150 & 16 & 0,103 & 12 \\
Kalkoolith & 0,762 & 56 & 0,676 & 23 & $-0,790$ & 52 & $-0,830$ & 20 \\
Dolomit & 0,483 & 14 & $-0,073$ & 7 & 0,993 & 3 & $-0,824$ & 3 \\
Travertin & $-0,190$ & 36 & 0,079 & 28 & 0,878 & 9 & 0,102 & 7 \\
\hline
\end{tabular}

Tab. 3.12: Datengrundlage zur Berechnung der Bereiche der bivariaten Aufenthaltswahrscheinlichkeit für die Untergruppen der Karbonatgesteine: Mittelwerte und Standardabweichungen $(\sigma)$ der Basiseigenschaften (Rohdichte $\rho_{\text {roh }}\left[\mathrm{g} / \mathrm{cm}^{3}\right]$; Porosität $\Phi$ [Vol.-\%]) und Festigkeitsparameter (jeweils in MPa).

\begin{tabular}{ccccccccc}
\hline \multirow{2}{*}{ Gruppe } & \multicolumn{2}{c}{ Rohdichte } & \multicolumn{2}{c}{ Porosität } & \multicolumn{2}{c}{ Druckfestigkeit } & \multicolumn{2}{c}{ Biegefestigkeit } \\
& $\rho_{\text {roh }}$ & $\sigma$ & $\Phi$ & $\sigma$ & St $_{\mathrm{C}}$ & $\sigma$ & St $_{\mathrm{F}}$ & $\sigma$ \\
\hline Kalkstein $\geq 2,6 \mathrm{~g} / \mathrm{cm}^{3}$ & 2,69 & 0,05 & 1,17 & 1,36 & 129,64 & 44,15 & 14,67 & 5,77 \\
Kalkstein $<2,6 \mathrm{~g} / \mathrm{cm}^{3}$ & 2,30 & 0,06 & 14,77 & 10,49 & 69,44 & 45,57 & 9,80 & 6,06 \\
Kalkbrekzie & 2,68 & 0,08 & 1,43 & 1,78 & 120,2 & 41,74 & 13,64 & 4,78 \\
Kalkoolith & 2,30 & 0,24 & 15,6 & 9,14 & 77,31 & 52,37 & 9,99 & 5,71 \\
Dolomit & 2,61 & 0,19 & 3,66 & 2,99 & 113,28 & 42,41 & 13,37 & 6,76 \\
Travertin & 2,51 & 0,11 & 4,12 & 2,86 & 71,58 & 36,75 & 11,60 & 3,18 \\
\hline
\end{tabular}



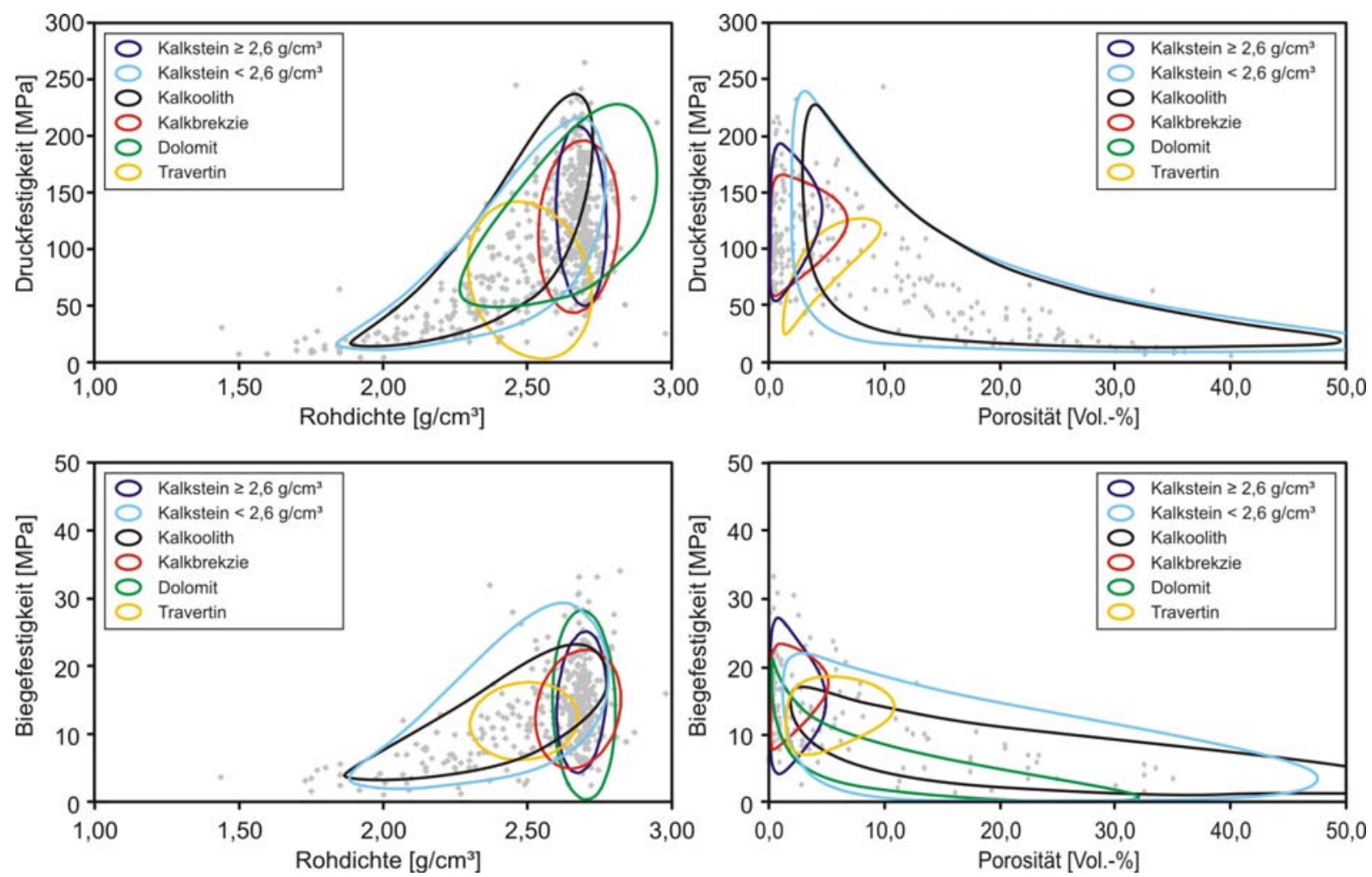

Abb. 3.61: Aufenthaltsbereiche für die verschiedenen Korrelationen der Basiseigenschaften mit den Festigkeitsparametern für die Untergruppen der Karbonatgesteine. Die Bereiche wurden aus den Daten der Tabellen 3.11 und 3.12 berechnet und beschreiben $80 \%$ der zu erwartenden Werte für die jeweilige Grundgesamtheit.

Für die Untergruppe /Travertin/ zeigt sich in der Darstellung der 80 \%-igen Aufenthaltswahrscheinlichkeit eine Gegenläufigkeit zur generellen Annahme einer verringerten mechanischen Stabilität mit zunehmender Porosität. Es ist anzunehmen, dass ein erhöhtes Datenaufkommen, welches auf einer repräsentativen Verteilung der Probekörper im Gestein beruhen muss, auch hier eine negative Korrelation nach sich ziehen würde. Denn gerade bei Travertinen können durch eine fehlerhafte Beprobung Testwerte erreicht werden, die mit dem infolge des häufig lagenweise deutlich wechselhaften, zum Teil extrem porösen Gefüges in keinem Zusammenhang stehen und damit nicht repräsentativ sind (vgl. Abb. 3.44). Aktuelle Untersuchungen am italienischen Travertin ROMANO CLASSICO haben darüber hinaus auch gezeigt, dass die allgemeine Annahme einer höheren Druck- und Biegefestigkeit gegen das sedimentäre Lager im Vergleich zu derjenigen gemessen im Lager, in manchen Fällen anscheinend nicht gehalten werden kann. So ergab sich für die Druckfestigkeit senkrecht zum Lagenbau lediglich ein Mittelwert von 18,40 MPa. Dagegen wurden für die Druckfestigkeit parallel zum Lagenbau deutlich höhere Werte ermittelt (Ø 49,57 MPa). Diese Tatsache ist durch eine gegen das sedimentäre Lager gerichtete Rekristallisation zu erklären, die eine stärkere Verbindung zwischen aufeinander folgenden Schichten und wahrscheinlich auch noch darüber hinaus bewirkt. Die technischen Eigenschaften werden in diesem Fall also durch ein sekundäres Gefüge kontrolliert. 


\subsubsection{Gruppe der metamorphen Gesteine}

In den Tabellen 3.13 und 3.14 sind die statistischen Kenndaten zur Berechnung der bivariaten Aufenthaltswahrscheinlichkeiten für die Untergruppen der metamorphen Gesteine (Abb. 3.62) zusammengefasst.

Hinsichtlich der Bereiche der 80 \%-igen Aufenthaltswahrscheinlichkeit der jeweiligen Merkmalspaare fällt die recht gute relative Übereinstimmung mit der Gruppe der plutonischen Magmatite auf. Diese ergibt sich infolge der Ähnlichkeiten im Charakter der Porosität und der Rohdichte, besonders in Bezug auf die Gruppe /Gneis, Migmatit/. Entscheidend ist jedoch, dass hier hinsichtlich der Korrelationen mit der Rohdichte die Längsachsen der Ellipsen in x-Richtung (Zunahme der Rohdichte) geringer ausgeprägt sind und damit rein visuell auf ungünstigere Bedingungen im Hinblick auf die Möglichkeit zur Prognose der Festigkeitsparameter schließen lässt.

Die Ellipsen zur Darstellung der Aufenthaltswahrscheinlichkeitsbereiche der Korrelationen weisen in den meisten Untergruppen der Metamorphite eine mehr ausgeprägte Längserstreckung in der y-Achse (Zunahme des jeweiligen Festigkeitsparameters) bei einer relativ geringeren Datenspannweite zur betrachteten Basiseigenschaft auf. Diese Ausbildung ergibt sich vermutlich durch die Auswirkungen der metamorphen Richtungsgefüge. Sehr anschaulich wird dies an der Untergruppe /Schiefer/ im Vergleich mit der Untergruppe /Marmor/. Während in Schiefern häufig extreme Texturen beziehungsweise Richtungsgefüge auftreten können, ist bei Marmoren meist eher eine Kornregelung ausgebildet, die auf einer gerichteten Kristallisation beruht. Diese Kornregelung resultiert auch in einem anisotropen Verhalten technischer und vor allem auch verschiedener physikalischer Parameter (z.B. thermisches und/oder thermohygrisches Dehnungsverhalten). Die unterschiedliche Widerstandsfähigkeit gegen mechanische Belastung tritt jedoch bei verschiedenen Einwirkungsrichtungen auf das Gefüge bei einer ausgeprägten Schieferung wesentlich deutlicher hervor.

Tab. 3.13: Datengrundlage zur Berechnung der Bereiche der bivariaten Aufenthaltswahrscheinlichkeit für die Untergruppe der metamorphen Gesteine: Lineare Korrelationskoeffizienten der Wechselbeziehungen zwischen den Basiseigenschaften (Rohdichte $\rho_{\text {roh }}$, Porosität $\Phi$ ) und den Festigkeitsparametern (Druckfestigkeit St $_{\mathrm{C}}$, Biegefestigkeit $\mathrm{St}_{\mathrm{F}}$ ).

\begin{tabular}{lcccccccc}
\hline \multicolumn{1}{c}{ Gruppe } & \multicolumn{7}{c}{ linearer Korrelationskoeffizient r für } \\
& $\rho_{\text {roh }} / \mathrm{St}_{\mathrm{C}}$ & $n$ & $\rho_{\text {roh }} / \mathrm{St}_{\mathrm{F}}$ & $n$ & $\Phi / \mathrm{St}_{\mathrm{C}}$ & $n$ & $\Phi / \mathrm{St}_{\mathrm{F}}$ & $n$ \\
\hline Gneis, Migmatit & 0,105 & 131 & 0,237 & 113 & $-0,582$ & 25 & $-0,283$ & 21 \\
Quarzit & $-0,313$ & 27 & $-0,124$ & 21 & $-0,188$ & 8 & $-0,383$ & 6 \\
Schiefer & 0,122 & 25 & 0,313 & 26 & $-0,914$ & 3 & 0,518 & 4 \\
Marmor & 0,287 & 223 & 0,045 & 190 & $-0,030$ & 42 & 0,184 & 30 \\
Serpentinit & $-0,155$ & 21 & 0,011 & 17 & 0,624 & 4 & $\mathrm{n} / \mathrm{d}$ & 2 \\
\hline
\end{tabular}


Tab. 3.14: Datengrundlage zur Berechnung der Bereiche der bivariaten Aufenthaltswahrscheinlichkeit für die Untergruppen der metamorphen Gesteine: Mittelwerte und Standardabweichungen $(\sigma)$ der Basiseigenschaften (Rohdichte $\rho_{\text {roh }}\left[\mathrm{g} / \mathrm{cm}^{3}\right]$; Porosität $\Phi[$ Vol.-\%]) und Festigkeitsparameter (jeweils in MPa).

\begin{tabular}{lcccccccc}
\hline \multicolumn{1}{c}{ Gruppe } & \multicolumn{2}{c}{ Rohdichte } & \multicolumn{2}{c}{ Porosität } & \multicolumn{2}{c}{ Druckfestigkeit } & \multicolumn{2}{c}{ Biegefestigkeit } \\
& $\rho_{\text {roh }}$ & $\sigma$ & $\Phi$ & $\sigma$ & St $_{\mathrm{C}}$ & $\sigma$ & St $_{\mathrm{F}}$ & $\sigma$ \\
\hline Gneis, Migmatit & 2,67 & 0,11 & 0,41 & 0,26 & 180,26 & 44,64 & 16,35 & 4,70 \\
Quarzit & 2,67 & 0,09 & 0,44 & 0,29 & 225,23 & 55,13 & 26,49 & 8,00 \\
Schiefer & 2,74 & 0,16 & 1,91 & 2,08 & 156,05 & 69,41 & 37,25 & 19,27 \\
Marmor & 2,73 & 0,06 & 0,54 & 0,85 & 106,50 & 36,51 & 15,57 & 5,22 \\
Serpentinit & 2,69 & 0,10 & 0,81 & 0,58 & 169,62 & 73,85 & 20,45 & 9,26 \\
\hline
\end{tabular}
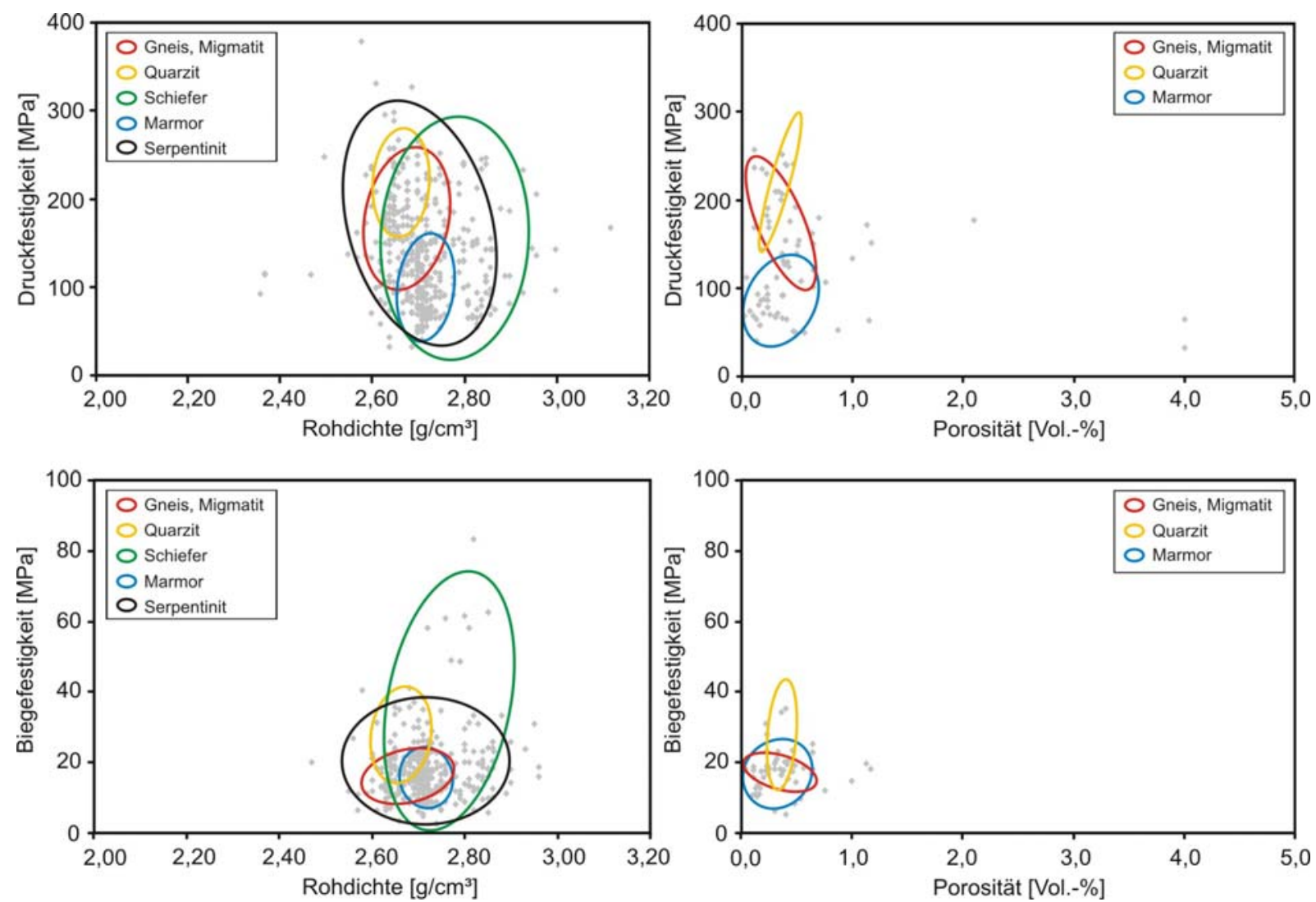

Abb. 3.62: Aufenthaltsbereiche für die verschiedenen Korrelationen der Basiseigenschaften mit den Festigkeitsparametern für die Untergruppen der metamorphen Gesteine. Die Bereiche wurden aus den Daten der Tabellen 3.13 und 3.14 berechnet und beschreiben $80 \%$ der zu erwartenden Werte für die jeweilige Grundgesamtheit.

\subsection{Ansätze zur Prognose technischer Eigenschaften}

In den vorherigen Kapiteln konnte gezeigt werden, dass die Zusammenhänge zwischen den Basiseigenschaften Rohdichte bzw. Porosität und den Parametern der Festigkeit innerhalb der unterschiedenen Gesteinsgruppen im Verteilungsmuster der jeweiligen Punktdiagramme zum Teil erheblich voneinander abweichen. Diese Differenzen begründen sich sowohl auf der Verschiedenheit hinsichtlich der Mineralogie als auch der Gefüge der Gesteine. Ersteres zeigt sich vor allem in der Gruppe der Plutonite (Kap. 3.5). Hier sind die Gesteine, die eine hohe Rohdichte infolge erhöhter Anteile an Mineralen mit einem relativ hohen spezifischen 
Gewicht aufweisen, im oberen Druckfestigkeitsbereich angesiedelt. In anderen Gruppen, so z.B. bei den klastischen und karbonatischen Sedimenten (Kap. 3.7, 3.8), ergibt sich aus der rein mineralogischen Betrachtung kein markanter Einfluss auf die mechanische Stabilität. Besonders deutlich wird diese Tatsache bei der Betrachtung der Karbonatgesteine in den separierten Rohdichtegruppen (Kap. 3.8.3). Obwohl eine mehr oder weniger monomineralische Zusammensetzung der Gesteine angenommen werden kann, sind hinsichtlich der Festigkeitseigenschaften weite Datenspektren dokumentiert. Somit ist als entscheidender Faktor für die mechanische Stabilität die gesteinsspezifische Ausbildung des Gefüges anzusehen. In dieser Hinsicht sind besonders die Verteilung des Porenraumvolumens, mikrostrukturelle Charaktere, wie die Ausbildung des Korngrenzgefüges, eine mögliche sekundäre Zementation oder die Art und Ausbildung von (Mikro-) Risssystemen von ausschlaggebender Bedeutung. Besonderes Augenmerk hinsichtlich der Anisotropie der Festigkeitsparameter kommt einem makroskopisch ausgebildetem Richtungsgefüge, so z. B. einem ausgeprägten Lagenbau, zu.

Die Gesamtheit dieser Vielzahl von Einzelkomponenten ist nur sehr schwer erfassbar. Daher sollen im Folgenden primär Möglichkeiten zur Prognose aus den Basiseigenschaften Rohdichte und Porosität aufgezeigt werden. In der Vielzahl der dargestellten Beziehungen zu den Parametern der Festigkeit hat sich gezeigt, dass besonders im Bereich der nicht hochkompakten Gesteine Korrelationen existieren, die zum Teil einen relativ hohen Grad des wechselseitigen Zusammenhangs aufweisen.

Wie schon beschrieben, stellen die Basiseigenschaften zwei richtungsunabhängige Gesteinseigenschaften dar, die sowohl auf der mineralogischen Zusammensetzung als auch auf der Ausbildung des Gesteinskörpers hinsichtlich dem Vorhandensein von Rissstrukturen und/oder Hohlräumen beruhen. Dabei ist jedoch zu beachten, dass in manchen Gesteinen auch eine gerichtete Porosität auftreten und dann Auswirkungen auf das mechanische Verhalten des Materials haben kann. Die Vielzahl der Korrelationen hat jedoch gezeigt, dass gerade der relative Hohlraumanteil, mit dem Ausdruck einer abnehmenden Rohdichte, lithologieübergreifend meist eng mit einer herabgesetzten mechanischen Stabilität des Mineralverbandes und damit des Gesteinskörpers einhergeht. Im Folgenden sollen innerhalb der verschiedenen Gesteinsgruppen mögliche Prognoseansätze dargestellt werden, die sich aus den Kapiteln 3.5 bis 3.8 ergeben haben. Mit Ausnahme der Gruppe der Plutonite (vgl. Kap. 3.11.1) wurden die ermittelten statistischen Ausreißer aus den Datensätzen der jeweiligen Gesteinsgruppen eliminiert, und gehen so nicht in die dargestellten Prognoseansätze ein. Die Gruppe der metamorphen Gesteine wird aufgrund der sehr komplexen Verschiedenheit in der Art und Ausbildung der Gefüge in diesem Kapitel nicht berücksichtigt.

\subsubsection{Gruppe der plutonischen Magmatite}

Im Bereich der hochkompakten Gesteine trifft die Aussage der verminderten Festigkeit mit abnehmender Rohdichte bzw. zunehmender Porosität nicht oder lediglich stark eingeschränkt zu. Daher ergeben sich in der linearen Regression erwartungsgemäß nur geringe Werte für 
den jeweiligen Determinationskoeffizienten. Da die Porosität hier dominant in Form von Rissstrukturen auftritt, soll zur Anpassung einer Abhängigkeit der technischen Eigenschaften in einem linearen Modell lediglich die Rohdichte als Prädiktorvariable angesetzt werden. Weiterhin ist zu beachten, dass lediglich als extreme Ausreißer eingestufte Werte und nicht sämtliche identifizierten Ausreißer eliminiert wurden. Dies deshalb, da eine Vielzahl höherer Werte auf die Untergruppe /Gabbro, Diorit/ bezogen sind, die in der Gesamtbetrachtung der Plutonite infolge der ungleichmäßigen Datenmengenverteilung (vgl. Kap. 3.5.3) als Ausreißer klassifiziert werden.

Für die vermuteten linearen Zusammenhänge zwischen der Rohdichte $\rho_{\text {roh }}$ (in $\mathrm{g} / \mathrm{cm}^{3}$ ) und den Parametern Druck- bzw. Biegefestigkeit ( $\mathrm{St}_{\mathrm{C}} \mathrm{bzw}_{\text {. }} \mathrm{St}_{\mathrm{F}}$ jeweils in MPa) ergeben sich in der Regressionsrechnung folgende Geradengleichungen mit Standardabweichung:

zu Abbildung 3.63 a) Druckfestigkeit in der Gruppe der plutonischen Magmatite:

$$
\mathrm{St}_{\mathrm{C}}=146,10 * \rho_{\mathrm{roh}}-225,28+/-45,91 \quad\left(\mathrm{R}^{2}=0,097\right)
$$

zu Abbildung 3.63 b) Biegefestigkeit in der Gruppe der plutonischen Magmatite:

$$
\mathrm{St}_{\mathrm{F}}=20,17 * \rho_{\mathrm{roh}}-39,18+/-4,91 \quad\left(\mathrm{R}^{2}=0,170\right)
$$
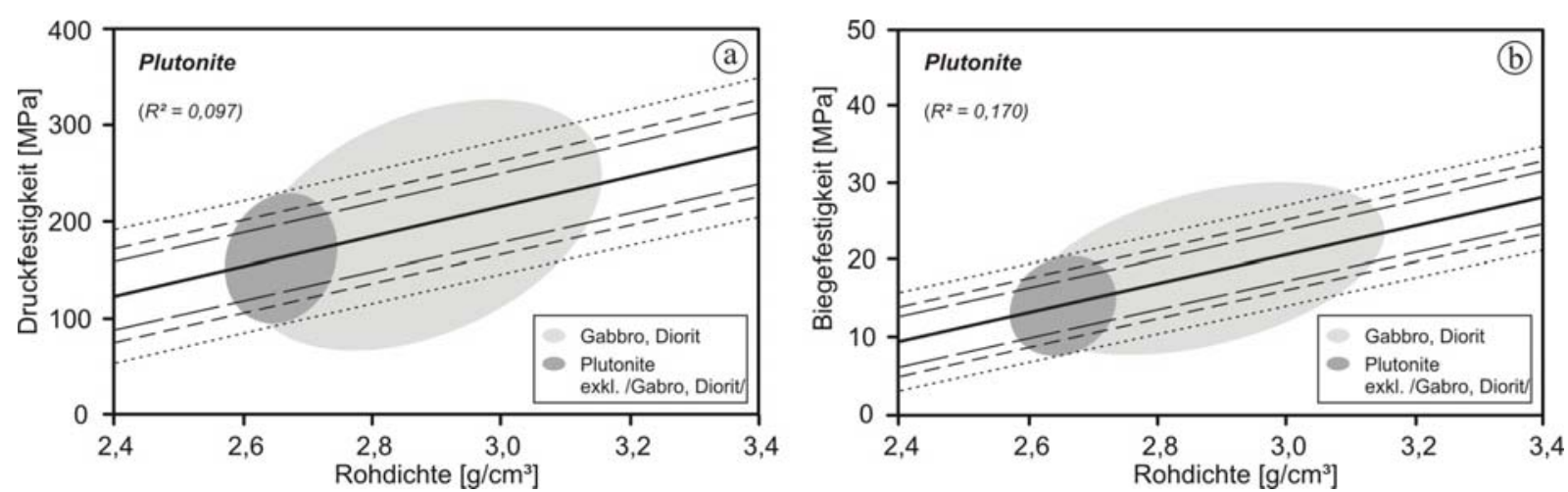

Abb. 3.63: Abhängigkeit der a) Druckfestigkeit sowie b) der Biegefestigkeit von der Rohdichte in der Gruppe der plutonischen Magmatite. Dargestellt ist die jeweilige Regressionsgerade mit den Bereichen, die 65 \% (lang gestrichelt), $80 \%$ (kurz gestrichelt) und $95 \%$ (gepunktet) der Streuung um die Gerade herum enthalten. Zusätzlich eingetragen sind die abgeschätzten $80 \%$-igen Aufenthaltswahrscheinlichkeitsbereiche für die jeweilige theoretische Grundgesamtheit der Datenpaare.

\subsubsection{Gruppe der vulkanischen Magmatite}

In der Gruppe der vulkanischen Gesteine ermöglicht der Datensatz lediglich die Verwendung der Rohdichte als Prädiktorvariable. Dabei ergeben sich hinsichtlich einer möglichen Prognose der Festigkeiten grundsätzlich zwei Gruppen: i) Tuffe und ii) übrige Vulkanite (vgl. Kap. 3.6.3). Zwar ist eine exponentielle Anpassung der Gesamtgruppe mit einem relativ hohen Determinationskoeffizienten belegt, doch soll den differenten Bildungsbedingungen und den daraus resultierenden Gefügecharakteren Rechnung getragen werden. In einer separaten Betrachtung erscheint dann eine Anpassung linearer Modelle angebracht, wobei diese im Falle der Tuffe durch eine sehr geringe Güte gekennzeichnet ist. Für die Gesamtheit der übrigen Vulkanite ist in der Regression eine relativ gute 
Anpassungsgüte gegeben. Der gerichtete Zusammenhang wird hier vor allem durch die Untergruppe /Andesit, Basalt/ geprägt, was auf den weiten Datenspektren zu den betrachteten Eigenschaften basiert. Als alleinige Untergruppe betrachtet, ergibt sich hier im Hinblick auf die Druckfestigkeit ein Determinationskoeffizient von $\mathrm{R}^{2}=0,808$, während für die Gesamtgruppe (exkl. Tuffe) lediglich ein Koeffizient von $\mathrm{R}^{2}=0,384$ beschrieben ist (Abb. 3.64). Relativ gleiche Verhältnisse sind im Bezug auf die Biegefestigkeit gegeben (Abb. 3.65).

Für die vermuteten linearen Zusammenhänge zwischen der Rohdichte $\rho_{\text {roh }}$ (in $\mathrm{g} / \mathrm{cm}^{3}$ ) und der Druckfestigkeit ( $\mathrm{St}_{\mathrm{C}}$ in $\mathrm{MPa}$ ) ergeben sich in der Regressionsrechnung folgende Geradengleichungen mit Standardabweichung:

zu Abbildung 3.64 a) Druckfestigkeit in der Gruppe der Vulkanite (exkl. Tuffe):

$$
\mathrm{St}_{\mathrm{C}}=156,02 * \rho_{\mathrm{roh}}-229,36+/-65,58 \quad\left(\mathrm{R}^{2}=0,384\right)
$$

zu Abbildung 3.64 b) Druckfestigkeit in der Untergruppe /Andesit, Basalt/:
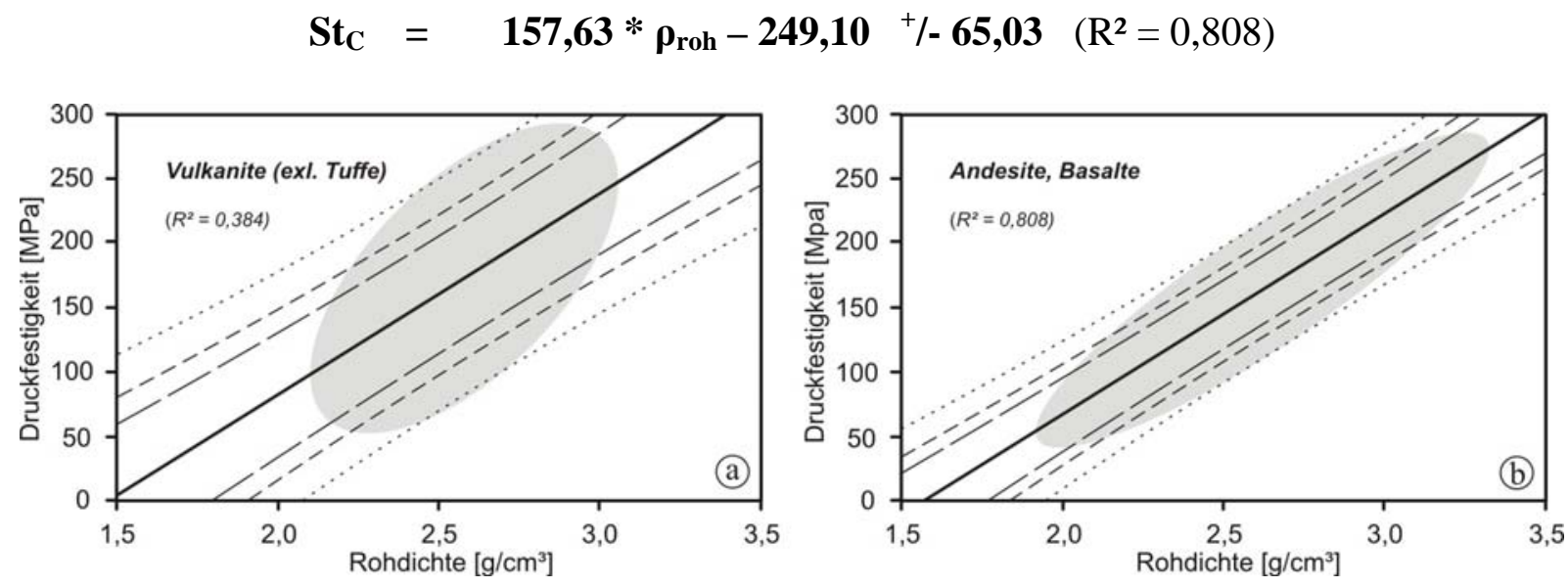

Abb. 3.64: Abhängigkeit der Druckfestigkeit von der Rohdichte in verschiedenen Gruppen der Vulkanite. Dargestellt ist die jeweilige Regressionsgerade mit den Bereichen, die 65 \% (lang gestrichelt), 80 \% (kurz gestrichelt) und $95 \%$ (gepunktet) der Streuung um die Gerade herum enthalten. Zusätzlich eingetragen sind die abgeschätzten 80 \%-igen Aufenthaltswahrscheinlichkeitsbereiche für die jeweilige theoretische Grundgesamtheit der Datenpaare.

Für die vermuteten linearen Zusammenhänge zwischen der Rohdichte $\rho_{\text {roh }}$ (in $\mathrm{g} / \mathrm{cm}^{3}$ ) und der Biegefestigkeit ( $\mathrm{St}_{\mathrm{F}}$ in $\mathrm{MPa}$ ) ergeben sich in der Regressionsrechnung folgende Geradengleichungen mit Standardabweichung:

zu Abbildung 3.65 a) Biegefestigkeit in der Gruppe der Vulkanite (exkl. Tuffe):

$$
\mathrm{St}_{\mathrm{F}}=16,02 * \rho_{\text {roh }}-24,37^{+} /-7,99 \quad\left(\mathrm{R}^{2}=0,283\right)
$$

zu Abbildung 3.65 b) Biegefestigkeit in der Untergruppe /Andesit, Basalt/:

$$
\mathrm{St}_{\mathrm{F}}=16,04 * \rho_{\text {roh }}-26,37^{+} /-\mathbf{8 , 0 0}\left(\mathrm{R}^{2}=0,529\right)
$$



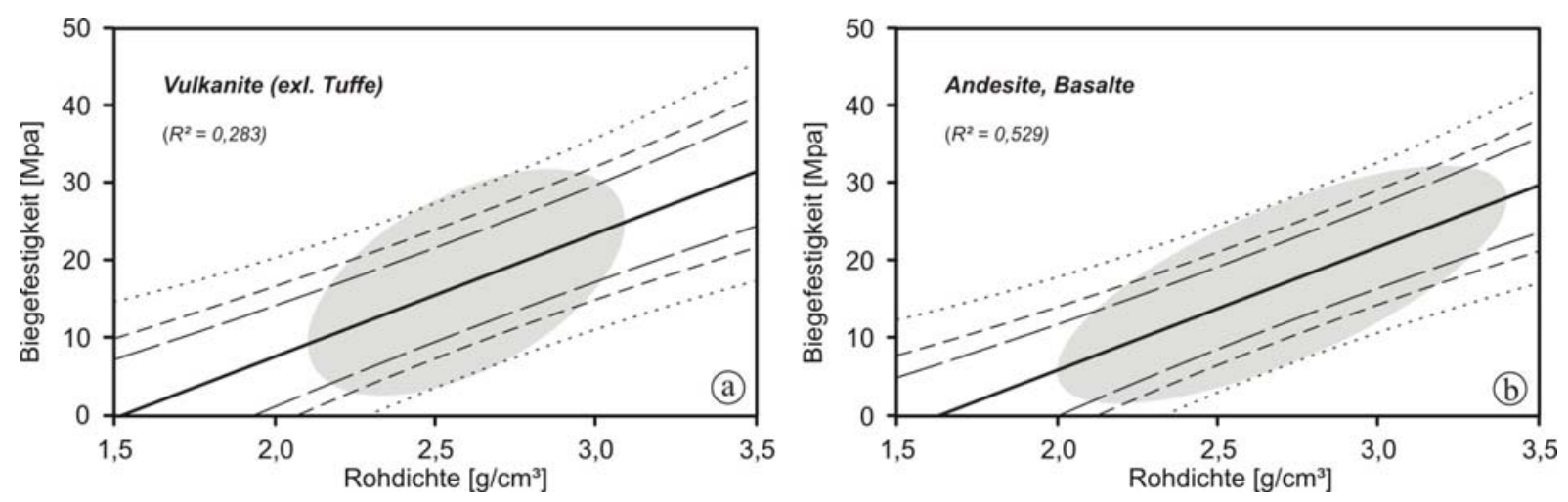

Abb. 3.65: Abhängigkeit der Biegefestigkeit von der Rohdichte in verschiedenen Gruppen der Vulkanite. Dargestellt ist die jeweilige Regressionsgerade mit den Bereichen, die 65 \% (lang gestrichelt), 80 \% (kurz gestrichelt) und $95 \%$ (gepunktet) der Streuung um die Gerade herum enthalten. Zusätzlich eingetragen sind die abgeschätzten 80 \%-igen Aufenthaltswahrscheinlichkeitsbereiche für die jeweilige theoretische Grundgesamtheit der Datenpaare.

In der Untergruppe /Tuff/ erscheint eine Prognosemöglichkeit der Festigkeitseigenschaften mit dem vorhandenen Datensatz nicht möglich. Diese Tatsache steht mit der Genese der Gesteine in Zusammenhang, die durch eine geringe, zum Teil sehr geringe Kompaktion des abgelagerten Materials gekennzeichnet ist. Daraus ergeben sich grundsätzlich unterschiedlichste Werte zu den Basiseigenschaften, die vor allem von der Art des Materials, der Partikelgröße etc. diktiert werden. Aus dem niedrigen Grad der Kompaktion ergibt sich jedoch zumeist eine insgesamt geringe Festigkeit.

\subsubsection{Gruppe der klastischen Sedimente}

In der Gruppe der klastischen Sedimente hat sich gezeigt, dass die Basiseigenschaften Rohdichte und Porosität eng miteinander verknüpft sind und dabei ein gerichteter Zusammenhang ausgebildet ist. Eine Abnahme der Rohdichte, gleichbedeutend mit einem Anstieg der Porosität, zieht dabei gleichzeitig eine Reduzierung der mechanischen Stabilität nach sich. Diese Tatsache resultiert aus der geringeren Anzahl an Bindungspunkten zwischen den aufbauenden Aggregaten, wodurch die Abstützung im Gesamtgerüst eines Gesteins erheblich geschwächt wird. Besonders quarzitische Bindemittel können wiederum die mechanische Stabilität positiv beeinflussen. Als Folge einer sekundären Zementation wird aber wiederum auch die Porosität herabgesetzt und folglich auch die Rohdichte angehoben. Daher kann davon ausgegangen werden, dass die Parameter der Festigkeit direkt aus den Basiseigenschaften ansatzweise abgeschätzt werden können.

Die Güte der Anpassung ist jedoch lediglich mit geringen Koeffizienten beschrieben. Daraus ist zu erkennen, dass die mechanische Stabilität nicht nur auf den Basiseigenschaften basiert. Neben dem Bindemittel sind auch Größe, Form und Sortierung der aufbauenden Kornaggregate als kritische Gefügeeigenschaften von Sandsteinen anzusehen. Abbildung 3.66 zeigt die Abhängigkeit der Druckfestigkeit von der Rohdichte. Dabei wurden die Sandsteine nach der mittleren Korngröße in zwei Gruppen gegliedert. Es zeigt sich, dass die Erwartungswerte zur Festigkeit für feinkörnigere Varietäten leicht höher anzusiedeln sind. Dies steht wiederum mit der Annahme im Einklang, dass aus der Sedimentation 
feinkörnigeren Materials im Allgemeinen geringere Porositäten resultieren (vgl. Kap. 3.7.1), was wiederum mit einer erhöhten Festigkeit assoziiert werden kann. Gleichzeitig lassen die Determinationskoeffizienten beider Regressionsgeraden im Falle der Auftrennung nach der Korngröße eine höher einzuschätzende Qualität der Anpassung annehmen, als dies bei der Gesamtbetrachtung der Fall ist.

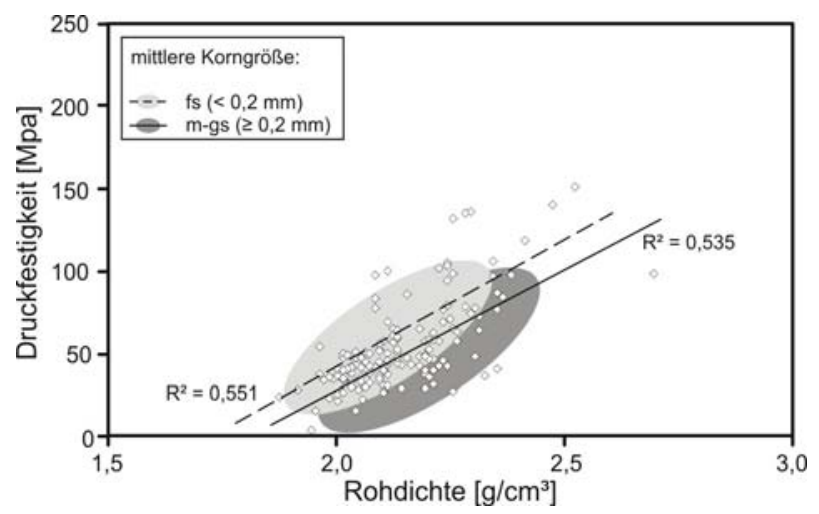

Abb. 3.66: Abhängigkeit der Druckfestigkeit von der Rohdichte unter Beachtung der mittleren Korngröße in der Gruppe der klastischen Sedimente.

Entsprechende Einflüsse sind für andere strukturelle (Korngrößenverteilung, Kornform) und texturelle Elemente (räumliche Anordnung) zu erwarten (z.B. Clemens et al. 1990). Entscheidende Bedeutung hat vor allem auch die Kornbindung. Kieslinger (1957) unterscheidet eine mittelbare Kornbindung über Bindemittel von einer unmittelbaren, die durch verzahnte Korngrenzen charakterisiert ist. Gerade im Hinblick auf die Nutzung als Naturwerkstein ergibt sich daraus ein wesentliches Element der Langzeitstabilität von Sandsteinen. Durch Lösungsprozesse kann es bei einer mittelbaren Bindung durch Lösungsprozesse zu einer Destabilisierung des Korngerüsts und damit einer fortschreitenden Reduktion der Verwitterungsresistenz kommen.

Für die vermuteten linearen Zusammenhänge zwischen der Rohdichte $\rho_{\text {roh }}$ (in $\mathrm{g} / \mathrm{cm}^{3}$ ) und den Parametern Druck- bzw. Biegefestigkeit ( $\mathrm{St}_{\mathrm{C}}$ bzw. $\mathrm{St}_{\mathrm{F}}$ jeweils in MPa) ergeben sich in der Regressionsrechnung folgende Geradengleichungen mit Standardabweichung:

zu Abbildung 3.67 a) Druckfestigkeit in der Gruppe der Sandsteine:

$$
\mathrm{St}_{\mathrm{C}}=\mathbf{8 7 , 7 9} * \rho_{\text {roh }}-\mathbf{1 1 9 , 7 3}+-28,84\left(\mathrm{R}^{2}=0,234\right)
$$

zu Abbildung 3.67 b) Biegefestigkeit in der Gruppe der Sandsteine:

$$
\mathrm{St}_{\mathrm{F}}=\mathbf{9 , 4 3} * \rho_{\mathrm{roh}}-\mathbf{1 4 , 1 6}+/-3,42 \quad\left(\mathrm{R}^{2}=0,210\right)
$$



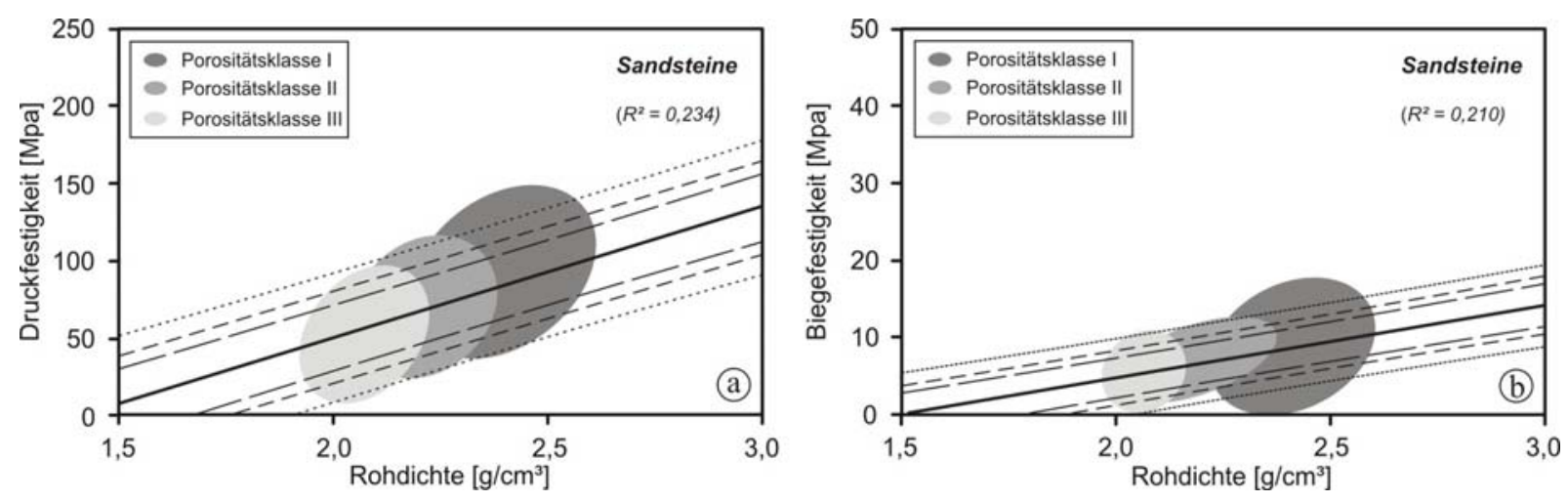

Abb. 3.67: Abhängigkeit a) der Druckfestigkeit sowie b) der Biegefestigkeit von der Rohdichte in der Gruppe der klastischen Sedimente. Dargestellt ist die jeweilige Regressionsgerade mit den Bereichen, die 65 \% (lang gestrichelt), $80 \%$ (kurz gestrichelt) und $95 \%$ (gepunktet) der Streuung um die Gerade herum enthalten. Zusätzlich eingetragen sind die abgeschätzten $80 \%$-igen Aufenthaltswahrscheinlichkeitsbereiche für die jeweilige theoretische Grundgesamtheit der Datenpaare in den definierten Porositätsklassen I ( $<13,4$ Vol.-\%), II ( $<$ 20,5 Vol.-\%) und III ( $\geq 20,5$ Vol.-\%).

Für die vermuteten linearen Zusammenhänge zwischen der Porosität $\Phi$ (in Vol.-\%) und den Parametern Druck- bzw. Biegefestigkeit (St $\mathrm{Cz}_{\mathrm{C}}$. $\mathrm{St}_{\mathrm{F}}$ jeweils in MPa) ergeben sich in der Regressionsrechnung folgende Geradengleichungen mit Standardabweichung:

zu Abbildung 3.68 a) Druckfestigkeit in der Gruppe der Sandsteine:

$$
\mathrm{St}_{\mathrm{C}}=-3,04 * \boldsymbol{\Phi}+\mathbf{1 2 1 , 7 8}+/-29,94 \quad\left(\mathrm{R}^{2}=0,349\right)
$$

zu Abbildung 3.68 b) Biegefestigkeit in der Gruppe der Sandsteine:

$$
\mathrm{St}_{\mathbf{F}}=-\mathbf{0 , 3 2} * \mathbf{\Phi}-\mathbf{1 2 , 4 4}{ }^{+} /-\mathbf{3 , 8 6}\left(\mathrm{R}^{2}=0,204\right)
$$
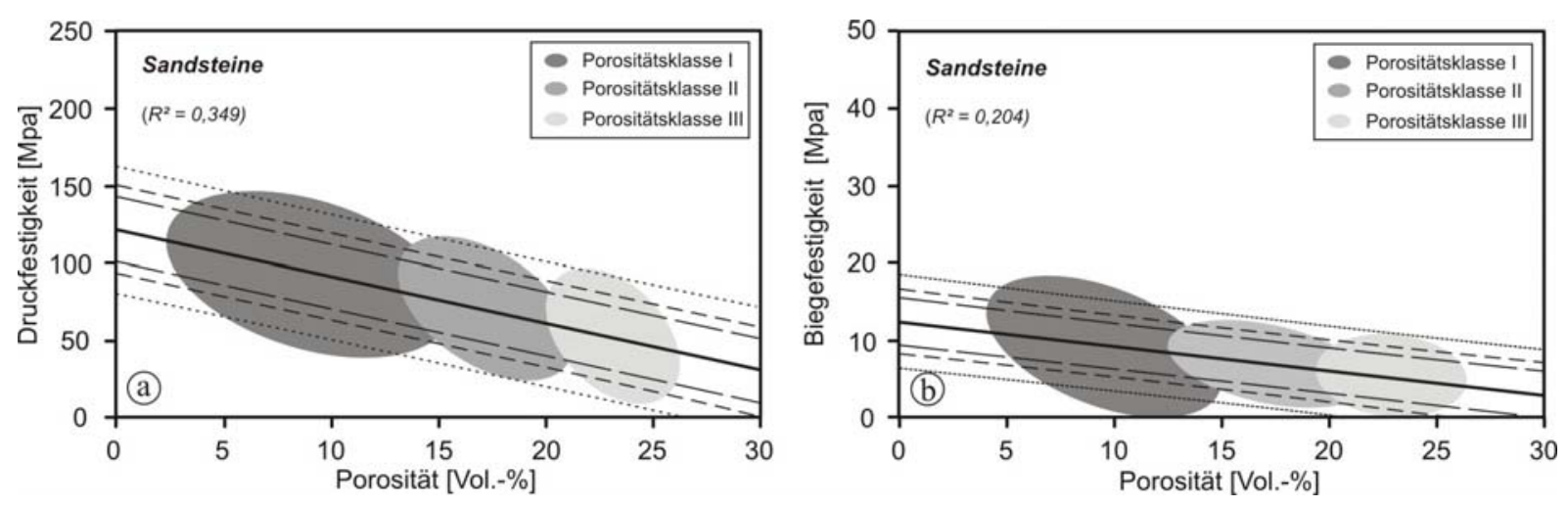

Abb. 3.68: Abhängigkeit a) der Druckfestigkeit sowie b) der Biegefestigkeit von der Porosität in der Gruppe der Sandsteine. Dargestellt ist die jeweilige Regressionsgerade mit den Bereichen, die 65 \% (lang gestrichelt), 80 \% (kurz gestrichelt) und $95 \%$ (gepunktet) der Streuung um die Gerade herum enthalten. Zusätzlich eingetragen sind die abgeschätzten $80 \%$-igen Aufenthaltswahrscheinlichkeitsbereiche für die jeweilige theoretische Grundgesamtheit der Datenpaare in den definierten Porositätsklassen I (< 13,4 Vol.-\%), II (< 20,5 Vol.-\%) und III ( $\geq 20,5$ Vol.-\%). 


\subsubsection{Gruppe der Karbonatgesteine}

In der Untergruppe /Kalkstein/ hat sich gezeigt, dass zu einer Berechnung und Darstellung einer theoretischen Grundgesamtheit die Aufspaltung des Datensatzes nach der Rohdichte sinnvoll erscheint. Diese Gliederung ist in den Abbildungen zur Abhängigkeit der Festigkeitsparameter von den Basiseigenschaften auf die Gesamtheit der Karbonatgesteine übertragen. Sowohl ausgehend von der Rohdichte als auch der Porosität als Prädiktorvariable soll ein nichtlineares Model in Form einer exponentiellen Anpassung zur Anwendung kommen. Zum einen ergibt sich nach dem Determinationskoeffizienten eine höher einzuschätzende Qualität der Anpassung im Vergleich mit einem linearen Modell. Zum anderen ist durch die niveauabhängige Fehlerstreuung die Tatsache des zunehmenden Fehlers bei höheren Rohdichten beziehungsweise geringeren Porositäten (vgl. Kap. 3.8.3) besser beschrieben. Im Gegensatz zu den Sandsteinen zeigt sich bei den chemisch-biogenen Sedimenten häufig eine deutlich heterogener ausgebildete Porosität (vgl. Kap. 3.8.1). Dadurch ergibt sich vielfach ein unregelmäßiges Gerüst, womit erhebliche Schwankungen der mechanischen Stabilität verbunden sein können. Parallel dazu treten häufig Gefügeelemente auf, von denen die Rohdichte beziehungsweise die Porosität unbeeinflusst bleiben, die aber potenzielle Schwächezonen im Gestein bilden (vgl. Kap. 3.8.3). Damit wird deutlich, dass die Kontrolle der Festigkeit zwar durch die Basiseigenschaften gegeben ist, im oberen Rohdichtebzw. unteren Porositätsbereich jedoch einer deutlichen Einschränkung unterliegt. Für eine genauere Abschätzung ist es so unerlässlich, das gesamte Gefügeinventar eines Gesteins mit in die Betrachtung einzubeziehen.

Für die vermuteten nichtlinearen Zusammenhänge zwischen der Rohdichte $\rho_{\text {roh }}$ (in $\mathrm{g} / \mathrm{cm}^{3}$ ) und den Parametern Druck- bzw. Biegefestigkeit (St $t_{C}$ bzw. $\mathrm{St}_{\mathrm{F}}$ jeweils in MPa) ergeben sich in der Regressionsrechnung folgende Gleichungen:

zu Abbildung 3.69 a) Druckfestigkeit in der Gruppe der Karbonatgesteine:

$$
\mathrm{St}_{\mathrm{C}}=0,25 * \mathrm{e}^{2,31 * \rho_{\text {roh }}}\left(\mathrm{R}^{2}=0,560\right)
$$

zu Abbildung 3.69 b) Biegefestigkeit in der Gruppe der Karbonatgesteine:

$$
\mathrm{St}_{\mathrm{F}}=0,20 * \mathbf{e}^{\mathbf{1 , 5 6} * \rho_{\mathrm{roh}}}\left(\mathrm{R}^{2}=0,338\right)
$$

Für die vermuteten nichtlinearen Zusammenhänge zwischen der Porosität (in Vol.-\%) und den Parametern Druck- bzw. Biegefestigkeit (St $t_{C} b z w$. St $t_{F}$ jeweils in MPa) ergeben sich in der Regressionsrechnung folgende Gleichungen:

zu Abbildung 3.70 a) Druckfestigkeit in der Gruppe der Karbonatgesteine:

$$
\mathrm{St}_{\mathrm{C}}=\mathbf{1 2 6 , 4 4 * \mathbf { e } ^ { - 0 , 0 6 * \Phi }}\left(\mathrm{R}^{2}=0,587\right)
$$

zu Abbildung 3.70 b) Biegefestigkeit in der Gruppe der Karbonatgesteine:

$$
\mathrm{St}_{\mathrm{F}}=14,45 * \mathbf{e}^{-0,05 * \Phi}\left(\mathrm{R}^{2}=0,346\right)
$$



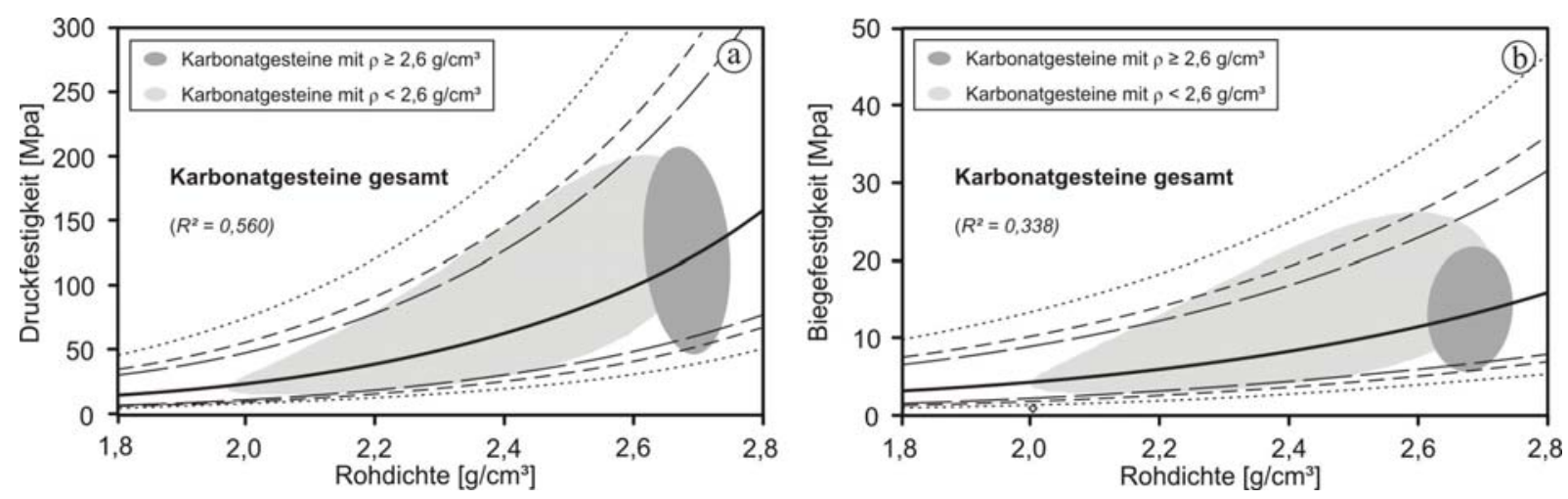

Abb. 3.69: Abhängigkeit der a) Druckfestigkeit sowie b) der Biegefestigkeit von der Rohdichte in der Gruppe der Karbonatgesteine. Dargestellt ist die jeweilige Regressionslinie mit den Bereichen, die 65 \% (lang gestrichelt), 80 \% (kurz gestrichelt) und $95 \%$ (gepunktet) der Streuung um die Linie herum enthalten. Zusätzlich eingetragen sind die abgeschätzten $80 \%$-igen Aufenthaltswahrscheinlichkeitsbereiche für die jeweilige theoretische Grundgesamtheit der Datenpaare in den definierten Dichtegruppen.
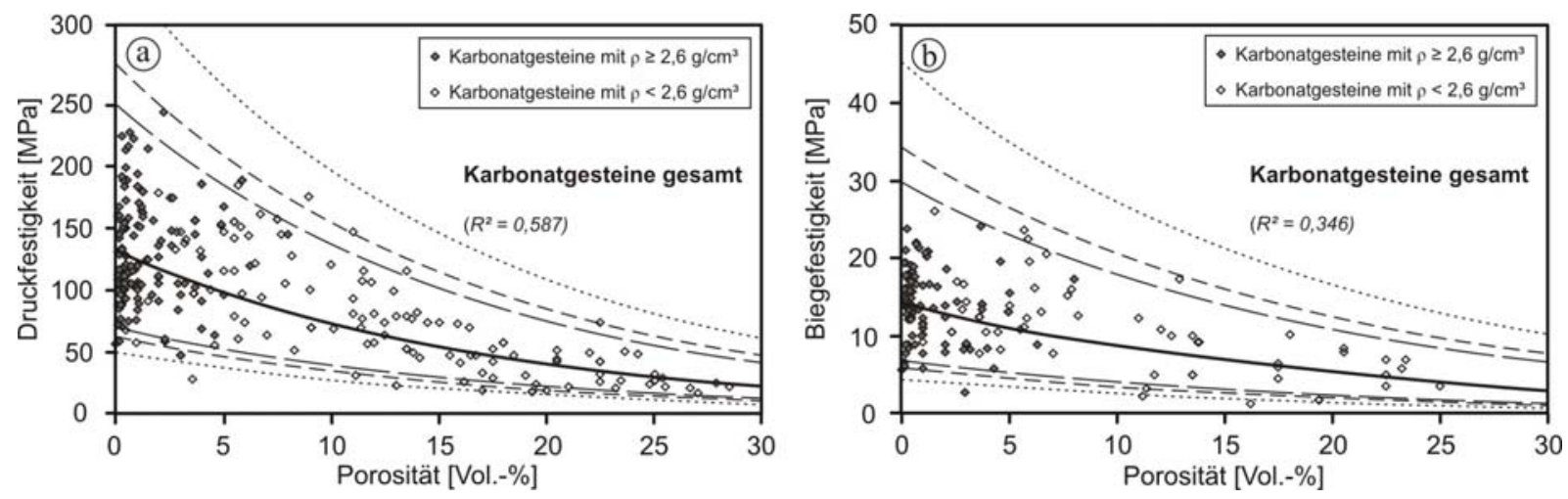

Abb. 3.70: Abhängigkeit a) der Druckfestigkeit sowie b) der Biegefestigkeit von der Porosität in der Gruppe der Karbonatgesteine. Dargestellt ist die jeweilige Regressionslinie mit den Bereichen, die 65 \% (lang gestrichelt), $80 \%$ (kurz gestrichelt) und 95 \% (gepunktet) der Streuung um die Linie herum enthalten.

Eine Anwendung und Diskussion der vorgestellten Regressionsmodelle erfolgt exemplarisch im Rahmen der überregionalen Erfassung von Naturwerksteinen mit dem Fallbeispiel Argentinien (Kap. 4 bzw. 4.8).

\subsection{Ultraschallanalytik - Korrelationen zu den elastischen Eigenschaften der Gesteine}

\subsubsection{Grundlagen und bautechnische Bedeutung}

Die Ultraschallanalytik ist eines der ältesten Verfahren der zerstörungsfreien Materialprüfung. Die an ein elastisches Medium gebundenen Schall- beziehungsweise mechanischen Schwingungen pflanzen sich im Raum fort, indem schwingende Teilchen im Erregungszustand ihre Energie auf benachbarte Teilchen übertragen (Kownatzki 1997). Primär erfolgt zumeist die Durchschallung mittels Kompressionswellen. Dabei resultiert die absolute Geschwindigkeitsverteilung der elastischen Wellen in Gesteinen aus der mineralogischen Zusammensetzung, dem Gefüge, dem Verfestigungsgrad sowie aus der Art und der Anordnung von Poren bzw. Rissen und unterliegt dabei einer gefüge- bzw. 
strukturbedingten Anisotropie (Schön 1983, Siegesmund 1996). Über eine multiple Messung der Kompressionswellengeschwindigkeiten an Kugelproben ist es darüber hinaus auch möglich, Aussagen über die räumliche Verteilung von Rissen bzw. Risssystemen zu machen. Im Gegensatz dazu beschreibt die intrinsische Ultraschallgeschwindigkeit die Geschwindigkeit, die rein auf den elastischen Eigenschaften des ein Gestein aufbauenden Mineralbestandes und dessen räumlicher Anordnung beruht. Die Differenz dieser beiden Parameter beruht darauf, dass die Fortpflanzungsgeschwindigkeit der Ultraschalwellen durch luftgefüllte Poren und Risse infolge der differenten spezifischen Fortpflanzungsgeschwindigkeiten der verschiedenen Medien (z.B. Quarz 6,050 km/s, Wasser $1,450 \mathrm{~km} / \mathrm{s}$, Luft $0,300 \mathrm{~km} / \mathrm{s}$ ) reduziert wird.

Die Methodik der Ultraschalldiagnostik basiert also auf der Annahme, dass eine Geschwindigkeitsänderung der Kompressionswellen mit der Rohdichte und der Porosität in enger Verbindung steht. Da diese Parameter wie beschrieben häufig mit der Gesteinsfestigkeit korrelieren, könnte die Ultraschalldiagnostik möglicherweise eine effektive und kostengünstige Alternative zur Bestimmung der Basiseigenschaften im Hinblick auf Prognoseansätze technischer Gesteinseigenschaften darstellen. Verwendung findet die Ultraschalldiagnostik derzeit sehr häufig bei der Dokumentation verwitterungsbedingter Gefügeauflockerungen beziehungsweise des Erhaltungszustandes von Skulpturen und Bauwerken (z.B. Köhler 1991, Siegesmund et al. 1997, Dürrast et al. 1999, Weiss et al. 2000). Außerdem wird das Verfahren auch für die Rissdetektion zur Klassifikation von Rohblöcken im Werksteinsektor genutzt. Ein weiteres breites Anwendungsgebiet findet sich in akustischen Messverfahren der Bohrlochgeophysik (z.B. Fricke \& Schön 1999).

\subsubsection{Gruppenspezifische Verteilungen und Beziehungen zu den Basiseigenschaften}

Im vorliegenden Datensatz sind für Plutonite, Sandsteine und Karbonatgesteine die Kompressionswellengeschwindigkeiten von insgesamt 245 Varietäten enthalten. In der explorativen Datenauswertung ergeben sich drei Gruppen, die sich deutlich in ihrer Verteilung über den absoluten Geschwindigkeitsbereich unterscheiden (Abb. 3.71 a). Für Sandsteine ist dabei exklusive weniger Ausreißer ein Wertebereich zwischen 1,86 km/s und $3,62 \mathrm{~km} / \mathrm{s}$ dokumentiert, während sich die Gruppe der Plutonite mit Werten zwischen 4,19 km/s und 6,05 km/s deutlich davon unterscheidet. Für die Gruppe der Karbonatgesteine ist dagegen ein umspannender Wertebereich von 2,06 km/s bis 6,47 km/s realisiert. 
(a)
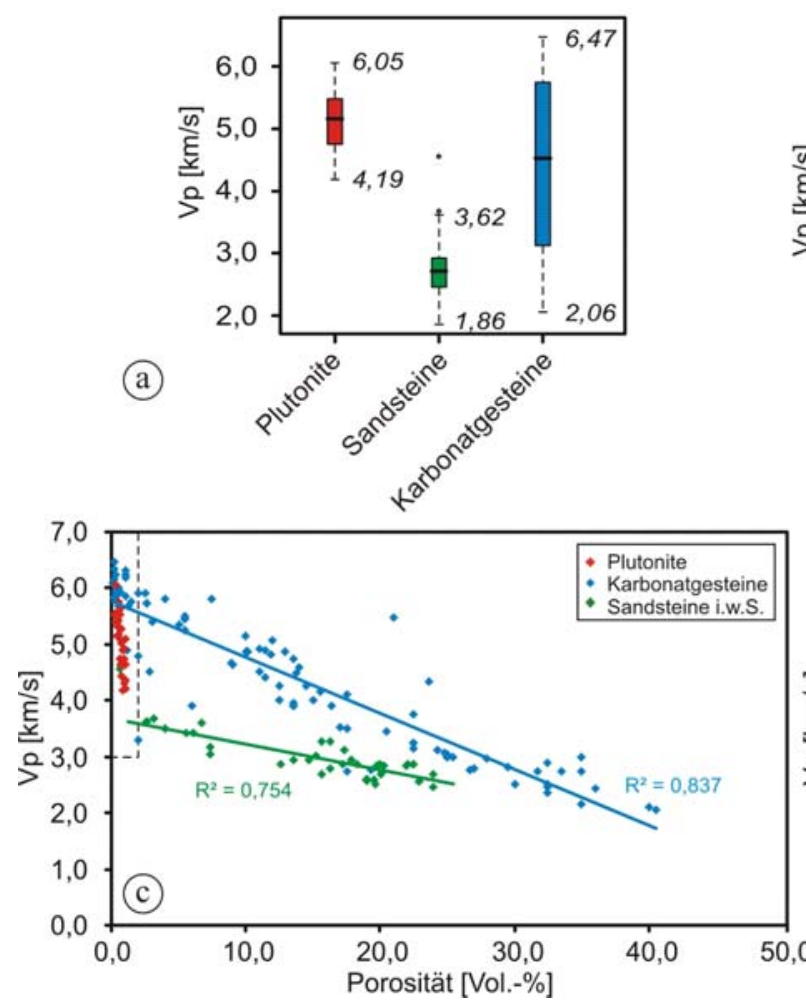
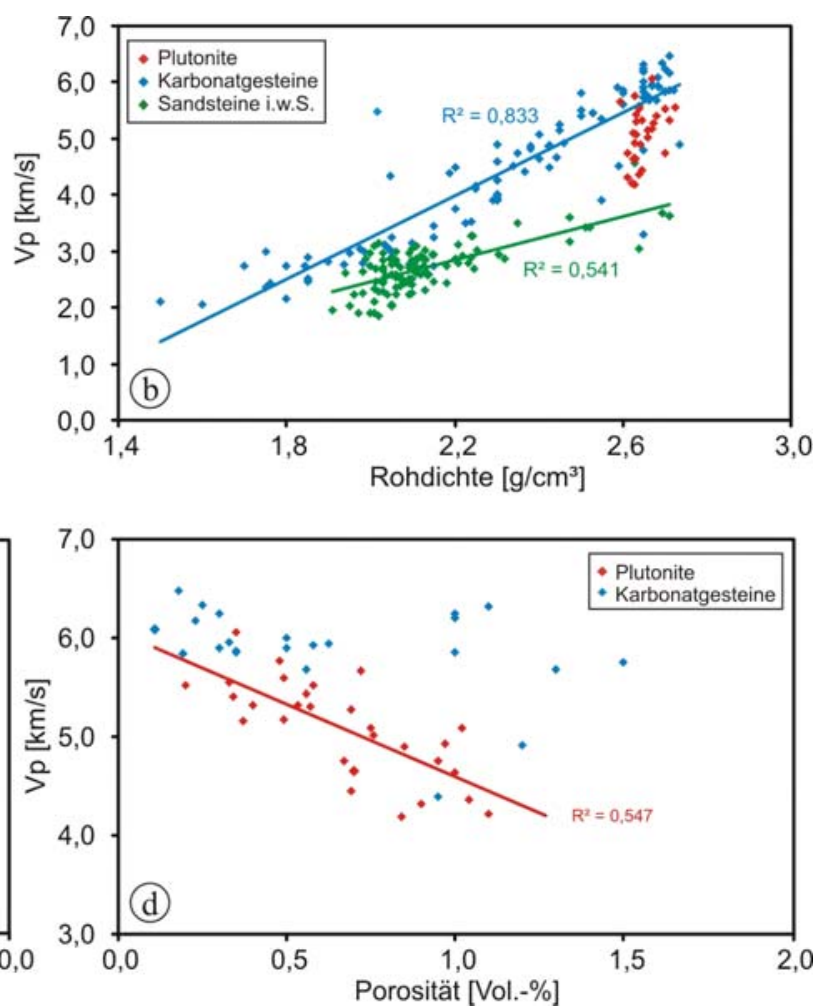

Abb. 3.71: a) Kompressionswellengeschwindigkeit Vp der drei unterschiedenen Hauptgesteinsgruppen. Angegeben sind die statistischen Kennwerte oberer und unterer Extremwert. b - c) Punktverteilungen der Korrelationen der Kompressionswellengeschwindigkeit mit den Basiseigenschaften Rohdichte und Porosität sowie die Determinationskoeffizienten $\mathrm{R}^{2}$ für den jeweiligen linearen Zusammenhang. d) Detail aus c im Bereich der niedrigporösen Gesteine mit $\Phi<2$ Vol.-\%.

Diese Teilung zeigt sich auch in den Streudiagrammen (Abb. 3.71) sowie den Aufenthaltswahrscheinlichkeiten (Abb. 3.72) zur Korrelation der Wellengeschwindigkeit mit den Basiseigenschaften. Für die verschiedenen Gesteine, die sich in ihrer mineralogischen Zusammensetzung und ihrem allgemeinen Gefügeinventar deutlich voneinander unterscheiden, kann keine einheitliche Beziehung etabliert werden. Zwar ist für Gesteine mit einer Rohdichte bis zu etwa 2,6 g/ $\mathrm{cm}^{3}$ beziehungsweise einer Porosität über etwa 5 Vol.-\% ein vergleichbarer Trend zu beobachten, doch werden bei Karbonatgesteinen bei gleicher Porosität höhere Werte als bei Sandsteinen erreicht. Hier zeichnet sich der Einfluss der mineralspezifischen elastischen Eigenschaften (Calcit: $\mathrm{Vp}=6,54 \mathrm{~km} / \mathrm{s}$, Quarz: $\mathrm{Vp}=6,05 \mathrm{~km} / \mathrm{s}$, Gebrande 1982) auf die absolute Ultraschallgeschwindigkeit ab. Weiterhin kann sich auch der Gehalt an Tonmineralen auf die Wellengeschwindigkeit auswirken (Han et al. 1986). Tonminerale zeigen deutlich geringere mineralspezifische elastische Eigenschaften als Quarz (1,1 - 2,8 km/s). So werden bei Sandsteinen, die eine tonige Matrix aufweisen, geringere Werte für die absolute Ultraschallgeschwindigkeit erreicht, als dies z.B. bei kieselig zementierten Sandsteinen der Fall ist. Ursächlich dafür ist die höhere Kompressibilität von Tonen, die so im Gestein hinsichtlich der Fortpflanzung von Kompressionswellen eine puffernde Wirkung haben (Schön 1996). Erhöhte Tongehalte resultieren folglich in geringeren Vp-Werten und können zudem, infolge der stark vom Wassergehalt abhängigen mineralspezifischen Eigenschaften, erhebliche Schwankungen im mechanischen und elastischen Verhalten eines Gesteins verursachen. 

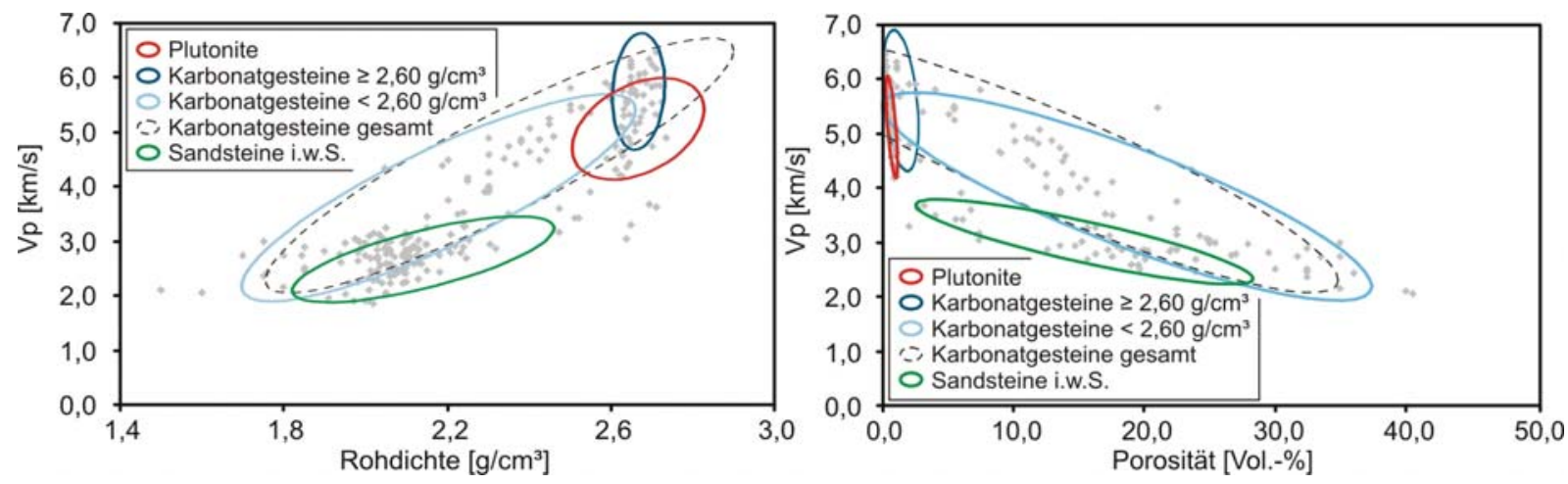

Abb. 3.72: Bereiche der 80 \%-igen Aufenthaltswahrscheinlichkeit für die Korrelation der Kompressionswellengeschwindigkeit Vp mit der Rohdichte bzw. der Porosität in den unterschiedenen Gesteinsgruppen.

Die mineralspezifische Abhängigkeit der absoluten Ultraschallgeschwindigkeit kann ansatzweise auch im niedrigporösen Bereich zwischen den Karbonatgesteinen und den Plutoniten erkannt werden, da Feldspäte im Allgemeinen geringere elastische Eigenschaften im Vergleich zum Calcit aufweisen. Höhere Werte wären lediglich von z.B. Peridotiten mit hohen Olivingehalten (Olivin: Vp = 8,42 km/s; Gebrande 1982) oder aber verschiedenen metamorphen Gesteinen (z.B. Eklogite, Gneise) zu erwarten, die jedoch nicht im hier zugrunde liegenden Datensatz enthalten sind. Geringere $\mathrm{SiO}_{2}$-Gehalte sind so zumeist mit einer Zunahme der Kompressionswellengeschwindigkeit verbunden (Schön 1996). Auch im Bereich niedrigporöser Gesteine ( $\Phi<1,5$ Vol.-\%) ist ein deutlicher, linearer Zusammenhang der Kompressionswellengeschwindigkeit und der Porosität ausgebildet. Die hochkompakten Karbonatgesteine lassen sich hier annähernd in den Trend der Plutonite einpassen. Der höchste Wert für Vp mit 6,472 km/s (bei $\Phi=0,18$ Vol.-\%) ist dabei für die Kalksteinvarietät MARBRE PALOMA (Frankreich) dokumentiert. Daraus lässt sich schließen, dass das Gestein eine annähernd perfekt monomineralische Zusammensetzung aufweisen muss.

\subsubsection{Beziehungen zu den Festigkeitseigenschaften}

Die Kenntnis über die regressive Verknüpfung der Porosität mit den Parametern der Festigkeit gerade im Bereich der Sandsteine und der nicht kompakten Karbonatgesteine lässt auch auf ein entsprechendes Verhalten gegenüber der Ultraschallgeschwindigkeit schließen. In der Korrelation zur Druckfestigkeit wird dies vor allem für die Karbonatgesteine deutlich (Abb. 3.73). Dabei ist mit zunehmender Vp eine weitere Datenspanne der Druckfestigkeit erkennbar. Diese Tatsache zeigt sich besonders in der Darstellung der Aufenthaltswahrscheinlichkeiten (Abb. 3.74). Dabei ergeben sich nach der Teilung aller Karbonatgesteine nach dem in Kapitel 3.8.3 definierten Schwellenwert der Rohdichte ( $\rho_{\text {roh }}<$ bzw. $\geq 2,60 \mathrm{~g} / \mathrm{cm}^{3}$ ) deutlich unterschiedliche Bereiche. Dadurch wird auch hier eine verbesserte beziehungsweise detailliertere Abschätzung der Grundgesamtheit erreicht. Zwar gehen aus dem errechneten Bereich für Karbonatgesteine mit $\rho_{\text {roh }} \geq 2,6 \mathrm{~g} / \mathrm{cm}^{3}$ für die Kompressionswellengeschwindigkeit Werte bis annähernd 7 km/s oder noch darüber hervor. Doch selbst für mehr oder weniger monomineralische Karbonatgesteine sind diese Werte praktisch nicht realisierbar. Für die Gruppe der Sandsteine ist die Korrelation infolge der 
geringeren Datenspannweite der verbundenen Parameter weniger gut ausgebildet. Beide Regressionsgeraden verlaufen annähernd parallel zueinander. In der Gruppe der Karbonatgesteine werden dabei gleiche Vp-Werte mit relativ geringeren Druckfestigkeiten verknüpft. Für die Gruppe der Plutonite ist eine Korrelation insgesamt schwach ausgebildet.

Die vorhandenen Daten zur Biegefestigkeit lassen erwartungsgemäß auf vergleichbare Zusammenhänge schließen. Bei Sandsteinen und Karbonatgesteinen ist jedoch der deutliche Steigungsunterschied der Regressionsgeraden auffällig. Dieser ist vermutlich auf die geringe Anzahl eingegangener Daten zurückzuführen. Aus Kapitel 3.7.2 ist bekannt, dass die Biegefestigkeit bei Sandsteinen scheinbar linear mit der Druckfestigkeit verknüpft ist. In der Folge wurden die Daten zur Korrelation $\mathrm{Vp} / \mathrm{St}_{\mathrm{F}}$ exemplarisch durch empirische Daten ergänzt. Im Resultat ergibt sich ein linearer Zusammenhang, der durch eine deutlich geringere Steigung der Regressionsgeraden beschrieben wird (Abb. 3.73).
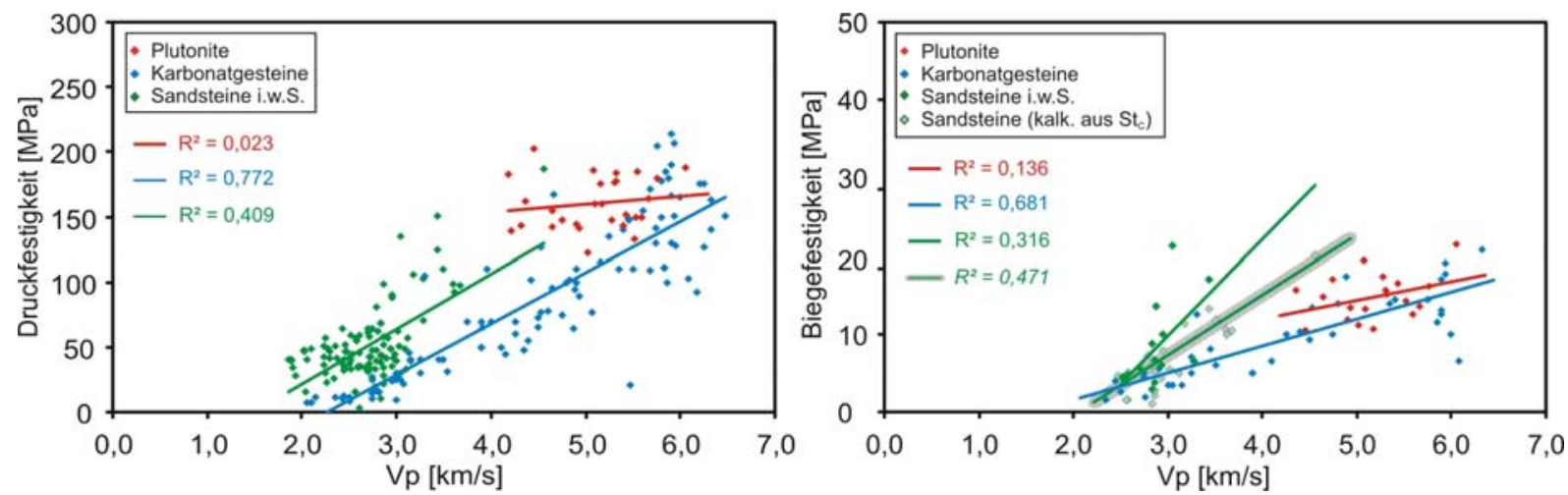

Abb. 3.73: Korrelationsdiagramme der Kompressionswellengeschwindigkeit Vp mit den mechanischen Festigkeitsparametern sowie die Determinationskoeffizienten $\mathrm{R}^{2}$ für den jeweiligen linearen Zusammenhang. Die im Korrelationsdiagramm $\mathrm{Vp} / \mathrm{St}_{\mathrm{F}}$ grau hinterlegte Regressionsgerade beruht auf einem durch empirische Daten aus der Druckfestigkeit erweiterten Datensatz zur Biegefestigkeit.
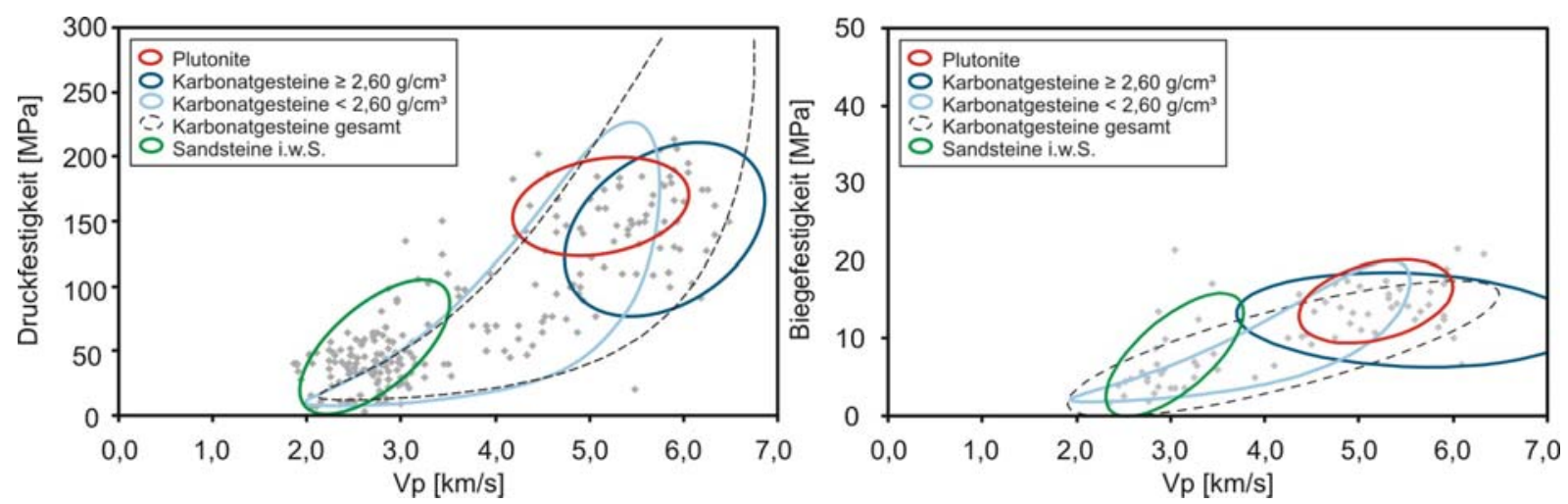

Abb. 3.74: Bereiche der 80 \%-igen Aufenthaltswahrscheinlichkeit für die Korrelation der Kompressionswellengeschwindigkeit Vp mit den Parametern Druck- bzw. Biegefestigkeit in den unterschiedenen Gesteinsgruppen.

\subsubsection{Ansätze zu Prognosen aus der Ultraschallanalytik}

Aus dem hier behandelten Datensatz ergibt sich für fast alle unterschiedenen Gesteinsgruppen ein mehr oder weniger ausgeprägter gerichteter Zusammenhang zwischen 
der Kompressionswellengeschwindigkeit und der Porosität. Eine Ausnahme bildet die Gruppe der Karbonatgesteine mit $\rho_{\text {roh }} \geq 2,60 \mathrm{~g} / \mathrm{cm}^{3}$. Die Ausbildung des Streudiagramms in Abbildung 3.71 d lässt zwar für die Gesamtheit hochdichter Gesteine (Plutonite, Karbonatgesteine mit $\rho_{\text {roh }} \geq 2,60 \mathrm{~g} / \mathrm{cm}^{3}$ ) ein statistisch gleichartiges Verhalten vermuten. Doch in der jeweiligen Anpassung an ein lineares Modell ergibt sich ein deutlich unterschiedliches Bild. Während für die Plutonite ein relativ guter gerichteter Zusammenhang mit gestreckten Prognosestreifen resultiert (Abb. 3.75 a), ergibt sich bei den Karbonatgesteinen ein sehr geringer Determinationskoeffizient nahe Null (Abb. 3.75 b). Die Vp-Werte für die nach der Rohdichte klassifizierten, hochkompakten Karbonatgesteine überspannen dabei lediglich einen Bereich von etwa $0,8 \mathrm{~km} / \mathrm{s}$, was bei angegebenen Porositätswerten zwischen 0,11 Vol.-\% und 2,6 Vol.-\% eine weite Öffnung der Streuungsbereiche um die angepasste Gerade herum zur Folge hat. Die hohen Werte zur Porosität sind zwar als Ausreißer einzustufen, doch bewirkt auch deren Eliminierung keine deutliche Verbesserung. Daher werden die Karbonatgesteine hinsichtlich eines Ansatzes zur Porosität im Folgenden als Gesamtgruppe betrachtet. Dann nämlich ergibt sich ein deutlicher, gerichteter Zusammenhang (Abb. 3.75 c), der jedoch mit einer relativ hohen Standardabweichung behaftet ist. Ein gleiches Verhalten kann auch für die Gruppe der klastischen Sedimente (Abb. 3.75 d) angenommen werden, wobei sich hier aber ein geringerer Fehlerbereich ergibt.

Zur Abschätzung eines regressiven Zusammenhangs bleibt $\mathrm{zu}$ vermerken, dass die Kompressionswellengeschwindigkeit in allen hier betrachteten Fällen über Linearfunktionen mit der Porosität verknüpft ist. Eine Anpassung des gerichteten Zusammenhangs über nichtlineare Funktionstypen bewirkt, wenn überhaupt, eine nur unbedeutende Verbesserung des Determinationskoeffizienten.

Für die vermuteten linearen Zusammenhänge zwischen der Kompressionswellengeschwindigkeit Vp (in km/s) und der Porosität $\Phi$ (in Vol.-\%) ergeben sich in der Regressionsrechnung folgende Geradengleichungen mit Standardabweichung:

zu Abbildung 3.75 a) Gruppe der Plutonite:

$$
\Phi=\quad-0,35 * \mathbf{V p}+\mathbf{6 1 , 4 9}+/-0,24 \quad\left(\mathrm{R}^{2}=0,512\right)
$$

zu Abbildung 3.75 c) Gruppe der karbonatischen Sedimente:

$$
\Phi=-\mathbf{8 , 3 3} * \mathrm{Vp}+\mathbf{5 0 , 4 9}+/-\mathbf{1 1 , 8 2}\left(\mathrm{R}^{2}=0,856\right)
$$

zu Abbildung 3.75 d) Gruppe der klastischen Sedimente:

$$
\Phi=-15,36 * V p+61,49+/-5,51 \quad\left(R^{2}=0,645\right)
$$



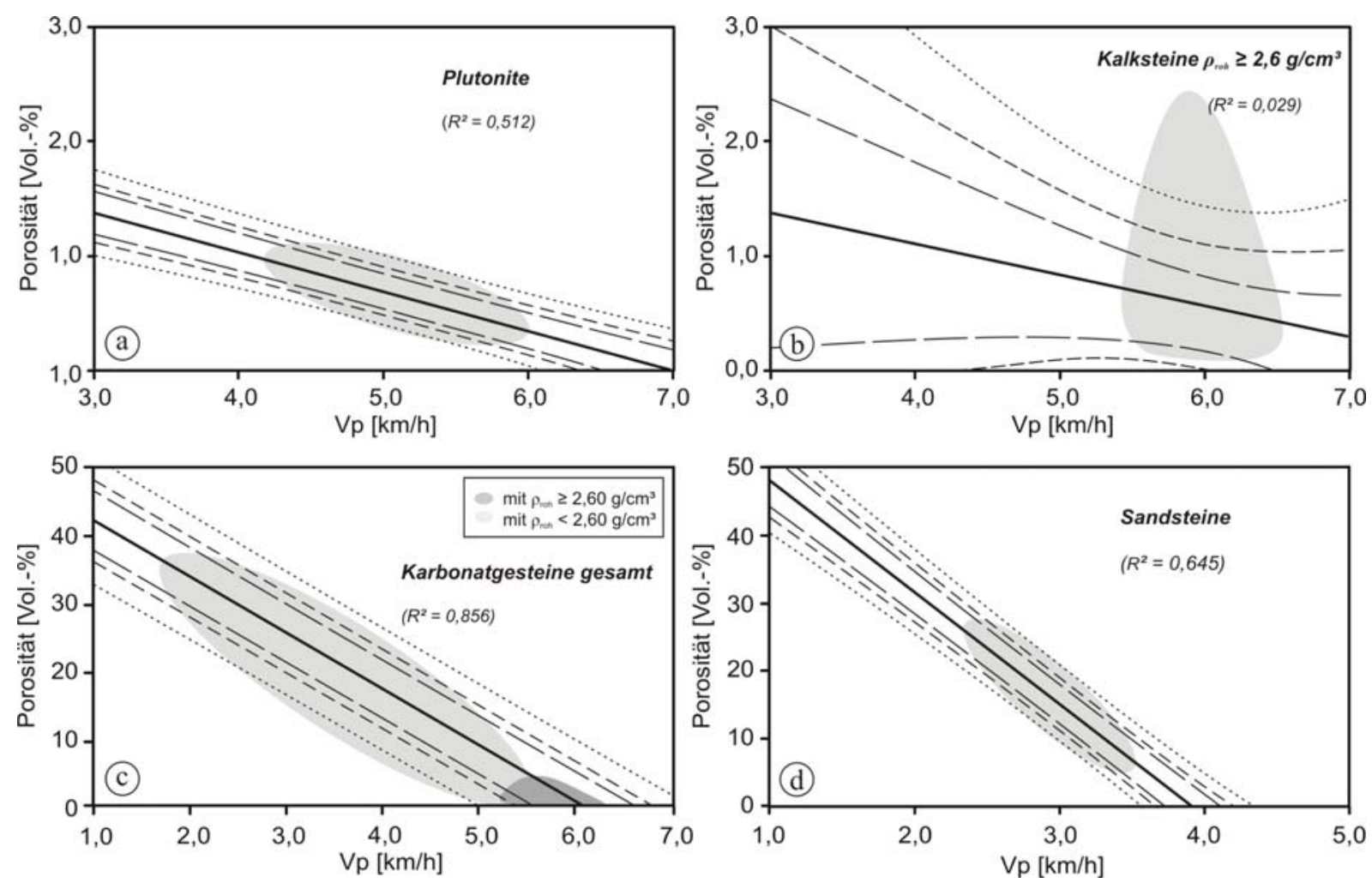

Abb. 3.75 Abhängigkeit der Porosität von der Kompressionswellengeschwindigkeit in den unterschiedenen Gesteinsgruppen. Dargestellt ist die jeweilige Regressionsgerade mit den Bereichen, die 65 \% (lang gestrichelt), $80 \%$ (kurz gestrichelt) und $95 \%$ (gepunktet) der Streuung um die Gerade herum enthalten. Zusätzlich eingetragen sind die abgeschätzten $80 \%$-igen Aufenthaltswahrscheinlichkeitsbereiche für die jeweilige theoretische Grundgesamtheit der Datenpaare.

Verschiedene Autoren stellen eine direkte Verbindung zwischen der Ultraschallgeschwindigkeit und den Parametern der Festigkeit für verschiedene Gesteinsgruppen her (z.B. Freyburg 1972, Golubev \& Rabinovic 1976, Peschel 1983). Neben den o.g. bautechnischen Verwendungen hinsichtlich der Charakterisierung des Schadenspotenzials wäre somit auch eine Anwendung der Ultraschallanalytik in der Lagerstättenerkundung zur ersten Einschätzung der mechanischen Stabilität eines Gesteins denkbar. Andere Ansätze beziehen sich z.B. auf die Ermittlung der Rückprallhärte (SchmidtHärte), um eine Aussage über die Druckfestigkeit eines Gesteins zu erlangen (Krauter et al. 1985, Dinçer et al. 2004). Doch liegt der Vorteil der Ultraschallanalytik in der volumenumfassenden Betrachtung eines Gesteins. Auch kann durch entsprechende Probengrößen beziehungsweise einer angepassten Messstrecke der jeweiligen Korngröße eines Gesteins Rechnung getragen werden.

Grundsätzlich lassen sich in den Beziehungen zwischen Vp und den Parametern der Festigkeit zwei verschiedene Modellanpassungen unterscheiden: (i) lineare Anpassung im Bereich der Plutonite und klastischen Sedimente und (ii) nichtlineare (exponentielle) Anpassung in der Gruppe der Karbonatgesteine.

Im Vergleich der linearen Beziehungen fällt auf, dass die Regressionsgeraden zu den Daten der Plutonite eine deutlich geringere Steigung aufweisen. Dementsprechend fallen auch die Determinationskoeffizienten derart gering aus, dass die Annahme einer linearen 
Abhängigkeit stark in Frage gestellt werden muss. Trotz dessen soll aber auch im Falle der Plutonite eine Anpassung an ein lineares Modell dargestellt werden. Im Zusammenhang mit der vorangegangenen Betrachtung zur Beziehung $\mathrm{Vp} / \Phi$ zeigen sich hier Art und Ausmaß des Einflusses der Porosität bei plutonischen Gesteinen. Während eine für Plutonite relativ hohe Porosität (bei 1 bis 1,5 Vol.-\%) eine Reduktion der Kompressionswellengeschwindigkeit infolge der puffernden Wirkung der Hohlräume bewirkt, wird die absolute mechanische Stabilität insgesamt wenig beeinflusst. Durch eine gerichtete Rissgeometrie kann es jedoch zu richtungsabhängigen Schwankungen im mechanischen Verhalten kommen (Strohmeyer 2003). Dieser Zusammenhang wäre über eine vollständige Bestimmung der Verteilung der Kompressionswellengeschwindigkeiten nach der Durchschallungsmethode (pulse transmission technique, Birch 1960, 1961) zu analysieren. Damit ist die Möglichkeit gegeben, tatsächliche Minima und Maxima der Wellengeschwindigkeiten zu detektieren (Siegesmund et al. 1993) und so Rückschlüsse auf eine Inhomogenität der mechanischen Stabilität hochkompakter Gesteine zu gewinnen.

Anders verhält es sich in der Gruppe der Sandsteine. Hier ist mit der Zunahme der Porosität zumeist eine Schwächung des Gesteins verbunden, da ein erhöhter Porenraum gleichbedeutend mit einer relativen Abnahme der Anzahl an Bindungspunkten im Gerüst der Aggregate ist. Dabei kann auch das jeweilige Bindemittel eines Sandsteins in der Geschwindigkeit der Ultraschallwellen zum Ausdruck kommen. Quarzitisch gebundene Sandsteine weisen im Allgemeinen eine höhere Festigkeit als tonig gebundene auf. Bei annähernd entsprechender Porosität ist aber hinsichtlich Vp eine Differenz festzustellen, die mit dem relativen Gehalt an Tonmineralen einhergeht (Han et al. 1986).

Für die vermuteten linearen Zusammenhänge zwischen der Kompressionswellengeschwindigkeit Vp (in km/s) und den Parametern Druck- bzw. Biegefestigkeit ( $\mathrm{St}_{\mathrm{C}}$ bzw. $\mathrm{St}_{\mathrm{F}}$ jeweils in $\mathrm{MPa}$ ) ergeben sich in der Regressionsrechnung folgende Geradengleichungen mit Standardabweichung:

zu Abbildung 3.76 a) Druckfestigkeit in der Gruppe der Plutonite:

$$
\mathrm{St}_{\mathrm{C}}=\mathbf{9 , 4 4} * \mathrm{Vp}+\mathbf{1 1 3 , 4 8}+/-20,43 \quad\left(\mathrm{R}^{2}=0,054\right)
$$

zu Abbildung 3.76 b) Biegefestigkeit in der Gruppe der Plutonite:

$$
\mathrm{St}_{\mathrm{F}}=2,30 * \mathrm{Vp}+2,96{ }^{+} /-2,87 \quad\left(\mathrm{R}^{2}=0,122\right)
$$

zu Abbildung 3.76 c) Druckfestigkeit in der Gruppe der Sandsteine:

$$
\mathrm{St}_{\mathrm{C}}=33,88 * \mathrm{Vp}-\mathbf{4 1 , 0 7}+/-24,62 \quad\left(\mathrm{R}^{2}=0,250\right)
$$

zu Abbildung 3.76 d) Biegefestigkeit in der Gruppe der Sandsteine:

$$
\mathrm{St}_{\mathbf{F}}=7,92 * \mathrm{Vp}-\mathbf{1 6 , 3 8}+/-4,75 \quad\left(\mathrm{R}^{2}=0,472\right)
$$



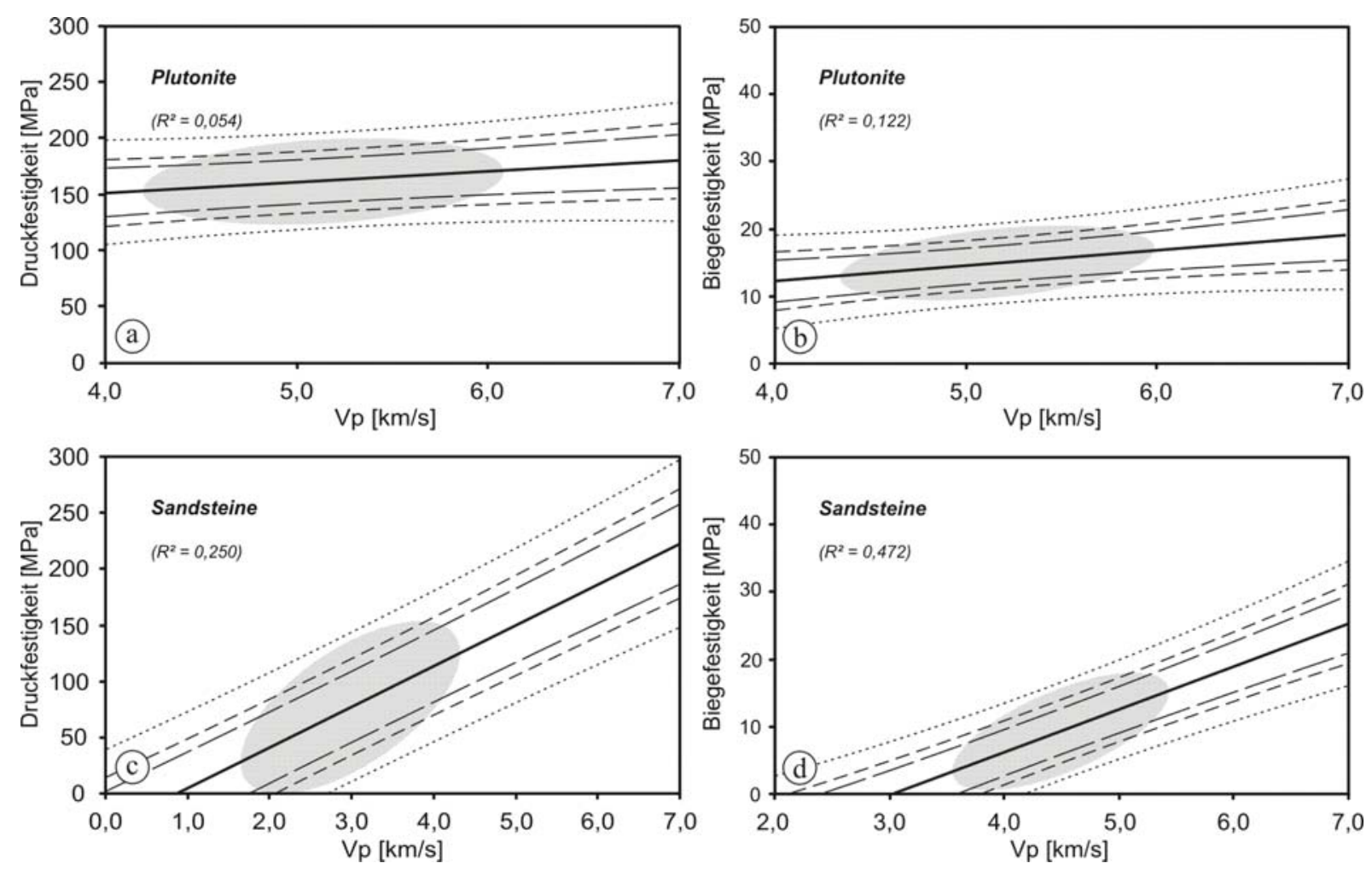

Abb. 3.76: Abhängigkeit der Druck- sowie Biegefestigkeit von der Kompressionswellengeschwindigkeit Vp in der Gruppe der Plutonite (a, b) und der Sandsteine (c, d). Dargestellt ist die jeweilige Regressionsgerade mit den Bereichen, die 65 \% (lang gestrichelt), 80 \% (kurz gestrichelt) und 95 \% (gepunktet) der Streuung um die Gerade herum enthalten. Zusätzlich eingetragen sind die abgeschätzten 80 \%-igen Aufenthaltswahrscheinlichkeitsbereiche für die jeweilige theoretische Grundgesamtheit der Datenpaare.

In der Gruppe der Karbonatgesteine ist die Anpassung einer linearen als auch nichtlinearen Funktion für die Beziehung $\mathrm{Vp} / \mathrm{St}_{\mathrm{C}}$ bzw. $\mathrm{Vp} / \mathrm{St}_{\mathrm{F}}$ mit einem recht hohen Determinationskoeffizienten belegt. Die nichtlineare Anpassung (Abb. 3.78) beschreibt jedoch mit den resultierenden Bereichen der niveauabhängigen Fehlerstreuung die Verteilung der zugrunde liegenden Punktwolke deutlich besser. Ausschlaggebend für den wachsenden Fehler in der Gesamtgruppe der Karbonatgesteine ist primär die oben beschriebene eingeschränkte Vp-Spannweite für Varietäten mit $\rho_{\text {roh }} \geq 2,60 \mathrm{~g} / \mathrm{cm}^{3}$. Aus Kapitel 3.11 ist bekannt, dass die Parameter der Festigkeit auch mit den Basiseigenschaften über eine Funktion nichtlinearen Charakters verbunden sind. Hinsichtlich der Kompressionswellengeschwindigkeit als Prädiktorvariablen werden dabei engere Bereiche der Streuung beschrieben. Die Tatsache des zunehmenden Fehlers ist wiederum mit Gefügefaktoren in Zusammenhang zu bringen, wie sie in Kapitel 3.8.3 beschrieben sind. Exemplarisch können die Handelssorten PIERRE DE HAUTVILLE ${ }^{1)}$ (Kalkstein, Frankreich, Abb. 3.77 a) und ROUGE ANTIQUE ${ }^{2)}$ (Kalkstein, Frankreich, Abb. 3.77 b) genannt werden. Für beide Varietäten sind hohe Rohdichten $\left({ }^{1)} 2,68 \mathrm{~g} / \mathrm{cm}^{3} ;{ }^{2)} 2,71 \mathrm{~g} / \mathrm{cm}^{3}\right)$ und Ultraschallgeschwindigkeiten $\left({ }^{1} 5,887 \mathrm{~km} / \mathrm{s} ;{ }^{2}{ }_{5,855} \mathrm{~km} / \mathrm{s}\right.$ ) bei geringen Porositäten $\left({ }^{1)} 0,60\right.$ Vol.-\%; ${ }^{2)} 0,35$ Vol.-\%) dokumentiert (Gargi et al. 1998). Hinsichtlich der Druckfestigkeit unterscheiden sie sich jedoch erheblich $\left({ }^{1)} 171,3 \mathrm{MPa} ;{ }^{2)} 100,0 \mathrm{MPa}\right.$; Gargi et al. 1998). Die Herabsetzung der Druckfestigkeit ist in einem verstärkten Auftreten einer sekundären, relativ grobkristallinen Calcitmineralisation in Bruchstrukturen der Varietät 
ROUGE ANTIQUE begründet. Die Auswirkungen auf die Rohdichte, die Porosität sowie die Ausbreitungsgeschwindigkeit der Kompressionswellen bleiben dabei gering. Bei der Bestimmung der Ultraschallgeschwindigkeit tritt ausschließlich eine elastische Deformationen von nur kurzer Dauer ein. Dagegen ist die mechanische Belastung bei der Ermittlung der Druckfestigkeit von längerer Dauer und führt in Ansätzen zu einer plastischen Verformung des Prüfkörpers und schließlich zum vollständigen Gesteinsversagen (Schön 1983, Strohmeyer 2003). Im Falle der kompressiven Belastung bilden die Calcitadern daher potenzielle mechanische Schwächezonen im Gestein.
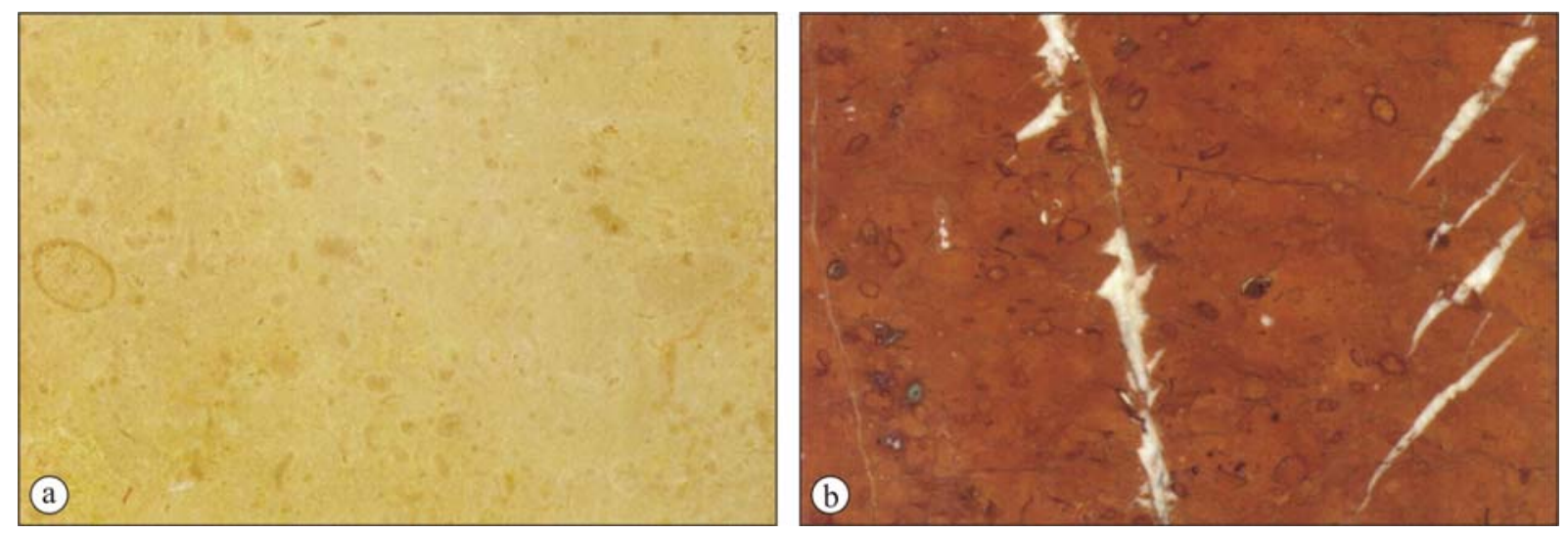

Abb. 3.77: Französische Kalksteine a) PIERRE DE HAUTVILLE und b) ROUGE ANTIQUE: Herabsetzung der Festigkeit durch eine relativ gröbere Calcitmineralisation in Bruchstrukturen im Falle der Varietät b bei gleicher Kompressionswellengeschwindigkeit sowie vergleichbarer Rohdichte und Porosität.

Für die vermuteten nichtlinearen Zusammenhänge zwischen der Kompressionswellengeschwindigkeit Vp (in km/s) und den Parametern Druck- bzw. Biegefestigkeit ( $\mathrm{St}_{\mathrm{C}}$ bzw. $\mathrm{St}_{\mathrm{F}}$ jeweils in $\mathrm{MPa}$ ) ergeben sich in der Regressionsrechnung folgende Gleichungen:

zu Abbildung 3.78 a) Druckfestigkeit in der Gruppe der Karbonatgesteine:

$$
\mathrm{St}_{\mathrm{C}}=3,17 * \mathbf{e}^{\mathbf{0 , 4 4 * V p}}\left(\mathrm{R}^{2}=0,824\right)
$$

zu Abbildung 3.78 b) Biegefestigkeit in der Gruppe der Karbonatgesteine:

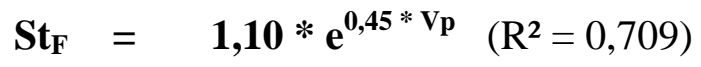



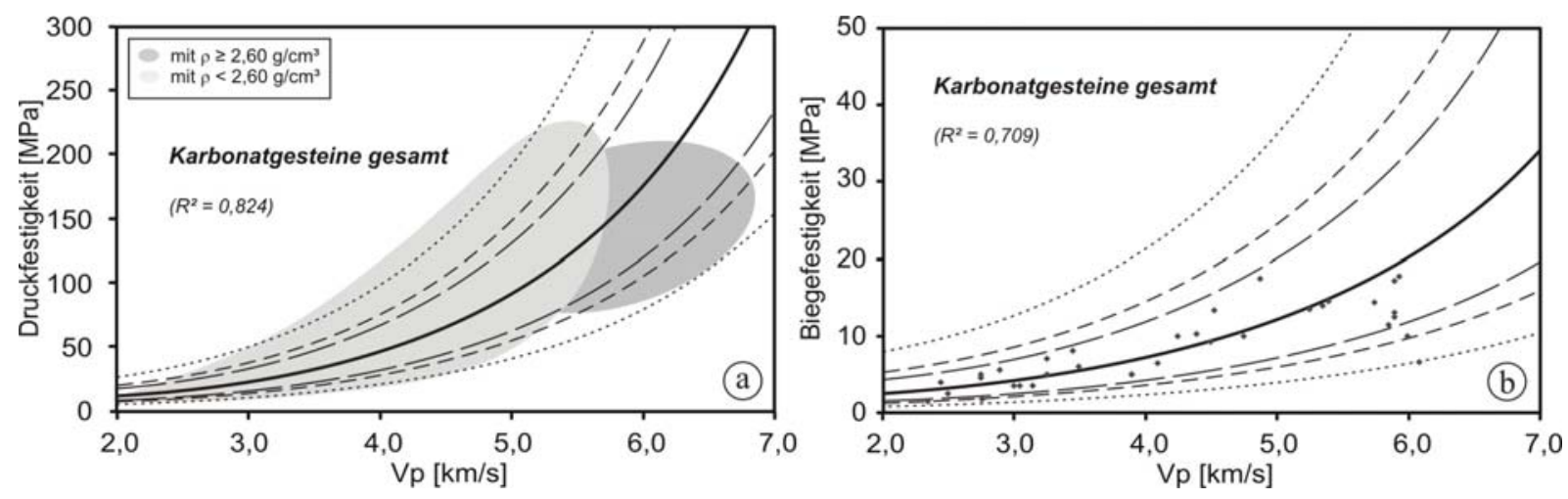

Abb. 3.78: Abhängigkeit a) der Druckfestigkeit sowie b) der Biegefestigkeit von der Kompressionswellengeschwindigkeit Vp in der Gruppe der Karbonatgesteine. Dargestellt ist die jeweilige Regressionslinie mit den Bereichen, die 65 \% (lang gestrichelt), 80 \% (kurz gestrichelt) und 95 \% (gepunktet) der Streuung um die Gerade herum enthalten. Zur Beziehung $\mathrm{Vp}_{\mathrm{St}}$ sind zusätzlich die abgeschätzten $80 \%$-igen Aufenthaltswahrscheinlichkeitsbereiche für die jeweilige theoretische Grundgesamtheit der Datenpaare eingetragen.

Einen Vergleich zwischen dem aus dem vorliegenden Datensatz ermittelten Zusammenhang von Ultraschallgeschwindigkeit und Druckfestigkeit mit der von Golubev \& Rabinovic (1976) für Kalkstein postulierten empirischen Beziehung zeigt Abbildung 3.79. Dabei ist zu vermuten, dass im Datensatz Golubev \& Rabinovic (1976) nur wenige niedrigporöse Gesteine mit annähernd monomineralischer Zusammensetzung und gleichzeitig homogenem Gefügecharakter vertreten und in die Berechnung eingegangen sind. Die exponentielle Anpassung der Beziehung $\mathrm{Vp} / \mathrm{St}_{\mathrm{C}}$ verhält sich für Karbonatgesteine mit $\rho_{\text {roh }}<2,60 \mathrm{~g} / \mathrm{cm}^{3}$ aus dem vorliegenden Datensatz annähernd kongruent mit der von o.g. Autoren gegebenen Beziehung. Eine separate Betrachtung der beiden definierten Untergruppen erscheint trotz allem nicht sinnvoll, da zum einen die Verknüpfung für die Gesamtgruppe einen deutlichen gerichteten Zusammenhang annehmen lässt. Zum anderen kann somit die Untergruppe der hochdichten Varietäten mit $\rho \geq 2,60 \mathrm{~g} / \mathrm{cm}^{3}$ in den Prognoseansatz miteinbezogen werden kann.

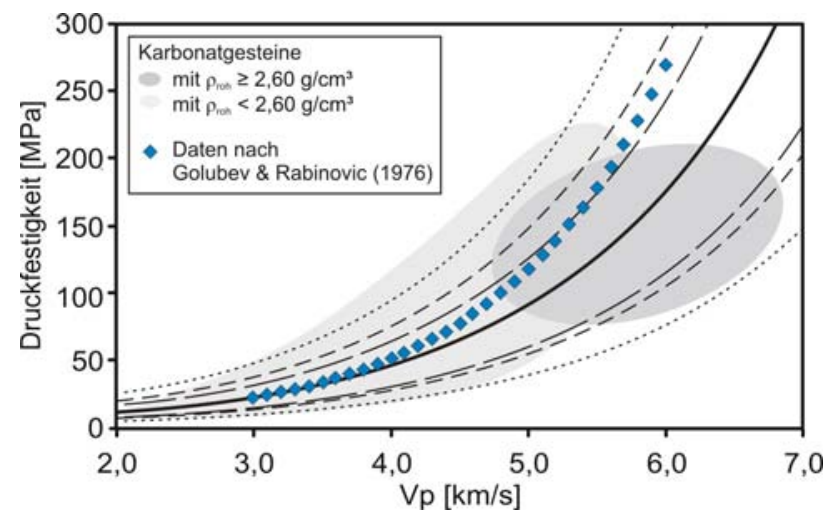

Abb. 3.79: Vergleich des aus dem vorliegenden Datensatz ermittelten regressiven Zusammenhangs zwischen der Kompressionswellengeschwindigkeit Vp und der Druckfestigkeit mit dem empirischen Zusammenhang für Kalkstein nach Golubev \& Rabinovic (1976). Nähere Erläuterungen siehe Text.

Zusammenfassend erscheint die Ultraschalldiagnostik eine sinnvolle Ergänzung zur primären Abschätzung von Festigkeiten eines Gesteins darzustellen. Die Ausnahme bildet dabei die Gruppe der Plutonite, bei der die Analyse der Kompressionswellengeschwindigkeit keine deutlichen Rückschlüsse auf die mechanische Stabilität erlaubt. Bei den verbleibenden, hier unterschiedenen Gesteinsgruppen sind bestimmte Faktoren, beispielsweise die 
Wellengeschwindigkeit beeinflussende Gefügeelemente, bei der Datenauswertung zu beachten.

\subsection{Feuchtehaushalt - Korrelationen zur Wasseraufnahmefähigkeit}

\subsubsection{Grundlagen und bautechnische Bedeutung}

Naturwerksteine unterliegen in der Anwendung als Baumaterial einer ständigen Beanspruchung durch verschiedene Verwitterungsprozesse. Neben der rein mechanischen Komponente, so z.B. direkte Belastung oder gravitativ induzierte Deformation, spielen viele Prozesse eine entscheidende Rolle, die durch den Wasserhaushalt beziehungsweise den Feuchtewechselgehalt der Gesteine in erheblichem Maße beeinflusst und gesteuert werden. Dabei sind die Auswirkungen von Porenwässern in keiner Weise auf bestimmte Gesteinsgruppen beschränkt. So können in porösen Karbonatgesteinen sowie auch in kompakten Marmoren Lösungsprozesse zur Karstbildung führen (vgl. Abb. 2.11). Verschiedene physikalische Verwitterungsphänomene, die in engem Zusammenhang mit der Gesteinsfeuchte stehen, können auch bei anderen hochkompakten Gesteinen, so z.B. Plutoniten, beobachtet werden. Beispielsweise konnte das seit langer Zeit für Marmore und Kalksteine bekannte Phänomen der Verbiegung von Fassadenplatten (z.B. Trewitt \& Tuchmann 1988, Winkler 1996, Grimm 1999, Koch \& Siegesmund 2004) auch für kompakte Granitoide nachgewiesen werden (Mauko et al. 2006, Siegesmund et al. 2007, Abb. 3.80). Als initiale Ursache dafür wird im Allgemeinen der thermische Eintrag verantwortlich gemacht. Zahlreiche Untersuchungen haben jedoch gezeigt, dass beim Einsetzen einer thermohygrischen Belastung ein progressiver Schadensprozess zu verzeichnen ist (z.B. Koch \& Siegesmund 2004). Als Folge dieser mangelnden Formbeständigkeit, die zudem mit einer Abnahme der mechanischen Stabilität einhergeht, kann es zu erheblichen Schädigungen in Form von Abplatzungen oder Ausbrüchen kommen (Abb. 3.80 b). Die Langzeitstabilität von Fassadenelementen kann dadurch stark herabgesetzt werden.
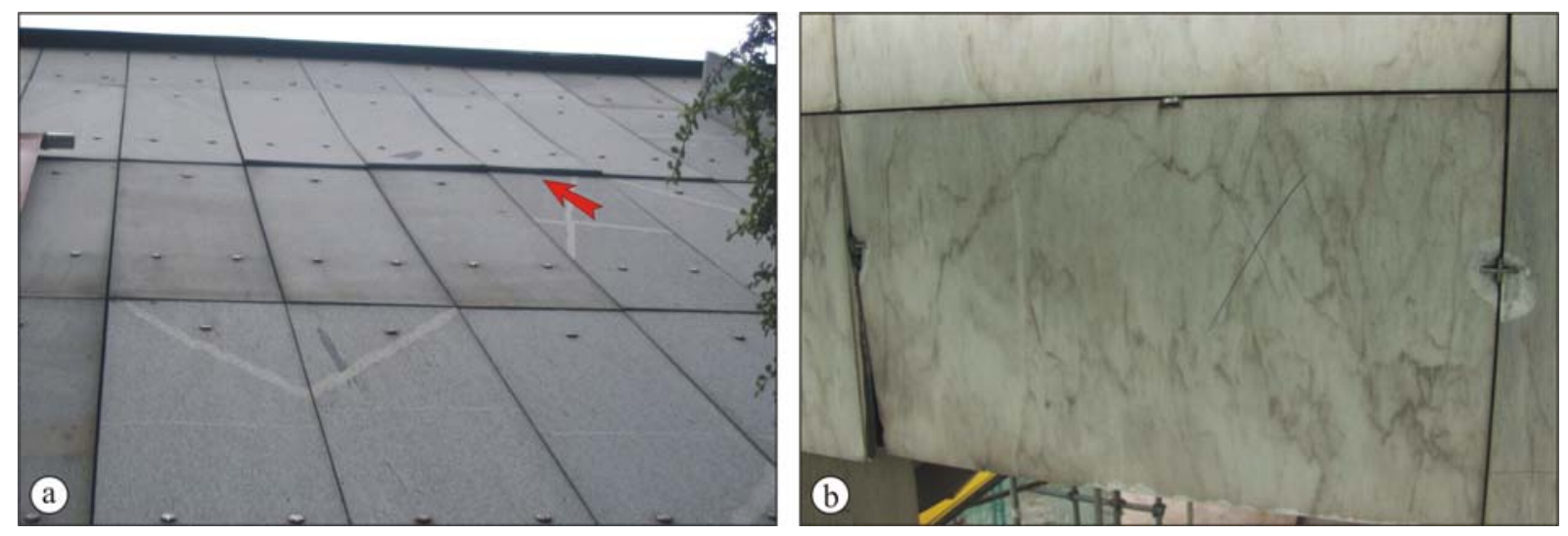

Abb. 3.80: Mangelnde Formbeständigkeit von Fassadenplatten: a) CEZLAK GRANODIORIT (Slowenien, Maxi-Market Ljubljana); b) Abplatzungen im Bereich der Ankerdorne infolge der Plattenverbiegung (Calcitmarmor ROSA ESTREMOZ, Portugal, Staatstheater Darmstadt). 
Die Art des Einflusses der Feuchtigkeit beziehungsweise des Wassergehaltes auf die physikalischen und technischen Eigenschaften von Naturwerksteinen kann verschiedenen Charakters sein. Neben der direkten Schädigung durch Lösungsprozesse kann z.B. die Quellfähigkeit von Tonmineralen zu erheblichen Spannungen im Gestein führen (z.B. Snethlage \& Wendler 1997). Eine erhebliche Belastung des Mineralverbandes kann auch aus der Kristallisation von Salzen oder Eis im Porenraum resultieren (Abb. 3.81). Dabei können Kräfte beziehungsweise Spannungen entstehen, die etwa die Spaltzugfestigkeit mancher Gesteine überschreiten (z.B. Mortensen 1933, Winkler 1968, Scherer 1999, Steiger 2005). Auch die mechanische Stabilität von Gesteinen kann durch den auftretenden Wassergehalt erheblich reduziert werden. So konnte beispielsweise bei verschiedenen Sandsteinen mit zunehmendem Feuchtegehalt eine Abnahme der einaxialen Druckfestigkeit um bis zu 50 \% nachgewiesen werden (Morales Demarco et al. 2007). Neben diesen destruktiven Auswirkungen stehen auch Verfärbungen und Fleckenbildungen bei der Anwendung von Naturwerksteinen in engem Zusammenhang mit dem Feuchtewechselgehalt, also der Fähigkeit zum Feuchtetransport und zur Feuchtespeicherung (Abb. 3.82)

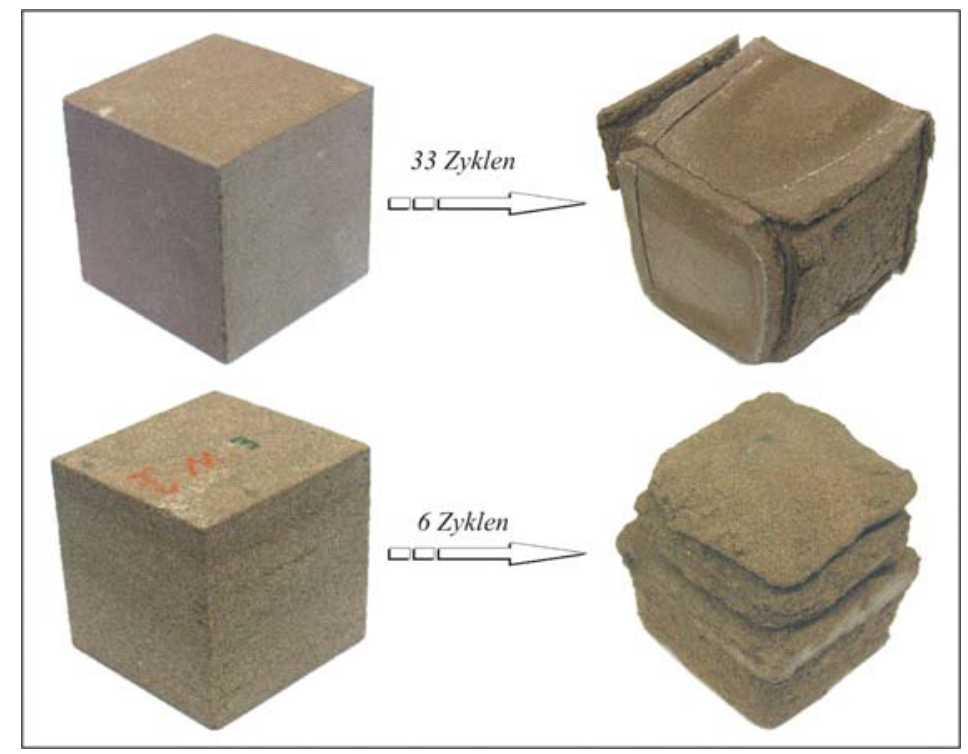

Abb. 3.81: Salzsprengversuch (nach VDI 3797) am ROTEN WESERSANDSTEIN Bad Karlshafen (oben) und Eddigehäuser Sandstein (unten). Deutliche Schadenscharakteristik in Form von Schalenbildungen mit Abplatzungen sowie gefügeabhängiger Reliefbildung mit jeweils erheblichem Materialverlust (verändert nach Kracke 2007).
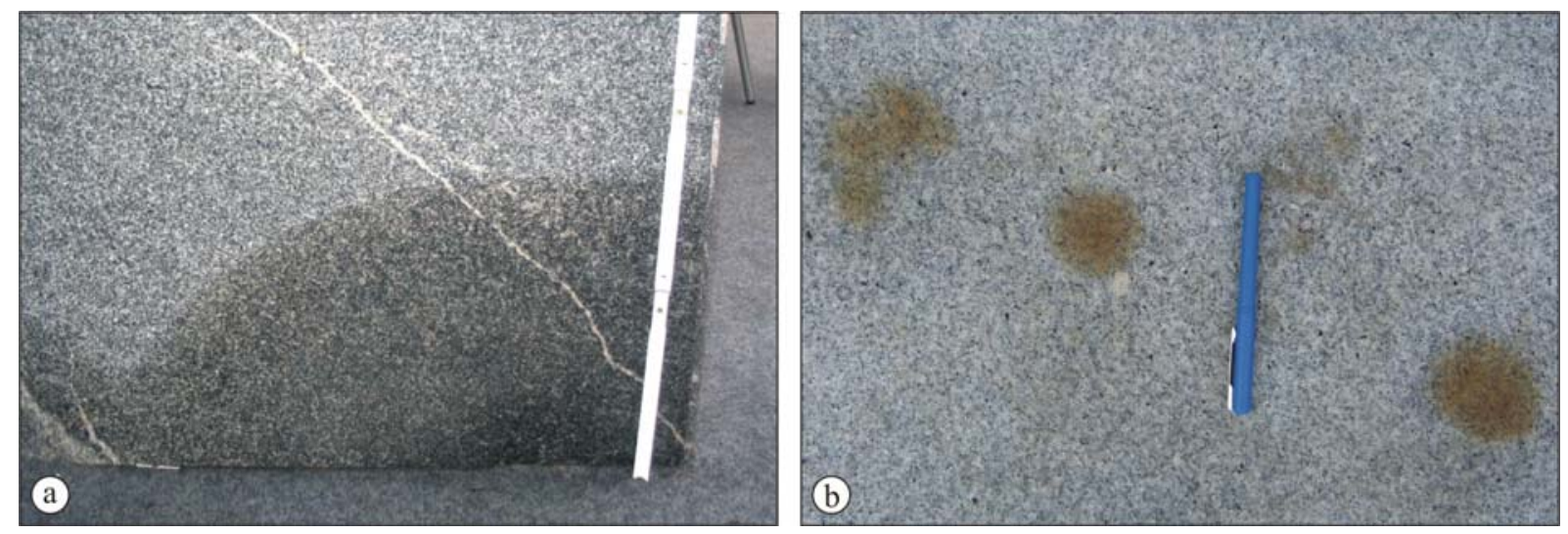

Abb. 3.82: a) Kapillar aufsteigende Feuchtigkeit (CEZLAK GRANODIORIT, Slowenien, Maxi-Market Ljubljana); b) Fleckenbildungen infolge von Ausrostungen (G 3503, Granit, China). 
Als primär relevanter Faktor hinsichtlich des Feuchtehaushalts eines Naturwerksteins gilt die kapillare Wasseraufnahmefähigkeit unter atmosphärischem Druck, deren Bestimmung in der DIN 52103 bzw. DIN EN 1097-06 geregelt ist. Die Berechnung erfolgt nach:

$$
\begin{array}{rll}
\text { WA } & =\frac{m_{f}-m_{t}}{m_{t}} \cdot 100 \\
\text { mit } \quad W A_{a t m} & =\text { Wasseraufnahme unter atm. Druck [Gew.-\%] } \\
m_{f} & =\text { Masse des Probekörpers nach freiwilliger Wasseraufnahme [g] } \\
m_{t} & =\text { Trockenmasse des Probekörpers [g] }
\end{array}
$$

Die Fähigkeit eines Gesteins zur freiwilligen Wasseraufnahme ist ursächlich an kapillare Saugkräfte gekoppelt und beruht dabei auf der physikalischen Erscheinung der Oberflächenspannung von Flüssigkeiten (Mirwald 1991). Die Intensität steht dabei in engem Zusammenhang mit der Charakteristik des Porenraums, also den Porengrößen und -formen, deren Verteilung sowie der Vernetzung untereinander. Die durchschnittliche Porengröße bedingt die verschiedenen Mechanismen des Wassertransportes, wobei die kapillare Wasseraufnahme überwiegend an Porenradien zwischen $1 \mu \mathrm{m}$ und $1 \mathrm{~mm}$, die so genannten Kapillarporen, gebunden ist (Klopfer 1985). Deutlich abzutrennen ist hierbei die „Totporosität“, also in sich abgeschlossene Porenräume, die somit keinen Beitrag zu den Prozessen des Feuchtetransports leisten. Auch verschiedene andere Elemente des Gesteinsgefüges (z.B. Schichtung, Foliation) üben einen Einfluss aus und führen so häufig zu einer Richtungsabhängigkeit der kapillaren Wasseraufnahme. So können z.B. parallel akkumulierte Tonminerale o.ä. eine sperrende Wirkung auf den Feuchtetransport haben.

\subsubsection{Gruppenspezifische Verteilungen und Beziehungen zu den Basiseigenschaften}

Für die Datenauswertung zur kapillaren Wasseraufnahme konnten rund 1650 Handelsvarietäten erfasst werden (Abb. 3.83 a, Tab. 3.15). Zwischen der kapillaren Wasseraufnahme und den Basiseigenschaften Rohdichte und effektive Porosität ist ein deutlicher Zusammenhang ausgebildet (Abb. 3.83). Wie zu vermuten, ist dieser vor allem in den Gruppen der stärker porösen Gesteine, also Sandsteine, Karbonatgesteine und auch Vulkanite, ausgebildet. Abgesehen von einigen Ausreißern, werden die weitaus höchsten Werte der freiwilligen Wasseraufnahme von Tuffen erreicht. Diese Tatsache steht mit der zumeist geringen bis sehr geringen Kompaktion der pyroklastischen Ablagerungen im Zusammenhang. Dadurch ergibt sich eine insgesamt hohe Porosität bei einer guten Vernetzung des Porenraumes. Die Gesamtheit der Datenpaare $\Phi / \mathrm{WA}_{\mathrm{atm}}$ der Vulkanite unterliegt jedoch einer erheblichen Streuung. Diese ist auf den zum Teil erheblich heterogenen Charakter des Porenraumes zurückzuführen (vgl. Kap. 3.6.1). Übergroße Poren leisten dabei keinen Beitrag zur kapillaren Wasseraufnahmefähigkeit. Auch im Bereich der niedrigporösen Gesteine kann ein ausgeprägter linearer Zusammenhang vermutet werden. Die Korrelation zwischen der Porosität und der kapillaren Wasseraufnahme ist für die Gruppen Plutonite und Metamorphite jeweils mit Koeffizienten über $r=0,85$ belegt, die Korrelation zur Rohdichte dagegen lediglich mit Koeffizienten um etwa $r=-0,15$. Für die Gruppe der Karbonatgesteine zeigt sich ein mit anderen physikalischen Eigenschaften vergleichbares 
Bild. Danach sind die Varietäten mit einer Rohdichte $\rho_{\text {roh }} \geq 2,6 \mathrm{~g} / \mathrm{cm}^{3}$ hinsichtlich der Wasseraufnahme mit den plutonischen und metamorphen Gesteinen vergleichbar. Mit abnehmender Rohdichte und zunehmender Porosität entsprechen die Werte beziehungsweise die Verteilung der Wertepaare der karbonatischen Gesteine eher denen der Sandsteinvarietäten.
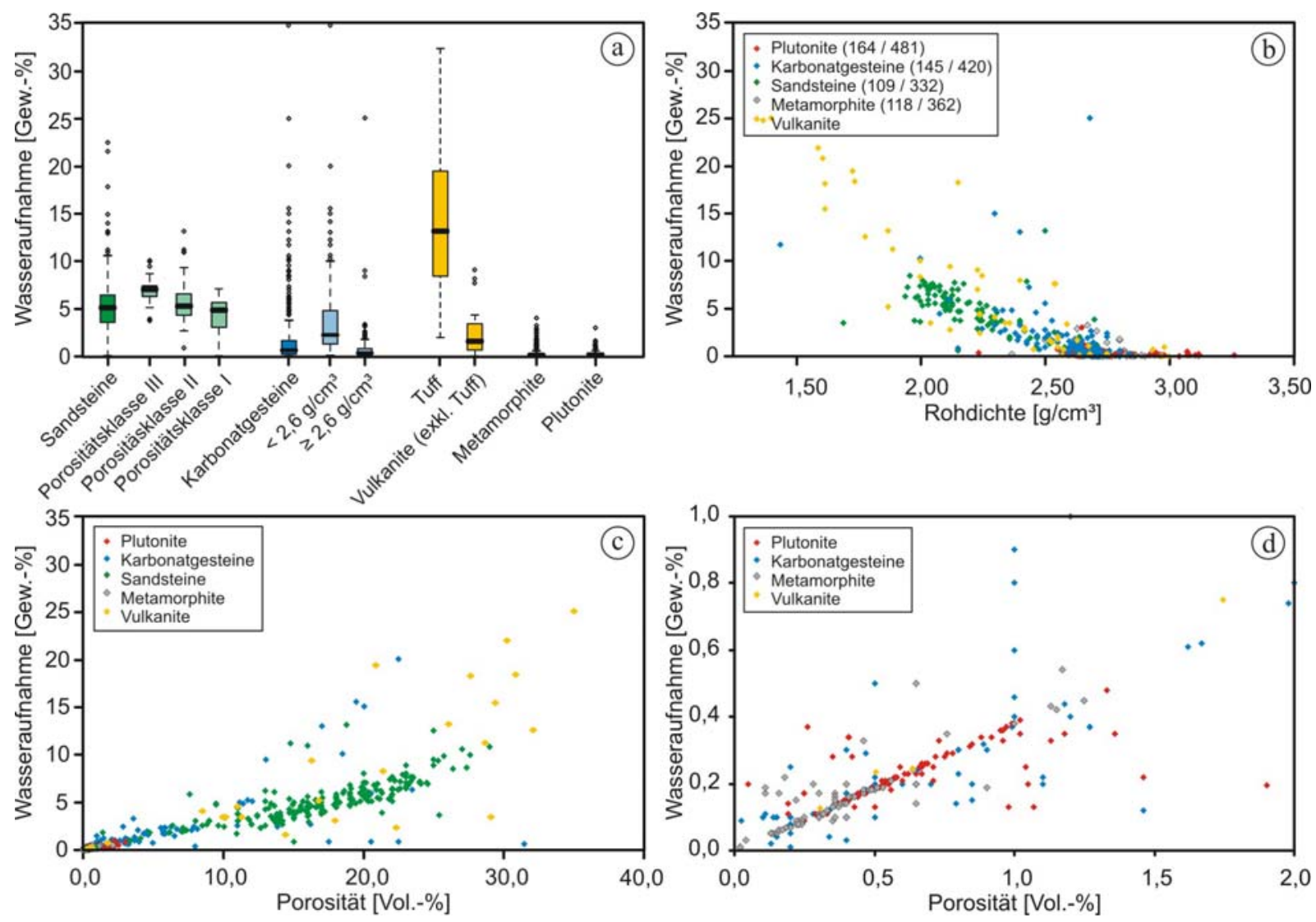

Abb. 3.83: a) Wertebereiche der kapillaren Wasseraufnahme; b - c) Punktverteilungen der Korrelationen der kapillaren Wasseraufnahme mit den Basiseigenschaften Rohdichte und Porosität; d) Detail aus c im Bereich der niedrigporösen Gesteine mit $\Phi<2$ Vol.-\%.

Die Intensität der kapillaren Wasseraufnahme steht folglich in engem Zusammenhang mit der Porosität. Anhand der explorativen Datenauswertung der Sandsteinvarietäten in den zuvor definierten Porositätsklassen (vgl. Kap. 3.7.1) wird deutlich, dass die Porosität jedoch nicht als alleiniger Faktor gelten kann. Zwar nimmt der Medianwert der Wasseraufnahme von der Klasse I $(\Phi<13,4$ Vol.-\%) zur Klasse III ( $\Phi \geq 20,5$ Vol.-\%) stetig zu, doch die zentralen Segmente zu den einzelnen Klassen überschneiden sich zum Teil deutlich (Abb. 3.83 a). Demnach kann die Porosität lediglich zu einer ersten Einschätzung der Fähigkeit eines Gesteins zur Wasseraufnahme herangezogen werden. Bei annähernd gleichen Gesamtporositäten wirken sich die spezifischen Porenradienverteilungen sowie die Porenformen aus, die sich, besonders bei den Sandsteinen, primär aus den Korn- bzw. Partikelgrößen und deren Sortierung ergeben (vgl. Kap. 3.7.1). Bei plutonischen und den meisten metamorphen Gesteinen kann (entsprechend Kap. 3.5 und Kap. 3.9) im Allgemeinen eine Rissporosität angenommen werden. Demnach sind für die kapillare Wasseraufnahme 
hier die Ausbildung der Korngrenzen mit den entsprechenden Riss- bzw. Öffnungsweiten von Bedeutung.

Tab. 3.15: Richtwerte für die kapillare Wasseraufnahme. Die Minimum- bzw. Maximumwerte entsprechen den statistischen Kennwerten oberer und unterer Extremwert aus der Datenauswertung mittels Boxplot. Ausreißer sind folglich nicht in die Übersicht mit eingegangen.

\begin{tabular}{lccc}
\hline Gruppe & \multicolumn{3}{c}{ kapillare Wasseraufnahme [Gew.-\%] } \\
& min & max & Median \\
\hline Plutonite & 0,01 & 0,60 & 0,23 \\
Metamorphite & 0,01 & 0,57 & 0,18 \\
$\quad$ Marmore & 0,01 & 0,38 & 0,15 \\
Gneise & 0,05 & 0,54 & 0,26 \\
Karbonatgesteine gesamt & 0,01 & 3,75 & 0,62 \\
$\quad$ Karbonatgesteine $\rho \geq 2,6 \mathrm{~g} / \mathrm{cm}^{3}$ & 0,01 & 1,75 & 0,30 \\
$\quad$ Karbonatgesteine $\rho<2,6 \mathrm{~g} / \mathrm{cm}^{3}$ & 0,05 & 10,00 & 2,21 \\
Sandsteine gesamt & 0,04 & 10,60 & 5,11 \\
$\quad$ Porositätsklasse I $(\Phi<13,4 \%)$ & 0,07 & 7,08 & 4,85 \\
$\quad$ Porositätsklasse II $(\Phi<20,5 \%)$ & 2,64 & 9,32 & 5,25 \\
$\quad$ Porositätsklasse III $(\Phi \geq 20,5 \%)$ & 5,12 & 8,65 & 7,04 \\
Vulkanite gesamt & 0,05 & 21,9 & 3,51 \\
Tuffe & 2,03 & 32,20 & 13,20 \\
Vulkanite exkl. Tuffe & 0,05 & 4,40 & 1,67 \\
\hline
\end{tabular}

Exemplarisch ist in Abbildung 3.84 anhand von 188 Datensätzen deutscher Sandsteine der Einfluss der zwei Faktoren Korngröße und -sortierung auf die kapillare Wasseraufnahmefähigkeit dargestellt. Zu beachten ist, dass andere Faktoren (Einfluss des Bindemittels, der Sphärizität, der Kornrundung etc.) nicht in die folgenden Betrachtungen mit einbezogen sind.

Untergliedert sind die Gesteine primär nach der mittleren Korngröße (fS: < 0,2 mm; mS: 0,2 - 0,63 mm) bzw. der Kornsortierung (/sehr gut, gut/; /gut-mäßig, mäßig/; /mäßig-schlecht, schlecht/) (Abb. 3.84 a). Trotz der Annahme, dass eine geringere mittlere Korngröße im Allgemeinen eine geringere Porosität nach sich zieht (vgl. Kap. 3.7.1), zeigt sich hier, dass der Medianwert der kapillaren Wasseraufnahme für die feinkörnigeren Varietäten (WA ${ }_{\text {atm }}=$ 6,00 Gew.-\%) gegenüber den gröberen Varietäten ( $\mathrm{WA}_{\mathrm{atm}}=$ 5,29 Gew.-\%) leicht höher liegt. Die Kornsortierung scheint hier keine direkte Auswirkung auf die Fähigkeit der Gesteine zur kapillaren Wasseraufnahme zu haben. Innerhalb der zwei Gruppen zur Korngröße lassen sich jedoch Trends nach der Sortierung erkennen (Abb. 3.84 b). Danach steigt die Fähigkeit zur kapillaren Wasseraufnahme bei den feinkörnigeren Sandsteinen (fS) mit zunehmender Sortierung an, während sie bei den gröberen Varietäten (mS) im gleichen Verlauf leicht abnimmt. 

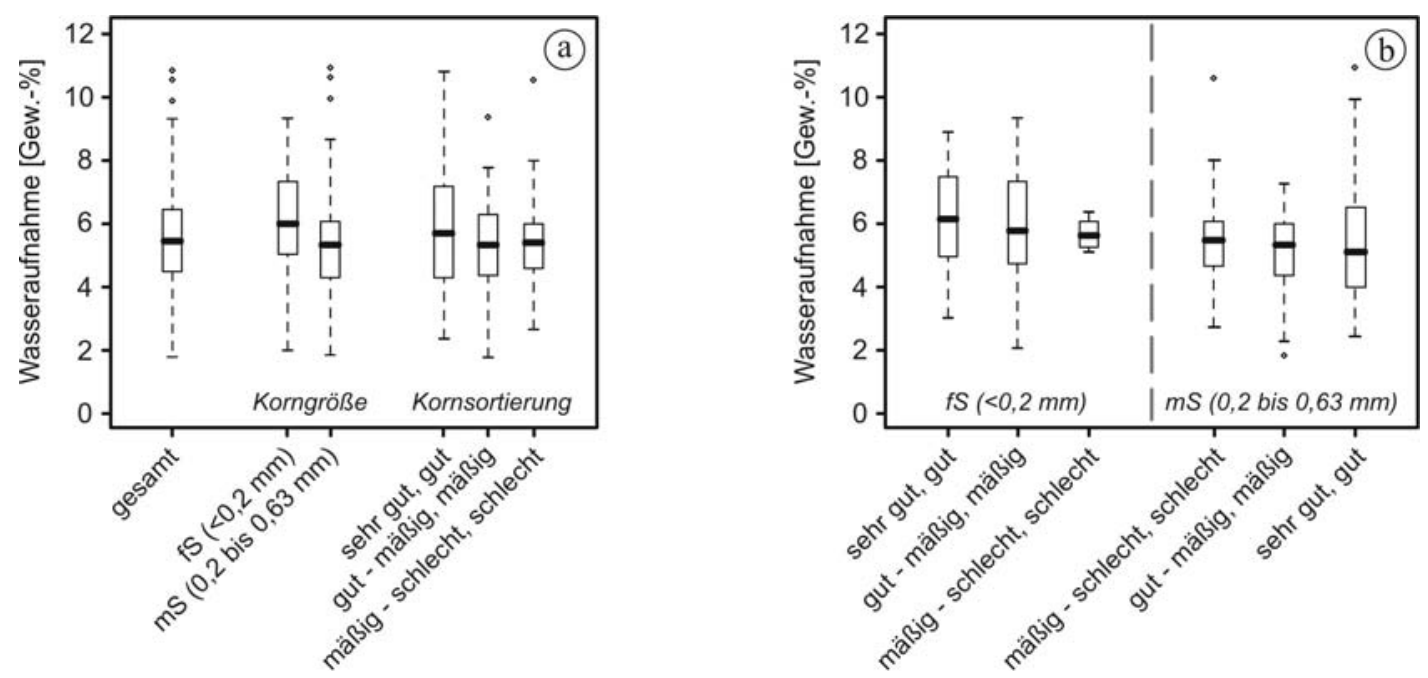

Abb. 3.84: Explorative Datenauswertung zur kapillaren Wasseraufnahmefähigkeit mit Bezug auf Korngröße und -sortierung an Daten zu 188 deutschen Sandsteinvarietäten (Daten aus Grimm 1990, Wenzel \& Häfner 2003, David 2006). Die geringe Spannweite in der Korngruppe „fS / mäßig-schlecht, schlecht“ (in b) ist lediglich auf die geringe Datenmenge von $n=4$ zurückzuführen.

Demnach ist für gut bis sehr gut sortierte, mittelkörnige Sandsteine der geringste und für sehr gut bis gut sortierte, feinkörnige Sandsteine der höchste Medianwert der kapillaren Wasseraufnahme zu erwarten. Vermutet werden kann daher, dass im Bereich der feinkörnigeren Varietäten die Porenräume durch eine Vermischung mit sehr feinen bis schluffigen Partikeln infolge einer schlechten Sortierung derart verkleinert werden, dass eine Kapillarität nur noch eingeschränkt gegeben ist. Ein gegenläufiger Effekt tritt demnach bei gröberen Sandsteinen bei einer schlechteren Sortierung auf. Hier bewirkt scheinbar die Ungleichheit der Korngrößen eine räumliche Verteilung der aufbauenden Körner, die eine gesteigerte Effektivität der kapillaren Wasseraufnahme infolge einer günstigeren Porengrößenverteilung nach sich zieht.

Die Trends, die sich anhand der explorativen Datenauswertung in Abbildung $3.84 \mathrm{~b}$ annehmen lassen, bestätigen sich in einer Aufschlüsselung hinsichtlich der Anisotropiewerte $\alpha$ (Abb. 3.85 a), die sich aus den minimalen und maximalen Korngrößen (KG) der jeweiligen Varietät errechnen $\left(\alpha=\left(K_{\max }-K_{\min }\right) / K_{\mathrm{max}}\right)$. Da die Werte der kapillaren Wasseraufnahme erhebliche Schwankungen zeigen, sind die jeweiligen Trends entsprechend schwach ausgebildet. Ob sich jedoch Tendenzen auch bei einer höheren Anzahl an Datensätzen bestätigen würden, erscheint fraglich. Denn eine Aneinanderreihung aller Daten geordnet nach der kapillaren Wasseraufnahmefähigkeit (3.85 b) beschreibt eine zunehmende Fähigkeit zur kapillaren Wasseraufnahme bei einer Annäherung von minimaler und maximaler Korngröße, gleichzusetzen mit einem Anisotropiewert gegen Null. Daran wird insgesamt die Komplexität der zugrunde liegenden Einflussparameter (Kornformen, Sphärizität etc.) deutlich. 

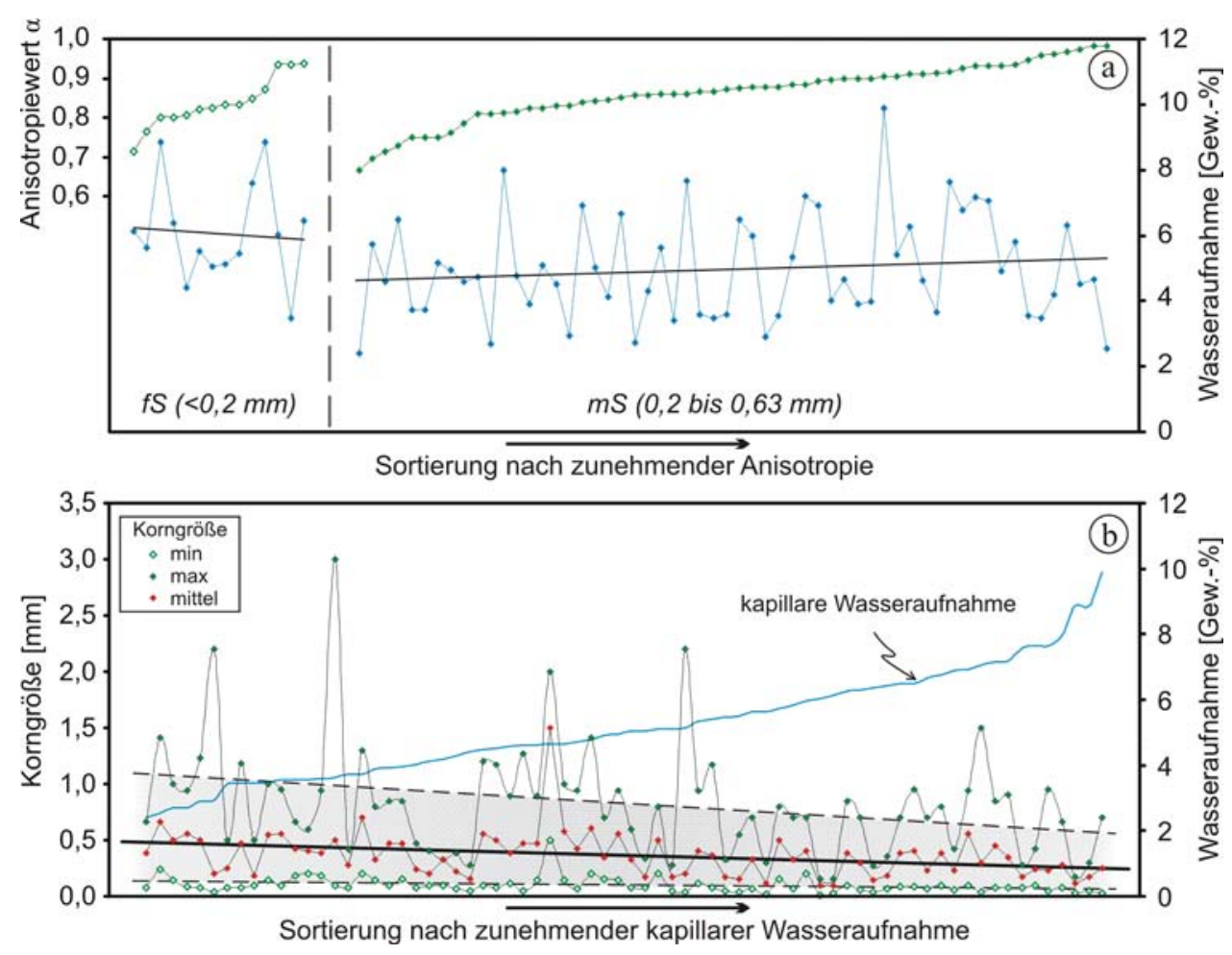

3.85: Die kapillare Wasseraufnahme von Sandsteinen in Bezug auf a) den Anisotropiewert der Korngrößenverteilung und b) die absoluten Werte für die jeweilige minimale, maximale und mittlere Korngröße (Daten aus Grimm 1990, David 2006). Nähere Erläuterungen im Text.

\subsubsection{Abschätzung bivariater Aufenthaltswahrscheinlichkeiten}

Es zeigt sich also, dass der Fähigkeit von Gesteinen zur kapillaren Wasseraufnahme ein komplexes System von Einzelfaktoren, die vielfach ineinander greifen, zugrunde liegt. Deutlich wird dies im Vergleich zwischen den Gruppen der klastischen und karbonatischen Sedimente. Während für Sandsteine über die Porosität die Möglichkeit einer recht sicheren Abschätzung der kapillaren Wasseraufnahme eines Gesteins gegeben ist, zeigt sich für Karbonatgesteine ein anderes Bild. Hinsichtlich der Gruppe der Vulkanite ist die Möglichkeit zur Visualisierung der Aufenthaltswahrscheinlichkeitsbereiche infolge der weiten Datenspannen stark eingegrenzt.

In Abbildung 3.86 sind die bivariaten Aufenthaltswahrscheinlichkeiten für den jeweiligen Zusammenhang zwischen den Basiseigenschaften Rohdichte und Porosität in Korrelation mit der kapillaren Wasseraufnahme dargestellt. Für den Vergleich der porösen Gesteinsgruppen, also Sandsteine und Karbonatgesteine, fällt deutlich auf, dass für die Gruppe der Karbonatgesteine mit zunehmender Porosität beziehungsweise abnehmender Rohdichte eine ansteigende Spannweite in den Verteilungen der entsprechenden Datenpaare zu beobachten ist. 

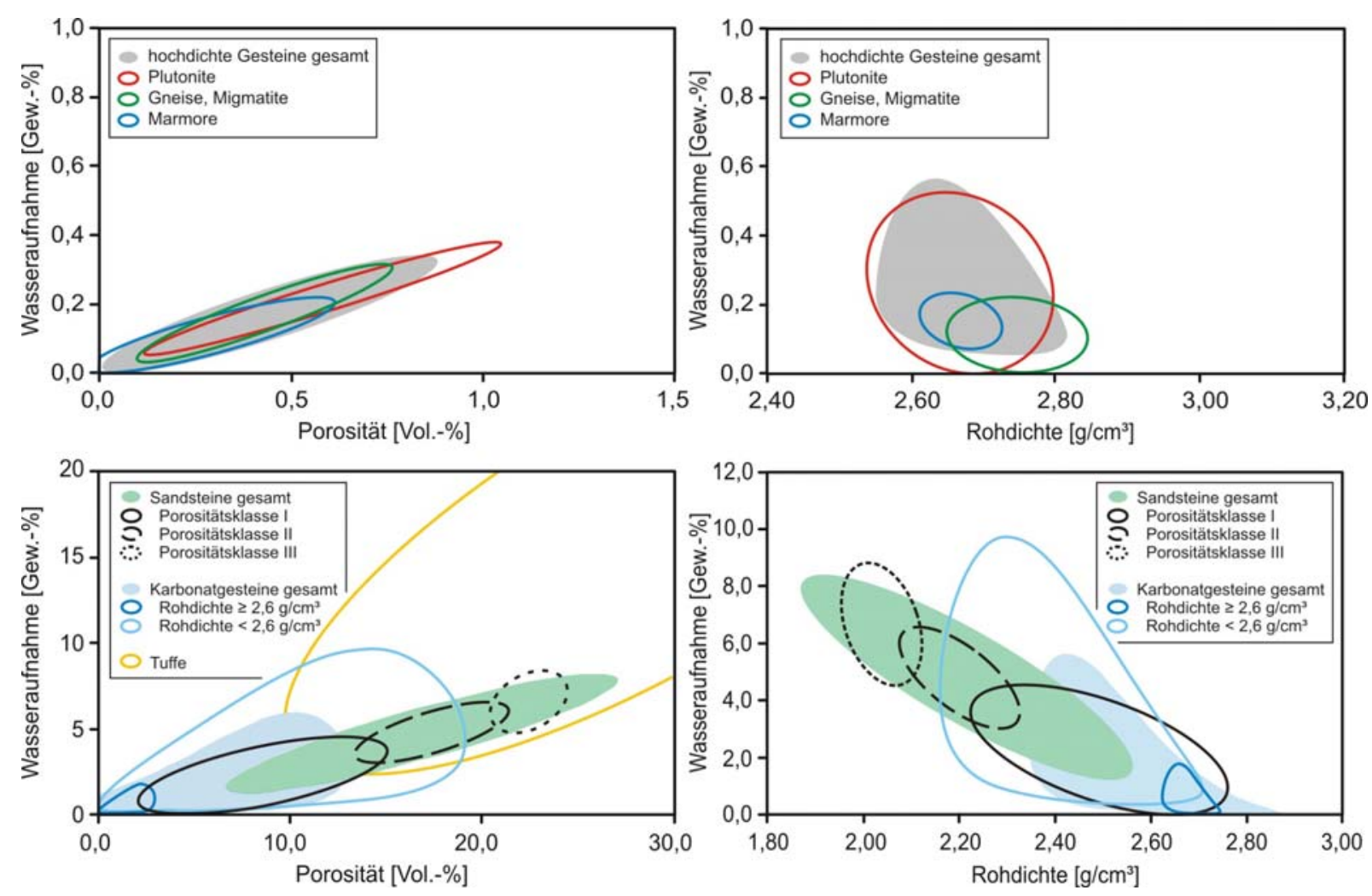

Abb. 3.86: Bereiche der 80 \%-igen Aufenthaltswahrscheinlichkeit für die Korrelation der kapillaren Wasseraufnahme mit der Porosität bzw. Rohdichte in den unterschiedenen Gesteinsgruppen.

Diese Verteilungen sind in der unterschiedlichen Porenraumcharakteristik begründet. Die beteiligten Komponenten bei der Bildung von klastischen und chemisch-biogenen Sedimenten unterscheiden sich zumeist erheblich voneinander.

Im Falle der klastischen Sedimente wiederholt sich eine „Grundform“, also Partikel mit vergleichbaren Formen und Größen. In der Folge bildet sich primär ein Gerüst aus diesen Partikeln, das je nach deren Grundformen sowie dem Energieeintrag bei der Sedimentation, einen zusammenhängenden Porenraum (Zwischenpartikel-Porosität) ergibt. Im Zuge der Diagenese kommt es durch die Kompaktion und Zementation des Sediments häufig zu einer Verringerung der Porosität, der Charakter bleibt aber zumeist erhalten. Zwar können je nach beteiligten Mineralphasen, so z.B. bei karbonatreichen Sandsteinen, auch Lösungsprozesse erfolgen, die jedoch eher von untergeordneter Bedeutung sind.

Auch bei Karbonatgesteinen, so z.B. Kalkoolithen, kann diese Zwischenpartikel-Porosität auftreten. Häufig ist die primäre Porenraumcharakteristik von Karbonatgesteinen jedoch deutlich heterogenener ausgebildet (z.B. Tucker 1985, Koch \& Sobott 2005). Infolge der am Aufbau des Karbonatgesteins beteiligten Organismen, der Formen, Größen und dem Zersetzungszustand der biogenen und nichtbiogenen Partikel, ergibt sich diese inhomogene Form- und Größenverteilung des Porensystems (z.B. Füchtbauer \& Richter 1988, vgl. Kap. 3.8.1, Abb. 3.39). Auch können in sich abgeschlossene Porenräume entstehen. So können Karbonatgesteine eine Ausgangsporosität von 70 bis 80 Vol.-\% aufweisen. Bei klastischen Sedimenten liegt dieser Wert je nach Ausbildung der Kornpackung deutlich niedriger. Außerdem kann es während der Bildung von Kalksteinen zu einer mehrfachen Verschiebung 
des Karbonatgleichgewichts kommen, was wiederholt sowohl Lösungs- als auch Fällungsprozesse hervorrufen kann (Koch \& Sobott 2005). Anders verhält es sich mit rein chemisch gefällten Kalksteinen, die eine Porosität gegen Null aufweisen können.

Als weiterer entscheidender Faktor steht die Relevanz der diagentisch-tektonischen sekundären Porosität bei Karbonatgesteinen im Gegensatz zu Sandsteinen. Entscheidend ist vor allem, dass das primäre Porensystem überlagert und verändert werden kann. So kann es beispielsweise zu einer Vernetzung einer primären Totporosität kommen, was sich positiv auf die Permeabilität eines Gesteins auswirken kann. Andererseits können isolierte Hohlräume durch die Lösung von individuellen Partikeln entstehen. So können bei Gesteinen mit vergleichbaren Werten der Gesamtporosität sowie der Rohdichte deutliche Unterschiede in der Porenradienverteilung auftreten. Sind in einem karbonatischen Gestein, das eine mikritische, dichte Matrix aufweist, lediglich größere sekundäre Lösungsporen vorhanden, kann die Fähigkeit zur kapillaren Wasseraufnahme sehr schwach ausgebildet sein, da der effektive Bereich der Porenradien überschritten ist. Bei gleicher Porosität kann aber auch eine hohe kapillare Wasseraufnahme auftreten, wenn die Porenräume gut vernetzt sind und entsprechend geringere Radien aufweisen.

Bei der Betrachtung der Aufenthaltsbereiche in den Korrelationen der freiwilligen Wasseraufnahme für hochkompakte Gesteine ( $\Phi<1,5$ Vol.-\%) zeigt sich besonders deutlich die unterschiedliche Ausprägung hinsichtlich der Basiseigenschaften Rohdichte und Porosität. Dabei unterliegt die Korrelation zur Porosität einem deutlichen, linear ausgebildeten Trend. Im Bereich der metamorphen Gesteine fällt auf, dass die Werte in der Gruppe der Marmore noch unter denen der Gneise bzw. Granite angesiedelt sind. Viele Marmore weisen im frischen, unverwitterten Zustand eine sehr geringe Primärporosität und auch eine entsprechend geringe Fähigkeit zur kapillaren Wasseraufnahme auf. Die physikalischen Eigenschaften unterliegen jedoch gerade häufig bei Marmoren verwitterungsbedingten Änderungen, die bei der Exposition am Bauwerk schon nach kurzer Dauer auftreten können. So kann bei Marmoren eine verstärkte Vernetzung von Kapillarporen gerade bei einer einsetzenden Verwitterung mit dem Effekt der Gefügeauflockerung eintreten. Dadurch kann die Fähigkeit zur freiwilligen Wasseraufnahme deutlich ansteigen. So konnte in Laboruntersuchungen für verschiedene Marmorvarietäten im leicht verwitterten Zustand eine vollständige Durchnässung der Probekörper festgestellt werden (Rüdrich 2003). In der Folge kann bei vielen Gesteinen bereits nach kurzer Zeit der Exposition eine erhöhte Wasseraufnahmefähigkeit resultieren. Destruktive Phänomene der Verwitterung (insbesondere das thermohygrische Dehnungsverhalten) können dadurch verstärkt werden. Entsprechende Schädigungen treten vor allem bei vielen Calcitmarmoren infolge des extrem anisotropen thermischen Verhaltens des Calcits auf (Erwärmung verursacht eine Dehnung parallel der c-Achse bei gleichzeitiger Kontraktion parallel der a-Achsen). Laborgestützte Verwitterungssimulationen an Granitoiden (Siegesmund et al. 2007) haben ergeben, dass vergleichbare Effekte für plutonische und möglicherweise auch andere, metamorphe Gesteine, wenn auch in geringerem Ausmaß, angenommen werden können. Hier sind besonders die Unterschiede der thermischen Eigenschaften der aufbauenden Minerale und deren Beziehungen untereinander von Bedeutung (Siegesmund et al. 2007). Deutlich wird der 
Einfluss einer zunehmenden Wasseraufnahme vor allem anhand der mangelnden Formbeständigkeit von Fassadenplatten aus Werkstein (vgl. Abb. 3.80). Anhand vieler Beispiele hat sich der Prozess der Verbiegung bei thermohygrischer Belastung als ein ständig fortschreitender erwiesen, der zu einem völligen Versagen des Gesteinsgefüges führen kann. Dabei hat sich gezeigt, dass diese Verformung mit einer Volumenzunahme des Gesteinskörpers im Zusammenhang steht. Diese ist wiederum durch eine Zunahme der Porosität beziehungsweise einer Verschiebung der Porenradienverteilung begründet (Abb. 3.87). Folglich kann die Effektivität der kapillaren Wasseraufnahme durch eine erhöhte Anzahl an Kapillarporen ansteigen und der Prozess wird weiter verstärkt.

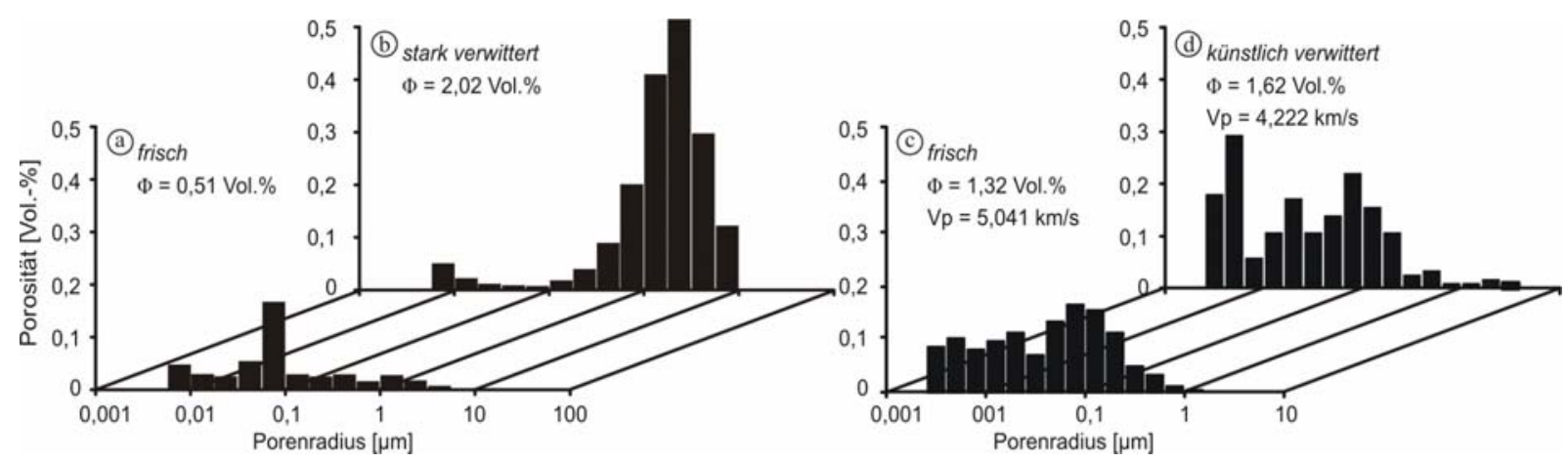

Abb. 3.87: Verteilung der Porenradien bei a) frischem und b) stark verwittertem Carrara Marmor (aus Rüdrich 2003) sowie c) frischem und d) künstlich verwittertem CEZLAK GRANODIORIT.

3.13.4 Sättigungsbeiwert - Zusammenhang zwischen freiwilliger und erzwungener Wasseraufnahme

Die kapillare Wasseraufnahme stellt eine relevante, wenn auch komplex zusammengesetzte Eigenschaft hinsichtlich des Verwitterungsverhaltens von Naturwerksteinen dar. Wie erwähnt, ist dieser Vorgang jedoch primär auf den Bereich der Kapillarporen beschränkt. Dem gegenüber steht demnach eine „absolute“ Sättigung. Dieser verlangsamte Vorgang wird im Allgemeinen mit einer allmählichen Füllung der Restporenräume infolge von Prozessen der Kapillarkondensation interpretiert (Mirwald 1991). Laborgestützt erreicht werden kann dieser Zustand annähernd über eine vorhergehende Evakuierung der Gesteinsprobe bis zum Erreichen eines Vakuums und einer anschließenden Tränkung. Durch diesen Vorgang entsteht ein erheblicher Saugeffekt. Doch haben Untersuchungen von Fitzner (1970) gezeigt, dass eine tatsächlich vollständige Sättigung mit annähernd konstanten Endwerten erst nach sehr langen Versuchszeiten von bestenfalls ein bis zwei Jahren erreicht wird.

Die Berechnung der erzwungenen Wasseraufnahme erfolgt nach:

$$
\begin{aligned}
W A_{v a k} & =\frac{m_{n}-m_{t}}{m_{t}} \cdot 100 \\
\text { mit } \quad W A_{v a k} & =\text { Wasseraufnahme unter Vakuum [Gew.-\%] } \\
m_{n} & =\text { Masse des Probekörpers nach erzwungener Wasseraufnahme [g] } \\
m_{t} & =\text { Trockenmasse des Probekörpers [g] }
\end{aligned}
$$


Zur Beurteilung der Frostbeständigkeit von Natursteinen ist der Quotient aus der freiwilligen, kapillaren Wasseraufnahme $\left(\mathrm{WA}_{\mathrm{atm}}\right)$ und der erzwungenen Wasseraufnahme ( $\mathrm{WA}_{\mathrm{vak}}$ ), der so genannte Sättigungsgrad oder Sättigungsbeiwert S (Hirschwald 1908) von Bedeutung. Er gibt an, welcher Anteil des Porenraums von kapillarem Wasser gefüllt wird. Ist $\mathrm{S}<0,75$, das heißt weniger als $75 \%$ des Porenraums kann über die kapillare Wasseraufnahme erreicht werden, gilt ein Gestein im Allgemeinen als frostunempfindlich (DIN 52106, Hirschwald 1908). Bei einem Sättigungsbeiwert von S > 0,75 ist die Eignung des Materials durch eine Prüfung nach dem Frost-Tauwechsel-Verfahren (DIN EN 1367-1) zu kontrollieren. Gesteine, die einen Sättigungsbeiwert von $\mathrm{S}>0,9$ aufweisen, sind hinsichtlich ihrer Frostbeständigkeit als besonders kritisch zu betrachten. Kommt es zu einer Eisbildung im Porenraum eines derart intensiv durchfeuchteten Gesteins, kann die Volumenexpansion infolge der Eiskristallisation durch den Restporenraum kaum kompensiert werden (Hörenbaum 2005). Die Folge sind Rissbildungen an Korngrenzen und damit eine möglicherweise erhebliche Schädigung des Gesteins durch die Reduzierung der mechanischen Stabilität.

Für plutonische und metamorphe Gesteine sowie auch Karbonatgesteine mit $\rho_{\text {roh }} \geq 2,6 \mathrm{~g} / \mathrm{cm}^{3}$ muss jedoch vermutet werden, dass die Aussagekraft des Sättigungsbeiwertes eingeschränkt ist. Bei diesen Gesteinen treten zum Teil sehr geringe Werte von $\mathrm{WA}_{\mathrm{atm}}$ und $\mathrm{WA}_{\mathrm{vak}}$ auf, wobei dann auch relative hohe Quotienten resultieren können. Dieser Umstand ist in vielen Fällen auf die Auflösungsgrenze des Messvorgangs zurück zu führen. So ergibt sich z.B. auch für den LICHTENBERGER DIORIT, einem mittelkörnigen Bt-Hbl-Diorit, ein Sättigungsbeiwert von $S=0,94$ (Grimm 1990). Eine Frostbeständigkeit des Gesteins sollte jedoch uneingeschränkt gegeben sein.

Im Bereich der Sandsteine sowie auch der porösen Kalksteine zeigt sich die höhere Variabilität der freiwilligen im Vergleich mit der erzwungenen Wasseraufnahme bei gleicher Porosität (Abb. 3.88). Es wird deutlich, dass die Effektivität der freiwilligen Wasseraufnahme auf bestimmte Porenradien bezogen ist. Hier zeigt sich auch deutlich die angesprochene Porengrößenvariabilität verschiedener Basaltlaven (vgl. Kap. 3.6.1). Bei Porositäten von 22,31/29,06 Vol.-\% sind zur freiwilligen Wasseraufnahme Werte von 2,36/3,50 Gew.-\% bei Sättigungsbeiwerten von 0,24/0,25 dokumentiert (Grimm 1990). Hinsichtlich eines Zusammenhanges zwischen dem Sättigungsgrad S und der Porosität lässt sich zwar ein relatives Ansteigen von S im Porositätsbereich von etwa 10 bis 30 Vol.-\% vermuten. Ein genereller Trend konnte aus dem vorliegenden Datensatz jedoch nicht ermittelt werden. 


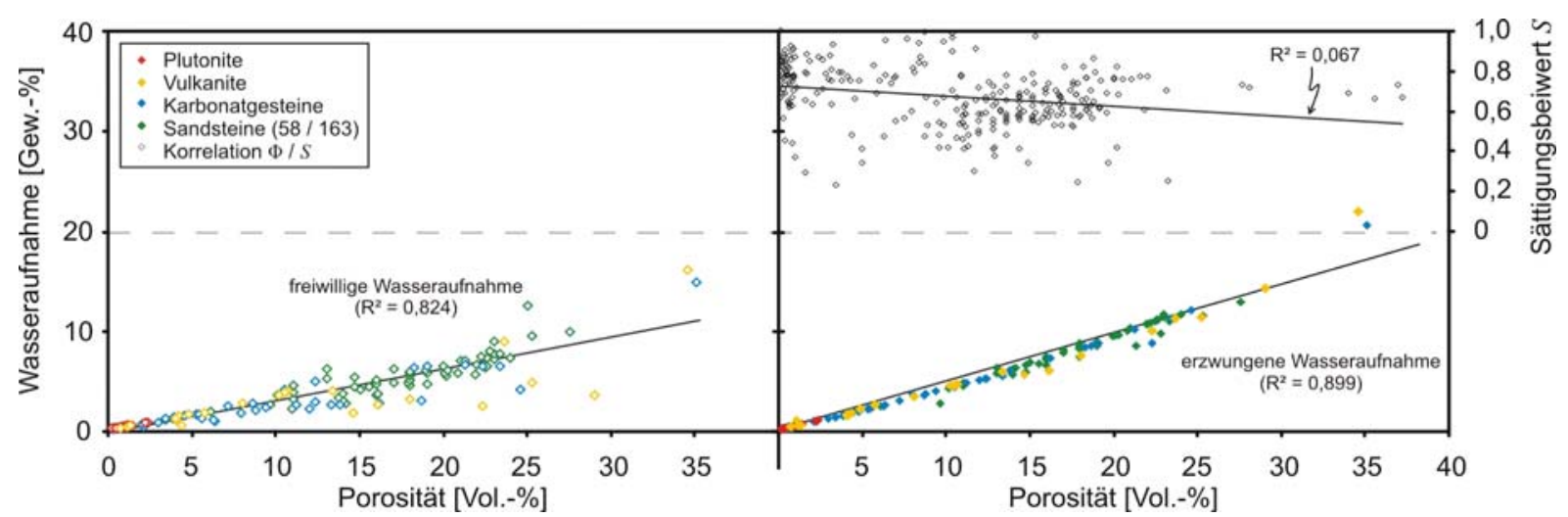

Abb. 3.88: Gruppenspezifische Punktverteilungen der Korrelation zwischen Porosität und freiwilliger (links) sowie erzwungener (rechts) Wasseraufnahme sowie dem Sättigungsbeiwert S. Angegeben ist die jeweils geschätzte Anpassung der Regressionsgerade mit dem Bestimmtheitsmaß R².

\subsubsection{Ansätze zur Prognose der freiwilligen Wasseraufnahme}

Die Berechnung der freiwilligen und der erzwungenen Wasseraufnahme kann an sich im Rahmen der Ermittlung von Porosität, Roh- und Matrixdichte mittels des Auftriebverfahrens mit eingeschlossen werden. Jedoch stehen für eine erste Einschätzung eines Gesteins hinsichtlich der Verwendungsmöglichkeit als Naturwerkstein häufig nur kleinere Proben oder polierte Musterplatten zur Verfügung. In diesem Falle kann so z.B. eine Porositätsmessung über die Methode der Quecksilberporosimetrie (Brakel et al. 1981) erfolgen, wofür nur sehr kleine Proben benötigt werden. Neben der Porenradienverteilung wird dabei auch eine Gesamtporosität gegeben. Zu beachten ist jedoch, dass sich hierbei häufig eine geringere Porosität als bei der Auftriebsmessung ergibt. Mit der Quecksilberporosimetrie kann lediglich der Bereich bis zu einem kreisäquivalenten Porenradius von etwa $50 \mu \mathrm{m}$ erfasst werden. Besonders bei Gesteinen, die eine extreme Heterogenität in der Porengrößenverteilung aufweisen oder aber insgesamt sehr grobporig sind, kann die sich ergebende Differenz zur tatsächlichen effektiven Porosität beträchtlich sein. Folglich wären die daraus abgeleiteten Werte der Wasseraufnahme als zu gering anzusehen, doch eine generelle Tendenz kann im Vergleich mit der Porenradienverteilung durchaus abgeleitet werden.

Hinsichtlich der Abschätzung von Möglichkeiten zur Prognose der freiwilligen Wasseraufnahme soll hier die Porosität als primäre Basiseigenschaft genutzt werden. Im Gegensatz zur Rohdichte lassen sich auch für die plutonischen und metamorphen Gesteine Trends vermuten. In den vorherigen Betrachtungen haben sich grundsätzlich zwei Typen herauskristallisiert. Dabei folgen sowohl die zusammengefassten Gruppen der hochdichten Gesteine Plutonite und Metamorphite (exklusive der Karbonatgesteine mit $\rho_{\text {roh }} \geq 2,6 \mathrm{~g} / \mathrm{cm}^{3}$ !) als auch die Gruppe der klastischen Sedimente eher einem linearen Trend in der Verbindung mit der Porosität als Prädiktorvariablen (Abb. 90 a, b). Hinsichtlich der Gruppe der Sandsteine erscheint hier eine Teilung in die zuvor definierten Porositätsklassen weniger sinnvoll. Zwar lässt die separate Betrachtung der drei Klassen eine Verknüpfung nichtlinearen Charakters vermuten, doch bestätigt sich dies nicht (Abb. 3.89). Erstaunlich ist dabei auch, dass die Determinationskoeffizienten innerhalb der drei Klassen lediglich Werte zwischen 
etwa 0,3 und 0,45 annehmen, die Gesamtgerade dagegen aber einen deutlichen linearen Zusammenhang annehmen lässt.

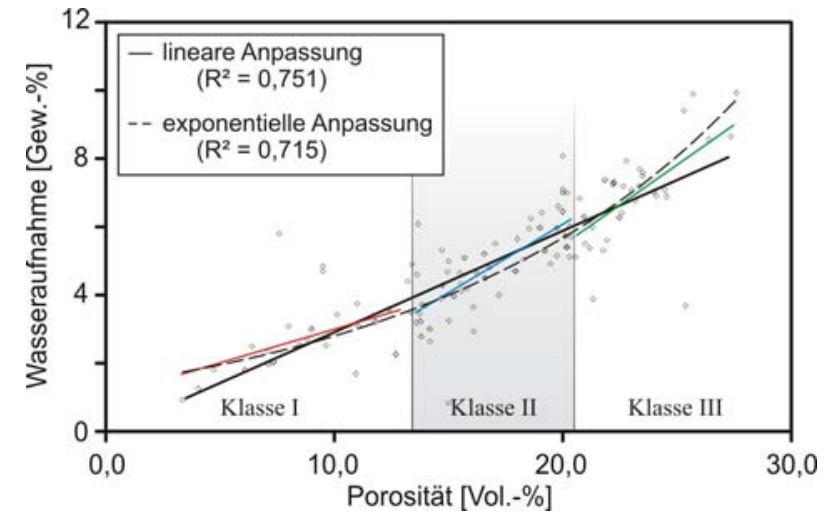

Abb. 3.89: Abhängigkeit der freiwilligen Wasseraufnahme von der Porosität der Sandsteine insgesamt sowie innerhalb der definierten Porositätsklassen.

Für die vermuteten linearen Zusammenhänge zwischen der Porosität $\Phi$ (in Vol.-\%) und der freiwilligen, kapillaren Wasseraufnahme $W_{a t m}$ (in Gew.-\%) ergeben sich in der Regressionsrechnung folgende Geradengleichungen mit Standardabweichung:

zu Abbildung 3.90 a) hochkompakte Gesteine mit $\Phi<1,5$ Vol.-\% (Plutonite, Metamorphite):

$$
\mathbf{W A}_{\mathrm{atm}}=\mathbf{0 , 3 4} * \mathbf{\Phi}+\mathbf{0 , 0 2}+/-\mathbf{0 , 0 9}\left(\mathrm{R}^{2}=0,846\right)
$$

zu Abbildung 3.90 b) Sandsteine (ohne Unterscheidung der Porositätsklasse):

$$
\mathrm{WA}_{\mathrm{atm}}=\mathbf{0 , 3 0} * \mathbf{\Phi}-\mathbf{0 , 1 3}{ }^{+} /-\mathbf{1 , 9 1}\left(\mathrm{R}^{2}=0,751\right)
$$
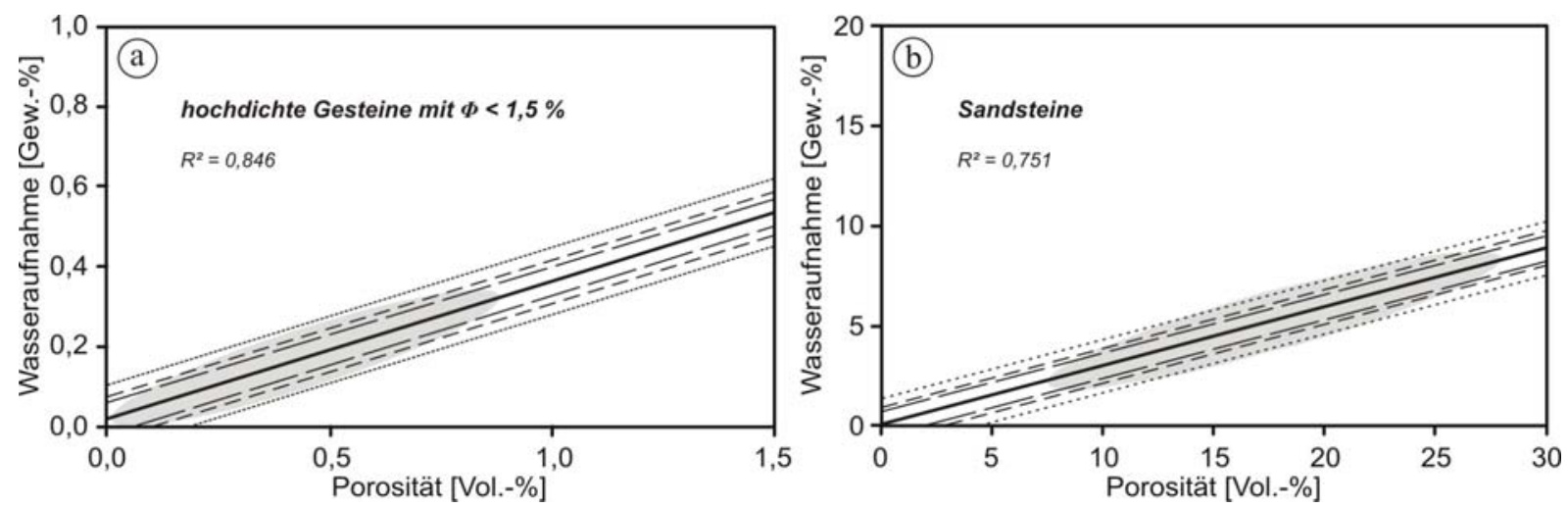

Abb. 3.90: Abhängigkeit der freiwilligen Wasseraufnahme $\mathrm{WA}_{\mathrm{atm}}$ von der Porosität im Bereich der a) hochdichten Gesteine (Plutonite, Metamorphite) und b) der Sandsteine. Dargestellt ist die jeweilige Regressionsgerade mit den Bereichen, die $65 \%$ (lang gestrichelt), $80 \%$ (kurz gestrichelt) und $95 \%$ (gepunktet) der Streuung um die Gerade herum enthalten. Zusätzlich eingetragen ist jeweils der abgeschätzte 80 \%-ige Aufenthaltswahrscheinlichkeitsbereich für die jeweilige theoretische Grundgesamtheit der Datenpaare.

Dagegen ergibt sich für die Gruppe der Karbonatgesteine hinsichtlich einer Möglichkeit zur Prognose wiederum das Problem des deutlich ansteigenden Fehlers mit zunehmender Porosität. Für die hochkompakten Karbonatgesteine mit $\rho_{\text {roh }} \geq 2,6 \mathrm{~g} / \mathrm{cm}^{3}$ zeigt sich jedoch ebenfalls ein guter linearer Zusammenhang. Dieser ist mit einem Determinationskoeffizienten von $\mathrm{R}^{2}=0,879$ beschrieben (Abb. 3.91 a). 
Hinsichtlich der Karbonatgesteine mit $\rho_{\text {roh }}<2,6 \mathrm{~g} / \mathrm{cm}^{3}$ ist ein vergleichbares Verhalten lediglich bis zu einer Porosität von etwa 8 Vol.-\% anzunehmen. Eine höhere Porosität resultiert in einer erheblichen Streuung der Daten. Demnach wäre eine lineare Anpassung im Falle der hochporösen Gesteine mit einem zu hohen Fehler behaftet. Stattdessen soll hier die Anpassung an ein nichtlineares Modell erfolgen (Abb. 3.91 b). Insgesamt erscheint die Möglichkeit der Prognose in dieser Untergruppe jedoch stark eingeschränkt, da sowohl für ein lineares als auch ein nichtlineares Modell relativ geringe Determinationskoeffizienten gegeben sind.

Für die vermuteten linearen und nichtlinearen Zusammenhänge zwischen der Porosität $\Phi$ (in Vol.-\%) und der freiwilligen, kapillaren Wasseraufnahme $W_{a t m}$ in (Gew.-\%) ergeben sich in der Regressionsrechnung folgende Gleichungen mit Standardabweichungen:

zu Abbildung 3.91 a) Karbonatgesteine mit $\rho_{\text {roh }} \geq 2,6 \mathrm{~g} / \mathrm{cm}^{3}$ :

$$
\mathrm{WA}_{\mathrm{atm}}=\mathbf{0 , 3 3} * \boldsymbol{\Phi}+\mathbf{0 , 0 3}{ }^{+} /-\mathbf{0 , 4 5}\left(\mathrm{R}^{2}=0,872\right)
$$

zu Abbildung 3.91 b) Karbonatgesteine mit $\rho_{\text {roh }}<2,6 \mathrm{~g} / \mathrm{cm}^{3}$ :

$$
\mathrm{WA}_{\mathrm{atm}}=1,31 * \mathbf{e}^{\mathbf{0 , 0 6} * \Phi} \quad\left(\mathrm{R}^{2}=0,267\right)
$$
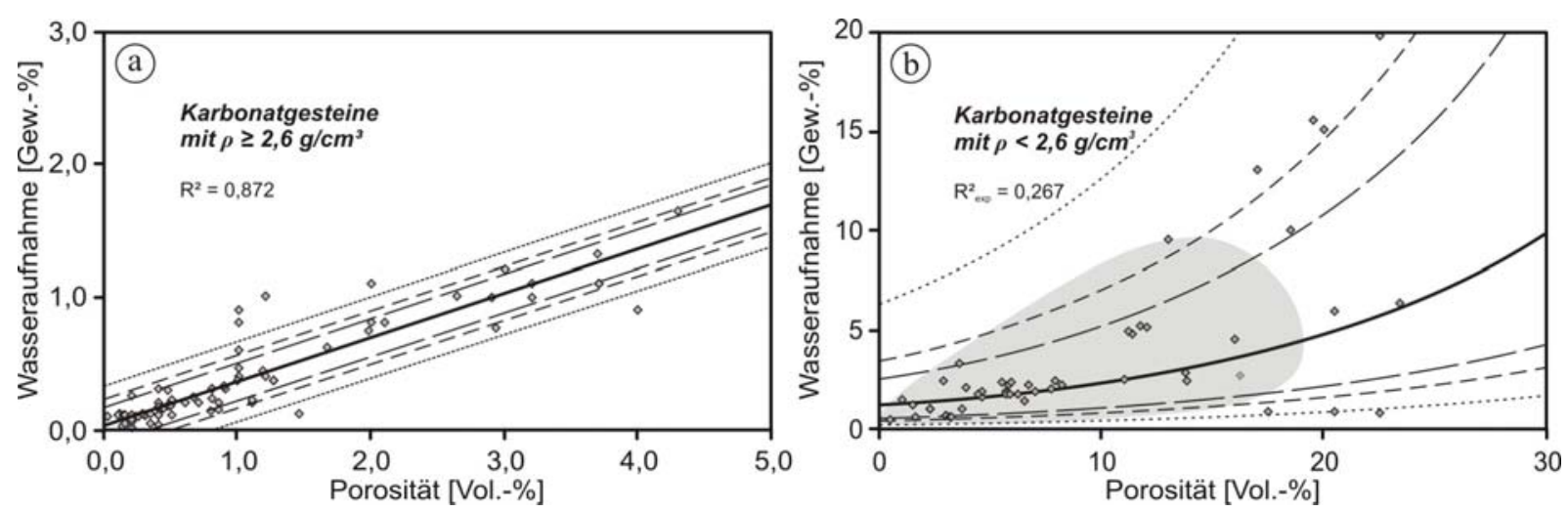

Abb. 3.91: Abhängigkeit der freiwilligen Wasseraufnahme $W_{\mathrm{atm}}$ von der Porosität $\Phi$ im Bereich der Karbonatgesteine mit a) $\rho_{\text {roh }} \geq 2,6 \mathrm{~g} / \mathrm{cm}^{3}$ und b) $\rho_{\text {roh }}<2,6 \mathrm{~g} / \mathrm{cm}^{3}$. Dargestellt ist die jeweilige Regressionsgerade bzw. -linie mit den Bereichen, die 65 \% (lang gestrichelt), 80 \% (kurz gestrichelt) und $95 \%$ (gepunktet) der Streuung um die Gerade bzw. Linie herum enthalten. Zusätzlich eingetragen ist in b) der abgeschätzte 80 \%-ige Aufenthaltswahrscheinlichkeitsbereich für die theoretische Grundgesamtheit der Datenpaare.

Auch hinsichtlich der Prognoseabschätzung der kapillaren Wasseraufnahme, stellt sich die Frage der Funktionalität der Ultraschalldiagnostik. Aus Kapitel 3.12 ist die gute Verbindung der Kompressionswellengeschwindigkeit mit der Porosität bekannt. Diese zeigt sowohl bei porösen Gesteinen (Sandsteine, Karbonatgesteine $<2,6 \mathrm{~g} / \mathrm{cm}^{3}$ ) als auch bei hochkompakten Gesteinsvarietäten einen linearen Charakter. Daraus lässt sich auch eine direkte Verbindung mit der Eigenschaft der kapillaren Wasseraufnahme ableiten.

Sowohl für die Gruppe der Plutonite als auch die der Sandsteine lässt sich eine lineare Abhängigkeit der kapillaren Wasseraufnahme vermuten. Im Falle der Plutonite fällt die Erklärungskraft der angepassten Funktion sehr gering aus (Abb. 3.92). Diese Tatsache steht damit in Verbindung, dass die Spannweite der Daten zur Wasseraufnahme der plutonischen Gesteine im relativen Vergleich mit der Datenbasis der Sandsteine deutlich geringer ist. Die 
Vp-Werte überspannen dagegen in beiden Fällen in etwa einen Bereich von 1,6 km/s. Die eingeschränkte Erklärungskraft des linearen Modells spiegelt sich zudem auch in der weiteren Öffnung der Konfidenzintervalle wider. Insgesamt erscheint die Anwendung der Ultraschallanalytik zur Vorhersage einer möglichen freiwilligen Wasseraufnahme jedoch als praktikabel. Die sich ergebenden Standardabweichungen fallen dabei sogar etwas geringer aus, als bei der Verwendung der Porosität als Ausgangsvariable.

Für die vermuteten linearen Zusammenhänge zwischen der Kompressionswellengeschwindigkeit $\mathrm{Vp}$ (in $\mathrm{km} / \mathrm{s}$ ) und der freiwilligen, kapillaren Wasseraufnahme $W_{a t m}$ (in Gew.-\%) ergeben sich in der Regressionsrechnung folgende Geradengleichungen mit Standardabweichungen:

zu Abbildung 3.92 a) Plutonite:

$$
\mathrm{WA}_{\mathrm{atm}}=-\mathbf{0 , 0 5} * \mathrm{Vp}+\mathbf{0 , 5 4}+-\mathbf{0 , 0 6}\left(\mathrm{R}^{2}=0,153\right)
$$

zu Abbildung 3.92 b) Sandsteine:

$$
\mathrm{WA}_{\mathrm{atm}}=-2,61 * \mathrm{Vp}+12,52{ }^{+} /-\mathbf{0 , 8 5}\left(\mathrm{R}^{2}=0,547\right)
$$
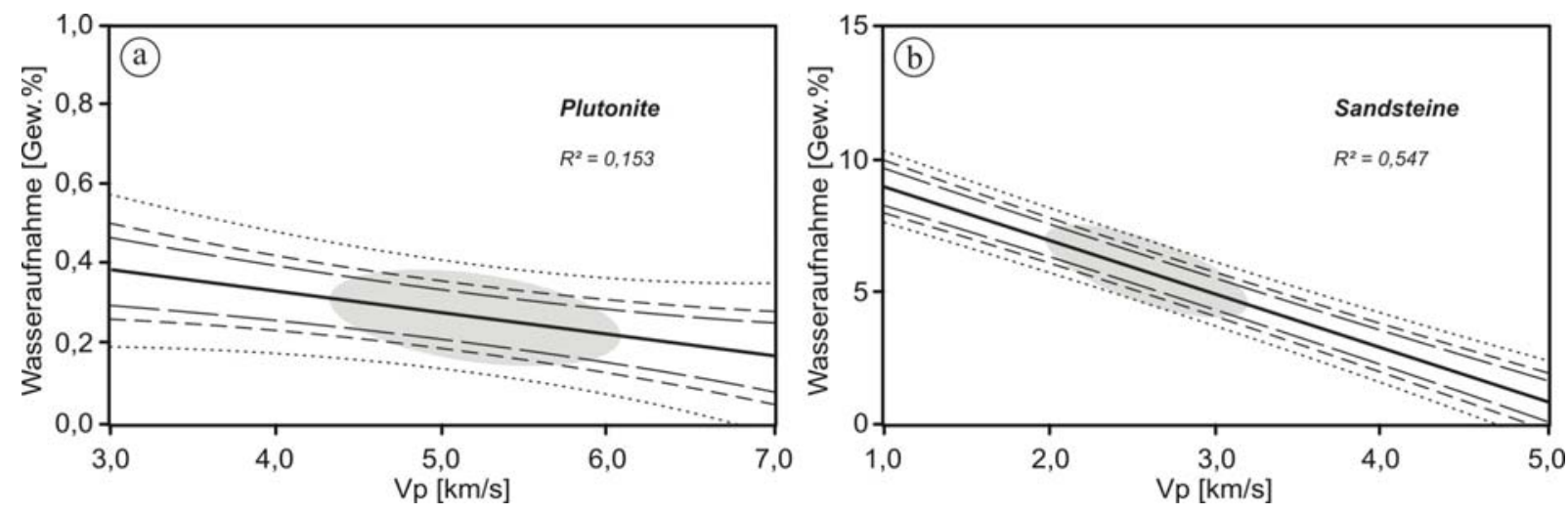

Abb. 3.92: Abhängigkeit der freiwilligen Wasseraufnahme $\mathrm{WA}_{\mathrm{atm}}$ von der Kompressionswellengeschwindigkeit Vp in der Gruppe der a) Plutonite und b) Sandsteine. Dargestellt ist die jeweilige Regressionsgerade mit den Bereichen, die 65 \% (lang gestrichelt), 80 \% (kurz gestrichelt) und 95 \% (gepunktet) der Streuung um die Gerade herum enthalten. Zusätzlich eingetragen ist der abgeschätzte 80 \%-ige Aufenthaltswahrscheinlichkeitsbereich für die theoretische Grundgesamtheit der Datenpaare.

Auch für die Gesamtheit der Karbonatgesteine konnte für die Porosität eine Abhängigkeit linearen Charakters von der Kompressionswellengeschwindigkeit Vp aufgezeigt werden (vgl. Kap. 3.12.4). Die geringe Datenmenge zur Verbindung $\mathrm{Vp} / \mathrm{WA}_{\mathrm{atm}}$ erlaubt jedoch keine Anpassung eines linearen oder nichtlinearen Modells. Es ist jedoch zu erwarten, dass eine ähnliche Verteilung der Streuung um eine angepasste Regressionslinie (steigender Fehler mit Zunahme der Kompressionswellengeschwindigkeit) infolge der Heterogenität der Porosität resultiert. 


\subsection{Zusammenfassende Betrachtung zur statistischen Untersuchung der Gesteinseigenschaften}

Bisher basiert die Entscheidungsfindung bei der Frage nach einem zu verwendenden Werkstein für ein Bauprojekt in vielen Fällen lediglich auf den optischen beziehungsweise ästhetischen Eigenschaften eines Gesteins. Die statistischen Auswertungen haben gezeigt, dass erhebliche Unterschiede der verschiedenen Gesteinseigenschaften bestehen, die in erheblichem Maße die bautechnische Eignung des jeweiligen Materials beeinflussen und diktieren. Mit Hilfe der statistischen Untersuchungen konnten für die unterschiedlichen Gesteinsgruppen lithologietypische Bereiche definiert werden. Dies gilt für Datenreihen zu einer Eigenschaft als auch für Datenpaare mit dem Ausdruck der Bereiche der 80 \%-igen Aufenthaltswahrscheinlichkeit einer Korrelation. Damit konnte gleichzeitig eine Werkzeug zur vergleichenden Bewertung physikalischer und technischer Daten eines Gesteins oder ganzer Datensätze gegeben werden. Außerdem konnten auf der breiten Datenbasis Möglichkeiten hergeleitet werden, Eigenschaften, deren experimentelle Bestimmung zeit- und kostenintensiv ist, aus einfach zu bestimmenden Gesteinseigenschaften (Rohdichte, effektive Porosität, Ultraschallanalytik) abschätzend zu prognostizieren.

In Verbindung mit der Trennflächenanalytik, die die Grundlage einer generellen Eignungsabschätzung eines Lagerstättenkörpers und auch Basis einer optimierten Gewinnung des Rohstoffs darstellt, ist mit der detaillierten Gesteinscharakterisierung die Grundlage zur Beschreibung der bautechnischen Verwendungsmöglichkeit eines Gesteins gegeben. Dabei muss es das Ziel sein, die Langzeitstabilität des Baustoffs Naturwerkstein optimal zu nutzen.

In den folgenden Kapiteln sollen die Erkenntnisse aus den methodischen Grundlagen in angewandten Beispielen zur überregionalen (Kap. 4, Argentinien) und regionalen (Kap. 5, Tansania) Erfassung, Erkundung und Bewertung von Werksteinlagerstätten umgesetzt werden. 


\section{4. ÜBERREGIONALE ERFASSUNG VON NATURWERKSTEINEN: FALLBEISPIEL ARGENTINIEN}

\subsection{Ansatz zur Werksteinerfassung Argentiniens}

Infolge der komplexen geologischen Entwicklung weist der gesamte südamerikanische Kontinent ein weites Spektrum an Gesteinen auf, die ein enormes Werksteinpotenzial darstellen. In Argentinien hat die Verwendung von Naturstein als natürlicher Baustoff eine lange Tradition. Insgesamt liegen jedoch über argentinische Werksteinvarietäten wenige Informationen vor. Ausnahmen bilden hierbei die wenigen Gesteine, die am Weltmarkt vertreten und etabliert sind. Ursächlich dafür ist zumeist, dass die entsprechenden Betriebe in internationale Firmenkonsortien involviert sind. Ein Beispiel dafür ist der Marmor AZUL CIELO, der seit mehreren Jahrzehnten weltweit als nahezu einziger blauer Marmor vertrieben wird. Dies geschieht dann zumeist über Generalunternehmen in Europa, namentlich Italien. Einige andere argentinische Gesteine gehen zwar ebenfalls in den Export, vornehmlich jedoch in die USA oder Asien.

Detaillierte Untersuchungen zur lagerstättengeologischen Situation eines Vorkommens liegen nur in sehr vereinzelten Fällen vor. Zumeist erfolgt der Abbau ohne fundierte Kenntnisse z.B. des Trennflächengefüges oder anderer, den Abbau kontrollierender Elemente. Ebenso liegen physikalische und technische Kenndaten zu den einzelnen Lithologien häufig nicht oder nur in eingeschränktem Maße vor. Auch sind gegebene Eigenschaften in manchen Prospekten unzureichend ausgewiesen oder beruhen auf schlecht zu identifizierenden Testverfahren.

An diesem Punkt setzt die regionale Erfassung des Werksteinpotenzials Argentiniens an. Zur Einschätzung der wirtschaftlichen Bedeutung der Vorkommen sind grundlegende geound materialwissenschaftliche Kenntnisse vonnöten. Auf entsprechenden Ausarbeitungen können dann gezielt Detailerkundungen einzelner Lagerstätten erfolgen, um zu einer wirtschaftlich sinnvollen Nutzung der Ressourcen zu gelangen.

Im Rahmen dieser Studie wurden magmatische, metamorphe und sedimentäre Vorkommen in acht argentinischen Provinzen befahren, um die jeweiligen lagerstättenrelevanten Aspekte zu erfassen (vgl. Mosch et al. 2007). Der Fokus wurde dabei auf lithologische und strukturelle Untersuchungen zur geowissenschaftlich basierten Charakterisierung der Lagerstätten beziehungsweise der jeweiligen Werksteinvarietäten einschließlich der repräsentativen Erfassung der Dekore gesetzt. Zusätzlich fanden auch wirtschaftliche Aspekte hinsichtlich Abbau und Verarbeitung Beachtung. Darüber hinaus erfolgte eine ausreichende Beprobung der verschiedenen Vorkommen, um zusätzlich physikalische und technische Analysen durchführen zu können. Die daraus resultierende Datensammlung dient einer umfassenden Charakterisierung und damit einer direkten Einschätzung der Qualität der Materialien.

Im Folgenden werden zunächst die wirtschaftlichen, geologischen und geographischen Rahmenbedingungen beschrieben, um dann auf den Werksteinsektor einzugehen. 


\subsection{Wirtschaftliche Situation des argentinischen Natursteinsektors}

Die bis dato positive, wenn auch schwache Entwicklung der Natursteinindustrie in Argentinien, erfuhr durch die nationale Wirtschaftskrise in den Jahren 2001/2002 einen herben Rückschlag. Bereits 1998/1999 kam es zu einer starken Rezession, die politische Instabilitäten und in der Folge den totalen Kollaps des Finanzsystems nach sich zog. Der Höhepunkte der „Argentinien-Krise“ wurde im Jahr 2002 erreicht, wobei es zu einem drastischen Rückgang des Bruttoinlandproduktes um 11,2 \% kam. Nach Angaben offizieller Stellen erreichte die Arbeitslosigkeit mit 21,5 \% ihren Maximalwert im Mai 2002, die Armutsrate überschritt dabei eine Grenze von $50 \%$.

Heute ist wieder eine Stabilisierung der Gesamtwirtschaft zu beobachten, deren Stärke und Aktivität mit der Zeit vor der Krise vergleichbar ist. Seit dem Tiefpunkt Mitte 2002 ist ein kontinuierlicher Zuwachs des Bruttoinlandproduktes zu verzeichnen. Für das Jahr 2005 hat das Auswärtige Amt der Bundesrepublik Deutschland eine Zunahme von 9,2 \% festgestellt.

Auch im Natursteinsektor spiegelt sich die wirtschaftliche Erholung, wenn auch schwach, wider (Montani 2005). So konnte im Jahr 2003 für den Granitsektor ein Ausbringungszuwachs von $16 \%$ im Vergleich zum Vorjahr festgestellt werden (Zolezzi et al. 2005). Dabei ist festzustellen, dass sich der Natursteinsektor in den letzten Jahren hauptsächlich auf den Abbau und die Verarbeitung granitoider Werksteinvarietäten konzentriert. In den Provinzen Patagoniens, hier vor allem in der Provinz Chubut, werden zudem große Mengen an Porphyren abgebaut. Insgesamt übersteigen die Produktionszahlen an karbonatischen Gesteinen (Kalkstein, Dolomit, Travertin) zwar bei weitem die Produktion an granitoiden Gesteinen, doch wird ein Großteil der ausgebrachten Karbonatgesteine für die Zementherstellung verwendet. Im Vergleich der Gesamtproduktion nichtmetallischer Rohstoffe machen die als Naturwerkstein genutzten Gesteine nicht mehr als $1 \%$ aus (Abb. 4.1, Zolezzi et al. 2005).

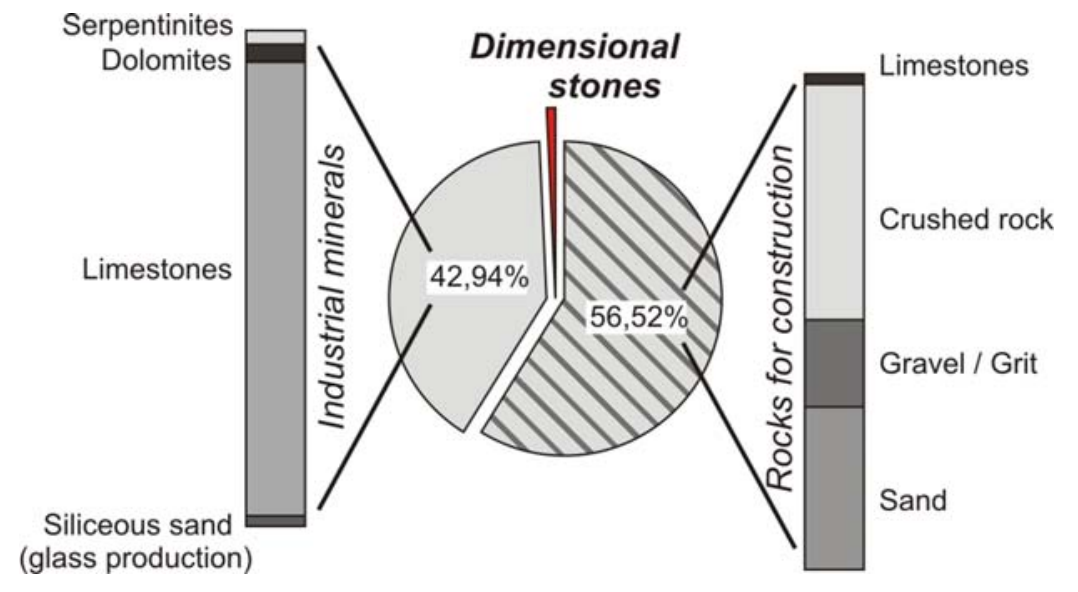

Abb. 4.1: Gesamtproduktion an Naturwertstein im Jahr 2003 mit der prozentualen Verteilung auf verschiedene Industriezweige (Zolezzi et al. 2005). 
Andere Rohstoffzweige der argentinischen Wirtschaft

Im Jahr 2006 wurde das Investitionsvolumen zur Exploration auf Industrieminerale mit 1,3 Mrd. US-\$ beziffert, wobei sich ein Großteil auf die Prospektion und frühe Phasen der Exploration bezog (Torres 2008). In gleichen Jahr zählte Argentinien zu den führenden Produzenten von Bor und Lithium (15 \% bzw. 12 \% der Weltproduktion). Insgesamt wurde der Wert der mineralischen Abbauprodukte im Jahr 2006 auf 3,15 Mrd. US-\$ geschätzt, was einen Anstieg von rund 67 \% gegenüber dem Vorjahr ausmacht. Diese erhebliche Zunahme ist zum größten Teil einer um 58 \% gewachsenen Goldproduktion zuzuschreiben, was mit der Öffnung der Goldmine Veladero im N der Provinz San Juan im Zusammenhang steht (Mining Press 2007). Andere wichtige metallische Rohstoffe sind Aluminium, Kupfer und Silber, deren Produktionszahlen im Jahr 2006 gegenüber dem Vorjahr keine bedeutenden Änderungen unterlagen (Torres 2008). Außerdem zählt Argentinien zu den wichtigsten Erzeugern fossiler Brennstoffe in Lateinamerika.

\subsection{Geographischer Überblick}

Mit einer N-S-Erstreckung von rund $3460 \mathrm{~km}$ und einer Gesamtfläche von etwa 3,8 Mio. km² ist Argentinien nach Brasilien das zweitgrößte Land des südamerikanischen Kontinents. Im N grenzen die Republiken Bolivien sowie Paraguay und im E Brasilien, Uruguay sowie der Atlantische Ozean an. Die Gebirgskette der Anden definiert die Grenze zum W' angrenzenden Chile.

Der nahe der chilenischen Grenze in der Provinz Mendoza gelegene Gipfel Cerro Aconcagua (Höhe 6952 mNN) ist nicht nur der höchste Punkt Argentiniens sondern auch der höchste Berg außerhalb Asiens. Obwohl auch in anderen Gebieten Argentiniens Gipfelhöhen über 6000 mNN erreicht werden (z.B. die Sierra de Famatina in der Provinz La Rioja), ist die Topographie großräumig als eher flach und eben zu bezeichnen. Zusammengefasst kann die physiographische Situation Argentiniens grob in drei Einheiten unterteilt werden:

1) Gebirge entlang der W' Grenze mit den höchsten Erhebungen in den zentralen Bereichen der Andenkette sowie einer Anzahl weniger markanter Gebirgs- bzw. Hügelketten, die in den N' und zentralen Regionen des Landes zumeist eine N-SOrientierung und in den außerandinen Gebieten Patagoniens eine WNW-ESE-Orientierung aufweisen.

2) Die Meseta (relativ karge Hochebenen): i) im NW des Landes im Bereich über 3000 mNN liegend (Altiplano de la Puna) und ii) S' des Rio Colorado mit einer Erstreckung von etwa $770000 \mathrm{~km}^{2}$ (Meseta Patagonia) zum Atlantik hin bis auf Meeresniveau seicht abfallend.

3) Die etwa $700000 \mathrm{~km}^{2}$ große Ebene (Ilanura), die sich im zentralen und E' Bereich des Landes ausbreitet und mit ausgedehnten Grasflächen auf fruchtbaren Böden die Grundlage der intensiven Viehwirtschaft bildet. Im SE' Teil der Ilanura wird das 
relativ gleichmäßige, sanfte Relief durch zwei Hügelketten (Sierra de Ventania und Sierra de Tandil) unterbrochen.

Klimatisch ergibt sich aus der flächenmäßigen Ausdehnung des Staates eine Vielfalt, die von subtropischen Bedingungen im $\mathrm{NE}$ des Landes über gemäßigte Zonen in Zentralargentinien bis zu den ariden bis semi-ariden kalten Klimaten Patagonien und entlang der Andenkette reicht.

\subsection{Geologischer Überblick über die für Naturwerkstein relevanten Regionen}

Die komplexe Geologie Argentiniens führt zu einer Vielzahl geologischer Provinzen (Abb. 4.2), von denen die im Rahmen dieser Erfassung von genutzten Vorkommen an Naturwerksteinen relevanten im Folgenden beschrieben werden.
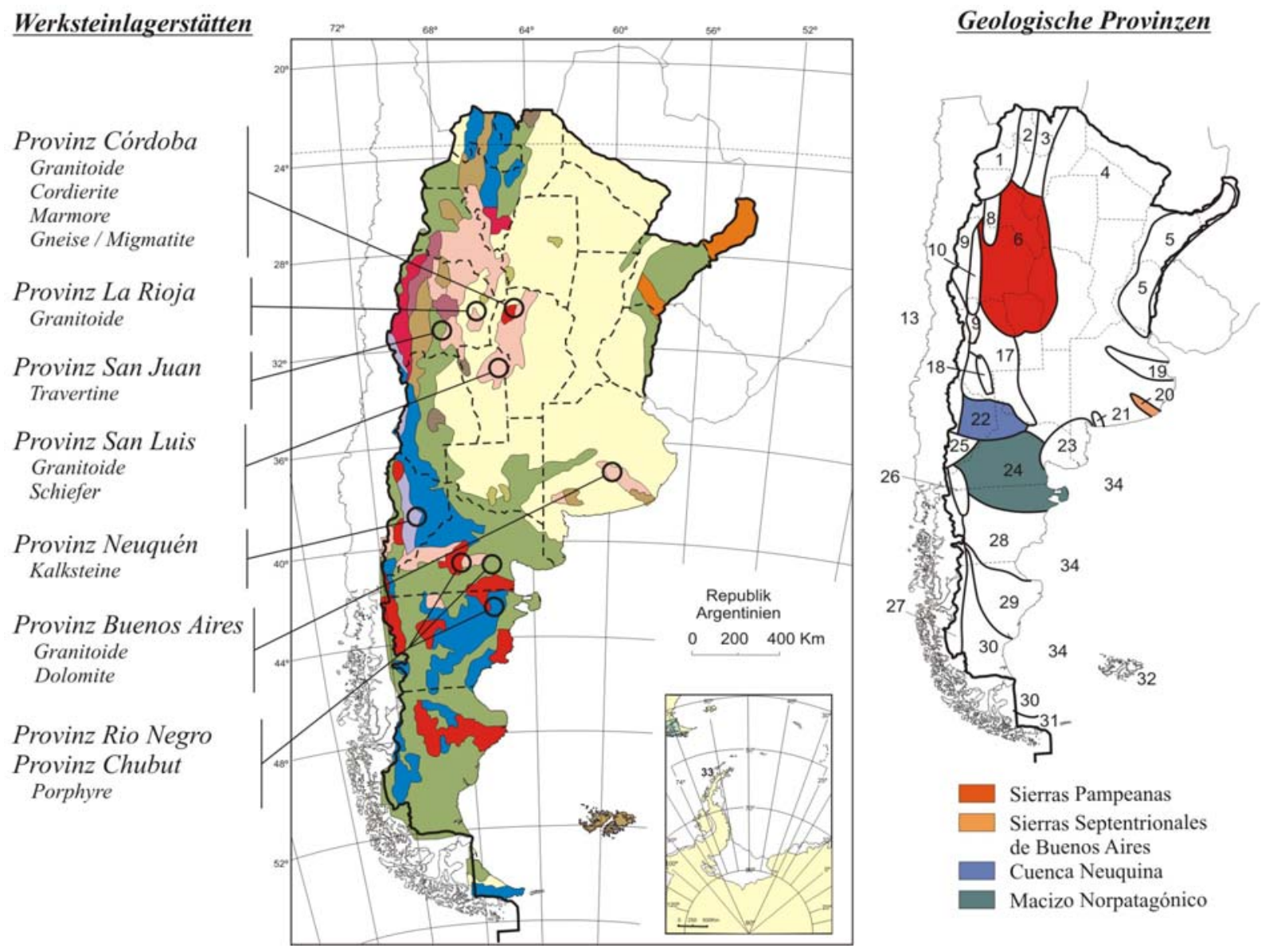

Abb. 4.2: Geologische Übersichtskarte Argentiniens und Lokalitäten der erfassten Naturwerksteinvorkommen. Rechts: Geologische Provinzen: 1-Puna, 2-Cordillera Oriental, 3-Sierras Subandinas, 4-Llanura Chaco Pampeana, 5-Mesopotamia, 6-Sierras Pampeanas, 8-Sistema del Famatina, 9-Cordillera Frontal, 10Precordillera, 13-Cordillera Principal, 17-Cuenca de Cuyo, 18-Bloque de San Rafael, 19-Cuenca del Salado, 20Sierras Septentrionales de Buenos Aires, 21-Sierras Australes de Buenos Aires, 22-Cuenca Neuquina, 23-Cuenca de Colorado, 24-Macizo Norpatagónico, 25-Cordillera Neuquina, 26-Precordillera Patagónica, 27-Cordillera Patagónica, 28-Meseta Patagónica Norte, 29-Macizo del Deseado, 30-Meseta Patagónica Sur, 31-Cordillera Fueguina, 32-Islas Malvinas, 33-Antártida Argentina e Islas del Atlántico del Sur, 34-Plataforma Continental Argentina. 
Die Sierras Pampeanas, im zentralen und NW' Argentinien (zwischen $24^{\circ}$ - $34^{\circ}$ S' Breite und $64^{\circ}-68^{\circ} \mathrm{W}$ ' Länge) gelegen, stellt eine morphotektonische Einheit dar, die im Allgemeinen durch mehrere in etwa N-S gerichtete Höhenzüge gekennzeichnet ist. Als Hauptgesteinsvarietäten treten neben Marmoren und Metabasiten vor allem präkambrische bis paläozoische Gneise, Migmatite, Schiefer und Phyllite auf. In dieses Basement kam es im Laufe von Kambrium bis Devon immer wieder zur Intrusion granitoider Körper (u.a. Rapela et al. 1998, Sims et al. 1998, Rapela 2000, López de Luchi et al. 2007). Caminos (1972, 1979) differenziert die Sierras Pampeanas, basierend auf den Unterschieden der lithologischen Einheiten und der magmatischen sowie metamorphen Entwicklung, in die Östlichen und Westlichen Sierras Pampeanas. In den Westlichen Sierras Pampeanas treten dabei dominant Gesteine schwach- bis mittelgradig metamorphen Charakters auf, in welche eine Anzahl kleiner granitischer Körper intrudierte. Ältere kristalline Gesteine, die mit dem Großkontinent Laurentia in Verbindung gebracht werden, konnten im äußersten W der Region nachgewiesen werden (Vujovich \& Kay 1998). Die Östlichen Sierras Pampeanas sind aus metasedimentären Serien ordovizischen, kambrischen und auch präkambrischen Alters aufgebaut, die durch unterschiedliche Metamorphosegrade gekennzeichnet sind. In den Sierras de Córdoba wurden granulitfazielle Metamorphosebedingungen erreicht (Gordillo 1984). Der regionale Metamorphosecharakter der Gesteine des Pringles Metamorphic Complex der Sierra de San Luis (Sims et al. 1998) im S der Sierras Pampeanas wird der Pampean Orogenese (Kambrium) sowie der Famatinian Orogenese (Ordovizium) zugeschrieben (Pankhurst et al. 1998, Rapela et al. 1998, Steenken et al. 2004). Die eher niedrigmetamorphen sedimentären Gesteine der Puncoviscana Formation sind in ihrer Zusammensetzung mit den hochmetamorphen Einheiten der Sierras de Córdoba vergleichbar, womit sich ähnliche primäre Bildungsbedingungen ableiten lassen (Rapela et al. 1998, Steenken et al. 2004).

Die Region Patagonien, S’ des Rio Colorado gelegen, wurde lange Zeit als „eigenständige Landmasse“ angesehen, die sich markant in den verschiedensten Faktoren vom Rest des südamerikanischen Kontinents unterscheidet. Dieser Unterschied wurde vor allem an der topographischen und klimatischen Situation, an Flora und Fauna sowie an paläontologischen Aufzeichnungen fixiert. Ramos (1984) postuliert die Kollision eines allochthonen Terrains (Patagonia) mit dem kratonischen Schild des südamerikanischen Kontinents (zum Superkontinent Gondwana gehörend) im Karbon und interpretiert die Rio Colorado Zone als Nahtstelle. Andere Autoren sehen dagegen eine Fortsetzung der Serien des zentralargentinischen Basements (Pankhurst et al. 2006 und darin). Primär kann das Basement der Region Patagonien in zwei Großeinheiten separiert werden: i) das Nordpatagonische Massiv (NPM, Macizo Norpatagónico) und ii) der Rio Deseado Komplex. Letzterer steht in Verbindung mit mehreren verstreut auftretenden metamorphen Einheiten, für die eine genaue Altersdatierung nur sehr schwer zugänglich ist. Die ältesten sedimentären Gesteine treten in der karbonischen Tequel-Gruppe im W' Abschnitt der NPM sowie in ausgedehnten Ablagerungen der silurischen Sierra Grande Formation auf.

Die mesozoische vulkanische Provinz Patagonia, die aus überwiegend sauren Vulkaniten gebildet wird, bedeckt eine Fläche von rund 1 Mio km². Dabei wird in manchen Gebieten eine nachweisliche Mächtigkeit von rund 500 m erreicht. Die lokalen Bezeichnungen einzelner 
Formationen (z.B. Marifil, Chon-Aike, El Quemado, Ibañez) beziehen sich dabei auf die Hauptaufschlussgebiete. Rhyolite treten im gesamten Gebiet auf, wobei eine deutliche Konzentration im NE zu vermerken ist. Der dortige Marifil-Komplex setzt sich überwiegend aus verschweißten, kristallinen Ignimbriten und subhorizontal eingeschalteten, massiven Asche- und Lavalagen zusammen. Auch die Chon-Aike Formation im SE ist aus rhyolitischen und trachydazitischen, pyroklastischen Lagen zusammengesetzt, wobei hier auch subvulkanische rhyolitische Körper eingeschaltet sind. In der Rio Chubut Region treten ebenfalls rhyolitische Gesteinsformationen auf, die hier jedoch eng mit den andesitischen Gesteinen der Lonco-Trapial Formation verflochten sind. Im Bereich der andinen Kordilleren sind sowohl rhyolitische als auch andesitische Gesteine vorhanden. In diesem Bereich lassen die Aufschlussverhältnisse jedoch eine erhebliche Zergliederung des Gesteins infolge der tektonischen Beanspruchung vermuten (El Quemado-, Ibañez- und Tobifera-Formation).

Im N bzw. NW grenzt an das Nordpatagonische Massiv eine als Neuquén Sequenz zusammengefasste, hauptsächlich sedimentäre Abfolge mesozoischen bis känozoischen Alters mit vereinzelten darin eingebetteten vulkanischen Lagen. Dieses Neuquén-Becken ist als back-arc Becken charakterisiert, dessen Bildung in Zusammenhang mit dem Auseinanderbrechen Gondwanas, zeitlich in der Trias angesiedelt, gebracht wird (Uliana \& Biddle 1988). Es erstreckt sich über eine Gesamtfläche von etwa $160000 \mathrm{~km}^{2}$ bei einer Sedimentmächtigkeit von rund $7 \mathrm{~km}$. Mit Beginn in der mittleren Trias bis in das Sinemurium (Untere Jura) wurden die Subsidenz des Beckens und die damit verbundene Sedimentation durch eine Anzahl eigenständiger Halbgrabenstrukturen gesteuert (Vergani et al. 1995). Primär treten dabei grobe vulkanische und kontinentale Ablagerungen auf. Gegen Ende der Trias kam es zu einer ersten Transgression des Pazifischen Ozeans, die den vulkanischen Bogen überwand. Mit dem Beginn der regionalen Absenkung kam es zu einer zunehmenden Vernetzung der Grabenstrukturen und in der Folge zu einer Überflutung des gesamten Beckenbereichs im Pliensbachium (Legarreta \& Gulisano 1989). Bis zur Mitteljura entwickelte sich eine westwärts fortschreitende Sedimentationsabfolge, die von küstennahen Sandsteinen bis zu Tiefseesedimenten reicht. Die gesamte Abfolge ist sporadisch durch vulkanische Lagen unterbrochen. In den jurassischen Gesteinen des Neuquén-Beckens sind lokal auch Ammoniten enthalten, die in die European Standard Zonation eingegliedert werden können (Iglesia Llanos et al. 2006).

Der Kern des Río de la Plata-Kratons wird von paläoproterozoischen Gesteinen gebildet, für die eine schwache metamorphe Beeinflussung durch neoproterozoische orogene Ereignisse nachgewiesen werden kann (Dalla Salda et al. 1988, Pankhurst et al. 2003 und darin). Durch die dominant auftretenden granitischen Gneise und Migmatite mit der Einschaltung anderer Lithologien, so Schiefer, Marmore und mafischer bis ultramafischer, magmatischer Gesteine, wird eine komplexe Krustenevolution beschrieben. Dabei sind sowohl Hinweise auf Subduktion als auch Kontinentkollision gegeben. Das kristalline Basement des Kratons wird primär durch neoproterozoische bis frühpaläozoische Sedimente, im W' Bereich durch die mächtigen phanerozoischen Sedimente des Chaco-Parana-Beckens, bedeckt. Kristalline Gesteine des Kratons konnten auf der Insel García Martin im Bereich der Mündung des Río de la Plata (Dalla Salda 1981), in verschiedenen Bohrungen im NE 
Argentiniens (Russo et al. 1979) sowie im Bereich Tandilia (Provinz Buenos Aires; Cingolani \& Dalla Salda 2000) nachgewiesen werden. Dabei sind vor allem im W'Bereich der Region Tandilia zwischen den Städten Olavarría und Azul verschiedene Granitoide und Gneise des Basements direkt aufgeschlossen.

\section{5 Überblick zur Abbau- und Verarbeitungstechnik}

Der Abbau von Naturwerksteinen wird zurzeit hauptsächlich von kleineren und mittleren Unternehmen durchgeführt. Zumeist treten diese, häufig aus nur wenigen Arbeitern bestehenden Kleinbetriebe als Subunternehmen größerer, marktführender Unternehmen (z.B. Onemar S.A., Genaro y Andrés De Stefano S.A., Kursaal S.A.) auf. Nur in wenigen Fällen wird in kleineren Betrieben die konsequente Linie von der Ausbringung des Rohblocks bis zur Herstellung von Endprodukten einschließlich der Vermarktung verfolgt. Die Eigenproduktion beschränkt sich dabei auf Kleinserien von Tisch- und Küchenarbeitsplatten, Boden- bzw. Wandfliesen oder aber Grab- und Ornamentsteinen. Dabei wird aber meist, je nach Bedarf und Auftragslage, nur ein Teil des ausgebrachten Materials der Eigenverwendung zugeführt. Überständige Rohblöcke werden von den marktführenden Unternehmen aufgekauft und gehen in die Produktion von Großserien ein.

In der Abbautechnik bzw. den eingesetzten technischen Mitteln treten keine entscheidenden Unterschiede im Hinblick auf die abzubauende Gesteinsart auf. Im Allgemeinen erfolgt die Ausbringung von Rohblöcken unter Verwendung von Pressluftbohrern und dem anschließenden, vorsichtigen Einsatz von Explosiva oder auch Expansionszementen (Abb. 4.3 a). In einigen größeren Brüchen granitoider Varietäten erfolgt beim Vorhandensein sehr weitständiger Trennflächengefüge die Separierung großvolumiger Blöcke über die Flammenschnitttechnik (vgl. Kap. 2.4.1). Die Formatierung zu Rohblöcken findet fast ausnahmslos unter Verwendung von Pressluftbohrer und dem manuellen Einsatz von Federkeilen statt. Im Bereich der Schiefer- und Porphyrproduktion ist zudem das manuelle Aufspalten zu Pflastersteinen, Platten und Fliesen gängig (Abb. 4.3 b). Moderne Diamantseilsägen werden im Allgemeinen nur selten eingesetzt, was zum einen mit der deutlich höheren finanziellen Belastung und zum anderen mit der unumgehbaren Notwendigkeit einer permanenten Wasserversorgung in Zusammenhang steht.

Zum Abbau von Travertin in der Provinz San Juan setzt die Firma NaturalStones S.A. neben Diamantseilsägen auch eine Schrämmsäge modernster Bauart ein. Mit dieser Technik kann eine unnötige Schwächung des relativ weichen Materials weitestgehend vermieden werden. Zur Konfektionierung der Rohblöcke wird sowohl eine Diamantseilsäge als auch die herkömmliche Methode mittels Pressluftbohrer und Federkeilen eingesetzt.

Die Großunternehmen sind dagegen fast ausnahmslos auf die Verarbeitung konzentriert. Das Angebot aus nationalen Natursteinvarietäten wird zudem durch Importe aus z.B. Südafrika, Brasilien wie auch Europa ergänzt. In der Produktion kommen moderne Verarbeitungstechniken meist italienischer (u.a. Gaspari Menotti) aber auch spezialisierter argentinischer Hersteller (Coch) zum Einsatz (Abb. 4.3 c). Das Aufschneiden der Rohblöcke erfolgt meist permanent an bis zu acht Gattersägen pro Fertigungsanlage. Zur Herstellung 
polierter Oberflächen werden ebenfalls moderne Polierstraßen mit automatischer Be- und Entladung eingesetzt. Da diese Gerätschaften selten älter als 10 oder 15 Jahre, meist jedoch deutlich jünger, sind, ist die Maßhaltigkeit der Rohplatten wie auch die Qualität der Oberflächenbearbeitung gegeben.

Einige wenige international tätige Unternehmen sind auf den Abbau sowie die Verarbeitung einer bestimmten Gesteinsart konzentriert (z.B. Piedra Púrpura S.A. - Porphyr; San Luis Slates S.R.L. - Schiefer). Dadurch sind die Produktion sowie das Qualitätsmanagement auf höchstem Niveau anzusiedeln. Als eine weitere Besonderheit ist der Einsatz moderner computergesteuerter Fräsmaschinen zu erwähnen (Fa. Marmolería Jarque, Tandil, Provinz Buenos Aires), wodurch die Fertigung fein strukturierter, filigraner Endprodukte (z.B. Vasen, Skulpturen usw.) in hoher Qualität möglich ist (Abb. 4.3 d).
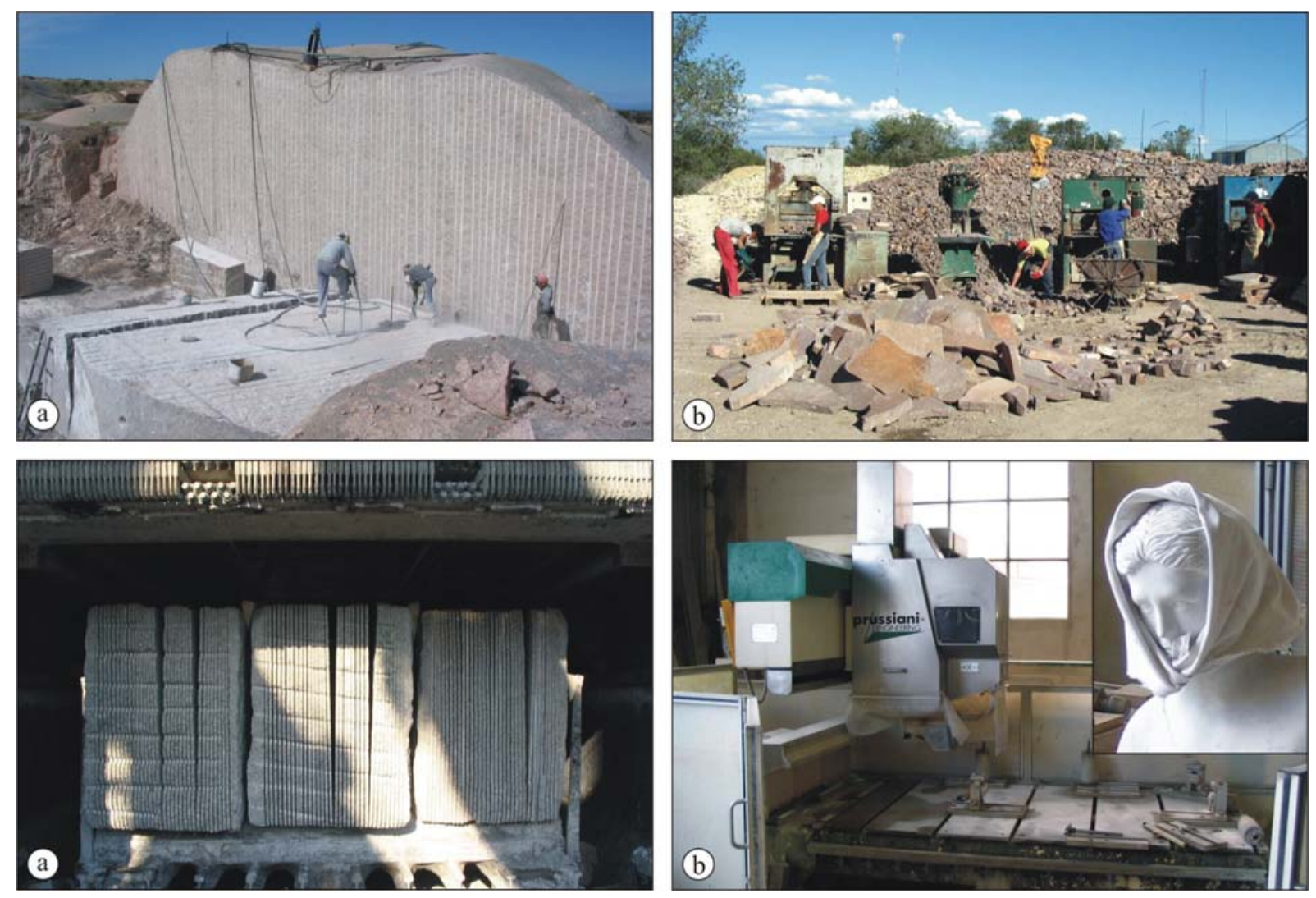

Abb. 4.3: Abbau und Verarbeitungstechnik in der argentinischen Naturwerksteinindustrie: a) Ausbringen und Abvieren von Rohblöcken unter Verwendung von Pressluftbohrern (Granitbruch MARRON COCO, Provinz San Luis); b) Manuelle Produktion von Pflastersteinen und Polygonalplatten (Porphyr, Fa. Lencina Layas y Porfidos, Los Menucos, Provinz Rio Negro); c) In Gattersäge aufgeschnittene Rohblöcke der granitischen Varietät GRIS PERLA (Fa. Onemar S.A., Villa de Soto, Provinz Córdoba); d) Computergesteuerte Fräsmaschine (Fa. Marmolería Jarque, Tandil, Provinz Buenos Aires).

Bei der Verarbeitung von Rohplatten granitoider Naturwerksteinvarietäten erfolgt zumeist eine Politur der Oberflächen. Seltener, meist für spezielle Zwecke, wird ein geflammtes oder rustikales, also sägeraues, Finish verwendet. Deutlich überwiegt die Herstellung polierter Rohplatten mit etwa 90 - 95 \% der Gesamtproduktion. Die verbleibenden Prozente entfallen primär auf die direkte Produktion von Wand- und Bodenfliesen. Als Standarddicken in der Rohplattenproduktion finden Stärken von $20 \mathrm{~mm}$ und $25 \mathrm{~mm}$, selten $15 \mathrm{~mm}$ und $30 \mathrm{~mm}$, 
Verwendung. Bodenfliesen werden auf ein Standardmaß von 305 x 305 mm bei einer Stärke von $10 \mathrm{~mm}$ zugeschnitten. Großformatige oder asymmetrische Fliesen werden lediglich auf Bestellung gefertigt. Zum überwiegenden Teil erfolgt die Fliesenproduktion aus dem Zuschnitt großformatiger Rohplatten nach der Politur, obwohl auch die technische Einrichtung zur Verwendung flacher bzw. schlanker Blöcke über Aufschneiden mit Mehrblattsägen (vgl. Kap. 2.1) vorhanden ist.

Ein mit entscheidender Kostenfaktor und auch von eminenter Relevanz für die Umweltverträglichkeit der Natursteinverarbeitung ist der verantwortungsbewusste Umgang mit dem im Produktionsprozess zirkulierenden Wasser. So konnte z.B. die Firma Genaro y Andrés De Stefano S.A. durch den Einsatz moderner Wasseraufbereitungstechnik die tägliche Zufuhr von Frischwasser auf etwa $5000 \mathrm{l}$ reduzieren, während täglich insgesamt etwa $50000 \mathrm{l}$ in den verschiedenen Produktionsstufen zirkulieren. Die traditionelle Trennung von Wasser und Bohr- bzw. Sägeschlamm wurde hier durch spezielle Druckfiltersysteme ergänzt. Dadurch kann der Restwassergehalt im Schlamm von 90 \% nach der Sedimentation auf 20 \% nach der Druckfilterung reduziert werden. Auch die Rückgewinnung des Sägegranulats, einer speziellen Körnung bestehend aus Karbonstahl zur Verwendung im Abrasivsägegatter, mittels Dichtetrennung während der Sedimentation trägt zu einer deutlichen Steigerung der Umweltverträglichkeit bei.

\subsection{Lithologische und lagerstättengeologische Charakterisierung des Werksteinpotenzials}

\subsubsection{Lagerstätten granitoider Gesteine}

Auf dem Sektor der granitoiden Naturwerksteine konzentriert sich der Abbau auf die zentralen Provinzen San Luis, Córdoba und La Rioja sowie die Provinz Buenos Aires. Daneben werden noch eine kleinere Anzahl an Vorkommen im Norden Patagoniens genutzt, die aber nicht im Rahmen dieser Übersicht aufgenommen werden konnten.

\subsubsection{Provinz San Luis}

Seit vielen Jahrzehnten stellt das Abbaugebiet Potrerillos, etwa $150 \mathrm{~km} \mathrm{NE}$ ' der Provinzhauptstadt San Luis gelegen, ein wichtiges Zentrum der argentinischen Naturwerksteinindustrie. Der Großteil der genutzten Vorkommen befindet sich im zentralen und S' Teil des Las Chacras-Potrerillos Batholithen. Dieser Pluton bildet mit einer Fläche von etwa $650 \mathrm{~km}^{2}$ den größten Komplex einer Anzahl postorogener Intrusiva (spätes Devon frühes Karbon), die über die gesamte Sierra de San Luis verteilt sind (Siegesmund et al. 2004). Es handelt sich um eine polyphase Intrusion überwiegend granodioritischer, monzogranitischer und syenogranitischer Gesteine (López de Luchi et al. 2001).

Die genutzten Granitoide zeigen verschiedene Varietäten in Struktur, Korngröße und Farbe (Abb. 4.4). Der zurzeit größte aktive Bruch mit einer Ausbringung von bis zu $400 \mathrm{~m}^{3} \mathrm{im}$ Monat befindet sich in einem grobkörnigen, porphyritischen (Monzo-) Granit. In Teilen weist das Gestein eine deutliche Foliation auf, die durch die parallele Einregelung von 
Kalifeldspäten definiert ist. Die als Mikroklin entwickelten K-Feldspäte erreichen eine Größe von 3-6 cm und sind in eine Matrix, die durch einen relativ hohen Biotitgehalt und Korngrößen bis zu $1 \mathrm{~cm}$ gekennzeichnet ist, eingebettet. Alle differenzierten Varietäten zeigen mikroskopisch Hinweise auf eine schwache kataklastische Überprägung, was an feinen Bruchstrukturen besonders in Quarzkristallen identifiziert werden kann. In Mikroklinen sind teilweise submagmatische Mikrorisse zu beobachten, die mit Quarz, selten auch Plagioklas verfüllt sind.
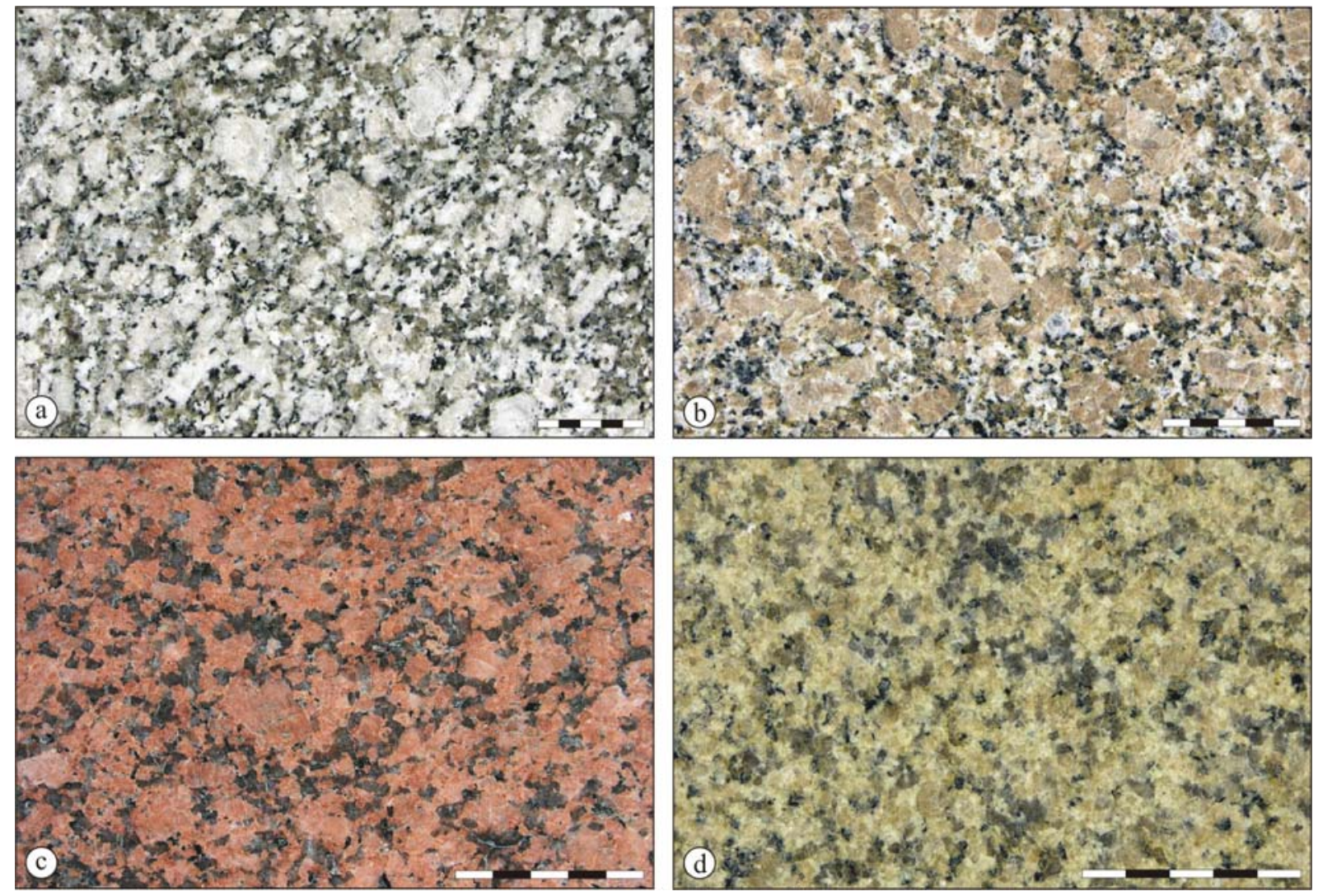

Abb. 4.4: Granitoide Naturwerksteinvarietäten der Provinz San Luis: a) GRIS PERLA, b) ROSA DEL SALTO, c) ROJO DRAGÓN, d) MARRON COCO (polierte Oberflächen, Maßstäbe in cm).

Die Farbpalette reicht von homogen grau (GRIS PERLA; Abb. 4.4 a) bis zu einem rötlichen grau-braun (ROSA DEL SALTO; Abb. 4.4 b). Der Farbübergang mit lachsfarbenen Tönen ist in der Varietät SALMON realisiert. Die Färbung des Gesteins wird dabei primär durch die Grundfarbe der Kalifeldspäte kontrolliert, die von hellgrau bis zu einem hellen rotbraun variiert, wobei die Rotfärbung durch das Auftreten von eingeschlossenem Hämatit verstärkt wird. In einigen Bereichen, besonders in der Varietät ROSA DEL SALTO, treten lokal begrenzt schlierige Biotitakkumulationen auf, die das Gesamterscheinungsbild des Gesteins hin zu dunkleren Tönen verändern. Der gegenteilige Effekt, ein helleres, aber im Allgemeinen damit verbunden auch deutlich heterogeneres Dekor, wird durch die Akkumulation von Kalifeldspäten zu flow-banding Strukturen verursacht. Diese Phänome, wie auch aplitische Gänge, die in ein Abbaukonzept als Ausschlussfaktor im Hinblick auf die Rohblockausbringung miteinbezogen werden müssen (vgl. Kap. 2.4), konnten im aufgeschlossenen Teil der Lagerstätte jedoch kaum beobachtet werden und unterbrechen somit nur relativ selten das sonst homogene Erscheinungsbild des Gesteins. 
Im S’ Stock des Batholithen können primär zwei Einheiten unterschieden werden (López de Luchi et al. 2001): i) ein äußerer, primär biotitführender Ring und ii) ein primär muskovitführender Kern, der zudem durch einen höheren Quarzgehalt gekennzeichnet ist. Die differenzierten Handelssorten wurden vor der Wirtschaftskrise 2001/2002 in einer Vielzahl von Brüchen auf einer Gesamtfläche von etwa 200 ha abgebaut. Die Hauptvarietät ROJO DRAGÓN (Abb. 4.4 c), ein equigranularer Monzogranit von roter Grundfarbe, zeigt insgesamt eine sehr homogene Strukturierung. Makroskopisch weisen alle unterschiedenen Varietäten ein richtungsloses, mittel- bis grobkörniges Gefüge auf. Die Intensität der Schattierung ist gewissen Schwankungen unterworfen, wobei generell zwischen einem dunklen und einem hellen Farbcharakter, der zumeist auch relativ feinkörniger ist, unterschieden wird. Die dunklen, dabei aber meist klaren Quarzkristalle weisen im Allgemeinen hypidiomorphe Formen auf. Dabei kann häufig eine schwache und nur schwer anhand elongierter Körner identifizierbare Foliation ausgebildet sein. Zum Teil weisen einzelne Quarzkristalle einen bläulichen Farbschimmer auf, was in der ausgeglichenen Grundfärbung des Gesteins zu einem interessanten Farbeffekt führt.

Rezent wird lediglich eine Varietät gewonnen, die im diffusen Übergangsbereich der beiden beschriebenen Haupteinheiten ansteht. Im Gegensatz zu den sonst dominierenden Rottönen weist diese eine warme, hellbräunliche Färbung auf (MARRON COCO, Abb. 4.4 d). Zudem sind die Korngrößen im Gegensatz zu der Varietät ROJO DRAGÓN leicht reduziert. Strukturen, die auf eine syn- oder postmagmatische Deformation hindeuten können, sind in den untersuchten Proben der Varietäten des S' Stocks nur in sehr geringem Umfang nachzuweisen.

Ausgehend von der strukturellen Entwicklung des Batholithen (Siegesmund et al. 2004) bestehen für das Trennflächengefüge in seiner Orientierung und Abstandsverteilung Unterschiede zwischen dem zentralen Teil (GRIS PERLA, SALMON, ROSA DEL SALTO) und dem S' Teil (ROJO DRAGÓN) (Abb. 4.5). In den Lagerstätten des zentralen Teils ist ein insgesamt weitständig ausgebildetes, annähernd orthogonales Kluftsystem gegeben. Dadurch ist zumeist eine gleichmäßige Ausbringung gut dimensionierter Rohblöcke möglich. In manchen Bereichen ist dabei der Abbau mit einer Abfallrate von weniger als $20 \%$ des Gesamtmaterials verbunden. Zum Zeitpunkt der Untersuchung betrug das mittlere Volumen der gewonnenen Rohblöcke etwa $5 \mathrm{~m}^{3}$ (vgl. Kap. 2.4.1, Abb. 2.24). Etwa gleiche Bedingungen sind auch im Abbau der Varietät MARRON COCO gegeben (vgl. Abb. $4.3 \mathrm{a}$ ).

Im Gegensatz dazu sind die Aufschlussbereiche der Varietät ROJO DRAGÓN durch ein im Hinblick auf die räumliche Anordnung der Trennflächen komplizierteres und gleichzeitig engständigeres System gekennzeichnet, in welchem keine Orthogonalität gegeben ist. Problematisch ist hierbei vor allem das stark variierende Einfallen der NNE-SSW streichenden Kluftschar (Abb. 4.5 c). Basierend auf dem natürlichen Trennflächengefüge ergeben sich häufig spitzwinklige in-situ Blöcke, wodurch ein Ausbau der Lagerstätte mit senkrechten Wänden zumeist nicht möglich ist. Die Heterogenität in der Form der Primärblöcke wurde augenscheinlich in manchen Bereichen durch eine vertikale Führung der Abbaubohrungen forciert, da intakte Gesteinspartien unkontrolliert zergliedert werden (vgl. Kap. 2.5). Nach Information eines ehemaligen Brucharbeiters ergaben sich zum Teil extrem 
hohe Abraumraten von mehr als $90 \%$. Darüber hinaus traten in der letzten Abbauphase immer häufiger Festigkeitsprobleme auf, die in einem erhöhten Auftreten von Mikrorissen begründet sind (vgl. 3.12.4). Daher wurde die Produktion in einer Vielzahl der Brüche in dieser Varietät bereits vor der landesweiten Wirtschaftskrise eingestellt.
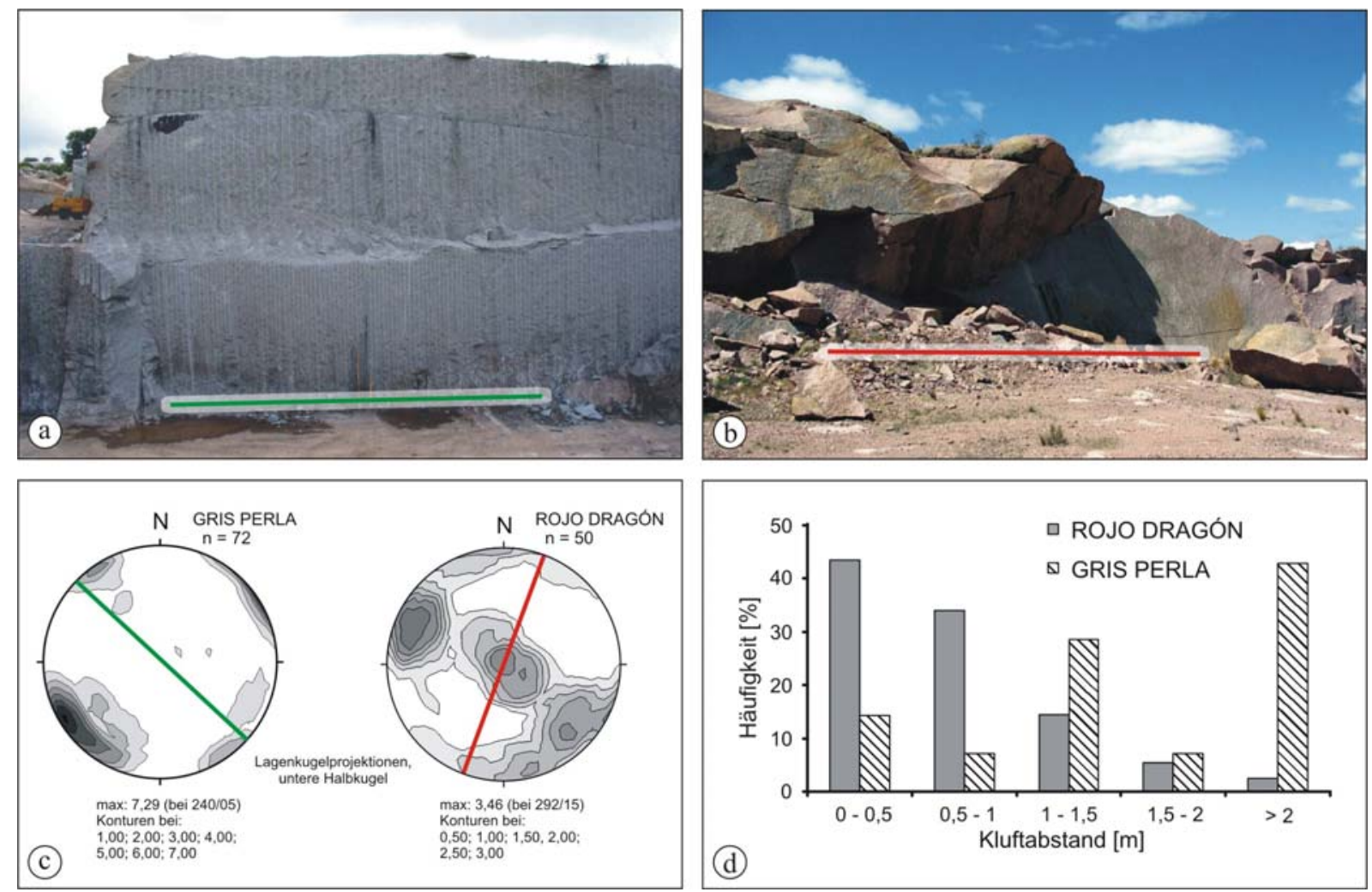

Abb. 4.5: Lagerstättengeologische Situation im Abbau der Varietäten a) GRIS PERLA (Steinbruch „Las Nietas“, Fa. P.M. Granitos; Höhe der Wand etwa $18 \mathrm{~m}$ ) und b) ROJO DRAGÓN. c) Kluftverteilungen in den Lagerstättenbereichen der Varietäten; d) Kluftabstandsverteilungen im Abbau der Varietäten ohne Richtungsbezug. Ein weitständigeres, orthogonales Kluftsystem ermöglicht die Ausbringung gut dimensionierter Rohblöcke der Varietät GRIS PERLA bei gleichzeitig deutlich geringerem Abraumanteil.

Ein weiterer Pluton dieser Serie großvolumiger Intrusivkörper devonischen Alters im S' Bereich der Sierras Pampeanas ist der Renca-Batholith (López de Luchi et al. 2002). Im NW' Teil dieses Batholithen wurde bis zu Beginn des Jahres 2005 ein grobkörniger Granodiorit / Monzogranit (SAN FELIPE, Abb. 4.6) gewonnen. Das Hauptmerkmal dieses an Biotit und Hornblende reichen Gesteins ist ein ausgeprägt porphyritisches Gefüge, in welchem hypidiomorphe bis idiomorphe Kalifeldspäte, die als Mikroklin entwickelt sind, in Größen bis über $10 \mathrm{~cm}$ auftreten. Das Gesamterscheinungsbild des Gesteins ist dabei durch das meist deutlich parallele Arrangement der Megakristalle in der Matrix gekennzeichnet, wodurch eine magmatische Foliation widergespiegelt wird. Im Kornverband der Matrix, in der die Korngrößen zwischen $1 \mathrm{~cm}$ und $3 \mathrm{~cm}$ liegen, sind häufig zu Chlorit alterierte Biotite zu beobachten. Das Auftreten von Serizit und Tonmineralen ist dagegen auf den verwitterungsbedingten Zerfall von Plagioklas zurückzuführen (siehe auch López de Luchi et al. 2002). Durch die verstärkte Alteration mafischer Minerale, die in Feldspatkristallen eingeschlossen sind, kann es zu einer offenen Porosität bei polierten Oberflächen kommen. 
Dieser Problematik versucht man mit einer Versiegelung der Oberfläche im Polierprozess entgegenzuwirken.

Aus der Mineralzusammensetzung ergibt sich im Allgemeinen eine hellrosa-graue Grundfarbe. Häufig ist jedoch eine Akkumulation von Feldspatphänokristallen zu verzeichnen, wodurch der Modalbestand zwischen etwa $15 \%$ und $40 \%$ schwanken kann. Gleichzeitig ist häufig in direkter Umgebung dieser deutlich helleren Bänder eine Anreicherung an Biotit gegeben. Das homogene Gesamtbild wird dadurch gravierend gestört (vgl. Kap. 2.4). Diese Bereiche müssen meist als Ausschussmaterial der weiteren Produktion von Rohblöcken entnommen werden. Eine weitere Problematik ergab sich im Abbau durch das gehäufte Auftreten aplitischer Gänge (Abb. 4.6 b), was dann insgesamt zur Einstellung des Abbaubetriebes führte.
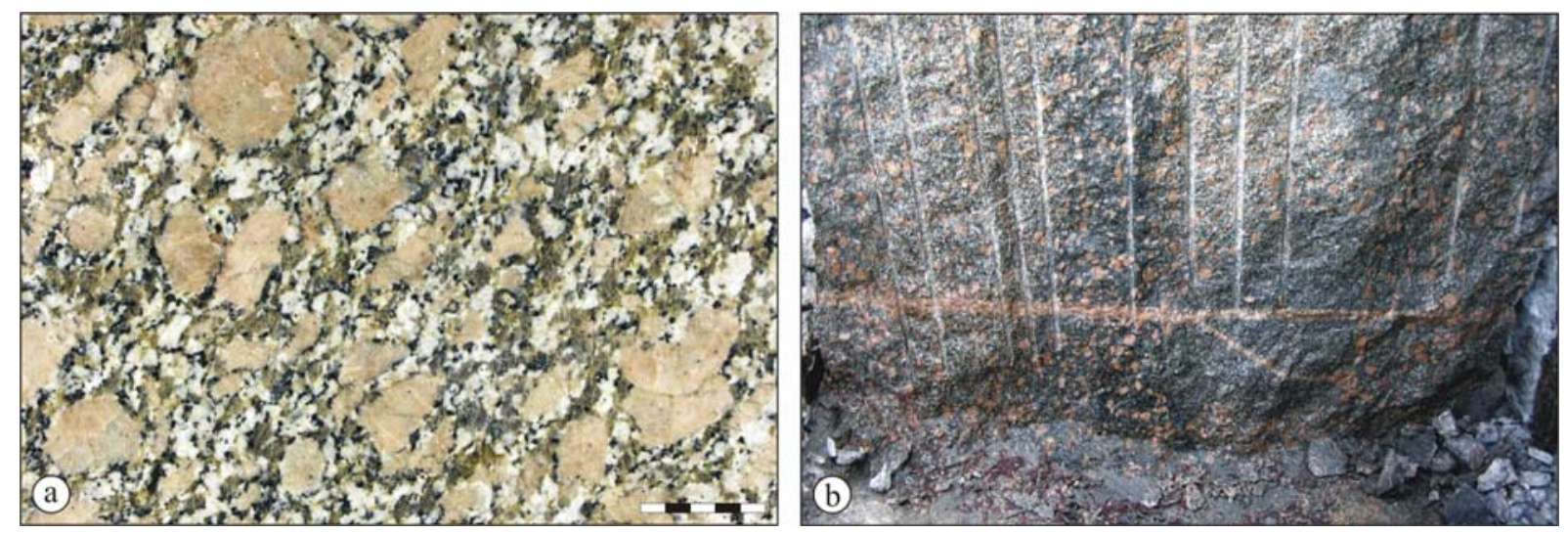

Abb. 4.6: a) Polierte Probe der Varietät SAN FELIPE (Provinz San Luis, Maßstab in cm); b) Detailansicht einer Abbauwand in der Lagerstätte mit deutlichen Akkumulationen von dominant Biotit sowie aplitischen Gängen (Bildbreite etwa $3 \mathrm{~m}$ ).

\subsubsection{Provinz Córdoba}

In der Provinz Córdoba konzentriert sich der Abbau granitoider Naturwerksteine weitestgehend auf das Departamento Cruz del Eje, etwa 150 km W' der Stadt Córdoba, im NW der Sierras de Córdoba gelegen. Dort werden im kambrischen Granitkomplex El Pilón verschiedene Varietäten mittel- bis grobkörniger, porphyritischer Gesteine granitischer bis granodioritischer Zusammensetzung als Naturwerkstein gewonnen. Aufgrund der Aufschlussverhältnisse ist hier jedoch im Gegensatz zum Abbaugebiet Potrerillos (Provinz San Luis) die Werksteingewinnung an das Aufarbeiten einzelner Boulder gebunden, was insgesamt auf ein relativ engständigeres Trennflächengefüge für diese Region hindeutet (Abb. 4.7, vgl. Kap. 2.2). Daher gestaltet sich die Rohblockgewinnung deutlich zeitintensiver und ist zudem mit einem erhöhten Abraumanteil verbunden. Inwieweit eine Tieferführung des Abbaus dem entgegenwirken könnte, ist aus der Oberflächensituation nur schwer abschätzbar. 

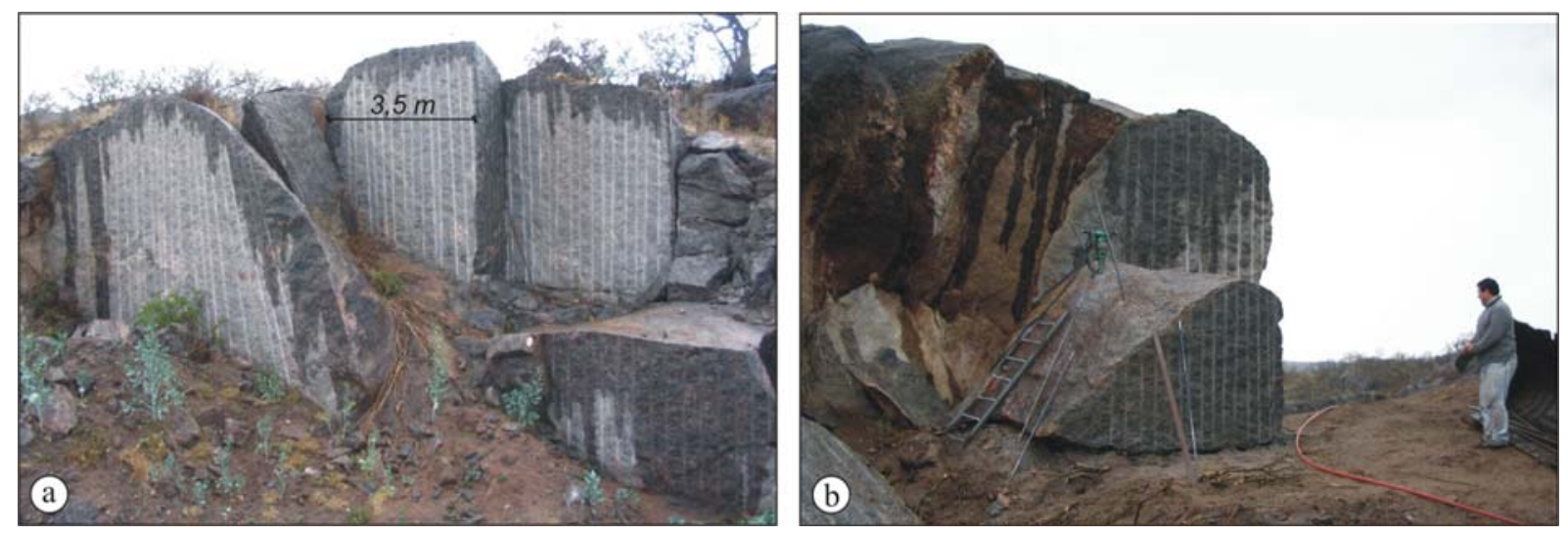

Abb. 4.7: a) Typische Aufschlusssituation im Granitkomplex El Pilón; b) die zeitintensive Aufarbeitung einzelner Boulder ist zumeist mit einem erhöhten Abraumanteil verbunden (Steinbruch Fa. Agrimar).

Die porphyritischen Granitoide, die in unterschiedlicher Menge Cordieritminerale aufweisen, werden in mehreren Brüchen für die Verwendung als Naturwerkstein gewonnen. Ein charakteristisches Merkmal der meisten der differenzierten Handelssorten sind schmale Feldspatleisten, die eine Länge bis zu $8 \mathrm{~cm}$ aufweisen können. Häufig ist durch die parallele Anordnung der Feldpäte eine deutliche Foliation definiert. Quarz, Biotit, Plagioklas und, zumeist zu hypidiomorpher Gestalt verwitterte, Cordierite treten als verbleibende Mineralphasen auf (Rapela et al. 2002).

Die Färbung der Gesteine variiert insgesamt stark zwischen rötlichen, braunen und beigen Grundtönen (ROJO ORCOLLANO, MARRON ORCOLLANO, BEIGE PUMA [Abb. 4.8 a]). Durch eine stärker heterogene Verteilung der Feldspatphänokristalle, bei der auch häufig die Parallelorientierung verloren geht, kommt es weiterhin lokal immer wieder zu Dekorwechseln. Dabei wirkt das Gesamtbild turbulenter und es zeigt sich meist ein Wechsel zu helleren Farbnuancen. Eine prinzipielle Abgrenzung der Gewinnungsgebiete der einzelnen Handelssorten ist somit kaum möglich. Infolgedessen erfolgt die Separierung zumeist durch Inaugenscheinnahme nach Fertigstellung der Rohblöcke.

In einem kleineren Bereich der Lagerstätte Orcollano wird neben den oben genannten Lithologien die Handelssorte ROJO PRINCIPE als vierte Varietät abgebaut. Der Hauptunterschied ist das Fehlen elongierter Feldspatkristalle, wodurch eine Foliation im Vergleich mit den drei anderen granitoiden Varietäten nicht so prägnant ausgebildet ist. Anstelle dessen ist durch das „Umfließen“ der scheinbar zum Teil gedrehten Feldspäte durch Biotit ein eher gneisiger bis schwach mylonitischer Charakter ausgebildet. Auch resultiert aus dem mit bis zu 20 \% etwas höheren Gehalt an mafischen Mineralen, vor allem Biotit und untergeordnet Hornblende, ein insgesamt etwas dunklerer Farbeffekt. Ob die abgrenzenden Merkmale dieser Varietät innerhalb der gesamten Lagerstätte einem lokalen Phänomen entsprechen oder ob auch in anderen Bereichen entsprechende Charakteristika auftreten, konnte im Rahmen dieser Studie nicht weiter untersucht werden. 

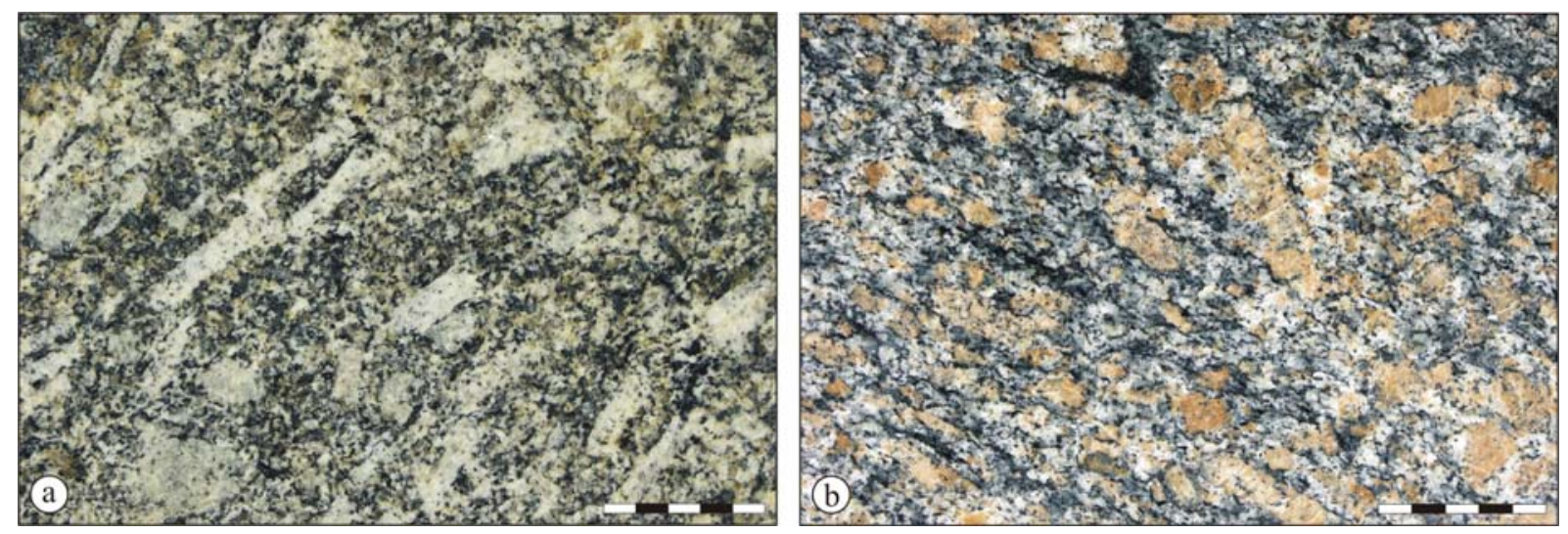

Abb. 4.8: Granitoide Werksteinvarietäten der Provinz Córdoba, Abbaugebiet Orcollano: a) Varietät BEIGE PUMA mit typischen Feldspatleisten; b) Varietät ROJO PRINCIPE mit stärker gneisartigem Charakter (polierte Oberflächen, Maßstäbe in cm).

Der Komplex El Pilón ist in kleineren Bereichen mit metamorphen Cordieritgesteinen assoziiert, die eine zum Teil annähernd monomineralische Zusammensetzung mit mehr als $90 \%$ Cordierit aufweisen (Rapela et al. 2002). Innerhalb der Cordieritgesteine werden aus wirtschaftlicher Sicht zwei Hauptvarietäten unterschieden: i) eine massige, dunkel graugrüne, mittel- bis grobkörnige Varietät mit unregelmäßig verteilten Spots meist weißer oder leicht rosaweißer Feldspat- und Quarzakkumulationen (NOCHE BUENA) und ii) eine sphärisch texturierte Varietät, deren Gefüge aus ovalförmigen, bis zu $20 \mathrm{~cm}$ großen Cordieritknollen, die im Kern deutlich dunklere Anreicherungen an hauptsächlich Biotit und Sillimanit zeigen, zusammengesetzt ist (VERDE ORCOLLANO, Abb. 4.9). Die zum Teil zentimetergroßen, klaren Cordieritminerale weisen meist einen leicht bläulichen Farbschimmer auf. Die Grünfärbung wird durch feine Pinitnadeln als typisches Alterationsprodukt des Cordierits hervorgerufen, die netzartig den Kristallverband durchwachsen. Besonders die Varietät VERDE ORCOLLANO fand aufgrund der tiefen grünbläulichen Färbung in Verbindung mit dem wohl einzigartigen, spektakulären Gefüge bis in die 1950er Jahre hinein als Naturwerkstein Verwendung (Gordillo 1974). Infolge eines sehr komplexen Trennflächengefüges und der kleinräumigen Ausdehnung musste der Abbau jedoch eingestellt werden. Heute stehen nur noch wenige Blöcke zur Verfügung, die dann für meist exklusive Bauvorhaben genutzt werden.
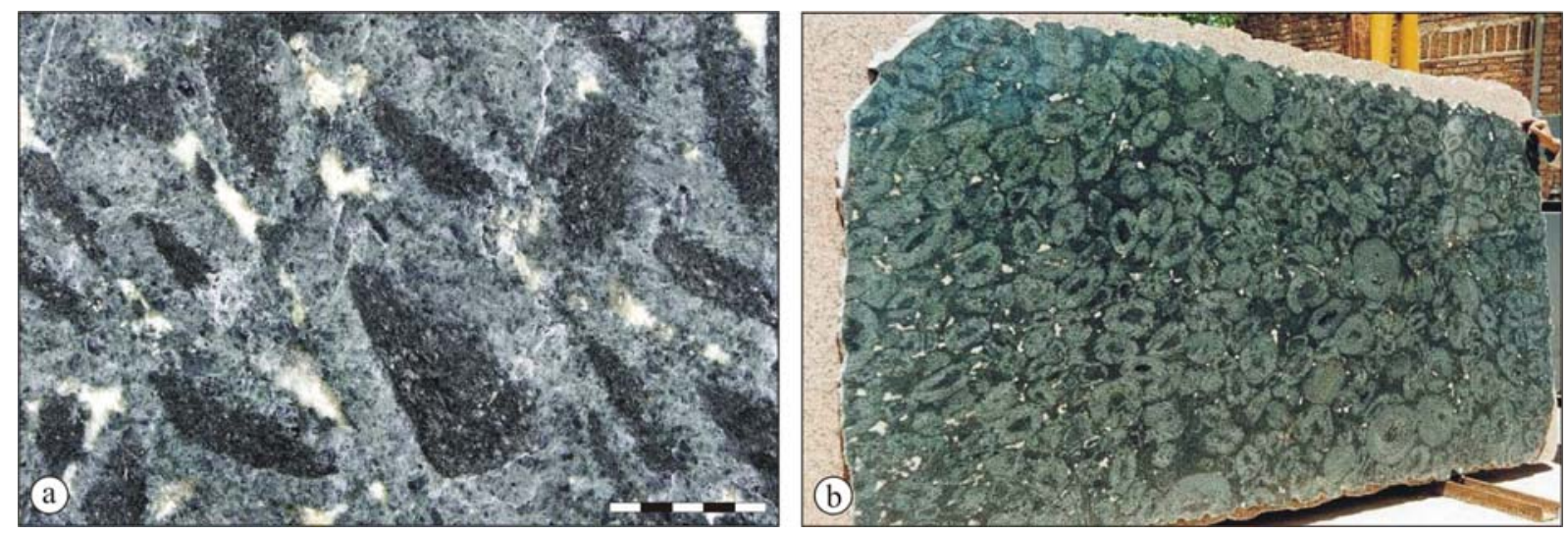

Abb. 4.9: Cordieritgestein VERDE ORCOLLANO (Prov. Córdoba, Abbaugebiet Orcollano) mit einzigartigem Dekor durch sphärische Akkumulation der Cordieritminerale (polierte Oberflächen, Maßstab in cm). 
Im Januar 2004 wurde das Vorkommen eines migmatitischen Paragneises (FRANCO VETEADO; Abb. 4.10) bei Ruma Huasi, etwa 50 km S' der Stadt Cruz del Eje, erschlossen. Das mittel- zum Teil grobkörnige Gestein weist eine intensiv gebänderte, foliierte Textur auf, die auf der Separierung dunkler, biotitreicher Lagen (Paläosom) von deutlich helleren, felsischen Partien (Leukosom) basiert. Insgesamt ergibt sich daraus eine mittelgraue Grundfarbe. Quarz, Plagioklas und Biotit, deren jeweiliger Anteil infolge des migmatischen Charakters auf kurzer Distanz stark schwanken kann, bilden die Hauptgemengteile. In einigen Bereichen ist der Biotit zudem mit geringen Mengen Muskovit vergesellschaftet. Nach der geochemischen Analyse ist insgesamt eine granodioritische Zusammensetzung gegeben. Zusätzlich treten akzessorisch Apatit und Zirkon auf. Quarz weist als Einzelkristall zumeist xenomorphe bis hypidiomorphe Formen auf, tritt aber ebenso als Zwickelfüllung oder als Sekundärfüllung in Mikrorissen bei Plagioklasen oder auch größeren Quarzkörnern auf. Die intensive Bänderung wird unregelmäßig durch grobkörnigere Bereiche unterbrochen, in denen kein gerichtetes Mineralgefüge erkennbar ist. Gleichzeitig kann in diesen Bereichen neben den genannten Mineralkomponenten auch Cordierit in Mengen von 5 - 10 \% nachgewiesen werden. Vermutlich lassen sich diese Erscheinungen auf eine verstärkte Anatexis zurückführen. Mikroskopisch zeigen die Cordieritminerale zumeist eine deutliche Alteration zu Pinit, was zu einem blassgrünen Farbstich führt. Zudem wird das strukturreiche Dekor kleinflächig nebulös verzerrt, was aber nicht unbedingt als Negativfaktor angesehen werden muss.

Die Lagerstätte wird durch großvolumige, massive Gesteinskörper gebildet, in denen nur sehr wenige Trennflächen erkannt werden können. Der zum Zeitpunkt der Studie aktuell im Abbau befindliche Boulder (Abb. 4.10 a) wies bei einer verbleibenden Ausbissbreite von etwa 40 m und einer Länge von etwa 50 m parallel zur Abbauwand Kluftabstände von nicht unter $6 \mathrm{~m}$ auf. In der orthogonalen Richtung dazu konnten an der Oberfläche lediglich fünf offene Klüfte im aufgeschlossenen Bereich des Boulders nachgewiesen werden. Tatsächlich ergaben die ersten $50 \mathrm{~m}$ in der Boulderlänge eine Ausbeute von etwa 60 \% formatierte Rohblockware. Dabei wurden lediglich Blöcke berücksichtigt, die für die Weiterverarbeitung in Großgattersägen im Werk der Firma Kursaal S.A. (Villa de Soto) nach Werksvorgaben vorbereitet wurden. Die Länge der Blöcke belief sich auf 2,5 m bis 3,0 m und die zwei verbleibenden Seiten auf 1,6 m bis 1,8 m bzw. 1,3 m bis 1,5 m. Die Blockvolumina der noch im Bruch und im Blocklager vorhandenen Rohblöcke lagen so zwischen etwa 6 und $7 \mathrm{~m}^{3}$ (im Mittel 6,6 m³), was nach Primavori (1999) dem Idealfall in der Ausbringung von Rohblöcken in der Naturwerksteingewinnung entspricht (vgl. Kap. 2.1). Ausgeschlossen wurden bei dieser Schätzung geringer dimensionierte Rohblöcke, die in einem gesonderten Stock eingelagert wurden und der Weiterverarbeitung in einer Fliesenstraße zugeführt werden sollten. Dabei handelt es sich um längliche Blöcke, die in der zweiten und dritten Dimension kürzere Abmessungen zwischen 0,5 m und 1,25 m aufwiesen. Mit Hinblick darauf, dass auf einer Fläche von mehreren $1000 \mathrm{~m}^{2}$ Ausdehnung das oberflächlich aufgeschlossene Gestein in Farbe und Struktur demjenigen im aktuellen Abbaubereich anzutreffenden mehr oder weniger ähnelt und zudem anhand einer einfachen Testbohrung mittels Pressluftbohrer ein Ende des 
massiven Gesteinskörpers bis etwa 7 m Teufe nicht abzusehen ist, erscheint ein als hoch einzuschätzendes wirtschaftliches Potenzial für diese Lagerstätte vorzuliegen.

In einem angrenzenden Bereich wurde zudem zu Beginn des Jahres 2005 ein Testabbau in einer farblichen Varietät (ROJO FRANCO VETEADO, Abb. 4.10 c) begonnen, wobei eine weitestgehend homogene Rotfärbung der leukosomatischen Bereiche auftritt. Hervorgerufen wird diese durch in Quarz und Plagioklas fein dispers verteilte Hämatitblättchen. Fraglich ist, ob es sich dabei um ein oberflächennahes Phänomen handelt oder ob die Farbcharakteristik auch in einem tieferen Anschnitt der Lagerstätte erhalten bleibt.
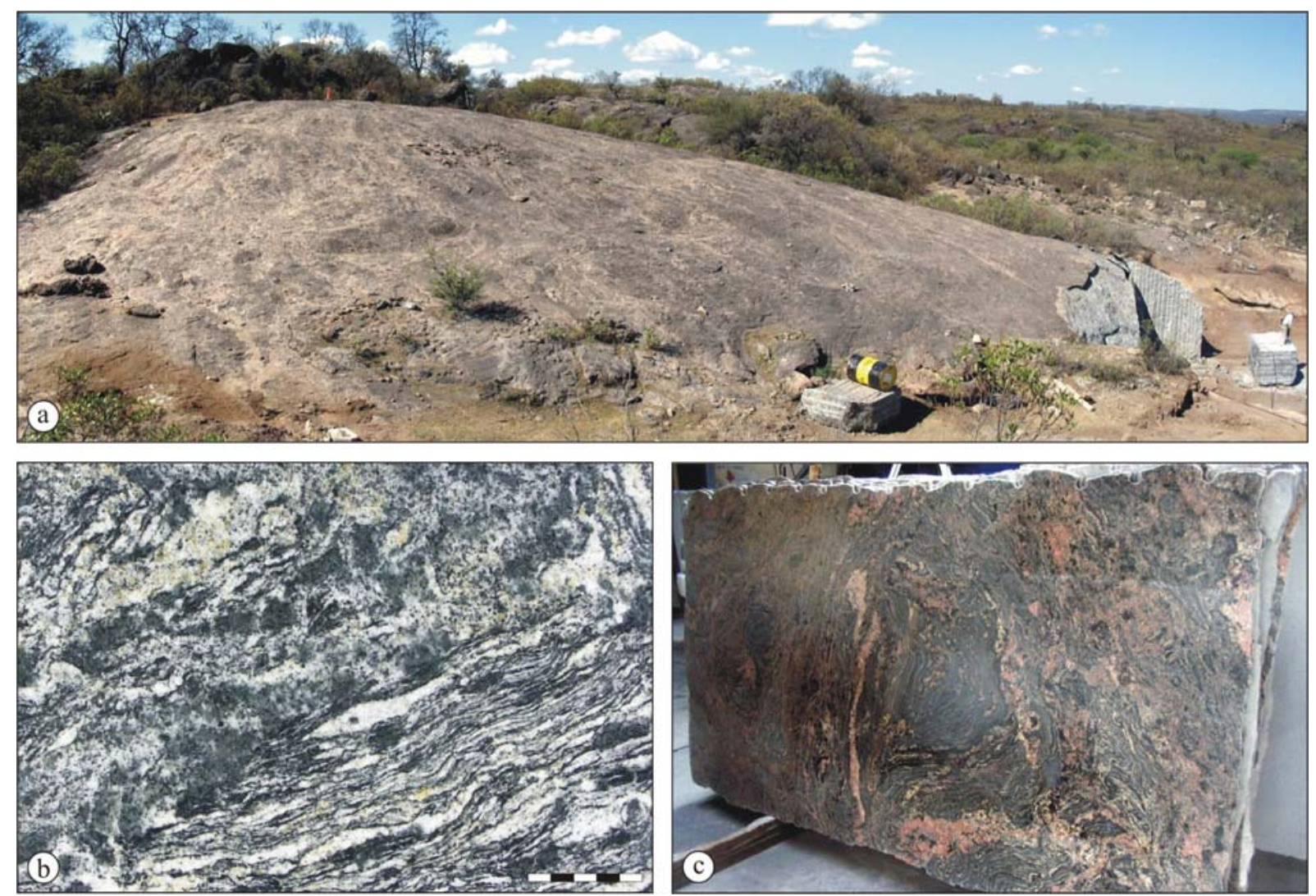

Abb. 4.10: a) Großvolumige, massive Boulder erlauben eine hohe Ausbringung gut dimensionierter Rohblöcke beim Abbau des migmatitischen Paragneises FRANCO VETEADO (Provinz Córdoba, Cruz del Eje, Steinbruch Fa. Minera Ruma Huasi). b) FRANCO VETEADO mit deutlichem Wechsel von Paläosom und Leukosom sowie Bereichen einer verstärkten Anatexis (Maßstab in $\mathrm{cm}$ ); c) Farbvarietät ROJO FRANCO VETEADO (polierte Oberflächen).

Im S’ Bereich der Sierras de Córdoba bei der Ortschaft Yacanto wurde bis vor wenigen Jahren im Steinbruch „La Cantera Kinzigita“ ein Cordierit-Granat-Gneis (AZUL TANGO, Abb. 4.11) abgebaut. Infolge zunehmender Probleme durch ein engständiges und in der Orientierung variables Kluftsystem musste der Abbau eingestellt werden (vgl. Kap. 2.5, Abb. 2.36). Zwar erfolgte der Abbau annähernd senkrecht zur Hauptkluftrichtung, doch erweist sich das Trennflächengefüge insgesamt als sehr wechselhaft. Dabei treten vor allem auch immer wieder dominante Klüfte auf, die spitzwinklig zum horizontalen Abbauvortrieb verlaufen. Der Abbau erfolgte auf drei Ebenen. Ob in einem tieferen Anschnitt das Gestein eine intaktere Struktur aufweist, ist hier schwer abzuschätzen. Trotz dessen ist aber noch allein im Bruch ein Stock von mehr als 150 Blöcken vorhanden, wobei einige durch deutliche 
Trennflächen oder aber durch spitzwinkliges Zulaufen der Seitenflächen erheblich wertgemindert sind (vgl. Kap. 2.5, Abb. 2.37).

Neben Quarz, Cordierit und akzessorisch auftretender Hornblende und Biotit ist die Mineralogie dieses hochmetamorphen Gesteins durch hohe Gehalte (bis zu 30 \%) an tiefrotem Granat gekennzeichnet. Mikroskopisch zeigen die bis zu $7 \mathrm{~mm}$ messenden Granate sehr häufig Alterationserscheinungen und sind zum Teil auch deutlich zerbrochen, wobei jedoch meist hypidiomorphe bis idiomorphe Formen bestehen bleiben. Die zumeist homogene Verteilung der dunkelroten Granate in der hellgrauen, bläulich schimmernden Matrix gibt dem Gestein ein dekorreiches, farbenprächtiges Erscheinungsbild, das durch das häufig ausgeprägte Richtungsgefüge unterstützt wird.

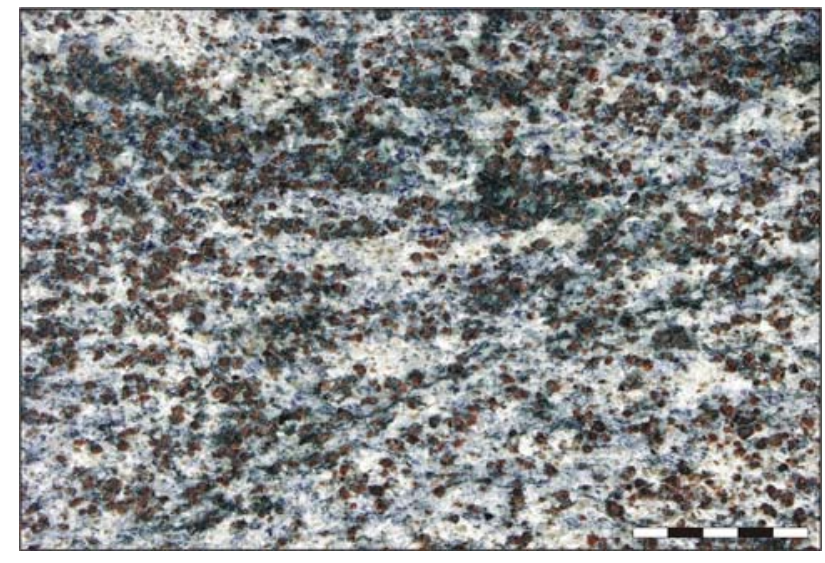

Abb. 4.11: Hochmetamorpher Cordierit-GranatGneis AZUL TANGO aus der Region Yacanto, Provinz Córdoba (polierte Oberfläche, Maßstab in $\mathrm{cm})$.

Etwa 20 km SE’ der Ortschaft Cruz del Eje ist ein weiterer silurischer / devonischer Pluton granodioritscher bis tonalitischer Zusammensetzung aufgeschlossen. Hier wird auf einer Fläche von aktuell 30 ha die Handelssorte SAN MARCOS BLANCO (Abb. 4.12) gewonnen. Der Abbau ist an die Marktsituation gebunden und wird daher je nach Nachfrage zeitweilig unterbrochen.

Insgesamt zeigt das equigranulare, mittelkörnige Gestein eine hell- bis mittelgraue Grundfärbung. Eine Vorzugsorientierung im Gefüge ist im Allgemeinen selten erkennbar. In der mineralogischen Zusammensetzung dominieren Quarz, Plagioklas, Glimmer und in geringeren Mengen auch Mikroklin. Hinsichtlich der Glimmerminerale übersteigt der Gehalt an Muskovit den von Biotit meist deutlich. Akzessorisch treten Serizit und Chlorit auf, die zumeist auf Alterationsprozesse zurückgeführt werden können. Aus werksteintechnischer Sicht ist der variable Gehalt an Hornblende von Interesse, welcher lokal bis zu $10 \%$ betragen kann. Die dunkelbraun gefärbten Hornblendeminerale weisen zumeist einen nadeligen Habitus auf und ergeben in ihrer homogenen, zum Teil gerichteten Verteilung in der Matrix ein ansprechendes Dekor. Neben einer möglichen Chloritisierung einhergehend mit grünlichen Verfärbungen, kann eine erhöhte Oxidationsanfälligkeit zur Bildung von Rostflecken führen (vgl. Kap. 3.13.1). Als limitierender Faktor hinsichtlich der Farbstabilität treten zudem in unregelmäßiger Verteilung im Gesteinskörper xenolithische Einschlüsse auf (Abb. 4.12 b). 

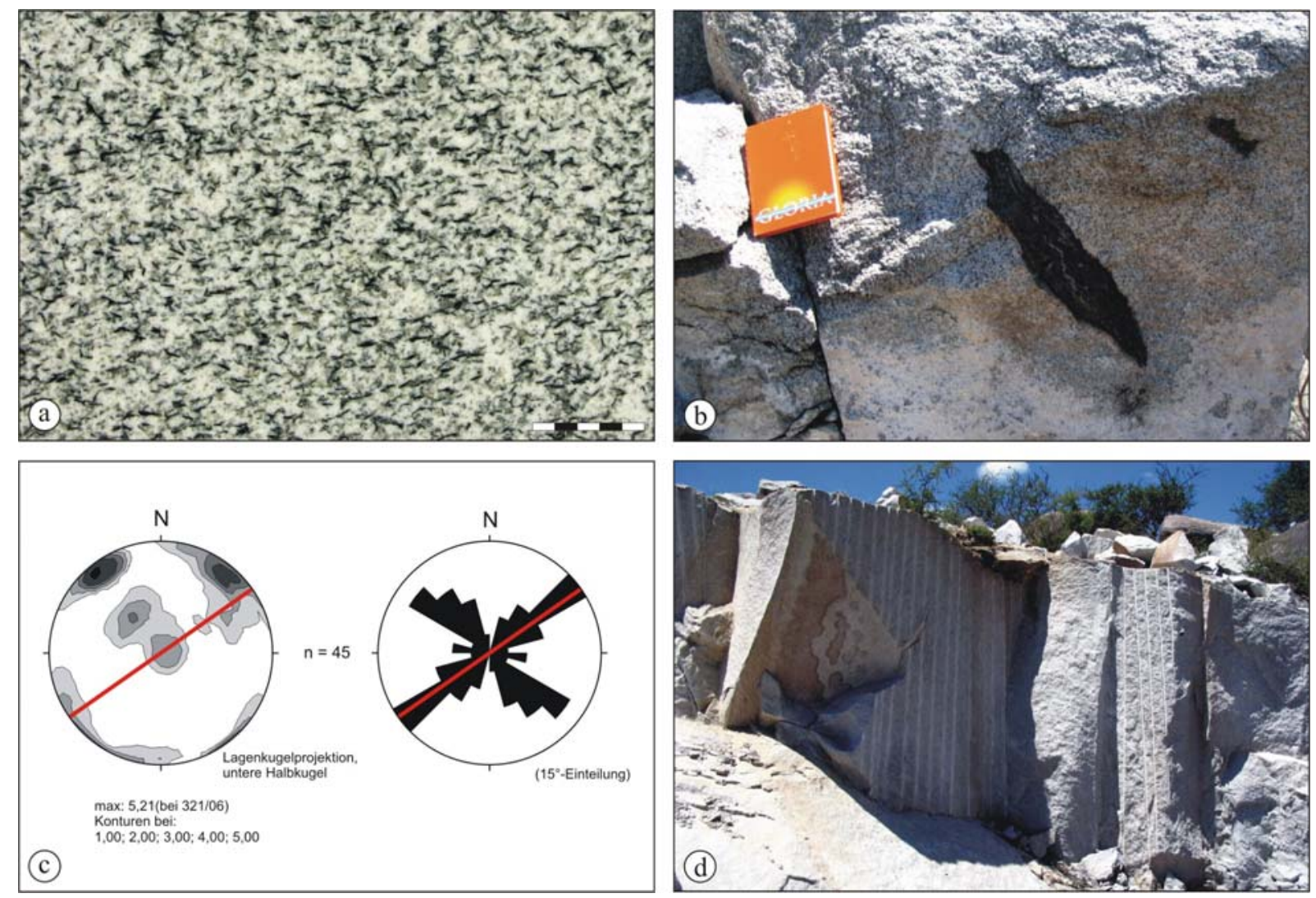

Abb. 4.12: Varietät SAN MARCOS BLANCO (Provinz Córdoba, Cruz del Eje): a) polierte Oberfläche (Maßstab in cm); b) Dekorabweichungen durch xenolithische Einschlüsse; c) Zwei annähernd orthogonal zueinander ausgerichtete Hauptkluftscharen werden von einer dritten Schar diagonal geschnitten, was zu d) schrägen Ausbrüchen im Abbau führt (Steinbruch der Fa. San Giovanni Bianco, La Falda).

Die Trennflächenverteilung im zuletzt bearbeiteten Abbaubereich weist im Allgemeinen Abstände von über $1 \mathrm{~m}$ und mehr auf. In der räumlichen Orientierung sind zwei annähernd orthogonal zueinander ausgerichtete Hauptkluftscharen ausgebildet, die jeweils ein Einfallen von etwa $80^{\circ}$ aufweisen (Abb. 4.12 c). Zusätzlich wird der Gesteinskörper durch ein diagonales Kluftsystem zergliedert, das im Streichen zwar parallel zur NE-SW streichenden Hauptkluftschar angelegt ist, dieses jedoch mit einem Einfallen von rund $35^{\circ}$ schneidet. Daraus ergeben sich zum Teil spitzwinklige in-situ Blöcke (vgl. Kap.2.4.2), wodurch sich in der letzten Phase vor der zeitweiligen Einstellung des Abbaus Abraumraten von rund 70 \% ergaben (pers. Mitt. Sr. L. Gervasoni, Fa. San Giovanni Bianco, La Falda).

\subsubsection{Provinz La Rioja}

Aus dieser Provinz sind drei Vorkommen an magmatischen Naturwerksteinen von Bedeutung und in diese Studie eingegangen. Dabei ist vor allem die Lagerstätte der Varietät NEGRO RIOJANO, ein fein- bis mittelkörniger Gabbro, infolge der weltweiten Nachfrage nach feinkörnigen, gleichmäßig dunkelgrauen bis schwarzen Gesteinen von hohem wirtschaftlichem Interesse.

Der Gabbro wird im NW' Bereich der Sierra de Los Llanos, etwa 20 km W' der Ortschaft Chamical, abgebaut. Der Bruch zeigt eine intensive Fragmentierung mit Klüften, 
Störungsbereichen und pegmatitischen Gängen als lagerstätten-kontrollierende Elemente (Abb. 4.13). Daher gestaltet sich die Ausbringung gut dimensionierter Rohblöcke schwierig und ist mit einem Materialverlust von bis zu 80 \% der bewegten Gesteinsmasse verbunden. Der Abbau konzentrierte sich daher zum Zeitpunkt der Studie auf drei massive Komplexe von insgesamt etwa $20 \mathrm{~m}$ Breite bei einer Wandhöhe von rund $6 \mathrm{~m}$ (Abb. 4.13 a), die durch deutlich geöffnete, senkrechte Klüfte voneinander getrennt sind. Innerhalb dieser Bereiche ist eine weitere Zergliederung gegeben, wobei zum Teil kataklastische Bahnen im cm- bis dm-Bereich auftreten (Abb. 4.13 b).
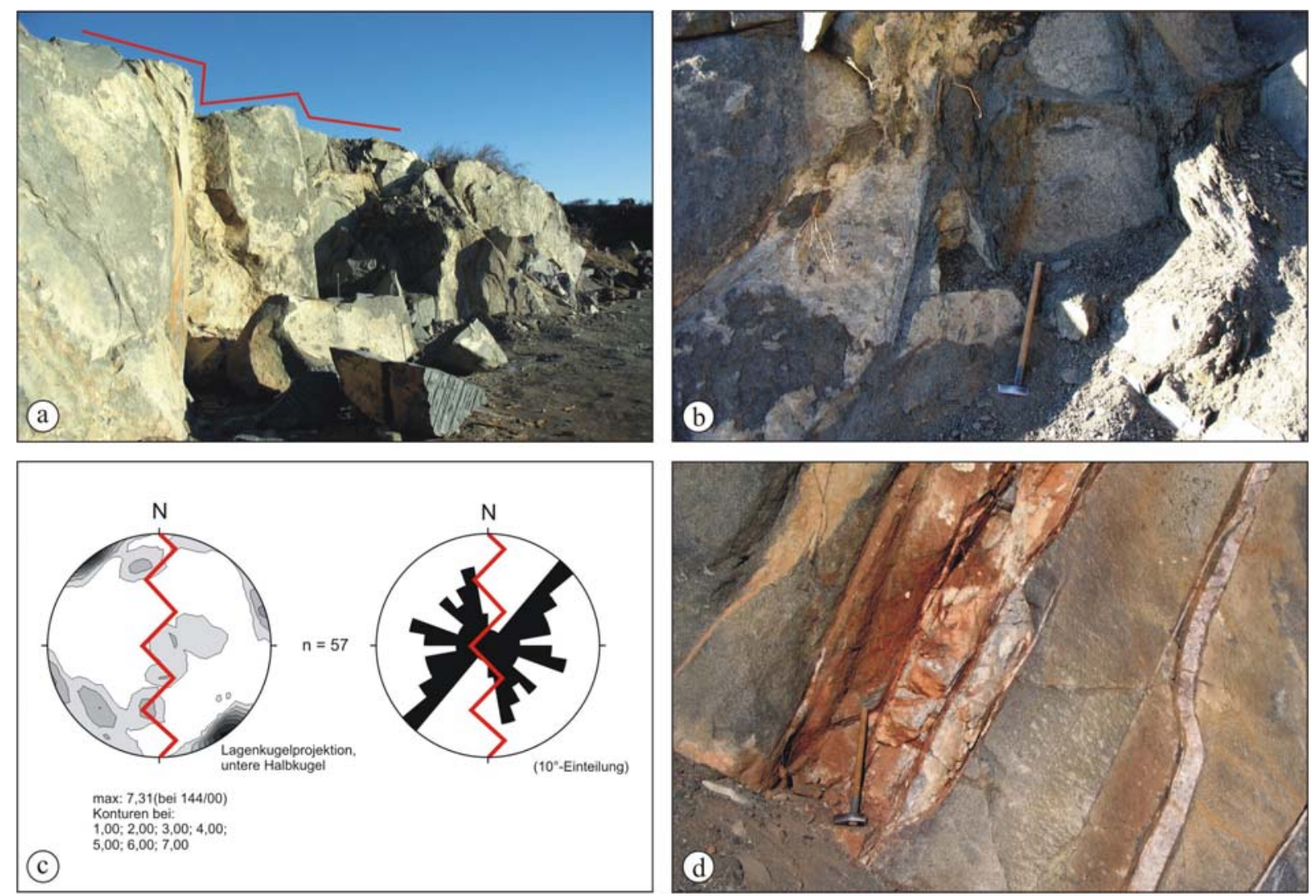

Abb. 4.13: a) Abbau des Gabbros NEGRO RIOJANO (Provinz La Rioja, Sierra de Los Llanos; Steinbruch der Fa. Granitos Alcazar S.A., La Rioja): Die Trennflächenverteilung (b) in Kombination mit dem Auftreten von kataklastisch ausgebildeten Klüften (c) und pegmatitischen Gängen (d) lässt auf eine leichte Scherung des Lagerstättenkörpers schließen.

Neben einer im Streichen etwa NE-SW ausgerichteten, steil stehenden Hauptkluftschar zeigt die Trennflächenverteilung kein weiteres, deutliches Maximum (Abb. $4.13 \mathrm{c}$ ). Ausgebildet sind lediglich mehrere Submaxima. Diese zeigen eine breitere Verteilung mit Einfallsrichtungen zwischen $0^{\circ}$ und $90^{\circ}$ bzw. $180^{\circ}$ und $270^{\circ}$ bei ebenfalls variableren Einfallswinkeln und beschreiben so die Zergliederung der Komplexe zu relativ unförmigen in-situ Blöcken, deren Abvierung die hohen Abraumraten nach sich zieht. Das Auftreten von Pegmatiten (Abb. 4.13 d) ist auf die stärker gestörten Randbereiche des Anschnitts konzentriert und hat zum aktuellen Zeitpunkt keinen direkt negativen Einfluss auf die Werksteingewinnung. Nach Auskunft des Bruchbesitzers soll mit dem Ausbau einer zweiten Abbauebene eine Erweiterung in die Teufe durchgeführt werden, um weniger gestörte Bereiche der Lagerstätte zu erschließen. Dies erscheint jedoch fraglich, da die durchgreifend 
auftretende Hauptkluftschar, die parallel auftretenden Gänge sowie auch die kataklastische Ausbildung vieler Klüfte auf eine leichte Scherung des Körpers zurückgeführt werden können.

Das fein- bis mittelkörnige Gestein weist eine massive Struktur auf, in der keine Vorzugsrichtung im Mineralverband zu erkennen ist. Die Politur der Oberfläche ergibt eine sehr dunkelgraue Grundfarbe (Abb. 4.14 a), die zum Teil einen deutlich olivgrünen Farbstich zeigt, was als nachteilig gegenüber möglichen Konkurrenzgesteinen bewertet werden muss. Die Farbschwankung resultiert aus einem leicht wechselhaften Augitgehalt. Stellenweise zeigen sich Anreicherungen an hellerem Feldspat, wobei die entsprechenden Bereiche auch etwas grobkörniger erscheinen. Darauf basierend ist die Varietät NEGRO RIOJANO CON NUBES (con nubes (span.) = mit Wolken) oder NEGRO OVERO definiert (Abb. 4.14 b). Das Dekor ist vorrangig auf Mingling- oder auch „Einfrierungs“-Prozesse (Koopmann 2004) zurückzuführen, bei der eine Schmelze mechanisch mit einer zweiten vermischt und dabei abgekühlt wird, woraus eine adernetzwerkartige Struktur resultiert
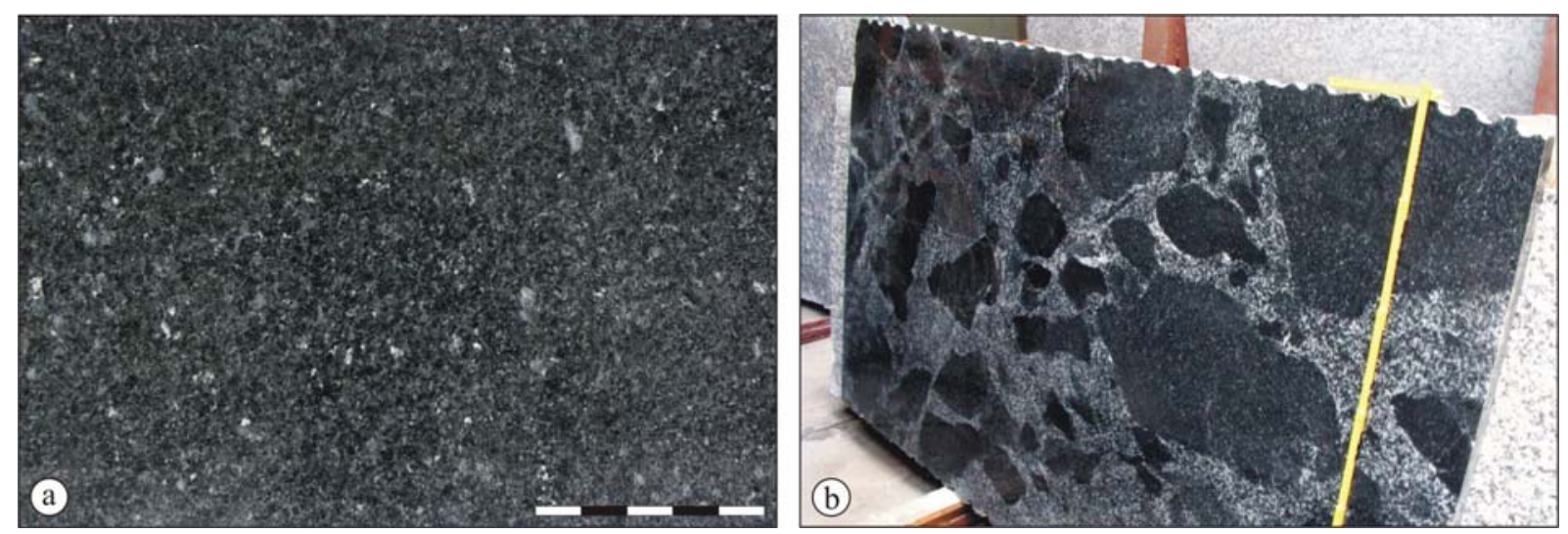

Abb. 4.14: a) Polierte Oberfläche der Varietät NEGRO RIOJANO (Gabbro, Provinz La Rioja, Maßstab in cm); b) Unmaßtafel der Dekorvarietät NEGRO OVERO mit adernetzwerkartiger Struktur infolge von Mingling- oder auch „Einfrierungs“-Prozessen.

In der Sierra de Los Quinteras, etwa $20 \mathrm{~km}$ S' der Ortschaft Chamical, ist ein Komplex eines mittelkörnigen Granodiorits (Handelsname AZUL MARA, Abb. 4.15 a) auf einer Fläche von mehreren Quadratkilometern aufgeschlossen. Die hellgraue Grundfärbung des Gesteins weist einen bläulichen Farbstich auf, die eventuell auf Gitterfehler im Quarz infolge einer Druckbeanspruchung oder auf eine Kristallisation feinster Rutilnadeln zurückgeführt werden kann. Selten wird das insgesamt sehr homogene, richtungslose Gefüge durch kleine xenolithische Einschlüsse unterbrochen.

Mikroskopisch sind in größeren Quarzkörnern, die randlich zumeist eine deutliche Rekristallisation zeigen, Deformationslamellen zu beobachten. Dagegen weisen Plagioklase überwiegend hypidiomorphe bis idiomorphe Kornformen bei Größen bis zu $5 \mathrm{~mm}$ auf und zeigen meist eine deutlich entwickelte Zonierung. Während Muskovit infolge der fein dispersen Verteilung und Bildung feinster Partikel auf bevorzugt Plagioklasen als Sekundärmineral anzusehen ist, tritt Biotit richtungslos gewachsen im primären Mineralverband mit Gehalten von zum Teil über $10 \%$ auf. Als weiteres mafisches Mineral ist 
untergeordnet Hornblende beteiligt. Akzessorisch ist Chlorit, meist als Alterationsprodukt des Biotits, Epidot und vereinzelte Erzminerale zu beobachten.

Das Landschaftsbild dieser Region ist durch die typische Wollsackverwitterung geprägt (vgl. Kap. 2.2, Abb. 2.4 b). Folglich ist der Abbau, vergleichbar mit der Situation in den Lagerstätten granitoider Gesteine in der Provinz Córdoba, an einzelne, großvolumige Boulder gebunden (Abb. 4.15 b). Eine reale Bruchentwicklung ist hier nicht anzutreffen. Vielmehr ist der Abbau mit einem ständigen Ortswechsel verbunden, wobei die anfallenden Abraummassen sowie auch häufig abgenutztes oder anderweitig unbrauchbar gewordenes Material zumeist am Ort belassen werden. Aus den gegebenen Aufschlussverhältnissen mit ausgeprägten Boulderfeldern lässt sich ein relativ engständiges Kluftsystem ableiten.
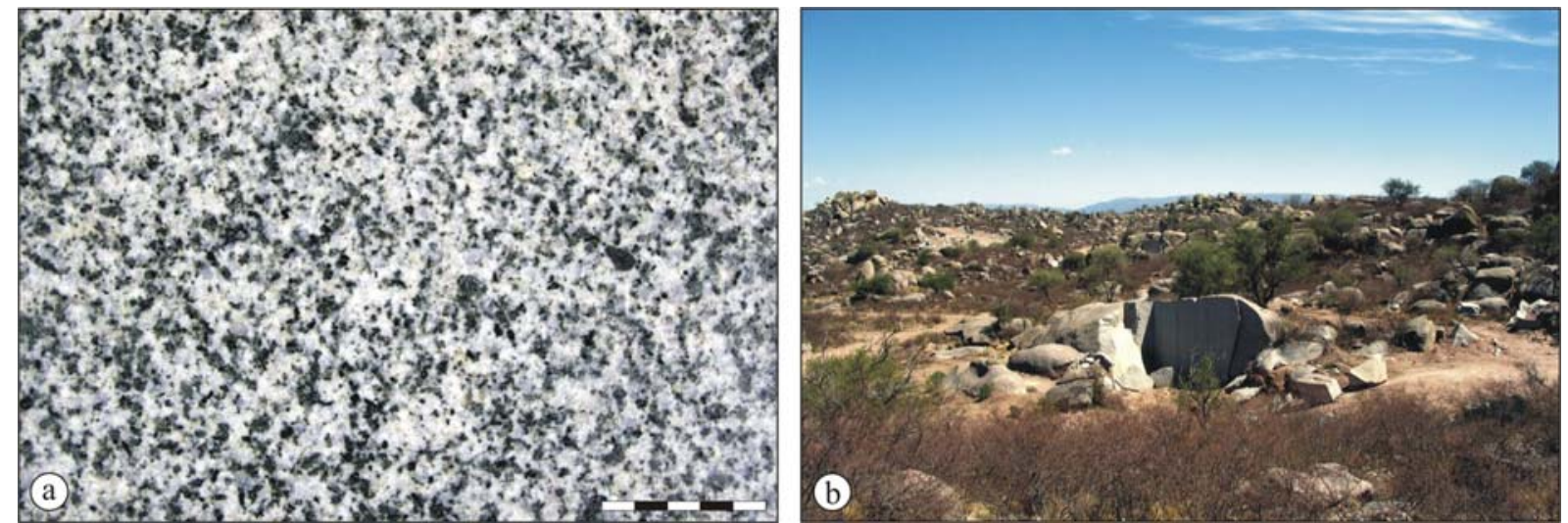

Abb. 4.15: a) Polierte Oberfläche der Varietät AZUL MARA (Granodiorit, Provinz La Rioja, Maßstab in cm); b) Infolge großflächiger Boulderfelder ist der Abbau an einzelne Großboulder ohne eine reale Bruchentwicklung gebunden.

Ein Vorkommen eines mittel- bis grobkörnigen Monzogranits (ROJO IMPERIAL) wurde in der Region La Aguadita in der Sierra de Malanzán, etwa 100 km SW' Chamical, erschlossen. Das massive Gestein setzt sich bei einer equigranularen Korngrößenverteilung dominant aus Quarz und Mikroklin, relativ geringeren Anteilen Plagioklas und bis zu 5 \% Biotit zusammen. Der Mineralbestand wird durch sehr geringe Anteile an Hornblende und verschiedener opaker Minerale vervollständigt. Es resultiert eine leicht gräuliche Rottönung als Grundfarbe (Abb. 4.16 a). Schlierenbildungen durch die Akkumulation von Biotit konnten im aufgeschlossenen Bereich der Lagerstätte nur sehr selten beobachtet werden. In dem ansonsten richtungslos erscheinenden Gefüge kann lediglich anhand einiger elongierter Quarzkörner eine schwache Foliation definiert werden.

Der zu Beginn auf mehrere Brüche verteilte und mit insgesamt bis zu 80 Arbeitern intensiv geführte Abbau wurde vor wenigen Jahren aufgrund einer zu geringen Ausbeute dimensionsgerechten Rohblockmaterials bei einem sehr hohen Abraumanteil eingestellt. Zurückzuführen ist dieser Umstand auf die Orientierung der Trennflächen und deren Abstandsverteilung (Abb. 4.16 c, d). Neben einer ungefähr horizontalen Entlastungsklüftung sind primär zwei deutliche Kluftscharen ausgebildet, die sich jedoch in einem Winkel von etwa $75^{\circ}$ schneiden. Weiterhin ist untergeordnet ein zweites, zueinander orthogonal gerichtetes Kluftsystem vorhanden, womit der Gesteinskörper eine erhebliche Zergliederung 
aufweist. Innerhalb der Abstandsverteilung, die im Bereich eines inaktiven Abbaus aufgenommen wurde, konnten in allen Raumrichtungen selten Abstände über $1 \mathrm{~m}$ festgestellt werden. Basierend auf dieser durchgreifenden Zergliederung brachten auch weitere Testabbaue in der näheren Umgebung keinen nennenswerten Erfolg. Auch eine Verbesserung der Situation durch eine Tieferführung des Abbaus erscheint fraglich. Zwar wären eventuell größere Trennflächenabstände im Bereich der Entlastungsklüfte (z-Richtung, Abb. 4.16 d) zu erwarten, jedoch keine grundlegenden Änderungen hinsichtlich der aus dem regionalen Spannungsfeld resultierenden Orientierung der Kluftsysteme zueinander.
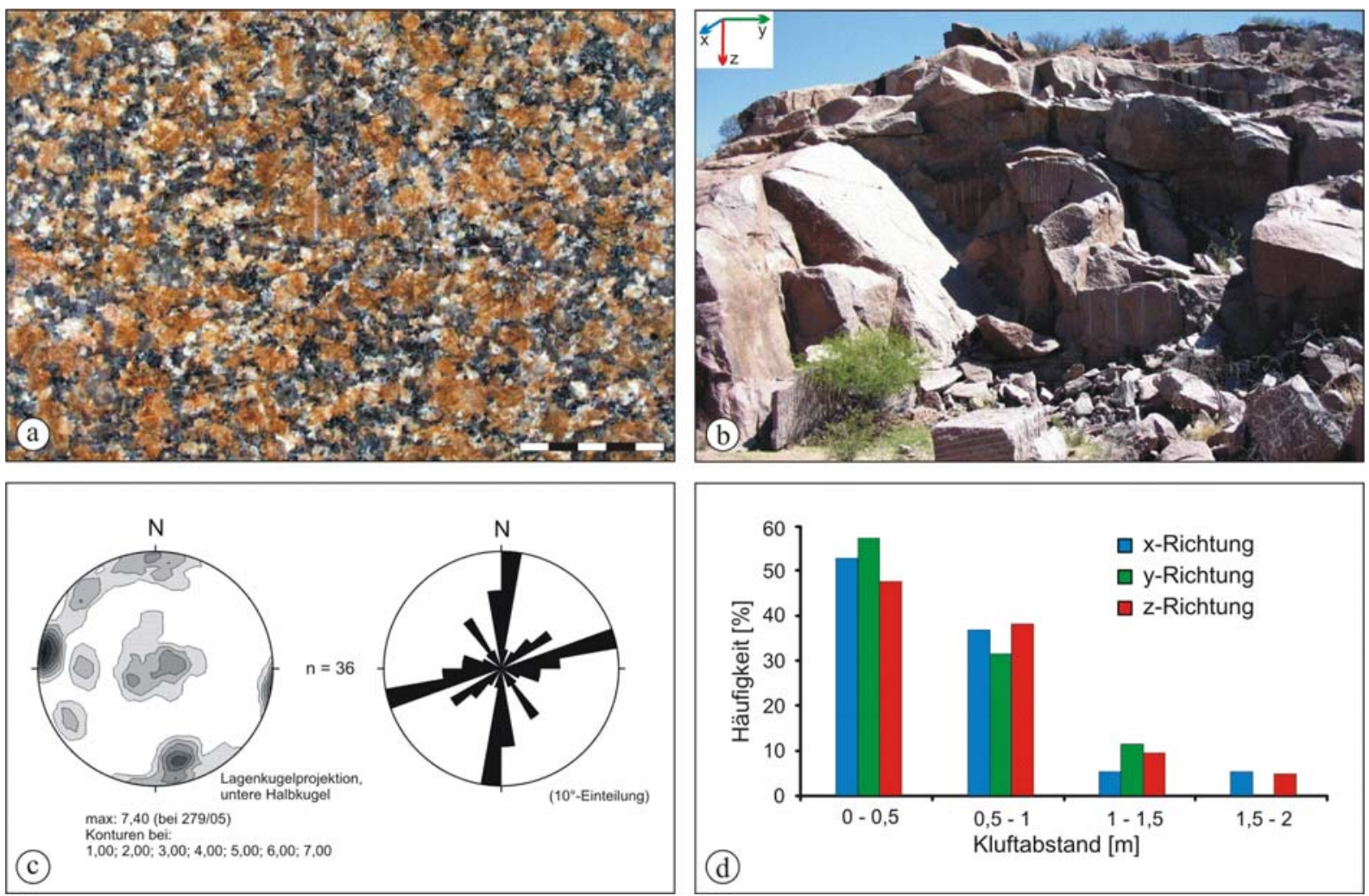

Abb. 4.16: a) Polierte Oberfläche der Varietät ROJO IMPERIAL (Monzogranit, Provinz La Rioja, Maßstab in $\mathrm{cm}$ ); b) Die Lagerstätte ist durch mehrere, spitzwinklig zueinander ausgerichtete Kluftscharen (c), die engständig ausgebildet sind (d), durchgreifend zergliedert.

\subsubsection{Provinz Buenos Aires}

Eine weitere auf dem internationalen Naturwerksteinmarkt vertretene granitische Handelsvarietät wird im Departamento Olavarría in der Sierra de Tandil gewonnen. Durch die für argentinische Verhältnisse sehr verkehrsgünstige Lage in einer Entfernung von etwa 300 km zur Hauptstadt Buenos Aires ist die problemlose Anbindung an den weltweiten Markt gegeben. Infolge der lediglich leicht hügeligen Landschaft, gestaltet sich hier, im Gegensatz zu verschiedenen zuvor beschriebenen Lagerstätten, sowohl die Extraktion als auch der Transport des zu bewegenden Materials einfacher. Nachteilig ist jedoch, dass die Neuerschließung oder Expansion eines Bruches mit dem Abtragen der quartären Sedimentbedeckung verbunden ist, die durchaus mehrere Meter Mächtigkeit aufweisen kann. Zudem ergibt sich aus der topographischen bzw. geographischen Situation, die vor allem durch einen relativ hohen Grundwasserstand gekennzeichnet, für die meisten Brüche in 
diesem Gebiet die Notwendigkeit einer nahezu permanenten Wasserhaltung, um einen ganzjährigen Abbau zu gewährleisten.

Gewonnen werden hier die farblich differenzierten Varietäten ROJO SIERRA CHICA (Abb. 4.17 a) und MARRON SIERRA CHICA (oder LABRADORITA, Abb. 4.17 b) in mehreren ausgedehnten Brüchen. Die Mineralogie dieses zumeist richtungslos grobkörnigen Alkalifeldspatgranits ist durch einen mit etwa 40 - 50 \% relativ hohen Anteil an Mikroklin, vervollständigt durch Quarz (etwa $25 \%$ ), Plagioklas (etwa $20 \%$ ) sowie Biotit und untergeordnet Hornblende als mafische Minerale (etwa $15 \%$ ) charakterisiert. Größere Mikrokline zeigen häufig bereits makroskopisch erkennbare Zwillingsbildungen. Infolge einer schwachen kataklastischen Überprägung weist das Gestein feine Risstrukturen mit sekundärem Wachstum von Chlorit, Muskovit und Eisenoxiden auf. Gleiches ist auch bei größeren Quarzkristallen zu beobachten. Darüber hinaus weisen diese auch mikroskopisch mit Schachbrettmustern und durch Rekristallisation verzahnten monophasen Korngrenzen Strukturen auf, die als Folge einer schwachen Deformation interpretiert werden können. Als Akzessorien treten Epidot, Zirkon und verschiedene opake Minerale auf.

Die Farbvarianz, als Basis zur Definition der beiden Handelssorten, wird dominant durch den Gehalt und die Art der Eisenoxide bestimmt, die vor allem in den hypidiomorphen bis xenomorphen Mikroklinen eingeschlossen auftreten. Die dunkelrote Färbung der Varietät ROJO SIERRA CHICA ist dabei auf einen erhöhten Anteil von Hämatit zurückzuführen, das entweder in Form einzelner Mineralblättchen oder als Akkumulation feiner Partikel auftreten kann. Dagegen spricht die eher bräunliche Färbung der Varietät MARRON SIERRA CHICA für Eisenoxide mit überwiegend limonitischem Charakter. Mikroskopische Untersuchungen zeigen einen eher nadeligen Habitus der Eisenoxide, was ebenfalls auf Limonit oder aber Goethit hindeuten kann.
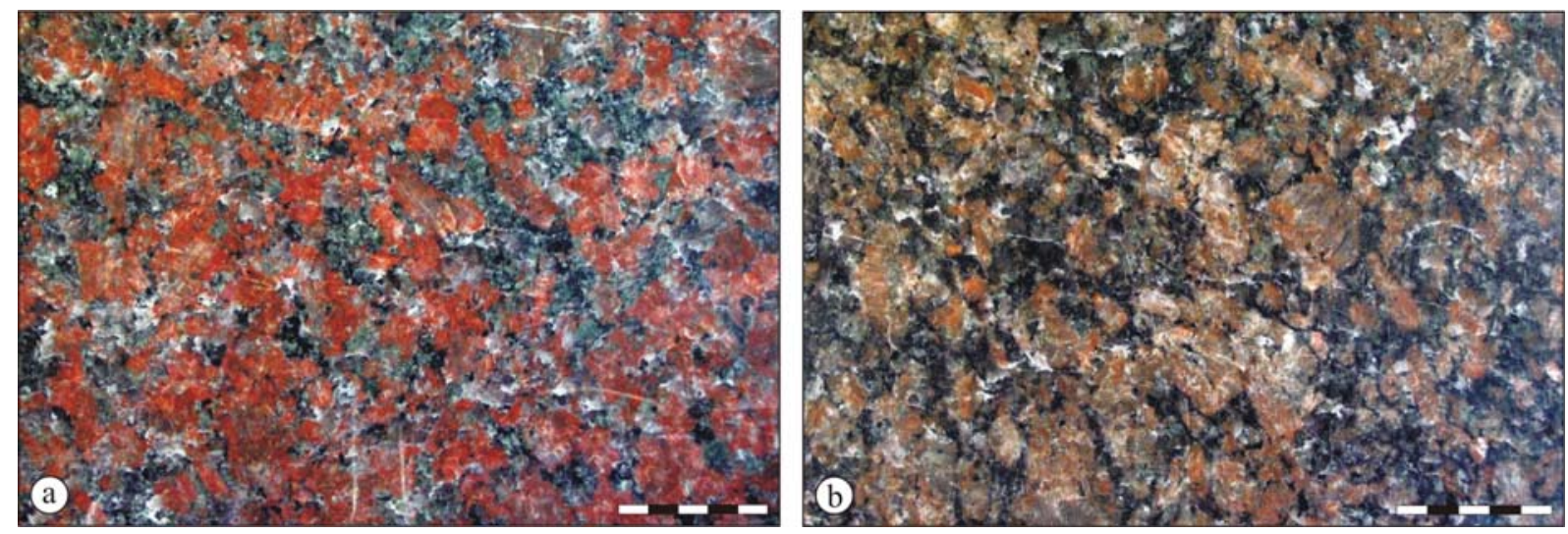

Abb. 4.17: Farbvarietäten a) ROJO SIERRA CHICA und b) LABRADORITA (Granit, Provinz Buenos Aires, Sierra de Tandil, polierte Oberfläche, Maßstab in cm).

Beide Lithologien, besonders die rote, weisen in unterschiedlicher Intensität einen grünlichen Schimmer auf, der mit einem variablen Auftreten von Epidot verbunden ist. Ursächlich dafür sind vermutlich zirkulierende Fluide in benachbarten Bereichen von Klüften und Störungen, da ein verstärkter Farbeffekt zumeist an NE-SW streichende, steil einfallende 
Störungen beziehungsweise damit assoziierten Kluftscharen (vgl. Kap. 2.2) gebunden ist (Abb. 4.18).

Neben diesen steil stehenden Störungsbereichen ist der Abbaukörper durch kataklastisch deformierte, horizontale Bewegungsbahnen zergliedert, die eine Mächtigkeit im cm- bis dm-Bereich aufweisen können. Die Lagerstättensituation, und damit das potenziell gewinnbare Volumen zur Fertigung von Rohblöcken, wird durch beide tektonischen Elemente in erheblichem Maße kontrolliert. Besonderes Augenmerk muss dabei auf die wellenförmige Dickenveränderung der Kataklasebereiche gelegt werden. Auch bei einem scheinbaren Auskeilen können verstärkt Mikrorissstrukturen in angrenzenden Bereichen vorhanden sein. Diese können nach der Ausbringung infolge der Druckentlastung reaktiviert werden und zu einem erhöhten und möglicherweise nicht kalkulierten Materialverlust führen.
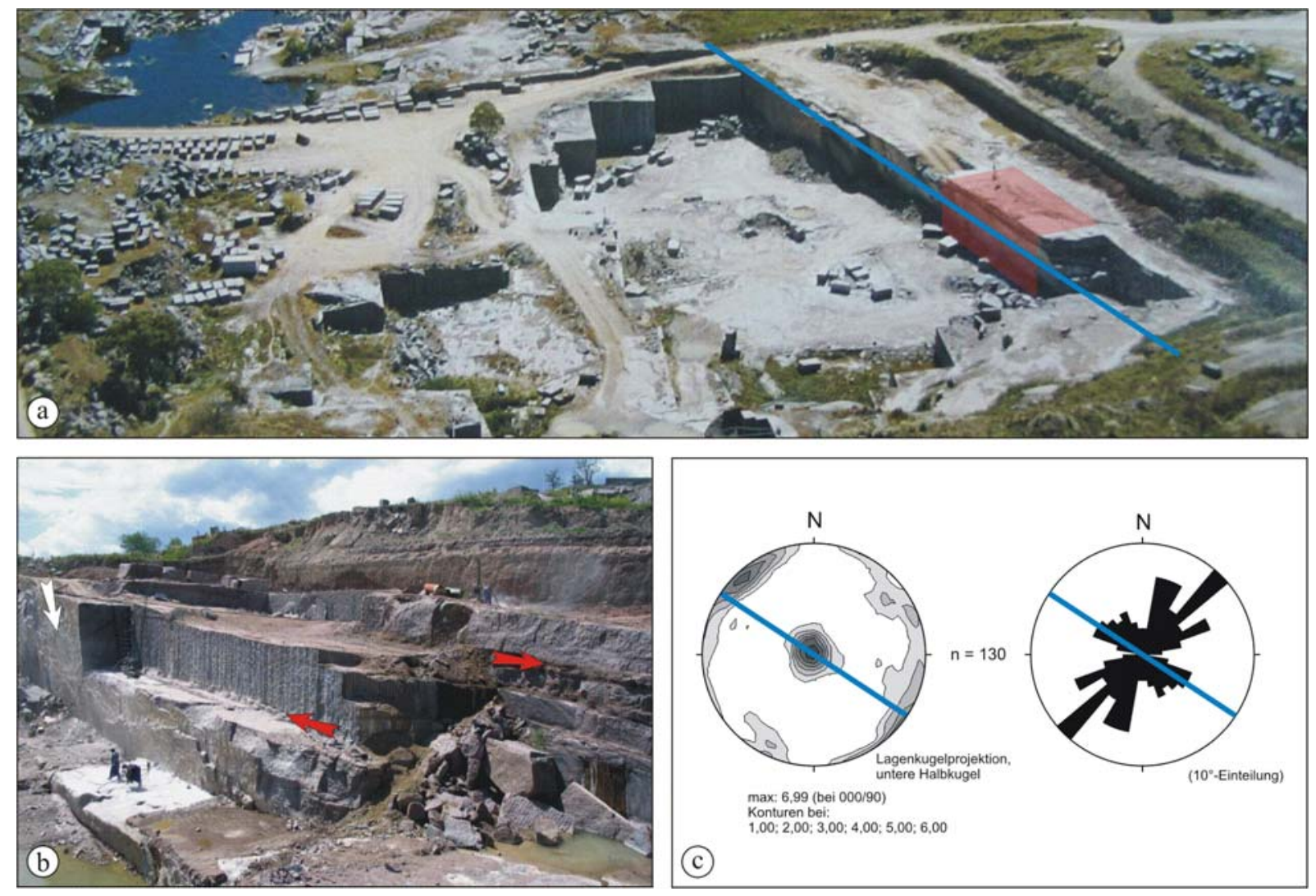

Abb. 4.18 a) Steinbruch der Fa. Marmoleria Sierra Chica S.A. b) Im aktuellen Abbaubereich (rot hinterlegt in a) ist die Lagerstätte von steil stehenden Klüften (weiße Pfeile) und horizontalen Kataklasebahnen (grüne Pfeile) durchzogen. c) Insgesamt ist ein annähernd orthogonales Trennflächengefüge mit horizontalen Entlastungsklüften ausgebildet.

\subsubsection{Lagerstätten karbonatischer Naturwerksteine}

\subsubsection{Provinz San Juan}

Neben den Provinzen Buenos Aires und Córdoba zählt die Provinz San Juan zu den wichtigsten Produzenten auf dem Sektor der karbonatischen Wertgesteine (Zolezzi et al. 2005). Dabei werden in großräumig erschlossenen Abbaugebieten intensiv verschiedene 
Kalksteine als Rohstoff zur Zementproduktion abgebaut. Die Gewinnung von Naturwerkstein beläuft sich lediglich auf einen verschwindend geringen Teil. Die im Rahmen dieser Studie untersuchte Lagerstätte eines Travertins befindet sich im Departamento Alberdon, etwa $30 \mathrm{~km}$ N' der Provinzhauptstadt San Juan. In dieser Region stehen 80 \% der gesamten Wirtschaft in enger Verbindung mit der Produktion oder Weiterverarbeitung des Rohstoffs Naturstein.

Im N und NW der Provinz San Juan ist das kambrische Basement von neogenen, kontinentalen Sedimenten überdeckt, auf denen unregelmäßig quartäre Schotterterrassen zur Ablagerung kamen (Colombo et al. 2000). Durch neotektonische Aktivität ist ein N-S streichendes Störungssystem ausgebildet (Bastías et al. 1990), das die quartäre Bedeckung durchzieht und so, gebunden an hydrothermale Quellen (Sánchez et al. 1986), den Aufstieg kalziumkarbonatreicher Lösungen begünstigt. Daraus resultiert die Bildung einer Vielzahl kleinerer und größerer Travertinkörper, die zumeist eine mit Stratovulkanen vergleichbare Form aufweisen. Einige wurden zur Gewinnung von Werkstein erschlossen. Der wichtigste Bruch „La Esmeralda“ (Abb. 4.19) befindet sich im größten dieser Körper im Gebiet La Laya. Anhand geologischer Untersuchungen durch die zuständige Bergbaubehörde konnten hier Reserven von mindestens $300.000 \mathrm{~m}^{3}$ Material nachgewiesen werden. In diesem Rahmen abgeteufte Bohrungen im N' Bereich der Lagerstätte (Abb. 4.19 c) zeigen bis in eine Teufe von etwa $25 \mathrm{~m}$ ein relativ gleichbleibendes Material. Lediglich die für Travertine zumeist übliche Variabilität hinsichtlich des Charakters und des Volumenanteils der Porosität ist zu beobachten (vgl. Kap. 3.8.1, Abb. 3.44).

Der Travertinkomplex wird durch zwei dominante Kluftgruppen segmentiert. Eine dieser Kluftscharen (rot in Abb. 4.19 c, d) verläuft zirkulär mit dem ehemaligen Aufstiegskanal als Zentrum. Die zweite Gruppe (grün in Abb. 4.19 c, d) zerschneidet den Komplex radialstrahlig vom Zentrum ausgehend. Dabei beträgt der Abstand der dominanten, bis zu $15 \mathrm{~cm}$ weit geöffneten Klüfte jeweils mehrere Meter.

Aktuell erfolgt der Abbau auf zwei Ebenen. Im unteren Bereich der Lagerstätte zeigt das Gestein einen deutlichen Lagenbau und wirkt insgesamt etwas grünlich oder bräunlich, was auf die Verunreinigung einzelner Lagen mit organischem Material zurückzuführen ist. In der oberen Partie erscheint das Gestein dagegen weniger lagenreich und damit massiver und homogener (Abb. 4.19 b). Diese Tatsache beruht auf der stetigen Ausdünnung der einzelnen Lagen mit zunehmendem Abstand vom ehemaligen Aufstiegskanal der $\mathrm{CaCO}_{3}$-reichen Lösungen. Zum Zeitpunkt dieser Studie war der Abbau auf die tiefer liegende Ebene konzentriert, wobei eine Schrämmsäge (Abrasivkettensäge) sowohl für die vertikale als auch horizontale Schnittführung eingesetzt ist. Für die Formatierung der Rohblöcke wird neben der konventionellen Methode (Pressluftbohrer, Federkeile) auch eine Diamantseilsäge eingesetzt. In der Folge werden zwei Rohblockqualitäten differenziert. In der Verarbeitung der Rohblöcke werden zwei Schnittrichtungen unterschieden: i) „a la veta“ -Schnittführung senkrecht zum Lagenbau und ii) „al agua“ - Schnittführung parallel zum Lagenbau. Besonders durch die parallele Schnittführung kann sich gerade bei der an Lagen reicheren Varietät ein abwechslungsreiches, teils nebulöses Dekor ergeben. Im Allgemeinen findet das Gestein sowohl im Innen- als auch im Außenbereich Verwendung, wobei sich jedoch gerade 
im Außenbereich eine möglicherweise schnelle und deutlich wertmindernde Verschmutzung infolge der offenen Porosität einstellt.
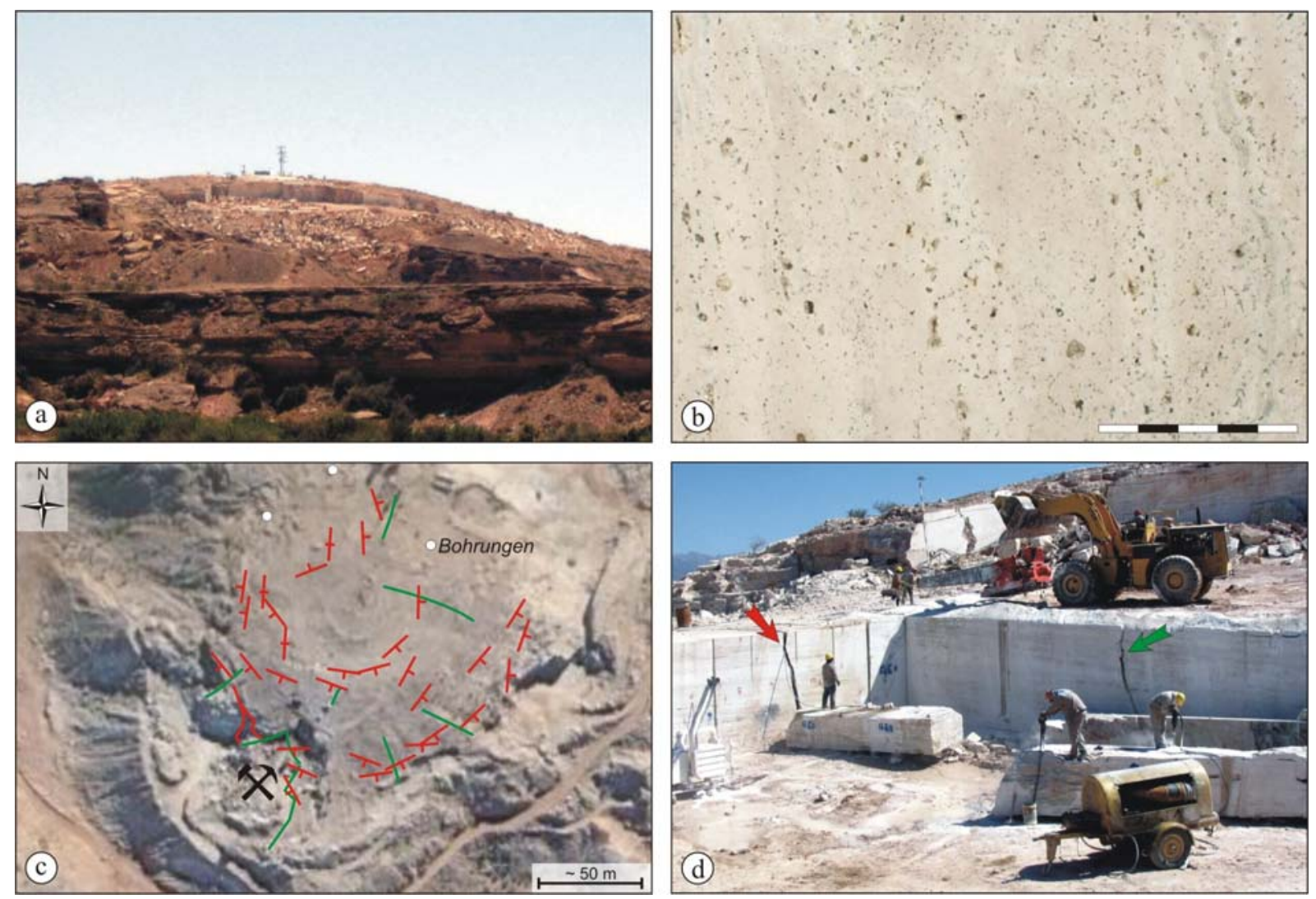

Abb. 4.19: a) Werksteinbruch „La Esmeralda“ (Travertin, Fa. NaturalStones S.A., San Juan): auf quartären Schotterterrassen sind entlang von N-S streichenden Störungssystemen Travertinkörper gebildet; b) Varietät TRAVERTINO BLANCO (polierte Oberfläche, Maßstab in $\mathrm{cm}$, Schichtung senkrecht); c) Luftbild des Travertinkörpers mit d) aktuellem Abbaubereich (Bergbausymbol in Bild c): die Lagerstätte ist durch zwei dominante Kluftscharen gegliedert. Nähere Erläuterungen im Text.

\subsubsection{Provinz Neuquén}

Die Provinz Neuquén liegt im NW' Bereich Patagoniens. Die Produktion von Naturwerkstein ist hier infolge der geologischen Gegebenheiten fast ausschließlich auf verschiedene karbonatische Varietäten fokussiert. Dabei bildet die Region Zapala, rund 180 km W' der Stadt Neuquén gelegen, ein wichtiges Zentrum. In einer Vielzahl der hier vorhandenen Lagerstätten wurde jedoch der Abbau gänzlich eingestellt oder kann, infolge der klimatischen Bedingungen, nicht ganzjährig geführt werden. In manchen Fällen stehen die Stilllegungen nicht in Zusammenhang mit der letzten Wirtschaftskrise, sondern sind in einem Wechsel des Tätigkeitsfeldes der betreibenden Firmen begründet (pers. Mitt. Lic. M.A. Bruna, Dirección General de Minería). Das Geologische Landesamt der Provinz Neuquén arbeitet an verschiedenen Projekten, die die Erkundung zur Wiederinbetriebe einiger Lagerstätten zum Ziel haben. Zudem sollen Untersuchungen zur Neuerschließung von Lagerstätten zur Gewinnung von Naturwerkstein durchgeführt werden.

Neben den zum Teil extremen klimatischen Bedingungen stellt die Anbindung an den nationalen und internationalen Markt für die gesamte Region ein grundlegendes Problem 
hinsichtlich des Managements der Vermarktung sowohl der Rohblockware als auch der Fertigprodukte dar. Die Entfernung zur Hauptstadt Buenos Aires beträgt etwa $1000 \mathrm{~km}$. Die nächstgelegene Möglichkeit zur Verschiffung gibt der internationale Seehafen in Corral, Chile. Die Entfernung beträgt hier lediglich etwa $300 \mathrm{~km}$, doch ist der Transport von Rohblöcken mit der schwierigen Passage der Gebirgskette der Anden verbunden. Daher geht das ausgebrachte Material fast ausschließlich in den regionalen und zum Teil auch nationalen Markt ein. Eine intensive Entwicklung umfangreicher Gewinnungs-, Verarbeitungs- und Vermarktungskonzepte gestaltet sich schwierig.

Zwischen der Stadt Zapala im S und der etwa 150 km N' gelegenen Stadt Curacó ist eine kontinuierliche Sequenz von Karbonatsedimenten nachgewiesen (Brodtkorb et al. 1982). Lokal sind Karbonatbänke jurassischen Alters mit Mächtigkeiten von mehreren Metern direkt aufgeschlossen. Die untersuchten Lithologien zeigen im Allgemeinen eine massive, feinstkörnige Struktur, wobei das Farbspektrum von homogenen graubraunen Tönen bis zu gelblichen und goldgelben Farben reicht. Der Fossilgehalt ist in seiner Menge und der Partikelgröße in der Vertikalen als auch Horizontalen variabel und reicht von mikroskopisch feinen Bruchstücken bis zu Einzelfunden im dm-Bereich.

Die erste aufgenommene Lagerstätte befindet sich im Departamento Catán Lil, etwa $60 \mathrm{~km}$ S’ der Stadt Zapala. Der dortige Abbau der Handelssorte CALIZA AMARILLA (Abb. 4.20) wurde, immer wieder zeitweise unterbrochen, für vier Jahre betrieben. Der Abbau erfolgte dabei nach der anfänglichen Entnahme einiger Probeblöcke lediglich auf direkte Anfrage, zumal es zu Komplikationen hinsichtlich der Konzession für den Betrieb kam. Der aktuelle Bruch erstreckt sich daher lediglich über wenig mehr als $100 \mathrm{~m}^{2}$ Grundfläche.

Die Extraktion der Blöcke beschränkte sich auf eine nahezu söhlig gelagerte Bank von etwa 1,4 m Mächtigkeit. Infolge einer geringen tektonischen Beeinflussung ist ein annähernd orthogonal ausgerichtetes Kluftsystem senkrecht zur sedimentären Schichtung ausgebildet (Abb. 4.20 a). Der auflagernde Abraum setzt sich aus mehreren dünnbankigen Lagen von insgesamt nicht mehr als $1 \mathrm{~m}$ zusammen (Abb. 4.20 b). Eine sedimentäre Bedeckung ist infolge der klimatischen Bedingungen nicht vorhanden. Ein optimaler Abbau wäre hier durch vollständiges Beräumen der bauwürdigen Bank zu erreichen (vgl. Kap. 2.3.2.1 und 2.5). Darauf aufbauend können unter Ausnutzung der natürlichen Trennflächen die in-situ Blöcke aus dem Gesteinsverband herausgelöst werden. Infolge der Rechtwinkligkeit des Kluftsystems sind dabei geringe Abraumraten bei der dimensionsgerechten Formatierung zu erwarten (vgl. Kap. 2.4, Abb. 2.22 b und 2.26)

Das Gestein weist eine mikritische Textur auf, wobei Fossilfragmente im mm-, selten im cm-Bereich in das Gefüge eingeschlossen sind (Abb. 4.20 c). Farblich dominieren warme Gelbtöne mit Farbübergängen zwischen leicht gräulich hellen $\mathrm{zu}$ bräunlichen, ins crémefarbene spielenden Nuancen. Wertmindernd sind vulkanische Bomben mit Durchmessern bis zu $20 \mathrm{~cm}$ eingeschlossen, deren Auftreten im aufgeschlossenen Bereich lediglich auf die direkt im Hangenden folgende Schicht beschränkt ist. In der abbauwürdigen Bank sind schmalere, vertikal begrenzte Zonen mit knollig gewachsenen Aggregaten von amorphem Quarz („Phtanit“) in Größen bis zu $15 \mathrm{~cm}$ zu beobachten (Abb. 4.20 d). Diese 
Akkumulationen sind auf unterschiedliche Druckbedingungen während der Diagenese zurückzuführen, wodurch eine Migration silikatreicher Fluidphasen und die lokale Anreicherung in weniger druckbelasteten Bereichen angeregt wurden. Diese Bereiche, die im aufgeschlossenen Teil nicht mehr als $1 \mathrm{~m}$ bis $2 \mathrm{~m}$ Breite aufweisen, sind bei der Verarbeitung des Gesteins auszuschließen.
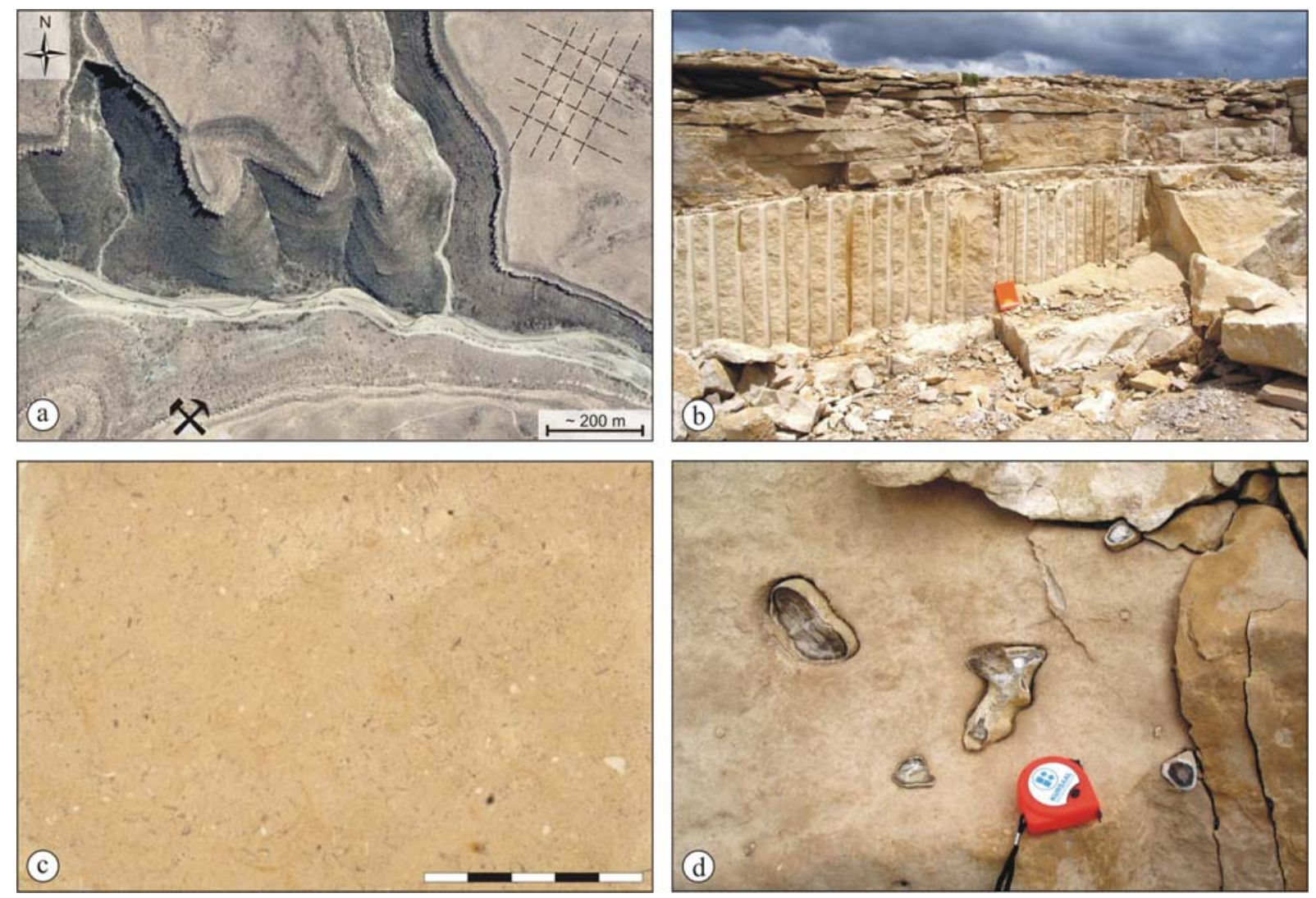

Abb. 4.20: Kalkstein CALIZA AMARILLA (Provinz Neuquén): a) Im Luftbild der näheren Umgebung des Werksteinbruchs (Bergbausymbol) wird die schematisch dargestellte Ausrichtung des orthogonalen Trennflächengefüges der bauwürdigen Bank deutlich. b) Dünnbankige Lagen bilden den aufliegenden Abraum. c) Polierte Oberfläche des Kalksteins (Maßstab in cm, Schnitt parallel zur Schichtung). d) Bildung von „Phtanit“-knollen infolge unterschiedlicher Druckbedingungen während der Diagenese.

Ein deutlich massigeres und dichteres Gefüge zeigt die Varietät LUNEL COVUNCO, die in einer Lagerstätte etwa $30 \mathrm{~km}$ NW' von Zapala abgebaut wurde. Bis zum Ende der 90er Jahre wurde dieser Werkstein über einen Zeitraum von fast 30 Jahren periodisch genutzt. Der dolomitische Kalkstein weist ein sparitisches, hochkompaktes Gefüge auf. Im Mineralbestand sind lediglich etwa 15 \% bis 20 \% mikritischen Calcits beinhaltet. Das sedimentäre Lager ist makroskopisch nur anhand schwacher Farbdifferenzen und horizontaler Anreicherungen feiner Fossilfragmente erkennbar. Hinsichtlich der Grundfarbe dominieren hier hellgräulich braune bis ockerfarbene Töne (Abb. $4.21 \mathrm{a}$ ).

Die angeschnittene Schicht fällt seicht nach E ein und weist im Bruch eine Mächtigkeit von rund 1,7 m auf (Abb. 4.21 b). Eine alternierende Schichtung aus dünneren Kalkstein- und Tonlagen im cm- bis dm-Bereich, decken die abbauwürdige Bank ab. Da eine sedimentäre Bedeckung nicht ansteht, beträgt die Gesamthöhe des Abraums etwa 1,0 m bis 1,2 m. Die Lage des Abbauortes am Rand eines über mehrere Quadratkilometer ausgedehnten 
Kalksteinplateaus führt verstärkt zur Öffnung des stratigraphischen Lagenbaus. Forciert wird dieser Prozess zudem durch die räumliche Nähe zu einer regionalen Scherzone. Mit zunehmender Entfernung in Richtung des Zentrums des Plateaus tritt dieser Effekt jedoch mehr und mehr zurück (pers. Mitt. Lic. M.A. Bruna, Dirreción General de Minería).

Die insgesamt sehr homogene Struktur des Gesteins wird durch dominante Klüfte, feine Fissuren und Rissstrukturen unterbrochen. Im aufgeschlossenen Bereich ist eine annähernd orthogonale Ausrichtung der Haupttrennflächen gegeben, die mit der Situation in der Lagerstätte der Varietät CALIZA AMARILLA vergleichbar ist. Zu beobachten ist jedoch häufiger ein bogenartiger Verlauf der die bauwürdige Bank zergliedernden Klüfte, was einen erhöhten Rauabfallanteil beim Aufsägen der Blöcke nach sich zieht (vgl. Kap. 2.1, Abb. 2.1 b). Die Klüfte, die im Bruch zumeist eine deutliche Öffnung von zum Teil mehreren Zentimetern aufweisen, zeigen häufig Kristallrasenbildungen von idiomorphen Calcitkristallen. Ein Großteil der Fissuren oder auch feinen Risse ist durch das sekundäre Wachstum des Calcits geschlossen (in Abb. 4.21 b).
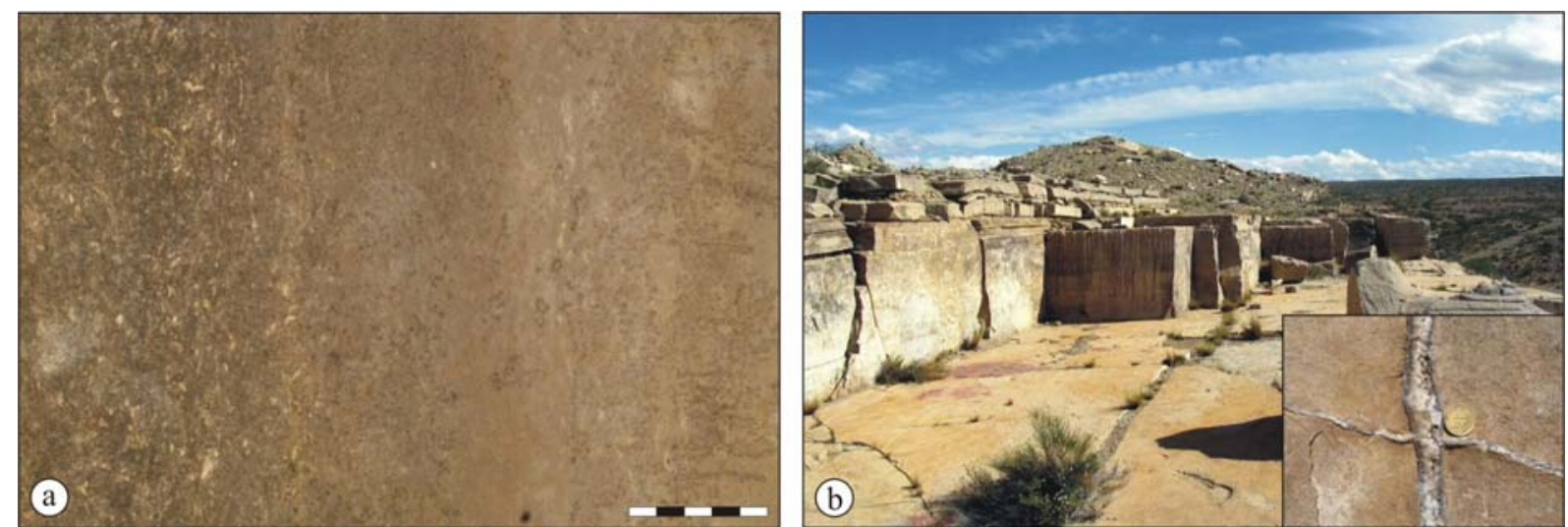

Abb. 4.21: Dolomitischer Kalkstein LUNEL COVUNCO (Provinz Neuquén): a) Der sedimentäre Lagenbau (hier senkrecht) kann anhand leichter Farbunterschiede und der Anreicherung feiner Fossilfragmente identifiziert werden (polierte Oberfläche, Maßstab in cm). b) Die Lagerstätte ist durch ein zumeist orthogonales Kluftsystem gegliedert. Kleines Bild: Klüfte bzw. Rissstrukturen mit der Mineralisation von z.T. idiomorphen Calcitkristallen.

Ein sehr eindrucksvolles Dekor bietet die Handelssorte PATAGONIA GOLDEN (Abb. 4.22). Es handelt sich um einen oberkretazischen Kalkstein (Formation Picún Leúfu), der eine Vielzahl mariner Fossilien und Fossilfragmente im mm- bis dm-Bereich eingebettet in eine mikritische Matrix aufweist. Auffällig sind dabei vor allem mehrere Dezimeter große Exemplare von Brachiopoden, Muscheln oder auch Cephalopoden in einem hervorragenden Erhaltungszustand (Abb. 4.22 a). Die Lagerstätte dieser Lithologie ist etwa 25 km SW' von Zapala erschlossen. Zum Zeitpunkt dieser Studie lag die Produktion bei etwa 15 bis $20 \mathrm{~m}^{3}$ pro Monat, wobei nach Auskunft des verarbeitenden Betriebes Blockgrößen zwischen $4 \mathrm{~m}^{3}$ und $7 \mathrm{~m}^{3}$ erreicht werden konnten. Der Abbau wird nur zeitlich unterbrochen in Abhängigkeit der direkten Nachfrage des Marktes durchgeführt. Außerdem wirken sich hier infolge der Höhenlage von etwa 1300 mNN die extremen klimatischen Bedingungen verstärkt aus, so dass über die Wintermonate der Bruchbetrieb vollständig eingestellt werden muss. 
Farblich dominiert in dieser Lithologie ein gelbliches Braun bis Ocker. In der gesamten Mächtigkeit von etwa 7,5 m dieser fossilreichen Bänke treten auch kleinere Bereiche mit einem deutlich rötlicheren Farbstich auf (Bruna 2001). Makroskopisch zeigt sich eine geringe Porosität. Das Gefüge ist jedoch von einem Netz feiner Rissstrukturen durchzogen, welches vermutlich infolge des hohen Gehalts an fossilen Fragmenten bei einer gleichzeitig relativ geringen diagenetischen Verfestigung des Sediments generiert wurde. Daher ist im Prozess der Verarbeitung die Stabilisierung mit hinterlegten Gewebematten und Kunstharz unumgänglich und wird ausnahmslos praktiziert. Größere Hohlräume oder auch Calcitaggregate treten dagegen nur selten in Erscheinung. Die im Bruch aufgeschlossenen, massiven Bänke weisen eine Schichtmächtigkeit von bis zu 1,3 m auf (Abb. 4.22 b). Dabei zeigt die obere Bank eine z.T. starke Verkarstung. Das Schichteinfallen beträgt etwa $15^{\circ}$ bis $20^{\circ}$, wodurch sich das Ausbringen von Rohblöcken relativ problemlos gestaltet. Zu Beginn des Abbaus wurden zum Lösen der Blöcke verschiedene Explosiva eingesetzt. Da die damit verbundenen Druckwellen eine weitere erhebliche Schwächung des Gesteinsgefüges nach sich zieht, erfolgt die Extraktion heute unter Verwendung von Expansionszementen. Bei einer intensiveren Nutzung dieser Lagerstätte wäre demnach der Einsatz einer Schrämm- oder Diamantseilsäge anzustreben, um den Abbau so schonend wie möglich zu gestalten.
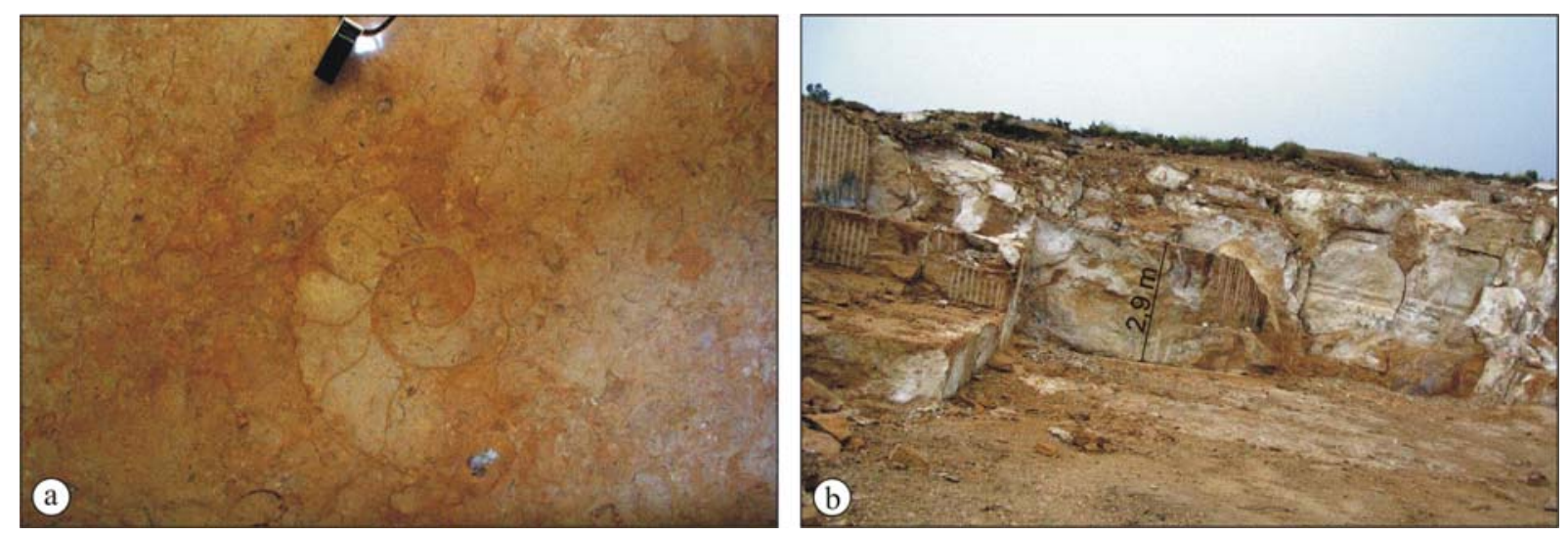

Abb. 4.22: Kalkstein PATAGONIA GOLDEN (Provinz Neuquén): a) Zahlreiche Fossile und Fossilfragmente sind in eine mikritische Matrix eingebettet (polierte Oberfläche). b) Der Abbau wird lotrecht zur sedimentären Schichtung unter weitgehender Ausnutzung des orthogonalen Trennflächensystems geführt (Steinbruch Fa. Patagonia Golden S.R.L., Neuquén).

\subsubsection{Provinz Buenos Aires}

In der geologischen Provinz „Tandilia“, die sich von der Stadt Mar del Plata in NE’ Richtung erstreckt, werden in einer Vielzahl an Lagerstätten verschiedene calcitische und dolomitische Kalksteine abgebaut. Mit einer Produktion von mehr als 9.000.000 t ausgebrachtem Material im Jahr 2003 stellt die Provinz Buenos Aires den wichtigsten Zulieferer für die Zementproduktion dar (Zolezzi et al. 2005). Die entsprechenden Lagerstätten zeigen zumeist eine erhebliche Zergliederung durch sehr engständige Trennflächensysteme. In wenigen Vorkommen ist jedoch auch die Ausbringung von werksteingerechten Rohblöcken möglich. 
In den Sierras Bayas, etwa 20 km W' der Stadt Olavarría ist die Lagerstätte eines Dolomits erschlossen (Abb. 4.23 a). Das gesamte Vorkommen setzt sich aus mehreren Rücken des Dolomits zusammen, die durch E-W verlaufende Störungen voneinander getrennt sind. In einem Bruch erfolgt hier seit dem Jahr 2002 die Gewinnung großvolumiger Blöcke, die als Werkstein Verwendung finden. Die Ausbringung ist auf zwei massive Bänke konzentriert, wobei Blockgrößen zwischen 3,5 $\mathrm{m}^{3}$ und 5,5 $\mathrm{m}^{3}$ realisiert sind.

Der im Hangenden anstehende Abraumbereich beträgt in der Gesamtmächtigkeit etwa 6 bis $7 \mathrm{~m}$. Dieser ist stärker gegliedert und zeigt eine Sequenz verschieden mächtiger Dolomitschichten in Wechsellagerung mit tonig-mergeligen Bändern (Abb. 4.23 b). Die oberen Dolomitbänke weisen dabei auch intern eine stärkere Zerklüftung auf. Die Aufnahme mehrerer Vertikalprofile, verteilt über die Breite des Bruches, ergab für die massiven Dolomitbänke Schichtmächtigkeiten zwischen 0,4 m und 1,9 m. Es ist davon auszugehen, dass auch in stärkeren Lagen eine makroskopisch nicht erkennbare Gliederung des Lagenbaus vorhanden ist. Trotz dessen sollte die Beräumung der zum Werksteinabbau genutzten Partie relativ schonend ausgeführt werden, um massivere, weniger zergliedert Bereiche nutzen zu können (s.a. Lorenz \& Gwosdz 2003). Hierbei wäre die Produktion von kleinvolumigeren Bauelementen, so z.B. für Trockenmauerwerke oder den Garten- und Landschaftsbau, denkbar (vgl. Kap. 2.1).
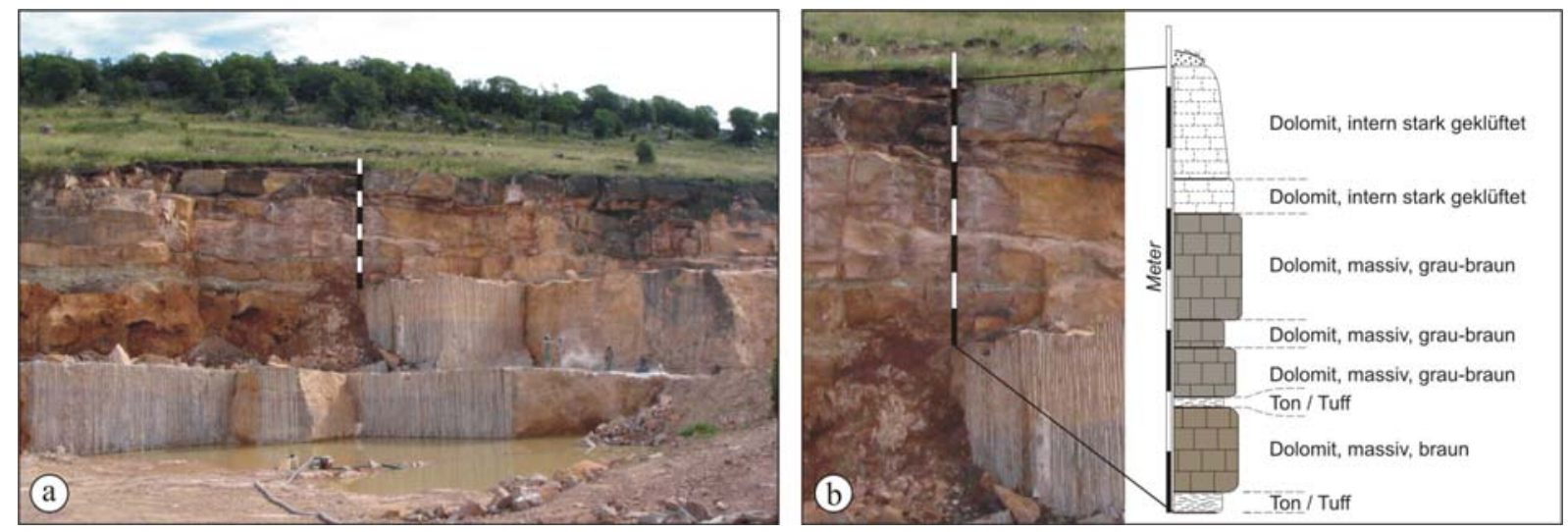

Abb. 4.23: a) Dolomit-Werksteinbruch der Fa. Terrum Minera S.R.L. (Olavarría, Provinz Buenos Aires; Maßstab in m); b) der aufliegende Abraumbereich ist aus z.T. massiven Dolomitbänken in Wechsellagerung mit tonig-mergeligen Schichten zusammengesetzt.

Beide angeschnittenen Werksteinbänke zeigen ein mikritisches, massives Gefüge. Während im Liegenden ein fein gebänderter Lagenbau ausgebildet ist, erscheint die obere Partie in ihrer Zusammensetzung relativ homogener und weist eine hellbraune bis cremefarbene Grundtönung auf. Diese Färbung ist auf eine durchgreifende Limonitisierung des Gesteinsmaterials zurückzuführen. Dagegen ist das Material der unteren Bank durch eine eher graubraune Grundfarbe, die teilweise einen leicht grünlichen Farbstich aufweisen kann, charakterisiert. Die dunklere Färbung ist aus einem alternierenden Wechsel bläulich grauer und hellbrauner Bänder entwickelt. Basierend auf diesen Farbdifferenzen definieren sich die Handelssorten DOLOMITA DORADA (gold, Abb. 4.24 a), DOLOMITA GRIS (grau) und DOLOMITA VERDE (grün). 

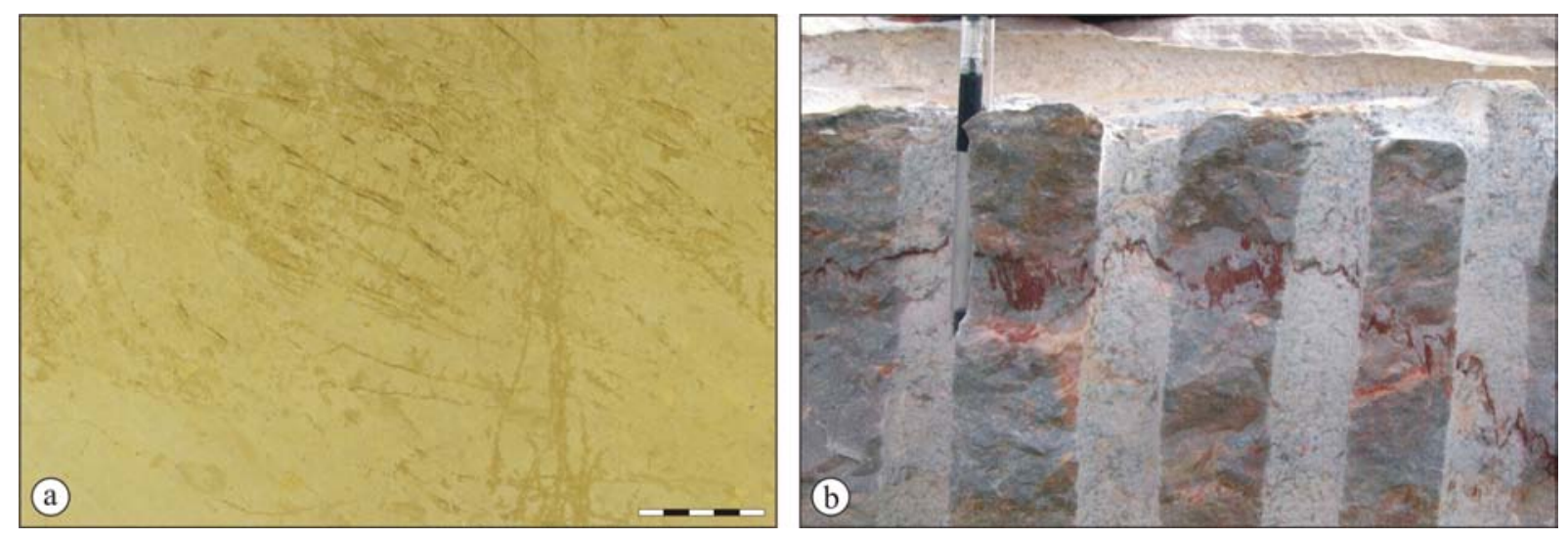

Abb. 4.24: a) polierte Oberfläche der Varietät DOLOMITA DORADA mit feinen, auskristallisierten Fissuren (Maßstab in cm); b) Stylolithenband in einem Rohblock der Varietät DOLOMITA VERDE.

Das ausgeprägte, annähernd orthogonal angelegte, steil einfallende Kluftsystem weist im Allgemeinen Abstände von mehreren Metern zwischen den individuellen Trennflächen auf und hat somit auf die potenzielle Gewinnung von Rohblöcken keinen direkten negativen Einfluss (vgl. Kap. 2.4). Dagegen begünstigt sowohl die geöffnete Klüftung als auch das Aufklaffen des sedimentären Lagers die Zirkulation von Bodenwässern. In der Folge kommt es zur Auslaugung des Gesteins, was die Bildung von Kavernen und Schlotten nach sich zieht. Vereinzelt sind auf manchen Kluftflächen auch Harnische erkennbar, die leichte Bewegungen im Gesteinskörper dokumentieren. Neben dem dominierenden Kluftsystem treten im aufgeschlossenen Bereich untergeordnet Drucklösungssuturen auf (Abb. 4.24 b). Diese können vor allem bei flächigen, dünnplattigen Werkstücken zu einer deutlichen Minderung der mechanischen Stabilität führen (vgl. Kap. 3.8.3). Dagegen erscheinen feine, meist vollständig auskristallisierte Fissuren, die anscheinend gehäuft in der Farbvarietät DORADA auftreten, keinen solchen Effekt zu haben.

\subsubsection{Provinz Córdoba (Marmor)}

Auch in der Provinz Córdoba ist eine Vielzahl von Lagerstätten in karbonatischen Gesteinen erschlossen, die der Rohstoffversorgung der Zementindustrie dienen. Im Jahr 2003 wurde dabei die Provinz als zweitgrößter Rohstoffproduzent erfasst (Zolezzi et al. 2005). Das Gebiet in den Sierras de Córdoba, auf die sich ein großer Teil der Produktion konzentriert, ist auch der Herkunftsort für das wahrscheinlich bekannteste Ornamentgestein Argentiniens, dem Marmor AZUL CIELO (Abb. 4.25). Das außergewöhnliche Dekor dieses Gesteins ist durch die pastellblaue Farbe und einer Vielzahl struktureller Elemente geprägt.

Die Genese des Gesteins steht im Zusammenhang mit der präkambrischen Metamorphose der Sierras Pampeanas. Der zumeist hochreine Marmor zeigt eine mittel- bis grobkörnige Textur. Neben Calcit können feinst verteilt geringe Anteile an Diopsid, Wollastonit, Apatit und auch Quarz als akzessorische Mineralbestandteile auftreten. Die Quarze sind dabei meist rekristallisiert und zu sehr feinen Bändern akkumuliert. Die beeindruckende Blaufärbung ist vermutlich auf den Effekt eines physikalischen Phänomens im Kristallgitter zurückzuführen, wodurch ein Blauschimmer im Kern der Calcitminerale entsteht. In umfangreiche Untersuchungen hinsichtlich des Chemismus konnten keine Elemente nachgewiesen werden, 
die eine derartige Färbung verursachen könnten. Außerdem hat sich gezeigt, dass die Blaufärbung bei einer Erhitzung des Gesteins über $200^{\circ} \mathrm{C}$ verloren geht (Lamberghini et al. 2000).
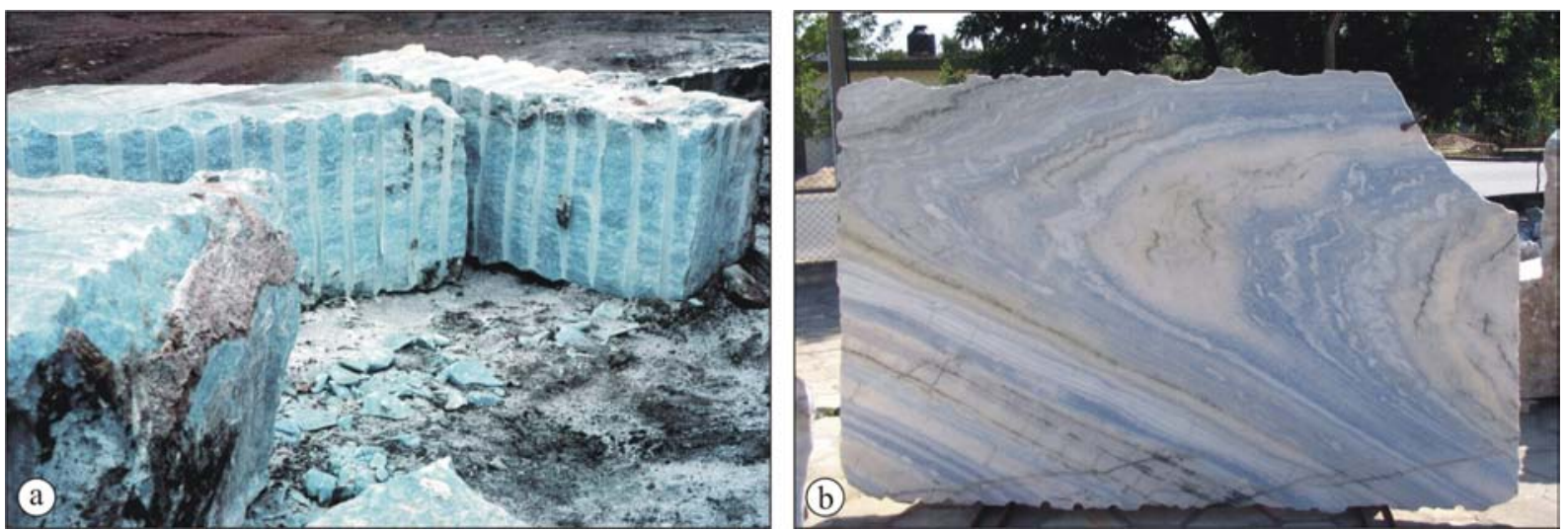

Abb. 4.25: Calcitische Marmorvarietät AZUL CIELO (Provinz Córdoba): a) ausgebrachte Rohblöcke (Foto: Fa. Altamar S.R.L.) und b) polierte Unmaßtafel.

In seiner Gesamtheit weist der Marmorkörper bei einer Fläche von rund 300 ha eine erhebliche Heterogenität sowohl in der Ausbildung des Trennflächengefüges als auch in der Verteilung verschiedener Farb- und Strukturvarietäten auf. Nach Firmeninformation können lediglich rund $20 \%$ des ausgebrachten Materials der Varietät AZUL CIELO zugeordnet werden. Der verbleibende Teil gliedert sich zu etwa gleichen Anteilen in weiße, graue und rosafarbene Marmore.

Zur Aufnahme und Bewertung des Trennflächengefüges des Lagerstättenkörpers stand lediglich ein seit mehreren Jahren stillgelegter Bruch zur Verfügung (Abb. 4.26 a). Hier werden bereits dessen Komplexität und die damit verbundenen Schwierigkeiten im Abbau des hochmetamorphen Marmors deutlich (vgl. Kap. 2.2). Im Streichen ergeben sich zwei Haupkluftscharen, die in einem Winkel von etwa $70^{\circ}$ bis $80^{\circ}$ zueinander ausgerichtet sind. Dabei sind in der NNW-SSE streichenden Schar deutliche Unterschiede im Einfallen gegeben, womit eine Überlagerung steil stehender Klüfte mit Diagonalklüften beschrieben ist. Die WSW-ENE streichende Kluftschar unterliegt insgesamt einer stärkeren Variation der Einfallswinkel. Die durchgreifende Zergliederung zeigt sich auch in der Abstandsverteilung der Trennflächen in allen drei Raumrichtungen (Abb. 4.26 c). Bezogen auf die Gesamtlänge aller Profillinien ( 53 m) entfallen lediglich rund $15 \%$ auf Abstände über 1,5 m zwischen erkennbaren Trennflächen. Das Aufmass gelagerter Rohblöcke ergab ein entsprechend geringes Rohblockvolumen von durchschnittlich 2,2 $\mathrm{m}^{3}$ (mittlere Blockgröße 1,90 x 1,23 x 0,87 m). Zusätzlich zu diesem komplexen und engständigen Trennflächengefüge treten in vielen Bereichen der Lagerstätte zum Teil erhebliche Karstbildungen in Erscheinung (vgl. Kap. 2.2, Abb. 2.11 b), wodurch der Abbau von werksteinfähigen Rohblöcken hier insgesamt mit einer hohen bis sehr hohen Abraumrate verbunden ist. 

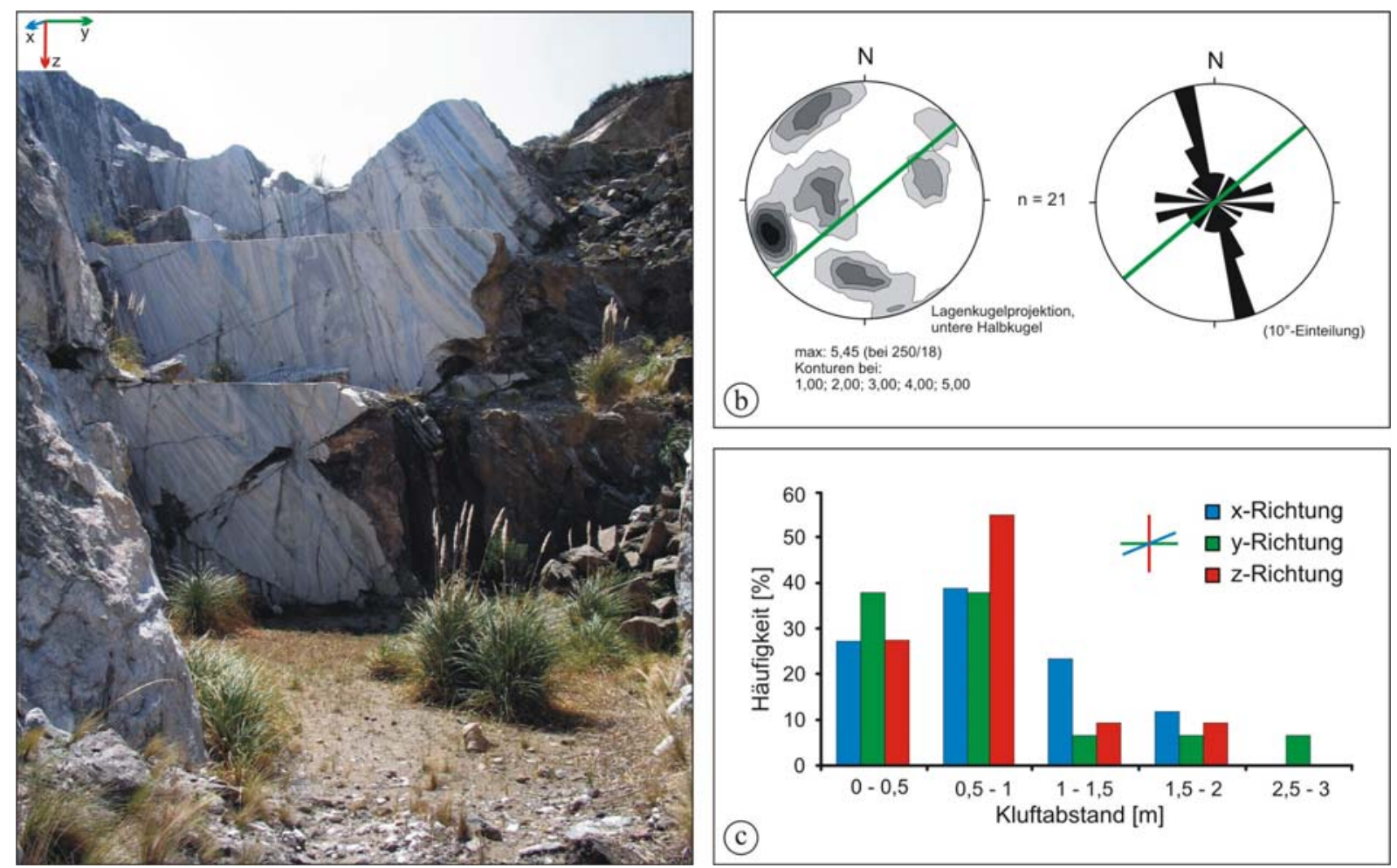

Abb. 4.26: a) Stillgelegter Werksteinbruch des Calcitmarmors AZUL CIELO (Fa. Campa S.R.L., Córdoba, Provinz Córdoba; Höhe der unteren Abbaustufe etwa 5,2 m); b) Der Lagerstättenkörper ist durch ein b) kompliziert verteiltes und c) zumeist relativ engständiges Trennflächengefüge charakterisiert.

\subsubsection{Lagerstätten von Porphyren in Nordpatagonien}

Das präkambrische Basement der Region Patagonien ist in vielen Bereichen von ausgedehnten Fließdecken intermediärer bis saurer Vulkanite bedeckt (z.B. Gust et al. 1985, Pankhurst et al. 2006). Diese stehen mit dem frühen Stadium des Auseinanderbrechens von Gondwana während des frühen und mittleren Jura in Zusammenhang. Die Gesteine weisen eine sehr feinkörnige, teils hyaline Matrix auf, in welche vor allem Quarz und Feldspäte als Einsprenglinge eingeschlossen sind. Im Bereich des Nordpatagonischen Massivs, besonders in den Provinzen Rio Negro und Chubut, werden diese Porphyrdecken seit vielen Jahrzehnten zur Verwendung als Werkstein abgebaut. Häufig erfolgt der Abbau im Klein- bis Kleinstbergbau. Dabei ermöglicht die zum Teil sehr gut entwickelte Spaltbarkeit des Gesteins eine rein manuelle Produktion.

Im Rahmen dieser Studie wurden verschiedene Lagerstätten mit einbezogen, die die hohe Variabilität in Art und Umfang der Erschließung und Produktion des Rohstoffs widerspiegeln. Dabei wurde die gesamte Spannweite vom manuellen Kleinstbergbau bis zum modernen Bruchmanagement mit hochtechnisierter Ausbringung und Verarbeitung des Werksteinmaterials beachtet.

In der Provinz Rio Negro erfolgt in einer Vielzahl kleiner bis kleinster Steinbrüche der Abbau des Materials mit einfachen Hilfsmitteln. Die Produktion ist dabei auf den oberflächlichen Bereich der Lagerstätten beschränkt. Ein exemplarisches Beispiel für diese primäre Stufe der Rohstoffförderung befindet sich im Departamento Valcheta (Abb. 4.27 a). 
Die Breite des Abbaus beträgt hier nicht mehr als $20 \mathrm{~m}$, wobei der abbauwürdige Bereich bis zu einer Teufe von maximal $4 \mathrm{~m}$ angeschnitten ist. Der Abbau erfolgt hier rein manuell unter Zuhilfenahme von einfachen Brecheisen. Trotz des relativ geringen Teufenanschnitts, zeigt sich hier bereits in der Vertikalen eine Veränderung in der Abstandsverteilung der annähernd senkrecht einfallenden Klüfte hin zu einer feineren Spaltbarkeit im oberen Bereich der Abbauwand (vgl. Kap. 2.2). Selten sind im aufgeschlossenen Bereich diagonal verlaufende Klüfte zu beobachten.

Als einziges Produkt werden hier polygonale, handgespaltene Platten ausgebracht. Von hervorzuhebendem Interesse ist dabei die beschriebene, zum Teil sehr gute Spaltbarkeit zu Stärken unter $10 \mathrm{~mm}$ (Abb. 4. 27 b). Damit prädestinieren sich die hier abgebauten Werksteine für eine feine Verarbeitung, gerade im Bereich von Zierelementen sowohl im Innen- als auch Außenbereich. Die individuelle Färbung beziehungsweise verschiedenen Farbschattierungen zwischen Rot und Rotbraun finden bei der Zusammenstellung der Chargen keine Beachtung. Eine grobe Sortierung erfolgt lediglich nach der Plattenstärke.
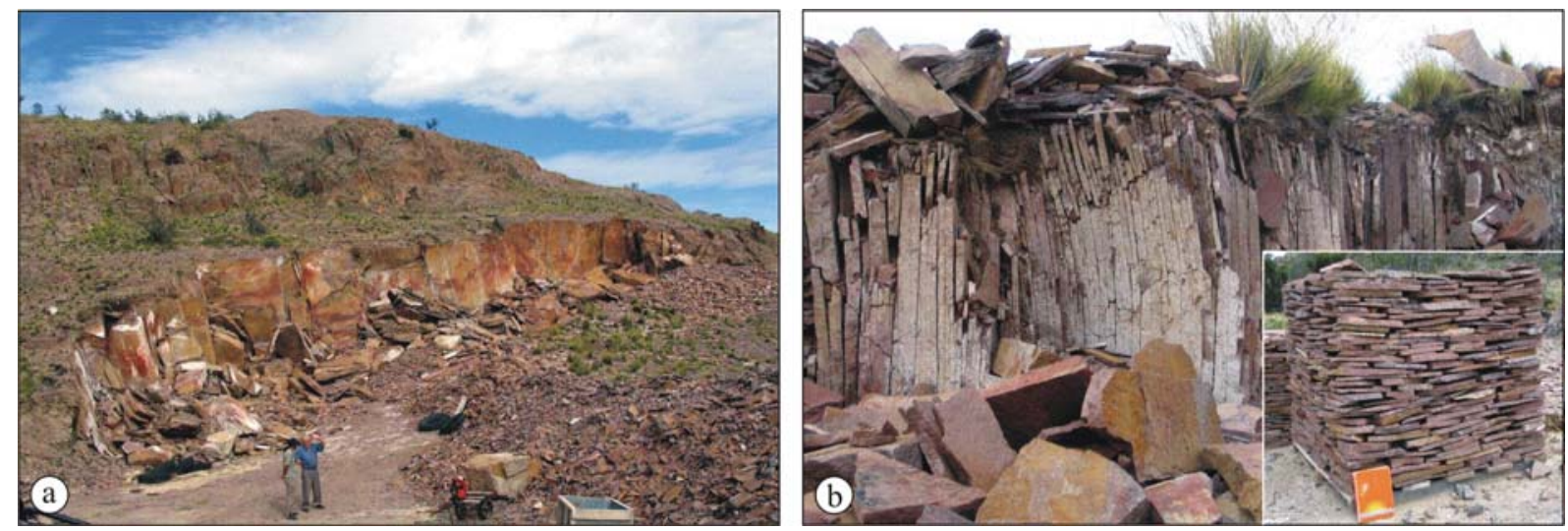

Abb. 4.27: a) Gewinnung von Porphyr im Kleinstbergbau (Departamento Valcheta, Provinz Rio Negro); b) Das Gestein dieser Lagerstätte weist infolge einer vor allem im Hangenden zum Teil sehr engständigen Klüftung eine Spaltbarkeit zu Plattenstärkern unter $10 \mathrm{~mm}$ auf.

Bei der Ortschaft Los Menucos im Departamento Veinticinco de Mayo, W' von Valcheta gelegen, ist eine Lagerstätte in einem fortgeschrittenerem Stadium erschlossen. Die Größe des abbauwürdigen Bereichs beträgt nach Auskunft des Besitzers etwa 300 x 150 m, wobei jedoch keine Informationen bezüglich einer möglichen Abbauteufe vorliegen. Die derzeitige Abbauwand weist eine Höhe von etwa $5 \mathrm{~m}$ auf. Die sedimentäre Bedeckung beträgt lediglich rund 0,5 m. Der Abbau wird hier unter Zuhilfenahme eines Baggers geführt. In kompakteren Partien erfolgt zudem die Gesteinslösung durch den Einsatz von Explosiva in Bohrlöchern.

Das Vorkommen weist im Vergleich eine mit der Region Valcheta vergleichbare lagerstättengeologische Ausgangssituation auf. Jedoch ist hier die Spaltbarkeit nach den Abkühlungsklüften etwas weitständiger ausgebildet, womit die Mächtigkeit der Lagerstätte etwas höher einzuschätzen ist. In der obersten Partie des Abbaus beträgt die Stärke der ausgebrachten Platten minimal etwa $30 \mathrm{~mm}$. Die Zunahme der Trennflächenabstände mit zunehmender Teufe ist auch hier deutlich ausgebildet. Senkrecht zu den mit etwa $80^{\circ}$ einfallenden Abkühlungsklüften ist eine zweite, weitständigere Hauptkluftschar ausgebildet. Dieses System wird von einem diagonalen Kluftsystem mit einem Einfallen von rund $45^{\circ}$ 
zerschnitten (Abb. 4.28 a). Für einen tieferen Abbau, mit der eventuellen Möglichkeit zur Ausbringung größerer Rohblöcke, ist folglich davon auszugehen, dass in manchen Bereichen spitzwinklige Blockgeometrien aus dem Trennflächengefüge hervorgehen (vgl. Kap. 2.4.2). Im erschlossenen Bereich der Lagerstätte sind die Diagonalklüfte jedoch selten zu beobachten ist. Als weiteres tektonisches Element durchzieht eine steil stehende Scherzone den Bruch. Im nahen umgebenden Bereich ist das Gestein deutlich stärker fragmentiert. Weiter Scherzonen sind aus der Aufschlusssituation nicht zu identifizieren, können jedoch für den Lagerstättenkörper keinesfalls ausgeschlossen werden.

Als Hauptprodukt fallen in dieser Lagerstätte polygonale Platten in Stärken von $5 \mathrm{~cm}$ bis $10 \mathrm{~cm}$ an (Abb. 4.28 b). Außerdem werden diese auch zu Pflastersteinen verarbeitet (vgl. Abb. 4.3 b). Die generelle Grundfarbe des Gesteins variiert von Rottönen im oberflächennahen Bereich, hin zu einer graubraunen Färbung im tieferen Anschnitt. Im direkt angrenzenden Bereich zur nachgewiesenen Scherzone weist das Gestein einen zum Teil deutlich rötlich-violetten Farbstich auf. Dieser Farbwechsel basiert auf der Migration fluider Phasen, die eine durchgreifende Kaolinitisierung des Gesteins bewirkte. Entsprechend farbliche Veränderungen sind jedoch im angeschnittenen Bereich lediglich auf einer Breite von rund 1 bis $2 \mathrm{~m}$ entlang der Störung zu beobachten.
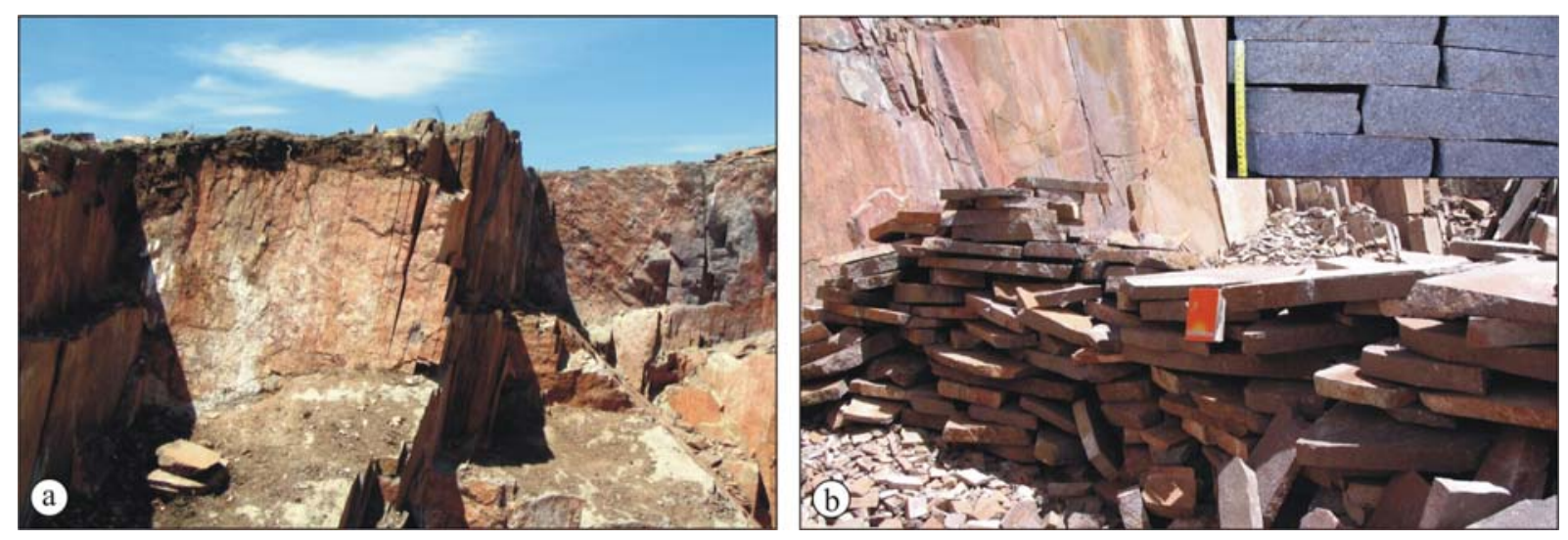

Abb. 4.28: Porphyrlagerstätte bei Los Menucos (Provinz Rio Negro; Steinbruch der Fa. Lencina Layas y Porfidos): a) Im Lagerstättenkörper ist neben zwei orthogonal zueinander stehenden Kluftscharen eine Diagonalklüftung ausgebildet. b) Polygonalplatten in Stärken zwischen 5 und $10 \mathrm{~cm}$ als Hauptprodukt des Bruchs. Kleines Bild: Farbliche Veränderung des Gesteins infolge einer durchgreifenden Kaolinitisierung im Bereich einer Scherzone.

Das relevanteste Vorkommen an Porphyren befindet sich in der Sierra Chata, in einer Entfernung von rund $80 \mathrm{~km} \mathrm{~W}$ ' der Stadt Puerto Madryn (Provinz Chubut). Der Steinbruch „Namuncurá“ erstreckt sich über eine Fläche von mehreren Hektar (Abb. 4.29 a). Im Gegensatz zu den vorherigen Beispielen zur Nutzung der Vulkanite, unterliegen der Abbau und die Produktion einem strikten Qualitätsmanagement. Die Sortierung des ausgebrachten Materials beginnt direkt nach dem Abbau mit der Klassifikation in Fertigprodukte wie spaltraue Polygonalplatten, die direkt in den Verkauf gehen, und in Halbfertigprodukte, die im Werk weiter bearbeitet werden (Frattari \& Stenico 2001). Hier erfolgt die Produktion von u.a. Pflaster-, Mauer- und Kantsteinen sowie von großformatigen Platten zur Herstellung stärker dimensionierter Bodenplatten mit gebrochenen Kanten. Die Fertigprodukte werden 
nach der Färbung in verschiedene Qualitäten klassifiziert und zudem detailliert nach Größe beziehungsweise Dicke sortiert (Abb. 4.29 b).
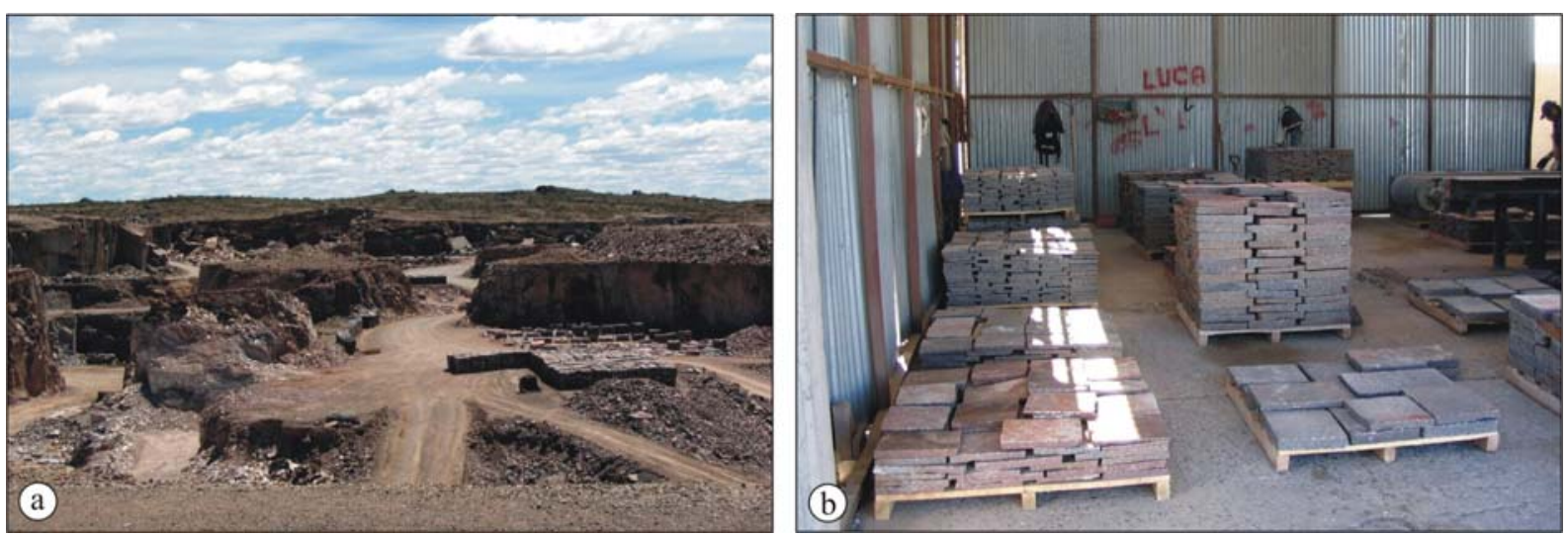

Abb. 4.29: a) Werksteinbruch „Namuncurá“ (Porphyr, Sierra Chata, Provinz Chubut): eine erste Auswahl und Sortierung des ausgebrachten Materials erfolgt bereits Vorort; b) die weitere Klassifikation (Färbung, Stärke, Größe) nach Bearbeitung im Werk.

Die zum Teil weitständig ausgebildete Klüftung (vgl. Kap. 2.2, Abb. 2.3 a) erlaubt zusätzlich den Abbau monolithischer Blöcke zur Gewinnung großvolumiger Werksteinblöcke (Abb. 4.30 a). Die Ausbringung erfolgt über eine selektive, gesteinsschonende Extraktion der in-situ Blöcke. Dazu werden nach der Freilegung eines entsprechenden Großblocks horizontale Bohrungen gesetzt, in denen eine sehr geringe Sprengladung gezündet wird, um den Block zu lösen. Die Abvierung zu quaderförmigen Idealblöcken erfolgt durch eng gesetzte Bohrungen und dem Einsatz von Federkeilen. Zur Verarbeitung dieser Blöcke steht mit Diamantseilsägen und Brückensägen modernes technisches Gerät zur Verfügung.

Das Produktionsgelände befindet sich in der Hafenstadt Puerto Madryn, womit die direkte Möglichkeit zum weltweiten Export gegeben ist. Auch das Abfallmaterial aus der Fliesenund Plattenproduktion wird noch zu feinerer Pflasterung gebrochen, um eine bestmögliche Materialnutzung zu erreichen. Dadurch konnte die Gesamtmenge an Ausschuss auf etwa 5 \% des ausgebrachten Materials reduziert werden.
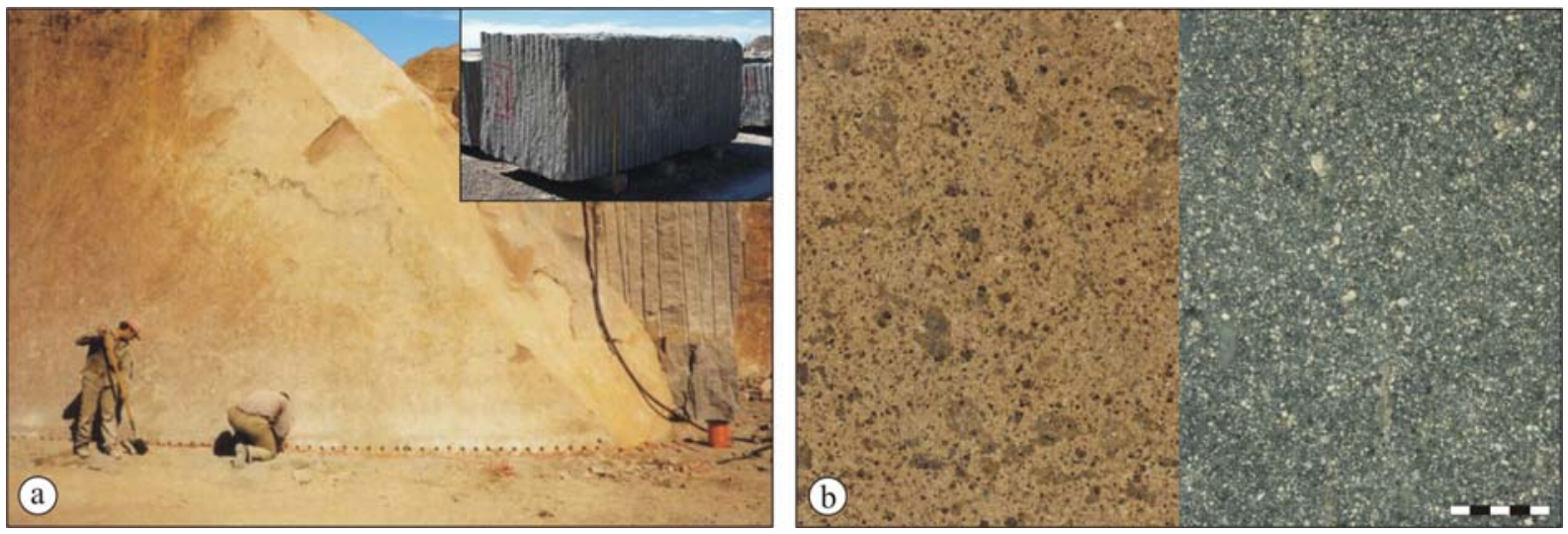

Abb. 4.30: a) Ein z.T. weitständiges Kluftsystem erlaubt die Ausbringung großvolumiger Rohblöcke über die schonende Loslösung unzergliederter Gesteinspartien (Abb. aus Frattari \& Stenico (2001); kleines Bild: Blockgröße 3,1 x 1,6 x 1,5 m); b) Rötliche und graue (STARDUST GREY) Farbvarietät des Prophyrs (polierte Oberflächen, Maßstab in cm). 


\subsubsection{Schiefergewinnung in der Provinz San Luis}

Das Basement der Sierra de San Luis setzt sich aus einer Sequenz paläozoischer Metamorphite zusammen. Dabei sind in die San Luis Formation (Kambrium - Ordovizium) abbauwürdige Schieferhorizonte eingeschlossen, die in einigen Bereichen seit mehreren Jahrzehnten intensiv als Naturwerkstein genutzt werden. In der Nähe der Stadt El Trapiche, etwa 35 km NW' der Provinzhauptstadt San Luis gelegen, befindet sich das wichtigste dieser Schiefervorkommen (Steinbruch „La Represa“, Abb. 4.31), das eine Ausdehnung von etwa 50 $\mathrm{km}$ in der Länge und rund $5 \mathrm{~km}$ in der Breite aufweist.
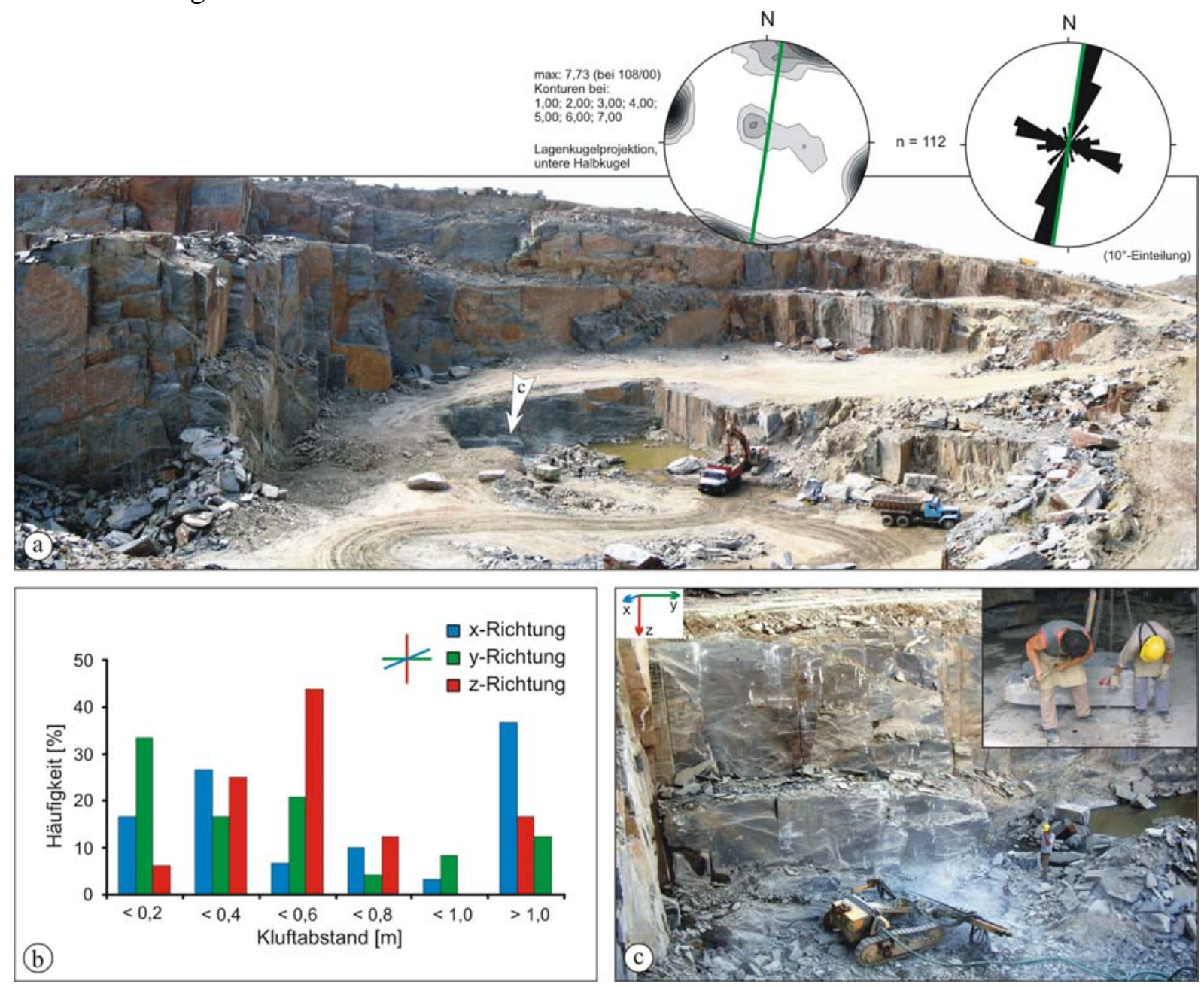

Abb. 4.31: a) Schiefergewinnung im Werksteinbruch „La Represa“ bei El Trapiche (Fa. San Luis Slates S.A., Provinz San Luis): Ein orthogonal angelegtes Kluftsystem mit b) einer für Schiefer relativ weitständigen Abstandsverteilung erlaubt c) eine Blockausbringung an der Bruchsohle unter Einsatz eines pneumatischen Raupenbohrgerät.

Zum Untersuchungszeitpunkt erfolgte der Abbau bis in eine Teufe von etwa $50 \mathrm{~m}$. Die Anlage des Abbaus ist dem orthogonal ausgerichteten Kluftsystem angepasst (Abb. $4.31 \mathrm{a}$ ), das durch die sedimentäre Schichtung und die Schieferung gegeben ist. Unterbrochen wird das regelmäßige Trennflächengefüge von wenigen diagonal verlaufenden Klüften. Begünstigt wird die Abbausituation dadurch, dass für Schiefer im Allgemeinen relative hohe Abstandsweiten zwischen den Trennflächen auftreten (Abb. 4.31 b), womit das Ausbringen von Blöcken in Größen bis zu $5 \mathrm{~m}^{3}$ möglich wird. Die entsprechenden Partien werden durch 
wenige Bohrungen aus dem Gesteinsverband gelöst und anschließend unter Ausnutzung der natürlichen Spaltbarkeit zu den für die weitere Produktion benötigten Maßen manuell gespalten (Abb. 4.31 c). In der oberflächennahen Partie ist das Gestein dagegen stark zerbrochen und zerklüftet, wodurch sich ein hoher Abraumanteil ergibt. Daraus resultiert ein enormer finanzieller Aufwand bei der Erweiterung des Abbaus in der Fläche. In der Folge wurde ein direkt angrenzender Bruch nach mehreren Jahrzehnten der Nutzung stillgelegt, da für eine Umlagerung beziehungsweise Beseitigung des Abraummaterials nach Firmeninformation Kosten in Höhe von etwa 250.000 US\$ kalkuliert wurden.

Der zumeist dunkelgraue, teils leicht grünliche Schiefer zeigt im aktuellen Abbaubereich zumeist eine homogene Struktur. Akzessorisch ist Pyrit im Mineralverband eingeschlossen (Detail in Abb. 4.32 a), selten zu einzelnen, dünnen Bändern akkumuliert. Diese können bei der Verwendung im Kontakt mit Wasser durch Ausrosten zu unattraktiven Fleckenbildungen führen (vgl. Kap. 3.13). Neben diesen Lagen wird das sonst homogene Gesamtbild des Gesteins durch selten auftretende Quarzgänge von maximal $15 \mathrm{~cm}$ Stärke unterbrochen. Petrologisch ist das Gestein als Phyllitschiefer zu betrachten, der einen geringen Quarzanteil aufweist. Dieser ist zumeist zu rekristallisierten, feinen Bändern akkumuliert.
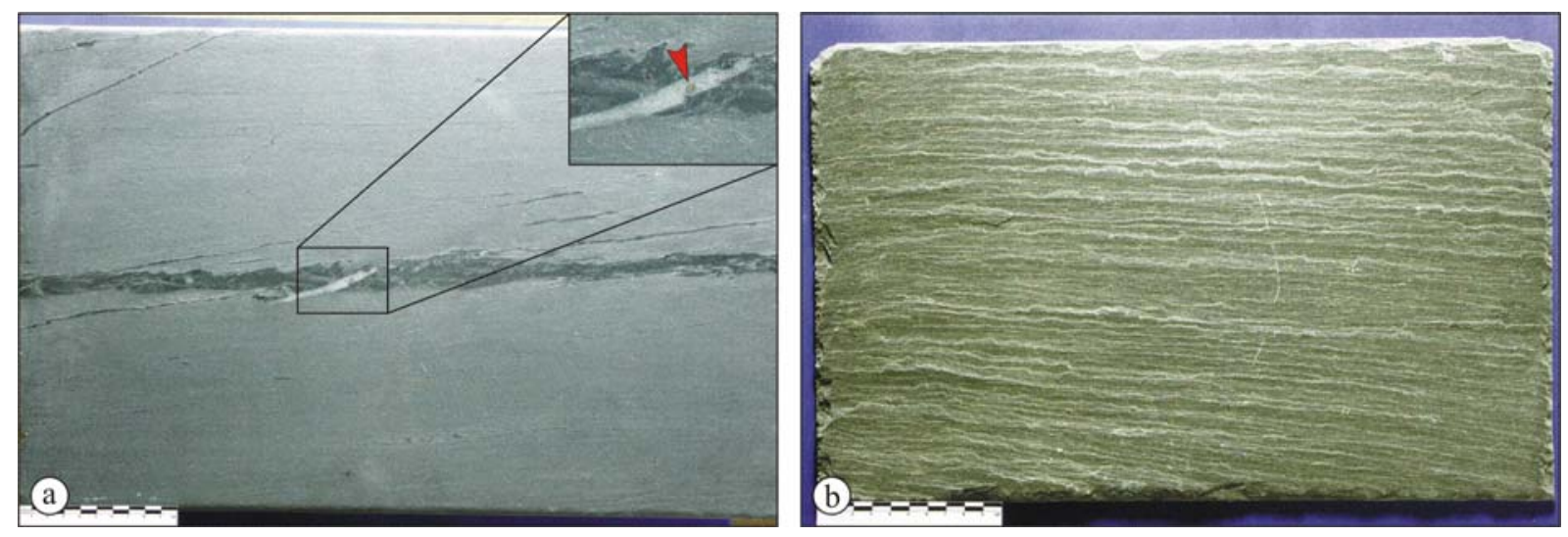

Abb. 4.32: a) Kalibrierte Bodenfliese mit polierter Oberfläche und gefräster Fase (Bildausschnitt: einzeln auftretende Pyrite); b) Dachschindel der Handelsklasse Montana (3. Qualität, Stärke > 9 mm) mit spaltrauer Oberfläche und manuell gebrochener Kante (Maßstäbe in cm).

Die Klassifikation zu Handelssorten orientiert sich sowohl an der Plattenstärke als auch an der Oberflächen- und Kantenbearbeitung. Die Produktion bruchrauer Platten erfolgt nach der manuellen Aufspaltung der Großblöcke zu Dicken von rund 20 - 25 cm, eine Formatierung in zwei Sägeschritten auf das jeweils gewünschte Flächenmaß. Die endgültige Plattenstärke (1. Qualität: < $6 \mathrm{~mm}$; 2. Qualität: 7 - $9 \mathrm{~mm}$; 3. Qualität: > 9 mm [Abb. 4.32 b]) wird ebenfalls durch manuelles Aufspalten erreicht. Kalibrierte Bodenfließen werden in Standardmaßen von 20, 30 bzw. $40 \mathrm{~cm}$ maschinell gefertigt, wobei der Hauptanteil an der Produktion auf eine Fliesenbreite von $30 \mathrm{~cm}$ entfällt. Die Kalibrierung auf eine Stärke von $1 \mathrm{~cm}$, der Schnitt auf die Länge (überwiegend 61,5 cm), das Oberflächenfinish (poliert oder antik „gebürstet“) bis zur Fräsung der Falz sowie der Mörtelrillen erfolgt in einer zusammenhängenden Produktionsstraße. Zum Teil werden auch geflammte Oberflächen produziert. Dabei erfolgt die Bearbeitung der Oberfläche unter Wasser, da ansonsten infolge der benötigten Temperatur die Gefahr des Zerbrechens des Schiefers zu hoch ist. 
Obwohl die Lagerstätte seit annähernd 50 Jahren permanent intensiv genutzt wurde, erfolgte eine Anpassung der technischen Ausrüstung sowohl im Abbau als auch in der Verarbeitung an einen modernen Standard erst innerhalb der letzten 5 Jahre. Die Firma San Luis Slates S.A., als Hauptpächter der Lagerstätte, ist auf die Produktion von Boden- und Dachbelägen spezialisiert. Im Firmenzusammenschluss der SSQ International werden die Produkte weltweit vertrieben und konnten am internationalen Natursteinmarkt etabliert werden.

\subsection{Physikalische und technische Charakterisierung der aufgenommenen Lithologien}

Sämtliche Untersuchungen zur physikalischen und technischen Charakterisierung der beprobten argentinischen Werksteinvarietäten wurden in Anlehnung an die standardisierten Prüfverfahren für Naturwerkstein (DIN bzw. EN) entsprechend dem Stand der Technik im Gesteinsphysikalischen Labor des GZG (Universität Göttingen) ausgeführt. Die entsprechenden Untersuchungsmethoden sind im folgenden Unterkapitel kurz beschrieben, sofern sie nicht bereits in Kapitel 3 dargestellt wurden. Die erhaltenen Daten werden im Anschließenden kurz dargelegt und vergleichend bzw. bewertend betrachtet.

\subsubsection{Untersuchungsmethoden}

Viele petrophysikalische und technische Eigenschaften unterliegen einer richtungsbedingten Anisotropie, die auf räumlich orientierten Gefügeelementen oder aber einer kristallographischen Vorzugsrichtung beruht (vgl. Kap. 3). Um das Ausmaß dieser Schwankungen zu erfassen, erfolgte die Durchführung der verschiedenen Eigenschaftsermittlungen nach Möglichkeit in drei zueinander senkrecht stehenden Raumrichtungen (x, y, z). Dabei erfolgte die Orientierung der xy-Ebene nach dem domierende Gefügeelement (Schichtung, Foliation). Wenn makroskopisch keine weiteren Elemente zum Richtungsgefüge, wie z.B. eine Lineation, festgestellt werden konnten, wurde das Referenz-Koordinatensystem nach der gegebenen Blockgeometrie ausgerichtet (Abb. 4.33).

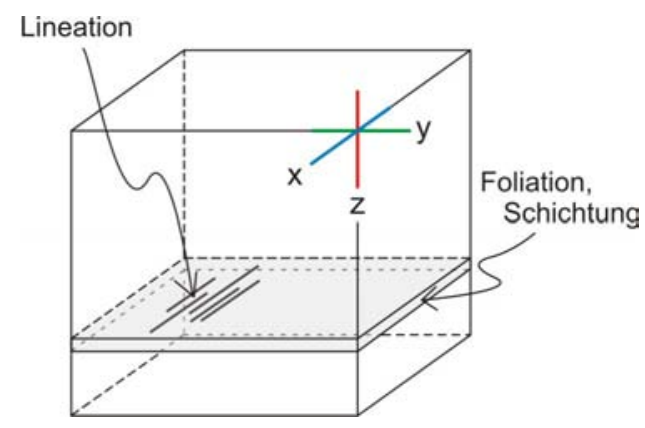

Abb. 4.33: Referenz-Koordinatensystem mit Bezug auf das Gesteingefüge zur richtungsabhängigen Ermittlung physikalischer und technischer Gesteinseigenschaften.

\subsubsection{Dichten, Porenraum und Wasseraufnahmeverhalten}

Die Bestimmung der effektiven Porosität $\Phi$ erfolgte in Anlehnung an EN 1936 über die verschiedenen Gewichtszustände Trockengewicht, Nassgewicht und Auftriebsgewicht an Würfelproben (Kantenlängen 65 bis 100 mm). Als porensättigendes Medium kam dabei 
demineralisiertes Wasser zum Einsatz. Die vollständige Wassersättigung wurde durch eine vorherige Evakuierung der Proben erreicht. Aus den ermittelten Daten erfolgt parallel die Berechnung der Rohdichte $\rho_{\text {roh }}$ sowie der Matrixdichte $\rho_{\text {matrix }}$ (vgl. Kap. 3.4.1).

Die Bestimmung der Porengrößenverteilung erfolgte mittels Quecksilberporosimetrie (Brakel et al. 1981) an zylindrischen Proben $(\varnothing=12,5 \mathrm{~mm}, \mathrm{~h}=40 \mathrm{~mm})$ an einem Hg-Porosimeter 2000 der Firma Carlo Elba. Die Kapillardruckkurven wurden bis zu einem Druck von 2 kbar gemessen, was die Erfassung bis zu einem idealisierten, kreisäquivalenten Porenradius von 3,8 nm ermöglicht.

Das Wasseraufnahmeverhalten wurde für alle beprobten Gesteine unter Beachtung des Gesteinsgefüges über die Dampfdiffusion in Anlehnung an DIN 52615 ermittelt. Für die Messungen wurden scheibenförmige Probekörper ( $\varnothing=40 \mathrm{~mm}, \mathrm{~h}=10 \mathrm{~mm}$ ) als Abdichtung auf einen mit demineralisiertem Wasser gefüllten Teflontiegel aufgesetzt. Durch regelmäßige Wägungen erfolgte die Bestimmung der Wassermenge, die während einer bestimmten Zeit durch die Gesteinsprobe diffundiert ist. Zwischen den einzelnen Messungen lagerten die aufgesetzten Proben in einem Klimaschrank (Luftfeuchtigkeit: $50 \%$, Temperatur: $20^{\circ} \mathrm{C}$ ), um ein definiertes Konzentrationsgefälle zwischen der Unter- und Oberseite der Probe zu gewährleisten. Die Ergebnisse der Wasserdampfdiffusion finden im dimensionslosen Koeffizienten $\mu$ (Wasserdampf-Diffusionswiderstandszahl) Ausdruck, der die Dichtigkeit des Gesteins gegenüber diffundierender Wassermoleküle beschreibt (Poschlod 1989).

Zusätzlich erfolgte für die Suite der Karbonatgesteine aufgrund der höheren Porosität die Ermittlung der kapillaren Wasseraufnahme in Anlehnung an prEN 1925 an kubischen Probekörpern (Kantenlängen 65 bis 100 mm). Die Proben wurden mit der jeweiligen Prüffläche in Kontakt mit der Wasseroberfläche gebracht, wodurch der Prozess des kapillaren Saugens einsetzt. Über eine Unterflurwaage wurde die Gewichtszunahme zeitabhängig registriert. Der Wasseraufnahmewert w (w-Wert) errechnet sich aus der über die definierte Prüffläche aufgenommenen Wassermenge pro Zeit.

\subsubsection{Ultraschallanalytik}

Die Ultraschallanalytik dient im Allgemeinen der Quantifizierung des Entfestigungszustandes von Naturwerksteinen (vgl. Kap. 3.12.1), der sich in der Auflockerung des Korngefüges beziehungsweise dem Aufbrechen der Korngrenzstrukturen äußert. Die Methodik basiert auf der Annahme, dass eine Geschwindigkeitsabnahme der Kompressionswellen auf eine Porositätszunahme bzw. eine Auflockerung des Kornaggregats zurückzuführen ist, was konsequenterweise mit dem Verwitterungszustand eines Gesteins in Verbindung gebracht werden kann.

Die Ermittlung der Kompressionswellengeschwindigkeit Vp des Materials erfolgte an zuvor ofengetrockneten Würfelproben mit Kantenlängen von 65 bis 100 mm. Neben der Messung an trockenem Material, erfolgte durch die vorherige Wassersättigung zudem die Abschätzung der intrinsischen Vp-Werte, die auf der mineralogischen Komposition sowie der Textur des Gesteins basiert. 


\subsubsection{Technische Eigenschaften}

Zur Charakterisierung der mechanischen Stabiltät der Gesteine erfolgte die Bestimmung der Druck-, Biege-, Spaltzug- und Abriebfestigkeit. In der Gesamtheit dient die Ermittlung der verschiedenen Parameter der Simulation von unterschiedlichen Belastungzuständen, denen ein Gestein in der bautechnischen Anwendung konstruktionsbedingt oder durch Verwitterungsprozesse unterliegt. Die Orientierung der verschiedenen Probekörper mit Bezug auf das Gesteinsgefüge bzw. im o.g. Referenz-Koordinatensystem, die jeweilige Belastungsrichtung sowie die resultierenden Spannungsrichtungen innerhalb der verschiedenen Probekörper sind aus Abbildung 4.34 zu ersehen.

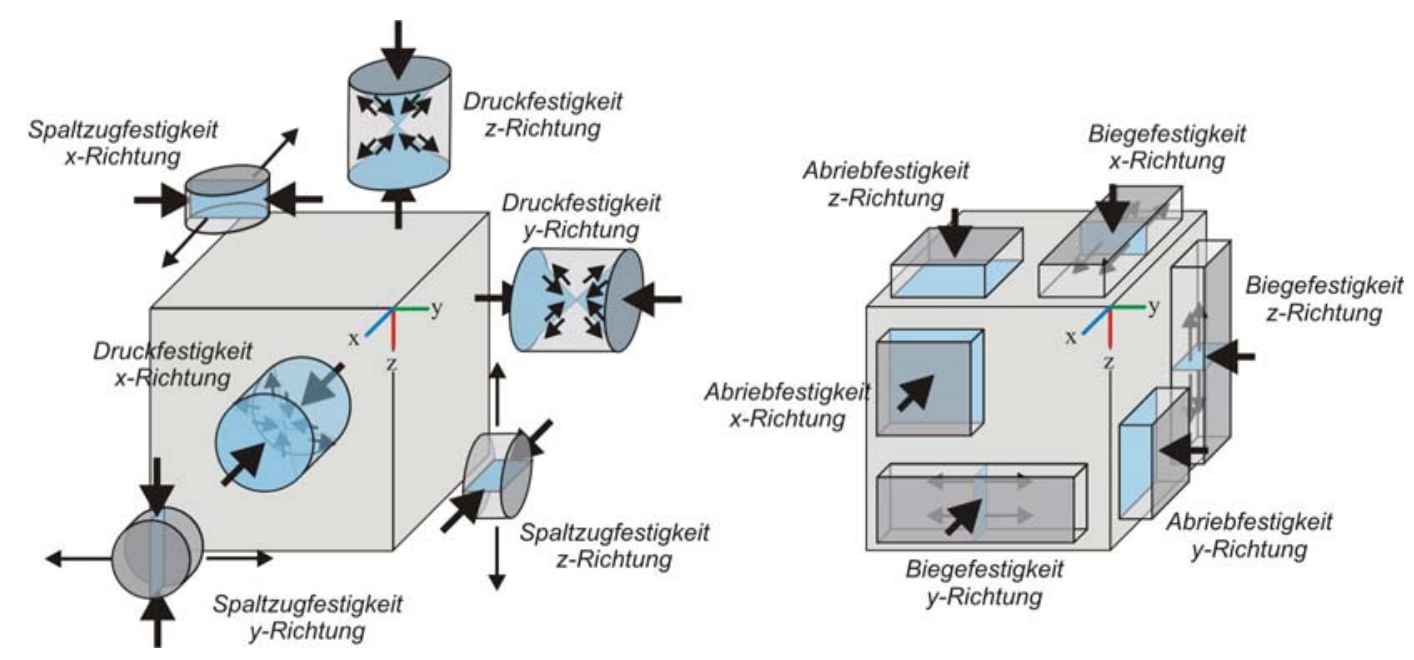

Abb. 4.34: Orientierung der Probekörper zur Bestimmung der verschiedenen technischen Eigenschaften mit Bezug auf das Gesteinsgefüge im Referenz-Koordinatensystem. Große Pfeile zeigen die Belastungsrichtung, kleine Pfeile die aus der jeweiligen Belastung resultierenden Spannungen im Probekörper.

Die Ermittlung der einaxialen Druckfestigkeit St $t_{C}$ erfolgte in Anlehnung an EN 1926 an zylindrischen Probekörpern mit einem Längen-Durchmesser-Verhältnis von $1: 1$ ( $\varnothing=50 \mathrm{~mm}, \mathrm{~h}=50 \mathrm{~mm}$ ). Die Probekörper wurden parallel zur jeweiligen Mantelfläche bei einer Kraftzunahme von 1000 N/s bis zum vollständigen Gesteinsversagen belastet, das aus einer Kombination aus Zug- und Scherrissen durch den aufgebrachten Druck initiiert wird. Die einaxiale Druckfestigkeit errechnet sich aus der maximalen Bruchlast und der Kopffläche des Zylinders.

Die Biegefestigkeit $\mathrm{St}_{\mathrm{F}}$ des Materials wurde in Anlehnung an die DIN EN 12372 bestimmt. Dabei wurden prismenförmige Probekörper (L 150 x B 50 x H 25 mm) auf zwei parallelen Walzen mit einem Abstand von 125 mm gelagert und über eine dritte Walze von oben entlang der Mittellinie belastet. Dadurch werden auf der konvexen Seite der Probe Druckspannungen und auf der konkaven Seite Zugspannungen generiert, die zum Gesteinsversagen führen. Die Belastungszunahme erfolgte ebenfalls Kraft-Zeit gesteuert entsprechend der Bestimmung der Spaltzugfestigkeit.

Die Bestimmung der Spaltzugfestigkeit $\mathrm{St}_{\mathrm{T}}$ wurde mit dem Brazil-Test (indirekte Zugspannung) in Anlehnung an DIN 22024 an zylindrischen Probekörpern ( 
h= $20 \mathrm{~mm}$ ) durchgeführt. Längs zweier gegenüberliegender Mantellinien wird auf den Probekörper eine Streifenlast mit einer Kraftzunahme von $30 \mathrm{~N} / \mathrm{s}$ übertragen. Aus der vertikalen Druckbelastung resultiert auf über 70 \% des Probekörperdurchmessers eine horizontale Zugspannung, die final das Gesteinsversagen herbeiführt.

Die Abriebfestigkeit wurde nach dem Böhme-Verfahren (DIN 52108) an quadratischen Probekörpern mit einer Kantenlänge von 71 mm und einer Höhe von 25 mm ermittelt. Mit der relieffreien Grundfläche wird der Probekörper mit einem definierten Andruck gegen die Schleifscheibe belastet und mit Normschleifmittel abgerieben. Der Materialverlust nach einer definierten Anzahl von Umdrehungen der Schleifscheibe gibt die Abriebfestigkeit [in $\mathrm{cm}^{3} / 50 \mathrm{~cm}^{2}$ ] des beprobten Materials.

\subsubsection{Physikalische Eigenschaften des Probenmaterials}

Die ermittelten Werte zu Dichten, Porenraumeigenschaften sowie Wasseraufnahmeverhalten der betrachteten Lithologien sind in den Tabellen 4.1 und 4.2 zusammengefasst dargestellt.

Die Matrixdichten der argentinischen Granitoide, einschließlich der metamorphen Varietäten (Migmatit, Cordierite und Cdr-Gr-Gneis) überspannen ein Spektrum von $2,63 \mathrm{~g} / \mathrm{cm}^{3}$ (ROJO DRAGÓN) bis 2,96 g/ $\mathrm{cm}^{3}$ (AZUL TANGO). Sämtliche Dichtewerte über 2,75 g/cm³ ordnen sich erwartungsgemäß den Cordierit-führenden Varietäten zu und geben so die Abhängigkeit der Matrixdichte von der Mineralogie wieder (vgl. Kap.3.4.1). Entsprechend ihrer mineralogischen Zusammensetzung aus mehrheitlich Quarz und Feldspat ergeben sich für die übrigen Varietäten Werte zwischen 2,64 g/ $\mathrm{cm}^{3}$ und 2,69 g/cm³ (vgl. Kap. 3.4.1). Für alle beprobten granitoiden Lithologien ergibt sich ein Porenraumvolumen von < 0,6 Vol-\%, womit insgesamt sehr dichte Gesteine beschrieben sind (vgl. Kap. 3.5.1). Dabei ist die überwiegende Mehrheit der Proben mit mittleren Porenradien $<0,15 \mu$ m oder wenig darüber gekennzeichnet. Die Verteilungen der Porenradien zeigen zumeist ein einheitliches Bild mit einer recht gleichmäßigen Verteilung und der Ausbildung eines schwachen Maximums. Das Wasseraufnahmeverhalten ist mehrheitlich mit Zahlenwerten zum Widerstand gegen Wasserdampfdiffusion von über 1000 beschrieben. Ausnahmen bilden hierbei die Varietäten ROJO ORCOLLANO, ROJO PRINCIPE und FRANCO VETEADO. Alle drei Lithologien sind durch einen relativ hohen Gehalt an Biotit gekennzeichnet, aus dem vermutlich eine relativ höhere Rissdichte resultiert, womit eine erhöhte Durchlässigkeit verbunden ist. Der im Mittel geringste Wert ist für die Varietät ROJO PRINCIPE dokumentiert. Zudem zeigt sich die Gefügeabhängigkeit des Wasseraufnahmeverhaltens. So ergibt sich für die Varietät ROJO ORCOLLANO eine Anisotropie von rund 75 \%. Diese Gefügeabhängigkeit muss vor allem im Bezug auf der Varietät FRANCO VETEADO beachtet werden, bei dem die Textur erheblichen Schwankungen unterliegt.

Hinsichtlich der Karbonatgesteine wird aus der Matrixdichte die dolomitische Mineralogie der Varietäten DOLOMITA DORADA $\left(2,84 \mathrm{~g} / \mathrm{cm}^{3}\right)$ und LUNEL COVUNCO $\left(2,83 \mathrm{~g} / \mathrm{cm}^{3}\right)$ gegenüber einer Matrixdichte von 2,70 g/ $\mathrm{cm}^{3}$ bei der Varietät CALIZA AMARILLA (vgl. Kap. 3.4.1). Bei allen drei Kalksteinen wird die Abhängigkeit der Rohdichte von der Porosität 
deutlich (vgl. Kap. 3.8.1). Auffällig ist, dass die Porenradienverteilungen der drei Gesteine deutlich unterschiedlichen Charakters sind (Abb. 4.35). Während die relativ poröse Varietät CALIZA AMARILLA ( $\rho_{\text {roh }} 2,39 \mathrm{~g} / \mathrm{cm}^{3} ; \quad \Phi$ 11,40 Vol.-\%) eine recht enge Porenradienverteilung mit einem deutlich ausgeprägten Maximum bei 0,225 $\mu \mathrm{m}$ aufweist, ist in der Varietät LUNEL COVUNCO ( $\rho_{\text {roh }} 2,69 \mathrm{~g} / \mathrm{cm}^{3}$, $\Phi$ 5,02 Vol.-\%) eine relativ breite Verteilung um etwa $0,1 \mu \mathrm{m}$ ausgebildet. Die geringere Porosität steht dabei im Zusammenhang mit einer stärkeren Kompaktion des Sediments. Der Dolomit DOLOMITA DORADA ( $\rho_{\text {roh }} 2,60 \mathrm{~g} / \mathrm{cm}^{3} ; \Phi$ 8,51 Vol.-\%) zeigt dagegen ein noch breiteres Spektrum an Porenradien. Dabei ist jedoch eine deutlich schiefe Verteilung mit einem Maximum bei annähernd $1 \mu \mathrm{m}$ gegeben. Es ist davon auszugehen, dass sekundäre Lösungsprozesse diese Verteilung hervorgerufen haben. Infolge der relativ hohen Porosität ist für alle betrachteten Kalksteinvarietäten eine verstärkte Wasseraufnahme im Vergleich mit den meisten plutonischen Varietäten gegeben. Dem Sättigungsbeiwert folgend, ist jedoch keines der Gesteine hinsichtlich der Frostbeständigkeit als besonders kritisch zu betrachten (vgl. Kap. 3.13.4).
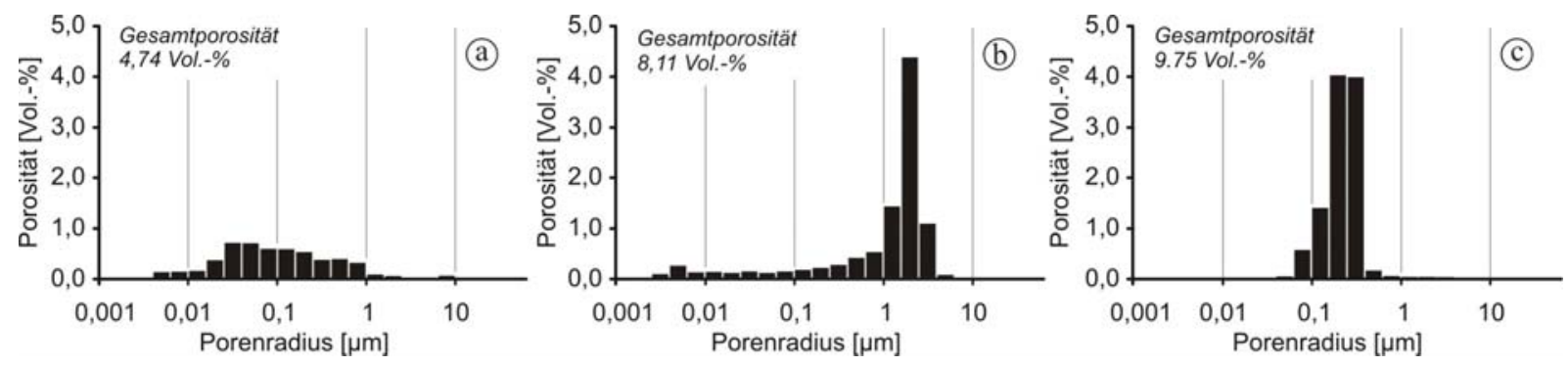

Abb. 4.35: Porenradienverteilungen der karbonatischen Werksteinvarietäten a) LUNEL COVUNCO, b) DOLOMITA DORATA und c) CALIZA AMARILLA mit den aus der Hg-Porosimetrie berechneten Gesamtporositäten.

Das für die Gesteinscharakterisierung des Marmors AZUL CIELO zur Verfügung stehende Material ist entsprechend dem Ergebnis der Ultraschallwellenanalyse (Kap. 4.7.3) als zunehmend porös $\left(\rho_{\text {roh }}\right.$ 0,41 Vol.-\%, $\Phi$ 2,71 g/ $\mathrm{cm}^{3}$ ) zu beschreiben und muss der von Köhler (1991) definierten Schadensklasse I zugeordnet werden. Dies wird auch an einem mittleren Porenradius von 0,658 $\mu \mathrm{m}$ deutlich, da frische Marmore im Allgemeinen Werte unter 0,2 $\mu \mathrm{m}$ aufweisen. Daher kann das vorhandene Material nur bedingt zu Aussagen über die bautechnische Eignung des Gesteins herangezogen werden. Der Vollständigkeit halber sind die ermittelten Daten in die Übersichtstabellen einbezogen. Da es sich nach Firmeninformation um relativ frisches Material handelt, zeigt sich hier bereits die Verwitterungsanfälligkeit dieses Calcitmarmors.

Das vorliegende Probenmaterials des Porphyrs ist der Handelssorte STARDUST GREY zugeordnet. Die Varietät, als einziges magmatisches Ergussgestein der Probensuite, weist eine Rohdichte von 2,53 g/cm³ bei einer effektiven Porosität von 0,60 Vol.-\% auf. Die Porenradien decken ein Spektrum von 0,002 $\mu \mathrm{m}$ bis 6,3 $\mu \mathrm{m}$ ab, wobei jedoch eine deutliche Schiefe mit einem ausgeprägtem Maximum bei 0,005 $\mu \mathrm{m}$ (mittlerer Porenradius 0,01 $\mu \mathrm{m}$ ) ausgebildet ist. Hinsichtlich der mittleren Diffusionswiderstandszahl $\mu$ ist der Porphyr im unteren Bereich der beprobten Granitvarietäten anzusiedeln, unterliegt dabei jedoch einer erheblichen Anistropie 
mit einem Minimum parallel der x-Achse. Dies kann mit der Ausbildung eines gerichteten Porengefüges infolge einer Fluidaltextur in Verbindung gebracht werden.

Für den Schiefer resultiert infolge der extrem geringen Porosität von 0,13 Vol.-\% für die Matrix- und auch Rohdichte ein gleicher Wert von 2,79 g/ $\mathrm{cm}^{3}$. Auffällig ist dabei ein relativ breites Spektrum an Porenradien zwischen 0,01 $\mu \mathrm{m}$ und annähernd $10 \mu \mathrm{m}$, wobei sich ein mittlerer Porenradius von 0,177 $\mu \mathrm{m}$ ergibt. Im Vergleich mit den ähnlich gering porösen Granitoiden resultiert daraus ein geringerer Widerstand gegen Wasserdampfdiffusion. Dabei unterliegt die Eigenschaft des Wasseraufnahmevermögens infolge der flächenhaften Paralleltextur des Gesteins einer deutlichen Anisotropie.

Tab. 4.1: Dichten, Porenraumcharakteristik und Sättigungsbeiwert der beprobten Lithologien argentinischer Naturwerksteine.

\begin{tabular}{|c|c|c|c|c|c|}
\hline & $\begin{array}{c}\text { Matrixdichte } \\
{\left[\mathrm{g} / \mathrm{cm}^{3}\right]}\end{array}$ & $\begin{array}{c}\text { Rohdichte } \\
\text { [g/cm³] }\end{array}$ & $\begin{array}{l}\text { Porosität } \\
\text { [Vol.-\%] }\end{array}$ & $\begin{array}{c}\text { mittlerer } \\
\text { Porenradius } \\
{[\mu \mathrm{m}]}\end{array}$ & $\begin{array}{c}\text { Sättigungs- } \\
\text { beiwert }\end{array}$ \\
\hline \multicolumn{6}{|l|}{ Granitoide } \\
\hline ROJO DRAGÓN & 2,63 & 2,62 & 0,45 & 0,088 & 0,81 \\
\hline MARRON COCO & 2,64 & 2,62 & 0,54 & 0,150 & 0,76 \\
\hline GRIS PERLA & 2,66 & 2,64 & 0,58 & 0,088 & 0,44 \\
\hline ROSA DEL SALTO & 2,66 & 2,64 & 0,59 & 0,031 & 0,61 \\
\hline SAN MARCOS BLANCO & 2,68 & 2,66 & 0,59 & 0,089 & 0,80 \\
\hline ROJO ORCOLLANO & 2,69 & 2,68 & 0,57 & 0,122 & 0,68 \\
\hline ROJO PRINCIPE & 2,69 & 2,68 & 0,43 & 0,098 & 0,87 \\
\hline AZUL HEMATITA & 2,69 & 2,69 & 0,07 & 0,213 & 0,70 \\
\hline FRANCO VETEADO & 2,75 & 2,75 & 0,33 & 0,204 & 0,51 \\
\hline NOCHE BUENA & 2,84 & 2,84 & 0,09 & n.d. & n.d. \\
\hline NEGRO RIOJANO & 2,91 & 2,91 & 0,04 & 0,007 & 0,53 \\
\hline AZUL TANGO & 2,96 & 2,96 & 0,16 & 0,097 & 0,95 \\
\hline \multicolumn{6}{|l|}{ Kalkstein } \\
\hline CALIZA AMARILLA & 2,70 & 2,39 & 11,40 & 0,225 & 0,75 \\
\hline LUNEL COVUNCO & 2,83 & 2,69 & 5,02 & 0,091 & 0,34 \\
\hline DOLOMITA DORADA & 2,84 & 2,60 & 8,51 & 0,968 & 0,44 \\
\hline \multicolumn{6}{|l|}{ Marmor } \\
\hline AZUL CIELO & 2,73 & 2,71 & 0,41 & 0,658 & 0,75 \\
\hline \multicolumn{6}{|l|}{ Vulkanit } \\
\hline STARDUST GREY & 2,54 & 2,53 & 0,60 & 0,010 & 0,85 \\
\hline \multicolumn{6}{|l|}{ Schiefer } \\
\hline SAN LUIS SLATE & 2,79 & 2,79 & 0,13 & 0,177 & 0,82 \\
\hline
\end{tabular}


Tab. 4.2: Wasseraufnahmeverhalten der beprobten Lithologien argentinischer Naturwerksteine (innerhalb der Gesteinsgruppen sortiert nach der Rohdichte) mit dem jeweiligen gefügebedingten Anistotropiewert $\alpha=(\max -\min ) / \max \cdot 100$.

\begin{tabular}{|c|c|c|c|c|c|c|c|c|}
\hline & \multicolumn{4}{|c|}{ Diffusionswiderstandszahl $\mu$} & \multicolumn{4}{|c|}{$\mathrm{w}$-Wert $\left[\mathrm{kg} / \mathrm{m}^{2} \cdot \sqrt{ } \mathrm{t}\right]$} \\
\hline & $\mathrm{x}$-Richtung & y-Richtung & z-Richtung & $\alpha$ & $\mathrm{x}$-Richtung & y-Richtung & z-Richtung & $\alpha$ \\
\hline \multicolumn{9}{|l|}{ Granitoide } \\
\hline ROJO DRAGÓN & 1516,53 & 1497,58 & 1234,10 & 18,6 & - & - & - & - \\
\hline MARRON COCO & 1909,39 & 2326,14 & 2575,98 & 25,9 & - & - & - & - \\
\hline GRIS PERLA & 1924,32 & 1246,29 & 1447,34 & 35,2 & - & - & - & - \\
\hline ROSA DEL SALTO & 1568,21 & 1809,04 & 1664,31 & 13,3 & - & - & - & - \\
\hline SAN MARCOS BLANCO & 1009,99 & 1094,58 & 1127,61 & 10,4 & - & - & - & - \\
\hline ROJO ORCOLLANO & 1957,35 & 506,02 & 920,57 & 74,2 & - & - & - & - \\
\hline ROJO PRINCIPE & 359,48 & 805,75 & 704,82 & 55,4 & - & - & - & - \\
\hline FRANCO VETEADO & 829,30 & 663,56 & 940,19 & 29,4 & - & - & - & - \\
\hline NEGRO RIOJANO & 5927,82 & 2321,00 & 3324,94 & 60,8 & - & - & - & - \\
\hline AZUL TANGO & 1479,04 & 1543,81 & 1305,76 & 15,4 & - & - & - & - \\
\hline \multicolumn{9}{|l|}{ Karbonatgesteine } \\
\hline CALIZA AMARILLA & 136,13 & 170,46 & 162,10 & 20,1 & 1,82 & 1,58 & 2,65 & 40,4 \\
\hline LUNEL COVUNCO & 255,42 & 160,35 & 196,89 & 37,2 & 0,71 & 1,12 & 0,97 & 36,6 \\
\hline DOLOMITA DORADA & 1126,19 & 1011,42 & 1072,86 & - & 1,10 & 1,01 & 0,75 & 31,8 \\
\hline \multicolumn{9}{|l|}{ Marmor } \\
\hline AZUL CIELO & 1424,12 & 787,27 & 1545,04 & 49,0 & - & - & - & - \\
\hline \multicolumn{9}{|l|}{ Vulkanit } \\
\hline STARDUST GREY & 510,62 & 1654,20 & 1396,30 & 69,1 & - & - & - & - \\
\hline \multicolumn{9}{|l|}{ Schiefer } \\
\hline SAN LUIS SLATE & 183,37 & 679,52 & 596,06 & 73,0 & - & - & - & - \\
\hline
\end{tabular}

\subsubsection{Elastische Eigenschaften des Probenmaterials}

Die Geschwindigkeiten der Kompressionswellen (Vp) für die betrachteten Lithologien sind in Tabelle 4.3 zusammengefasst dargestellt.

Nach Iliev (1967) sollten frische, unverwitterte Granitoide im trockenen Zustand einen VpWert von mindestens 5 km/s aufweisen. Im Rahmen der hier beprobten Granitoide wird dieser Wert lediglich von den granitischen Varietäten MARRON COCO und ROJO DRAGÓN sowie dem Gabbro NEGRO RIOJANO und dem Cdr-Gt-Gneis AZUL TANGO erreicht. Die Werte zu den verbleibenden magmatischen Gesteinen liegen im Mittel zwischen 4,4 km/s und 4,9 km/s. Die Minimalwerte werden bei den relativ biotitreicheren Granitoiden des Abbaugebietes Orcollano (Provinz Córdoba) erreicht. Da keine erheblichen Porositätsunterschiede bestehen, kann der Kopplungsgrad der Mineralkörner, also eine Zunahme der Rissdichte, für eine Verringerung der Laufzeiten verantwortlich gemacht werden (Schön 1983).

In der Gruppe der Kalksteine wir der geringste Werte der Kompressionswellengeschwindigkeit bei der vergleichsweiße hochporösen Varietät CALIZA AMARILLA erreicht. In den an den gesättigten Proben ermittelten Werten spiegeln sich 
möglicherweise die Unterschiede in der mineralogischen Zusammensetzung der Gesteine wieder. Der geringste Wert wird auch hier bei der calcitischen Varietät erreicht. Bei den verbleibenden zwei Varietäten nimmt der Vp-Wert mit zunehmendem Dolomitgehalt ebenfalls zu. Für den reinen Dolomit ist auch trotz einer etwas höheren Porosität im Vergleich zum dolomitischen Kalkstein der höchste Vp-Wert für die getrockneten Proben beschrieben. Andererseits können hier auch Unterschiede in der Porengeometrie zum Ausdruck kommen. Der Vergleich der gefügebedingten Laufzeitenunterschiede zeigt insgesamt die Homogenität der Materialien, da sich lediglich sehr geringe Anisotropiewerte von maximal 2,1\% (DOLOMITA DORADA) ergeben.

Für den Calcitmarmor AZUL CIELO werden richtungsbezogene Werte zwischen $3,58 \mathrm{~km} / \mathrm{s}$ und 3,72 km/s erreicht. Damit ist eine nicht unerhebliche Gesteinsschädigung beschrieben, die nach der Klassifikation von Köhler (1991) mit „zunehmend porös“ bewertet werden muss. Eine fortgeschrittene Gefügeentfestigung des vorliegenden Probematerials konnte bereits aus der physikalischen Charakterisierung des Calcitmarmors erkannt werden (Kap. 4.7.2).

Die für den Porphyr STARDUST GREY ermittelten Vp-Werte (im Mittel 5,13 km/s) liegen im oberen Bereich der von Gebrande (1982) gegebenen Erwartungswerte für saure Vulkanite. Eine Richtungsabhängigkeit durch mögliche Vorzugsorientierung in der Anordnung von Riss- oder Porensystemen infolge einer Fluidaltextur konnte dabei nicht festgestellt werden.

Für den Phyllitschiefer ist eine deutliche Richtungsabhängigkeit der P-Wellengeschwindigkeit ausgebildet ( $\alpha=28,5 \%$ ). Der Minimalwert wird dabei parallel der z-Richtung, also senkrecht zum metamorphen Lagenbau, erreicht, während sich für die verbleibenden Gefügerichtungen in etwa gleiche Vp-Werte ergeben. Ursächlich dafür ist die ausgeprägte Paralleltextur des Schiefers mit der Einregelung der Phyllosilikate. Besonders die Betrachtung der intrinsichen Kompressionswellengeschwindigkeiten verdeutlicht die ausgeprägte Textur des Gesteins, da sich auch hier ein Anisotropiewert von 25,3 \% errechnet.

\subsubsection{Technische Eigenschaften des Probenmaterials}

Die ermittelten Werte zu den Parametern der mechanischen Stabilität der betrachteten Lithologien sind in der Tabelle 4.4 zusammengefasst dargestellt.

In Bezug auf die mittleren Druckfestigkeiten der Granitoide und Gneise sind für den für den Cdr-Gt-Gneis AZUL TANGO sowie für den Gabbro NEGRO RIOJANO mit über 200 MPa die Maximalwerte dieser Probencharge beschrieben. Deutlich geringere Werte im Bereich zwischen 110 und $140 \mathrm{MPa}$ ergeben sich für die Gesteine, die mit einem relativ höheren Biotitgehalt gekennzeichnet sind (FRANCO VETEADO, ROJO ORCOLLANO, ROJO PRINCIPE, vgl. Kap. 3.3.5). Die übrigen Gesteine sind in einem Druckfestigkeitsbereich um 160 bis 170 MPa angesiedelt. Anhand der Granitoide aus der Provinz San Luis wird eine Abhängigkeit der mechanischen Stabilität von der Korngröße deutlich. Hier übersteigen die Werte der relativ feinkörnigeren Varietät MARRON COCO die der übrigen Litholgien um etwa $20 \mathrm{MPa}$. Grundsätzlich können auch für die Biege- und 
Spaltzugfestigkeit gleiche Annahmen gemacht werden. Die Maximalwerte sind auch hier durch die Cordierit-führenden Varietäten sowie durch den Gabbro gegeben. Auffällig ist jedoch, dass für die Gesteine, die mit einer relativ geringen Druckfestigkeit gekennzeichnet sind, hier erhöhte Durchschnittswerte erreicht werden. Der Materialverlust durch abrasive Belastung fällt für alle Granitoide sehr gering aus. Der Minimalwert wird hier von der Varietät AZUL TANGO erreicht, was in der mineralspezifischen Härte der Hauptgemengteile, hier Cordierit und Granat, begründet ist.

Tab. 4.3: Kompressionswellengeschwindigkeiten Vp der beprobten Lithologien argentinischer Naturwerksteine (innerhalb der Gesteinsgruppen sortiert nach der Rohdichte) mit dem jeweiligen gefügebedingten Anistotropiewert $\alpha=(\max -\min ) / \max \cdot 100$.

\begin{tabular}{|c|c|c|c|c|c|c|c|c|}
\hline & \multicolumn{4}{|c|}{ Vp (trocken) $[\mathrm{km} / \mathrm{s}]$} & \multicolumn{4}{|c|}{ Vp (nass) $[\mathrm{km} / \mathrm{s}]$} \\
\hline & x-Richtung & y-Richtung & z-Richtung & $\alpha$ & x-Richtung & y-Richtung & z-Richtung & $\alpha$ \\
\hline \multicolumn{9}{|l|}{ Granitoide } \\
\hline ROJO DRAGÓN & 5,06 & 5,16 & 5,10 & 2,0 & & & & \\
\hline MARRON COCO & 4,98 & 5,06 & 5,23 & 4,7 & 5,99 & 5,93 & 5,87 & 2,0 \\
\hline GRIS PERLA & 4,68 & 4,97 & 4,70 & 5,8 & 5,93 & 5,92 & 6,15 & 3,7 \\
\hline ROSA DEL SALTO & 4,83 & 5,12 & 4,75 & 7,1 & 5,73 & 6,07 & 5,71 & 5,9 \\
\hline ROJO ORCOLLANO & 4,24 & 4,52 & 4,42 & 6,2 & & 5,61 & 5,65 & 0,6 \\
\hline ROJO PRINCIPE & 4,53 & 4,55 & 4,23 & 7,0 & 5,70 & 5,50 & 5,49 & 3,7 \\
\hline SAN MARCOS BLANCO & 4,63 & 4,78 & 4,51 & 5,8 & 5,72 & 5,66 & 5,51 & 3,7 \\
\hline FRANCO VETEADO & 4,42 & 4,28 & 4,57 & 6,5 & 6,04 & 6,08 & 5,96 & 2,0 \\
\hline NEGRO RIOJANO & 6,31 & 6,49 & 6,34 & 2,7 & 6,52 & 6,66 & 6,58 & 2,1 \\
\hline AZUL TANGO & 6,22 & 6,24 & 5,67 & 9,1 & 6,76 & 6,86 & 6,58 & 4,0 \\
\hline \multicolumn{9}{|l|}{ Karbonatgesteine } \\
\hline CALIZA AMARILLA & 4,83 & 4,83 & 4,84 & 0,2 & 5,01 & 5,14 & 5,06 & 2,5 \\
\hline LUNEL COVUNCO & 5,86 & 5,93 & 5,84 & 1,5 & 5,86 & 5,93 & 5,84 & 1,5 \\
\hline DOLOMITA DORADA & 6,02 & 5,97 & 6,10 & 2,1 & 6,15 & 6,11 & 6,21 & 1,5 \\
\hline \multicolumn{9}{|l|}{ Marmor } \\
\hline AZUL CIELO & 3,72 & 3,64 & 3,58 & 3,9 & 6,24 & 6,30 & 6,16 & 2,4 \\
\hline \multicolumn{9}{|l|}{ Vulkanit } \\
\hline STARDUST GREY & 5,16 & 5,10 & 5,14 & 1,2 & 5,28 & 5,25 & 5,26 & 0,6 \\
\hline \multicolumn{9}{|l|}{ Schiefer } \\
\hline SAN LUIS SLATE & 6,76 & 6,30 & 4,84 & 28,5 & 6,82 & 6,40 & 5,09 & 25,3 \\
\hline
\end{tabular}

Innerhalb der untersuchten Kalksteine ist für die dolomitischen Varietäten LUNEL COVUNCO und DOLOMITA DORADA die mechanische Stabilität im Vergleich mit der calcitischen Varietät CALIZA AMARILLA mit deutlich höheren Werten beschrieben. Die Werte zur Druck-, Biege- und Spaltzugfestigkeit für die calcitische Handelssorte fallen in allen Fällen um annähernd 50 \% und mehr geringer aus. Der abrasive Materialverlust ist für die Karbonatgesteine mit Werten zwischen etwa 19 und $25 \mathrm{~cm}^{3} / 50 \mathrm{~cm}^{2}$ dokumentiert und fällt somit naturgemäß im Vergleich mit den beprobten granitoiden Varietäten deutlich höher aus.

Auch wenn die beschriebenen Werte zur mechanischen Stabilität der beprobten Karbonatgesteine in den lithologietypischen Bereichen liegen (vgl. Kap. 3.8.2, 3.10.3), kann 
die mechanische Stabilität durch verschiedene strukturelle Elemente herabgesetzt sein. Besonders sehr feine, makroskopisch nur schwer erkennbare Risssysteme in der Varietät LUNEL COVUNCO stellen potenzielle Schwächezonen im Hinblick auf die mechanische Stabilität dar. Insgesamt traten diese Elemente in der Probensuite zur gesteinstechnischen Charakterisierung sehr selten auf. Doch gerade hinsichtlich der Biege- und Spaltzugfestigkeit können entsprechende Gefügeelemente zu deutlichen Ausreißerwerten führen (vgl. Kap. 3.8.3 und 3.12.4). Dagegen konnten verstärkte Anisotropieeffekte in den Parametern der Festigkeit infolge des primären Lagenbaus nicht festgestellt werden. Lediglich bei der Ermittlung der Biegefestigkeit zeigte sich eine etwas erhöhte Anisotropie von 26 \% (LUNEL COVUNCO). Die Herabsetzung dieses Festigkeitsparameters ist vermutlich in einem Korngrößenwechsel hin zu einem höheren Anteil mikritischen anstelle des sparitischen Materials begründet. Dabei spiegeln aber selbst die geringsten an dieser Varietät ermittelten Werte im Vergleich mit den übrigen untersuchten Lithologien karbonatischer Werksteine eine deutlich höhere mechanische Stabilität wider (vgl. Kap. 3.8.2, 3.10.3).

Hinsichtlich der Varietät DOLOMITA DORADA ist davon auszugehen, dass sich besonders durch auftretende Stylolithenbahnen, die auf syndiagenetische, druckinduzierte Lösungsprozesse zurückgehen, eine Schwächung der mechanischen Widerstandskraft des Gesteins ergibt (vgl. Kap. 3.8.3). Feine, oft schwer erkennbare Mikrorissstrukturen können einen ähnlichen Effekt haben, besonders wenn anstelle der vollständigen Verheilung eine feinblättrige Kristallisation von Eisenoxiden stattgefunden hat. Trotz allem erweist sich die beprobte Varietät DOLOMITA DORADA bei den Untersuchungen zur Ermittlung der gesteinstechnischen Parameter im Allgemeinen als ein hochstabiles Material mit annähernd homogenen Verhalten bei mechanischer Belastung. Lediglich bei der Bestimmung der Biegezugfestigkeit sind wenige Negativausreißer aufgetreten, die dann mit oben erwähnten Mikrorissstrukturen erklärt werden konnten.

Die Untersuchungen der physikalischen und elastischen Eigenschaften haben gezeigt, dass für den Marmor AZUL CIELO lediglich Proben vorlagen, die eine verwitterungsbedingte Vorschädigung in Form einer Gefügeentfestigung aufweisen (vgl. Kap. 4.7.2, 4.7.3). Daher sind die ermittelten Werte, vor allem zu den Parametern der Festigkeit, mit einem daraus resultierenden Fehler behaftet. So hat sich bereits bei der Präparation einzelner Probekörper die fortgeschrittene Entfestigung des Gefüges durch ein deutliches Absanden bemerkbar gemacht. Der Vollständigkeit halber sind die erhaltenen Testresultate in der Tabelle 4.4 mit beinhaltet. Nach Firmeninformation liegen die Werte jedoch um ein Vielfaches höher (Druckfestigkeit 167 MPa, Biegefestigkeit $37 \mathrm{MPa}$ ).

Für die Porphyr-Varietät STARDUST GREY wurden hinsichtlich der mechanischen Stabilität Werte ermittelt, die lithologieübergreifend zu den Maximalwerten zu zählen sind. Das dichte, sehr kompakte Gefüge resultiert in Druckfestigkeiten, die zum Teil jenseits $200 \mathrm{MPa}$ liegen. Entsprechend hohe Werte sind auch für die verbleibenden Parameter der Festigkeit gegeben $\left(\mathrm{St}_{\mathrm{F}}\right.$ 21,5 $\mathrm{MPa}$; $\mathrm{St}_{\mathrm{T}}$ 10,4 MPa). Hinsichtlich des Widerstands gegen abrasive Kräfte zeigt der Porphyr ein ausgesprochen anisotropes Verhalten. Die Spanne zwischen Minimalwert $\left(4,7 \mathrm{~cm}^{3} / 50 \mathrm{~cm}^{2}\right)$ und Maximalwert $\left(12,6 \mathrm{~cm}^{3} / 50 \mathrm{~cm}^{2}\right)$ beschreibt dabei den von Peschel (1983) genannten lithologietypischen Wertebereich für dazitische Gesteine. 
In der Verwendung von Schiefern als Werkstein ist generell das charakteristische, zumeist extreme Richtungsgefüge zu beachten (vgl. Kap. 3.9). Diese Anisotropie ist in allen untersuchten Festigkeitseigenschaften gegeben, wobei sich bei der Ermittlung der Biegefestigkeit eine maximale Wertdifferenz von annähernd 100 \% (min. 0,5 MPa; max. 72,0 $\mathrm{MPa}$ ) ergibt. Die Gefügerichtung, für die der Minimalwert gegeben ist und die sehr gute, natürliche Spaltbarkeit des Schiefers wiedergibt, ist aus bautechnischer Sicht jedoch vernachlässigbar (vgl. Kap. 3.2 und 3.9.2). Von Vorteil ist vor allem auch der für Schiefer im Allgemeinen relativ geringe Materialverlust durch Abrasivkräfte. Diese Resistenz resultiert aus einem recht hohen Quarzgehalt in Verbindung mit der sehr geringen Porosität des Gesteins. Die richtungsabhängigen Werte liegen im Bereich zwischen 7 und $21 \mathrm{~cm}^{3} / 50 \mathrm{~cm}^{2}$ und somit kann der argentinische Schiefer in den Wertebereich der z.B. von Peschel (1983) für Monzonite beschriebenen Abriebfestigkeit eingegliedert werden.

\subsection{Exemplarischer Vergleich experimenteller und prognostizierter Daten}

Häufig erfolgt im Rahmen einer regionalen Erfassung eine Beprobung von deutlich geringerem Umfang. Üblicherweise liegen lediglich Handstücke zur petrographischen Analyse mittels Dünnschliffmikroskopie oder verschiedene Musterplatten eines Gesteins vor. An diesen kann bereits die Bestimmung der Basiseigenschaften Rohdichte und Porosität des Materials erfolgen. Außerdem ist die Ermittlung des elastischen Verhaltens mit dem Ausdruck der Kompressionswellengeschwindigkeit Vp möglich. Die entsprechenden Untersuchungen stellen relativ einfache und kostengünstige Versuche dar. Die detaillierte Analyse der mechanischen Festigkeitsparameter ist dagegen mit einem hohen Zeit- und Kostenaufwand verbunden. Zudem ist ein erheblich umfangreicheres Probeninventar nötig, um zu einer repräsentativen und umfassenden Charakterisierung der technischen Eigenschaften zu gelangen.

Wie in Kapitel 3 gezeigt, lassen sich aus den Basiseigenschaften Rohdichte und effektive Porosität sowie auch aus der Kompressionswellengeschwindigkeit in der gemeinsamen Betrachtung mit den Ergebnissen der lithologischen Untersuchungen erste Eindrücke hinsichtlich der mechanischen Stabilität eines Gesteins gewinnen. Im Folgenden werden anhand verschiedener argentinischer Granitoide und Karbonatgesteine unter Ausnutzung der in Kapitel 3.11 und 3.12.4 beschriebenen regressiven Zusammenhänge exemplarisch Daten zur Druckfestigkeit sowie zur Biegefestigkeit errechnet und mit den ermittelten Daten verglichen.

Generell ist zu beachten, dass bei Daten zur mechanischen Stabilität, die sich aus den Basiseigenschaften errechnen, kein Bezug zu gerichteten Gefügeelemente wie Foliation oder sedimentärer Lagenbau besteht (vgl. Kap. 3.11). Da im Falle der hier betrachteten Gesteinsproben keine erheblichen richtungsbezogenen Stabilitätsunterschiede bei der Prüfung der Druckfestigkeit festgestellt werden konnten, wird auf diesen Aspekt im Kapitel zur Betrachtung der Biegefestigkeit näher eingegangen. 


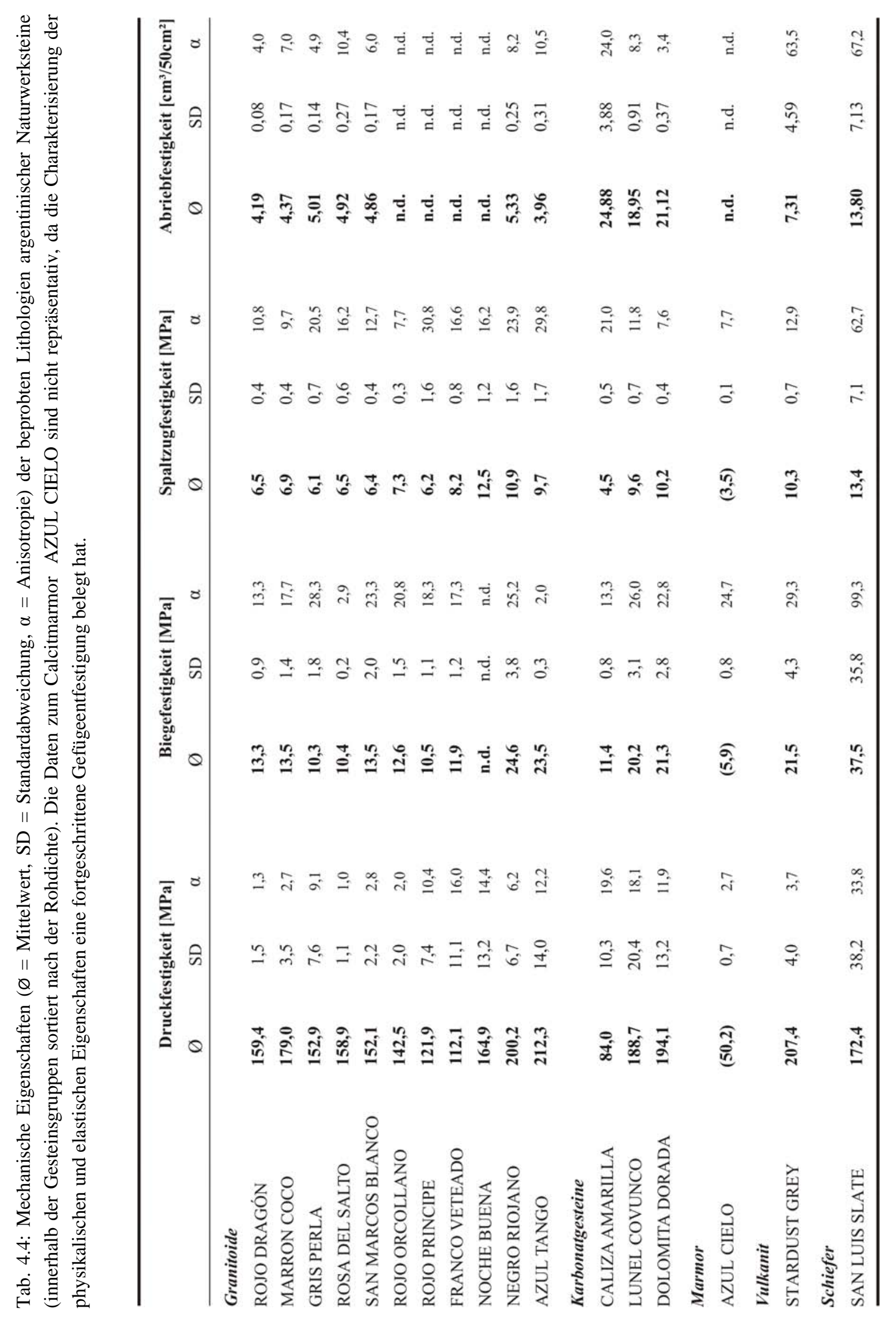




\subsubsection{Prognose der Druckfestigkeit}

Die Prognose der Druckfestigkeiten zu den granitoiden Werksteinvarietäten beruht auf linearen, die der Karbonatgesteine auf exponentiellen Funktionen (vgl. Kap. 3.11.1, 3.11.4 und 3.12.4). Im Vergleich mit den experimentell bestimmten Mittelwerten ergibt sich in der Gruppe der Granitoide in der Mehrheit eine relativ gute Deckung (Abb. 4.36). Abweichend davon sind für die Gesteine ROJO PRINCIPE und ROJO ORCOLLANO geringere und für die Varietät MARRON COCO höhere Werte aus den Prüfungen hervorgegangen. Die größte Abweichung ist mit $44 \mathrm{MPa}$ für den Granit ROJO PRINCIPE zu verzeichnen, womit der experimentelle Mittelwert von 121,9 MPa in den unteren Bereich der 80 \%-igen Streuung um die Regressionsgerade fällt. Diese deutliche Abweichung resultiert aus dem erhöhten Biotitgehalt im Mineralbestand, da sich die mineralspezifisch schwache Stabilität auf das Gesamtgefüge des Gesteins auswirkt. Relativ gleiche Annahmen können zur Handelssorte ROJO ORCOLLANO gemacht werden, wobei hier der im Allgemeinen geringere Biotitgehalt jedoch zu einer etwas höheren Druckfestigkeit führt. Im Falle des Granits MARRON COCO kann die Höhe der festgestellten Druckfestigkeit, die rund 20 MPa über dem prognostizierten Wert liegt, ebenfalls durch das Gefüge erklärt werden. Im Gegensatz zu den anderen Varietäten des Abbauareals (GRIS PERLA, ROSA DEL SALTO, ROJO DRAGÓN) ist die Korngröße bei einem gleichkörnigen Gefüge leicht reduziert. Gleichzeitig ist der Quarzgehalt etwas höher anzusetzen. Insgesamt resultiert daraus eine erhöhte Stabilität bei einer statischen Belastung trotz der Tatsache, dass mit einem Wert von 2,62 g/ $\mathrm{cm}^{3}$ die geringste Rohdichte in der Gruppe der Granitoide beschrieben ist. Die Werte zur Druckfestigkeit basierend auf der Kompressionswellengeschwindigkeit Vp sind im Vergleich mit denen aus der Rohdichte errechneten mit einer Ausnahme in etwa gleich angeordnet. Die Abweichung zwischen beiden Werten liegt im Mittel bei etwa $3 \%$. Die Ausnahme ergibt sich für den Gabbro NEGRO RIOJANO, bei dem sich ein Unterschied von 13 \% ergibt. Doch auch der aus Vp errechnete Wert liegt im zentralen Bereich der lithologietypischen Festigkeit.

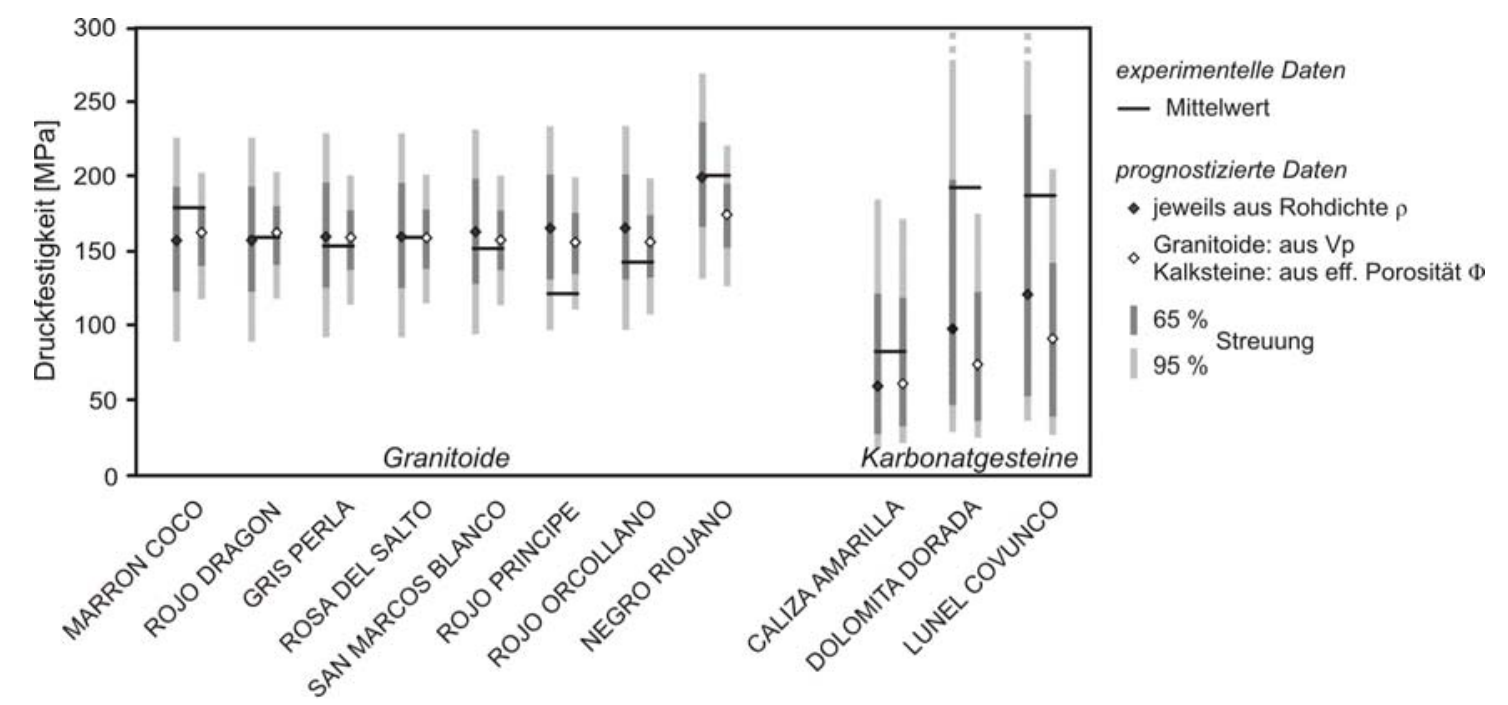

Abb. 4.36: Vergleich von experimentell ermittelten und prognostizierten Daten zur Druckfestigkeit argentinischer Granitoide und Karbonatgesteine. Die Gesteinsgruppen sind jeweils nach zunehmender Rohdichte sortiert. 
Für die verschiedenen Karbonatgesteine liegen die prognostizierten Werte zumeist deutlich unterhalb der experimentell ermittelten Druckfestigkeiten (Abb. 4.36). Dabei errechnen sich im Extremfall (DOLOMITA DORADA, $\mathrm{St}_{\mathrm{F}}$ aus $\Phi$ ) lediglich $39 \%$ der bestimmten Druckfestigkeit. Trotz der ebenfalls hohen Abweichungen zu den tatsächlichen Werten, ergeben sich aus der Rohdichte bessere Näherungen. Der jeweilige untere Wert der 65 \%-igen Streuung um die Regressionslinie herum ist noch im unteren Bereich der lithologiebezogenen 80 \%-igen Aufenthaltswahrscheinlichkeit der jeweiligen Korrelation eingeordnet (Abb. 4.37). Die zumeist erheblichen Abweichungen basieren auf einem insgesamt sehr homogenen Gefüge, dass bei allen hier betrachteten Karbonatgesteinen ausgebildet ist. Wie zuvor beschrieben, wird die mechanische Stabilität im Falle der Varietäten DOLOMITA DORADA und LUNEL COVUNCO lediglich durch feine Mikrorissstrukturen negativ beeinflusst, die jedoch bei der Druckbelastung lediglich untergeordnet zur Wirkung kommen.

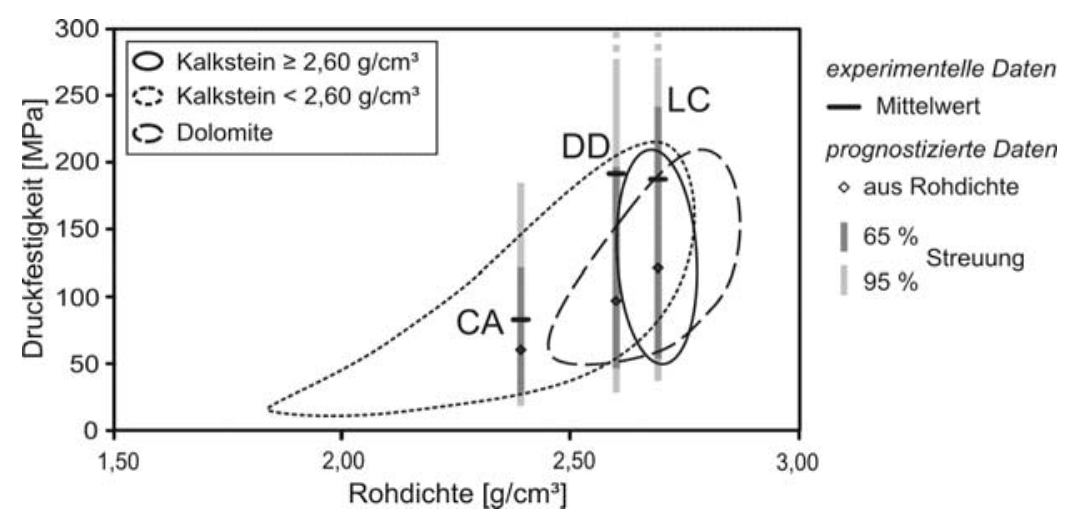

Abb. 4.37: Vergleich experimentell bestimmter Daten mit prognostizierten Daten zur Druckfestigkeit der argentinischen Karbonatgesteinvarietäten CALIZA AMARILLA (CA), DOLOMITA DORADA (DD) und LUNEL COVUNCO (LC) in Korrelation mit der Rohdichte. Zusätzlich sind die Bereiche der 80 \%-igen Aufenthaltswahrscheinlichkeit der jeweiligen Grundgesamtheit der Untergruppen eingetragen.

\subsubsection{Prognose der Biegefestigkeit}

Entsprechend der Druckfestigkeit beruht auch die Prognose der Biegefestigkeit der granitoiden Werksteinvarietäten auf linearen, die der Kalksteine auf exponentiellen Funktionen (vgl. Kap. 3.11.1, 3.11.3 und 3.12.4). Die aus den verschiedenen Parametern prognostizierten Daten zur Biegefestigkeit der Granitoide und Karbonatgesteine sind dabei in ihrer relativen Höhe mit den Werten der Druckfestigkeit vergleichbar (Abb. 4.38, vgl. Abb. 4.36). Innerhalb der Granitoide zeigen sich die deutlichsten Abweichungen wiederum bei der gneisartigen Varietät ROJO PRINCIPE und zudem bei den Graniten GRIS PERLA und ROSA DEL SALTO sowie beim Gabbro NEGRO RIOJANO.

Anhand der Abweichungen zwischen den experimentell bestimmten und errechneten Werten in der Gruppe der Granitoide ist deutlich die Abhängigkeit der mechanischen Stabilität von dem gesteinstypischen Korngrößenspektrum erkennbar. Bei den Varietäten, die eine homogene Korngrößenverteilung ohne eine mineralogische Vorzugsorientierung bei gleichzeitig fein- bis mittelkörnigen Korngrößen aufweisen (NEGRO RIOJANO, MARRON COCO), liegen die ermittelten Werte im vorhergesagten Bereich oder deutlich darüber. Die granitischen Varietäten GRIS PERLA und ROSA DEL SALTO sind dagegen durch ein 
porphyritisches Gefüge mit einer grobkörnigen Matrix charakterisiert. Daraus ergibt sich, vor allem im Vergleich mit der equigranular mittelkörnigen Varietät MARRON COCO mit etwa gleicher Rohdichte, eine geringere Biegefestigkeit. Für die relativ geringe Höhe der experimentell bestimmten Werte zu den Handelssorten ROJO ORCOLLANO und vor allem ROJO PRINCIPE muss, wie auch bei der Druckfestigkeit, die mechanische Instabilität der verstärkt auftretenden Biotite verantwortlich gemacht werden.

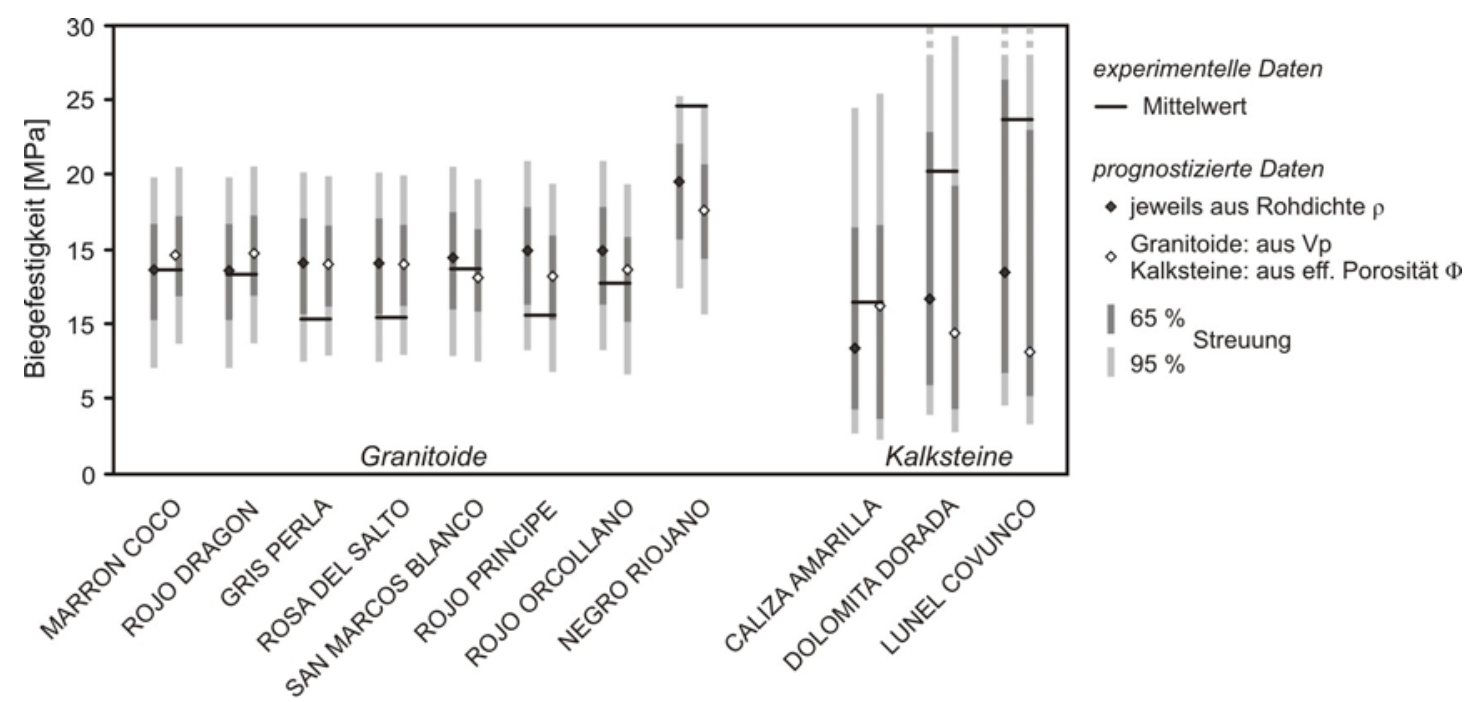

Abb. 4.38: Vergleich von experimentell ermittelten und prognostizierten Daten zur Biegefestigkeit argentinischer Granitoide und Karbonatgesteine. Die Gesteinsgruppen sind jeweils nach zunehmender Rohdichte sortiert.

In der Gruppe der Karbonatgesteine zeigt sich bei der Betrachtung der experimentell bestimmten Mittelwerte wiederum die niveauabhängige Fehlerstreuung infolge der exponentiellen Verknüpfung der betrachteten Parameter. Dabei ist wie auch bei der Druckfestigkeit die deutlichste Abweichung bei der Varietät DOLOMITA DORADA beschrieben. Hier erreicht der aus der Porosität berechnete Wert lediglich $46 \%$ der tatsächlichen mittleren Biegefestigkeit.

Für die im Rahmen der mechanischen Stabilitätsmessungen durchgeführten Versuche haben sich bei der Bestimmung der Biegefestigkeit der argentinischen Werksteinvarietäten die höchsten Anisotropiewerte aller betrachteten Festigkeitsparameter ergeben. Diese richtungsabhängigen Differenzen im mechanischen Verhalten ergeben sich aus der räumlichen Anordnung mechanisch schwacher Gefügeelemente, so z.B. die parallele oder subparallele Orientierung von Glimmermineralen, Mikrorisssysteme oder auch ein sedimentärer Lagenbau. In Abbildung 4.39 sind die in Abhängigkeit vom jeweiligen Gefüge ermittelten Wertebereiche der Biegefestigkeit sowie die daraus resultierenden, richtungsbedingten Anisotropien der argentinischen Granitoide und Karbonatgesteine dargestellt. Neben den zum Teil erheblichen Schwankungen innerhalb einer Prüfrichtung, können auch die Abweichungen der prognostizierten Werte von den tatsächlichen Werten auf verschiedene Elemente des Gefüges zurückgeführt werden. 
In der Suite der beprobten granitoiden Gesteine ist für den Granit GRIS PERLA mit 28,3 \% die höchste Anisotropie ausgebildet. Dies überrascht deshalb, da die Varietät ROSA DEL SALTO, die lediglich eine Farbvarietät darstellt, gleichzeitig den geringsten Anisotropiewert aufweist. Zurückgeführt werden kann dies eventuell darauf, dass in den Lagerstättenbereichen, in denen die Handelssorte ROSA DEL SALTO abgebaut wird, das porphyrische Gefüge nicht so extrem ausgebildet erscheint. Folglich sind die verstärkten Schwankungen der grauen Farbvarietät infolge der größeren Phänokristalle zu verstehen. Bei einzelnen Prüfkörpern erfolgte der Bruch bei Biegebelastung fast ausschließlich in diesen Großkristallen (Abb. 4.40 a, vgl. Abb. 4.40 c), wobei dann auch häufig die Bruchfläche von der eigentlichen Belastungslinie abweicht. Neben der geringen mineralspezifischen Stabilität wird dabei deutlich, dass, wie oben beschrieben, mit zunehmender Korngröße eine Herabsetzung der Festigkeit einhergeht. Zum anderen zeigt sich die Notwendigkeit, die Probengröße an das Korngrößenspektrum des zu untersuchenden Gesteins anzupassen, wie es auch die entsprechende DIN EN 12372 vorsieht. In diesem Fall wären für eine im Detail normgerechte Prüfung jedoch Probekörper von mindestens $100 \mathrm{~mm}$ Höhe und damit einer Länge von $600 \mathrm{~mm}$ notwendig. Entsprechend dimensionierte Prüfeinrichtungen stehen jedoch zumeist nicht zur Verfügung.

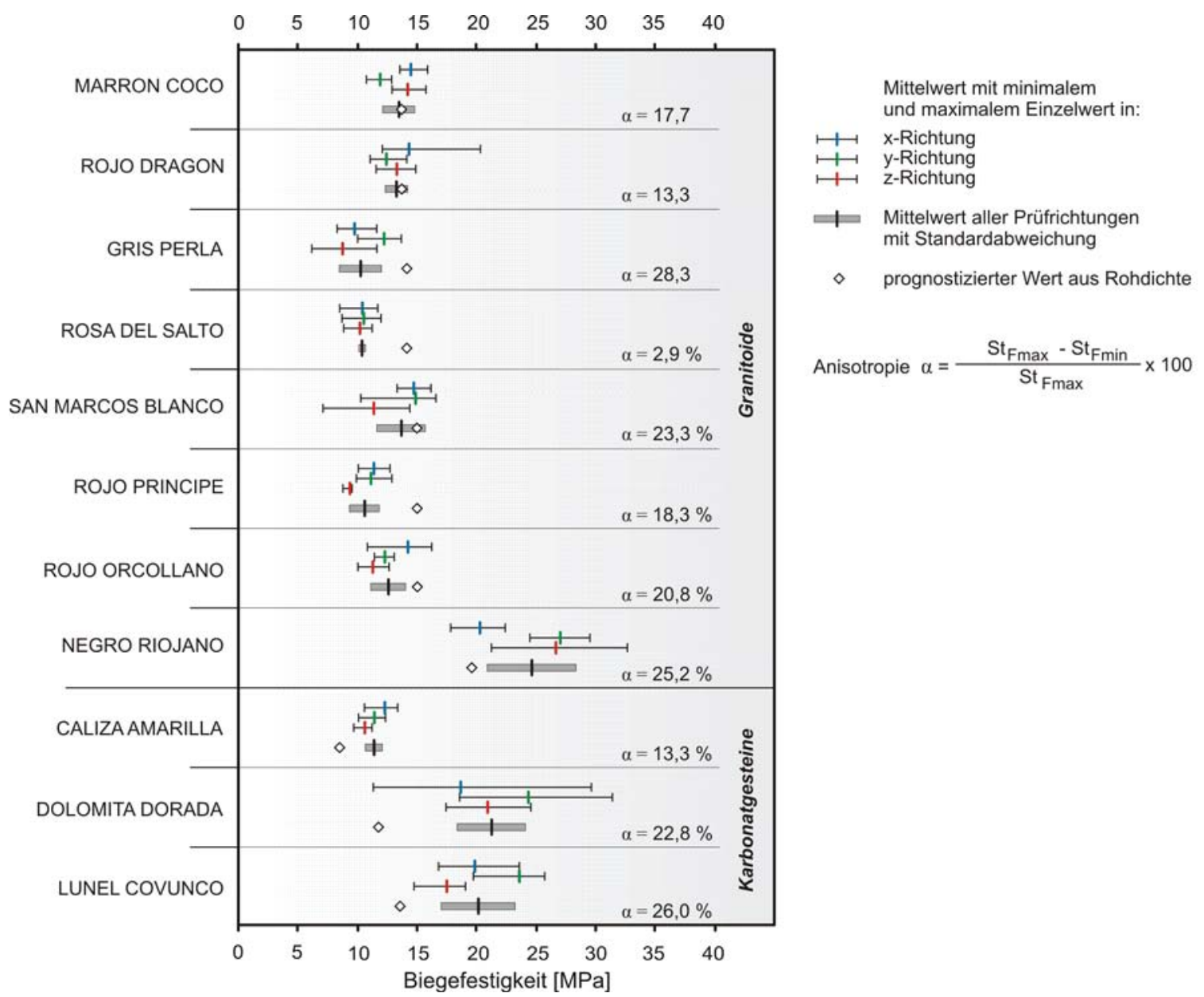

Abb. 4.39: Vergleich der gefügeabhängig experimentell bestimmten Werte der Biegefestigkeit argentinischer Werksteinvarietäten mit aus der Rohdichte errechneten Werten. 
Hohe Anisotropien zeigen sich weiterhin bei den Varietäten ROJO ORCOLLANO, ROJO PRINCIPE und SAN MARCOS BLANCO. In allen drei Fällen können diese auf Glimmerminerale zurückgeführt werden, doch unterscheiden sich die einzelnen Prüfrichtungen in ihrem internen Datenspektrum zum Teil deutlich voneinander. Für die Varietät ROJO PRINCIPE ist in allen Prüfrichtungen ein relativ enger Datenbereich beschrieben, was auf ein deutliches Parallelgefüge der Biotite in der xy-Ebene zurückzuführen ist. Dagegen ist eine derartige Vorzugsorientierung im Gefüge des Granodiorits SAN MARCOS BLANCO nicht ausgebildet. Die Glimmerminerale, zumeist Muskovit, sind eher regellos im Mineralverband verteilt. Dadurch kommt es zu stärkeren Schwankungen in den Einzelwerten, wenn im Probekörper ein gehäuftes Auftreten von Glimmermineralen parallel zur Belastungsrichtung vorhanden ist (Abb. 4.40 b, d).
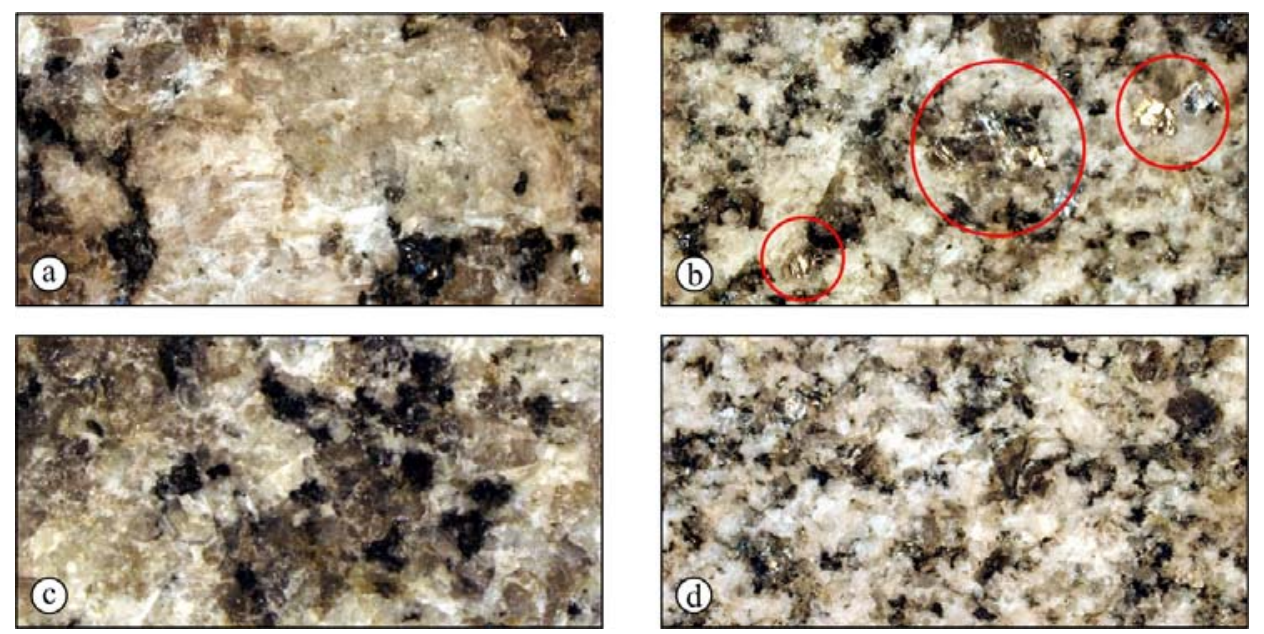

Abb. 4.40: Bruchflächen von Prüfkörpern nach Biegebelastung (Ausschnittgröße jeweils 50 x 25 mm): a, c) GRIS PERLA (Granit) mit herabgesetzter mechanischer Stabilität durch Feldspatphänokristalle (Bild a); b, d): SAN MARCOS BLANCO (Granodiorit) mit herabgesetzter mechanischer Stabilität durch Muskovitakkumulation parallel zur Belastungsrichtung (Bild b) im sonst richtungslosen Korngefüge (Bild d).

Besonders bei sedimentären Gesteinen ist ein Augenmerk auf mögliche Wechsel in der mechanischen Stabilität zu legen, die mit dem sedimentären Lagenbau einhergehen (vgl. Kap. 3.2 und Kap. 3.8.2). Für die Suite der argentinischen Karbonatgesteine ist im Allgemeinen eine massige, mikritische Struktur beschrieben, bei der kein deutlicher Schichtwechsel erkennbar ist (vgl. Kap. 4.6.2.2). Ausgenommen ist hierbei die Varietät LUNEL COVUNCO, bei der ein sedimentäres Lager anhand eines wechselnden Fossilgehaltes gegeben ist. Hier ist folglich auch ein anisotropes Verhalten bei Biegebeanspruchung mit dem Minimum senkrecht zum Lagenbau (z-Richtung) ausgebildet (Abb. 4.39). Auf die gleiche Prüfrichtung entfällt auch der Minimalwert bei der Varietät CALIZA AMARILLA, womit auch hier trotz der geringeren Anisotropie die sedimentäre Schichtung zum Ausdruck kommt.

Ein relativ hoher Anisotropiewert ergibt sich auch bei der Prüfung der Varietät DOLOMITA DORADA, obwohl ein ausgeprägter Lagenbau nicht erkannt werden konnte. Eventuell könnte eine gerichtete Mineralisation aus dem Prozess der Dolomitisierung hervorgegangen sein, was jedoch selbst bei mikroskopischer Betrachtung infolge des mikritischen Gefüges nicht identifiziert werden kann. Auffallend sind zudem die hohen 
Wertebereiche zu den einzelnen Prüfrichtungen. So liegen z.B. die Einzelwerte bei der Prüfung in x-Richtung zwischen 11,5 und 29,8 MPa (im Mittel 18,8 MPa). Entsprechend weite Datenspektren sind auch für die übrigen Prüfrichtungen gegeben. Außerdem resultiert aus der Belastung parallel der Mittellinie bei mehreren Proben ein unregelmäßiger Bruch (Abb. 4.41 a). Das mechanische Versagen des Gesteins ist in diesen Fällen an makroskopisch kaum erkennbare Haarrisse gebunden, die erst nach dem Bruch anhand einer oft deutlichen, feinen Mineralisation von Eisenoxid identifiziert werden können (Abb. 4.41 b, c).

Wie bereits in den Kapiteln 3.8 .3 beschrieben, wird insgesamt deutlich, dass bei Karbonatgesteinen aus einer stark heterogenen Gefügeausbildung eine niveauabhängige Fehlerstreuung mit Zunahme bei höheren Rohdichten beziehungsweise geringeren Porositäten resultiert. Bei der Prüfung der Biegefestigkeit des Dolomits DOLOMITA DORADA wurde jedoch lediglich bei einer Einzelprobe der prognostizierte Wert nicht erreicht $\left(\mathrm{St}_{\mathrm{Fmin}}\right.$ 11,5 MPa; prognostizierter Wert 11,7 MPa). Die Mittelwerte in allen drei Prüfrichtungen liegen jedoch deutlich über dem prognostizierten Wert.

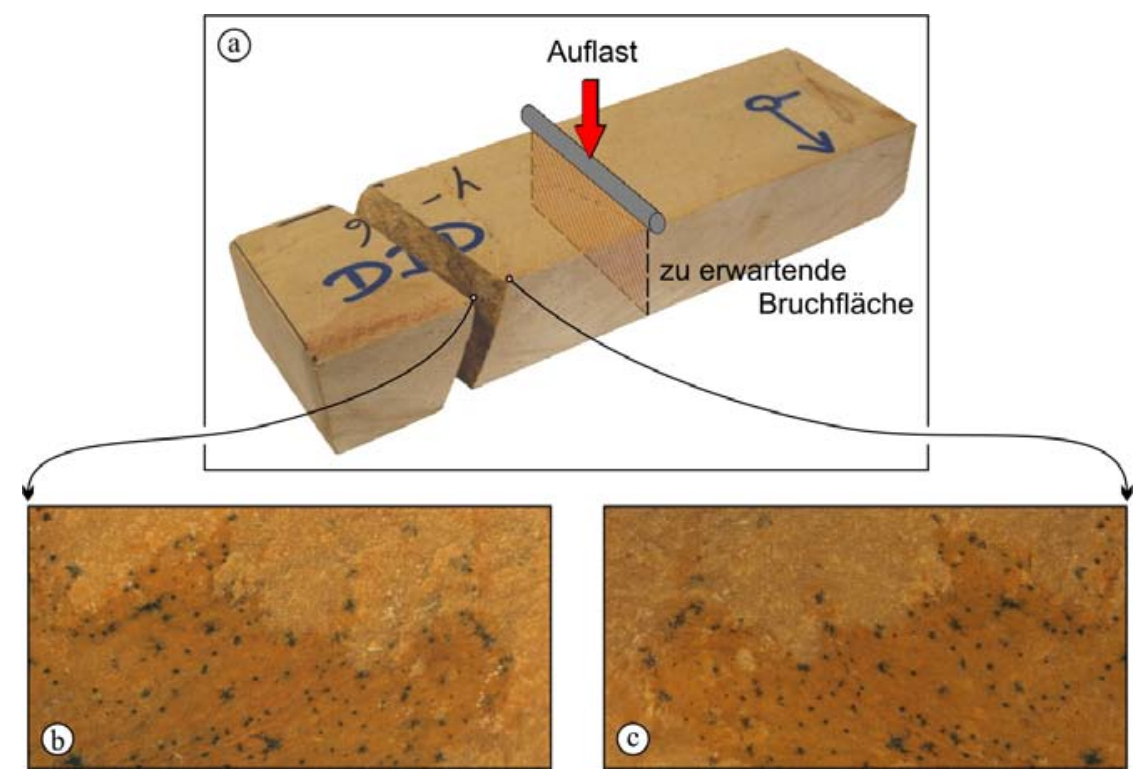

Abb. 4.41: a) Prüfung der Biegefestigkeit in y-Richtung am argentinischen Dolomit DOLOMITA DORADA mit unregelmäßiger Ausbildung der Bruchfläche infolge b) und c) makroskopisch kaum erkennbaren Mikrorissen, die nach dem Bruch an einer Mineralisation identifiziert werden können (Ausschnittgröße in b und c jeweils $50 \mathrm{x}$ $25 \mathrm{~mm})$.

Der Vergleich der experimentell bestimmten Werte für die Parameter Druck- und Biegefestigkeit mit den aus der Rohdichte, der effektiven Porosität sowie der Kompressionswellengeschwindigkeit kalkulierten Daten hat gezeigt, dass eine generelle Einschätzung der mechanischen Stabilität möglich erscheint. Dies gilt vor allem in einer vergleichenden Betrachtung unterschiedlicher Gesteine einer lithologischen Gruppe. Herauszustellen ist dabei, dass eine Einbeziehung des gesteinsspezifischen Gefügeinventars in die Interpretation prognostizierter Daten unbedingt erfolgen muss. 


\subsection{Zusammenfassende Bewertung des Werksteinpotenzials und Schlussfolgerungen}

Im Verlauf der letzten Jahrzehnte stieg die weltweite Nachfrage nach hochqualitativen Naturwerksteinen mehr und mehr an. Basierend auf der komplexen geologischen Vielfalt des südamerikanischen Kontinents, verfügt auch Argentinien über eine Vielzahl von Varietäten, die ein breites Spektrum an Strukturen und Farben abdeckt. So kann Argentinien verschiedene Gesteine anbieten, die am weltweiten Natursteinmarkt permanent von Interesse sind. Dies gilt z.B. für die Granitvarietät GRIS PERLA (Provinz San Luis), für die im Hinblick auf die technischen Eigenschaften eine Konkurrenzfähigkeit am weltweiten Markt gegeben ist. Andere Varietäten, wie die Porphyr-Handelssorte STARDUST GREY bzw. die Gesamtheit der patagonischen Porphyre oder auch der Phyllitschiefer aus der Provinz San Luis, konnten bereits auf dem Weltmarkt etabliert werden. Hier ist zumeist eine Zusammenarbeit mit europäischen Firmen oder Firmenkonsortien vorhanden. Zudem konnten verschiedene Lagerstätten aufgenommen werden, für die, basierend auf den spezifischen Dekor- und Materialeigenschaften, ein hohes wirtschaftliches Interesse resultieren könnte. Dies bezieht sich z.B. auf den Gabbro NEGRO RIOJANO (Provinz La Rioja) oder auch die verschiedenen Kalksteine der Provinz Neuquén.

Sowohl der Abbau als auch die Verwendung von Naturwerksteinen bei Bau- sowie Monumentalwerken hat in Argentinien eine lange Tradition. Im Vergleich mit anderen Ländern Südamerikas, insbesondere des direkten Nachbarn Brasilien, ist eine detaillierte Erfassung zur intensiven Nutzung des hochqualitativen Rohstoffs Naturstein bisher lediglich in Ansätzen erfolgt. Sicherlich auch dadurch bedingt, gehen lediglich $20 \%$ der Gesamtproduktion in den Export, während z.B. 45 \% der brasilianischen Gesamtproduktion in den weltweiten Natursteinmarkt einfließen (Montani 2003). Diese Situation steht zum Teil auch mit der letzten Wirtschaftskrise im Zusammenhang. Zurzeit ist eine leichte Erholung der Werksteinindustrie Argentiniens zu beobachten. Einige der infolge der Wirtschaftskrise stillgelegten Brüche konnten wieder in Betrieb genommen werden und auch einige wenige Vorkommen konnten neu erschlossen werden. Die im Vorangegangen beschriebenen Natursteinvarietäten geben nur einen Ausschnitt aus dem auf der komplexen geologischen Vorgeschichte beruhenden möglichen Potenzial mit einer Vielfalt an Varietäten wieder. Leider lag bisher für viele Lagerstätten keine oder eine nicht ausreichende geologische Erkundung hinsichtlich der Lagerstättensituation vor. Zudem existierte für nur wenige Werksteinvarietäten ein ausreichender Kenntnisstand hinsichtlich der physikalischen und technischen Gesteinseigenschaften.

Die hier vorgestellte Aufnahme verschiedener Werksteinvorkommen Argentiniens stellt zum einen grundlegende lagerstätten-kontrollierende Elemente dar. Diese können als Basis für detaillierte Erkundungen zur kontrollierten Gewinnung des Rohstoffs herangezogen werden. Ferner konnte exemplarisch gezeigt werden, dass auch bei sehr geringen Probenmengen unter Beachtung der Mineralogie sowie der charakteristischen Gefügemerkmale eines Gesteins eine generelle Einschätzung hinsichtlich der mechanischen Stabilität erfolgen kann. Somit kann der bautechnische Verwendungsbereich eines Gesteins tendenziell eingegrenzt werden. Durch eine ungefähre Kenntnis der gesteinsspezifischen 
Eigenschaften können auch Rückschlüsse gezogen werden, die sich auf die generelle Qualität eines Gesteins beziehen. Somit kann unter Einbeziehung des charakteristischen Dekors eines Werksteins eine Einschätzung des wirtschaftlichen Potenzials im Hinblick auf die Absatzfähigkeit verschiedener Produktgruppen erfolgen. Unter Beachtung der spezifischen Trennflächengefüge der Lagerstätten können wiederum auch Rückschlüsse auf die Abbauplanung beziehungsweise Abbauführung gezogen werden.

Eine entsprechend durchgeführte überregionale Erfassung von Werksteinlagerstätten kann somit als Orientierung und Grundlage für weitere Entscheidungen im Hinblick auf die Entwicklung und den Ausbau des Natursteinsektors dienen. Neben den Erkenntnissen aus den methodischen Grundlagen zur Relevanz des Trennflächengefüges und der umfassenden Gesteinscharakterisierung müssen hinsichtlich einer Bewertung des wirtschaftlichen Potenzials einer Lagerstätte jedoch weitere Faktoren Beachtung finden. Dazu zählen vor allem auch Faktoren, die nicht beziehungsweise nur in sehr geringem Maße numerisch erfasst und ausgewertet werden können. Beispiele dafür sind etwa strukturelle Änderungen (Dekoränderungen) im Gestein, mineralogische Variabilitäten auf der Fläche eines Vorkommens oder auch die sich daraus ergebende Farbvarianz. Dabei muss weiterhin auch auf ökonomische Aspekte eingegangen werden, die sich z.B. mit der Definition von Handelssorten und der damit verbundenen Notwendigkeit einer langfristigen Verfügbarkeit ergeben.

Exemplarisch sollen im Folgenden die verschiedenen, relevanten Punkte am Beispiel eines Dolomitmarmorvorkommens im S der Republik Tansania dargestellt werden. Marmorkörper können infolge einer möglicherweise wechselhaften tektonischen und strukturellen Entwicklung kompliziert gebaute Lagerstättenkörper bilden (vgl. Kap. 2.2, Kap. 4.6.2.4). Ein weiteres Problem, dass anhand des gewählten Fallbeispiels dargestellt werden soll, ist der oftmals wechselnde Chemismus, der sich in einer differenzierten mineralogischen Zergliederung des Lagerstättenkörpers äußern kann. Im Rahmen der generellen Gesteinscharakterisierung soll außerdem das oft typische, thermohygrisch induzierte Expansionsverhalten von Marmor berücksichtigt werden, womit ein enormes Schadenspotenzial in der bautechnischen Anwendung verbunden sein kann (vgl. Kap. 3.13.1, Abb. 3.80). Da eine Normierung entsprechender Untersuchungen bisher aussteht, müssen die diesbezüglichen Analysen an den Stand der Wissenschaft angepasst sein. Somit kann im Rahmen der regionalen Erfassung, die die Basis zur Detailexploration darstellen muss, u.a. auch verdeutlicht werden, dass eine detaillierte Gesteinscharakterisierung vor allem auch an die mögliche Verwendung eines Gesteins angepasst werden muss (vgl. Kap.1). 


\section{REGIONALE ERFASSUNG VON NATURWERKSTEIN: \\ DER DOLOMITMARMOR VON MTESA, TANSANIA}

\subsection{Grundlagen und Vorgehensweise}

Die fundierte Bewertung einer Werksteinlagerstätte nach geowissenschaftlichen Gesichtspunkten erfordert die Zusammenführung der methodischen Grundlagen, um zu einer Einschätzung des wirtschaftlichen Potenzials zu gelangen. Neben dem generellen Volumen des Lagerstättenkörpers bestimmt die Genese des Primärgesteins als auch die sich anschließende metamorphe und tektonische Entwicklung eines Gesteinskomplexes zum einen den Grad der Verfügbarkeit bzw. die generelle Möglichkeit zur Gewinnung werksteinfähiger Blockware. In gleichem Zusammenhang steht zudem auch die technische Umsetzbarkeit eines Rohstoffabbaus im Sinne einer optimierten und nachhaltigen Nutzung. Diesem Aspekt ist sowohl bei geologisch einfach strukturierten Lagerstättenkörpern und insbesondere bei tektonisch komplizierten Lagerstätten unbedingte Beachtung $\mathrm{zu}$ schenken. Zum anderen gehen aus der mineralogischen und strukturellen Zusammensetzung eines Gesteins die physikalischen und technischen Eigenschaften des Materials hervor, die wiederum eine Abgrenzung der bautechnischen Anwendungsmöglichkeit bedingen.

Die geologischen Erkundung einer Lagerstätte verfolgt somit das Ziel einer Einschätzung der Rohstoffvorräte innerhalb eines Lagerstättenkörpers und deren technologische Gewinnbarkeit sowie einer Aussage über die Qualität des Rohstoffs und deren Veränderlichkeit innerhalb der Lagerstätte als auch im Hinblick auf spezifische Gefüge- oder Strukturelemente. Daher wurden exemplarisch am Marmorvorkommen von Mtesa, Tansania, folgende Untersuchungen durchgeführt (vgl. Mosch \& Siegesmund 2008):

a) Erfassung und kartographische Darstellung des Vorkommens anhand bekannter Abbaue sowie weiterer Aufschlusspunkte im Oberflächenausbiss.

b) Erfassung und Vermessung der Lagerungsverhältnisse, des Trennflächengefüges und der Verteilung der Farbvarianz im Vorkommen.

c) Erfassung und Bewertung des Einflusses möglichen Nebengesteins auf die Lagerstättensituation.

d) Erfassung des Einflusses großräumiger Störungen auf die Ausbildung des Trennflächengefüges.

e) Orientierte Probennahme zur umfassenden Gesteinscharakterisierung entsprechend den mineralogischen und gefügekundlichen Aspekten der auftretenden Varietäten, sofern sie von wirtschaftlicher Bedeutung sind.

Im Gegensatz zum Fallbeispiel der überregionalen Erfassung von Naturwerksteinen in Argentinien, soll hier in der Gesamtheit eine detaillierte Bewertung der Marmorlagerstätte nach marktwirtschaftlichen Kriterien erfolgen. Dabei kommt die „Internationale RahmenVorratsklassifikation der Vereinten Nationen / UNFCR“ (UNO 1997) zur Anwendung (Abb. 5.1). Diese Klassifikation wurde ursprünglich zur Beurteilung fester fossiler Brennstoffe und 
mineralischer Rohstoffen entwickelt und den Aspekten der Naturwerksteinerkundung und -bewertung angepasst (Stein 2007).

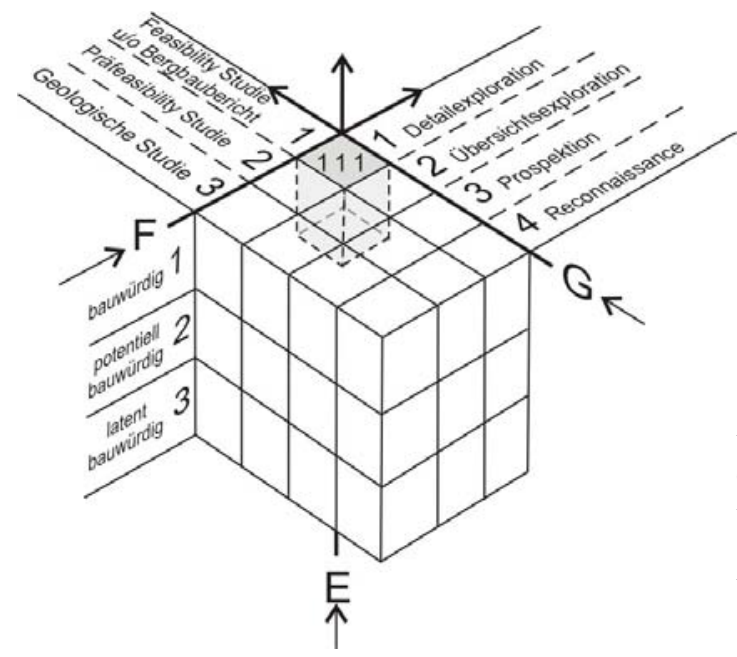

Abb. 5.1: Schematische Darstellung zur Bewertung nach der „Internationale Rahmen-Vorratsklassifikation der Vereinten Nationen“ (UNO 1997) mit: Geologischer Achse, Feasibility Achse, Bauwürdigkeits-(Economic) -achse. Nähere Erläuterungen im Text.

Die Bewertung wird dabei in drei Teilaspekten zum geologischen Untersuchungsstand (G), zum bergtechnisch-bergwirtschaftlichen Untersuchungsstand ( $\mathrm{F}=$ Feasibility) und zum Grad der Bauwürdigkeit ( $\mathrm{E}=$ Economic) vorgenommen, woraus eine Beschreibung der Vorratsklasse in einem dreistelligen Zahlencode (E-F-G, in Abb. 5.1 folglich 1-1-1) resultiert. Dabei steht jeweils die kleinste Ziffer für den höchsten Grad. Verschiedene Aspekte der Bewertung von Massenrohstoffen lassen sich dabei auf den Werksteinsektor übertragen. Wie in Kapitel 2 beschrieben, müssen jedoch Werksteinlagerstätten besonderen Anforderungen genügen, die sich vor allem auf die Ausbildung und Stabilität im Dekor und der gewinnbaren Blockgrößen beziehen. Die finale Bewertung der Lagerstätte von Mtesa ist daher an den von Stein (2007) zur Bewertung nach dem UNFCR von Werksteinvorkommen in Benin vorgestellten Kriterien ausgerichtet. Der entscheidende Vorteil, der sich aus der Anwendung dieser Klassifikation ergibt, ist die Möglichkeit, eine internationale Vergleichbarkeit zu erreichen.

\subsection{Geographische Einordnung und infrastrukturelle Anbindung des Arbeitsgebietes}

Das betrachtete Marmorvorkommen befindet sich im S der Republik Tansania rund 30 km N' der Grenze zur Republik Mozambique in der Region Mtwara (Abb. 5.2). Topographisch ist ein etwa ENE-WSW ausgerichtetes Tal ausgebildet, dass eine Länge von rund $8 \mathrm{~km}$ bei einer Breite zwischen 1 und 1,5 km aufweist. Die mittlere Höhenlage beträgt etwa 300 bis $450 \mathrm{mNN}$, wobei das Gelände insgesamt von W nach $\mathrm{E}$ leicht ansteigt. Intern ist das Vorkommen durch differenzierte Gesteinsformationen und Täler temporärer Flüsse gegliedert und weist ein leicht hügeliges Relief auf. Dabei sind besonders die Kernzonen des Vorkommens gegenüber der gesamten Tal-Lage leicht erhöht. Begrenzt wird das Tal durch parallele, besonders im N ausgeprägte Höhenzüge, die eine Höhe bis zu etwa 550 mNN erreichen. Daran schließt sich jeweils ein bis zu mehrere Kilometer breites Tal an. 
Im Jahresverlauf treten zwei Regenzeiten auf: (i) eine schwächere in den Monaten November / Dezember und (ii) eine ausgeprägte zwischen März und Mai. Die Niederschläge können innerhalb der Regenzeiten sehr variabel ausfallen, wodurch die nicht oder nur wenig befestigten Straßen möglicherweise zeitweise einer Nutzungseinschränkung unterliegen (Abb. 5.3 a). Zum Zeitpunkt der Untersuchung führten die Flüsse kein Wasser. Demnach wäre für einen möglichen Abbau des Marmors zu überprüfen, ob eine Wasserversorgung in ausreichendem Maße über Brunnenbohrungen im Bereich der Flussläufe der Region zu realisieren wäre (vgl. Kap. 4.5).

Das gesamte Vorkommen wird in seiner E-W-Erstreckung von der Straße NambungaNagaga durchzogen. Die Entfernung vom Vorkommen mit der zentral gelegenen Ortschaft Mtesa zum nächstgelegenen Seehafen in Mtwara beträgt rund $160 \mathrm{~km}$ in direkter Verbindung bei Nutzung einer nur abschnittsweise befestigten Straße. Die Anfahrt über eine befestigte Allwetterstraße ergibt sich ab der Ortschaft Masasi, dem nächstgelegenen Ort mit städtischer Infrastruktur, in einer Entfernung von rund $45 \mathrm{~km} \mathrm{NW}$ ' Mtesa. Eine Begrenzung der Transportmöglichkeit ist dabei jedoch durch eine Anzahl an Brücken gegeben, die vor allem im Streckenabschnitt bis Masasi als Metallkonstruktionen mit losem Holzbohlenbelag ausgebaut sind (Abb. 5.3 b). In einem Fall zwischen den Ortschaften Masasi und Ndanda lag die Belastungsgrenze einer Brücke nach Ausweisung bei $20 \mathrm{t}$.

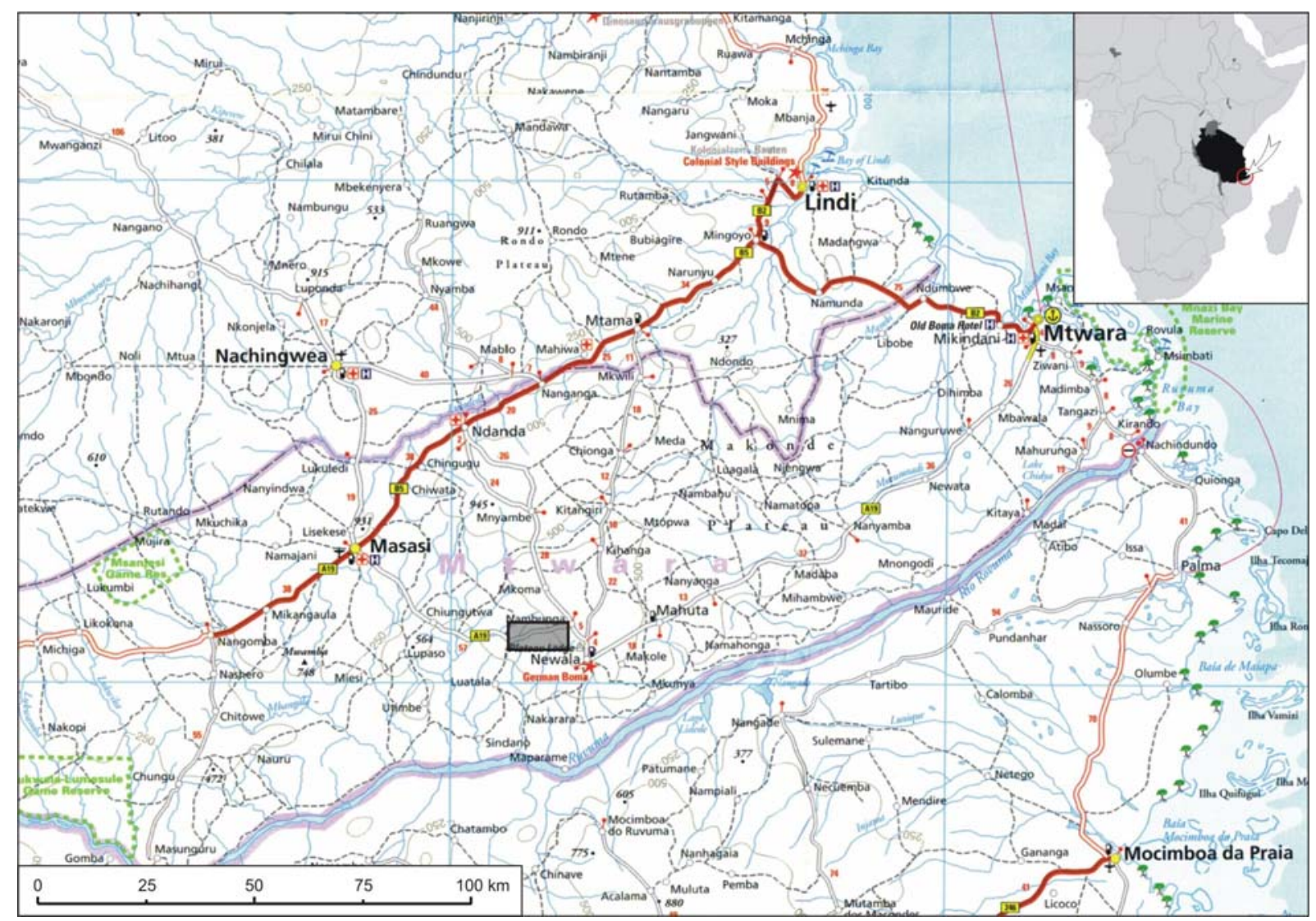

Abb. 5.2: Topographische Lage des untersuchten Marmorvorkommens im S der Republik Tansania (Arbeitsgebiet grau unterlegt).

Die Hauptgebiete des Vorkommens selbst sind nicht besiedelt und nur sehr gering durch Landwirtschaft genutzt. Durch einen zum Teil engräumigen Gesteinswechsel grenzen jedoch 
in einigen Bereichen landwirtschaftlich genutzte Flächen direkt an den offen anstehenden oder sedimentär bedeckten Marmor an. Die zentral im Vorkommen gelegene Ortschaft Mtesa und die damit verbundenen landwirtschaftlichen Flächen würden durch einen möglichen Abbau in keiner Weise beeinträchtigt, da sowohl im E als auch im W der Ortschaft ein Abstand von rund $1 \mathrm{~km}$ zu abbauwürdigen Bereichen des anstehenden Marmors gegeben ist. Der nächstgelegene ausgeprägtere Industriestandort, die Stadt Newala, befindet sich in einer Entfernung von rund $25 \mathrm{~km}$, womit auch eine Anbindung der Ortschaft Mtesa an das Stromnetz gegeben ist.
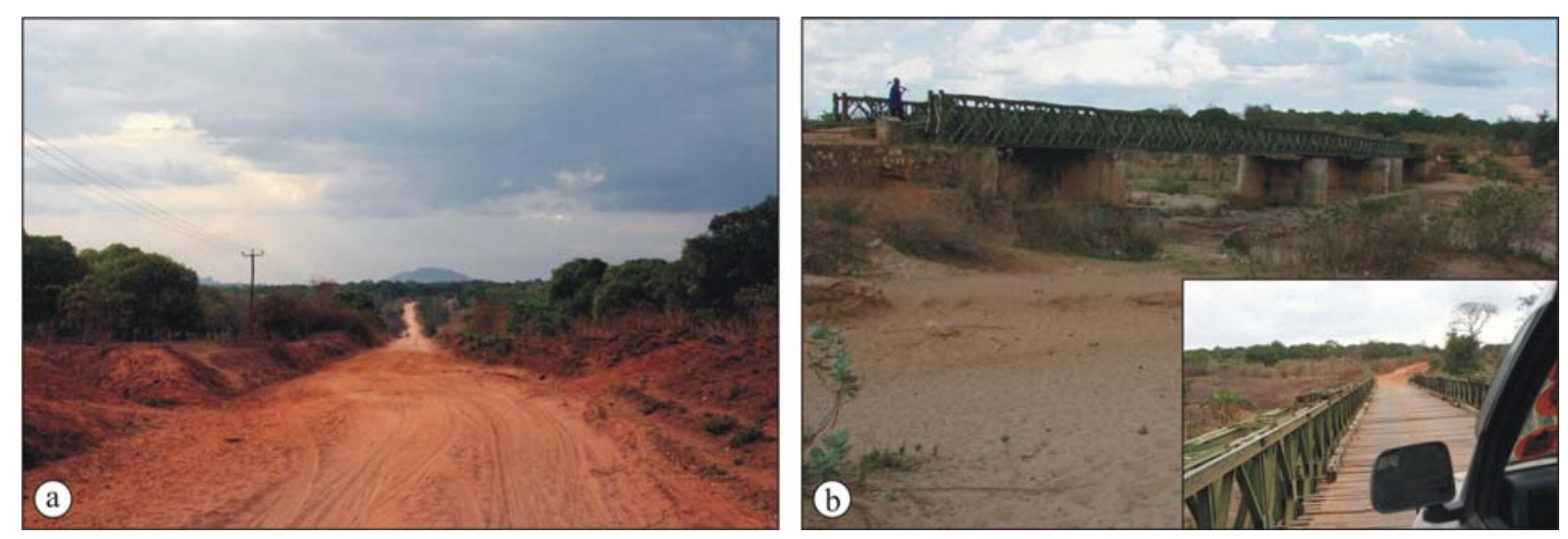

Abb. 5.3: a) Typische Wegeverhältnisse im Bereich des Vorkommens, hier W' Mtesa; b) Metallbrückenkonstruktion mit losen Holzbohlenbelag auf der Strecke Mtesa - Masasi.

\subsection{Aktuelle Abbausituation}

Im Gebiet des anstehenden Marmors erfolgt in mehreren Bereichen ein zum Teil intensiver Kleinstbergbau für die Gewinnung als Zuschlagstoff (Abb. 5.4 a). Dabei werden oberflächennahe Boulder in manuell angelegten Vertiefungen bis etwa $2 \mathrm{~m}$ freigelegt und mittels Feuersetzen in kleinere Brocken bis zu einer Größe von rund $50 \mathrm{~cm}$ zerlegt (Abb. 5.4 b). Diese werden anschließend in reiner Handarbeit zu Körnungen von etwa 3 bis $5 \mathrm{~cm}$ Größe gebrochen. Dieses Endprodukt findet Verwendung als Schottermaterial oder zur Herstellung von Terrazzoplatten. Der Erlös beträgt für etwa 5 t gebrochenen Materials 30.000 TSH (ca. $20 €$ ). Die Gewinnung erfolgt meist durch kleinere Personengruppen ohne jegliche Arbeits- und Umweltschutzmaßnahmen. Durch die gegebene Abbausituation ergab sich eine zum Teil recht gute Aufschlusslage.

Im Falle einer Nutzung des Marmors als Werkstein wäre eine industrielle Weiterverarbeitung in Kleinserien im etwa $60 \mathrm{~km}$ entfernten Ndanda denkbar. In der dortigen Kirchenmission (Building Department) wird eine moderne Blockseilsäge italienischen Fabrikats sowie eine Brückensäge und eine Wandarmschleifmaschine älterer Herstellung zur Verarbeitung eines Marmors einer nahe gelegenen Lagerstätte betrieben (Abb. 5.5). Die Schritte zur Oberflächenbearbeitung erfolgen außerdem unter Verwendung von Winkelschleifern, die Endpolitur dagegen rein manuell. 

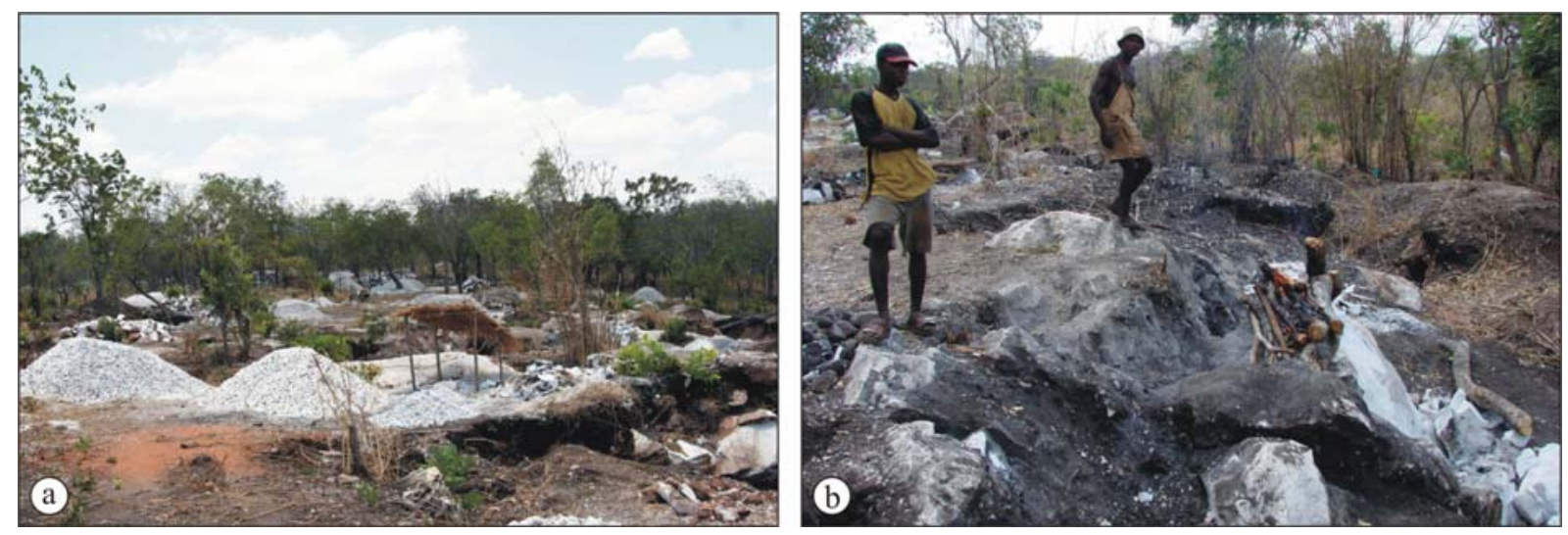

Abb. 5.4: a) Intensiver Kleinstbergbau im Lagerstättenbereich E’ Mtesa. b) Zerkleinerung freigelegter massiver Bereiche mittels Feuersetzen.
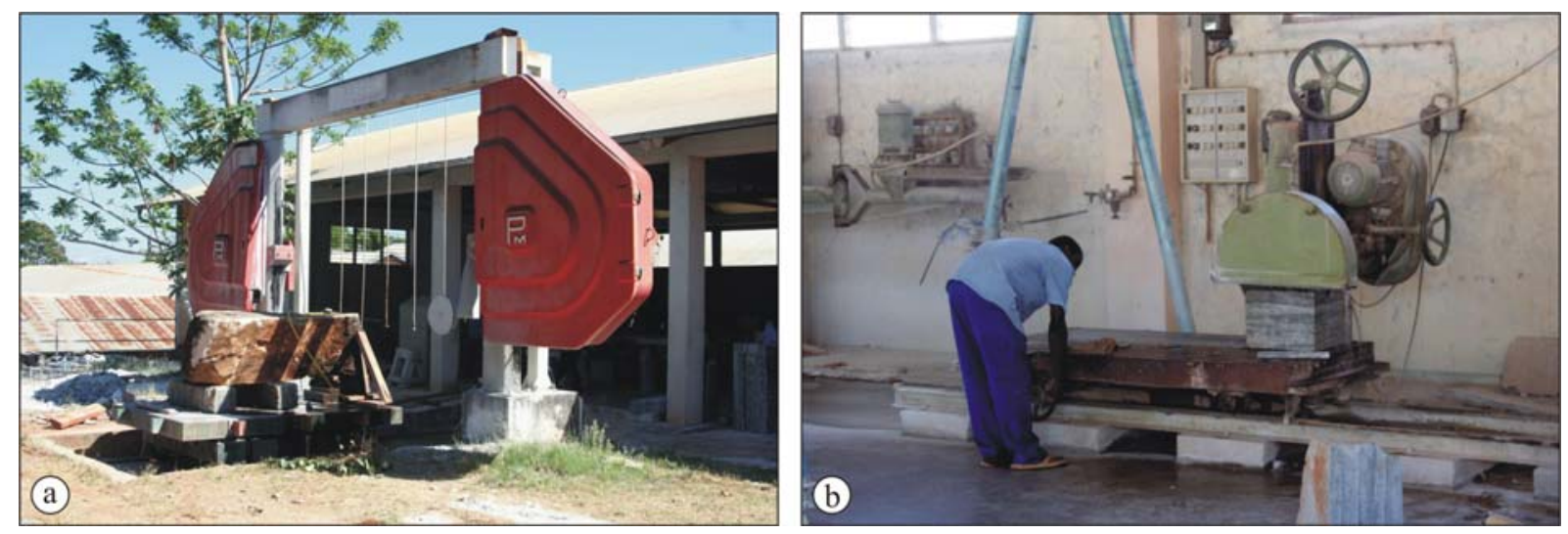

Abb. 5.5: Marmorverarbeitung in der Kirchenmission Ndanda mit a) Blockseilsäge und b) manuell gesteuerter Brückensäge. Links im Hintergrund ist die Wandarmschleifmaschine montiert.

\section{4 Überblick zur regionalen und lokalen Geologie}

Das Gebiet Tansanias ist geologisch in drei Einheiten gegliedert. Dabei ist der zentral gelegene archaische Tansania-Kraton von zum Teil hochmetamorphen Gesteinsabfolgen umgeben. Im SW ist dies die Ubendian-Folge, im E die Usagaran-Folge. In beiden Folgen weisen die Gesteine frühproterozoische Alter auf (Meinhold 1970, Wendt et al. 1972, Priem et al. 1979). Eine Beeinflussung durch die Pan-Afrikanische Orogenese konzentriert sich auf die Usagaran-Folge und verliert in Richtung W an Bedeutung (Gabert et al. 1978, Priem et al. 1979). Das untersuchte Marmorvorkommen befindet sich innerhalb der Usagaran-Folge, am E’ Rand des Masasi Basement Komplexes im Übergang zum Makonde Plateau. Dieses flach zum Indischen Ozean einfallende Plateau ist durch eine Abfolge kretazischer Sedimente gebildet. Im W schließen sich ausgedehnte Bereiche hochmetamorpher Gneise an, in die kristalline Schiefer, Quarzite und Marmore eingeschaltet sind (Nagaga Beds, Stockley \& Oates 1931). Dadurch ergibt sich im Bereich W' des Makonde Plateaus eine relativ tiefer liegende ausgedehnten Ebene, die mit schroffen Höhenzügen und Inselbergen durchsetzt ist. Die Wechsellagerungen sind in mehreren Phasen intensiv lateral und vertikal verfaltet. In diese Faltenstrukturen ist eine Vielzahl kleinerer granitischer Intrusionen paläozoischen Alters eingeschlossen. 
Insgesamt kann der neoproterozoische Masasi Basement Komplex als N’ Fortsetzung des Mozambique Belts angesehen werden. Die geologische Großstruktur stellt eine Suturzone dar, die sich während der Pan-Afrikanischen Orogenese bildete. Auch in der N' Fortsetzung bis Kenia und der SW' Fortsetzung im NW von Mozambique treten zahlreiche Karbonatlinsen auf, die teilweise als Marmorlagerstätten genutzt werden.

Detailliertes geologisches Kartenmaterial liegt für den Bereich Tansanias nur sehr vereinzelt vor. Für das Arbeitsgebiet im S der Republik standen lediglich topographische Karten im Maßstab 1 : 50.000, sowie eine begonnene Kartierung der State Mining Corporation (STAMICO, Dar es Salaam, Tansania) im gleichen Maßstab zur Verfügung. Zur Darstellung des geologischen Einheiten des Marmorvorkommens wurde ein Ausschnitt der topographischen Karte „Lulindi“ (Series Y742; Sheet 306/3) in einer Vergrößerung auf $1: 10.000$ verwendet.

\subsection{Lithologische Charakterisierung der auftretenden Gesteine im Vorkommen}

\subsubsection{Marmorvarietäten}

\subsubsection{Mineralogie und Dekoreigenschaften}

Als Hauptgestein des Vorkommens (Abb. 5.6) tritt ein weißer Dolomitmarmor auf, wobei neben Dolomit als dominierenden Hauptgemengteil röntgenographisch (RDA) noch Magnesit und zum Teil sehr geringe Anteile an Quarz im Mineralbestand nachgewiesen werden können (Abb. 5.7 a, 5.8 a, s.a. Tafel 1, S. 215). Der Marmor ist zumeist von mittel- bis feinkörniger Struktur. Lediglich in Bereichen kontaktmetamorpher Überprägung kann ein Kristallwachstum von bis zu mehreren Zentimetern auftreten. Eine reliktische Schichtung konnte anhand des Kristallgefüges nicht beobachtet werden. In der vertikalen Abfolge treten jedoch in unregelmäßiger Verteilung leicht dunklere Bänder und Lagen mit Mächtigkeiten im cm- bis dm-Bereich auf, die eine Anreicherung mafischer Minerale (Amphibol, Chlorit) aufweisen. Diese entsprechen offensichtlich einer primären Schichtung mit differenzierten Anteilen von Peliten und weisen im Lagerstättenkörper ein Einfallen von $30^{\circ}$ bis $40^{\circ}$ nach S auf. Akzessorisch treten auch Graphit, Pyrit, Muskovit und Hämatit auf. Pyrit und Hämatit können sowohl in idiomorpher Kristallform in Größen bis $2 \mathrm{~mm}$ als auch feinstverteilt in Lagen beobachtet werden. Dagegen tritt Graphit nur sehr unregelmäßig und dabei sehr selten akkumuliert auf. Das Auftreten von hellblauem Apatit in bis zu 5 cm großen Aggregaten ist scheinbar auf Linsen von wenigen Metern begrenzt. Diese sind ohne erkennbares Muster über das gesamte Vorkommen verteilt. Trotz der begleitenden Mineralphasen konnte die reinweiße Färbung des Dolomitmarmors in Dimensionen von über $1 \mathrm{~m}$ nachgewiesen werden. 


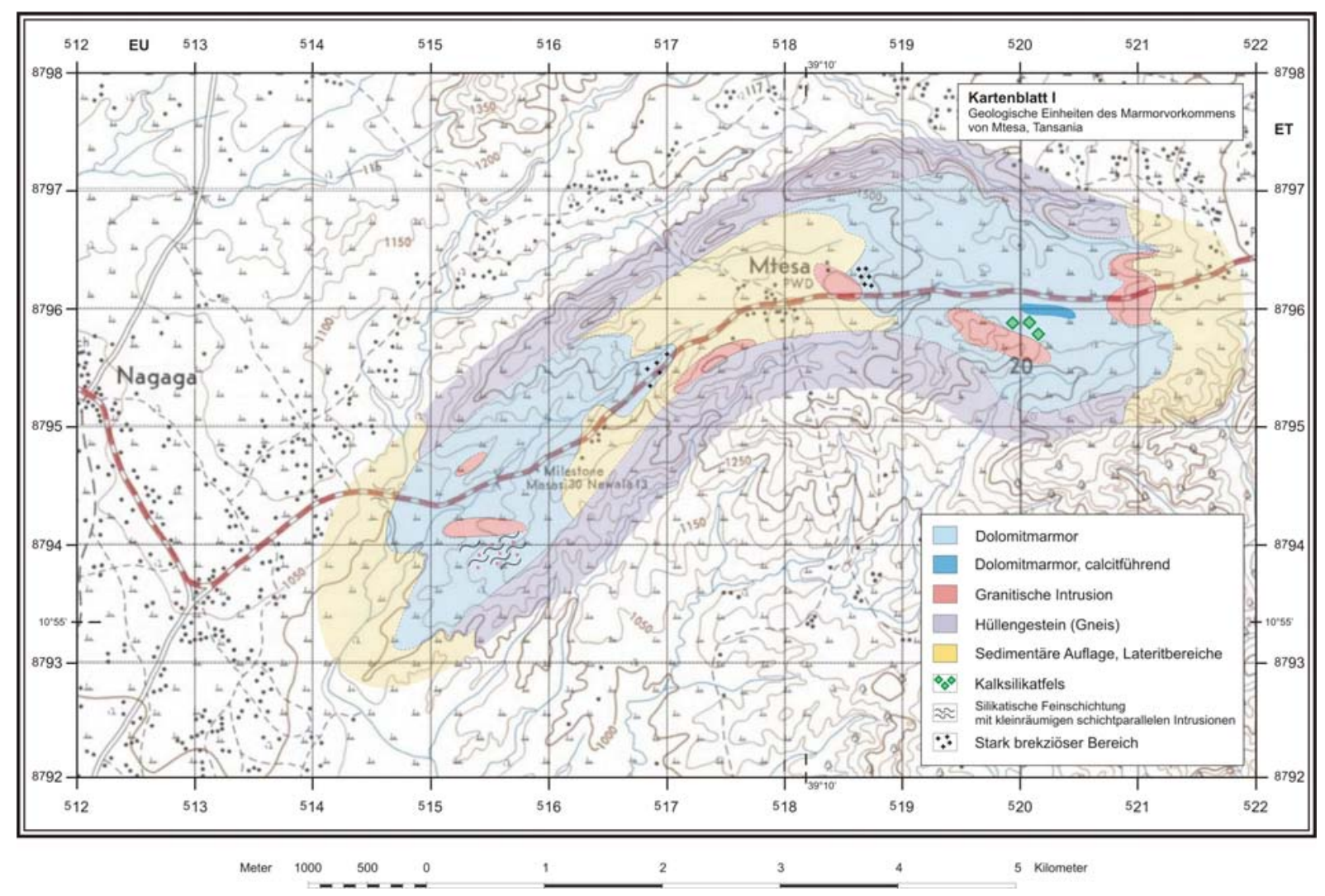

Abb. 5.6: Geologische Einheiten des Marmorvorkommens von Mtesa, Tansania.

Als weitere Marmorvarietät konnte lokal im E' Teil des Vorkommens eine hell- bis mittelgraue Marmorvarietät von mittel- bis grobkristalliner Struktur nachgewiesen werden (Abb. 5.7 b). Neben dem auch hier als Hauptgemengteil auftretenden Dolomit treten Calcit und Serpentin (Lizardit) als Nebenkomponenten auf. Der Vergleich der röntgenographischen Analysen der Varietäten (Abb. 5.8) zeigt jedoch bei einem vergleichbaren Dolomitanteil für diesen calcitführenden Marmor das Fehlen von Magnesit. Neben Calcit tritt dabei ein höherer Quarzanteil sowie Serpentin und Muskovit in der Mineralparagenese auf (s.a. Tafel 1, S. 215). In der Folge zeigt sich auch in der chemischen Zusammensetzung eine Abnahme des MgOGehaltes zugunsten erhöhter Anteile an $\mathrm{SiO}_{2}$ und $\mathrm{CaO}$ (Tab. 5.1). Die im Aufschluss erkennbaren mafischen Lagen weisen eine Orientierung entsprechend derer im Dolomitmarmor auf. Auch der begleitende akzessorische Mineralbestand entspricht dem der dominanten Hauptvarietät.

Tab. 5.1: Chemische Zusammensetzung der Marmorvarietäten auf Grundlage der röntgenographischen Analyse (RFA). Angaben in Gew.-\%.

\begin{tabular}{lcccccccccccc}
\hline & $\mathrm{SiO}_{2}$ & $\mathrm{TiO}_{2}$ & $\mathrm{Al}_{2} \mathbf{O}_{3}$ & $\mathbf{F e}_{2} \mathbf{O}_{3}$ & $\mathbf{M n O}$ & $\mathbf{M g O}$ & $\mathbf{C a O}$ & $\mathbf{N a}_{2} \mathbf{O}$ & $\mathbf{K}_{2} \mathbf{O}$ & $\mathbf{P}_{2} \mathbf{O}_{5}$ & $\mathbf{H}_{2} \mathbf{O}$ & $\mathbf{C O}_{2}$ \\
\hline Dolomitmarmor & 0,56 & $<0.01$ & 0,01 & 0,02 & 0,01 & 25,43 & 27,36 & 0,07 & $<0.01$ & 0,02 & 0,37 & 46,12 \\
Dol-Marmor, calcitfhr. & 3,92 & 0,03 & 0,56 & 0,18 & 0,01 & 20,76 & 29,52 & 0,06 & 0,02 & $<0.01$ & 1,50 & 43,26 \\
\hline
\end{tabular}



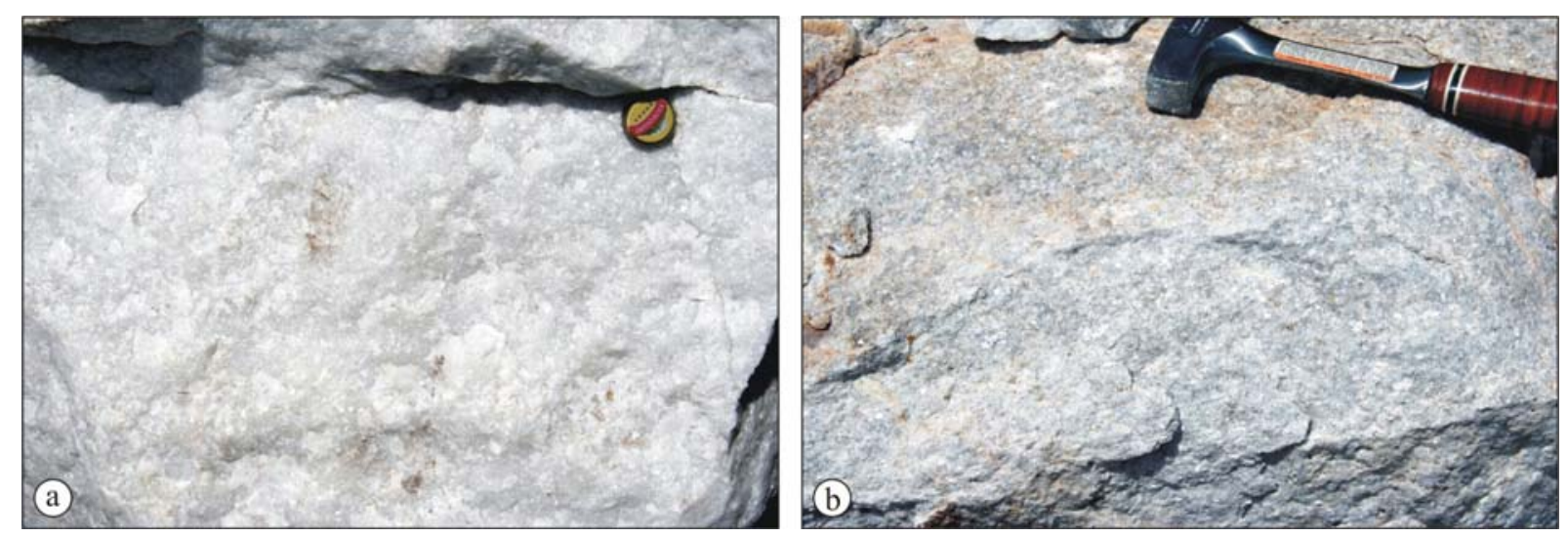

Abb. 5.7: Marmorvarietäten des Vorkommens: a) weißer Dolomitmarmor, b) grauer calcitführender Dolomitmarmor.

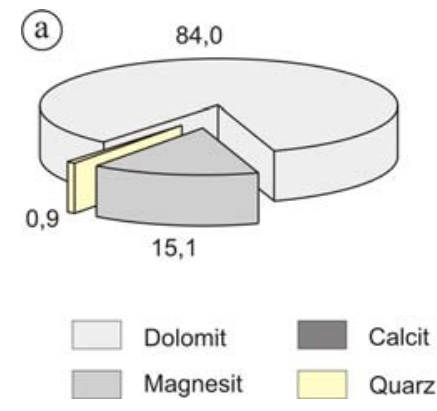

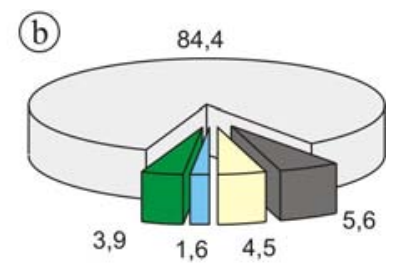

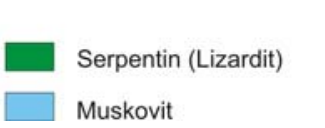

Abb. 5.8: Mineralogische Zusammensetzung a) des Dolomitmarmors und b) des calcitführenden Dolomitmarmors auf Grundlage der röntgenographischen Analysen (RDA). Angaben in Vol.-\%.

In unregelmäßigen Abständen, die zwischen $10 \mathrm{~cm}$ bis über $1 \mathrm{~m}$ betragen können, wird das Gestein sowohl vertikal als auch diagonal von bis $\mathrm{zu} 5 \mathrm{~mm}$ breiten Bruchstrukturen durchzogen, die eine dichte Mineralisation kryptokristallinem Calcits hell reinweißer Färbung aufweisen (Abb. 5.9 a). Trotz der weißen Färbung des Dolomitmarmors ist dabei ein markanter Farbunterschied festzustellen (vgl. Kap. 2.4). Die scheinbare Reduzierung der weißen Farbintensität des Dolomitmarmors lässt darauf schließen, dass auch in Lagen ohne deutliche Bänderungen mafischer Minerale ein akzessorischer Anteil von Metapeliten vorhanden sein könnte. Im oberflächennahen Bereich von wenigen Dezimetern ist die feine Klüftung infolge des Herauswitterns des Calcits meist geöffnet. Vereinzelt treten auch wenige Zentimeter breite kavernöse Bereiche auf.

Überwiegend in kontaktnahen Bereich zu granitischen Intrusionen oder angrenzenden brekziierten Zonen (vgl. Abb. 5.6) ist häufig eine auffällige Durchdringung des weißen Dolomitmarmors mit grauem Calcit in Kristallgrößen bis zu $2 \mathrm{~cm} \mathrm{zu}$ beobachten. Oberflächennah sind dadurch häufig Bereiche mit schwammartiger Gerüststruktur vorhanden (Abb. 5.9 b). Da beim Aufbringen von 5 \%-iger Salzsäure lediglich eine leichte Reaktion im Bereich der offenen Poren in Erscheinung trat, ist zu vermuten, dass der calcitische Anteil gelöst wurde und ein dolomitisches Skelett bestehen geblieben ist. Der durch Auslaugung erzeugte Porenraum beträgt etwa 20 bis $30 \%$.

In kleineren Bereichen im SW' Teil des Vorkommens weist die Aufschlusslage darauf hin, dass in den karbonatischen Serien in eingeschränktem Maße auch psammitische Lagen auftreten können. Sowohl in ihrer Zusammensetzung, dem Metamorphosegrad als auch in der Farb- und Gefügeausbildung ähneln diese Lagen makroskopisch dem feinkörnigen, das 
Vorkommen begrenzenden Gneis. In diesen Bereichen können intensive Kleinfaltungen der Schichtpakete aus Gneis und Marmor nachgewiesen werden. (Abb. 5.9 c, d).
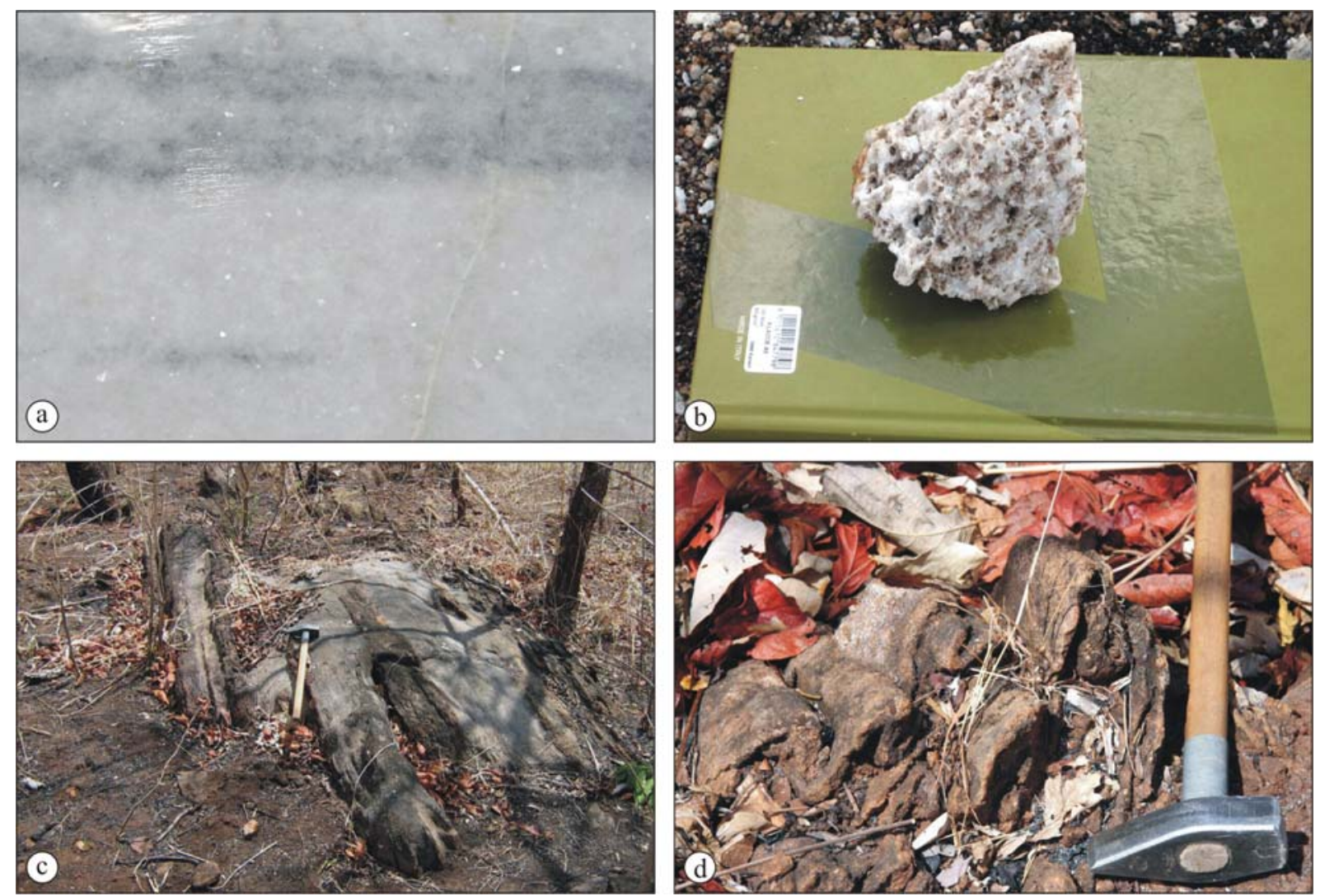

Abb. 5.9: a) Feine kryptokristalline Calcitader im weißen Dolomitmarmor (hier mit mafischem Band, Bildhöhe ca. $15 \mathrm{~cm}$ ); b) schwammartige Gerüststruktur von Dolomitmarmor vermutlich infolge Auslaugung eines Calcitanteils; c) Wechsellagerung karbonatischer und psammitischer Lagen mit d) zum Teil intensiver Kleinfältelung der Schichtpakete.

\subsubsection{Mikrogefüge}

Dem Mikrogefüge von Marmoren kommt im Hinblick auf die physikalischen und technischen Eigenschaften des Gesteins eine bedeutende Rolle zu (vgl. Kap. 3.9, 3.13). Elementare Parameter sind dabei die Kornform, die Korngrößenverteilung sowie vor allem die Korngrenzengeometrie. Gerade die Korngrenzengeometrie ist als entscheidender Faktor hinsichtlich der Verwitterungsprozesse zu sehen, da mit zunehmender Unregelmäßigkeit die Länge bzw. im Dreidimensionalen die Fläche der Korngrenzen zunimmt. Daraus resultiert im Allgemeinen eine stärkere Bindung zwischen den einzelnen Kristallen, was sich in einer erhöhten Festigkeit des Kornverbandes äußert (Rüdrich 2003).

Das Mikrogefüge des reinen Dolomitmarmors ist durch ein ungleichmäßiges (seriates) Korngefüge gekennzeichnet, in welchem Korngrößen von etwa 0,2 bis $2 \mathrm{~mm}$ bei hypidiomorphen bis xenomorphen Kornformen erreicht werden (Abb. 5.10 a). Somit kann der Marmor nach der Skala für kristalline Gesteine nach Teuscher als fein- bis mittelkörnig beschrieben werden (Wimmenauer 1985). Vereinzelt treten auch deutlich größere Einzelkristalle bis über $5 \mathrm{~mm}$ auf, die den Marmor makroskopisch grobkörniger erscheinen 
lassen. In diesen sind mit einer undulösen Auslöschung lokal Anzeichen auf eine kaltplastische Deformation erkennbar. Ebenso sind feine, intra- und intergranulare Rissstrukturen zu beobachten, die eine vollständige Verheilung aufweisen. Hinsichtlich der Geometrie der Korngrenzen sind vor allem buchtige oder gezackte, vereinzelt auch stärker verzahnte Formen ausgebildet, woraus ein interlobates Kornaggregat resultiert (Abb. 5.10 b). Auch die oben beschriebenen Rissstrukturen weisen gezackte Ränder auf. Insgesamt kann im mikroskopischen Skalenbereich keine Vorzugsorientierung der Kristalle festgestellt werden.
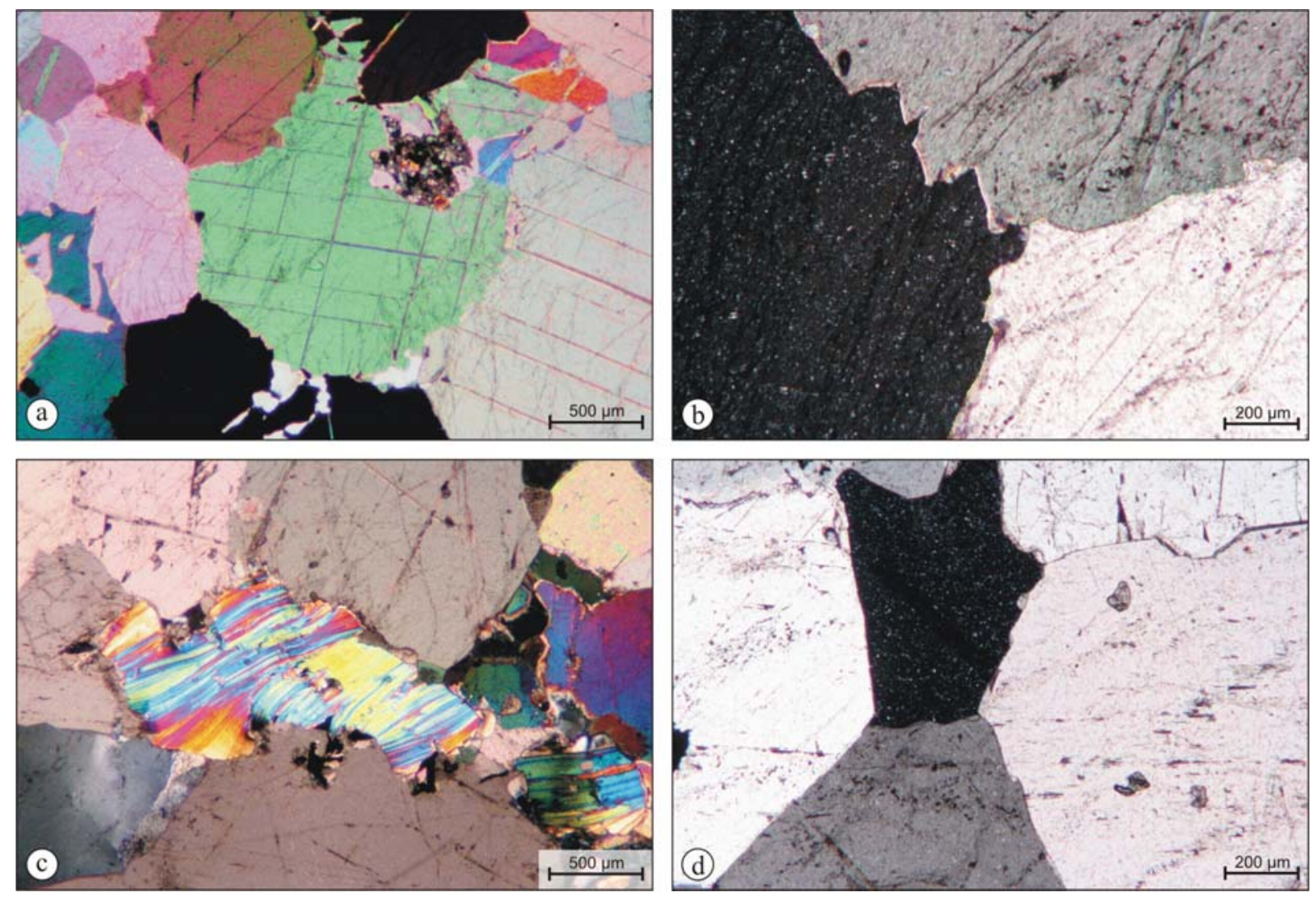

Abb. 5.10: a) Seriates, interlobates Korngefüge des Dolomitmarmors mit b) ausgeprägter Kornverzahnung. c) Korngefüge des calcitführenden Marmors mit Deformationsstrukturen im Muskovit und d) zumeist geradlinigerer Korngrenzgeometrie.

Die calcitführende Varietät erscheint im mikroskopischen Skalenbereich dagegen insgesamt etwas grobkörniger, was vor allem auf einem geringeren Anteil feinkörniger Kristalle beruht. An auftretenden Muskoviten können zudem vergleichbare Deformationsphänomene wie im reinen Dolomitmarmor festgestellt werden (Abb. 5.10 c). Hinsichtlich ihrer Geometrie weisen die Korngrenzen im Allgemeinen gestrecktere, weniger verzahnte oder gebuchtete Formen auf, womit eine Minimierung der Kornoberflächen durch Prozesse wie die Korngrenzenflächenreduktion (Passchier \& Trouw 1996) beschrieben ist. Dadurch ergeben sich mehrfach Bereiche eines eher polygonalen Kornaggregats (Abb. $5.10 \mathrm{~d}$ ). 


\subsubsection{Das Marmorvorkommen begleitende Gesteine}

Das betrachtete Marmorvorkommen wird sowohl im $\mathrm{N}$ als auch im S von einem feinkörnigen, meist feinlagigen, häufig quarzitreichen Gneis von hellgrauer Färbung begrenzt. Der Gneis tritt morphologisch hervor und bildet insbesondere in der N' Begrenzung des Vorkommens ein ausgeprägtes, lang gestrecktes Relief aus (Abb. 5.11 a, b). In der Südbegrenzung beginnt der Gneis dabei überwiegend erst im Scheitel des Höhenrückens. Das Einfallen der Foliation beträgt sowohl im $\mathrm{N}$ als auch im $\mathrm{S}$ etwa $60^{\circ}$, wobei die Einfallsrichtung im W-E-Verlauf des Vorkommens von ESE nach SSE wechselt. Dabei spiegelt sich im Hüllengestein die ungefähre Orientierung des reliktischen Schichtungsgefüges des Marmors bei etwas steilerem Einfallen wider.
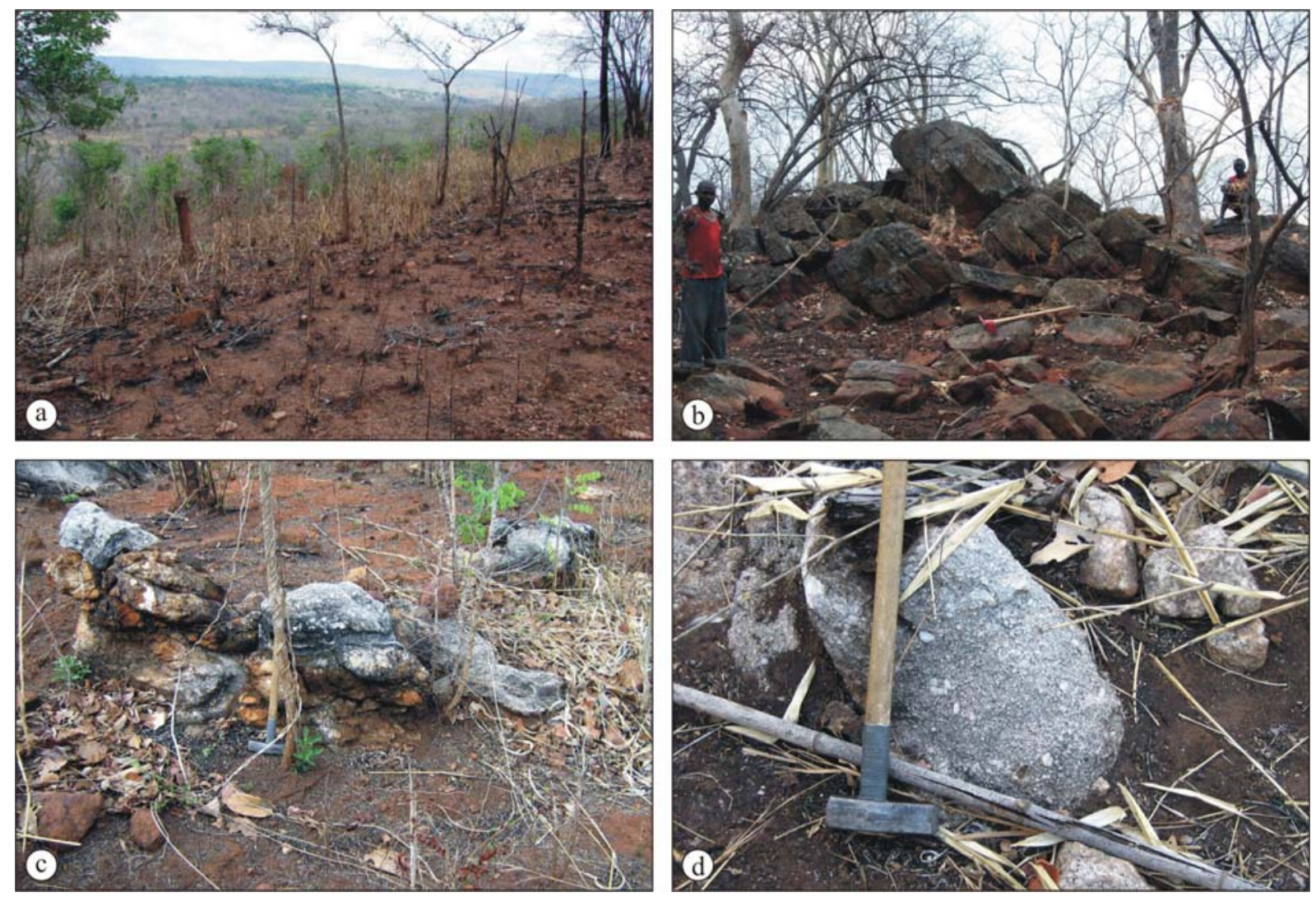

Abb. 5.11: a) Blick vom SE' gelegenen Höhenrücken über das Vorkommen. Im Hintergrund der die Morphologie bestimmende Gneisrücken, der das Vorkommen im N begrenzt. b) Aufschluss im anstehenden Gneis. Kontaktbereich des Marmors zum Gneis mit c) wulstartigen Einpressungen und d) vereinzelt auftretendem überdurchschnittlichen Kristallwachstum.

Makroskopisch weist das Gestein folgende Zusammensetzung auf (in \%): Quarz 50, Kalifeldspat 20, Plagioklas 20 und Biotit 10. Akzessorisch treten zudem Epidot und Hämatit (besonders auf Kluftflächen) auf. Das Gestein ist insgesamt in Folge der Verwitterung von einer lateritischen Krustenbildung sowie Blockfeldern überzogen.

In der Nordbegrenzung konnte an einer Position der direkte Grenzbereich zwischen Gneis und Marmor am Fuß des dortigen Höhenzuges beobachtet werden, wobei sich eine linsenund taschenförmige Einpressung des Marmors in den Gneis zeigt (Abb. 5.11 c). Vereinzelt ist 
dabei ein überdurchschnittliches Größenwachstum einzelner Kristalle im Marmor zu beobachten (Abb. 5.11 d), was jedoch auf den Einfluss kleinerer pegmatitischer Gänge zurückzuführen ist.

Das Vorkommen wird geographisch durch die Ortslage Mtesa in zwei Bereiche gegliedert. In diesem zentralen Bereich des Gesamtvorkommens treten anstelle des W' bzw. SE' aufgeschlossenen Marmors im Anschluss einer Granitintrusion in wechselnder Abfolge granitische Gneise, Kalksilikatfelse, Hornfelse, Rhyolite und Marmorlagen auf, die zum Teil auch von Quarzgängen durchzogen werden. Insgesamt umfasst dieser Bereich eine E-W-Ausdehnung von rund $2 \mathrm{~km}$ und wird im $\mathrm{N}$ und $\mathrm{S}$ durch die das gesamte Marmorvorkommen umgebenden Gneise begrenzt. Die zum Teil deutlich kataklastisch überprägte Zone weist zumeist eine starke Bedeckung mit Lateritböden auf. Die direkten granitischen Bereiche sind dagegen in Folge der starken Zersetzung von einer kaolinitisch-sandigen Bodenbildung überzogen (Abb. 5.12).

Der hellgraue, leicht rötliche Granit weist eine grobkristalline Struktur auf. Häufig ist dabei ein deutlich schriftgranitisches Gefüge ausgebildet, wobei teilweise auch eine Foliation anhand gerichteter Feldspäte oder auch einer orientierten Quarzblastese erkennbar ist. Makroskopisch zeigt sich eine monzogranitische Zusammensetzung mit (in \%): Quarz 35, Kalifeldspat 30, Plagioklas 25 und Biotit, Muskovit 10. Entsprechende Intrusionen existieren an mehreren Positionen im Vorkommen (vgl. Abb. 5.6), wobei die Komplexe in ihrer Ausdehnung in etwa dem Streichen der Gesamtstruktur folgen. Oberflächennah sind keine kataklastisch überprägten Zonen direkt $\mathrm{zu}$ beobachten. Eventuell ist anhand lokaler Vorkommen an Lateritböden eine kleinräumige Ausbildung dieser bruchhaft deformierten Bereiche zu vermuten.
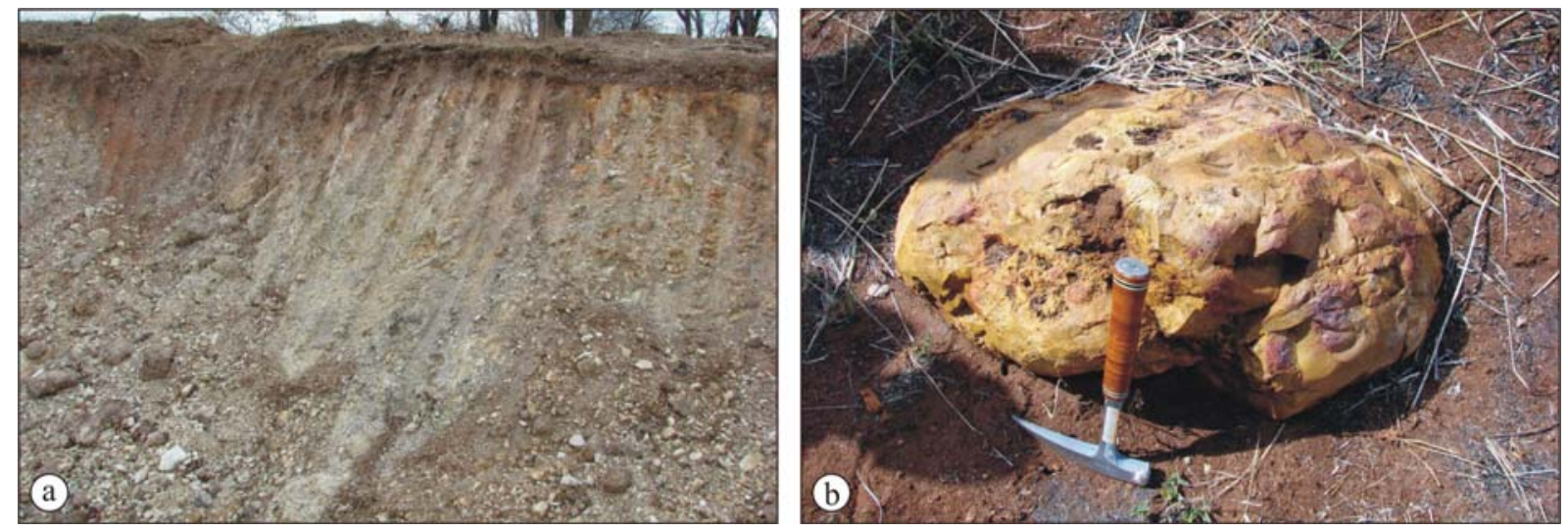

Abb. 5.12: a) Eine starke Zersetzung der granitischen Intrusivkörper führt zu einer kaolinitisch-sandigen Bodenbildung. b) Hornfelsbildung im Bereich einer vermuteten Scherzone im Übergangsbereich Granit - Marmor E’ Mtesa.

Im S' Abschnitt des E' Lagerstättenbereichs parallel zur dortigen Intrusion ist ein durchgreifend metasomatisch überprägter Körper mit einer Länge von maximal $1 \mathrm{~km}$ und einer Breite von rund 30 bis $50 \mathrm{~m}$ aufgeschlossen. Die intensive Metamorphose des Marmors durch die thermische Induktion ausgehend vom Intrusivkörper führte zur Bildung eines komplexen Kalksilikatfelses (Abb. 5.13, s.a. Tafel 1, S. 215). Die röntgenographische 
Untersuchung (RDA) weist einen Calcitanteil von über 50 Vol.-\% aus (Abb. 5.14). Komplettiert wird der Mineralbestand durch rund 40 Vol.-\% Serpentin (Lizardit) sowie sehr geringe Mengen Dolomit. Infolge der Mineralneubildungen ergibt sich ein Gestein von hellgrünlich grauer bis gelblich graugrüner Grundfärbung mit einer erheblichen Farbheterogenität. Infolge der vermutlich intrusionskontrollierenden Scherung ergibt sich im Kalksilikatfels ein brekziöses Gefüge, wobei die zum Teil dm-großen Klasten in einer feinkristallinen Matrix aus Kalksilikatfels verheilt sind. Die Kluftverhältnisse lassen sich im aufgeschlossenen Bereich des Körpers nicht eindeutig erkennen bzw. abschätzen. Aufgrund der Geländesituation mit dem Auftreten einzelner Boulder im m-Bereich sind jedoch Abstände von mehr als einem Meter für das Trennflächengefüge zu vermuten.
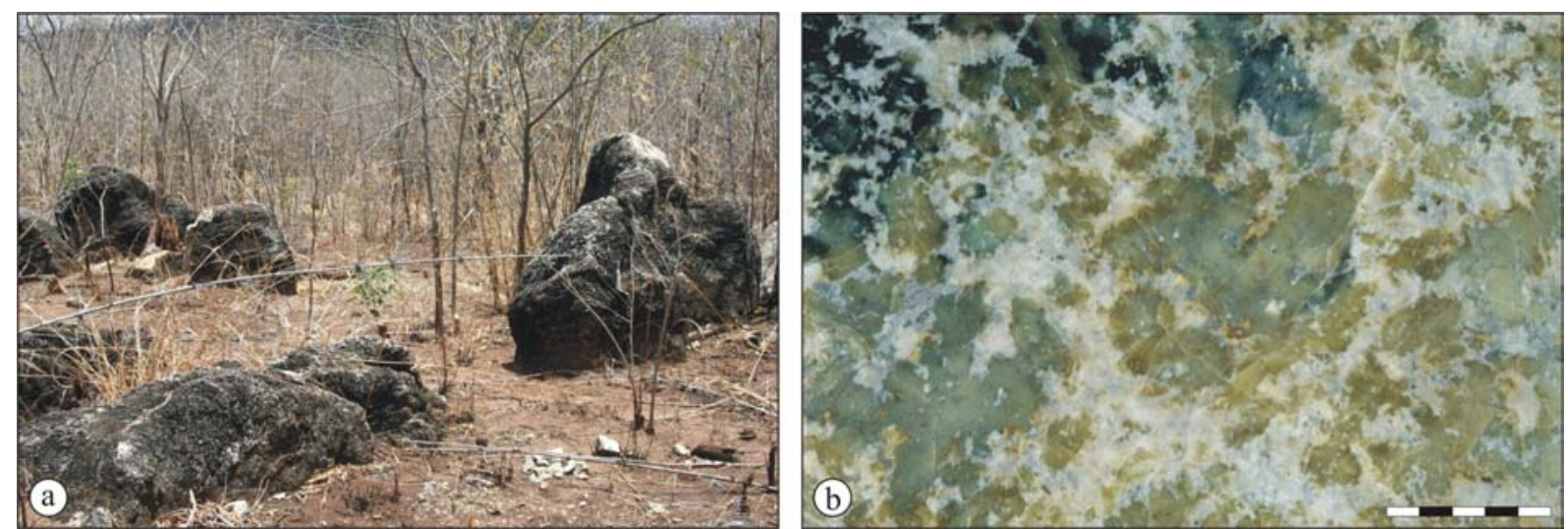

Abb. 5.13: Ausbildung von Kalksilikatfels im Kontaktbereich zur granitischen Intrusion im SE des Vorkommens (in b polierte Oberfläche, Maßstab in $\mathrm{cm}$ ).

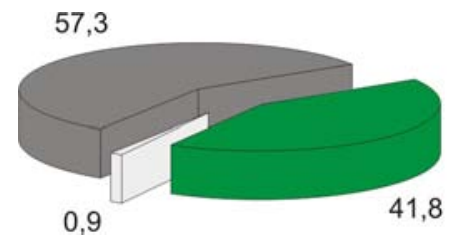

Dolomit

Calcit
Abb. 5.14: Mineralogische Zusammensetzung des Kalksilkatfels auf Grundlage der röntgenographischen Analysen (RDA). Angaben in Vol.-\%.

\subsubsection{Metamorphe Entwicklung des Marmorkörpers}

Neben den Intrusionskörpern können im Vorkommen drei Gesteinsbereiche differenziert werden: i) ein Dolomitmarmor mit geringen bis sehr geringen Beimengungen von Magnesit, Quarz sowie Kalksilikatmineralen, ii) ein calcitführender Dolomitmarmor mit Beimengung von Serpentin, Quarz und Muskovit sowie ein Kalksilkatfels aus primär Calcit und Serpentin und geringen bis sehr geringen Beimengungen von Dolomit (Tafel 1, S. 215). Es ist davon auszugehen, dass das primäre Karbonatgestein eine gewisse Verunreinigung durch detritisches Material aufwies. Beleg dafür ist durch das Vorhandensein psammitischer Lagen und durch das Auftreten von Graphit gegeben. 
Tafel 1: Kathodolumineszenz-Mikroskopie
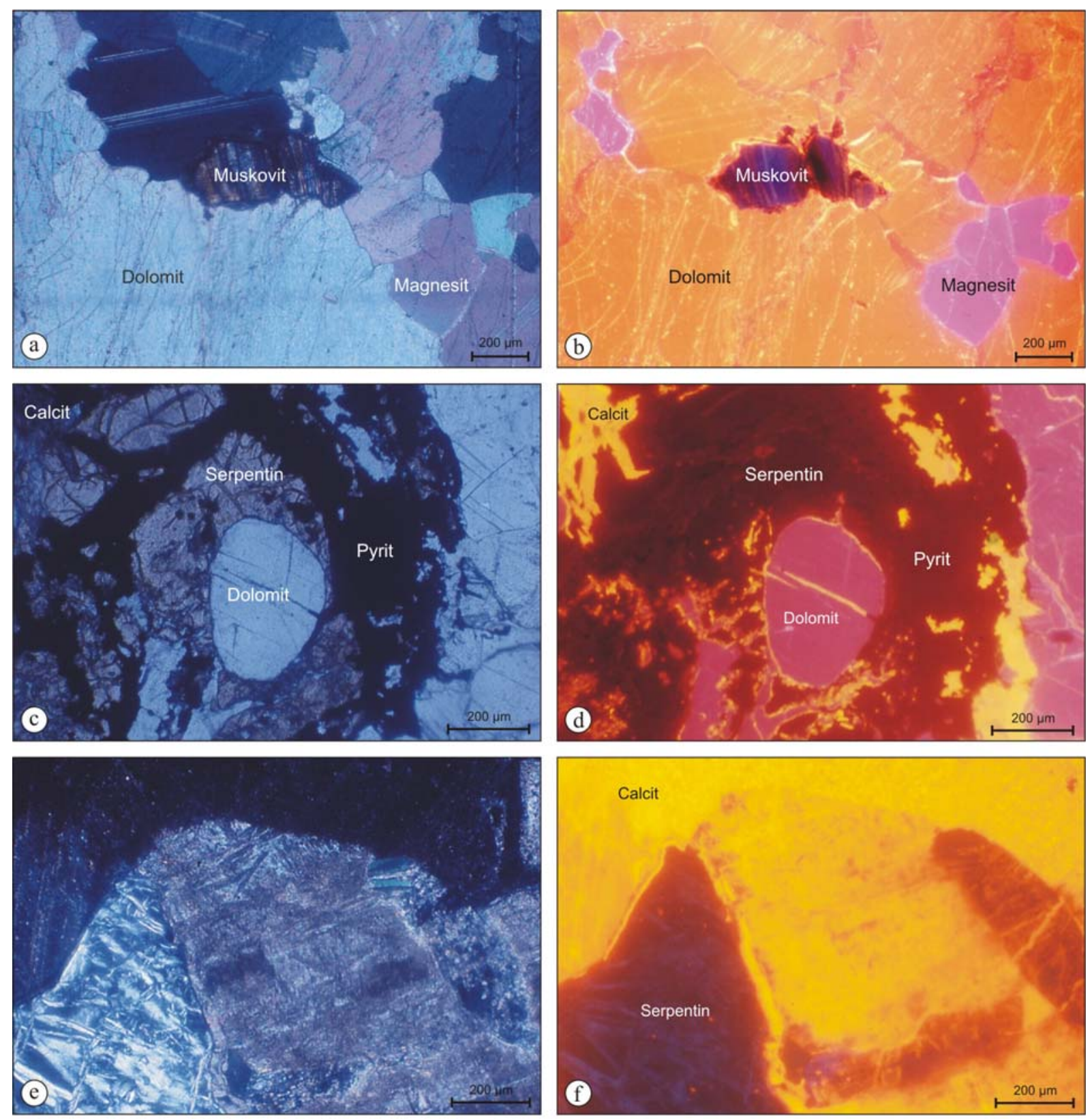

Durchlichtmikroskopische Abbildungen mit dem jeweiligen Äquivalent der Kathodolumineszenz-Mikroskopie:

Abb. a, b: Dolomitmarmor mit Beimengungen von Magnesit und akzessorisch auftretendem Muskovit.

Abb. c, d: Calcitführender Dolomitmarmor mit erhöhter Beimengung von Serpentin (Lizardit) und akzessorisch auftretendem Pyrit.

Abb. e, f: Kalksilikatfels mit Calcit und Serpentin (Lizardit) als Hauptgemengteile. 
Hinsichtlich der metamorphen Entwicklung des Marmorkörpers können aus dem Mineralbestand verschiedene Szenarien vermutet werden. Dabei ist vor allem die relative zeitliche Bildung der Kalksilikatminerale besonders im Mineralbestand des Dolomitmarmors von Bedeutung. Diese können zum einen durch eine retrograde Metamorphose aus der Reaktion von primär gebildetem Diopsid oder Forsterit hervorgegangen sein. Für deren Genese wäre eine Regionalmetamorphose unter amphibolitfaziellen Bedingungen anzusetzen. Ein Hinweis auf entsprechende Verhältnisse kann mit dem Auftreten von Granat im begrenzenden Gneisgürtel gegeben sein. Ein retrograder Prozess, der mit einer regionalen Hebung einhergehen würde, könnte mit der Bildung von Tremolit, Calcit und Quarz aus Diopsid beschrieben werden (Yardley 1997). Diese Rückreaktion benötigt das Vorhandensein von $\mathrm{H}_{2} \mathrm{O}$ sowie einen Temperatureintrag während der Hebungsphase, was durch die Intrusion der Magmen gegeben wäre.

Zum anderen kann eine direkte Bildung von Tremolit unter grünschieferfaziellen Bedingungen vermutet werden. Aus der Reaktion von Dolomit und Quarz folgt dabei in einem ersten Reaktionsschritt die Bildung von Talk und Calcit. Sollte dabei der Silikatgehalt drastisch gegen Null gesengt werden, kann aus der Reaktion von Talk und Calcit eine Mineralparagenese aus Tremolit und Dolomit resultieren (Yardley 1997). Im Mikroskop konnten jedoch Einzelkörner beobachtet werden, die dem Habitus nach Diopsid oder Forsterit zugeordnet werden können, jedoch eine Umwandlung zu Calcit und einem Kalksilikat, eventuell Tremolit, aufweisen. Demnach ist grundsätzlich eher dem ersten Ansatz zu entsprechen, da zudem ein Einfluss der Intrusionen auf die Mineralogie des karbonatischen Gesteins in jedem Fall anzunehmen ist.

In diesem Zusammenhang bleibt jedoch der Umfang bzw. die Intensität des Einflusses der magmatischen Intrusionen auf die durchgreifende Metamorphose im SE der Lagerstätte offen. Yardley (1997) nennt als spektakulärstes Beispiel, das zur Bildung von Kalksilikat-Gesteinen bzw. Skarnen führen kann, die Intrusion von Graniten in Marmor, wie es in diesem Falle vorliegt. Es ist davon auszugehen, dass sämtliche vorhandenen Intrusionskörper relativ zeitgleich einzuordnen sind. Folglich könnten auch an anderen Lokalitäten im Vorkommen ähnliche Gesteinsbildungen nachzuweisen sein. Daher ist anzunehmen, dass eine etwas veränderte Zusammensetzung der Magma dieser Intrusion, geringe Unterschiede im Stockwerk oder aber eine lokal stärkere Verunreinigung des primären Sediments die erhebliche Bildung von Kalksilikaten, in diesem Falle vor allem Serpentin (Lizardit), zur Folge hatte. Diese Mineralneubildungen können infolge der damit verbundenen Schwankungen in der Färbung und der Gesteinshärte einen erheblichen Einfluss auf die Nutzung des Dolomitmarmors als Werkstein ausüben, worauf in Kapitel 5.6.3 eingegangen wird. 


\subsection{Lagerstätten-kontrollierende Elemente}

\subsubsection{Lithologische Aspekte}

Grundsätzlich ergeben sich besonders im Abbau metamorpher Werksteinvarietäten häufig stark unterschiedliche strukturelle und mineralogische Ausbildungen, die auf variierenden Metamorphosebedingungen beruhen (vgl. Kap. 2.2). Diese Veränderungen können unter Umständen auch kleinräumige Bereiche erfassen bzw. auf kurzen bis sehr kurzen Distanzen auftreten. In der Folge muss zu jedem Zeitpunkt im laufenden Abbaubetrieb mit Variationen in Dekor und Muster gerechnet werden, was zu einer gesonderten Aushaltung anderer als primär definierter Sorten führen kann. Aufgrund der häufig zu beobachtenden, verschwommen gegeneinander abgegrenzten Vielfalt an Strukturen auf möglicherweise kleinem Raum, können sich demzufolge Probleme hinsichtlich der längerfristigen wirtschaftlichen Verfügbarkeit primär definierter Handelssorten ergeben. Daraus kann folglich eine Instabilität in der Bedienung des Marktes nach der Sortendefinition resultieren.

Anhand der makroskopischen und mikroskopischen Charakterisierung des Dolomitmarmors wurde deutlich, dass in die Sedimentation des primären Karbonatgesteins vereinzelte Schüttungsphasen mit metapelitischen Edukten eingeschlossen waren (vgl. Kap. 5.5.1.1). Diese äußern sich im Dekor in Lagen und Bändern mafischer Minerale, wobei auch vereinzelt Pyrite auftreten können. Diese Bändern sind im aufgeschlossenen Bereich meist über mehrere Meter zu verfolgen, erreichen dabei Mächtigkeiten im cm- bis dm-Bereich, keilen jedoch dann häufig aus. Öfters sind lediglich linsenartige Akkumulationen mafischer Minerale zu beobachten, wobei diese dann meist fein dispers verteilt in der Dolomitmatrix auftreten.

Basierend auf der farblichen Divergenz und des variierenden Auftretens im Lagerstättenkörper können in dieser Phase der Erkundung mindestens 3 Farbqualitäten unterschieden werden (Abb. 5.15):

- Dolomitmarmor reinweiß bzw. reinweiß mit einer vernachlässigbarer Beimengung dispers verteilter mafischer Minerale oder einer entsprechenden Bänderung

- Dolomitmarmor weiß bzw. weiß/reinweiß mit einem verstärkten Auftreten dunkel abgesetzter Bänder und/oder entsprechend farblich abgesetzter Bereiche

- Dolomitmarmor grau/weiß bzw. weiß/reinweiß mit einem erheblichen Auftreten dunkel abgesetzter Bänder und/oder entsprechend farblich abgesetzter Bereiche

Einer anzunehmenden Preisreduktion, die mit einer Herabsetzung in der Farbqualität bzw. einer verstärkten Heterogenität im Dekor einhergeht, könnte mit einer individuellen Abstimmung des Aufschneidens entsprechender Rohblöcke entgegengewirkt werden. In der Produktion von Rohplatten können durch verschiedene Schnittrichtungen dekorative Anschnittseffekte entstehen, wodurch eine erhöhte positive Variabilität hinsichtlich des Dekors der Endprodukte erreicht werden könnte (vgl. Kap. 4.6.2.1). So kann beispielsweise aus der Bänderung im Anschnitt parallel zum Lager eine flächig bis wolkig-nebulöse Textur 
resultieren. Diesem Aspekt sollte bereits bei der Planung einer möglichen Abbauführung Rechnung getragen werden (vgl. Kap. 2.5).
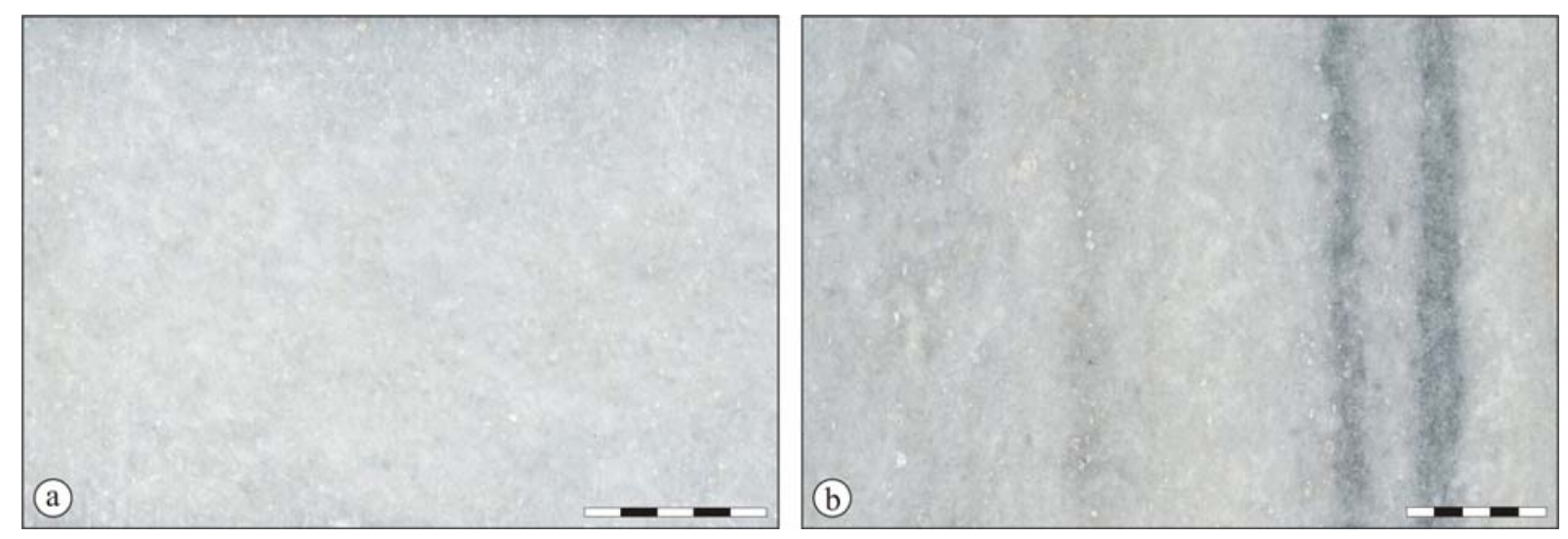

Abb. 5.15: Bandbreite des farblichen Dekors im Dolomitmarmor des Vorkommens von Mtesa: a) reinweiß bzw. reinweiß mit vernachlässigbarer Beimengung dispers verteilter mafischer Minerale oder einer entsprechenden Bänderung. b) grau/weiß bzw. weiß/reinweiß mit einem erheblichen Auftreten dunkel abgesetzter Bänder und/oder entsprechend farblich abgesetzter Bereiche.

Als weitere farbliche Veränderungen in der relativ monochromatischen Matrix des Dolomitmarmors tritt vereinzelt Apatit und untergeordnet Hämatit auf. Zumeist sind diese Minerale nur sporadisch und dann fein im Gestein verteilt, wodurch keine erhebliche, durchgreifende Veränderung in der Homogenität des Grundfarbtons zu erwarten ist. Lediglich stark begrenzt konnte im aufgeschlossenen Bereich lokal ein verstärktes Auftreten von Apatit in akkumulierter Form im cm-Maßstab beobachtet werden (Abb. 5.16 a). Eine gezielte Aushaltung einer weiteren Farbqualität in einer wirtschaftlichen Größenordnung ist dabei jedoch auszuschließen. Auch das Auftreten von Graphit ist weitestgehend als unproblematisch zu erachten, da der Gesamtgehalt als sehr gering einzuschätzen ist (Abb. 5.16 b). Im aufgeschlossenen Bereich konnten Mineralakkumulationen zu Bändern oder Linsen nicht beobachtet werden.

Für die Gesamtheit der akzessorisch auftretenden Minerale ist infolge der zumeist fein dispersen Verteilung im Dolomitmarmor kein entscheidender Einfluss im Hinblick auf eine Definition der Dekorqualitäten zu erwarten. Zu beachten ist jedoch, dass vor allem Pyritkristalle in der Anwendung bei Kontakt mit Wasser durch Oxidationserscheinungen zu unästhetischen Fleckenbildungen führen können (vgl. Kap. 3. 13.1). Ein verstärktes Auftreten ist demnach als limitierender Faktor anzusehen. 

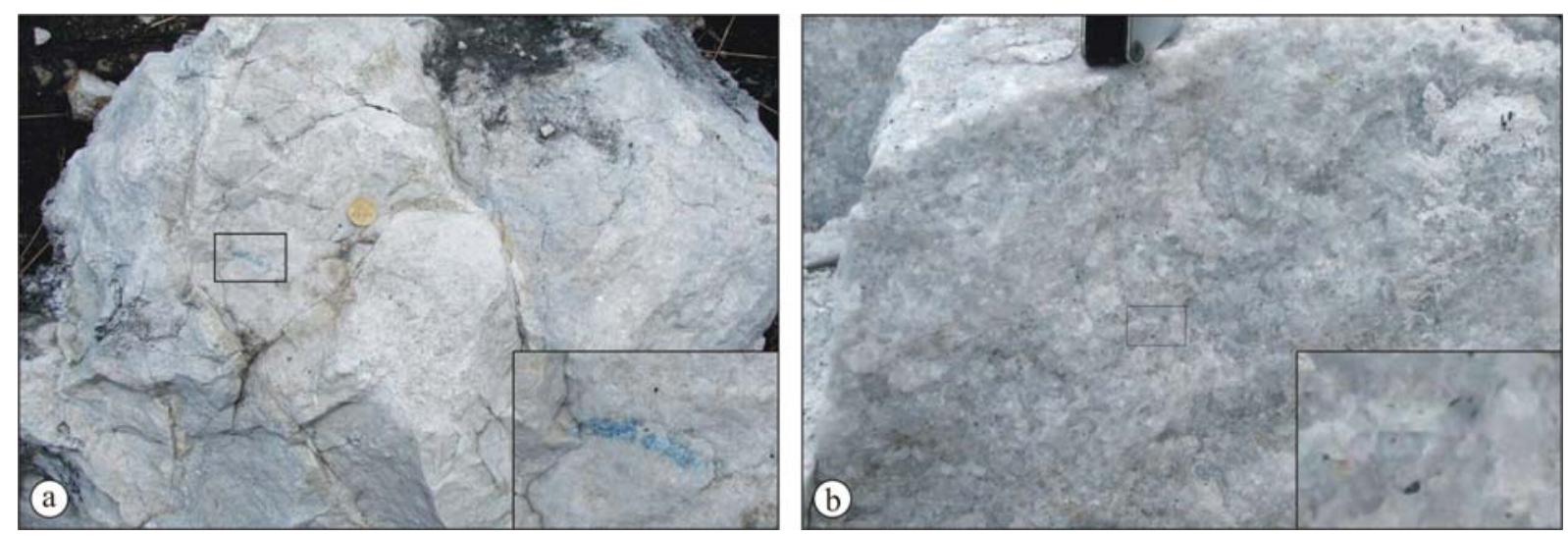

Abb. 5.16: a) Fleckig akkumuliertes Auftreten von Apatit und b) disperse Verteilung von Graphitschuppen im Dolomitmarmor.

\subsubsection{Tektonische Überprägung, Trennflächengefüge}

Dem Gefügeinventar nach zu urteilen unterlag der Lagerstättenkörper einer geringen, dominant duktilen Überprägung. Demzufolge ist eine intensive Vermischung von differenzierten Schichtpaketen nicht anzunehmen, womit größere Partien mit gleich bleibendem Dekor zu vermuten sind. Im Zuge dieser primären tektonischen Phase, die vermutlich einer regionalen Faltung entspricht, erfolgte auch die Anlage eines zumeist weitständigen, annähernd orthogonalen Kluftsystems (Abb. 5.17 a). Hinsichtlich der Abstandsverteilung innerhalb des Trennflächensystems treten nicht selten Werte zwischen 1 bis $1,5 \mathrm{~m}$ und darüber auf (Abb. $5.17 \mathrm{~b}$ ), womit grundsätzlich gute Voraussetzungen zur Ausbringung von Werksteinrohblöcken gegeben sind (vgl. Kap. 2.1 und 2.3.2.2). So konnten bereits im oberflächennahen Bereich einzelne größere, quaderförmige in-situ Blöcke beobachtet werden (Abb. 5.18 a). Eine Einschränkung ist dabei durch wiederholt in Abständen von vermutlich 20 bis $30 \mathrm{~m}$ auftretende engständige Kluftscharen gegeben (Abb. 5.18 a, vgl. Kap. 2.4.1).
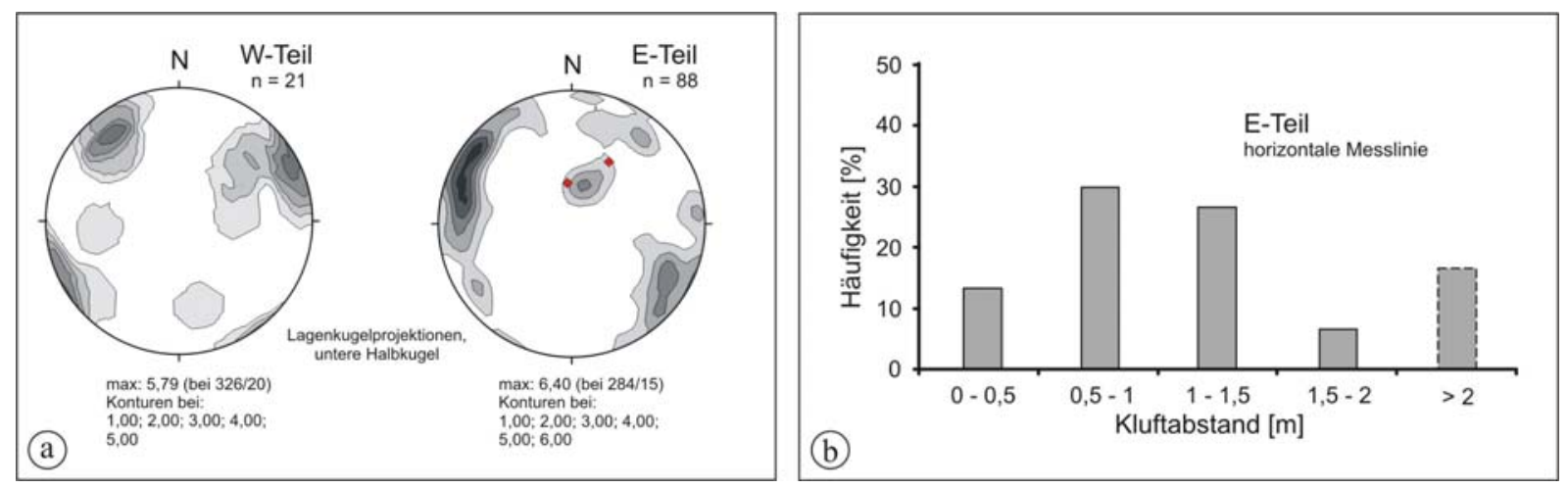

Abb. 5.17: a) Lagenkugelprojektion der Trennflächensysteme im Marmorkörper für die zwei Hauptbereiche der Lagerstätte von Mtesa (rote Rauten: Polpunkten zweier festgestellter Scherbahnen); b) Abstandsverteilung im Trennflächensystem am Beispiel einer Traverse im E-Teil der Lagerstätte (die relative Häufigkeit für Abstände $>2 \mathrm{~m}$ ist vermutlich geringer, da anzunehmen ist, dass infolge der Aufschlusslage nicht jede Kluft aufgenommen werden konnte). 

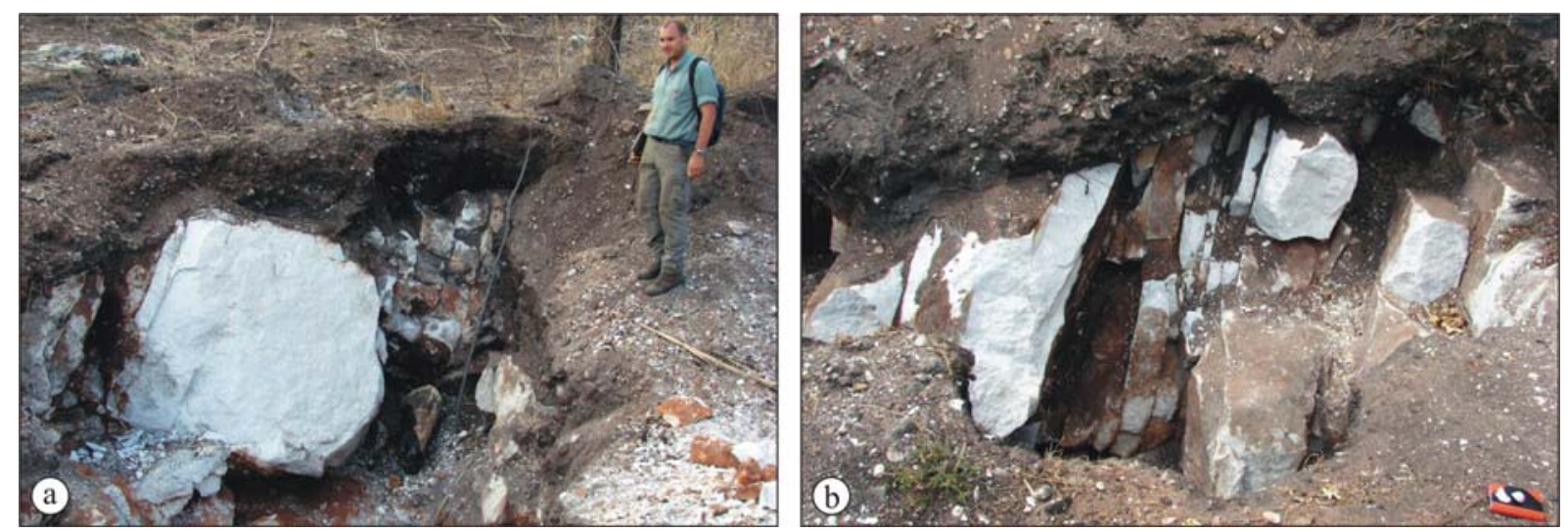

Abb. 5.18: a) Bereits oberflächennah treten vereinzelt größere, annähernd quaderförmige in-situ Blöcke auf. b) Die Rohblockhöffigkeit wird durch wiederholt auftretende engständige Kluftscharen herabgesetzt.

Dieser massiv erscheinende Lagerstättenkörper wurde jedoch durch eine zweite tektonische Phase, die vermutlich einer regionalen Einengung mit Scheransätzen entspricht, weiter zergliedert. Die Überprägung bewirkte ein diagonal verlaufendes Kluftsystem mit einem Einfallen zwischen 35 und $55^{\circ}$. Durch den abweichenden Winkel zum Hauptkluftsystem werden die aus dem primären Trennflächengefüge resultierenden, relativ gleichmäßigen in-situ Blöcke zerschnitten (vgl. Kap. 2.4.2). Die Diagonalklüftung tritt jedoch im beobachtbaren Bereich der Lagerstätte relativ selten auf (Abb. 5.19). Gleichzeitig ist nach dem Geländebefund die Tiefenwirkung des Kluftsystems fraglich, da vielfach eine vollständige Verheilung durch Mineralisation zu beobachten ist. Erkennbare Alterationen, die zum Teil auch einen kavernösen Charakter besitzen, sind vermutlich auf oberflächennahe Bereiche mit einer Tiefenwirkung von einigen Dezimetern beschränkt. Zudem sind auch im aufgeschlossenen Bereich Gesteinspartien von mehr als einem Meter ohne erkennbare Trennfläche zu beobachten. Folglich ist mit zunehmender Teufe mit größeren, relativ soliden Partien zu rechnen. Dabei ist jedoch bei entsprechender Belastung in der bautechnischen Anwendung die Ausbildung von möglichen Sollbruchstellen infolge der feinen Fissuren zu beachten (vgl. Kap. 2.4 und 4.8).

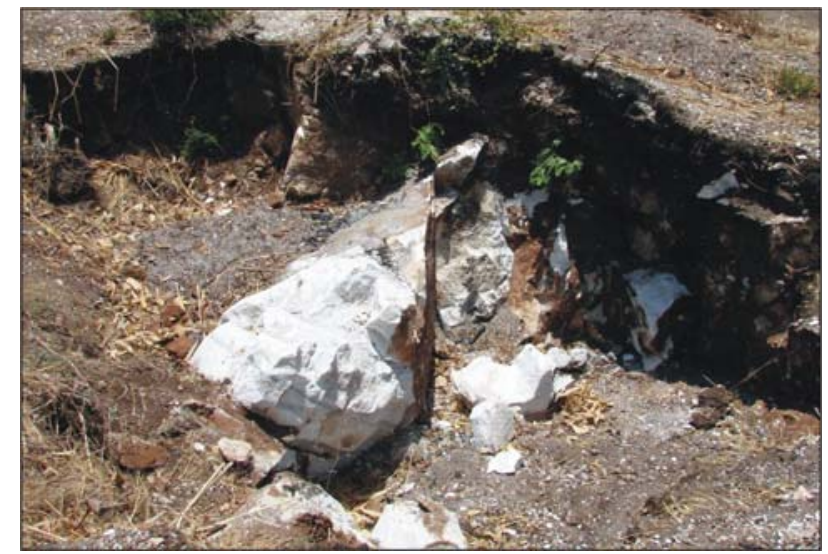

Abb. 5.19: Das primäre annähernd orthogonal ausgerichtete Trennflächengefüge des Marmorkörpers wird durch ein diagonales Trennflächensystem zerschnitten.

Als weiteres lagerstätten-kontrollierendes Element konnten im NE-Teil der Lagerstätte zwei Scherbahnen festgestellt werden (Abb. 5.20, vgl. Abb. 5.17). Die kataklastisch deformierten Bewegungsbahnen zeigen ein Einfallen von $25^{\circ}$ bzw. $45^{\circ}$ nach S bzw. SW und 
folgen somit in etwa dem primären Schichtungsgefüge. In der Mächtigkeit zeigen die Scherbahnen eine wellenförmige Variabilität mit Ausmaßen zwischen wenigen Dezimetern bis rund einem Meter. In den intensiv zerriebenen Bereichen liegt insgesamt kein geschlossener Kornverband mehr vor. Lediglich einzelne dünnere Bänke sind bestehen geblieben, die jedoch ebenfalls in kleinere Blöcke zergliedert und von kataklastischem Material vollständig umgeben sind. Inwieweit die Gefügeentfestigung auf das direkt angrenzende, scheinbar intakte Gestein übergegriffen hat, ist nur schwer abzuschätzen (vgl. Kap. 4.6.1.4). Auf Basis der aktuellen Aufschlusslage konnten keine weiteren Scherzonen nachgewiesen werden. Anhand des Reliefs innerhalb des Marmorkörpers, mit entsprechenden seichten Absenkungen in der Längserstreckung $(\sim \mathrm{E}-\mathrm{W})$, ist das Auftreten paralleler Scherzonen jedoch nicht auszuschließen.

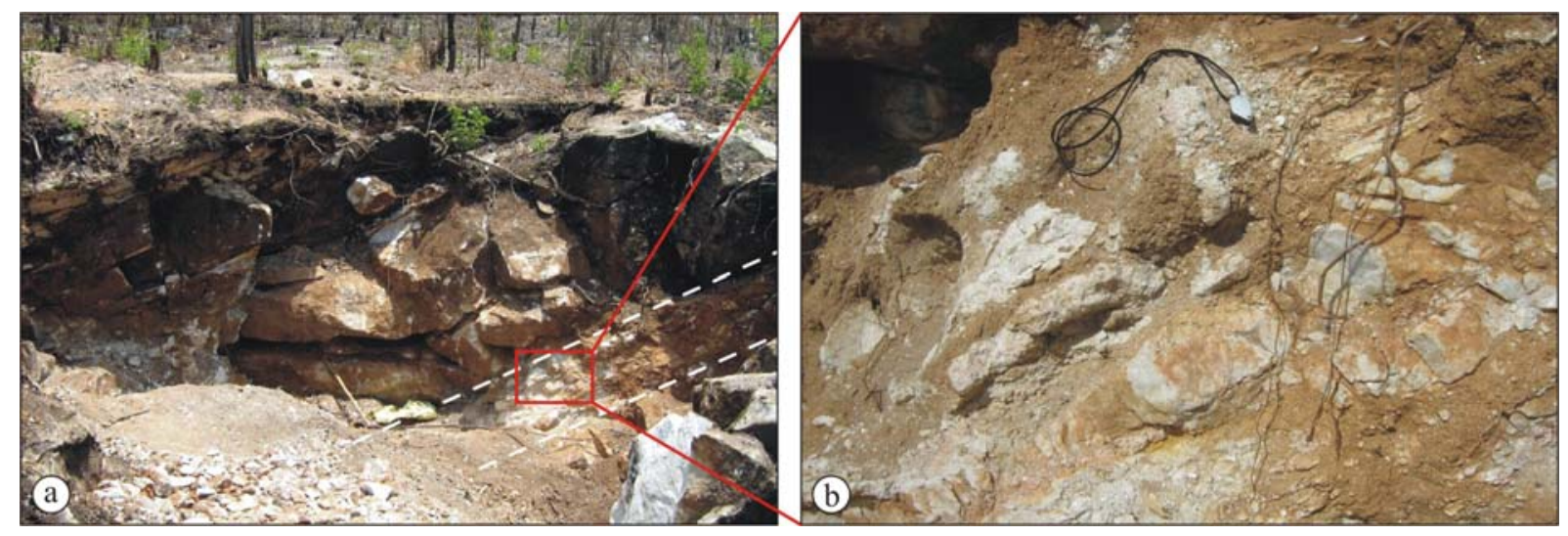

Abb. 5.20: a) Aufschluss einer Scherbahn im NE-Teil der Lagerstätte; b) boudinageartig gegliederte intakte Bereiche des Marmors im intensiv zerriebenen Material.

Für das Vorkommen sind aus der geologischen Situation im W' sowie E' Kontaktbereich zwischen der sedimentbedeckten Zone um Mtesa und dem angrenzenden Marmor weiterhin zwei größere Störungsbereiche zu vermuten. Vor allem nach der gerichteten Form und dem Ausmaß der Intrusionskörper E' Mtesa sowie im SE' Bereich des Vorkommens kann eine raumgreifende Zone kataklastischer Deformation mit einem WNW-ENE gerichteten Streichen nicht ausgeschlossen werden. Eine ähnliche Situation ist im Bereich W' Mtesa gegeben. Hier kann parallel zu einer angenommenen Störung im E' Teil des Vorkommens eine weitere großräumige Diskontinuität angelegt sein, die über die dortige brekziöse Zone und den sich im SW anschließenden Intrusionskörper verläuft. Die Ausrichtung dieser Intrusion weist zwar eine zur vermuteten Störung winkelabweichende Erstreckung aus, doch könnte diese auch auf einem zum umgebenden Hüllengestein schichtparallelen Eindringen des Intrusionskörpers basieren. Auch anhand von Lesesteinen in der Verlängerung nach WNW, im Bereich des Abtauchens des Marmors unter die sedimentäre Bedeckung, ist das mögliche Vorhandensein einer breiter angelegten Störungszone dokumentiert. In den Grenzbereichen zu diesen Störungszonen ist im Hinblick auf einen möglichen Abbau des Dolomitmarmors eine generell höhere Trennflächendichte anzunehmen (vgl. Kap. 2.2). 


\subsubsection{Thermische Beeinflussung durch granitische Intrusionen}

Durch die verschiedenen Granitintrusionen bzw. die in den Brekzienzonen auftretenden pegmatitischen Körper werden lokal gefügebezogene und/oder mineralogische Veränderungen des Dolomitmarmors hervorgerufen (vgl. Kap. 5.5.3). Diesbezüglich konnten im aufgeschlossenen Bereich partielle stoffliche Umwandlungen sowie kleinräumige, flächige Injektionen von Silikatmineralen beobachtet werden.

Mit Ausnahme des komplexen Kalksilikatfelses im S' Abschnitt des E' Lagerstättenbereichs erscheint die metasomatische Beeinflussung des Dolomitmarmors auf einen Bereich von rund $30 \mathrm{~m}$ im Kontakt zu den granitoiden bzw. pegmatitischen Körpern begrenzt zu sein. In diesen Säumen erfolgte die Bildung verschiedener Kalksilikatminerale (Abb. 5.21). Zumeist treten diese metasomatischen Mineralneubildungen zu Lagen oder auch Linsen akkumuliert auf, wobei häufig Restkerne des Dolomitmarmors von kalksilikatischen Bändern umflossen werden, was eine erhöhte Farbdivergenz zur Folge hat. Dabei treten farbliche Veränderungen hin zu hellgräulichen, meist leicht grünlichen Farbstichen auf. Verbunden sind diese Bereiche zudem häufig mit einer erhöhten Heterogenität hinsichtlich der Textur, woraus eine verstärkte Unregelmäßigkeit und Unruhe im Dekor resultiert. Auf der Variabilität im Mineralbestand können zudem auf kurzer bis sehr kurzer Distanz Differenzen in der Gesteinshärte basieren. Diese Schwankungen können zu einer gewissen Problematik in der Seilführung im Abbau mit Diamantseilsägen führen. Diese können sich vor allem in einem erhöhten Verschleiß oder in einem unregelmäßigen, schwer zu kontrollierenden Lauf des Diamantseils äußern. Auch in den weiteren Schritten der Rohblockformatierung und Endproduktfertigung können durch divergierende Gesteinshärten Probleme bzw. Unregelmäßigkeiten in der Maßhaltung hervorgerufen werden. Die Möglichkeit zur Ausweisung einer oder mehrerer eigenständiger Handelssorten kann nur im laufenden Abbaubetrieb geklärt werden. Aus dem reinen Oberflächenbefund ist jedoch eine Aussage über die Tiefenwirkung und damit über eine mögliche Vorratslage entsprechend definierter Handelssorten nicht möglich.

Infolge des Auftretens mehrerer granitoider bzw. pegmatitischer Körper (vgl. Abb. 5.6) ist eine intensive Durchdringung des gesamten Lagerstättenkörpers nicht auszuschließen. Dies gilt besonders für weitere mögliche Granitoide in der Teufe. Auch daraus ergibt sich die Notwendigkeit der geowissenschaftlichen Begleitung eines möglichen Abbaus. Durch eine kontinuierliche Analyse des ausgebrachten Materials können Schwankungen im Mineralbestand, die durch eine verstärkte thermische Beeinflussung des Marmors hervorgerufen wurden, erfasst werden. Gegebenenfalls kann dann eine Änderung der Abbauführung dazu beitragen, im Spektrum der definierten Handelssorten zu bleiben. Neben der rein wirtschaftlichen Komponente ist so vor allem auch die Umweltbilanz der Marmorgewinnung positiv zu beeinflussen, da unnötige Materialbewegungen, einhergehend mit der Erweiterung des Abbaus, vermieden werden können. 

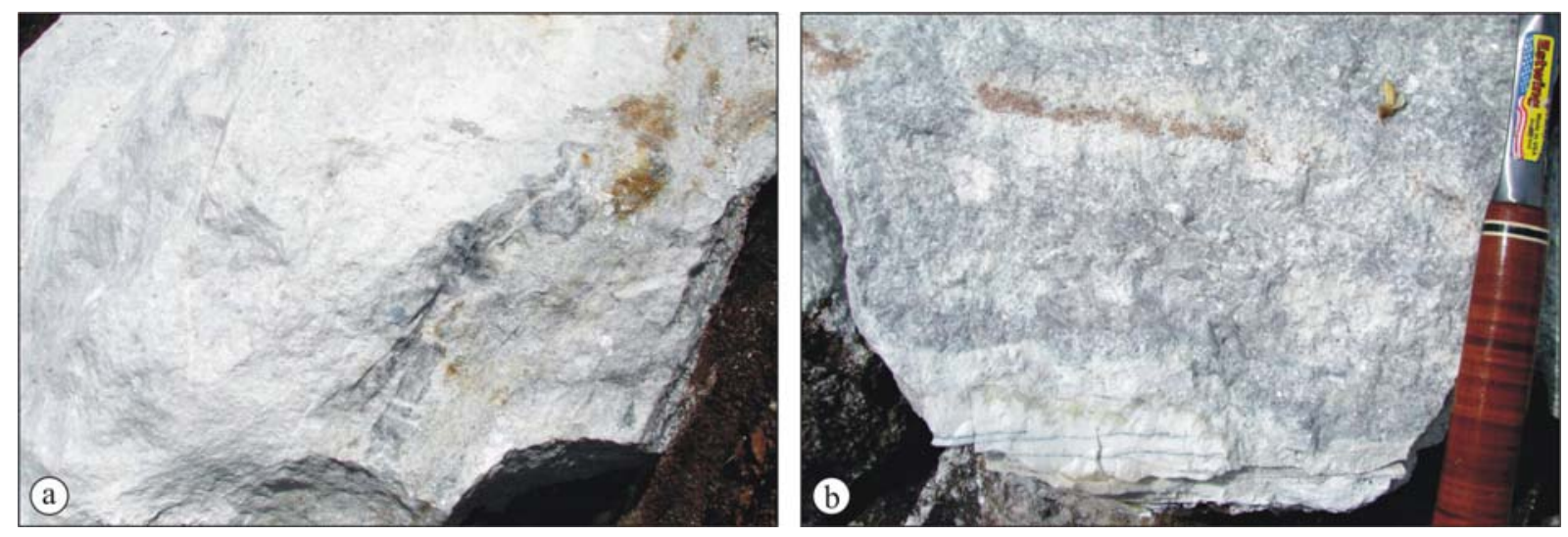

Abb. 5.21: Thermische Beeinflussung des Dolomitmarmors mit kalksilikatischen Reaktionssäumen oder -bändern (a und b) und überdurchschnittlichem Kristallwachstum (Bildmitte in b).

Als weitere thermisch induzierte Gefügeveränderung tritt lokal begrenzt eine Blastese des Dolomitmarmors auf (Abb. 5.21 b, s.a. Abb. 5.11). Diese Bereiche können vermutlich Ausmaße im dm-Bereich bis zu wenigen Metern umfassen und weisen dabei keine erkennbar regelmäßige Form auf. Dominante Erscheinung ist dabei ein überdurchschnittliches Kristallwachstum, wobei Größen von über $1 \mathrm{~cm}$, teilweise über $3 \mathrm{~cm}$, bei idiomorphem Kristallhabitus und polygonalen Korngrenzen beobachtet wurden. Gleichzeitig sind entsprechende Bereiche meist mit dem Auftreten von Kalksilikatmineralen sowie teils auch Feldspäten oder Trümmer von Feldspäten in verheilten Kluft- bzw. Risssysthemen charakterisiert. Insgesamt ist im Allgemeinen infolge der Korngröße mit einer Herabsetzung der mechanischen Stabilität zu rechnen (vgl. Kap. 3.9.2).

Eine mineralogische Abweichung im Dolomitmarmor stellt zudem die Kristallisation von Feldspäten auf Kluftflächen bzw. eine entsprechende Verheilung von Klüften dar. Diese beruhen auf der Injektion durch Restfluide und ist augenscheinlich dominant an das sekundäre Trennflächensystem der Diagonalklüfte gebunden. Neben einem möglichen Wechsel in der Gesteinshärte im Abbau ist vor allem auch hier zu beachten, dass diese Bänder mögliche potenzielle Schwächezonen hinsichtlich der bautechnischen Verwendung darstellen.

Das Gestein des komplexen Kalksilkatfelskörpers, der in der Kontaktaureole der Intrusion im SE' Bereich der Lagerstätte gebildet ist, erscheint primär insgesamt zäh. Damit ist im Vergleich mit dem Abbau des Marmors von einem erhöhten Maschinen- bzw. Energieeinsatz auszugehen. Hinsichtlich der physikalischen und technischen Parameter ist jedoch infolge des divergierenden Gefügebildes bzw. des brekziösen Charakters mit gewissen Schwankungen zu rechnen. Zudem können aus der Randlage am Gesamtvorkommen und dem direkten Kontakt zum Intrusionskörper mögliche Fazieswechsel auf kurzen Distanzen resultieren. Daraus ergeben sich im Abbau sowie in der Verarbeitung vergleichbare Problematiken hinsichtlich einer verstärkten Variabilität der Färbung und Gesteinshärte. 


\subsection{Lagerstättenökonomie}

\subsubsection{Charakterisierung einzelner Teillagerstätten}

Aus der Gesamtheit der lagerstätten-kontrollierenden Elemente lassen sich auf der Fläche des Vorkommens mehrere Teilbereiche separieren (Abb. 5.22). Diese Zergliederung ist primär durch die verschiedenen, zum Teil raumgreifenden Intrusionen sowie durch die sedimentäre Bedeckung gegeben. In Folgenden sollen die differenzierten Teilbereiche in ihrer räumlichen Ausdehnung sowie den charakteristischen Elementen beschrieben werden. Die primäre Viertelung des Vorkommens folgt dem Hauptstraßenverlauf sowie in der Vertikalen der Ortslage Mtesa. Die Benennung der Bereiche richtet sich nach den Himmelsrichtungen, womit die Lagerstättenbereiche NW, NE, SE, SW gebildet werden. Erfolgt eine weiter differenzierte Zergliederung, so sind die einzelnen Teilbereiche mit der entsprechenden Himmelsrichtung sowie einer aufsteigenden Nummerierung versehen.

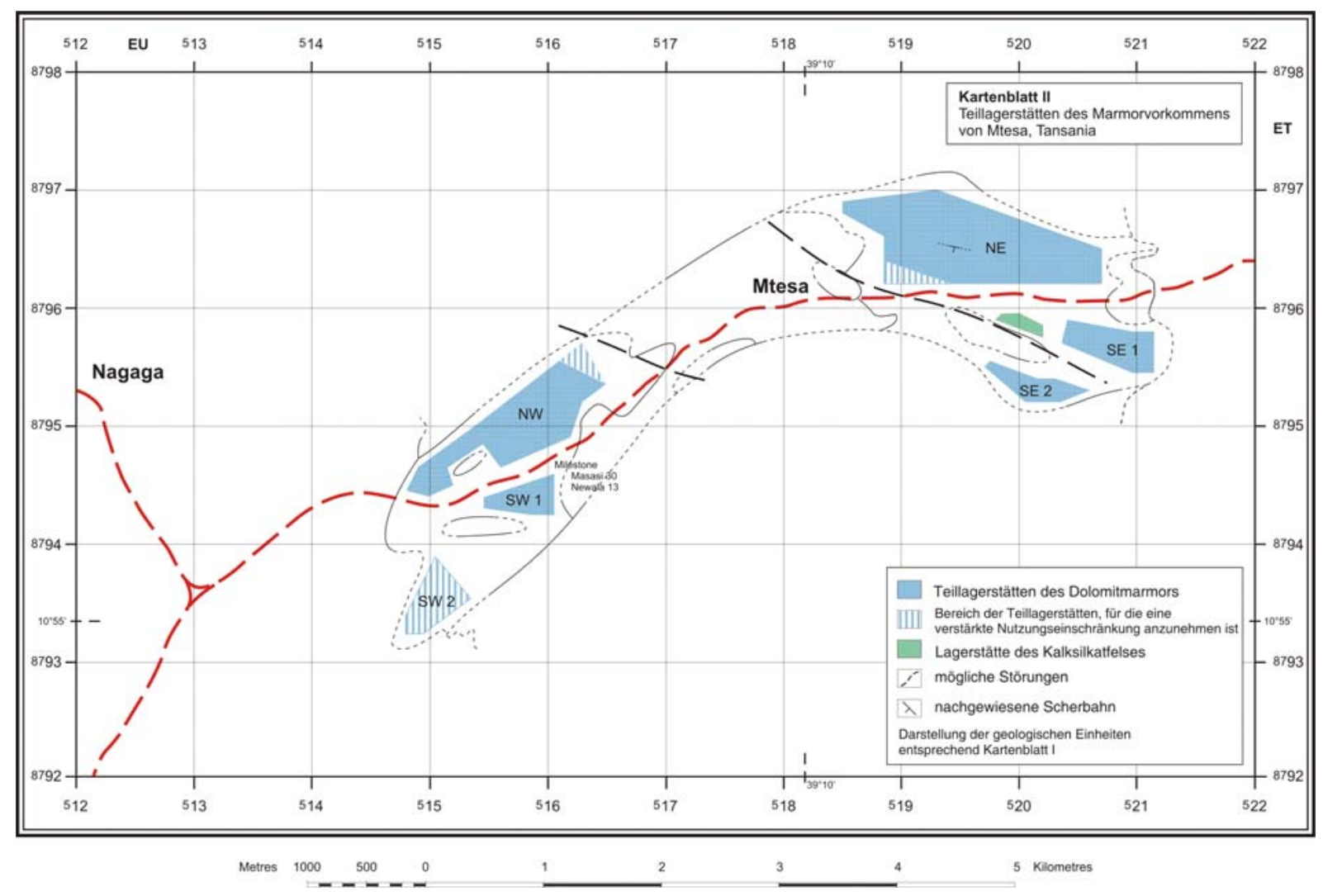

Abb. 22.: Teillagerstätten im Marmorvorkommen von Mtesa, Tansania.

\section{Teillagerstätte NW}

Nach der vorhandenen Aufschlusslage ist der Lagerstättenbereich NW im W durch überlagernde jüngere Sedimente begrenzt, wobei eine weitere Ausdehnung in der Teufe nicht auszuschließen ist. Für den NE’ Bereich dieser Teillagerstätte könnte jedoch die Möglichkeit einer ökonomisch sicheren Vorratslage stark eingeschränkt sein, da die räumliche Ausdehnung der dortigen Brekzienzone aus dem Geländebefund nicht eindeutig belegbar ist. 
Intern ist die Geometrie der Teillagerstätte durch einen, im Ausstrich kleineren Intrusionskörper zerschnitten, der hier als Härtling die Morphologie bestimmt. Es ist nicht auszuschließen, dass sich dieser Körper in der Teufe fortsetzt, da zumindest in der NW' Verlängerung vereinzelt kleinere Partien beobachtet werden konnten, die eine metasomatische Überprägung mit der Bildung von Kalksilikatmineralen aufweisen. Hinweise auf Störungsbereiche als Basis für die Intrusion konnten in diesem Bereich des Vorkommens nicht nachgewiesen werden. Dementsprechend könnte neben dem Hauptgebiet der Teillagerstätte NW auch die flächenmäßig kleineren Bereiche NW' der Intrusion sowie im N' Anschluss an den Straßenverlauf im kleineren Maßstab als Lagerstätte genutzt werden. Voraussetzung dafür ist, dass sich der nachteilig wirkende Einfluss des granitischen Körpers weitestgehend auf den sichtbaren Abschnitt beschränkt.

Abgesehen von den metasomatisch geprägten Säumen oder Bändern, ist der Marmor dieses Bereichs von überwiegend weißer bis hellgrauweißer, lokal reinweißer Färbung. Unregelmäßig treten dabei cm- bis dm- mächtige grau, teils grünlich grau abgesetzter Lagen und Bänder auf.

\section{Teillagerstätte NE}

Dieser flächenmäßig größte Bereich des Vorkommens ist durch das Fehlen einer internen Zergliederung durch Intrusionskörper charakterisiert. Doch durch die im E am Marmor angrenzende Intrusion sind hinsichtlich der Begrenzung im Abbau gleiche Annahmen zu möglichen metasomatisch bedingten Veränderungen im Gefüge und/oder der mineralogischen Komposition des Dolomitmarmors zu beachten. Gleiches gilt für den kontaktnahen Bereich zur granitischen Intrusion E' der Ortschaft Mtesa. Inwieweit die kataklastische Zone in diesem Bereich möglicherweise in dem zu vermutenden etwa ESE-WNW-verlaufenden Störungsbereich einzugliedern ist, ist aus der gegebenen Aufschlusssituation nicht sicher belegbar. Für eine Abschätzung der Vorratslage kann sich für diesen Bereich in der Folge eine gewisse Einschränkung ergeben. Gleiches gilt für die Frage, ob und in welchem Maße ein mögliches wiederholtes Auftreten der in diesem Teilbereich nachgewiesenen Scherbahnen eine weitere Zergliederung bzw. Verkleinerung der Teilfläche nach sich ziehen würde.

Im Allgemeinen kann angenommen werden, dass für das ausgewiesene Gebiet hinsichtlich der farblichen, texturellen und mineralogischen Varietät des Marmors eine mit dem Kernbereich der Teillagerstätte NW vergleichbare Qualität vorliegen wird.

\section{Teillagerstätten SW 1 und SW 2}

Die Zergliederung der Teillagerstätte SW ergibt sich primär aus der dortigen Intrusion. Auf dem N' bis zum Bereich des Straßenverlaufs gelegenen Abschnitt (Teillagerstätte SW 1) konnten keine Hinweise auf kataklastisch ausgeprägte Bewegungsbahnen erkannt werden. Hinsichtlich der Qualität des Dolomitmarmors bestehen Bereiche, die überwiegend ein nahezu reinweißes Farbdekor aufweisen. Wiederholt zeigen sich jedoch auch auf kurzer 
Distanz Oberflächen, die durch deutliche Farbschwankungen infolge einer metasomatischen Beeinflussung charakterisiert sind.

Im Falle der Nutzung der Teillagerstätte SW 2 ist in jedem Fall mit einem wiederholten Auftreten psammitischer Lagen zu rechnen (vgl. Abb. 5.9). Wie weit der im S an die Intrusion angrenzende Bereich einer ausgeprägten silikatischen Feinschichtung mit kleinräumigen schichtparallelen Intrusionen tatsächlich ausgebildet ist, erscheint fraglich. Zudem sind im Bereich S’ der Intrusion Hinweise auf mögliche pegmatitische Gänge in der Teufe gegeben, deren räumliche Ausbreitung nicht abzuschätzen ist.

Teillagerstätten SE 1 und SE 2

Dieser Bereich des Vorkommens wird von einem raumgreifenden Intrusionskörper mit einer Längserstreckung in ENE/WSW' Richtung von rund $1000 \mathrm{~m}$ bei einer ungefähren Breite von $250 \mathrm{~m}$ dominiert. Bedingt durch die Intrusion und des $\mathrm{zu}$ vermutenden Störungsbereiches, der in Verbindung mit der für die Teillagerstätte NE beschriebenen Nutzungseinschränkung steht, ergeben sich für diesen Teilbereich des Vorkommens zwei relativ kleinräumige potenzielle Abbauflächen.

Hinsichtlich der Qualität des Dolomitmarmors ist von einer relativ hohen Variabilität in Dekor und Farbe auszugehen. Dies beruht auf der kurzen Distanz zum beherrschenden Granitkörper sowie zu einer weiteren Intrusion, die den Teilbereich SE 1 im N begrenzt. Dementsprechend sind häufig kalksilikatische Mineralbildungen in Bändern oder Linsen für beide Teilbereiche charakteristisch. Für den Bereich SE 1 ist zudem die Begrenzung im NW, die mit dem Wechsel vom Dolomitmarmor zur calcitführenden Marmorvarietät gegeben ist, auf Basis der aktuellen Aufschlusssituation nicht eindeutig ersichtlich.

\section{Teillagerstätte eines Kalksilikatfelses}

Im direkten Kontakt der zentralen Intrusion im Bereich der Teillagerstätten SE 1 und SE 2 ist auf mindestens $50 \mathrm{~m}$ Distanz mit einer durchgreifenden metasomatischen Überprägung mit der Bildung von Kalksilikatfels zu rechnen (vgl. Kap. 5.5.2). Nachweislich ist dieser Bereich im N der Intrusion ausgebildet, wobei eine gesonderte Gesteins- und Farbqualität ausgewiesen werden muss. Die wirtschaftliche Vorratslage ist jedoch als sehr begrenzt anzusehen, woraus vor allem die Frage nach einer Aushaltung als Handelssorte mit einem gezielten Abbau oder aber der Einstufung als mögliches Nebenprodukt im Abbau des Dolomitmarmors resultiert. 


\subsubsection{Abschätzung der Gesamtvorratslage des Dolomitmarmors}

Für die Teillagerstätten in ihrer Gesamtheit ergibt sich für den Dolomitmarmor bei einer Fläche von rund 2,6 $\mathrm{km}^{2}$ und einer anzunehmenden Abbauteufe von etwa $40 \mathrm{~m}$ ein Gesamtvolumen von (in $\mathrm{m}^{3}$ ):

\begin{tabular}{|c|c|}
\hline NW & $47.800 .000 \mathrm{~m}^{3}$ \\
\hline $\mathrm{NE}$ & $27.850 .000 \mathrm{~m}^{3}$ \\
\hline SW 1 & $5.600 .000 \mathrm{~m}^{3}$ \\
\hline SW 2 & $7.300 .000 \mathrm{~m}^{3}$ \\
\hline SE 1 & $9.300 .000 \mathrm{~m}^{3}$ \\
\hline SE 2 & $5.440 .000 \mathrm{~m}^{3}$ \\
\hline$\sum$ & $103.290 .000 \mathrm{~m}^{3}$ \\
\hline
\end{tabular}

Bei der Aufstellung wurden die Abschnitte der Teillagerstätten NW und NE, welche einer möglichen Nutzungseinschränkung infolge eines raumgreifenden Störungsbereiches unterliegen, mit einbezogen. Sollte eine weitere Erkundung das Vorhandensein stärker kataklastisch überprägter Zonen bestätigen, muss durch die Reduktion der nutzbaren Fläche eine Herabsetzung des Gesamtvolumens im Falle der Teillagerstätte NW von mindestens 5 \% sowie im Falle der Teillagerstätte NE von mindestens $7 \%$ angenommen werden.

Aus der aktuellen Aufschlusssituation kann insgesamt kann davon ausgegangen werden, dass mit mindestens $50 \%$ als dominante Farb- und Dekorqualität des Dolomitmarmors eine weiße bis hellgräulich weiße, in kleineren Bereichen reinweiße Varietät mit hellgrauen Schlieren oder feineren Lagen auftritt. Die Vorratslage für eine nahezu reinweiße Varietät, die nur zu sehr geringen Anteilen einen hellgräulichen Farbstich durch feinstschlierig oder dispers verteilte mafitische Minerale aufweist, ist mit maximal $10 \%$ anzusetzen. Weiterhin ist anzunehmen, dass sich das Restvolumen relativ gleichmäßig auf Farbqualitäten verteilt, die weiße bis hellgraue Grundfarben aufweisen und dabei durch in unterschiedlichem Maße auftretende, hellgraue zum Teil graugrünlichen Lagen und Bänder in variierender Stärke charakterisiert sind. Als Folge der möglicherweise verstärkten Variabilität in der Stärke, der Verteilung und der Intensität farblich abgesetzter Bereiche, sollte eine Zuordnung fertig gestellter Rohblöcke zu definierten Handelssorten nach Inaugenscheinnahme erfolgen (vgl. Kap. 4.6.1.2).

\subsubsection{Charakterisierung des Abraummaterials}

Die Erfassung der lagerstätten-kontrollierende Elemente hat ergeben, dass sich Art und Umfang des Abraummaterials aus folgenden Aspekten zusammensetzt:

- $\quad$ sedimentäre Überdeckung

- Auswirkungen des Trennflächengefüges

- Auswirkungen der metasomatischen Einflüsse

Die Mächtigkeit der sedimentären Bedeckung ist für den gesamten Lagerstättenbereich als gering bis sehr gering zu betrachten (Abb. 5.23). Im E’ Bereich des Vorkommens ist dabei ein 
etwas erhöhter Anteil von bis zu $1 \mathrm{~m}$ anzunehmen. Auf der gesamten Fläche ist jedoch das Vorhandensein einzelner kavernöser Lösungshohlräume zu beachten (vgl. Kap. 2.2), deren Auftreten hier jedoch vermutlich auf oberflächennahe Bereiche begrenzt ist. Tieferreichende Karstbildungen sind anscheinend nicht vorhanden, da keine Hinweise auf lokale Einsturzdolinen aus der Geländesituation beobachtet werden konnten.

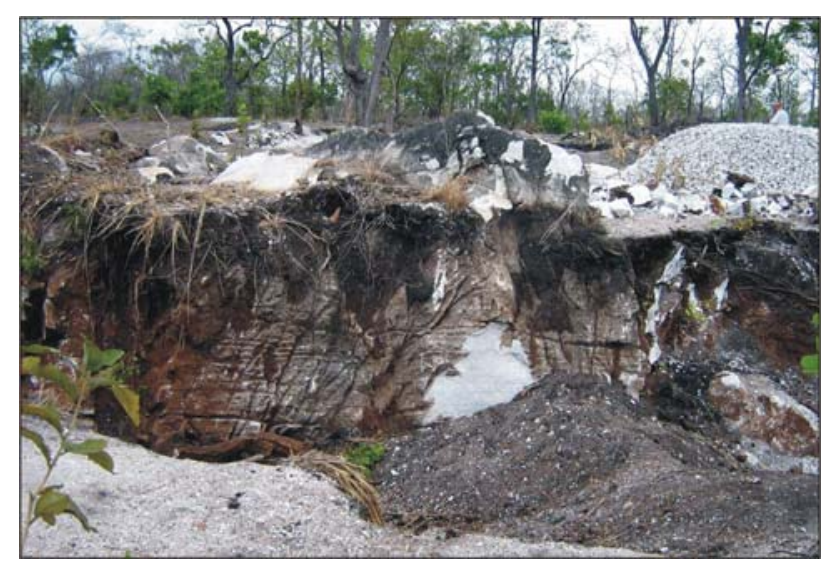

Abb. 5.23: Im Allgemeinen ist auf dem Gebiet der Lagerstätte, hier im Teilbereich NE, eine relativ geringe sedimentäre Bedeckung ausgebildet.

Da die potenziell gewinnbaren Rohblöcke einer Naturwerksteinlagerstätte in ihrer Form und ihrem Volumen durch das zugrunde liegende Trennflächengefüge definiert werden (vgl. Kap. 2.4), ist entsprechend dem primären, annähernd orthogonalen Kluftsystem eine relative hohe Rohblockhöffigkeit anzusetzen. Gemindert wird diese zum einen durch die wiederholt auftretenden Kluftscharen von rund 1 bis 2 m Breite (vgl. Abb. 5.18 b, vgl. Kap. 2.4.1). Das Gestein dieser Bereiche ist als Abraummaterial zu charakterisieren, da ein Ausbringen von gut dimensionierten Blöcken zum Zwecke der industriellen Aufarbeitung nicht möglich ist. Eine weitere Minderung hinsichtlich der Ausbringungsrate bewirkt das diagonal verlaufende Trennflächensystem (vgl. Abb. 5.19, vgl. Kap. 2.4.2). Hierdurch ist eine weitere Zergliederung primär großvolumiger in-situ Blöcke zu erwarten, aus dem sich ein Anteil an nicht verwertbarem Gestein von rund 40 bis $60 \%$ ergeben dürfte.

Hinsichtlich des klüftungsbedingten Abraumanteils ist zudem im oberflächennahen Bereich eine stärkere Ausprägung des Trennflächengefüges anzunehmen. Diese beruht zum einen auf der entlastungsmäßigen Öffnung von Diskontinuitäten (vgl. Kap. 2.2). Zum anderen ist das Gestein durch Oberflächenwässer bereichsweise kavernös zerlegt, womit eine deutliche weitere Öffnung der Klüfte zu beobachten ist. Das derzeitige Bild wird dabei durch den aktuellen Kleinstbergbau geprägt, wobei anzunehmen ist, dass dieser sich infolge der leichteren Abbaubarkeit häufig auf stärker geklüftete Bereiche konzentriert bzw. erweitert wurde. Doch konnten auch im direkten Oberflächenanschnitt einzelne großvolumigere, annähernd quaderförmige in-situ Blöcke identifiziert werden (vgl. Abb. 5.18 a). Für eine regelmäßige Ausbringung gut dimensionierter, industriell verwertbarer Großblöcke ist jedoch vermutlich ein Bereich von bis zu 5 m unter Geländeoberkante auszuschließen.

Außerdem muss der Materialanteil im Bereich möglicher Scherzonen dem verwertbaren Rohstoffanteil entzogen werden (vgl. Abb. 5.20). Auch für die direkt angrenzenden Bereiche und für Bereiche des Ausdünnens der Kataklasebänder ist eine verstärkte, primär nicht erkennbare Mikrorissbildung möglich, die bei Druckentlastung im Abbau oder bei der 
Formatierung von Rohblöcken reaktiviert werden kann, was zu einem möglicherweise erhöhten Materialverlust führen kann (vgl. Kap. 2.4.1 und 4.6.1.4). Eine exakte Abschätzung hinsichtlich der Häufigkeit dieser kataklastischen Bereiche und deren Mächtigkeit in der Teufe sind aus der Geländebeobachtung nur in unzureichendem Maße möglich. Sofern dies nicht durch die Anlage von Tiefenschürfen zu klären ist, wären im Rahmen einer Detailexploration Sondierbohrungen anzusetzen. Diese sollten im NE’ Bereich der Lagerstätte in einem Winkel von etwa $60^{\circ}$ gegen NNE, also in etwa lotrecht zu den dort nachgewiesenen Scherbahnen, niedergebracht werden, um eine Verfälschung durch schräge Anschnitte zu mindern (Palmström 1995). Für den W' Teil wäre eine dem Unterschied in der Anlage des Trennflächensystems entsprechende Veränderung der Bohrrichtung zu überdenken. Im Rahmen der Geländeerkundung konnten im aufgeschlossenen Bereich zwar keine Hinweise auf weitere Scherbahnen festgestellt werden, ein möglicher Abraumanteil von rund 10 bis $20 \%$ sollte jedoch nicht ausgeschlossen werden.

Hinsichtlich der metasomatischen Beeinflussung des Marmors und der sich daraus ergebenden Materialmengen, die von der Produktion von Rohblöcken ausgeschlossen werden müssen, ist im Vorfeld eine exaktere Einschätzung nur schwer möglich. Es ist daher zu beachten, dass darauf basierende Wechsel im Dekor oder der Gesteinsfestigkeit zu jedem Zeitpunkt im laufenden Abbaubetrieb auftreten können. Verstärkt wird dieser Umstand dadurch, dass eine vollständige Erfassung granitischer oder pegmatitischer Intrusionskörper im tatsächlich Umfang aus der Oberflächenkartierung gerade in den Kernbereichen des Lagerstättenkörpers nicht möglich ist. In der Ausweisung von potenziellen Abbaugebieten muss daher ein gewisser Mindestabstand $\mathrm{zu}$ den beschriebenen Intrusionen eingehalten werden. Trotz dessen sollte nach den Geländebeobachtungen eine mögliche Rate für metasomatisch bedingten Abraum von etwa 10 bis $15 \%$ angesetzt werden.

In der Gewinnung des Dolomitmarmors in Form von werksteinfähigen Rohblöcken muss somit unter Einbeziehung aller beschriebenen lagerstätten-kontrollierenden Elemente bei einer angenommenen Abbauteufe von $40 \mathrm{~m}$ insgesamt eine Abraumrate von mindestens 60 bis $80 \%$ angenommen werden. Unter Annahme einer Gesamtvorratslage des Dolomitmarmors von rund 103 Mio. $\mathrm{m}^{3}$ (vgl. Kap. 5.7.2) ergibt sich somit ein auszubringendes Rohblockvolumen von etwa 20 bis 40 Mio. $\mathrm{m}^{3}$. Eine Abschätzung möglicher Blockgrößen ist aus dem jetzigen Untersuchungsstand nur sehr eingeschränkt möglich, da eine detaillierte Analyse des Trennflächengefüges zur Berechnung eines Durchschnittsblocks (vgl. Kap. 2.3.2.2) infolge der Aufschlusslage nicht möglich ist. Aus der Oberflächensituation und der horizontalen Abstandsverteilung (vgl. Abb. 17 b) ist jedoch zu erwarten, dass der von Selonen \& Suominen (2003) beschriebene Minimalblock zur Aufarbeitung in modernen Sägeeinrichtungen (vgl. Kap. 2.1) in der Gewinnung des Dolomitmarmors erreicht werden kann.

Das Abraummaterial, vor allem im Bereich der engständigeren Kluftzonen (vgl. Kap. 5.6.2), könnte auch in kleineren Blöcken zur Verwendung als z.B. Trockenmauerwerk oder anderen kleinvolumigeren Elementen kommen oder aber den Belangen des Kleinstbergbaus zugeführt und zur Produktion von Zuschlagsstoffen verwendet werden. Damit könnten die 
Abraumrate vermindert und gleichzeitig auch lokale Interessen unterstützt und gefördert werden.

\subsection{Physikalische und technische Charakterisierung des Werksteinpotenzials}

Im Folgenden sollen die verschiedenen physikalischen und technischen Parameter, die Dehnungseigenschaften sowie die Verwitterungssensibilität des Marmors charakterisiert werden. Im Falle des Marmorvorkommens von Mtesa zielen die Eigenschaftsbestimmungen dominant auf die dolomitische Varietät ab. Dabei kamen Probekörper zur Anwendung, die aus einem Großblock des Dolomitmarmors präpariert wurden, welcher im Rahmen eines Kleintestabbaus gewonnen wurde. Zwar ist die Prüfung einer Anzahl, über das gesamte Vorkommen verteilter Proben als repräsentativer einzuschätzen (Reinsch 1991, vgl. Kap. 3.2), doch weisen die verschiedenen Probekörper mit reinweißen Bereichen, Bereichen mit einer mafischen Bänderung sowie einzelnen kryptokristallinen Calcitadern alle entscheidenden Elemente auf, die Auswirkungen auf die mechanische Stabilität des Gesteins ausüben könnten. Damit ist vor allem die Abschätzung einer möglichen negativen Einflussnahme der Gefügeelemente hinsichtlich der bautechnischen Verwendung gewährleistet (vgl. Kap. 2.4).

Sämtliche Untersuchungen erfolgten in Anlehnung an die standardisierten Prüfverfahren für Naturwerkstein (DIN bzw. EN), wie sie in Kapitel 4.7.1 beschrieben sind. Weiterhin wurde zu ausgewählten Fragestellungen, insbesondere der eventuell mangelnden Formbeständigkeit des Marmors hinsichtlich der Verwendbarkeit als Fassadenelement (vgl. Kap. 3.13.1), eine detaillierte Analyse nach dem Stand der Wissenschaft durchgeführt. Darin eingeschlossen sind die Charakterisierung der thermischen und thermohygrischen Dehnungseigenschaften des Dolomitmarmors, die Ermittlung des Verbiegepotenzials über Verwitterungssimulationsversuche („Biegeversuch“), sowie die Untersuchung der Auswirkungen bei einer Frost/Tau-Wechselbeanspruchung. Neben den standardmäßigen Untersuchungen der mechanischen Stabilität erfolgte auch die anwendungsspezifische Analyse der Ankerdornausbruchfestigkeit. Die angewandten Methoden werden in den entsprechenden Kapiteln aufgeführt.

Die Durchführung der verschiedenen Eigenschaftsermittlungen erfolgte in drei aufeinander senkrecht stehenden Raumrichtungen (vgl. Kap. 4.7.1), um mögliche gefügebedingte Anisotropien der verschiedenen Parameter zu erfassen (vgl. Kap. 3). Dabei wurde die xy-Ebene des Referenzsytems nach dem reliktischen Schichtungsgefüge orientiert (Abb. 5.24). Da makroskopisch keine weiteren Elemente zum Richtungsgefüge festgestellt werden konnten, wurde das Referenz-Koordinatensystem nach der gegebenen Blockgeometrie, die durch Kluftflächen definiert ist, ausgerichtet. 


\section{(a)}

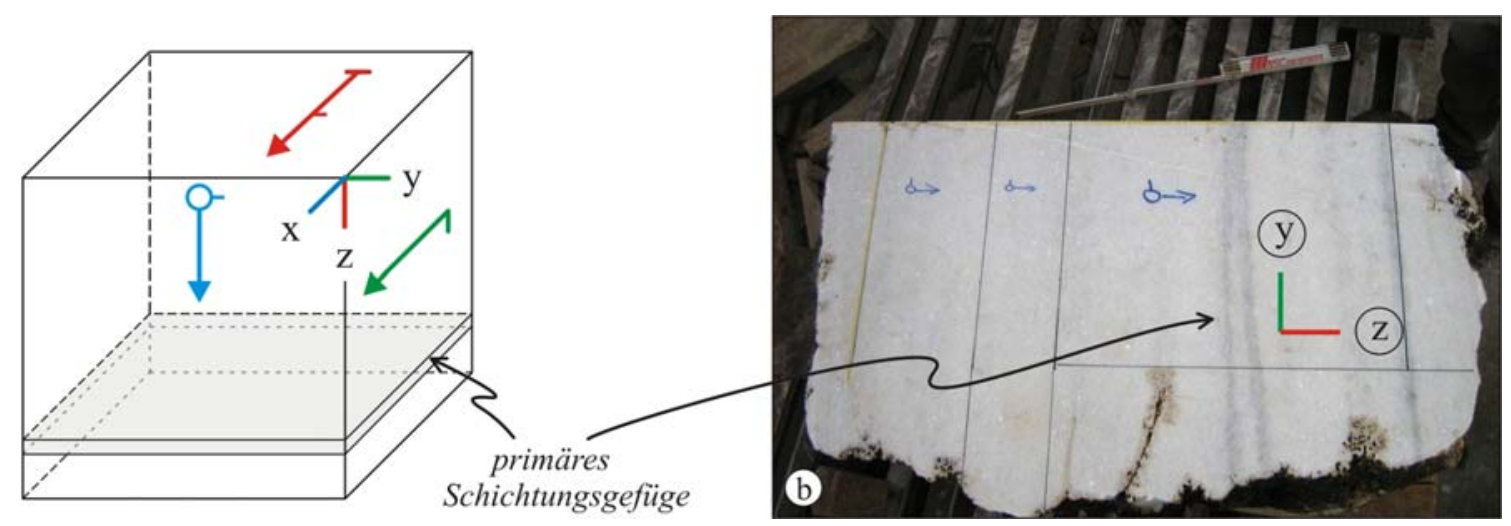

Abb. 5.24: a) Referenz-Koordinatensystem in Bezug auf das b) reliktische Schichtungsgefüge des Dolomitmarmors zur gefügebezogenen Ermittlung physikalischer und technischer Eigenschaften.

Parallel erfolgte eine eingeschränkte Charakterisierung der Parameter für den ausgewiesenen Bereich des komplexen Kalksilikatfelses sowie für die graue, calcitführende Marmorvarietät. Im Falle des Marmors konnte das Referenz-Koordinatensystem ebenfalls an eine metamorphe Schichtung angelehnt werde, die im Probeblock durch parallel angelegte kalksilikatische Reaktionsbänder beschrieben ist. Dagegen konnte hinsichtlich der Probe des Kalksilkatfelses kein Richtungselement definiert werden, so dass hier eine willkürliche Probenrichtung festgelegt wurde, um zu einer generellen Einschätzung der Eigenschaften zu gelangen.

\subsubsection{Marmorvarietäten}

\subsubsection{Dichten, Porenraumeigenschaften und Wasseraufnahmeverhalten}

Für die Hauptvarietät, den Dolomitmarmor, ergibt sich eine sehr geringe effektive Porosität von 0,16 Vol.-\% (vgl. Kap. 3.9.1), was sich dementsprechend auch darin niederschlägt, dass die Werte zur Roh- und Matrixdichte identisch sind (Tab. 5.2). Dabei spiegelt ein ermittelter Wert von 2,88 g/ $\mathrm{cm}^{3}$ den dolomitischen Charakter des Gesteins wider (vgl. Kap. 3.4.1). Aus der Hg-Porosimetrie (vgl. Brakel et al. 1981) ergibt sich für diese Varietät ein mittlerer Porenradius von 0,205 $\mu \mathrm{m}$ (Abb. 5.25 a). Im Allgemeinen zeigen sich für kristalline Marmore häufig Porengrößenmaxima im Bereich um 0,1 $\mu$ m (Rüdrich 2003), also dem Bereich, der den Übergang zwischen Wasserdampfdiffusion und Kapillarwirkung als wesentlicher Wassertransportmechanismus, darstellt (Snethlage 1984, Klopfer 1985, vgl. Kap. 3.13.1).

Für den calcitführenden Marmor ergibt sich, bedingt durch eine relativ höhere Porosität (0,65 Vol.-\%), eine etwas geringere Rohdichte von 2,78 g/ $\mathrm{cm}^{3}$. Die spezifische Mineraldichte des Dolomits wird dabei durch die Beimengung von Calcit und Serpentin überlagert. Auch die Hg-Porosimetrie zeigt eine relativ zum reinen Dolomitmarmor höhere Porosität (Abb. 5.25 b). Es ist jedoch ein deutliches Maximum der Porengrößen bei 0,025 $\mu \mathrm{m}$ ausgebildet, wobei gleichzeitig eine erheblich breitere Verteilung der Porengrößen mit Öffnungsweiten bis zu $10 \mu \mathrm{m}$ registriert wird. 

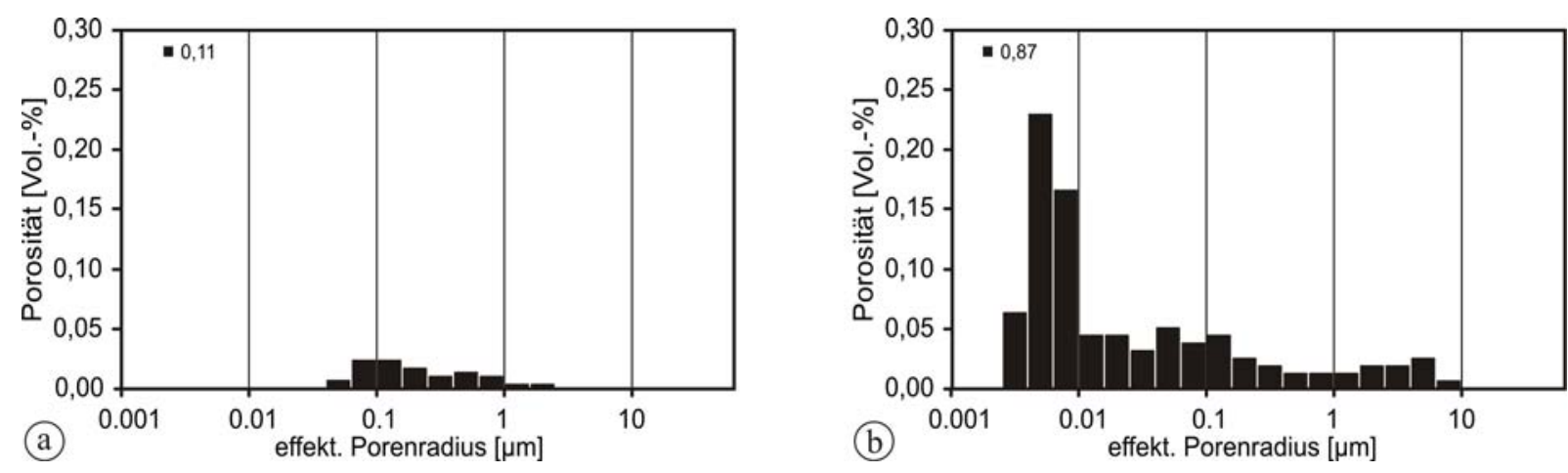

Abb. 5.25: Verteilung der Porenradien von a) der rein dolomitischen und b) der calcitführenden Marmorvarietät aus dem Vorkommen von Mtesa, Tansania.

Infolge der geringen Porosität erfolgte zur Charakterisierung des Wasseraufnahmeverhaltens die Bestimmung der Wasserdampf-Diffusionswiderstandszahl mit dem Ausdruck des dimensionslosen Koeffizienten $\mu$ (vgl. Kap. 4.7.1.1). Im Allgemeinen werden für die Hauptvarietät Werte zwischen 2000 und 5300 erreicht, wie sie auch für andere Marmore beschrieben sind (z.B. Hoffmann 2006). Dabei ist eine gefügebezogene Anisotropie zu verzeichnen (Abb. 5.26), bei der der geringste Koeffizient parallel der x-Achse aufgetreten ist. Grundsätzlich sind die erreichten Werte im Vergleich mit anderen Naturwerksteinen als relativ hoch einzustufen. So liegen die Diffusionswiderstandszahlen von Sandsteinen z.B. im Bereich zwischen 10 und 60 (Snethlage 1984), womit im Falle des Marmors eine Schädigung durch diffundierendes Wasser primär als recht unwahrscheinlich anzusehen ist.

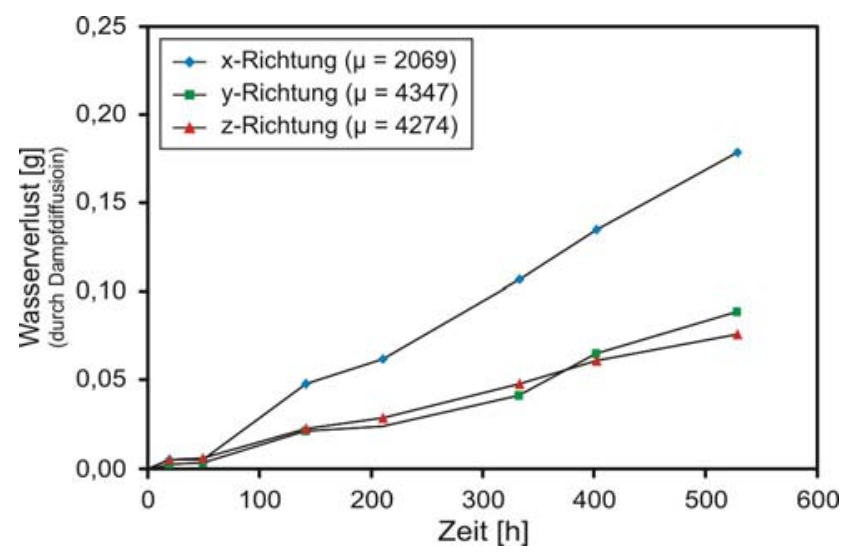

Abb. 5.26: Zeitlicher Verlauf der WasserdampfDiffusion zur Bestimmung der WasserdampfDiffusionswiderstandszahl $\mu$ an einem Probensatz des Dolomitmarmors von Mtesa.

Als weiterer relevanter Aspekt zur Charakterisierung des Wasseraufnahmevermögens erfolgte die Berechnung des Sättigungsbeiwertes S (vgl. Kap. 3.13.4), der die Frostbeständigkeit von Naturwerksteinen beschreibt (Hirschwald 1908, 1912). Die für die Marmorvarietäten erreichten Sättigungsbeiwerte liegen bei $S=0,79$ (Dolomitmarmor) bzw. $S$ = 0,52 (calcitführender Dolomitmarmor). Gesteine, deren S-Wert < 0,75 ausfällt, gelten in der Regel als frostunempfindlich (DIN 52106-1994, Hirschwald 1908). Liegt der S-Wert oberhalb 0,9, ist mit einer erheblichen Frostschädigung des Gesteins zu rechnen. Somit ist für die hier untersuchten Marmore eine Beeinträchtigung durch Temperaturwechsel nicht auszuschließen, was durch weiterführende Versuche hinsichtlich einer Gefügeauflockerung durch Frosteinwirkung erfasst werden muss (s. Kap. 5.8.1.4). 
Tab. 5.2: Dichten, Porenraumcharakteristik und Wasseraufnahmeverhalten der Marmorvarietäten aus der Lagerstätte Mtesa, Tansania.

\begin{tabular}{|c|c|c|c|c|c|c|c|c|}
\hline & \multirow{2}{*}{$\begin{array}{c}\text { Matrixdichte } \\
{\left[\mathrm{g} / \mathrm{cm}^{3}\right]}\end{array}$} & \multirow{2}{*}{$\begin{array}{l}\text { Rohdichte } \\
{\left[\mathrm{g} / \mathrm{cm}^{3}\right]}\end{array}$} & \multicolumn{2}{|c|}{ Porosität [\%] } & \multirow{2}{*}{$\begin{array}{c}\text { mittlerer } \\
\text { Porenradius } \\
{[\mu \mathrm{m}]}\end{array}$} & \multicolumn{2}{|c|}{$\begin{array}{c}\text { Wasseraufnahme } \\
\text { [Gew.-\%] }\end{array}$} & \multirow{2}{*}{$\begin{array}{c}\text { Sättigungs- } \\
\text { beiwert }\end{array}$} \\
\hline & & & Auftrieb & Hg & & $\mathbf{W A}_{\mathbf{a t m}}$ & $\mathbf{W A}_{\text {vak }}$ & \\
\hline Dolomitmarmor & 2,87 & 2,87 & 0,16 & 0,29 & 0,895 & 0,044 & 0,56 & 0,79 \\
\hline Dol-Marmor, calcitfhr. & 2,80 & 2,78 & 0,71 & 0,87 & 0,025 & 0,132 & 0,255 & 0,52 \\
\hline
\end{tabular}

\subsubsection{Ultraschallanalytik}

Die Ermittlung der Kompressionswellengeschwindigkeit des Materials (vgl. Kap. 3.12) erfolgte an Würfelproben mit einer Kantenlänge von 100 mm. Dabei wurden die Laufzeiten sowohl an Proben im trockenen als auch wassergesättigten Zustand bestimmt (vgl. Kap. 4.7.1.2)

Für den Dolomitmarmor bewegen sich die Kompressionswellengeschwindigkeiten in einem Wertebereich zwischen 6,11 und 6,42 km/s (Tab. 5.3). Damit spiegelt sich hier das kompakte Gefüge mit einer geringen Porosität wider. Die relativ höhere Porosität der calcitführenden Varietät zeigt sich konsequenterweise in geringeren Vp-Werten, die zwischen 4,21 und 4,81 km/s angesiedelt sind. Nach Köhler (1991) wäre das Gestein somit in den obersten Bereich der Schadensklasse I (zunehmend porös, Porosität: 1,3 - 0,5) im Übergang zur Klasse 0 (frisch) einzuordnen. Ausschlaggebend dafür ist jedoch vermutlich die Probenahme an der direkten Oberfläche unter Zuhilfenahme eines Vorschlaghammers, womit das Gestein einem gewissen Grad der Verwitterung unterlag bzw. eine mechanisch herbeigeführte Gefügeauflockerung aufweist. Die Abschätzung der intrinsischen Ultraschallgeschwindigkeit an wassergesättigten Proben ergibt mit mittleren Werten von $7,05 \mathrm{~km} / \mathrm{s}$ für die dolomitische und $6,52 \mathrm{~km} / \mathrm{s}$ für die calcitführende Varietät deutlich erhöhte Geschwindigkeiten. Vor allem Calcit weist ein enorm anisotropes elastisches Verhalten auf, wobei die Werte zwischen 5,7 km/s parallel und 7,7 km/s senkrecht zur c-Achse variieren können (Dreyer 1974). Bei den untersuchten Marmoren sind jedoch lediglich geringe Anisotropien ausgebildet (3,96 \% bzw. 7,28 \%), womit eine nur schwache Textur nachgezeichnet wird.

Tab. 5.3: Laufzeiten der Kompressionswellen für die Marmorvarietäten der Lagerstätte Mtesa, Tansania, gemessen in trockenem und wassergesättigtem Zustand.

\begin{tabular}{lcccccc}
\hline & \multicolumn{3}{c}{ Vp (trocken) [km/s] } & \multicolumn{3}{c}{ Vp (nass) [km/s] } \\
& $x$-Richtung & $y$-Richtung & z-Richtung & x-Richtung & $y$-Richtung & z-Richtung \\
\hline Dolomitmarmor & 6,416 & 6,369 & 6,108 & 7,196 & 6,911 & 7,038 \\
Dol-Marmor, Calcitfhr. & 4,810 & 4,738 & 4,206 & 6,680 & 6,687 & 6,200 \\
\hline
\end{tabular}




\subsubsection{Thermische und thermohygrische Dehnungseigenschaft}

Die Ermittlung des thermischen bzw. thermohygrischen Expansionsverhalten erfolgte richtungsabhängig in einem 6-Stangen-Dilatometer (Strohmeyer 2003) an zylindrischen Proben $(\varnothing=15 \mathrm{~mm}, \mathrm{~L}=50 \mathrm{~mm}$ ). Der Messaufbau ermöglicht dabei die simultane Messung von sechs Probekörpern, um einen systematischen Fehler, wie er aus mehreren Messdurchgängen durch geringfügig abweichende Labor- bzw. Versuchsbedingungen resultieren kann (vgl. Kap. 3.2), zu minimieren. Es hat sich vielfach gezeigt, dass nach einer initial thermisch bedingten Gefügeauflockerung, die eine erhöhte Fähigkeit zur kapillaren Wasseraufnahme nach sich zieht (vgl. Kap. 3.13.3), der Prozess der Verwitterung durch die Zunahme des hygrischen Einflusses massiv verstärkt werden kann. Daher wurden die Probekörper primär zehn Heizzyklen mit einem Temperaturintervall von jeweils 20 bis $90{ }^{\circ} \mathrm{C}$ bei einer Heiz-/Abkühlrate von $0,5^{\circ} \mathrm{C} / \mathrm{min}$ ausgesetzt. Daran schließen sich zehn Zyklen mit gleichem Temperaturintervall an, wobei die Probenkammer des Dilatometers vor jedem Zyklus mit Wasser geflutet wird. Während eines jeden Zyklus verdampft das Wasser, so dass die eingebauten Proben jeweils bei $90^{\circ} \mathrm{C}$ trocken fallen. Aus den gewonnenen Daten lässt sich neben dem Ausdehnungskoeffizient $\alpha$ auch die relative Längenänderung $\varepsilon[\mathrm{mm} / \mathrm{m}]$ sowie die Restdehnung $\varepsilon_{\mathrm{RD}}[\mathrm{mm} / \mathrm{m}]$, als irreversible Längenänderung nach der Wiederabkühlung, errechnen (Koch 2005).

Es zeigt sich, dass das thermisch induzierte Expansionsverhalten des dolomitischen Marmors eine Anisotropie aufweist (Abb. 5.27 a). So ergibt sich für den Ausdehnungskoeffizienten parallel der z-Richtung ein Wert von 2,09*10 $10^{-5} \mathrm{~K}^{-1}$, während die beiden übrigen Richtungen mit einem Koeffizienten von rund $1,2 * 10^{-5} \mathrm{~K}^{-1}$ belegt sind. Für die relative Längenänderung $\varepsilon$ ergibt sich dabei ein Maximalwert von $1,076 \mathrm{~mm} / \mathrm{m}$ in z-Richtung. Hinsichtlich der Restdehnung ergeben sich Beträge zwischen 0,05 und $0,12 \mathrm{~mm} / \mathrm{m}$ mit dem Maximalwert parallel der x-Richtung. Diese Restdehnung ist auf die thermisch induzierte Bildung von Mikrorissen zurückzuführen, wobei die Höchstbeträge bereits nach den ersten Trockenzyklen erreicht werden. Eine fortschreitende Schädigung durch wiederholtes Aufheizen ist unabhängig von der Probenrichtung nicht zu verzeichnen, da die Expansion der Kristalle bei erneuter thermischer Beanspruchung vermutlich durch präexistierende sowie während der primären thermischen Beeinflussung gebildete Risse gepuffert wird (Zeisig et al. 2002).

Beim Einsetzen einer thermohygrischen Belastung zeigen verschiedene calcitische Marmore einen fundamentalen Zuwachs der Restdehnung, der auch nach mehreren Zyklen kontinuierlich anhält (Koch \& Siegesmund 2004). Der hier beprobte dolomitische Marmor zeigt ein differentes Verhalten (Abb. 5.27 b). Parallel der x- und y-Achse wird zwar in den ersten Zyklen ein sprunghafter Anstieg der Restdehnung registriert, im weiteren Verlauf stagnieren die Werte jedoch. Dabei sind für die maximale Restdehnung Werte unter $0,2 \mathrm{~mm} / \mathrm{m}$ dokumentiert. Bei anderen Marmorsorten wurden dagegen Restdehnungsbeträge bis zu $1 \mathrm{~mm} / \mathrm{m}$ und auch weit darüber registriert (Koch 2005). Die Probe parallel der z-Achse zeigt sogar ein gegenläufiges Verhalten. Hier wird die primär gebildete Restdehnung sukkzesiv abgebaut, so dass bereits nach wenigen Zyklen annähernd die Ausgangslänge der 
Probe erreicht ist. Insgesamt ist daher eine progressive Gefügeauflockerung für den beprobten dolomitischen Marmor in nur sehr geringem Maße zu erwarten.
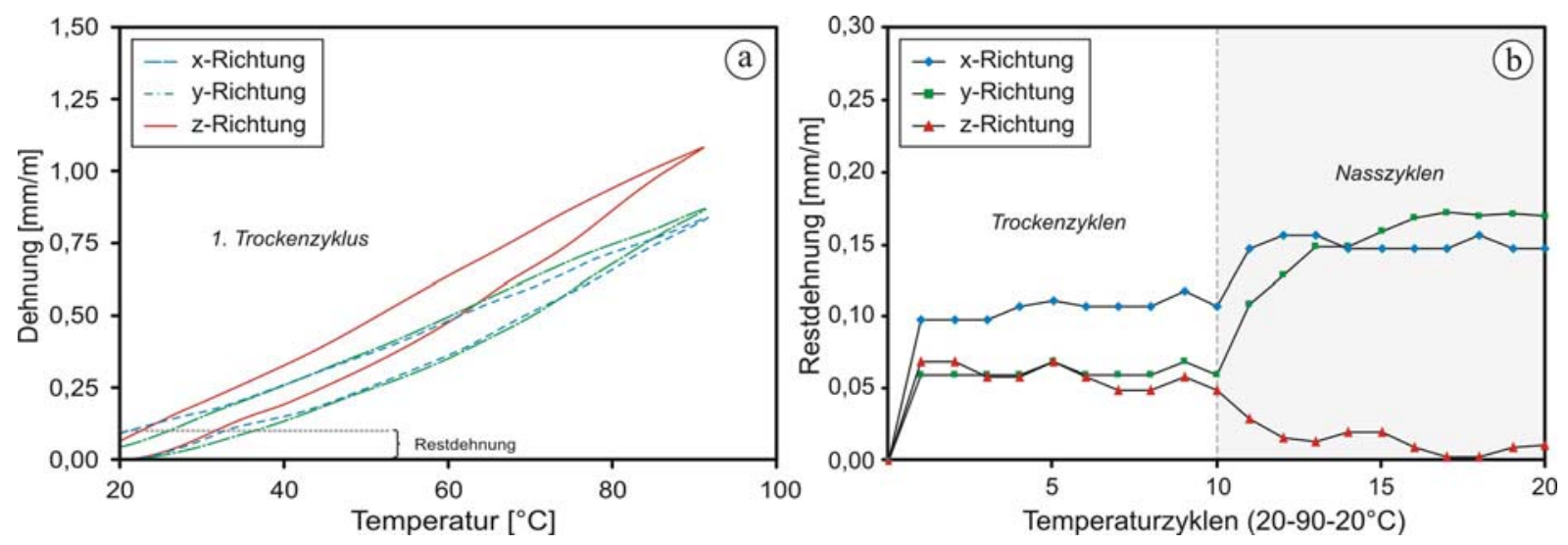

Abb. 5.27: Thermisch bzw. thermohygrisches Dehnungsverhalten des reinen Dolomitmarmors: a) Thermisch induzierte Restdehnung aus der ersten Temperaturbelastung; b) Restdehnung des Marmors als Funktion der Anzahl der Heizzyklen unter trockenen und nassen Bedingungen.

\subsubsection{Frost/Tau-Wechselverhalten}

Um Veränderungen zu erfassen, die aus einer möglichen Materialschädigung durch eine Frost/Tau-Wechselbeanspruchung resultieren können (vgl. Kap. 3.13.1), wurden am Dolomitmarmor polyzyklische Langzeitversuche in Anlehnung an DIN 52104 (Verfahren Z) durchgeführt. Dabei kamen quaderförmige Proben $(\mathrm{L}=160 \mathrm{~mm}, \mathrm{H}=\mathrm{B}=40 \mathrm{~mm}$ ) unterschiedlicher Orientierung (parallel x, parallel z) zur Anwendung. Nach einer primären Trocknung der Probekörper bei $60^{\circ} \mathrm{C}$ erfolgte ein Langzeitversuch aus 48 Frost/Tau-Zyklen. Jeder Zyklus setzt sich aus einer sechsstündigen Frostphase in einer Klimaprüftruhe bei $-20^{\circ} \mathrm{C}$ und einer sich anschließenden Auftauphase von zwei Stunden in einem auf $20^{\circ} \mathrm{C}$ temperiertem Wasserbad zusammen. Zur Erfassung möglicher Gefügeschädigungen erfolgte nach jeweils 10 Zyklen bzw. nach 48 Zyklen neben der Wägung der Proben die Bestimmung der Ultraschallwellengeschwindigkeit, um eine mögliche Gefügeentfestigung zu dokumentieren (vgl. Kap. 3.12.1). Die Ergebnisse zeigen, dass trotz einer längeren Belastung eine nur unerhebliche Schädigung des Gefüges zu verzeichnen ist (Abb. 5.28). So liegen die Vp-Werte zumeist noch im Bereich der Klasse I (frisch; nach Köhler 1991). Der Masseverlust liegt bei allen Proben unter 0,1\% des Ausgangsgewichts.

Ergänzend wurde ein CIF-Test („Capillary suction, Internal damage and Freeze thaw test“, z.B. Auberg et al. 1999) an zwei Würfelproben (Kantenlänge $10 \mathrm{~cm}$ ) durchgeführt. Auch dabei fand das reliktische Schichtungsgefüge als Probenreferenzsystem Beachtung. Nach insgesamt 56 Frost-Tau-Wechseln konnte hier lediglich eine Gewichtsreduktion von weniger als 0,1\% der Ausgangsmasse der Prüfkörper festgestellt werden. Sowohl die anschließende Bestimmung der Kompressionswellengeschwindigkeiten, bei der keine durchgreifende Reduktion festgestellt werden konnte, als auch die Bestimmung der Porenradienverteilung (Abb. 5.29) zeigt, dass es durch die Frostbelastung nicht oder nur in sehr geringem Maße zu einer Schädigung des Korngefüges gekommen ist. Vor allem im Bereich der Kapillarporen, 
an die die kapillare Wasseraufnahme hauptsächlich gebunden ist (vgl. Kap. 3.13.1), ist keine nennenswerte Änderung anhand der Porenradienverteilung erkennbar.
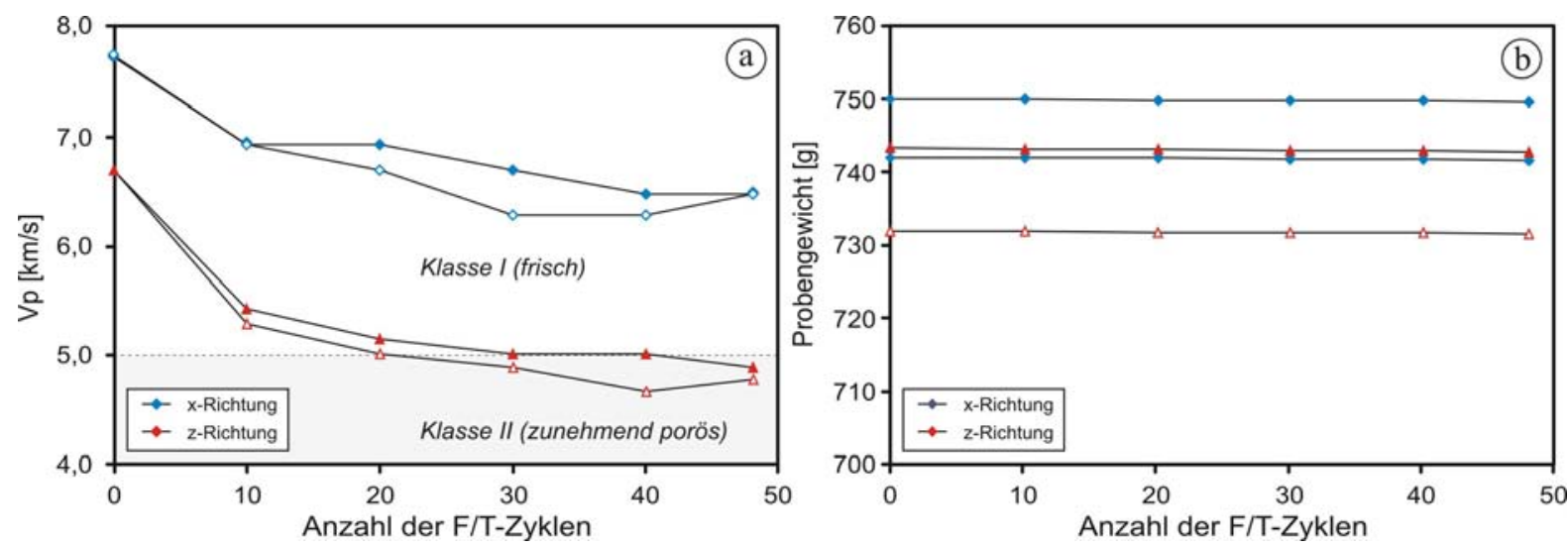

Abb. 5.28: Frost/Tau-Wechselverhalten des Dolomitmarmors ermittelt nach DIN 52104 (Verfahren Z): a) Veränderung der Ultraschallgeschwindigkeit (Vp) und b) Masseverlust als Funktion der Anzahl durchlaufener Frost/Tau-Zyklen.
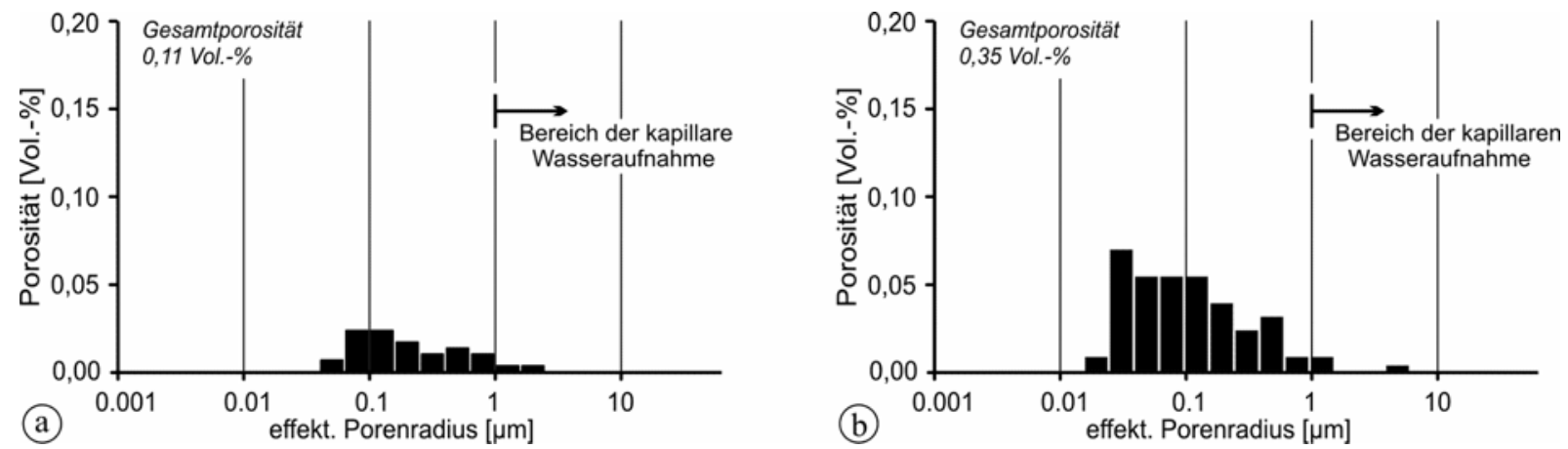

Abb. 5.29: Porenradienverteilung des Dolomitmarmors aus der Lagerstätte Mtesa, Tansania, a) vor und b) nach dem CIF-Test (56 Frost-Tau-Wechsel).

\subsubsection{Verbiegepotenzial}

Um die klimatischen Bedingungen am Bauwerk zu simulieren und daraus das Verbiegepotentials des Marmors abzuleiten, erfolgte die Untersuchung des Gesteins mittels eines Biegeversuchs in Anlehnung an die Nordtest Method NT BUILD 499 (2002). Dafür wurden längliche Platten verwendet (L 400 x B 100 x H $30 \mathrm{~mm}$ ), die in einen etwa $5 \mathrm{~mm}$ hohen Wasserfilm platziert und von oben mittels einer Heizmatte aus etwa $5 \mathrm{~cm}$ Entfernung zyklisch bestrahlt wurden. Das Temperaturintervall reichte auf der Oberseite von 20 bis $80^{\circ} \mathrm{C}$, wobei nach etwa zwei bis drei Stunden Maximalwerte erreicht und für weitere drei Stunden gehalten wurden. Die Phase der Abkühlung erstreckt sich über etwa 16 Stunden. Für die Charakterisierung des Verbiegeverhaltens wurden 30 aufeinander folgende Zyklen durchlaufen. Zur Datenerfassung wurden die Probekörper jeweils nach einer definierten Anzahl an Temperaturzyklen mit einer Spezialvorrichtung vermessen, deren Eichmaß bis in den Mikrometer-Bereich reicht und eine Genauigkeit von +/- $1 \mu \mathrm{m}$ aufweist.

Für den Dolomitmarmor ist kein Trend zu einer deutlichen Verformung der Probeplatten entwickelt (Abb. 5.30). Dies zeigt sich besonders im Vergleich mit der parallel gemessenen 
Probe eines Calcitmarmors. Der Kurvenverlauf lässt vermuten, dass sich eine anfänglich schwach konkave Biegung (Plattenmitte wölbt sich nach unten) von maximal -0,306 mm/m nach bereits 15 Zyklen bei weiterer thermohygrischer Belastung annähernd ausgleicht. Ein gleichartiges Verhalten konnte bereits bei der Untersuchung zum Dehnungsverhalten des Materials beobachtet werden. Die Vergleichsprobe (Calcitmarmor, Carrara) zeigt dagegen schon nach relativ kurzer Versuchsdauer eine deutliche Durchbiegung von annähernd $1 \mathrm{~mm} / \mathrm{m}$, wobei insgesamt eine zwar geringe, dabei aber kontinuierlich zunehmende Verformung zu verzeichnen ist.

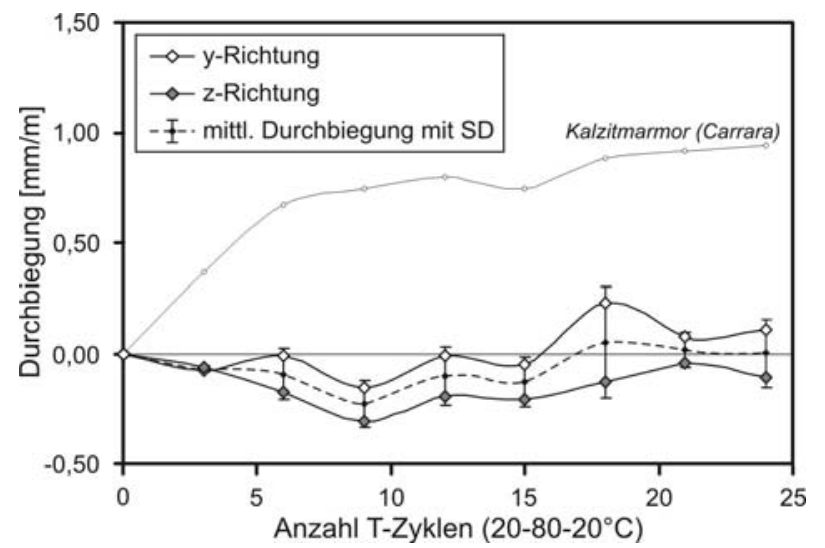

Abb. 5.30: Experimentell ermittelte Durchbiegung des Dolomitmarmors als Funktion der durchlaufenen Temperaturzyklen. Der Calcitmarmor (Carrara) wurde als Vergleichsmaterial parallel gemessen.

\subsubsection{Technische Eigenschaften}

Die Beschreibung der angewandten Verfahren sowie der Orientierung der jeweiligen Prüfkörper ist in Kap. 4.7.1.3 gegeben.

Die angegebenen Werte zur einaxialen Druckfestigkeit des Dolomitmarmors (Abb. 5.31 a, Tab. 5.4) beziehen sich für jede der drei Raumrichtungen auf eine Mittelwertbildung aus mindestens fünf Einzelergebnissen. Insgesamt werden bei der dolomitischen Varietät mit Werten zwischen 164,1 ${ }^{+} /-6,3 \mathrm{MPa}$ (parallel x) und 168,7 ${ }^{+} /-15,8 \mathrm{MPa}$ (parallel z) relativ zur dominant calcitführenden Varietät höhere Werte erreicht. Wenige Proben zeigen deutlichere Abweichungen hinsichtlich der mechanischen Stabilität. Ein Zusammenhang mit dem Auftreten mineralisierter Fissuren konnte dabei nicht festgestellt werden. Die geringere Druckfestigkeit der calcitführenden Varietät kann auf die insgesamt etwas gröbere Struktur sowie die Korngrenzenflächenreduktion zurückgeführt werden (vgl. Kap. 5.2). Beide Marmorvarietäten zeigen bezogen auf das Richtungsgefüge eine geringe Anisotropie von 2,8 \% (dolomitisch) bzw. 3,9\% (calcitführend).

Die Bestimmung der Biegefestigkeit des Dolomitmarmors basiert auf mindestens sechs Einzelversuchen je Richtung (Abb. 5.31 b). Der Mittelwert der Biegefestigkeit liegt bei 24,7 MPa. Für die calcitführende Varietät wird dagegen lediglich ein Wert von 13,4 MPa erreicht. Für beide Gesteine ergibt sich eine richtungsbedingte Anisotropie von rund $12 \%$, wobei jeweils der Minimalwert parallel der x-Richtung ermittelt wurde. Für beide Marmorvarietäten zeigt sich in der statistischen Auswertung bezogen auf die jeweilige Prüfrichtung, dass die Biegefestigkeit senkrecht zum Lager deutlich höheren Schwankungen unterliegt. So sind für die entsprechenden Prüfungen (Proben in $\mathrm{x}$ - und y-Richtung) für den 
reinen Dolomitmarmor Werte zwischen 14,4 und 30,6 $\mathrm{MPa}(\sigma=4,1 \mathrm{MPa})$ sowie für die calcitführende Varietät Werte zwischen 8,4 und 18,2 $\mathrm{MPa}(\sigma=4,0 \mathrm{MPa})$ realisiert, wobei jedoch keine statistischen Ausreißer identifiziert sind. Dagegen ergeben sich in der Prüfung senkrecht zum reliktischen Lagenbau (z-Richtung) bei Mittelwerten von 26,0 MPa bzw. 14,3 MPa geringe Standardabweichungen von jeweils 0,9 MPa. Daraus ist ersichtlich, dass scheinbar kein Einfluss des reliktischen Schichtungsgefüges hinsichtlich der Reduktion oder Variation der mechanischen Stabilität besteht. In diesem Fall wären erhöhte Abweichungen sowie vor allem Minimalwerte bei der Prüfung in z-Richtung infolge eines möglicherweise makroskopisch nicht erkennbaren Material- oder Gefügewechsels zu erwarten. Ein Zusammenhang zwischen der weiten Datenspannweite und dem Auftreten feiner Calcitgänge konnte nicht festgestellt werden.

Die Bestimmung der Spaltzugfestigkeit erfolgte für beide Marmorvarietäten an mindestens sechs Parallelmessungen je Raumrichtung, auf denen die Mittelung beruht (Abb. 5.31 c). Sowohl für die rein dolomitische als auch die calcitführende Marmorvarietät ergibt sich für die Spaltzugfestigkeit ein Mittelwert von rund $10 \mathrm{MPa}$. Die Varietäten unterscheiden sich darin, dass beim Dolomitmarmor die Maximalwerte bei parallel zum reliktischen Lagenbau aufgebrachter Last, dagegen bei der calcitführenden Varietät bei senkrechter Auflast erreicht werden.

Die Bestimmung der Abriebsfestigkeit des Dolomitmarmors nach dem Böhme-Verfahren erfolgte an jeweils zwei Parallelproben pro Prüfrichtung. Der Dolomitmarmor zeigt nach Beendigung des Versuchs einen mittleren Materialverlust von 18,70 cm $3 / 50 \mathrm{~cm}^{2}$ (Abb. $5.31 \mathrm{~d}$ ). Richtungsbedingte Abweichungen der Abriebfestigkeit konnten dabei nicht festgestellt werden. Für die Gesamtheit der drei differenzierten Proberichtungen ergab sich lediglich ein Anisotropiewert von unter $2 \%$.

Der Vergleich der drei technischen Eigenschaften Druck-, Biege- und Spaltzugfestigkeit mit den in Kapitel 3.9.2 statistisch ermittelten lithologietypischen Wertebereichen zeigt, dass der Dolomitmarmor eine ausgesprochen hohe mechanische Stabilität aufweist (Abb. 5.31). Einzig die Werte zur Spaltzugfestigkeit fallen relativ gering aus, liegen aber dennoch deutlich im zentralen Segment des beschreibenden Boxplots. Hinsichtlich der Abriebfestigkeit nennt Peschel (1983) für Marmore einen Wertebereich von 6,5 bis $32,5 \mathrm{~cm}^{3} / 50 \mathrm{~cm}^{2}$, womit der am Dolomitmarmor ermittelte Materialverlust durch abrasive Belastung ebenfalls positiv bewertet werden kann. 

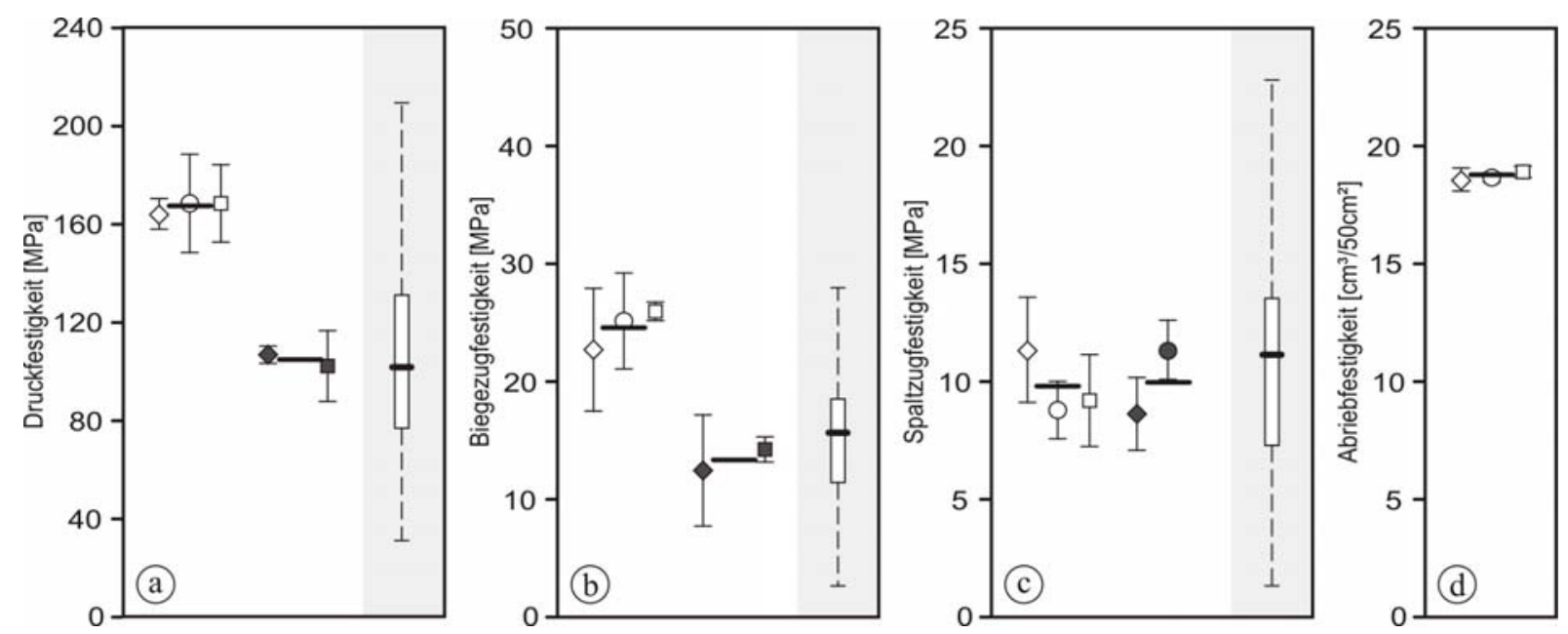

Abb. 5.31: Technische Eigenschaften der dolomitischen (leere Sym.) sowie der calcitführenden Marmorvarietät (gefüllte Sym.). Prüfrichtungen: Raute $=\mathrm{x}$, Kreis $=\mathrm{y}$, Quadrat $=\mathrm{z}$, Balken $=$ richtungsunabhängiger Mittelwert. Die Boxplots beschreiben die lithologietypischen Bereiche aus der statistischen Auswertung in Kapitel 3.9.2 dieser Arbeit.

Hinsichtlich einer möglichen Verwendung des Materials im Fassadenbau erfolgte zudem die Bestimmung der Ausbruchlast am Ankerdornloch in Anlehnung an DIN EN 13364. Dieser Wert gibt die Kraft an, bei der ein Ankerdorn bei senkrechter Belastung aus dem Ankerdornloch ausbricht. Neben Proben mit einer Kantenlänge von 200 mm (DIN EN 13364) erfolgte die Durchführung auch an Probekörpern mit einer Kantenlänge von $150 \mathrm{~mm}$ und einer Dicke von $30 \mathrm{~mm}$, da eine entsprechende Reduktion keine nennenswerten Unterschiede ergibt (Koch 2005). Zudem wurde die von der DIN EN 13364 vorgesehene Prüfgeschwindigkeit von 50 N/s auf 10 N/s reduziert, da sonst die Versuchsdauer infolge der relativ geringen Ausbruchlast im Falle des Marmors im Vergleich mit anderen Natursteinvarietäten zu kurz ausfallen würde. Die Wirkungsweise am Ankerdornloch kann infolge einer hohen Komplexität keiner bestimmten Raumrichtung zugeordnet werden. Infolgedessen werden in der o.g. Norm verschiedene Typen hinsichtlich der Lage des Gefüges im Probekörper differenziert.

Zur Beurteilung des reinen Dolomitmarmors hinsichtlich der Ausbruchlast am Ankerdornloch wurden Proben vom Typ 1, 2a und 2b getestet (Abb. 5.32 a). Dabei ergab sich eine Gesamtanisotropie von 17,2 \%. Die größte Streuweite mit einer Standardabweichung von $1,4 \mathrm{kN}$ zeigte sich beim Typ 1 . Dadurch ergibt sich hier auch mit 1,8 kN der geringste untere Erwartungswert, wohingegen sich für die verbleibenden Probentypen Werte von 2,7 kN (Typ 2a) und 2,8 kN (Typ 2 b) errechnen. Ursächlich für den geringen Festigkeitswert sind die abweichenden Werte, die sich an den Ankerdornlöchern 7 und 8 ergeben haben (Abb. 5.32 b). Für beide Testpunkte sind deutlich geringere Bruchlasten verzeichnet, die auf eine feinstkörnige Ader in Verlauf der diagonalen Verbindung beider Ankerdornlöcher zurückzuführen sind. Während am Ankerdornloch 8 im Test noch ein regelmäßiger Ausbruch auf der unteren Seite des Prüfkörpers erfolgte, kam es bei Belastung des Ankers 7 zum vollständigen mechanischen Versagen und somit zum Bruch der Platte. Unter Ausschluss dieser Daten ergibt sich auch für den Probentyp 1 ein unterer Erwartungswert von 2,3 kN bei einem Mittelwert von 4,1 kN. Für die zwei verbleibenden Typen ergeben sich im Mittel 
Werte von 3,9 $9^{+}-$0,9 $\mathrm{kN}$ (Typ 2a) und 4,1 ${ }^{+} /-0,7 \mathrm{kN}$ (Typ 2b), womit der obere Bereich des vom SFHF (Schweizerischer Fachverband für hinterlüftete Fassaden) genannten lithologietypischen Wertebereichs beschrieben ist.
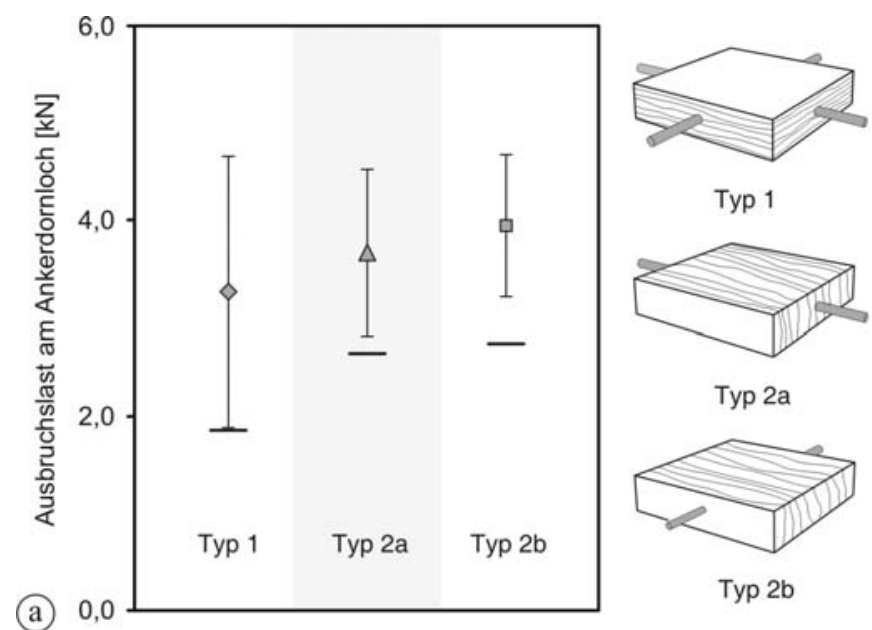

Typ 1

Typ 1
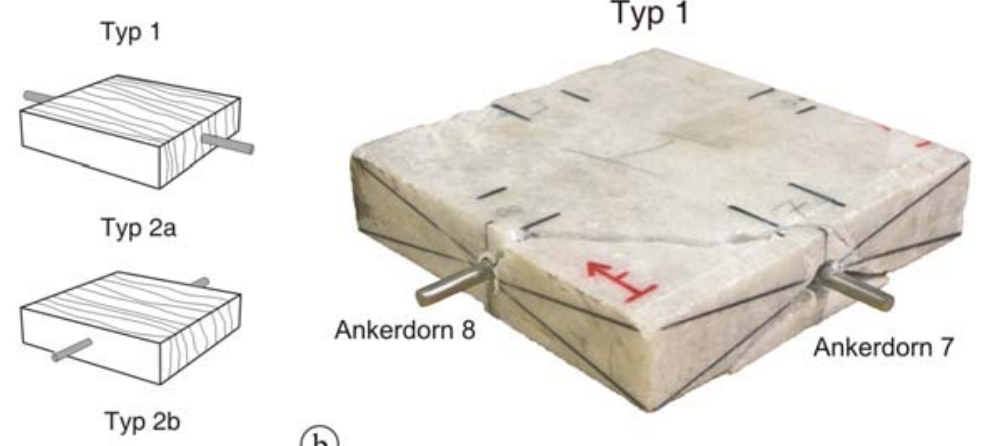

(b)

Abb. 5.32: Prüfung der Ausbruchlast am Ankerdornloch: a) Mittelwerte mit Standardabweichungen und unteren Erwartungswerten (Balken) des Dolomitmarmors sowie die entsprechenden Prüftypen nach DIN EN 13364. b) Unregelmäßiger Bruch der Probe bei Auflast am Ankerdorn 8.

Tab. 5.4: Übersicht zu den technischen Eigenschaften der Dolomitmarmorvarietäten der Lagerstätte Mtesa, Tansania, mit Bezug zum Richtungsgefüge.

\begin{tabular}{|c|c|c|c|c|c|}
\hline \multirow{2}{*}{ Prüfung } & \multirow{2}{*}{ Prüfrichtung } & \multicolumn{2}{|c|}{ Dolomitmarmor } & \multicolumn{2}{|c|}{ Dol.-marmor, calcitfhr. } \\
\hline & & $\varnothing$ & $\sigma$ & $\varnothing$ & $\sigma$ \\
\hline \multirow{3}{*}{ Druckfestigkeit [MPa] } & $\mathrm{x}$ & 164,1 & 6,3 & 106,6 & 2,1 \\
\hline & $\mathrm{y}$ & 168,4 & 20,1 & n.d. & n.d. \\
\hline & $\mathrm{z}$ & 168,7 & 15,8 & 102,5 & 14,7 \\
\hline \multirow{3}{*}{ Biegefestigkeit [MPa] } & $\mathrm{x}$ & 22,1 & 4,5 & 12,5 & 4,7 \\
\hline & $\mathrm{y}$ & 24,5 & 3,5 & 14,3 & 1,1 \\
\hline & $\mathrm{z}$ & 26,0 & 0,9 & n.d. & n.d. \\
\hline \multirow{3}{*}{ Spaltzugfestigkeit [MPa] } & $\mathrm{x}$ & 11,3 & 2,2 & 8,6 & 1,6 \\
\hline & $\mathrm{y}$ & 8,8 & 1,2 & 11,3 & 1,3 \\
\hline & $\mathrm{z}$ & 9,2 & 1,9 & n.d. & n.d. \\
\hline \multirow{3}{*}{ Abriebfestigkeit $\left[\mathrm{cm}^{3} / 50 \mathrm{~cm}^{2}\right]$} & $\mathrm{x}$ & 18,6 & 0,5 & n.d. & n.d. \\
\hline & $\mathrm{y}$ & 18,6 & 0,2 & n.d. & n.d. \\
\hline & $\mathrm{z}$ & 18,9 & 0,3 & n.d. & n.d. \\
\hline \multirow{3}{*}{$\begin{array}{l}\text { Ausbruchlast am } \\
\text { Ankerdornloch [kN] }\end{array}$} & Typ 1 & 3,65 & 1,05 & n.d. & n.d. \\
\hline & Typ 2a & 3,87 & 0,54 & n.d. & n.d. \\
\hline & Тур 2b & 4,14 & 0,63 & n.d. & n.d. \\
\hline
\end{tabular}




\subsubsection{Kalksilikatfels}

Für den Kalksilikatfels wurden Untersuchungen in nur geringem Maße durchgeführt, um eine generelle Charakterisierung des Gesteins vornehmen zu können. Auch bei einem möglichen Abbau des Gesteins ist in Folge der geringen Vorratslage vor allem die Verwendung im Bereich exklusiver Einzelobjekte, also die Fertigung von Elementen in Klein- bis Kleinstserien, anzunehmen. Insgesamt unterliegen die verschiedenen physikalischen und technischen Parameter des Kalksilikatfels einer relativ hohen Heterogenität. Dieser Umstand ist vor allem auf das sehr unregelmäßige Kristallwachstum zurückzuführen, wobei Korngrößen von mehreren Zentimetern auftreten können.

Aus der Auftriebswägung ergibt sich eine Porosität von 0,14 Vol.-\% bei einem für Rohund Matrixdichte äquivalenten Wert von 2,61 g/ $\mathrm{cm}^{3}$. Dementsprechend gering fällt auch der Wert zur freiwilligen Wasseraufnahme mit $W_{a t m}=0,04$ Gew.- $\%$ aus. In der Porengrößenverteilung zeigt sich im Mirkoporenbereich ein deutliches Maximum bei $0,016 \mu \mathrm{m}$. Daneben wird deutlich, dass auch die Porosität einer gewissen Heterogenität unterliegen kann, da sich aus der Hg-Porosimetrie eine Gesamtporosität von 0,50 Vol.-\% errechnet, was vor allem auf das Auftreten größerer Porenöffnungen bis zu $10 \mu \mathrm{m}$ zurückgeführt werden kann. Dem Sättigungsbeiwert folgend $(S=0,82)$ ist auch für den Kalksilikatfels eine gewisse Frostanfälligkeit zu erwarten.

Hinsichtlich der mechanischen Stabilität erfolgte die Untersuchung der Biege- und Spaltzugfestigkeit. Insbesondere in der Analyse der Biegefestigkeit zeigt sich eine enorme Heterogenität, die von Werten zwischen 2,6 bis 17,6 MPa (im Mittel 12,1 +/- 5,4 MPa) beschrieben ist. Bei Betrachtung des Minimalwertes als statistischen Ausreißer ergibt sich jedoch ein Mittelwert von 14,5 MPa. Der Bruch erfolgte bei keiner Probe entlang der Mittellinienbelastung, sondern folgte den dominanten Kontakten größerer Kristalle. Die Spaltzugfestigkeit dagegen zeigt bei einem Mittelwert von 6,2 ${ }^{+} /-0,7 \mathrm{MPa}$ ein relativ homogenes Bild. Bei einer erhöhten Probenanzahl ist jedoch auch hier eine Zunahme der Datenspannweite anzunehmen.

\subsection{Zusammenfassende Bewertung des Lagerstättenpotenzials}

Im Folgenden sollen die aus der Befahrung des Vorkommens gewonnenen Erkenntnisse mit den Ergebnissen der umfassenden Gesteinscharakterisierung zu einer Bewertung des wirtschaftlichen Potenzials der Lagerstätte zusammengeführt werden. Dabei müssen neben der Möglichkeit des Ausbringens gut dimensionierter, defektfreier Rohblöcke und der Eingrenzung der bautechnischen Anwendung des Materials auch Aspekte berücksichtigt werden, die die Einführung am internationalen Natursteinmarkt betreffen. So muss z.B. für eine definierte Handelssorte eine langfristige Verfügbarkeit in relativ gleich bleibender Qualität gegeben sein. Dies betrifft zum einen die physikalischen und technischen Eigenschaften, zum anderen aber auch die Stabilität der jeweiligen Farbnuancen. 
Die Marmorlagerstätte von Mtesa weist ein Gesamtvolumen von rund 100 Mio. $\mathrm{m}^{3}$ auf (vgl. Kap. 5.7.2). Unter Beachtung des zu erwartenden Abraums (vgl. Kap. 5.7.3) liegt ein verwendungsfähiges Volumen von rund 20 bis 40 Mio. $\mathrm{m}^{3}$ des Dolomitmarmors in unterschiedlichen Farb- und Dekorqualitäten vor (Tab. 5.5). Als gewinnbare Mindestvorratsmenge für Werksteinlagerstätten werden in der Literatur unterschiedliche Angaben gemacht. So nennt Singewald (1992) ein Volumen von mindestens 30.000 bis $50.000 \mathrm{~m}^{3}$. Berton \& Le Berre (1983) fordern sogar eine minimale Menge von $500.000 \mathrm{t}$, was im Falle des Dolomitmarmors einem Volumen von rund $174.000 \mathrm{~m}^{3}$ entspricht. In jedem Fall stellt somit die Lagerstätte von Mtesa sowohl für den regionalen als auch internationalen Markt ein hohes wirtschaftliches Potenzial dar.

Tab. 5.5 Zusammenfassende Übersicht zur Aufteilung des nutzbaren Rohblockvolumens ( 20 bis 40 Mio. t) der Dolomitmarmorlagerstätte von Mtesa, Tansania, in die definierten Farb- und Dekorqualitäten.

\begin{tabular}{lcc}
\hline \multicolumn{1}{c}{ Farb- / Dekorqualität } & $\begin{array}{c}\text { Anteil an zu erwartender } \\
\text { Rohblockmenge } \\
{[\%]}\end{array}$ & $\begin{array}{c}\text { Volumen der } \\
\text { Rohblockware } \\
{\left[\text { Mio. }^{3}\right]}\end{array}$ \\
\hline $\begin{array}{l}\text { reinweiß (mit vernachlässigbarer Beimengung dispers verteilter } \\
\text { mafischer Minerale oder entsprechender Bänderung) } \\
\text { weiß bzw. weiß/reinweiß (mit verstärktem Auftreten dunkel abgesetzter }\end{array}$ & $\sim 10$ & $\sim 2$ bis 4 \\
$\begin{array}{l}\text { Bänder und/oder entsprechend farblich abgesetzter Bereiche) } \\
\text { grau/weiß mit erheblicher Dekoration dunkel oder graugrün abgesetzter } \\
\text { Bänder und/oder entsprechend farblich abgesetzter Bereiche }\end{array}$ & $\sim 50$ & $\sim 10$ bis 20 \\
\hline
\end{tabular}

Basierend auf der Erfassung und Bewertung der lagerstätten-kontrollierenden Elemente ist die Möglichkeit zum Ausbringen industriell zu verarbeitender Rohblöcke mit anhaltend konstanten physikalischen, technischen und bedingt auch optischen Eigenschaften zu erwarten. Eine Sortendefinition muss sich hier nach Stärke und Anzahl dunkel abgesetzter Bänder und der farblichen Intensität richten (vgl. Tab. 5.5), wobei die entsprechende Zuordnung fertig gestellter Rohblöcke nach Inaugenscheinnahme durchgeführt werden muss.
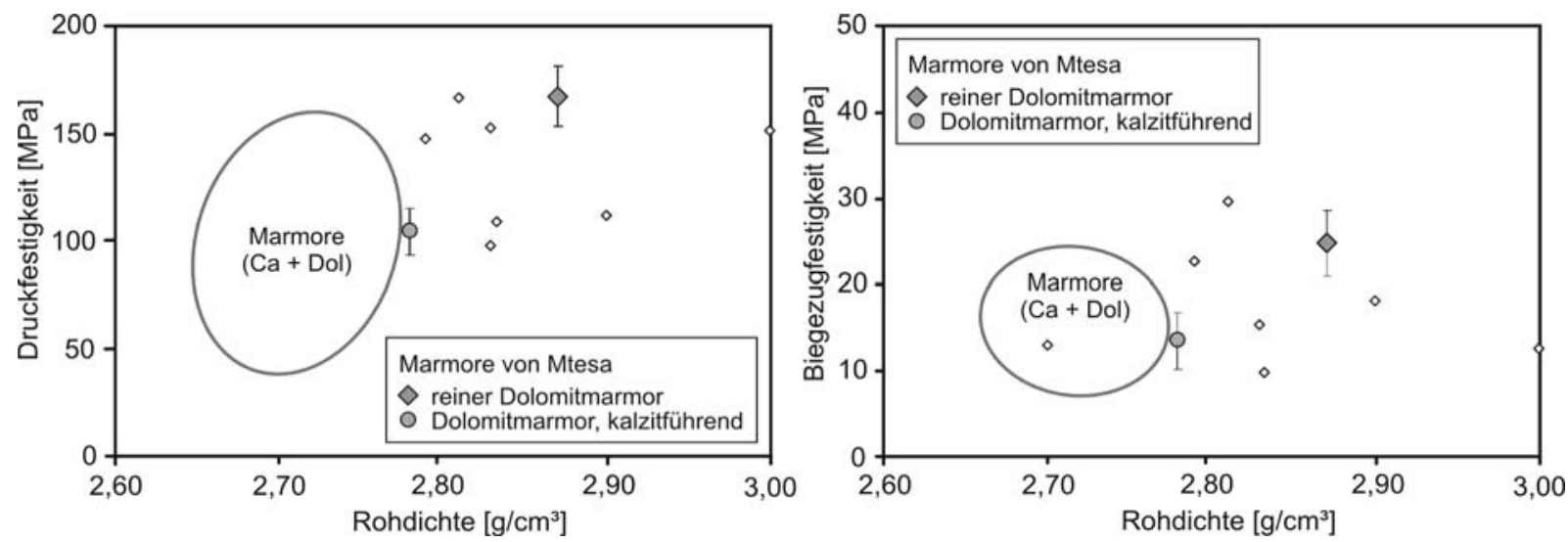

Abb. 5.33: Vergleich der Druck- bzw. Biegefestigkeit der Marmorvarietäten des Vorkommens Mtesa, Tansania, mit auf dem internationalen Markt vertretenden calcitischen und dolomitischen (leere Rauten) Marmoren. Die Ellipsen beschreiben Bereiche der 80 \%-igen Aufenthaltswahrscheinlichkeit für die jeweilige Korrelation, die sich aus der statistischen Auswertung in Kapitel 3.10.4 dieser Arbeit ergeben haben. 
Im Vergleich mit anderen am Markt vorhandenen Marmorvarietäten (vgl. Kap. 3.9) weist der Dolomitmarmor hinsichtlich der bautechnischen Anwendung sehr gute Eigenschaften auf (Abb. 5.33). Insbesondere ist hier die sehr hohe mechanische Stabilität zu nennen (vgl. Abb. 5.31, Tab. 5.4), die auf dem festen Kornverband beruht. Positiv erscheint dabei auch die geringe Anisotropie, womit eine richtungsorientierte Fertigung im Hinblick auf eine Optimierung der Abbauführung sowie auch bezüglich einer individuellen Abstimmung des Blockaufschnitts (vgl. Kap. 2.5) keinen Einschränkungen unterliegt.

Insgesamt ergeben sich in der Anwendung des Dolomitmarmors im Innenbereich, so als Boden- und Wandbeläge als auch Massivteile, keine Einschränkungen. Die relativ hohen Werte zur Biege- und bedingt auch der Spaltzugfestigkeit lassen auch den Einsatz als Treppenstufen möglich erscheinen, doch sind hierbei, infolge des mittel- bis z.T. grobkristallinen Gefüges, Materialverluste an den Kanten zu erwarten. Hinsichtlich der Anwendung des Dolomitmarmors im Außenbereich zeigen die Ergebnisse zum thermohygrischen Dehnungsverhalten des Materials (vgl. Kap. 5.8.1.3), dass auch bei wiederholter Beanspruchung keine progressive Längenänderung auftritt. Daher sollte auch bei Fassadenelementen in exponierter Lage (Sonnenbestrahlung und Niederschlag) eine langfristige Formbeständigkeit der Platten gegeben sein (vgl. 5.8.1.5). Dabei ist jedoch mit einer Minderung der Oberflächenpolitur sowie mit sekundären Farbänderungen durch Oxidationserscheinungen zu rechnen (Kap. 5.6.1 und 3.13.1). Auch die Untersuchung des Frost/Tau-Wechselverhaltens (vgl. Kap. 5.1.4) hat keine nennenswerten Gefügeveränderungen hervorgerufen.

Verglichen mit calcitischen Marmorvarietäten weisen Dolomitmarmore im Allgemeinen eine höhere Beständigkeit hinsichtlich des Mineralgefüges und damit der Gesamtheit der physikalischen und technischen Eigenschaften auf. Daher sollten Rohblöcke aus den Bereichen der Kontaktmetamorphose aus der Fertigung von Bauelementen für den Außenbereich ausgeschlossen werden, da mit einem erhöhten Calcitanteil gerechnet werden muss. Die Differenzen der mineralspezifischen Eigenschaften von Calcit und Dolomit und die daraus resultierenden Unterschiede in der mechanischen Stabilität sowie dem thermohygrischen Verhalten können die Langzeitstabiltität der Bauteile deutlich herabsetzen. Ebenso ist im laufenden Abbau durch eine fortlaufende Erkundung ein ausreichender Abstand zu tektonisch beanspruchten Bereichen wie Scherbahnen oder ausgeprägten Kluftzonen zu gewährleisten. Anderenfalls kann das Auftreten von Mikroklüften bzw. feineren Rissstrukturen ebenso die technischen Eigenschaften des Materials negativ beeinflussen. Dies gilt insbesondere unter dem Einfluss von Prozessen der Verwitterung in der Anwendung der Bauteile.

Der Vertrieb und die Anwendung von Naturwerkstein setzt für bestimmte Einsatzbereiche die CE-Kennzeichnung voraus, die dem Nachweis der Übereinstimmung mit den für den jeweiligen Einsatzbereich relevanten europäischen Normen dient (Brändlein \& Deppisch 2007, vgl. Kap. 1). Für den Dolomitmarmor Mtesa wurde eine Vielzahl physikalischer und technischer Eigenschaften nach dem Stand der Technik (DIN/EN) bzw. in besonderen Fragestellungen nach dem Stand der Wissenschaft erfasst. Zusätzlich erfolgte auch eine detaillierte petrographische Analyse des Gesteins. Im Rahmen der statistischen Auswertungen 
in Kapitel 3 dieser Arbeit wurde jedoch deutlich, dass ein entsprechend detaillierter Datensatz zu einer Werksteinhandelssorte nur in wenigen Ausnahmefällen zur Verfügung steht. Dementsprechend sind für den Dolomitmarmor die Grundlagen für eine CE-Kennzeichnung für viele Produktgruppen (Rohblöcke, Rohplatten, Fassadenplatten, Fliesen, Bodenplatten, Treppenstufen, Massivteile, Mauerwerk, Pflastersteine) gegeben.

Der Gesamtbewertung des Lagerstättenpotenzials erfolgt nach der „Internationale RahmenVorratsklassifikation der Vereinten Nationen / UNFCR“ (UNO 1997) in den Punkten i)geologischer Untersuchungsstand (G), ii) bautechnischer Untersuchungsstand (F) und iii) Grad der Bauwürdigkeit (E) (Abb. 5.34, vgl. Kap. 5.1).

Mit der durchgeführten geologischen Erkundung der Lagerstätte durch sachkundiges geologisches Personal ist die primäre Forderung zur Bewertung gegeben. Aufgrund der recht günstigen Aufschlusslage kann der Erkundungsstand in Anlehnung an vergleichbare Verhältnisse an entsprechenden Lagerstätten in Benin (Stein 2007) nach der UNFCR als „Übersichtsexploration“ (G 2) eingestuft werden. Die gewonnenen Ergebnisse können somit als Entscheidungsgrundlage für eine Detailexploration herangezogen werden. Die umfassenden labortechnischen Untersuchungen haben die bautechnische Anwendungsmöglichkeit des Gesteins gezeigt. Ausgenommen davon sind zwangsläufig Erkenntnisse zum Langzeitverhalten in der bautechnischen Exposition. Mit den gegebenen Randbedingungen kann im Vergleich mit bestehenden Abbauen in anderen Staaten Afrikas die Bauwürdigkeit der Lagerstätte Mtesa hinsichtlich der Ausbringung von Rohblöcken in wirtschaftlichem Maße als möglich bewertet werden. Nach der UNFCR entspricht die Lagerstätte somit der Stufe F 2 („Präfeasibility Studie“). Die Vorratslage kann in die Stufe E 1 („,bauwürdige Reserve“) eingegliedert werden. Dies bezieht sich insbesondere auf die Teillagerstätte NE, die mit einem Volumen von gut 45 Mio. $\mathrm{m}^{3}$ den umfangreichsten Teilbereich bei einer günstigen Aufschlusslage stellt und aus welcher zudem das detailliert untersuchte Probenmaterial entnommen wurde. Die übrigen Bereiche können der Stufe E 2 (,potentiell bauwürdig“) zugeordnet werden.

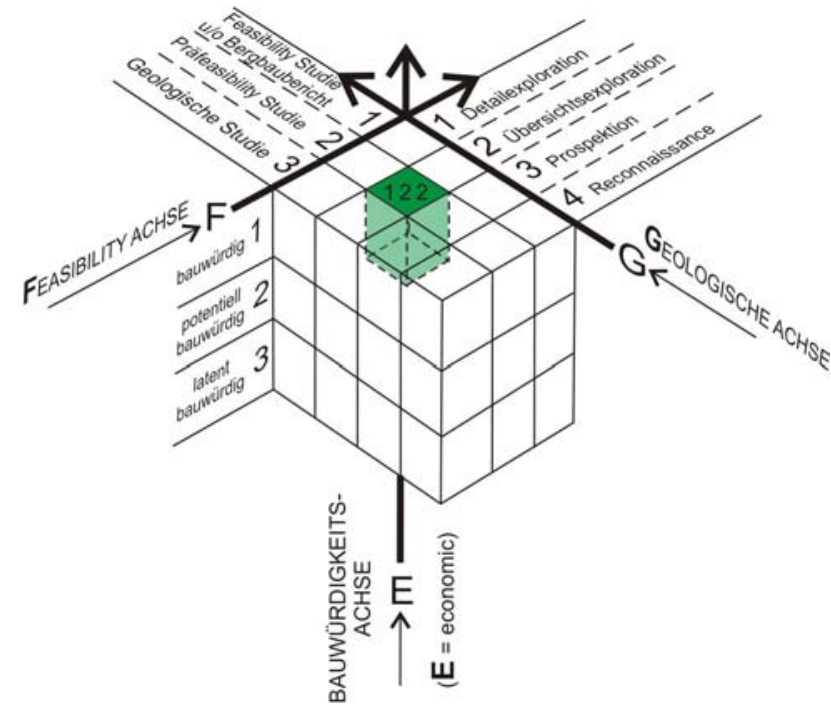

Abb. 5.34: Finale Bewertung der Lagerstätte des Dolomitmarmors von Mtesa, Tansania, nach der „Internationale Rahmen-Vorratsklassifikation der Vereinten Nationen / UNFCR“ (UNO 1997). 


\section{ZUSAMMENFASSUNG UND SCHLUSSFOLGERUNGEN}

Der Rohstoff Naturwerkstein erfreut sich einer zunehmenden Beliebtheit und wird aufgrund der vorhandenen Bandbreite an Farben und Strukturen in vielen Bereichen der Bautechnik und Architektur eingesetzt. Seit 1990 ist die Produktion jährlich um durchschnittlich 7,5 \% gestiegen und hat bereits einen Umfang von rund 210 Mio. t erreicht. Statistiken folgend, ist weltweit in den nächsten Jahren und Jahrzehnten mit einem weiteren überproportionalen Wachstum der Nettoproduktion zu rechnen. So beschreibt z.B. Montani (2007) einen möglichen Bedarf von 490 Mio. t um das Jahr 2025. Schon heute zeigt sich in Ländern wie Indien, China oder Brasilien, dass die Werksteinindustrie und die damit verbundene Zulieferindustrie, z.B. Konstruktion, Herstellung und Vertrieb von Abbau- und Verarbeitungstechnik, ein hohes wirtschaftliches Potenzial aufweisen, da an den Hauptmärkten gleichzeitig ansteigende Preise registriert werden. So sind Naturwerksteinprodukte aus China mit einem durchschnittlichen Preis von 17,50 US-\$/m² zwar noch am günstigsten (Italien: 43,40 US- $\$ / \mathrm{m}^{2}$ ), doch auch dort sind die Preise in den letzten drei Jahren kontinuierlich angestiegen (Montani 2007).

Sollte sich eine derart rasante Entwicklung bestätigen, ist eine Deckung der geforderten Rohstoffmengen kaum aus den derzeitig erschlossenen Lagerstätten möglich. Zudem wird auch das Verlangen nach Individualität in der baulichen und architektonischen Gestaltung nicht abnehmen. Als Konsequenz dieser Entwicklung wird in absehbarer Zeit eine Knappheit des Rohstoffs Naturwerkstein resultieren. Folglich ist eine Prospektion und Erschließung neuer Lagerstätten sowie eine Erweiterung der derzeitigen Abbauareale unumgänglich. Die Einbeziehung moderner wissenschaftlicher Erkenntnisse für die Analyse über die aus der geologischen Situation eines Vorkommens resultierenden lagerstätten-kontrollierenden Elemente ist unabdingbar. Die sich daraus ergebenden Rückschlüsse zur wirtschaftlichen Nutzbarkeit einer Lagerstätte müssen dabei unbedingte Beachtung finden. Dies gilt vor allem auch im Hinblick auf die Abbauplanung und -führung. Gleichzeitig muss mit den zurzeit zur Verfügung stehenden Rohstoffmengen verantwortungsbewusst und zielorientiert umgegangen werden. Hierbei ist vor allem der gesteinsspezifischen Eingrenzung der jeweiligen Anwendungsmöglichkeiten Rechnung zu tragen, die sich aus den physikalischen und technischen Eigenschaften eines Materials ergeben.

Im Folgenden werden zunächst die in der Arbeit gewonnenen Erkenntnisse zur Relevanz des Trennflächengefüges (Kap. 2) und zur Bedeutung der umfassenden Gesteinscharakterisierung (Kap. 3) kurz dargestellt. Nach einer kurzen Zusammenfassung der Ergebnisse der überregionalen (Kap. 4, Argentinien) und regionalen (Kap. 5, Tansania) Erfassung und Erkundung von Werksteinlagerstätten, werden anschließend die sich aus den methodischen Grundlagen ergebenden Rückschlüsse und Möglichkeiten unter Berücksichtung der notwendigen ökonomischen Effizienz im Abbau des Rohstoffs Naturwerkstein anhand ausgewählter Beispiele vorgestellt. 


\subsection{Relevanz der Trennflächenanalytik}

Die Aufnahme des strukturellen Inventars in einer Vielzahl von Werksteinvorkommen hat gezeigt, dass das Trennflächengefüge einer Lagerstätte in Abhängigkeit von der Lithologie und der Gesteinsgenese von unterschiedlicher Ausbildung hinsichtlich der Orientierung, Verteilung und Regelmäßigkeit sein kann (vgl. Kap. 2.2). Es konnte gezeigt werden, dass daher der detaillierten Erfassung und Analyse des strukturellen und tektonischen Inventars eines Lagerstättenkörpers in jeder Phase der Lagerstättenerkundung und -bewertung sowie auch der Abbauplanung und -führung eine enorme Bedeutung zukommt. Bereits in der ersten Erkundungsphase ist, basierend auf der Trennflächenverteilung im Gesteinskörper, eine generelle Nutzbarkeit abzuschätzen (vgl. Kap. 2.3.2). Dabei ist sowohl die Abstandsverteilung der Diskontinuitäten als auch deren Orientierung zueinander von Bedeutung, da sich daraus die Größe und Form der sich ergebenden in-situ Blöcke ableiten lässt (vgl. Kap. 2.4). Im gegebenen Fall einer potenziellen Werksteinlagerstätte muss in weiteren Schritten die Wahl und Anlage spezifischer Explorationsmethoden, aufbauend auf dem jeweils erweiterten Kenntnisstand, dem strukturellen Inventar angepasst werden, um Fehlinterpretationen zu vermeiden. Diese können z.B. aus einer Winkelabweichung zwischen Bohrungsrichtung und den zu erfassenden, strukturellen Elementen resultieren (Palmström 1995). Im Fallbeispiel der Dolomitmarmorlagerstätte von Mtesa, Tansania, sollten z.B. Sondierbohrungen rechtwinklig zu den nachgewiesenen Scherbahnen abgeteuft werden, um deren Häufigkeit und vor allem deren vertikale Mächtigkeit abschätzen zu können (vgl. Kap. 5.7.3). In der finalen Phase der Exploration, deren Erkenntnisse gegebenenfalls zu einer direkten Planung zur Erschließung eines Abbauareals zusammengeführt werden, muss basierend auf dem Trennflächengefüge eine Abbauführung und -methode definiert werden, die die Anforderungen an Blockgröße und Blockform (vgl. Kap. 2.1) berücksichtigt. Dabei muss vorrangig dem natürlichen Trennflächengefüge gefolgt werden, um eine zusätzliche, maschinell herbeigeführte Zergliederung des Wertgesteins zu vermeiden (vgl. Kap. 2.5 und 4.6.1.2). Natürlich ist dabei eine dem jeweiligen Gestein angepasste Abbaumethode zu wählen. Außerdem ist die Ausrichtung der Rohblöcke im Abbau so anzusetzen, dass eventuelle Farb- und Dekorerscheinungen, beispielsweise ein dekorativer Faltenbau, bestmöglich zur Geltung kommen (vgl. Kap.2.5). Generell ist aus dem Trennflächengefüge auch die Berechnung der zu erwartenden Blockgrößen von Bedeutung, da diese entscheidend die mögliche Verwendbarkeit und damit den ökonomischen Erfolg diktieren (vgl. Kap. 2.3.2.2).

Unter Beachtung der natürlichen Vorgaben können weiterhin entscheidende Parameter hinsichtlich der wirtschaftlichen Nutzung einer Lagerstätte positiv beeinflusst werden. Wesentliche Faktoren dabei sind zum einen die Minimierung der Produktionskosten bei der Ausbringung dimensionsgerechter Werksteinrohblöcke und zum anderen die Konzentration auf ungestörte Bereiche eines Lagerstättenkörpers. Unter Ausnutzung der natürlichen Trennflächen durch eine optimierte Anpassung des Abbaus kann so vor allem der generelle Energie-, Material- und Arbeitseinsatz gesenkt werden. Parallel ermöglicht die Detektion ungestörter Wertgesteinsbereiche bei gleichzeitigem Ausschluss nicht bauwürdiger Zonen 
eine positive Beeinflussung der Wertgestein-Abraum-Bilanz. In diesem Zusammenhang konnte an mehreren Beispielen gezeigt werden, dass zudem eine enge Verknüpfung zu materialwissenschaftlichen Grundlagen notwendig ist, um alternative Nutzungsmöglichkeiten abzuwägen (vgl. Kap. 2.1, 4.6.2.3 und 5.7.3).

Als ein wesentlicher Faktor konnte vor allem die Optimierung der Blockdimensionierung und Blockverteilung beim Abvieren der in-situ Blöcke beziehungsweise der Abbauscheiben herausgestellt werden (vgl. Kap. 2.5). Zur Zeit wird an einer Erweiterung der vorgestellten Software 3D-BlockExpert gearbeitet, die eine rechnergestützte Optimierung der Flächennutzung, wie sie in Kapitel 2.5 beschrieben ist, unter Vorgabe definierter Mindestund Maximalblockgrößen erlaubt. Damit können auch prognostische Aussagen bezüglich zu erwartender Blockgrößen im weiteren Abbauverlauf gemacht werden, um auf individuelle Forderungen oder Wünsche der verarbeitenden Industrie gezielt reagieren zu können.

\subsection{Relevanz der physikalischen und technischen Gesteinscharakterisierung}

Ursächlich für viele Schadensfälle bei der bautechnischen Anwendung von Naturwerkstein ist eine mangelnde oder ungenügende Kenntnis des spezifischen Materialverhaltens der Gesteine durch baukonstruktive oder verwitterungsbedingte Beanspruchungen. Grund dafür ist, dass in vielen Fällen die Entscheidungsfindung im Rahmen eines Bauvorhabens lediglich auf den optischen Eigenschaften eines Gesteins beruht. Zwar ist daher die Marktfähigkeit eines Produktes eng mit dem ästhetischen Erscheinungsbild verknüpft (Selonen et al. 2000, vgl. Kap. 1), doch spielen die individuellen physikalischen und bautechnischen Parameter eine entscheidende Rolle hinsichtlich der möglichen Verwendung. Denn viele Gesteine unterscheiden sich hinsichtlich Farbe und Struktur nur unwesentlich voneinander, zeigen jedoch infolge der spezifischen Eigenschaften (Abb. 6.1) bei gleicher bautechnischer Anwendung z.B. ein deutlich voneinander abweichendes Verwitterungsverhalten.

Die statistische Auswertung von rund 10.000 recherchierten Einzeldaten zu etwa 2.300 Werksteinvarietäten unterschiedlicher Lithologien hat insgesamt gezeigt, das bei der Ermittlung der verschiedenen Parameter zur Gesteinscharakterisierung zudem eine Vergleichbarkeit der Ergebnisse gegeben sein muss. Dies ist nur durch die Anwendung normierter Standardversuche realisierbar. Darauf aufbauend kann eine bewertende Gegenüberstellung verschiedener Handelssorten durchgeführt werden. Zusätzlich müssen vor allem auch spezifische Materialverhalten berücksichtigt werden. So ist z.B. die mangelnde Formbeständigkeit infolge des thermischen beziehungsweise thermohygrischen Dehnungsverhaltens vieler Marmore gezielt zu erfassen (vgl. Kap. 5.8.1.3). 

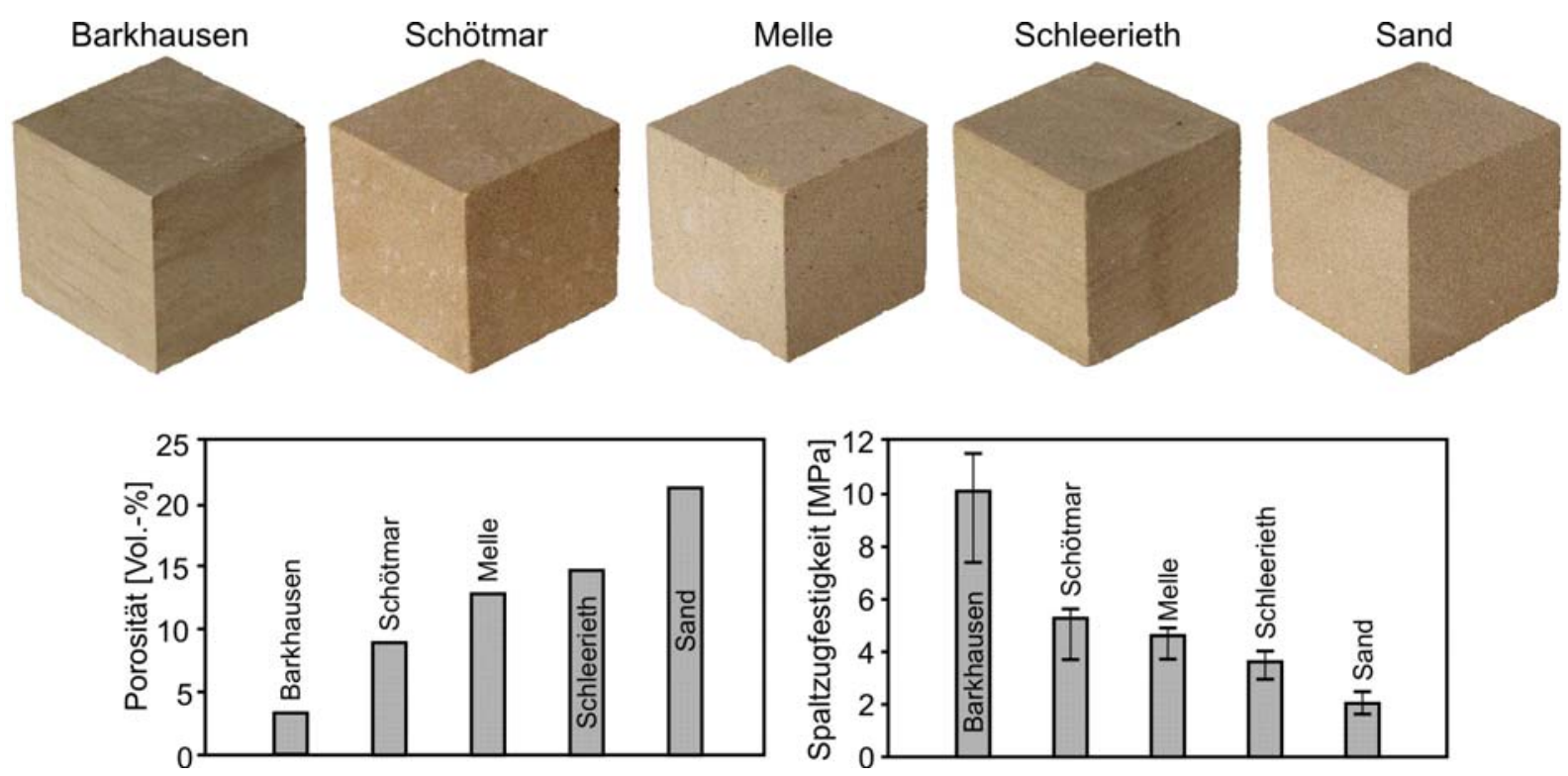

Abb. 6.1: Die annähernde Farb- und Strukturgleichheit der verschiedenen Schilfsandsteine (Mittlerer Keuper) bei deutlich voneinander abweichenden Gesteinseigenschaften verdeutlicht die Notwendigkeit der detaillierten Gesteinscharakterisierung zur Erfassung des Verwitterungsverhaltens bzw. -zustands, um die Anwendungsmöglichkeiten definieren zu können (Kantenlänge der Würfel 65 mm).

Die Auswertung der für diese Arbeit zusammengestellten Daten ermöglichte primär die Definition lithologietypischer Bereiche sowohl für die einzelnen Eigenschaften (Abb. 6.2 und 6.3) als auch für verschiedene Korrelationen der Parameter (Kap. 3.10, 3.12 und 3.13.3). Dabei erfolgte zunächst eine Darstellung der fünf Hauptgesteinsgruppen Plutonite, Vulkanite, klastische Sedimente, Karbonatgesteine und Metamorphite (Kap. 3.4). Durch die separate Betrachtung in Untergruppen zu den Hauptgesteinsgruppen, die sich aus Unterschieden in der Mineralogie oder der Genese der Gesteine ergeben (Kap. 3.5 bis 3.9), und auch an der Diskussion verschiedener statistischer Ausreißer konnte die Notwendigkeit einer detaillierten petrographischen Beschreibung und Zuordnung in die wissenschaftliche Terminologie deutlich gemacht werden. Dies gilt sowohl für die als Basiseigenschaften zusammengefassten Parameter effektive Porosität und Rohdichte als auch für die verschiedenen Parameter der mechanischen Stabilität, für die elastischen Eigenschaften mit dem Ausdruck der Kompressionswellengeschwindigkeit als auch für die Parameter zum Feuchtehaushalt der Gesteine. Mit der Schätzung der wahren Grundgesamtheiten für die verschiedenen Korrelationen mit der Darstellung von Aufenthaltswahrscheinlichkeiten konnte zudem ein einfaches Hilfsmittel aufgestellt werden, Einzeldaten oder auch Datensätze bewertend betrachten zu können (vgl. Kap. 5.8.1.6 und 5.9). 


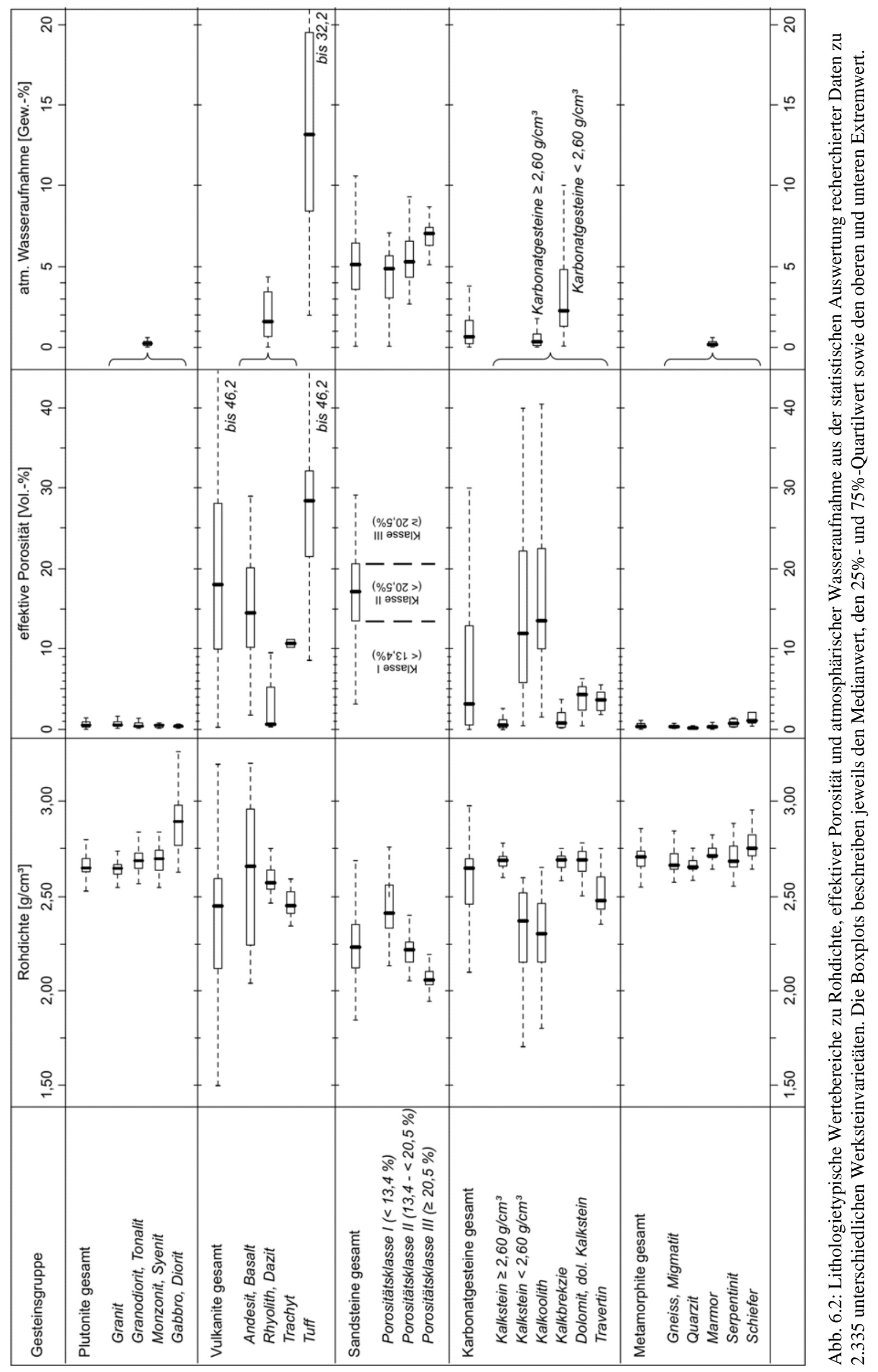




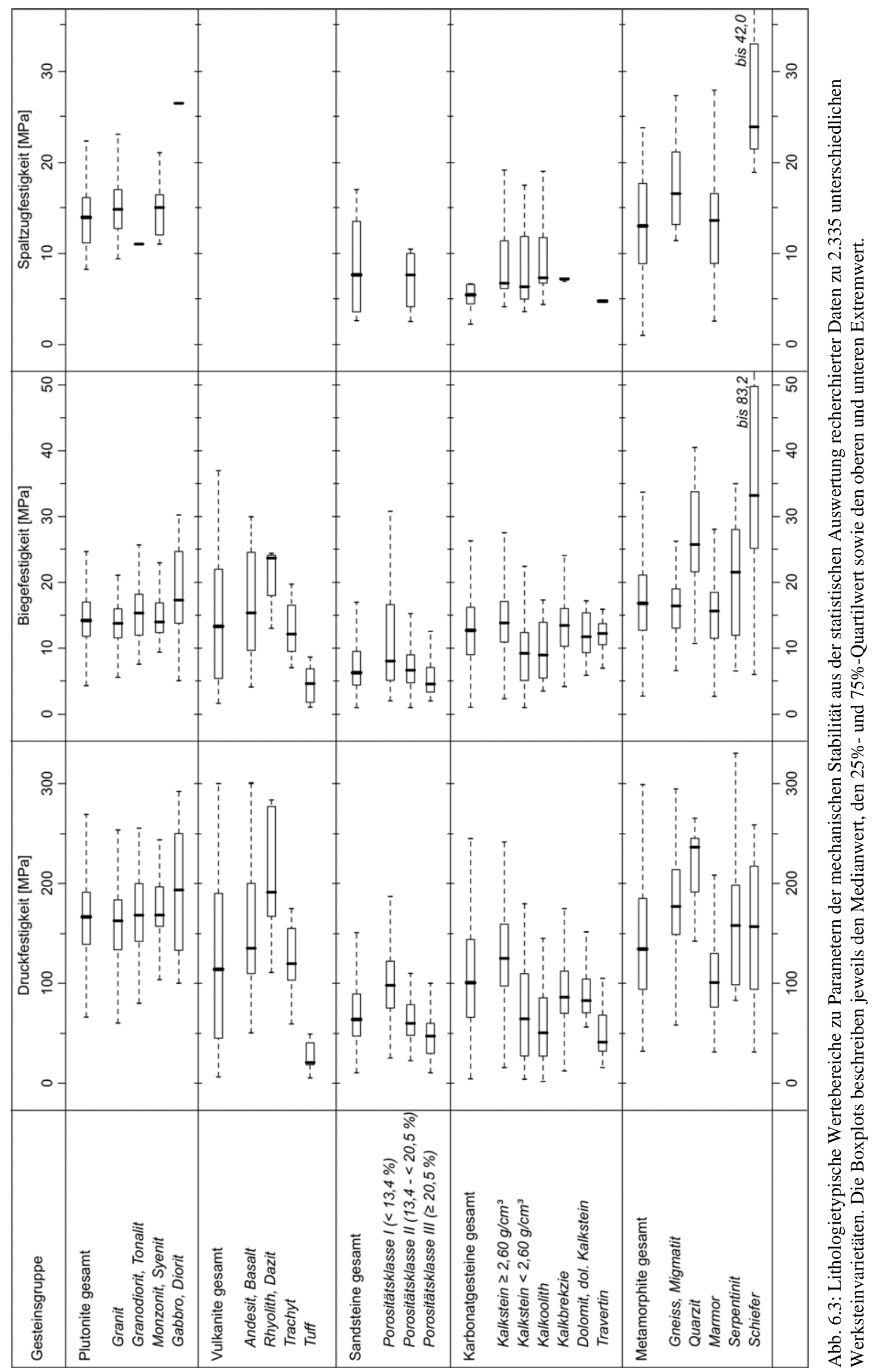


Hinsichtlich der Verhältnisse zwischen den Festigkeitseigenschaften wurden bisher relativ gleich bleibende Faktoren beschrieben (Hirschwald 1912, Peschel 1983), wobei jedoch keine eindeutige petrographische Unterscheidung aufgezeigt ist. Die Vielzahl der Daten hat dagegen gezeigt, dass die Verhältniswerte gesteinsspezifisch leicht variieren (Abb. 6.4). Die Druckfestigkeit erreicht über alle Gesteinsgruppen etwa den 5 bis 15 fachen Wert der Biegefestigkeit. Dabei ergeben sich im Mittel bei den Karbonatgesteinen die geringsten Faktoren, was vermutlich auf die relativ geringe mechanische Stabilität des Calcits, als dominantes Hauptgemengteil, zurückzuführen ist. Die sowohl höchsten als auch niedrigsten absoluten Faktoren sind für Metamorphite dokumentiert. Sehr geringe Verhältnisse (z.B. für verschiedene Schiefer oder stark mylonitische Gneise) sind vermutlich darauf zurückzuführen, dass bei der Ermittlung der Festigkeitseigenschaften extrem ausgebildete Richtungsgefüge keine ausreichende Beachtung gefunden haben. Bei vielen entsprechenden Werksteinvarietäten, so z.B. Schiefer, ist jedoch die Anwendungsmöglichkeit häufig bereits durch das Gefüge diktiert.

\begin{tabular}{|c|c|c|c|c|}
\hline & \multicolumn{3}{|c|}{ Verhältnis $S t_{C}: S t_{F}$} & \multirow[b]{2}{*}{ Median } \\
\hline & $5: 1$ & $10: 1$ & $15: 1$ & \\
\hline \multirow{5}{*}{$\begin{array}{l}\text { Plutonite gesamt } \\
\text { Gabbro, Diorit } \\
\text { Granit } \\
\text { Granodiorit, Tonalit } \\
\text { Monzonit, Syenit }\end{array}$} & & 1 & : & 11,3 \\
\hline & 1 & & ? & 10,5 \\
\hline & & & & 11,4 \\
\hline & & & & 10,1 \\
\hline & & & & 13,4 \\
\hline \multirow{3}{*}{$\begin{array}{l}\text { Vulkanite gesamt } \\
\text { Vulkanite exkl. Tuffe } \\
\text { Tuffe }\end{array}$} & & 1 & & 10,2 \\
\hline & & & & 10,6 \\
\hline & & & & 8,5 \\
\hline \multirow{4}{*}{$\begin{array}{l}\text { Sandsteine gesamt } \\
\text { Porositätsklasse I (<13,4\%) } \\
\text { Porositätsklasse II (13,4-<20,5\%) } \\
\text { Porositätsklasse III ( } \geq 20,5 \%)\end{array}$} & & & & 10,5 \\
\hline & & & & 9,3 \\
\hline & & & & 11,3 \\
\hline & & & & 10,6 \\
\hline \multirow{7}{*}{$\begin{array}{l}\text { Karbonatgesteine gesamt } \\
\text { Kalkstein } \geq 2,60 \mathrm{~g} / \mathrm{cm}^{3} \\
\text { Kalkstein }<2,60 \mathrm{~g} / \mathrm{cm}^{3} \\
\text { Kalkoolith } \\
\text { Kalkbrekzie } \\
\text { Dolomit, dol. Kalkstein } \\
\text { Travertin }\end{array}$} & ; & & & 8,0 \\
\hline & & & & 9,4 \\
\hline & & 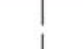 & & 6,3 \\
\hline & & 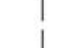 & & 7,3 \\
\hline & & & & 8,8 \\
\hline & & & & 11,5 \\
\hline & & 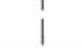 & & 7,3 \\
\hline \multirow{6}{*}{$\begin{array}{l}\text { Metamorphite gesamt } \\
\text { Gneiss } \\
\text { Migmatit, migmat. Gneiss } \\
\text { Marmor } \\
\text { Serpentinit } \\
\text { Schiefer }\end{array}$} & 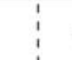 & & & 10,6 \\
\hline & $\vdots$ & & & 8,3 \\
\hline & 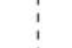 & & & 12,4 \\
\hline & 1 & & & 6,6 \\
\hline & I & & & 8,8 \\
\hline & $\frac{1}{1}$ & 1 & 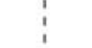 & 4,5 \\
\hline
\end{tabular}

Abb. 6.4: Verhältnisse zwischen den Parametern Druck- $\left(\mathrm{St}_{\mathrm{C}}\right)$ und Biegefestigkeit $\left(\mathrm{St}_{\mathrm{B}}\right)$ in den verschiedenen Gesteinsgruppen. Die eingetragenen Wertebereiche ergeben sich aus den 25 \%- und 75 \%-Quartilwerten der jeweiligen Datenmenge.

Insgesamt konnte verdeutlicht werden, dass für Gesteine, die eine große Spannweite in der Rohdichte beziehungsweise der effektiven Porosität aufweisen, gute bis sehr gute Möglichkeiten zur Prognose gegeben sind. Somit können die vorgestellten 
Regressionsmodelle (vgl. Kap. 3.11, 3.12.4 und 3.13.5) zu einer einschätzenden Betrachtung der Materialeigenschaften eines Gesteins bereits in einer frühen Phase der Lagerstättenerkundung herangezogen werden. Besonders im beispielhaften Vergleich prognostizierter Daten mit tatsächlich ermittelten Eigenschaftswerten (vgl. Kap. 4.8) wird deutlich, dass dabei neben der mineralogischen Komposition auch die gesteinsspezifische Gefügeausbildung berücksichtigt werden muss. So konnte sowohl in der Gruppe der Plutonite als auch der Sandsteine eine Zunahme der mechanischen Stabilität bei Abnahme der Korngröße festgestellt werden. Die betrachteten regressiven Zusammenhänge sind dabei zumeist linearen Charakters. Die Ausnahme bildet hierbei die Gruppe der Karbonatgesteine, in der für alle betrachteten Beziehungen ein systematischer Trend nichtlinearen Charakters ausgebildet ist (vgl. Kap. 3.11.4 und 3.12.4). Außerdem konnte festgestellt werden, dass bei Karbonatgesteinen eine niveauabhängige Streuung der Daten auftritt. Sowohl bei der Prognose der Festigkeitsparameter als auch der kapillaren Wasseraufnahme ist eine deutliche Zunahme der jeweiligen Fehlerbereiche mit zunehmender Rohdichte beziehungsweise abnehmender Porosität zu verzeichnen. Daher können bei gleicher Rohdichte in Abhängigkeit von der Homogenität des Gesteins erhebliche Unterschiede auftreten (Abb. 6.5). Bezüglich der mechanischen Stabilität sind neben der Schichtung vor allem Gefügeelemente wie Drucklösungssuturen, feine Haarrisse oder lokal gröbere Calcitkristallisationen zu beachten. Auch mineralogische Einflüsse, so z.B. ein erhöhter Tonmineralgehalt, können zu einer Herabsetzung der Festigkeit führen. Hohe Festigkeiten sind dagegen für Karbonatgesteine beschrieben, die ein homogenes, mikritisches Gefüge aufweisen. Durch die entsprechende Anpassung an exponentielle Modelle kann diese niveauabhängige Streuung der Daten besser beschrieben werden.

Bezüglich der kapillaren Wasseraufnahme, als entscheidender Parameter für viele Prozesse der Verwitterung (vgl. Kap. 3.13.1), ist die unterschiedliche Porenraumcharakterisitik der verschiedenen Gesteinsgruppen zu beachten (vgl. Kap. 3.13.3). Dabei weisen Plutonite und Metamorphite infolge der Kristallisation (bzw. Rekristallisation) dichte Gefüge mit einer Rissporosität auf, die lediglich geringe bis sehr geringe Öffnungsweiten zeigt. Sandsteine besitzen im Allgemeinen höhere Porositäten, wobei der Porenraum durch relativ gleich bleibende Formen der aufbauenden Partikel zumeist homogen ausgebildet ist. Dagegen zeigen viele Karbonatgesteine infolge der oft heterogenen Zusammensetzung aus klastischen, chemischen und organogenen Anteilen zum Teil sehr breite und unregelmäßige Porenradienverteilungen. Infolge dessen kann bei gleichen Porositätswerten die Fähigkeit zur Wasseraufnahme unterschiedlich ausgeprägt sein, da diese vorrangig an bestimmte Porenradien gebunden ist. Ein ähnliches Verhalten konnte auch bei verschiedenen Basaltlaven festgestellt werden. Hier liegt die Heterogenität des Porenraums in unterschiedlich starken Entgasungprozessen des Magmas begründet, die zu lokalen Schwankungen des Blasengefüges führen können. 

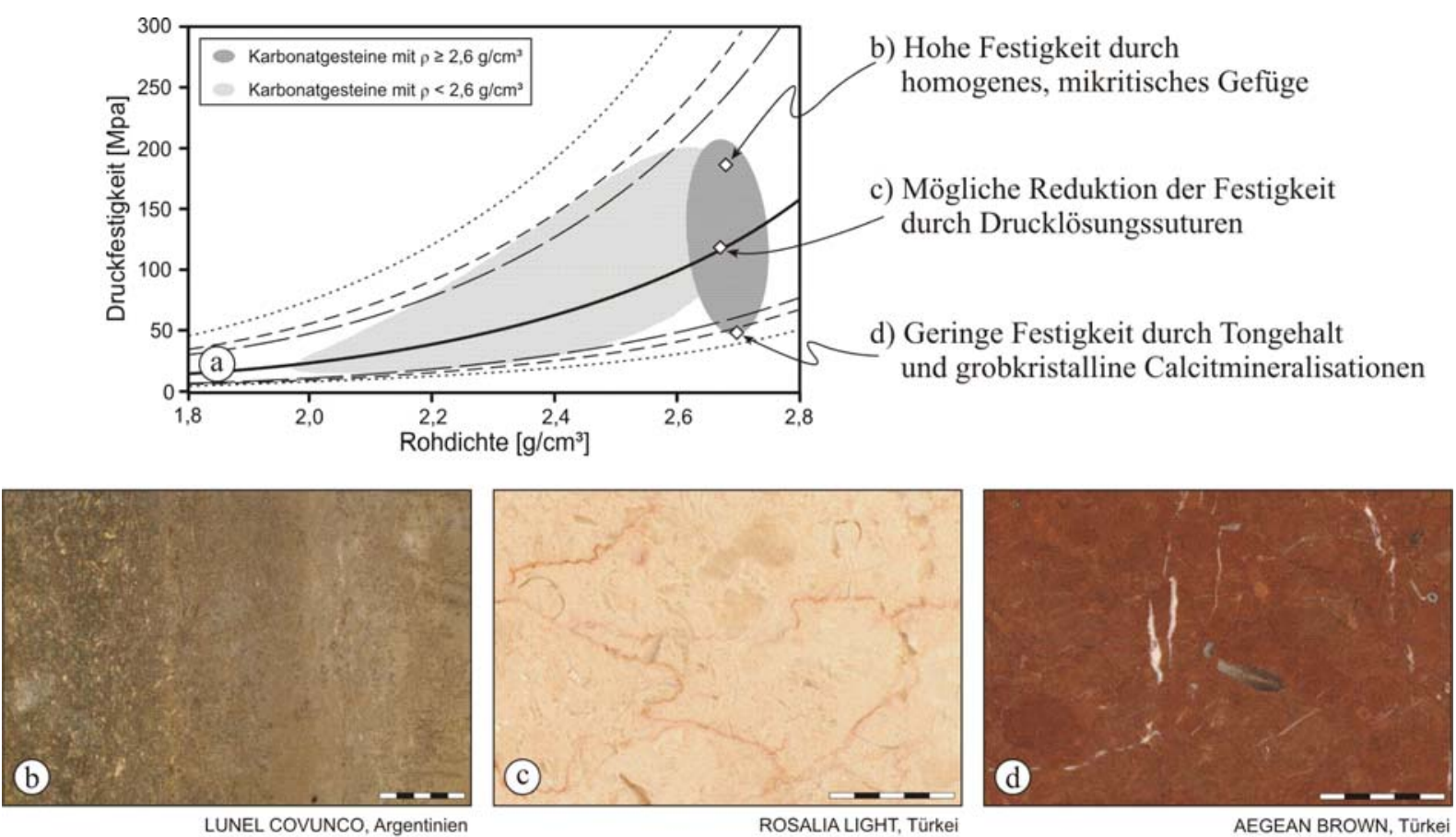

Abb. 6.5: Beeinflussung der mechanischen Stabilität durch die gesteinsspezifische Gefügeausbildung am Beispiel von drei Kalksteinen. In a) sind die abgeschätzten 80 \%-igen Aufenthaltswahrscheinlichkeitsbereiche für die jeweilige theoretische Grundgesamtheit der Datenpaare in den definierten Dichtegruppen dargestellt. Zusätzlich eingetragen ist die Regressionslinie mit den Bereichen, die 65 \% (lang gestrichelt), 80 \% (kurz gestrichelt) und $95 \%$ (gepunktet) der Streuung um die Linie herum enthalten.

Eine detaillierte Gesteinscharakterisierung und daraus abgeleitete Verwendungseinschränkungen sind insgesamt als Grundvoraussetzung für die Dauerhaftigkeit von Naturwerkstein in der bautechnischen Anwendung anzusehen. Die statistischen Auswertungen haben gezeigt, dass dabei eine lithologisch detaillierte Zuordnung unumgänglich ist. Weiterhin konnte aufgezeigt werden, dass unter Berücksichtigung des spezifischen Gefügeinventars die Möglichkeit besteht, eine generelle Einschätzung verschiedener Gesteinseigenschaften bereits in der Frühphase der Lagerstättenerkundung vorzunehmen. Zusammen mit der Betrachtung des generellen Trennflächengefüges eines Lagerstättenkörpers kann so frühzeitig die Eignung eines Gesteins im Hinblick auf die Nutzung als Werkstein beurteilt werden.

\subsection{Erkenntnisse aus der Zusammenführung der methodischen Grundlagen}

Im Hinblick auf die Einführung eines Gesteins als Werksteinvarietät am internationalen Markt werden bestimmte Anforderungen an das Material gestellt. Dies betrifft zum einen die Dekorstabilität einer definierten Handelssorte. Zwar sind Schwankungen im Rahmen der bemusterten Dekorbreite zulässig (vgl. Kap. 1), für das generelle Erscheinungsbild muss jedoch eine langfristige Verfügbarkeit gewährleistet sein (vgl. z.B. Kap. 5.6.1). Zum anderen gilt es, optimale Blockgrößen im Hinblick auf Volumen und Dimension zu gewinnen, was vor allem bei einem möglichen Export zum Tragen kommt (vgl. Kap. 2.1). Dabei werden die möglichen Blockgrößen in erster Linie aus der geologischen Situation beziehungsweise dem daraus hervorgehenden Trennflächengefüge bestimmt (Kap. 2.4). Als zweiter Faktor ist hier 
jedoch auch das potenzielle bautechnische Einsatzgebiet eines Gesteins relevant, welches durch die physikalischen und bautechnischen Eigenschaften diktiert wird (vgl. Kap. 3). Folglich ergeben sich differenzierte Anforderungen aus dem Fertigungsprofils eines natursteinverarbeitenden Betriebes. So können für eine Konzentration auf die Produktion von Bodenfliesen infolge der Fertigungstechnik andere Mindestblockmaße angesetzt werden, als z.B. bei polierten Unmaßtafeln als Hauptanteil der Produktion.

Im Allgemeinen werden für Naturwerksteine drei grundlegende Qualitätsstufen unterschieden. Höchste Anforderungen werden an Solitärblöcke gestellt, die in der Bildhauerei Verwendung finden sollen. Wenn nicht ausdrücklich anders erwünscht, muss dabei eine vollständige Homogenität in Farbe und Dekor gewährleistet sein. Form und Größe der hierfür verwendeten Gesteinsblöcke unterliegen einer spezifischen Auswahl und sind an individuelle Ansprüche gebunden. Die zweite Grundqualität bezieht sich auf den Grabmalsektor. Hierbei wird eine fehlerlose petrographische und strukturelle Ausbildung des Gesteins erwartet. Zudem ist aber auch ein hohes Maß an Individualität sowie ein hoher ästhetischer Wert gefordert, womit ein generell hohes Preissegment erreicht wird. In der dritten Gruppe sind sämtliche Qualitäten zusammengefasst, die im Bauwesen zur Anwendung kommen. Eine Unterscheidung ist hier durch unterschiedliche Produktgruppen gegeben, die verschiedenen Forderungen an die physikalischen und bautechnischen Eigenschaften unterliegen. So können Gesteine, die z.B. eine geringe Biegefestigkeit aufweisen, bei entsprechendem Einbau im Vollmörtelbett zwar problemlos als Bodenfliesen verwendet werden. Den hohen technischen Anforderungen im Bereich des Fassadenbaus wird ein entsprechendes Gestein jedoch nicht genügen. Damit verbunden ist zwangsläufig auch das zu erreichende Preissegment eines Gesteins am Markt.

Die umfassende Gesteinscharakterisierung ermöglicht mit der Erfassung des Verwitterungsverhaltens eine Eingrenzung der Einsatzmöglichkeiten und damit auch eine Abschätzung erforderlicher Blockgrößen zur Fertigung entsprechender Endprodukte. Außerdem ist ein bewertender Vergleich zwischen potenziellen Konkurrenzgesteinen durchführbar (vgl. Kap. 3.10 und 5.9). Aus der Trennflächenanalyse kann eine Einschätzung der zu erwartenden Blockgrößen erfolgen sowie ein entscheidender Beitrag zur Ausrichtung und Kontrolle der Abbauführung geleistet werden. In der Zusammenführung der methodischen Grundlagen können damit Anforderungen im Hinblick auf die Neuerschließung oder auch Neubewertung von Werksteinlagerstätten beschrieben werden, die sowohl den direkten Abbau und die Anwendung als auch damit verbundene Faktoren, wie z.B. Verarbeitungstechnik oder Logistik, betreffen.

Im Rahmen der Arbeit wurden die aus den methodischen Grundlagen gewonnenen Erkenntnisse an zwei Fallbeispielen der Erfassung, Erkundung und Bewertung von Werksteinlagerstätten umgesetzt. Abbildung 6.6 gibt einen methodischen Überblick über die Zusammenführung der geowissenschaftlichen Grundlagen mit dem Ziel einer optimierten Nutzung des Rohstoffs Naturwerkstein. Im Folgenden sollen die Fallbeispiele zusammengefasst dargestellt und die aufgezeigten methodischen Grundlagen und Möglichkeiten anhand ausgewählter Lagerstätten exemplarisch diskutiert werden. Dabei soll vor allem auch die ökonomische Effizienz im Abbau der Gesteine berücksichtigt werden. 


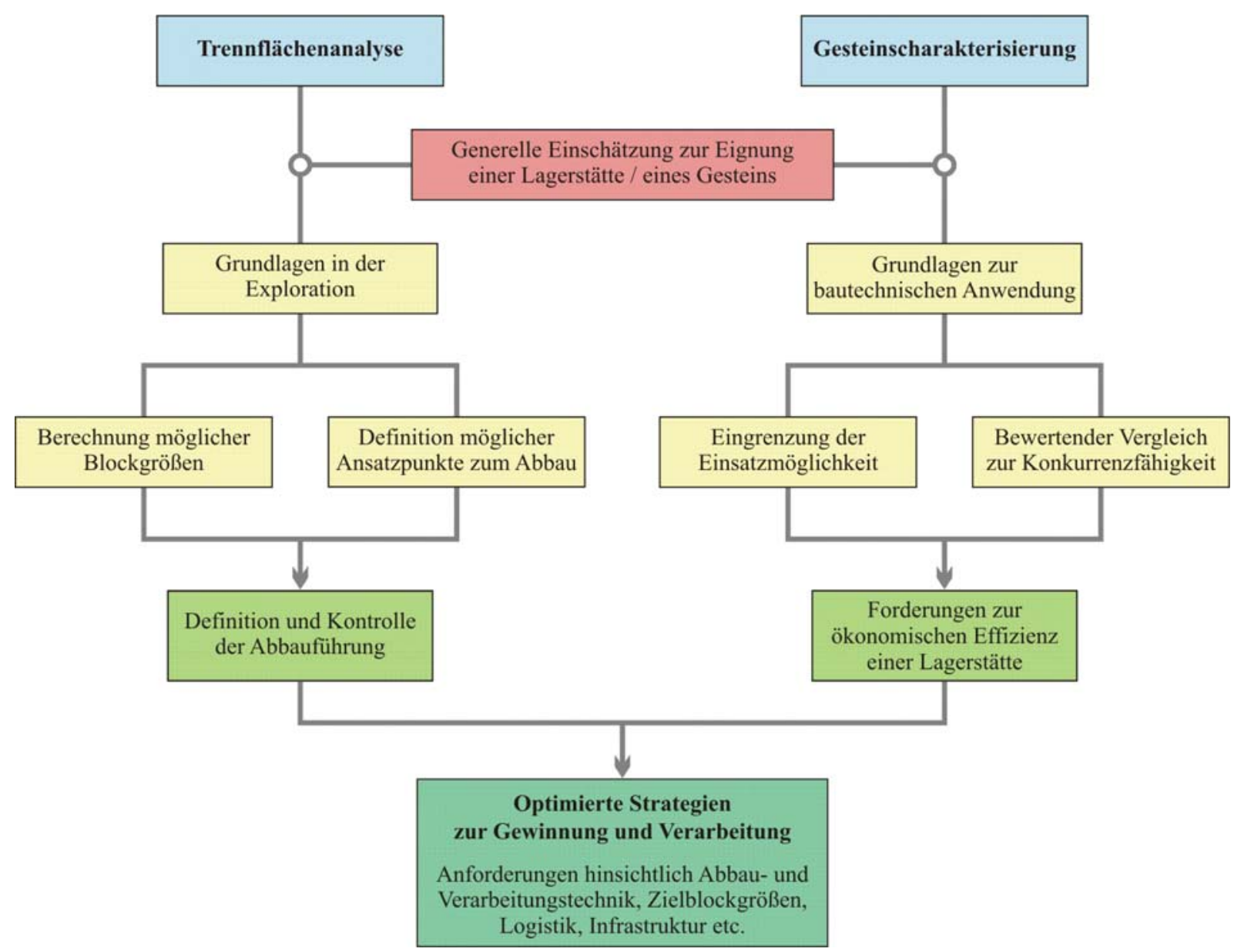

Abb. 6.6: Zusammenführung der methodischen Grundlagen der Trennflächenanalyse und der Gesteinscharakterisierung mit dem Ziel optimierter Gewinnungs- und Verarbeitungsstrategien.

\section{Fallbeispiel 1: Überregionale Erfassung von Naturwerksteinen, Argentinien}

Mit der überregionalen Erfassung am Beispiel Argentiniens (Kap. 4) wurde eine grundlegende Katalogisierung vorhandener Lagerstätten verfolgt. Argentinien verfügt infolge der komplexen geologischen Entwicklung des südamerikanischen Kontinents über eine Vielzahl von Gesteinen, die als Naturwerkstein genutzt werden oder wurden. Im Rahmen eines sechsmonatigen Geländeaufenthaltes wurde eine Vielzahl von magmatischen, metamorphen und sedimentären Lagerstätten in acht Provinzen befahren. Im Vordergrund standen dabei die lithologische und strukturelle Charakterisierung der ausgebrachten Materialien sowie eine repräsentative Dekorerfassung von etwa 40 aufgenommenen Handelssorten. In der Suite der beprobten Werksteinvarietäten sind sowohl Gesteine vertreten, für die ein breites Spektrum vergleichbarer Angebote am internationalen Natursteinmarkt gegeben ist (so z.B. verschiedene Granitoide, Kalksteine und Porphyre unterschiedlicher Farb- und Korngrößenspektren), als auch Gesteine, die nur mit sehr wenigen etablierten Handelssorten vergleichbar sind und somit ein hohes wirtschaftliches Potenzial darstellen. In dieser Gruppe sind vor allem die Vorkommen der Cordieritite bzw. Cordierit-führenden Gesteine der Provinz Córdoba (z.B. VERDE ORCOLLANO, AZUL TANGO) oder auch der Marmor AZUL CIELO zu nennen. 
Neben der Aufnahme der lagerstättengeologischen Situation der verschiedenen Vorkommen wurden zudem Informationen über wirtschaftliche Kenndaten zum Ausbringen und der Verarbeitung (z.B. Blockgrößen, Wertgestein-Abraum-Verhältnisse etc.) recherchiert. Unter Beachtung der lagerstättenspezifischen Trennflächengefüge konnten so die bestehenden Abbauverfahren dahingehend überprüft werden, ob diese an die jeweilige Lagerstättensituation angepasst sind oder ob z.B. Änderungen in der Abbauführung höhere Rohblockmengen ermöglichen können.

Insgesamt stehen über argentinische Werksteinvarietäten wenige Informationen zu technischen und physikalischen Eigenschaften zur Verfügung, sofern sie nicht bereits am internationalen Markt etabliert sind. Basierend auf einer umfangreichen Probennahme erfolgte daher an 18 Varietäten des Probeninventars eine umfassende Gesteinscharakterisierung, was im Rahmen einer überregionalen Erfassung von Werksteinen nicht der Regelfall ist. Zusätzlich wurden die experimentell ermittelten Daten genutzt, um die Kenntnisse über Prognosemöglichkeiten bezüglich der mechanischen Stabilität (Kap. 3.10) zu überprüfen und zu verifizieren.

Auf Basis der spezifischen Dekore, der generellen lagerstätten-kontrollierenden Elementen sowie auch der Gesteinseigenschaften kann die überregionale Erfassung zu einer Entscheidungshilfe im Hinblick auf detaillierte Erkundungen und zielorientierte Verwendung eines Werksteins herangezogen werden. Aus den gewonnenen Erkenntnissen zu den verschiedenen Vorkommen kann somit eine Neubewertung einzelner Lagerstätten erfolgen. Dies soll im Folgenden beispielhaft an zwei der argentinischen Werksteinvarietäten (Cdr-GtGneis AZUL TANGO, Granit GRIS PERLA) dargestellt werden.

Der Abbau der Varietät AZUL TANGO wurde infolge eines zu geringen Ausbringens defektfreier, großdimensionierter Rohblöcke eingestellt (vgl. Kap. 4.6.1.2 und 2.5). Den gesteinsspezifischen Eigenschaften (vgl. Kap. 4.7) folgend, stellt das Gestein jedoch ein qualitativ hochwertiges Material dar, das durch eine hohe mechanische Stabilität und eine hohe Verwitterungsresistenz ausgezeichnet ist. Zudem weist das Gestein durch die Färbung und das ansprechende Dekor ein überdurchschnittliches Maß an Individualität auf, womit insgesamt ein hohes Preissegment, angelehnt an den Bereich der Grabmalindustrie, angesetzt werden könnte. Durch die wenigen potenziellen Konkurrenzgesteine resultiert zudem ein geringer Preisdruck. Aufbauend auf einer Bewertung der strukturellen und bautechnischen Konkurrenzfähigkeit erscheint insgesamt eine Neubewertung der Lagerstätte sinnvoll beziehungsweise erforderlich. Der Fokus ist dabei vor allem auf die generelle Ausbringungsmöglichkeit defektfreier Rohblöcke zu setzen.

Die Trennflächenanalyse im Steinbruch als auch die Vermessung gelagerter Blöcke hat gezeigt, dass mit der bisherigen Abbauführung infolge der komplizierten lagerstättengeologischen Situation lediglich kleine Rohblöcke gewonnen werden konnten. Anzustreben wäre daher eine Optimierung der Abbauführung und der Abbaumethode, für die als Basis eine detaillierte Analyse des Trennflächengefüges unumgänglich ist. Aufgrund des möglicherweise erreichbaren Preissegments können dabei auch höhere Kosten für die Erkundung, die Gewinnung und die Verarbeitung toleriert werden. Sinnvoll wären so z.B. auch vertikale und horizontale Sondierbohrungen, um zu einem Gesamtbild des 
Lagerstättenkörpers zu gelangen. Ist abzusehen, dass auch durch eine Änderung der Abbauführung eine Verbesserung der Ausbringungsrate nicht zu erreichen ist, sollten auch mögliche Alternativprodukte in Erwägung gezogen werden. Hier wäre trotz höherer Produktionskosten vor allem die Ausbringung von Kleinblöcken denkbar, die die Forderungen der Grabmalindustrie erfüllen könnten. In diesem Zusammenhang wäre auch das Gewinnungsverfahren zu überdenken. Der bisherige Abbau erfolgte im Handbohrverfahren, da es die kostengünstigste Methode darstellt. Denkbar wäre jedoch auch der Einsatz einer Diamantseilsäge. Zwar sind die damit verbundenen Kosten infolge des Seilverschleißes gerade in diesem Hartgestein (Cordierit, Granat, Quarz) deutlich höher anzusetzen, doch der Materialverlust könnte bei der Blockabvierung minimiert und gleichzeitig die Blockqualität erhöht werden (Singewald 1992).

Der Granit GRIS PERLA (vgl. Kap. 4.6.1.2 und 2.4) ist dagegen als Werksteinvarietät in eine Gesteinsgruppe einzugliedern, für die eine Vielzahl an vergleichbaren Handelssorten (mittelgraue und mittel- bis grobkörnige Granitoide) am internationalen Markt vertreten ist. Daraus resultieren zum einen ein relativ niedriges Preissegment und zum anderen ein hoher Konkurrenzdruck. Dieser verstärkt sich umso mehr, als dass der argentinische Granit keine außergewöhnlichen Eigenschaften im Bezug auf die bautechnische Stabilität besitzt (Abb. 6.7). Die geologische Situation der Lagerstätte ermöglicht jedoch die gleichmäßige Gewinnung großdimensionierter Rohblöcke, wobei auch ein hohes Maß an Homogenität realisiert ist. Im Gegensatz zur Varietät AZUL TANGO ist im Falle des Granits GRIS PERLA daher unbedingt eine Kostenminimierung bei der Gewinnung notwendig, um eine gute Position am internationalen Markt schaffen zu können.

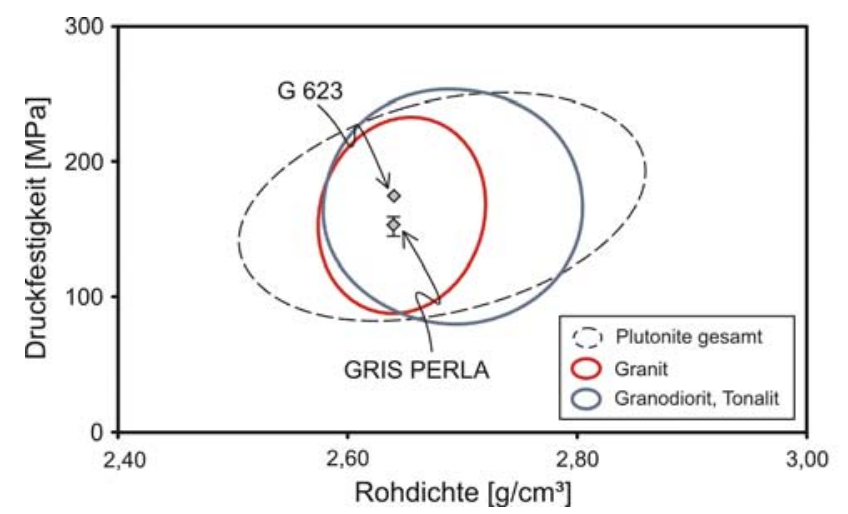

\begin{tabular}{lcc}
\hline & GRIS PERLA & G 623 \\
\hline Rohdichte [g/cm $\mathrm{cm}^{3}$ & 2,64 & 2,64 \\
Porosität [Vol.-\%] & 0,58 & 0,24 \\
Druckfestigkeit [MPa] & 152,9 & 174,2 \\
Biegefestigkeit [MPa] & 10,4 & 10,8 \\
\hline
\end{tabular}

Abb. 6.7: Vergleich der granitischen Handelssorte GRIS PERLA (Argentinien) mit einem potenziellen Konkurrenzgestein am internationalen Markt, dem Granodiorit G 623 (China), anhand ausgewählter spezifischer Kenndaten. Die Ellipsen beschreiben Bereiche der 80 \%-igen Aufenthaltswahrscheinlichkeit der Korrelation für die jeweilige Gesteinsgruppe, die sich aus der statistischen Auswertung in Kapitel 3.10.1 dieser Arbeit ergeben haben. Für die Varietät GRIS PERLA ist zudem die gefügebedingte Anisotropie im Diagramm angegeben.

Im Hauptbruch zur Gewinnung der Handelssorte GRIS PERLA wird neben Handbohrgeräten auch eine Diamantseilsäge eingesetzt. Höchster Kostenfaktor dabei ist der Seilverschleiß. Durch eine Optimierung im Abbau beziehungsweise der Abbauführung sollte dieser so gering wie möglich gehalten werden. Neben dem Trennflächengefüge ist daher auch die Ausbildung möglicher Strukturelemente im Gestein zu beachten. Der Granit GRIS PERLA weist zum Teil eine ausgeprägte Foliation auf, die anhand einer parallelen 
Anordnung porphyritischer Feldspäte definiert ist. Daher ist zu ermitteln, ob durch eine Schnittführung parallel der Foliation ein geringerer Verschleiß des Diamantseils erreicht werden kann. Bezüglich der Standzeit der Diamantseile ist zudem zu ermitteln, ob vorgezeichnete Spaltflächen des Gesteins, wie sie infolge residualer Gebirgsspannungen auftreten können (vgl. Kap. 2.4.1), für die Schnittführung genutzt werden können. Bei der Gesteinscharakterisierung wurde eine geringe Anisotropie der mechanischen Stabilität festgestellt (Kap. 4.7). Änderungen in der bautechnischen Eignung des Gesteins wären durch einen Wechsel der Schnittführung im Falle der Varietät GRIS PERLA jedoch nicht zu erwarten. Zum einen liegen die ermittelten Minimalwerte noch im lithologietypischen Bereich (Abb. 6.6), zum anderen entfallen sie auf die z-Richtung, also senkrecht zur Foliation. Als fertigungstechnische Konsequenz sollte daher das Blockaufschneiden im Idealfall parallel der Foliation erfolgen. Entsprechende Kraftrichtungen können so nicht zur Wirkung kommen. Weiterhin wäre zur Rohblockformatierung der Einsatz von Reihenbohrgeräten denkbar. Trotz höherer Anschaffungskosten im Vergleich zu Handbohrgeräten können die laufenden Gewinnungskosten durch die deutlich höhere Schneidleistung niedriger angesetzt werden (Singewald 1992).

Im Hinblick auf die Formatierung von Rohblöcken aus den Abbauscheiben sollte hier eine Optimierung nach dem Prinzip der Flächenausnutzung (vgl. Kap. 2.5) konsequent verfolgt werden. Damit ist vor allem die bestmögliche Ausnutzung des Materials gewährleistet. Des Weiteren können so auch prognostische Aussagen über den fortschreitenden Abbauverlauf gemacht werden. Dies würde eine Reaktion auf kurzfristige oder individuelle Kundenanfragen oder aktuelle Marktsituationen ermöglichen. Wie im Allgemeinen, muss auch gerade in diesem Beispiel die positive Gestaltung der Umweltbilanz in der Gewinnung des Werksteins besondere Beachtung finden. Das Prädikat „Naturprodukt“ muss durch ökologisches Denken bei der Festlegung von Abbau- und Verarbeitungsstrategien besonders hervorgehoben werden. Dies führt in der Gesamtheit $\mathrm{zu}$ einem Vorsprung gegenüber den Konkurrenzgesteinen, da so die relative Qualität des Gesteins gesteigert wird.

\section{Fallbeispiel 2: Regionale Erfassung und Bewertung von Naturwerksteinen, Tansania}

Ausgangssituation für das zweite Fallsbeispiel (Kap. 5) waren Kenntnisse über ein mögliches Marmorvorkommen im S der Republik Tansania anhand weniger Handstücke. Zudem lagen Informationen über bestehende Kleinstabbaue vor, die zur Gewinnung des Marmors als Zuschlagstoff für den regionalen Bedarf betrieben werden. Obwohl das Vorkommen seit vielen Jahrzehnten bekannt ist und eine eventuelle Nutzbarkeit als Naturwerksteinlagerstätte beschrieben wird (Stockley \& Oates 1931), fehlten eine Erfassung und Bewertung des Vorkommens sowie auch physikalische und technische Gesteinsuntersuchungen vollständig. Ziel der sich anschließenden Begutachtung des Vorkommens war die Ausweisung einer potenziellen Lagerstätte durch die bewertende Abschätzung der generellen Verwendbarkeit des Marmors als Werkstein. Neben der kartographischen Erfassung des Marmorkörpers sowie der begleitenden Gesteine (vgl. Kap. 5.5), wurden daher die lagerstätten-kontrollierenden Elemente erfasst und deren 
Auswirkungen auf die mögliche Nutzung des Gesteins untersucht (Kap. 5.6). Die Trennflächenanalyse hat gezeigt, dass das Ausbringen von Rohblöcken in verarbeitungsgerechten Dimensionen grundsätzlich möglich ist (vgl. Kap. 5.6.2). Als weiteres entscheidendes Element ist bei diesem Dolomitmarmor eine farbliche Variabilität ausgebildet, auf deren Basis auch eine Definition möglicher, zu unterscheidender Handelssorten beruhen muss (vgl. Kap. 5.6.1). Diese farbliche Variabilität ergibt sich zum einen aus einer unregelmäßigen Verteilung dunklerer Bänder oder Lagen, die einen erheblichen Einfluss auf das Dekor des Gesteins ausüben. Zum anderen ist eine metasomatische Beeinflussung des Marmorkörpers durch auftretende Intrusionen gegeben, die ebenfalls farbliche Veränderungen durch kalksilikatische Mineralneubildungen hervorgerufen hat (vgl. Kap. 5.6.3).

Aufbauend auf der Kenntnis über die Möglichkeit des Rohblockausbringens erfolgte exemplarisch eine umfassende Gesteinscharakterisierung des Dolomitmarmors zur Beschreibung seiner bautechnischen Anwendbarkeit (vgl. Kap. 5.8). Diese erfolgte nach dem Stand der Technik (DIN / EN) bzw. im Hinblick auf spezielle Fragestellungen nach dem aktuellen Stand der Wissenschaft. Hierbei konnte vor allem eine hohe mechanische Stabilität sowie eine Formbeständigkeit bei thermohygrischer Belastung festgestellt werden (vgl. Kap. 5.8.1.5 und 5.8.1.6). Insgesamt stellt der Dolomitmarmor von Mtesa, Tansania, somit eine hoch belastbare Werksteinvarietät dar.

Aus der Kenntnis über das Volumen und die allgemeinen strukturellen Gegebenheiten des Lagerstättenkörpers konnte eine Einschätzung der Bauwürdigkeit der Lagerstätte erfolgen (vgl. Kap. 5.9). Im derzeitigen geologischen Untersuchungsstand der Lagerstätte, der nach der UNFCR (UNO 1997, vgl. Kap. 5.1) mit G 2 angesetzt werden kann, konnten die beschriebenen Methoden der Trennflächenanalyse und der Gesteinscharakterisierung gezielt Anwendung finden. Aus der Betrachtung der Trennflächenverteilung geht hervor, wie und in welchem Winkel zu den strukturellen Elementen eventuelle Tiefenschürfe oder Sondierbohrungen angesetzt werden sollten. Neben diesen direkten Aussagen zu weiteren notwendigen Explorationsschritten beziehungsweise über erforderliche Maßnahmen zur Detailexploration, lassen sich auch Rückschlüsse auf die Abbaurichtung ziehen.

Im betrachteten Fall sollte der Abbau parallel zum Hauptkluftstreichen geführt werden (Abb. 6.8). Aus dem aktuellen Kenntnisstand zum Trennflächengefüge ist abzuleiten, dass in Streichrichtung NNE - SSW eine erhöhte Anzahl an Klüften existiert. Demnach wäre hier auch der notwendige Maschineneinsatz zum Lösen des Gesteins geringer anzusetzen. Neben dem eigentlichen verschleißbedingten Kostenaufwand für das technische Inventar, ließe sich damit auch die Umweltbilanz durch eine Reduktion der benötigten Wassermenge im Falle des Einsatzes einer Diamantseilsäge positiv beeinflussen. Dies erfordert vor allem auch die geographische Lage des Vorkommens. Außerdem ist es erforderlich, gegen das Einfallen der Klüftung, also in Richtung ESE, zu arbeiten, um ein Herabstürzen von größeren Blöcken zu vermeiden (vgl. Kap. 2.5). Bei einer entsprechenden Anlage des Abbaus ist wiederholt mit Bereichen zu rechnen, die durch eine engständigere Klüftung stärker fragmentiert sind. Diese Bereiche können gezielt beräumt und den Belangen des regionalen Kleinstbergbaus zugeführt werden. 

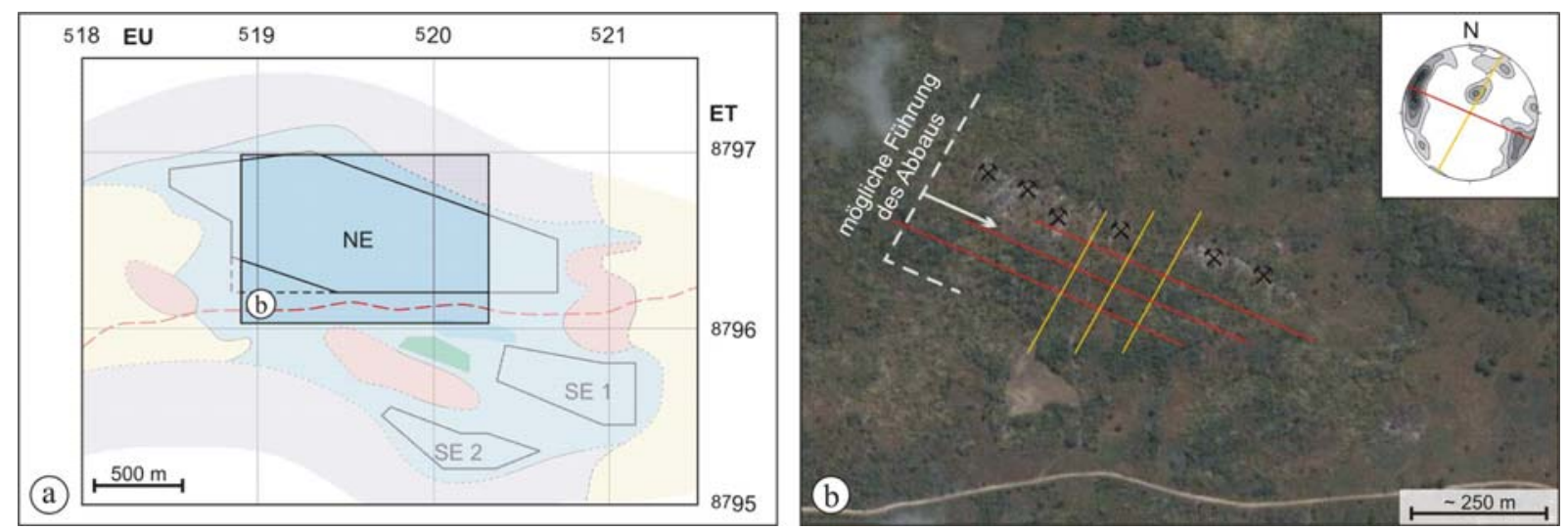

Abb. 6.8: a) Teillagerstätte NE des Dolomitmarmors von Mtesa, Tansania; b) Luftbild des Kartenausschnitts mit den Hauptkluftrichtungen und der daran ausgerichteten, möglichen Abbauführung.

Die Erschließung einer Lagerstätte ist zudem mit einer notwendigen infrastrukturellen Anbindung des Abraumareals verbunden. Neben den allgemeinen Wegeverhältnissen ist im Beispiel des Marmorvorkommens von Mtesa vor allem auf die Belastungsfähigkeit einer Vielzahl von Brückenbauten auf den Hauptverbindungswegen zu achten. Aus der Gesteinscharakterisierung des Dolomitmarmors geht hervor, dass sich das Material infolge der hohen bautechnischen Qualität insbesondere für den Fassadenbau eignen könnte. Im Gegensatz zu verschiedenen Calcitmarmoren tritt eine mangelnde Formbeständigkeit nicht oder in nur sehr geringem Maße auf (vgl. Kap. 5.8.1.5. Unter Annahme der von Selonen \& Suominen (2003) gegebenen Blockdimensionen (vgl. Kap. 1) ist ein Minimalblock von 2,5 x 1,3 x 1,0 m anzustreben. Aus der Trennflächenanalyse ist eine entsprechende Blockgröße durchaus realisierbar. Infolge der festgestellten Rohdichte von $2,87 \mathrm{~g} / \mathrm{cm}^{3}$ ist dabei von einem Mindestblockgewicht von etwa 9,3 t auszugehen. Dementsprechend wären in der Kostenaufstellung zur Erschließung der Lagerstätte für den Blocktransport Fahrzeuge einzukalkulieren, die eine Nutzlast von mindestens 9 bis $11 \mathrm{t}$ aufweisen. Das Gesamtgewicht wäre demnach mit etwa 20 bis $25 \mathrm{t}$ anzusetzen. Aus der Bewertung der Anwendungsmöglichkeit des Materials und den daraus resultierenden (maximal möglichen) Blockgrößen zeigt sich bereits, dass die vorhandene Infrastruktur nicht diesen Belastungen entsprechend ausgelegt ist (vgl. Kap. 5.2). Als Folge müssen in den Erschließungsplan entsprechende Änderungen der Infrastruktur aufgenommen werden. Alternativ wäre eine Anpassung der Größenvorgabe zur Rohblockgewinnung im Abbau hin zu geringeren Blockvolumina vorzunehmen oder aber das notwendige technische Inventar zur Aufarbeitung größerer Rohblöcke vor Ort zu installieren.

In der Gesamtheit wird deutlich, dass ohne eine intensivere Berücksichtigung der Materialund Geowissenschaften bei fortschreitender Entwicklung des Industriesektors Werkstein die gestellten Forderungen langfristig kaum erfüllt werden können. Dabei ist vor allem auch der Faktor Umwelt von enormer Bedeutung. Gerade in der heutigen Zeit, mit einer immer stärker werdenden Globalisierung und einem wachsenden Umweltbewusstsein ist es von großer Bedeutung, das Potenzial des Naturprodukts Werkstein voll auszuschöpfen. In vielen Bereichen von der Exploration bis zum fertigen Produkt ist daher ein Umdenken erforderlich. 
Abweichend von dem bisherigen Vorgehen ist ein nachhaltiger Umgang durch eine effiziente Nutzung der Lagerstätten auf wissenschaftlicher Basis konsequent zu verfolgen. Die verschiedenen Beispiele zeigen deutlich, dass die gemeinsame Betrachtung der Trennflächenanalyse und der detaillierten physikalischen, bautechnischen und strukturellen Gesteinscharakterisierung eine Optimierung der Gewinnungs- und Verarbeitungsstrategien zur Effizienzsteigerung einer Lagerstätte ermöglicht. 


\section{LITERATURVERZEICHNIS}

Adamini, R., Friese, P. \& Protz, A. (2005): Beseitigung von Verfärbungen auf Natursteinoberflächen. In: Siegesmund, S., Auras, M. \& Snethlage, R. (Hrsg.): Stein Zerfall und Konservierung. - Edition Leipzig, S. 96-101.

Auberg, R., Palecki, S. \& Setzer, M.J. (1999): Zerstörungsfreie Prüfung der inneren Schädigung von Beton durch Frostangriff. - DGZfP, Fachtagung Bauwerksdiagnose Praktische Anwendungen Zerstörungsfreier Prüfungen, München.

Auras, M. \& Steindlberger, E. (2005): Verwitterung und Festigung vulkanischer Tuffe. - Z. dt. Geol. Ges., 156 (1): 167-175.

Backhaus, K., Erichson, B., Plinke, W. \& Weiber, R. (1996): Multivariate Analysemethoden: eine anwendungsorientierte Einführung. - 8. Auflage, Springer, Berlin, 591 S.

Bamberg, G. \& Baur, F. (2002): Statistik. - R. Oldenbourg Verlag, 12. Auflage, München, Wien, $342 \mathrm{~S}$.

Bastías, H.E., Uliarte, E., Paredes, J.D., Sánchez, A., Bastías, J.A., Ruycki, L. \& Perucca, P. (1990): Neotectónica de la provincia de San Luis. In: Bordonaro, O. (ed.): Geología y Recursos Naturales de la Provincia San Juan. - XI Congreso Geológico Argentino, Relatorio, pp. 228-244.

Bebout, D. G., Davies, G. R., Moore, C. H., Scholle, P. A. \& Wardlaw, N. C. (1979): Geology of carbonate porosity. - Amer. Ass. Petrol. Geol. Continuing Course Notes, 11: 247 pp., Tulsa.

Berton, Y. \& Le Berre, P. (1983): Guides de Prospection des Matériaux de Carrière. - BRGM Manuels \& Methodes, 5, 160 S., Orléans.

Birch, F. (1960): The velocity of compressional waves in rocks to 10 kilobars, Part 1. - J. Geophys. Res., 66: 1083-1102.

Birch, F. (1961): The velocity of compressional waves in rocks to 10 kilobars, Part 1. - J. Geophys. Res., 66: 2199-2224.

Bock, H. (1971): Über die Abhängigkeit von Kluftabständen und Schichtmächtigkeiten. - N. Jb. Geol. Paläont., Mh., 8: 517-531.

Börner, K. \& Hill, D. (2007): Große Enzyklopädie der Steine: CD-ROM, Hasede (Abraxas Verlag).

Bortz, J. (1985): Lehrbuch der Statistik für Sozialwissenschaftler. - Springer-Verlag.

Brändlein, P. \& Deppisch, J. (2007): CE-Kennzeichnung von Naturstein. - Z. dt. Ges. Geowiss., 158/4: 709-717, Stuttgart.

Brakel, J. van, Modry, S. \& Svata, M. (1981): Mercury porosimetry: State of the art. Powder technology, 29: 1-12. 
Brodtkorb, M.K. de, Ramos, V., Barbieri, M. \& Ametrano, S. (1982): The Evaporitic Celestite-Barite Deposits of Neuquén, Argentina. - Mineralium Deposita, 17: 423-436.

Brosch, F.J., Schachner, K., Blümel, M., Fasching, A. \& Fritz, H. (2000): Preliminary investigation results on fabrics and related physical properties of an anisotropic gneiss. Journal of Structural Geology, 22: 1-12.

Bruna, M.A. (2001): Algunas rocas ornamentals en Neuquén. - Dirrecíon General de Minería de la Provincia Neuquén, Argentina, 14 S.

Büttgenbach, T. (1990): Statistische Untersuchungen der Geschwindigkeits-Dichte Relation in Kristallinen Gesteinen auf der Basis einer Datenbank. - Diss. Univ. Köln, 145 S.

Caminos, R. (1979): Sierras Pampeanas Noroccidentales Salta, Tucumán, Catamarca, La Rioja y San Juan. - $2^{\circ}$ Simposio de Geología Regional Argentina, Acad. Nac. Cs. Córdoba, 1, pp. 225-291.

Chitsazian, H. A. (1985): Beziehungen zwischen Mineralbestand, Gefüge und technologischen Eigenschaften der Niedersächsischen "Wealdon"-Sandsteine (Unterkreide). - Mitteilungen des Geologischen Instituts der Universität Hannover, 25: $103 \mathrm{~S}$.

Cingolani, C.A. \& Dalla Salda, L.H. (2000): Buenos Aires cratonic region. In: Cordani, U.G., Milani, E.J., Thomas Filho, A. and Campos, D.A., Editors, 2000. - XXXI International Geological Congress, Rio de Janeiro XXXI International Geological Congress, Rio de Janeiro, pp. 139-146.

Clemens, K., Grimm, W.D. \& Poschlod, K. (1990): Zur Kennzeichnung des Korngefüges und des Porenraumes der Naturwerksteine. In: Grimm, W.D.: Bildatlas wichtiger Denkmalgesteine der Bundesrepublik Deutschland. - Arbeitsheft Nr. 50, Bayerisches Landesamt für Denkmalpflege, München: 65-94.

Colombo, F., Busquets, P., Ramos, E., Vergés, J. \& Ragona, D. (2000): Quaternary alluvial terraces in an active tectonic region: the San Juan River Valley, Andean Ranges, San Juan Province, Argentina. - Journal of South American Earth Sciences, 3: 611-626.

Dalla Salda, L.H., (1981): The Precambrian geology of El Cristo, southern Tandilia region, Argentina. - Geologische Rundschau 70: 1030-1042.

Dalla Salda, L.H., Bossi, J. \& Cingolani, C.A. (1988): The Río de la Plata cratonic region of southwestern Gondwanaland. - Episodes 11: 263-269.

David, Chr. (2006): Buntsandsteine - Bausandsteine, Marburger Bausandstein unter der Lupe. - Marburger Geowissenschaften 3: 1-129, Marburg.

Davis, G.H. \& Reynolds, S.J. (1996): Structural Geology of Rocks and Regions. - John Wiley \& Sons, New York, 776 S.

Dinçer, I., Acar, A., Çobanoğlu, I. \& Uras, Y. (2004): Correlation between Schmidt hardness, uniaxial compressive strength and Young's modulus for andesites, basalts and tuffs. - Bull. Eng. Geol. Env., 63: 141-148. 
DIN EN 1097-06 (2001): Prüfung von Naturstein und Gesteinskörnungen: Bestimmung der Rohdichte und der Wasseraufnahme. - Beuth Verlag, Berlin.

DIN EN 12372 (1999): Prüfverfahren für Naturstein - Bestimmung der Biegefestigkeit unter Mittellinienlast. - Beuth Verlag, Berlin.

DIN EN 12670 (2002): Naturstein - Terminologie. - Beuth Verlag, Berlin.

DIN EN 1367-1 (2007): Prüfverfahren für thermische Eigenschaften und Verwitterungsbeständigkeit von Gesteinskörnungen - Teil 1: Bestimmung des Widerstands gegen Frost-Tau-Wechsel. - Beuth Verlag, Berlin.

DIN EN 13364 (2001): Prüfung von Naturstein - Bestimmung der Ausbruchlast am Ankerdornloch. - Beuth Verlag, Berlin.

DIN EN 13775 (2001): Prüfverfahren für Naturstein - Bestimmung der Wasseraufnahme bei atmosphärischem Druck. - Beuth Verlag, Berlin.

DIN EN 18332 (2002): VOB Vergabe- und Vertragsordnung für Bauleistungen - Teil C: Allgemeine Technische Vertragsbedingungen (ATV; Naturwerksteinarbeiten). - Beuth Verlag, Berlin.

DIN EN 1926 (1999): Prüfverfahren für Naturstein - Bestimmung der Druckfestigkeit. Beuth Verlag, Berlin.

DIN EN 1936 (1999): Prüfung von Naturstein - Bestimmung der Reindichte, der Rohdichte, der offenen Porosität und der Gesamtporosität. - Beuth Verlag, Berlin.

DIN 22024 (1989): Rohstoffuntersuchung im Steinkohlebergbau - Bestimmung der Spaltzugfestigkeit von Festgestein. - Beuth Verlag, Berlin.

DIN 52103 (1988): Prüfung von Naturstein und Gesteinskörnungen: Bestimmung von Wasseraufnahme und Sättigungswert (zurückgezogene Norm; ersetzt durch DIN EN 10976 (2001-01) und DIN EN 13755 (2002-03)). - Beuth Verlag, Berlin.

DIN 52104 (2004): Prüfung von Gesteinskörnungen - Untersuchung zur Beurteilung der Verwitterungsbeständigkeit. - Beuth Verlag, Berlin.

DIN 52106 (1994): Prüfung von Naturstein und Gesteinskörnungen: Untersuchungsverfahren zur Beurteilung der Verwitterungsbeständigkeit. - Beuth Verlag, Berlin.

DIN 52108 (1988): Prüfung anorganischer nichtmetallischer Werkstoffe; Verschleißprüfung mit der Schleifscheibe nach Böhme. - Beuth Verlag, Berlin.

DIN 52615 (1987): Bestimmung der Wasserdampfdurchlässigkeit von Bau- und Dämmstoffen. - Beuth Verlag, Berlin.

Dreyer, W. (1974): Materialverhalten anisotroper Festkörper: Thermische und elektrische Eigenschaften. - Springer Verlag, Wien, 295 S.

Dürrast, H., Siegesmund, S. \& Prasad, M. (1999): Die Schadensanalyse von Naturwerksteinen mittels Ultraschalldiagnostik: Möglichkeiten und Grenzen. - Z. dt. Ges. Geowiss., 150/2: 359-374. 
Eggloffstein, P. (1998): Vulkanische Tuffsteine als Naturwerkstein an historischen Bauwerken in Ungarn und Deutschland - Verwitterungsverhalten und Konservierungskonzepte. - Diss. Univ. Karlsruhe.

Eisbacher, G.H. (1991): Einführung in die Tektonik. - Ferdinand Enke Verlag, 310 S.

Fisher, R.V. (1961): Proposed classification of volcaniclastic sediments and rocks. - Bull. Geol. Soc. Amer., 72: 1409-1414, Boulder, Colorado.

Fisher, R.V. (1966): Proposed classification of volcanic fragments. - Earth-Sci. Rev., 1: 287298, Amsterdam.

Fitzner, B. (1970): Die Prüfung der Frostbeständigkeit von Natursteinen. - Geol. Mitt., Aachen, 10: 205-296.

Fitzner, B. (1988): Untersuchung der Zusammenhänge zwischen dem Hohlraumgefüge von Natursteinen und physikalischen Verwitterungsvorgängen. - Mitteilungen zur Ingenieurgeologie und Hydrogeologie, 29: $217 \mathrm{~S}$.

Fitzner, B. \& Basten, D. (1994): Gesteinsporosität - Klassifizierung, messtechnische Erfassung und Bewertung ihrer Verwitterungsrelevanz. - Jahresberichte Steinzerfall Steinkonservierung, 1992: 19-32.

Fleischer, G. (2002): Beurteilung von Ultraschalluntersuchungen an Natursteinobjekten in der Denkmalpflege. - Diss. Techn. Univ. Wien, 182 S.

Flick, H., Quade, H. \& Stache, G.A. (1991): Einführung in die tektonischen Arbeitsmethoden: Schichtenlagerung und bruchlose Verformung. - Clausthaler Tektonische Hefte 12, Köln, 94 S.

Frattari, A. \& Stenico, F. (2001): Porphyr - Architektur und Technik. - Gruppo Editoriale Faenza Editrice S.p.A., Italien, 127 S.

Freyburg, E. (1972): Der untere und mittlere Buntsandstein SW-Thüringens in seinen gesteinstechnischen Eigenschaften. - Berichte der deutschen Gesellschaft der geologischen Wissenschaften, 17: 735-743.

Fricke, S. \& Schön, J. (1999): Praktische Bohrlochgeophysik. - ENKE im Georg Thieme Verlag, Stuttgart, $254 \mathrm{~S}$.

Friedman, G. M. (1972): Coral reef rock from Red Sea: Sequence and time scale for progressive diagenesis and its effect on porosity and permeability. - Amer. Ass. Petrol. Geol. Bull., 56: 678 - 692, Tulsa.

Füchtbauer, H. (1959): Zur Nomenklatur der Sedimentgesteine. - Erdöl und Kohle 8, S. 605613, Hamburg.

Gabert, G. (1978): Vergleich zweier präkambrischer Orogene in Ost Afrika. - Geol. Jb. B. 30: 3-25.

Gargi, C., Benharrous, J., Bouineau, A., Michel, F. \& Platroz, P. (1998): Roches de France. Edition Pro Roc, Ternay, Frankreich, 226 S. 
Gebrande, H. (1982): Elastic wave velocities and constants of elasticity at normal conditions. In: Angenheister, G. (ed.): Physical properties of rocks. Landolt-Börnstein-Numerical Data and Functional Relationships in Science and Technology. Group V: Geophysics and Space Research. - Volume 1b, Springer-Verlag, Berlin, 8-35.

Golubev, A.A. \& Rabinovic, G.J. (1976): Resultaty primenenia apparatury akusticeskogo karostasa dlja opredelenia proenostych svoistv gornych porod na mestorosdeniach tverdych iskopaemych. - Pridkladnaja Geofizika, 73: 109-116.

Gordillo, C.E. (1974): Las rocas cordieríticas de Orcoyana y Cerro Negro - Soto (Córdoba). Bolétin de la Asociación Geológica de Córdoba, 2: 90 - 100.

Gordillo, C. (1984): Migmatitas cordieríticas de la Sierra de Córdoba, condiciones físicas de la migmatización. Misceláneas de la Academia Nacional de Ciencias de Córdoba, Córdoba. - Argentina, 68: 1-40.

Grimm, W.D. (1990): Bildatlas wichtiger Denkmalgesteine der Bundesrepublik Deutschland. - Bayerisches Landesamt für Denkmalpflege, Arbeitsheft 50, 250 S.

Grimm, W.D. (1999): Beobachtungen und Überlegungen zur Verformung von Marmorobjekten durch Gefügeauflockerung. - Z. dt. geol. Ges., 150 (2): 195-235.

Gross, M.R., Fischer, M.P., Engelder, T. \& Greenfield, R.J. (1995): Factors controlling joint spacing in interbedded sedimentary rocks: integrating numerical models with field observations from the Monterey Formation, USA. - Geological Society London Special Publications, 92/1, 215 S.

Gust, D.A., Biddle, K.T., Phelps, D.W. \& Uliana, M.A. (1985): Associated middle to late Jurassic volcanism and extension in southern South America. - Tectonophysics, 116: 223253.

Hair, J.F., Anderson, R.E., Tatham, R.L. \& Black, W.C. (1992): Multivariate data analysis. Macmillian Publishing Co, New York.

Han, D., Nur, A. \& Morgan, D. (1986): Effects of porosity and clay contant on wave velocities in sandstone. - Geophysics, 51 (11): 2093-2107.

Hill, D. (2007): Werkstoff Naturstein aus Sicht der Architekten. - Z. dt. Ges. Geowiss., 158/4: 701-707, Stuttgart

Hirschwald, J. (1908): Die Prüfung der natürlichen Bausteine auf ihre Wetterbeständigkeit. 675 S., Berlin.

Hirschwald, J. (1912): Handbuch der bautechnischen Gesteinsprüfung. Verlag Gebr. Bornträger, Berlin.

Hörenbaum, W. (2005): Verwitterungsmechanismen und Dauerhaftigkeit von Sandsteinsichtmauerwerk. - Diss. Univ. Karlsruhe, Schriftenreihe des Instituts für Massivbau und Baustofftechnologie, Heft 54, 357 S.

Hoffmann, A. (2006): Naturwerksteine Thailands: Lagerstättenerkundung und Bewertung. Diss. Univ. Göttingen, 242 S. 
Huang, Q. \& Angelier, J. (1989): Fracture spacing and its relation to bed thickness. Geological Magazine, 4: 355-362.

Hubert, M.K. \& Rubey, W.W. (1959): Role of fluid pressure in mechanics of overthrust faulting, I. Mechanics of fluid-fillied porous solids and its application to overthrustfaulting. - Bull. Geol. Soc. Am., 70: 115-166, New York.

Iglesia Llanos, M.P., Riccardi, A.C. \& Singer S. E. (2006): Palaeomagnetic study of Lower Jurassic marine strata from the Neuquén Basin, Argentina: A new Jurassic apparent polar wander path for South America. - Earth and Planetary Science Letters, 252: 379-397.

Iliev, I.G. (1967): An attempt to estimate the degree of weathering of intrusive rocks from their physico-mechanical properties. In: Proceedings of the $1^{\text {st }}$ International Congress of the International Society of Rock Mechanism, Lisbon, Vol. 1: 109-114.

ISRM (1978): Suggested methods for the quantitative description of discontinuities in rock masses. International Society for Rock Mechanics Commission on Standards of Laboratory and Field Tests. - International Journal of Rock Mechanics and Mining Sciences \& Geodynamics Abstracts, Vol. 15/6: 319-368.

Katzschmann, L., Aselmeyer, G. \& Auras, M. (2006): Natursteinkataster Thüringen. - IFSBericht Nr. 23 - 2006, 196 S.

Kieslinger, A. (1959): Rahmenverwitterung. - Geologie und Bauwesen, 24: 171-186, Wien.

Koch, A. (2005): Deformation von Fassadenplatten aus Marmor: Schadenskartierungen und gesteinstechnische Untersuchungen zur Verwitterungsdynamik von Marmorfassaden. Diss. Univ. Göttingen, 174 S.

Koch, A. \& Siegesmund, S. (2004): The combined effect of moisture and temperature on the anomalous expansion behaviour of marble. - Environmental Geology, 46: 350-363.

Koch, R. \& Sobott, R. (2005): Porosität in Karbonatgesteinen - Genese, Morphologie und Einfluss auf Verwitterung und Konservierungsmaßnahmen. - Z. dt. Geol. Ges., 156 (1): 33-55.

Koch-Moeck, M. (2005): Computergestützte geowissenschaftliche Entscheidungshilfe bei Planung und Optimierung der untertägigen Gewinnung von Marmor. - Diss. Techn. Univ. Berlin, 134 S.

Koopmann, A. (2004): Magma Mingling: Die hydrodynamische Genese magmatischer Dispersionen. - Diss. Univ. Würzburg, $148 \mathrm{~S}$.

Kownatzki, R. (1997): Verwitterungszustandserfassung von Natursteinbauwerken unter besonderer Berücksichtigung phänomenologischer Verfahren. - Aachener Geow. Beiträge, 22, $256 \mathrm{~S}$.

Klopfer, H. (1985): Lehrbuch der Bauphysik. - Teubner Verlag, Stuttgart, 335-336.

Köhler, W. (1991): Untersuchungen zu Verwitterungsvorgängen an Carrara-Marmor in Potsdam-Sanscouci. - Berichte zu Forschung und Praxis der Denkmalpflege in Deutschland. Steinschäden - Steinkonservierung, 2: 50-55. 
Kracke, T. (2007): Göttinger Buntsandsteine - Verwitterungsdynamik und Konservierungsstrategien: Fallbeispiel Bartholomäus Friedhof Göttingen. - Dipl.-Arbeit Univ. Göttingen, 213 S.

Krauter, H., Woszidlo, H. \& Büdinger, H. (1985): Festigkeitsuntersuchungen an Gesteinen mit dem Prallhammer nach Schmidt. In: Heitfeld, H.-H. (Hrsg.): Ingenieurgeologische Probleme im Grenzbereich zwischen Locker- und Festgestein. - Springer Verlag, Berlin, 658-671.

Kreyszig, E. (1977): Statistische Methoden und ihre Anwendungen. - Vandenhoeck und Rupprecht, Göttingen.

Kürzl, H. (1988): Exploratory data analysis: Recent advances for the interpretation of geochemical data. - J. Geochem. Explor., 30: 309-322.

Ladeira, F.L. \& Price, N.J. (1981): Relationship between fracture spacing and bed thickness. J. Struct. Geol., 3: 179-183.

Lamberghini, A. Fiora, L. \& Alciati, L. (2000): Il cielo dell’ Argentina nel marmo Azul. L'informatore del marmista, $N^{0} 464$, pp. 6-12.

Legarreta L. \& Gulisano C.A. (1989): Análisis estratigráfico de la Cuenca Neuquina (Triásico superior-Terciario inferior). In: Chebli, G.A. \& Spalletti L.A, (Eds): Cuencas Sedimentarias Argentinas. - Serie Correlación Geológica, 6, Instituto Miguel Lillo, Univ. Nac. de Tucumán, San Miguel de Tucumán, pp. 221-244.

Lepper, J. (2007): Naturwerkstein - kein Baurohstoff wie all die anderen! - Z. dt. Ges. Geowiss., 158/4: 685-693.

Lippmann, F. (1977): Diagenese und beginnende Metamorphose bei Sedimenten. - Bull T LVI Acad Serbe Sci Arts Sci Nat No 15.

López de Luchi, M.G., Siegesmund, S. Hulka, C., Hübner, H., Hoffmann, A. \& Mosch, S. (2001): Geological setting and composition of the Las Chacras-Potrerillos Batholith, Sierras Pampeanas, Argentina: First results. - Z. dt. Geol. Ges., 152 (2-4): 325-350.

López de Luchi, M.G., Rapalini, M.A., Rossello, E. \& Geuna, S. (2002): Rock fabric and magnetic fabric of the Renca batholith (Sierra de San Luis, Argentina). - Lithos, 61 (3-4): 161-186.

López de Luchi, M.G., Siegesmund, S., Wemmer, K., Steenken, A. and Naumann, R. (2007): Geochemical constraints on the petrogenesis of the Paleozoic granitoids of the Sierra de San Luis, Sierras Pampeanas, Argentina. - Journal of South America Earth Sciences (in press).

Lorenz, Walter \& Gwosdz, Werner (2003): Handbuch zur geologisch-technischen Bewertung von mineralischen Baurohstoffen. - Geol. Jahrbuch, Sonderhefte Reihe H, Heft SH 16, Hannover, 498 S.

Lu, P. \& Latham, J.P. (1999): Developments in the assessment of in-situ block size distributions of rock masses. - Rock Mechanics and Rock Engineering, 32 (1): 29 - 49. 
Matthes, N. (1990): Mineralogie. - Springer, Berlin, Heidelberg, New York, 461 S.

Mauko, A., Mirtic, B., Mladenovic, A \& Grelk, B. (2006): Deterioration of the granodiorite façade - case sample Maximarket, Ljubljana. - RMZ-Materials and Geoenvironment, 53 (1): 23-37.

McBride, E. F. (1963): A classification of common sandstones. - Journal of Sedimentary Petrology, 33/3: 664-669.

Meier, D. \& Kronberg, P. (1989): Klüftung in Sedimentgestein: Erscheinungsformen, Datenaufnahme, Datenbearbeitung, Interpretation. - Ferdinand Enke Verlag, Stuttgart, $116 \mathrm{~S}$.

Meinhold, K.D. (1970): Petrographie, Metamorphose, Tektonik und stratigraphische Stellung der Konse-Serie in Zentral-Tansania (Ostafrika). - Beih. Geol. Jb., 91: 137 S.

Merke, G. (2007): Die globale Welt: der Markt, der internationale Natursteinhandel und seine Auswirkungen auf Rohstoffe. - Z. dt. Ges. Geowiss., 158/4: 679-683.

Mining Press (2007): Argentina - La minería del siglo XXI (Argentina: Mining in the $21^{\text {st }}$ century). - Mining Press, Buenos Aires, Argentina, year 4, no. 21: 9-12.

Mirwald, P.W. (1991): Physikalische Eigenschaften der Gesteine. In: Naturwerksteine und Umweltschutz in der Denkmalpflege. - Ebner-Verlag, Ulm, S. 283-308.

Montani, C. (2003): Stone 2002 - World marketing handbook. - Faenza, Gruppo Editoriale Faenza Editrice.

Montani, C. (2005): Stone 2004 - World marketing handbook. - Faenza, Gruppo Editoriale Faenza Editrice, $249 \mathrm{~S}$.

Montani, C. (2007): Stone 2006 - World marketing handbook. - Faenza, Gruppo Editoriale Faenza Editrice.

Moore, C.H. (1989): Carbonate diagenesis and porosity. - Sedimentol. Min., spec. Publ., Gulf Coast Section: $283-308$.

Morales Demarco, M., Jahns, E., Rüdrich, J. Oyhantcabal, P. \& Siegesmund, S. (2007): The impact of partial water saturation in rock strength: an experimental study on sandstone. Z. dt. Ges. Geowiss., 158/4: 869-882, Stuttgart

Mortensen, H. (1933): Die Salzsprengung und ihre Bedeutung für die regionalklimatische Gliederung der Wüsten. - Petermann’s Mitteilungen aus Justus Perthes geographischer Anstalt, 79: 130-135.

Mosch, S. López de Luchi, M.G. \& Siegesmund, S. (2007): Dimensional stones in Argentina. - Z. dt. Ges. Geowiss., 158/3: 447-469, Stuttgart (Schweizerbart).

Mosch, S. \& Siegesmund, S. (2007): Statistisches Verhalten petrophysikalischer und technischer Eigenschaften von Naturwerksteinen. - Z. dt. Ges. Geowiss., 158/4: 821-868, Stuttgart (Schweizerbart). 
Mosch, S. \& Siegesmund, S. (2008): Lagerstättenerfassung und -bewertung von Naturwerkstein am Beispiel des Dolomitmarmors von Mtesa, Tansania. - In: Siegesmund, S. \& Snethlage, R. (Hrsg.): Denkmalgesteine - Festschrift - Wolf-Dieter Grimm. - S. dt. Ges. Geowiss., 59: 291-318, Hannover.

Müller, F.M. (2001): Gesteinskunde. - Ebner-Verlag, Ulm, 276 S.

Nikolayev, D.I., Siegesmund, S., Mosch, S. \& Hoffmann, A. (2007): Modell-based prediction of unfractured rock masses. - Z. dt. Ges. Geowiss., 158/3: 483-490, Stuttgart.

Nockolds, S.R. (1954): Average chemical composition of some igneous rocks. - Bull. Geol. Soc. Am., 65: 1007-1032.

Nordtest Method NT BUILD 499 (2002): Cladding panels: Test for Bowing. - Nordtest project $1443-99 / 1$.

O.T.C (2006): Análisis del sector de las canteras de la Sierra de Macaél y de las actuaciones realizadas dentro del plan director. - Oficina Tecnica Colaboradora Marmoles de Macaél, Espana.

Pätzold, J., Bickert, T. \& Fischer, G. (2004): Skript VAK 5-102 Ü Gesteinsbestimmung. Universität Bremen, 65 S.

Palmström, A. (1995): RMi - a rock mass characterisation system for rock engineering purposes. - Unpublished Ph.D. thesis of the University of Oslo, Norway, 211 p.

Pankhurst, R J., Rapela, C.W., Saavedra, J., Baldo, E., Dahlquist, J, Pascua, I. \& Fanning, C.M. (1998): The Famatinian magmatic arc in the central Sierras Pampeanas: an Early to Mid-Ordovician continental arc on the Gondwana. In: Pankhurst R.J., Rapela C.W. (Eds). The Proto-Andean Margin of Gondwana. - Geological Society of London Special Publication, 142: 343-367.

Pankhurst, R.J., Ramos, A. \& Linares, E. (2003): Antiquity of the Río de la Plata craton in Tandilia, southern Buenos Aires province, Argentina. - Journal of South American Earth Sciences, 16: 5-13.

Pankhurst, R.J., Rapela, C.R., Fanning C.M. \& Márquez, M. (2006). Gondwanide continental collision and the origin of Patagonia. - Earth-Science Reviews, 76: 235-257.

Passchier, C.W: \& Trouw, R.A.J. (1996): Microtectonics. - Springer Verlag, Berlin, 289 S.

Peschel, A. (1983): Natursteine. - VEB Deutscher Verlag für Grundstoffindustrie, Leipzig, $448 \mathrm{~S}$.

Pettijohn, F.J., Potter, P.E. \& Siever, R. (1973): Sand and Sandstone. - Springer Verlag, New York, 553 S.

Pollard, D.D. \& Aydin, A. (1988): Progress in understanding jointing over the past century. GSA Bulletin, 100: 1181-1204.

Popp, T. (1994): Der Einfluß von Gesteinsmatrix, Mikrorißgefügen und intergranularen Fluiden auf die elastischen Wellengeschwindigkeiten und die elektrische Leitfähigkeit 
krustenrelevanter Gesteine unter PT-Bedingungen - Eine experimentelle Studie. - Diss. Univ. Kiel, 176 S.

Poschlod, K. (1989): Das Wasser im Porenraum kristalliner Naturwerksteine und sein Einfluß auf die Verwitterung. - Münchner Geowissenschaftliche Abhandlungen 7, 62 S.

Priem, H.N.A., Boelrijk, N.A.I.M., Hebeda, E.H., Verdurmen, E.A.T., Verschure, R.H., Oen, I.S. \& Westra, L. (1979): Isotopic age determinations on granitic and gneissic rocks from the Ubendian-Usaragan System in southern Tanzania. - Prec. Res., 9: 227-239.

Priest, S.D. (1993): Discontinuity analysis for rock engineering. - Chapman and Hall, London, $473 \mathrm{~S}$.

Primavori, P. (1999): Planet Stone. - Giorgio Zusi Editore S.A.S., Verona, 326 S.

Prissang, R., Hellä, P., Lehtimäki, T., Saksa, P., Nummela, J. \& Vuento, A. (2007): Localisation of undisturbed blocks in larger dimension stone rock masses. - Z. dt. Ges. Geowiss., 158/3: 471-482.

Quervain, F. de (1967): Technische Gesteinskunde. - 2. Aufl., Basel, Stuttgart (Birkhäuser), $267 \mathrm{~S}$.

Rac, M.V. \& Chernyshev, S.N. (1970): Klüftigkeit und Eigenschaften geklüfteter Gesteine (russ.). - Verlag Nedra, Moskau.

Ramos, V.A. (1984): Patagonia: ¿Un continente paleozoico a la deriva? - $9^{\circ}$ Congreso Geológico Argentino, Actas 2: 311-325.

Rapela, C.W., Pankhurst, R.J., Casquet C., Baldo, E., Saavedra, J., Galindo, C. \& Fanning, C.M. (1998): The Pampean Orogeny of the southern proto-Andes: Cambrian continental collision in the Sierras de Córdoba. In: Pankhurst R.J., Rapela, C.W. (Eds): The ProtoAndean Margin of Gondwana. - Geological Society of London Special Publication, 142: 181-217.

Rapela, C.W. (2000): The Sierras Pampeanas of Argentina: Paleozoic Building of the Southern Proto-Andes. In: Cordani, U.G., Milani, E.J., Thomaz-Filho, A., Campos, D.A. (Eds.): Tectonic Evolution of South America. - $31^{\text {st }}$ International Geological Congress, Río de Janeiro, Brazil, 381-387.

Rapela, C.W., Baldo, E.G., Pankhurst, R.J. \& Saavedra, J. (2002): Cordieritite and Leucogranite Formation during Emplacement of Highly Peraluminous Magma: the El Pilón Granite Complex (Sierras Pampeanas, Argentina). - Journal of Petrology, 43/6: 1003-1028.

Rapela, C.W., Pankhurst, R.J., Casquet C., Fanning C.M., Baldo, E., González-Casado, J.M., Galindo, C. \& Dahlquist, J. (2007): The Río de la Plata craton and the assembly of SW Gondwana. - Earth-Science Reviews, 83 (1-2): 49-82.

R Development Core Team (2005): R: A language and environment for statistical computing. - R Foundation for Statistical Computing, Vienna, Austria, ISBN 3-900051-07-0. 
Reinsch, D. (1991): Natursteinkunde: Eine Einführung für Bauingenieure, Architekten, Denkmalpfleger und Steinmetze. - Ferdinand Enke Verlag, Stuttgart, 315 S.

Rüdrich, J. (2003): Gefügekontrollierte Verwitterung natürlicher und konservierter Marmore. - Diss. Univ. Göttingen, 158 S.

Rüdrich, J. \& Siegesmund, S. (2006): Salt and Ice crystallization in porous sandstones. In: Siegesmund, S. \& Steiger, M. (Hrsg.): Salt decay. - Environmental Geology, 52/2: 225249.

Russo, A., Ferello, A. \& Chebli, G. (1979): Llanura Chaco Pampeana. - Segundo Simposio de Geología Regional Argentina 1: 139-183.

Sánchez, V.H., Damiani, O. \& Guimaraes, R. (1986): Investigación hidrogeológica preliminar para abastecimiento de agua a los centros mineros de la Sierra de Villicum-Río Salado. Unpubl. Report, CRAS, IT-59: 1-31.

Scherer, G.W. (1999): Crystallization in pores. - Cement and Concrete Research 29: 13471358.

Schlittgen, R. (2000): Einführung in die Statistik: Analyse und Modellierung von Daten. - 9. Auflage, München - Wien, 518 S.

Schön, J.H. (1983): Petrophysik: physikalische Eigenschaften von Gesteinen und Mineralen. - Akademie-Verlag, Berlin, 405 S.

Schön, J.H. (1996): Physical properties of rocks: fundamentals and principles of petrophysics. In: Helbig, K. \& Treitel, S. (ed.): Handbook of geophysical Exploration. - Section I, Seismic exploration; Vol. 18, Verlag Elsevier, Oxford.

Schöne, D. \& Peschel, A. (1974): Die Gewinnbarkeit von Rohblöcken für die Naturwerksteinindustrie und ihr Einfluß auf das Abbausystem. - Neue Bergbautechnik, 4: 214-223, Leipzig.

Schuh, H. (1987): Physikalische Eigenschaften von Sandsteinen und ihren verwitterten Oberflächen. - Münchner Geowissenschaftliche Abhandlungen Reihe B, Band 6: 66 S., München.

Selonen, O., Luodes, H. \& Ehlers, C. (2000): Exploration for dimensional stone implications and examples from the Precambrian of Southern Finland. - Engineering Geology, 56: 275-291.

Selonen, O. \& Suominen, V. (Eds.) (2003): Nordic Stone. - Paris: UNESCO: IAEG. 64 S.

SFHF-Schweizerischer Fachverband für hinterlüftete Fassaden (2003): Baustoffe-Kennwerte. - http://www.sfhf.ch.

Secor, D.T. (1965): The role of fluid pressure in jointing. - Amer. J. Sci., 263: 633-646, New Haven. 
Siegesmund, S. (1989): Texturelle und strukturelle Eigenschaften mylonitischer Gesteine der Insubrischen Linie (Ivrea-Zone, Italien) und ihr Einfluss auf die elastischen Gesteinseigenschaften. - Diss. Univ. Kiel, 172 S.

Siegesmund, S., Vollbrecht, A., Chlupac, T., Nover, G., Dürrast, H., Müller, J. \& Weber, K.: (1993): Fabric-controlled anisotropy of petrophysical properties observed in KTB-core samples. - Sci. Drilling, 4: 111-125.

Siegesmund, S. (1996): The significance of rock fabrics for the geological interpretation of geophysical anisotropies. - Geotekt. Forsch., 85, $123 \mathrm{~S}$.

Siegesmund, S., Vollbrecht, A., Ullemeyer, K. Weiss, T. \& Sobott, R. (1997): Application of geological fabric analyses for the characterisation of natural building stone - case study Kaufung marble (in german). - International Journal for Restoration of Buildings and Monuments 3: 269-292.

Siegesmund, S., Steenken, A., López de Luchi, M.G., Wemmer, K., Hoffmann, A. \& Mosch, S. (2004): The Las Chacras-Potrerillos batholith (Sierras Pampeanas, Argentina): structural evidences, emplacement and timing of intrusion. - Int. J. Earth Sci., 93: 23-43.

Siegesmund, S., Mosch, S., Scheffzük, C. \& Nikolayew, D. (2007): The bowing potential of granitic rocks: Rock fabrics, thermal properties and residual strain. - Environmental Geology, 55/7: 1437-1448.

Sims, J.P., Ireland, T.R., Camacho, A., Lyons, P., Pieters, P.E., Skirrow, R.G., Stuart-Smith, P.G. \& Miró, R. (1998): U-Pb, Th-Pb and Ar-Ar geochronology from the southern Sierras Pampeanas, Argentina: implications for the Paleozoic evolution of the western Gondwana margin. In: Pankhurst J.R., Rapela. C.W. (Eds). The Proto-Andean Margin of Gondwana. - Geological Society of London Special Publication, 142: 259-281.

Singewald, C. (1992): Naturstein - Exploration und Gewinnung. - Rudolf Mueller, Bad Bentheim, $260 \mathrm{~S}$.

Smith, M.R. (Ed) (1999): Stone: Building stone, rock fill and armour stone in construction. Geological Society of London, Engineering Geology Special Publications, Vol 16.

Snethlage, R. (1984): Steinkonservierung. - Arbeitshefte des Bayrischen Landesamtes für Denkmalpflege, 22: 203 S.

Snethlage, R \& Wendler, E. (1997): Moisture cycles and sandstone degradation. In: Baer, N.S. \& Snethlage, R. (Eds.): Saving our Architectural Heritage: The Conservation of Historic Stone Structures. S. 7-24.

Steiger, M. (2005): Crystal growth in porous materials - I: The crystallisation pressure of large crystals. - J. of Crystal Growth, 282: 455-469.

Steenken, A., Wemmer, K., López de Luchi, M. G., Siegesmund, S. \& Pawlig, S. (2004): Crustal provenance and cooling of the basement complexes of the Sierra de San Luis: An insight into the tectonic history of the proto-Andean margin of Gondwana. - Gondwana Research, 7/4: 1171-1195. 
Stein, K.J. (2007): Vorkommen an Naturwerksteinen in Benin und deren Klassifikation nach der UNFCR. - Z. dt. Ges. Geowiss., 158/3: 429-446, Stuttgart.

Stockley, G.M. \& Oates, M.B.E. (1931): Notes on the Mineral Deposits in the Newala-Lindi Area. - Geological Survey Department Tanganyika Territory, Dar es Salaam.

Streckeisen, A. (1974): Classification and nomenclature of igneous rocks. - Geologische Rundschau, 63: 773-786.

Streckeisen, A. (1980): Classification and nomenclature of igneous rocks. - Geologische Rundschau, 69: 194-207.

Strohmeyer, D. \& Siegesmund, S. (2002): Anisotropic technical properties of building stones and their developement due to fabric changes. In: Siegesmund, S., Weiss, T. \& Vollbrecht, A. (eds.): Natural stones, weathering phenomena, conservation strategies and case studies. - Geological Society Special Publications, 205: 107-127.

Strohmeyer, D. (2003): Gefügeabhängigkeit technischer Gesteinseigenschaften. - Diss. Univ. Göttingen, 254 S.

Thuro, K. (1996): Bohrbarkeit beim konventionellen Sprengvortrieb. - Münchener Geol. Hefte, B 1, 145 S.

Torres, I.E. (2008): The mineral industry of Argentina. In: Minerals Yearbook 2006. - U.S. Department of the Interior, U.S. Geological Survey.

Trewitt, T.J. \& Tuchmann, J. (1988): Amoco may replace marble on Chicago headquarters. ENR March, 11-12.

Tucker, M. E. (1985): Einführung in die Sedimentpetrologie. Ferdinand Enke Verlag, Stuttgart, 265 S.

Uliana, M.A. \& Biddle, K. (1988): Mesozoic-Cenozoic paleogeographical and geodynamic evolution of southern South America. - Rev. Bras. Geoscienc., 18: 172-190.

UNO (1997): United Nations international framework classification for reserves/resources solid fuels and mineral commodities. Geneva 1997 - UNFC - Code.

Vereinte Nationen (1976): The development potential of dimension stone. - Department of Economic and Social Affairs, ST/ESA/34, New York, 239 S.

VDI 3797 (1980): Prüfung des zu erwartenden Resistenzverhaltens von konservierten und nicht konservierten Natursteinen gegenüber Immisionen; Salzsprengtest. - Kommission Reinhaltung der Luft im VDI und DIN - Normenausschuss KRdL.

Vergani G.D, Tankard A.J, Belotti H.J. \& Welsink H.J. (1995): Tectonic evolution and paleogeography of the Neuquén Basin, Argentina. In: Tankard, A.J., Suárez Soruco R., Welsink, H.J. (Eds), Petroleum Basins of South America. - Am. Assoc. Petr. Geol., Memoir vol. 62, The American Association of Petroleum Geologists, Tulsa pp. 383-402.

Vilwock, R. (1966): Industriegesteinskunde. - Offenbach/Main: Stein-Verlag. 
Vujovich, G.I. \& Kay, S.M. (1998), A Laurentian? Grenville-age oceanic/back-arc terrane in the Sierra de Pie de Palo, western Sierras Pampeanas, Argentina. In: Pankhurst J.R., Rapela. C.W. (Eds). The Proto-Andean Margin of Gondwana. - Geological Society of London Special Publication, 142: 159-179

Wanless, H. R. (1979): Limestone response to stress: Pressure solution and dolomitiziation. J. Sedim. Petrol., 49: 437-462.

Weiss, T., Siegesmund, S. \& Rasolofosaon, P.N.J. (2000): The relationship between deterioration, fabric, velocity and porosity constraint. - Proceedings $9^{\text {th }}$ International congress on deterioration and conservation of stone, Venice, Vol. 1: 215-223.

Wendt, I. Besang, C. Harre, W. Kreuzer, H. Lenz, H. \& Müller, P. (1972): Age determinations of granitic intrusions and metamorphic events in the Early Precambrium of Tanzania. $-24^{\text {th }}$ Int. Geol. Congr., Montreal, section 1, 295-314.

Wenzel, A. \& Häfner, F. (2003): Die roten Werksandsteine der Westpfalz. - IFS-Bericht Nr. 15 - 2003: 27 S.

Wimmenauer, W. (1985): Petrographie der magmatischen und metamorphen Gesteine. Ferdinand Enke Verlag, Stuttgart, 381 S.

Winkler, E.M. (1968): Frost damage to stone and concrete: Geological considerations. Engineering Geology, 2, 315-323.

Winkler, E.M. (1994): Stone in Architecture: Properties and Durability. - Springer, New York, $303 \mathrm{~S}$.

Winkler, E.M. (1996): Technical note; properties of marble as building veneer. International Journal of Rock Mechanics, Mineral Science and Geomechanics, 33/2: 215-218.

Wu, H. \& Pollard, D.D. (1995): An experimental study of the relationship between joint spacing and layer thickness. - Journal of Structural Geology, 17/6: 887-905.

Yardley, B.W.D. 1997: Einführung in die Petrologie metamorpher Gesteine. - Ferdinand Enke Verlag, Stuttgart, 253 S.

Zeisig, A., Siegesmund, S. \& Weiss, T. (2002): Thermal expansion and its control on the durability of marbles. - Geological Society, London, Special Publications, 205: 65-80.

Zolezzi, R. E., Miró, R. C. \& Munizaga, H. R. (2005): Censo Nacional Minero 2003-2004: Evaluación Estadística. - Secretaría de Minería de la Nación, Fundación Empremin, 74 p. 


\section{Danksagung}

Die vorliegende Arbeit entstand auf Anregung und unter Betreuung von Prof. Dr. Siegfried Siegesmund (GZG, Universität Göttingen). Für das überaus interessante Thema und das rege Interesse am Fortgang der Arbeit möchte ich mich hier bedanken. Das Korreferat übernahm Prof. Dr. Klaus Germann (TU Berlin), bei dem ich mich ebenfalls herzlich für sein Interesse und die kritische Betrachtung des Manuskripts bedanken möchten.

Finanziell wurde die vorliegende Arbeit durch ein Kurzstipendium des Deutschen Akademischen Austausch Diensts (DAAD), in dessen Rahmen die Geländearbeit in Argentinien durchgeführt wurde, sowie durch ein Hochschulvergabeprojekt der Bundesanstalt für Geowissenschaften und Rohstoffe (BGR) zur Erfassung und Bewertung des Marmorvorkommens in Tansania unterstützt. Dem DAAD und der BGR (namentlich Dr. Simone Röhling und Dr. Harald Elsner) seien an dieser Stelle herzlich gedankt.

Des Weiteren gilt mein herzlicher Dank:

- Dr. Jörg Rüdrich für die gute Zusammenarbeit im Tektoniklabor und seine andauernde Hilfsund Diskussionsbereitschaft

- Jochen Stein für seine ständige Bereitschaft, mir alle Fragen aus der Welt der Naturwerksteine zu beantworten.

- Dra. Monica López de Luchi für ihre ständige Unterstützung in Argentinien und auch später.

- Federico Roffé für seine Begleitung bei der langen Geländearbeit in Argentinien.

- Gideon Mwaya für die Unterstützung bei der Geländearbeit in Tansania (vor allem was die Sprache anbelangte...).

- Dr. Dmitry Nikolayev für seine Kenntnisse im Programmieren und die entstandene Software.

- Dr. Andreas Hoffmann für die gemeinsame Zeit in der Arbeitsgruppe sowie die Diskussion zum Manuskript.

- Reinhard Kögler für die Bereitstellung von umfangreichem Daten- und Bildmaterial.

- Dr. Günther Braun für die kritische Durchsicht des Manuskripts mit vielen Hinweisen.

- $\quad$ PD Dr. Werner Nagel und Dr. Oleg Nenadic für die Hilfe im Umgang mit dem Datensatz.

- Dr. Alfons van der Kerkhoff und Christian Gross für ihre Hilfe am KL-Mikroskop.

- Dr. Till Heinrichs für seine Hilfe im Bezug auf Tansania.

- Dr. Gerald Hartmann und Rudolf Naumann für die röntgenographischen Analysen.

- Stefan Pirskawetz und Dirk Kirchner für die Unterstützung bei der gesteinstechnischen Untersuchung.

- Michael Sosnitza, Günther Tondock und Holger Schwanke für ihre ständige und spontane Hilfsbereitschaft bei allen technischen Fragen.

- Friedrich-Georg Benne für die umfangreiche Unterstützung beim Probentransport.

- den vielen argentinischen Naturwerkstein-Firmen für das zur Verfügung gestellte Probenmaterial und die logistische Unterstützung.

- allen Firmen, die mir die Arbeit in den verschiedenen Steinbrüchen ermöglicht haben.

Zum Schluss möchte ich mich besonders bei meinen Eltern und auch bei meinen Geschwistern bedanken, deren Unterstützung ich mir zu jeder Zeit sicher sein konnte. Bei meiner Schwester Eva Christine vor allem auch für das unermüdliche Korrekturlesen. Und Silvia....Danke! 


\section{Lebenslauf}

Stephan Mosch

geboren am 21.09.1975 in Unterlüß

Staatsangehörigkeit deutsch

\section{Hochschulausbildung und wissenschaftlicher / beruflicher Werdegang}

Seit 01/2006

$01 / 2006-07 / 2008$

06/2005-09/2005

und

$04 / 2003-09 / 2004$

$10 / 2004-03 / 2005$

$08 / 2002$

$05 / 2001-10 / 2002$

$05 / 2000$

08/1997-09/1997

$10 / 1996-06 / 2002$
Promotion

am Geowissenschaftlichen Zentrum der Universität Göttingen (GZG)

- Thema der Dissertation:

„Optimierung der Exploration, Gewinnung und Materialcharakterisierung von Naturwerksteinen“

Wissenschaftlicher Mitarbeiter am Geowissenschaftlichen Zentrum der Universität Göttingen (GZG) Mitarbeit in den Projekten:

- Textur und Spannung als Ursache mangelnder Formbeständigkeit von Fassadenplatten aus Naturstein, BMBF

- Marmore im Südosten Tansanias (Region Mtwara) - Bewertung des Lagerstättenpotenzials, BGR

Geologe im Ingenieurbüro Dr.-Ing. Meihorst \& Partner, Beratende Ingenieure für Bauwesen $\mathrm{GmbH}$, Hannover

Gastwissenschaftler im Rahmen eines DAAD-Stipendiums Instituto de Geochronología y Geología Isotópica (INGEIS), Geologische Fakultät der Univ. Buenos Aires, Argentinien

Praktikum: SINTEF Civil and Environmental Engineering, Rock and Soil Mechanics, Trondheim, Norwegen

\section{Studentische bzw. wissenschaftliche Hilfskraft}

am Geowissenschaftlichen Zentrum der Universität Göttingen (GZG)

- Durchführung und Interpretation von petrophysikalischen, gesteinstechnischen und gefügekundlichen Analysen

Praktikum: Instituto de Geochronología y Geología Isotópica (INGEIS), Geologische Fakultät der Univ. Buenos Aires, Argentinien

Praktikum: Celler Brunnenbau GmbH, Celle

Diplomstudium Geologie / Paläontologie am Geowissenschaftlichen Zentrum der Universität Göttingen (GZG)

Thema der Diplomarbeit: „Strukturelle und gesteinsmagnetische Untersuchungen an den Granitoiden des Las Chacras-Potrerillos Batholithen (Sierra de San Luis, Argentinien)“

Geologische Kartierung des Las Chacras-Potrerillos Batholithen (Sierra de San Luis, Argentinien) 